\title{
ÉTUDES ÉCOLOGIQUES
}

SUR LES

\section{ASSOCIATIONS FORESTIEERES \\ DE LA}

\section{HAUTE-MAURIENNE}

PAR

\author{
Ch. BARTOLI \\ Ingénieur agronome \\ Ingénieur en Chef du Génie Rural, \\ des Eaux et des Forêts \\ à Grenoble
}




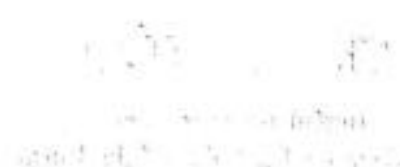

(1) $167+1):$ 


\section{TABLE DES MATIERES}

Avant-pkopos

$1^{\text {re PARTIE. }-B u t ~ d e ~ c e ~ t r a v a i l ~ e t ~ m e ́ t h o d e s ~ s t i v i e s ~}$

I.1. - Objet de ce mémoire ............................ 439

1.2. - Méthodes d'étude des associations .................. 444

I.3. - Méthodes d'étude des sols .......................... 486

I.4. - Méthodes d'étude des climats locaux et des microclimats .. 491

I.5. - Méthodes d'étude auxométrique .................... 494

2 PaRTIF. - Caractères généraux de la Haute-Maurienne .......... 497

II.1. - Le climat ................................... 499

II.2. - Les roches-mères ............................ 519

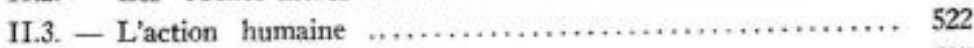

II.4. - Eléments floristiques ......................... 527

3 PARtIE. - Les associations forestières. ..................... 537

III.1. - Préambule ................................. 539

III.2. - Les pineraies de Pin sylvestre .................... 540

III.3. - Les pineraies de Pin à crochets ................... 580

III.4. - Les sapinières . ............................... 603

III.5. - Les pessières ................................ 618

III.6. - Les pineraies de Pin Cembro ..................... 638

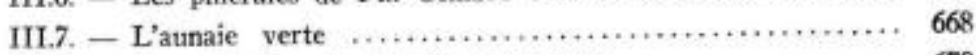

III.8. - Ies hétraies et chẻnaie pubescente ................. 678

4. PARtie. - Conclusions ................................... 695

IV.1. - Les facteurs écologiques décisifs .................. 697

IV.2. - Le problème du climax ....................... 713

IV.3. - Le problème de la nomenclature et de la classification. .. 716

IV.4. - Les problèmes sylvicoles ...................... 724

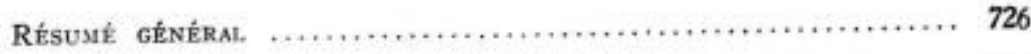

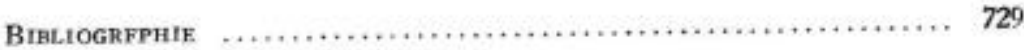

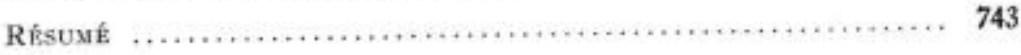

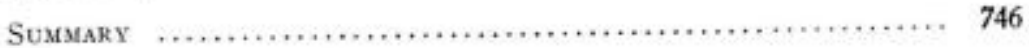

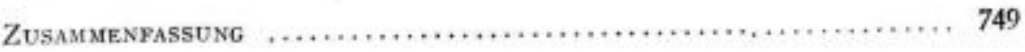





\section{AVANT-PROPOS}

Dans tous les pays du monde - les exemples à citer seraient très nombreux - l'étude des " stations ", dans le domaine forestier, prend une importance chaque jour plus grande.

On s'est, en effet, rendu compte que, si la forêt est une « usine à bois n- encore que son rôle ne doive point se limiter à celui-là cette usine est vivante et doit être traitée comme telle, et si le forestier doit avoir, au premier chef, des préoccupations économiques, son action s'insère dans un monde où la biologie joue un rôle capital et toujours contraignant.

La connaissance des lois biologiques - et, singulièrement celle du milieu, dans lequel et par lequel elles se manifestent - s'impose done au forestier, non seulement lorsqu'il pratique une sylviculture traditionnelle dans des forêts équilibrées, plus ou moins proches de leur état naturel, et pourtant parfaitement satisfaisantes du point de vue économique, mais encore et surtout, lorsqu'il cherche à modifier, à son profit, la concurrence vitale, les conditions écologiques ou le matériel génétique, ou lorsqu'il s'emploie à créer de nouvelles forêts.

Dans cette recherche de la connaissance du milieu, la Phytosociologie apporte un concours particulièrement précieux, car les plantes, comme chacun sait, constituent, en définitive, les meilleurs " intégrateurs n des conditions écologiques, qui sont toujours fort complexes.

Le présent travail est donc à la fois un travail de phytosociologie et d'écologie; il est le fruit de tournées effectuées surtout dans un but d'aménagement de forêts.

Si divers obstacles indépendants de notre volonté l'ont empêché de voir le jour plus tôt, il n'aurait pu être mené à son terme sans l'aide précieuse que m'ont apportée divers membres éminents de l'Université et de l'Administration forestière.

Je sais tout particulièrement gré à M. le Professeur EMBerger, Correspondant de l'Institut, Professeur à la Faculté des Sciences de Montpellier. d'avoir bien voulu diriger mes travaux et de m'avoir fait profiter de sa remarquable compétence. 
Je dois remercier vivement $M$. le Professeur Mor.rnier, de la Faculté des Sciences de Marseille, qui, lorsque je préparais le concours d'entrée à l'Institut National Agronomique, m'a initié à la Phytosociologie et qui, plus tard, est venu en Maurienne, à plusieurs reprises, pour me guider dans mes investigations, sans ménager sa peine ni son temps.

J'ai contracté une grande dette de reconnaissance envers M. DuChaufour, Professeur à la Faculté des Sciences de Nancy, et Directeur de l'Institut de Pédologie du C.N.R.S., qui, lorsqu'il était Professeur de Pédologie à l'Ecole Nationale des Eaux et Forêts de Nancy, m'a prodigué de nombreux conseils, est venu plusieurs fois sur le terrain, a fait faire les analyses des échantillons de sols que je lui avais expédiés; l'esprit de ce travail doit beaucoup à son enseignement.

Une part de cette reconnaissance va à son adjoint, M. Bonneau, Ingénieur des Eaux et Forêts, actuellement Directeur de la Station de Pédologie au Centre National de la Recherche forestière, à Nancy, qui m’a accompagné également dans certaines de mes tournées et a fait procéder aux analyses de sols que nous avions vus ensemble.

Toute ma gratitude va également à M. SAuvage, Professeur à la Faculté des Sciences de Montpellier et à M. Lossarnt, Maitre de Recherches au C.N.R.S. à Montpellier, qui ont hien voulu accepter de faire partie de mon jury de thèse.

M. RoL, aujourd'hui décédé, ancien Directeur de l'Ecole Nationale des Eaux et Forêts, avait bien voulu, en accord avec son adjoint, M. JaCamon, me prêter divers instruments de mesures écologiques et faciliter la rédaction de ce travail, lors de mon séjour ¿̇ l'Ecole de Nancy, comme chargé du cours d'Aménagement. Je remercie également M. VINEY, son successeur, pour sa parfaite compréhension,

M. le Professeur Gaume, puis M. Hamant, ce dernier professeur à l'Ecole Supérieure d'Agronomie de Toulouse, ont accepté d'examiner mes récoltes de mousses: je leur en suis tr.s reconnaissant; toute ma gratitude va également à M. Pierre LE Brun. du C.N.R.S., attaché à la Faculté des Sciences de Toulouse, qui a bien voulu confirmer quelques-unes, parmi les plus difficiles, de mes déterminations de Phanérogames,

M. Braun-Blanguet, en m'adressant ses deux otivrages fondamentaux sur la flore du Parc National suisse et sur la végétation xérophile des vallées internes des Alpes, a beaucoup contribué à me faire mieux comprendre la haute vallée de l'Arc. Qu'il en soit ici vivement remercié.

Je n'aurai garde, enfin, d'oublier combien m’a été précieuse la collaboration de certains de mes adjoints: Madame Lfeeux, qui a 
bien voulu procéder à la frappe, souvent délicate, de mon manuscrit: Madame Casy-PaLov, qui m'a transmis les renseignements météorologiques fournir par 1'O.N.E.R.A., à Avrieux; M. Charvoz, Chef de district des Eaux et Foréts à Termignon, qui m'a donné d'utiles renseignements sur l'enneigement; M. Grosse, Agent technique, qui a effectué des observations directes de durée d'ensoleillement: M. Bors, Agent technique, qui a eu la charge de relever les mesures données par le pédohygromètre.

Les subventions qui miont été accordées par le Centre national de la Recherche forestière et par le Comité Scientifique du Parc national de la Vanoise mont permis de faire face aux frais de publication de cet ouvrage; je prie tous leurs membres, de trouver ici. l'expression de ma très vive gratitude.

Grâce à l'extrème obligeance de $\mathrm{M}$, le Professeur Ozends, de la Faculté des Sciences de Grenoble, et à celle de ses collaborateurs, comme au concours financier que son Service m'a apporté, la carte phytosociologique en couleurs jointe à ce mémoire, dont elle constitue un élément indispensable, a pu être dessinée et reproduite dans les meilleures conditions. Je leur exprime mes remerciements chaleureux. 

PREMIÈRE PARTIE

But de ce travail et méthodes suivies 
La Haute-Maurienne dans les Alpes occidentales frangaises

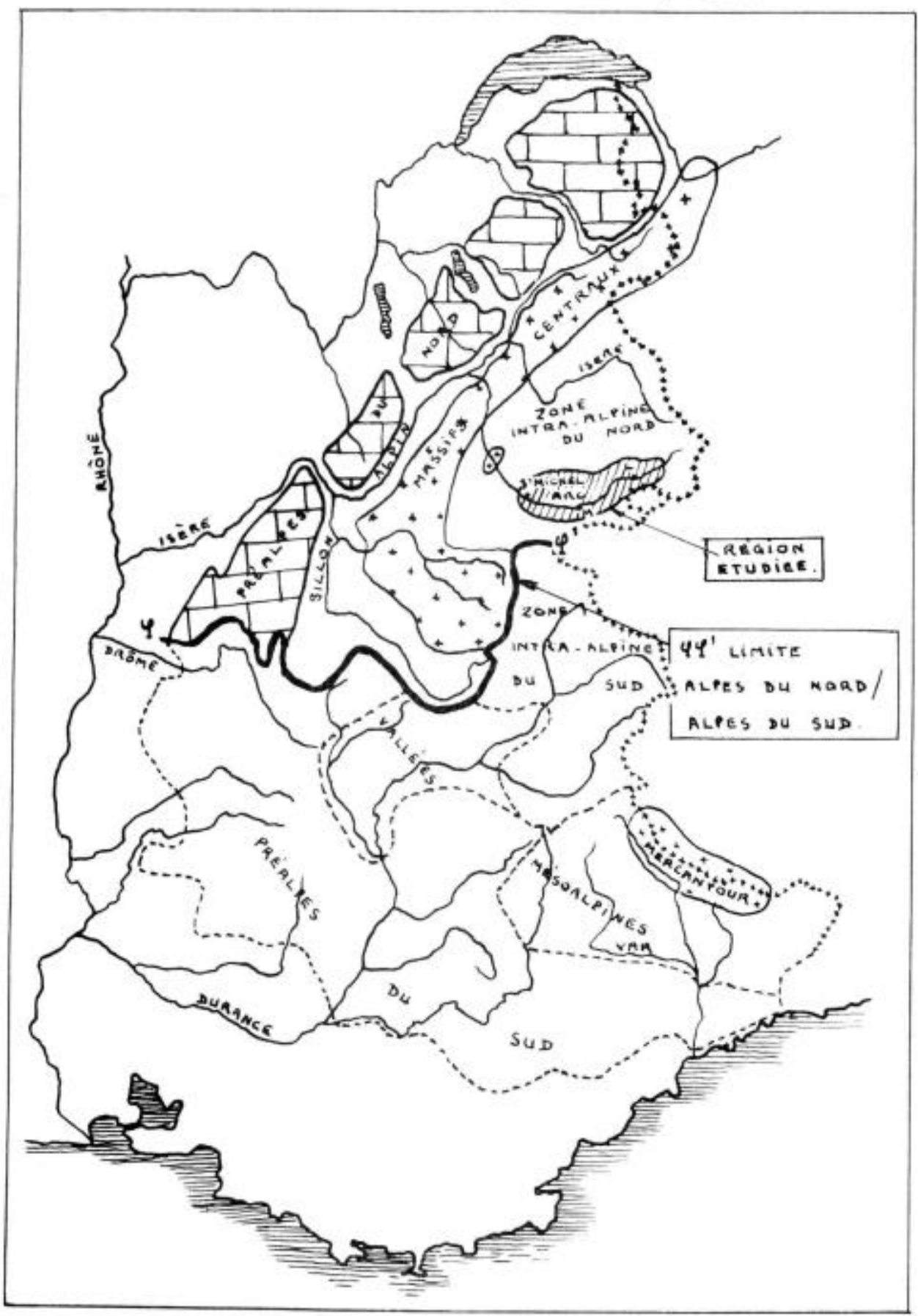




\section{1. - OBJET DE CE MEMOIRE}

Beaucoup de phytogéographes ou de phytosociologues, ont souligné, après Flahault (Ch.) notamment (1901), l'intérêt que présentaient, dans le domaine de la Géographie botanique ou dans celui de la Phytosociologie, les études de petites régions naturelles, ou mème, de stations bien choisies, car elles ouvrent la voie à des comparaisons de plus en plus nombreuses, et à des synthèses de plus en plus surres.

Dans les Alpes occidentales françaises, relativement peu connues, et qui, d'une façon générale, offrent des conditions favorables à ce genre d'étude: flore assez riche, contrastes écologiques nets, action perturbatrice de l'homme, sensible sins doute, mais non écrasante il nous a done semblé que la haute vallée de la Maurienne constituait une région particulièrement privilégiée.

Les géographes, comme les botanistes qui l'ont parcourue, ont toujours souligné son caractère intra-alpin qui se traduit par une sécheresse dont les conséquences sur la végétation sont considérables et qui en fait une des provinces géographiques et biologiques les plus originales des Alpes. Communiquant, en outre, avec l'est, par le bas créneau du col du Mont Cenis; avec le sud, par les vallées latérales qui la rapprochent du Briançonnais; avec l'ouest, par la vallée de l'Arc qui s'est frayé un chemin à travers les Massifs Centraux, elle se présente comme un carrefour d'influences diverses: piémontaises, briançonnaises, rhodaniennes... qui expliquent Y'existence d'espèces médioeuropéennes, subméditerranéennes, subatlantiques, sarmatiques...

Son orientation générale est-ouest, crée, d’autre part, deux versants principaux d'expositions opposées: I" " adret " ou " endroit " à exposition sud et l' a envers o à exposition nord (1). Cette opposition des versants, accentuće encore par la raideur des pentes, se reflète nettement dans la végétation, ce qui permet d'établir de suggestives comparaisons.

En Maurienne enfin, les grandes zones tectoniques et pétrographiques coupent la vallée à peu près perpendiculairement à son axe. Il en résulte qu'une même roche-mère - dont l'influence est grande

(1) Le terme $d^{\prime}$ e ubac s ne figure pas dans la toponymic locale, 
sur la végétation, puisque l'érosion freine sensiblement l'évolution des sols - occupe, à des altitudes diverses, les deux versants d'exposition opposées. Ces roches-mères ont, en outre, une composition assez variée: les unes sont très siliceuses, comme les quartzites du Trias ou - quoique à moindre degré - les grès du Houiller; les autres, au contraire, sont riches en carbonates de chaux (ou en sulfates), comme les calcaires du Lias, du Dogger ou du Flysch de la zone subbriançonnaise, les calcaires, les gypses et les cargneules de la zone de la Vanoise; d'autres enfin, présentent un caractère mixte, comme les schistes du Houiller et, surtout les schistes lustrés.

On conçoit donc que, en Maurienne, puissent ètre observées des interférences relativement peu nombreuses et, en tout cas assez nettement contrastées, des grands complexes de facteurs écologiques, comme la nature de la roche-mère, l'exposition, l'altitude, et que ces interférences entrainent l'existence de combinaisons écologiques générales, simples dans l'ensemble, facilitant l'étude des corrélations qu'elles peuvent présenter avec la végétation.

Sans doute ne faudrait-il pas simaginer que le recoupement de ces divers facteurs aboutit à un véritable quadrillage: en effet, la vallée principale dessine une courbe concave, et non rectiligne, qui donne des expositions tranchées, surtout dans sa partie médiane la plus au sud -, mais plus nuancées vers les extrémités; chaque versant principal présente, en outre, des expositions secondaires très diverses, dues à l'existence de vallées latérales ou d'ondulations du relief; toutes les grandes zones tectoniques et pétrographiques ne se reproduisent pas, de façon absolument symétrique, de part et d'autre de l'axe de lis vallée; d'autre part - et surtout - bien que les sols soient, en général, peu évolués et portent très souvent la marque de la roche-mère, l'action de la végétation et du climat local, jointe à celle du relief, n'en a pas moins joué, produisant sur des roches-mères de nature différente des sols " analogues $n$ au moins dans leurs horizons supérieurs; l'action humaine, enfin, ne s'est pas exercée partout avec la mème intensité, ni pendant une même durée.

C'est dire que, dans le détail, la réalité reste complexe; mais elle présente, toutefois, dans l'ensemble, d'excellentes conditions, pour l'étude comparative des groupements végétaux.

Cette région n'a pas encore donné lieu, à notre connaissance, à un travail phytosociologique de détail (1). Sans doute a-t-elle été bien exploríe et est-elle bien commue, quant à sa flore phanéroga-

(1) L'étude de Stavinsky (W.) (1933), outre qu'elle porte essentiellement sur l'histoire, la géographie et le tourisme, ne contient, malgré son titre, aucun relevé floristique et ne correspond pas, à la lettre, à un travail de phy-
tosociologie. 
mique, tout au moins. Mais les botanistes qui, à la suite d'AlloNI (C.) (1785), lont parcourue, à la fin du siècle dernier et au début de celui-ci, se sont surtont attachés à en dresser l'inventaire floristique. Huguenin et Bonjean, Chabert (1859 - 1860 - 1883), Convert (1896 - 1921), Saint-Lager (1887), Verlot (1863 1865 - 1896), GANDoger $(1890)$, Meyran $(1891-1900)$, plus près de nous I.e Brun (P.) (1919 - 1923 - 1957), Offrer (J.) (1920), Denarié (M.) (1920), Leredde (Cl.) ont été les principaux artisans de cette reuvre. D'autres, comme Perrier de la BATHIE (E.) $.1863-1909-1910-1911-1917-28$ ), Petrtannar (M.) (1907), Brauverd (1911 - 1921), Guyot (H.) (1921), de Litardik̀re (1934), lont explorée davantage en phytogéographes, en montrant, par une étude comparative des éléments floraux, les affinités qu'elle offre avec d'autres régions des Alpes, comme le Valais, le Briançonnais ou la région de Suse (2). Mais, bien que ces études présentent le plus grand intérèt - nous aurons l'occasion de nous y référer à maintes reprises - elles ne mettent en évidence aucun groupement végétal et ne relèvent donc pas, à proprement parler, de lat phytosociologie (3).

Il faut ajouter, enfin, que nos obligations professionnelles nous ont imposé l'étucle cl'un domaine qui nous parait avoir été moins exploré que tout autre: les étages silvatiques, en effet, présentent, en général, une flore "banale " que les floristes de naguère ont négligée. Leur attitude se conçoit: c'est essentiellement dans l'étage alpin ou dans les " garides ", vers le fond du talweg que s'observent ces espèces rares - relativement au moins - de caractère arctique ou steppique, qui donnent à la flore de la vallée une grande partie de son originalité et permettent de la rapprocher de celle d'autres vallées des Alpes centrales ou méridionales. Le développement de la Phytosociologie a heureusement donné un nouvel essor à Pétude de la flore des forêts: l'objet même du présent mémoire est de montrer tout l'intérêt qu'elle présente, pour la région qui nous occupe, intérêt accru du fait que les groupements forestiers des Alpes suisses et autrichiennes ont déjà fait l'objet, pour la plupart, d'analyses phytosociologiques détaillées.

L'idéal eut été d'étudier les association forestières de toute la vallée: si petite que puisse paraitre la Maurienne sur une carte gé-

(2) Bouvir. (1863) avait dressé la liste des botanistes qui avaient visité la Savoie Récemment. I. Brun et OFFNER (1956) ont établi un index bibliographique concernant \& un siècle de floristique à travers les Alpes Françaises \& où Yon trouvera la référence complète de travaux botaniques intéressant la Maurienne depuis cent ans.

(3) Au moment où nous achevions ce travail, paraissait le monumental ouvrage de Bravn-Blanoquer (1961) sur la végétation xérophile des vallées intra-alpines. La Maurienne y a trouvé une place de choix. L'auteur n'a donné qu'un bref aperçu des forêts qui, au demeurant, ne sont pas toutes xérophiles, loin de lì, et échappaient par conséquent à son enquéte. 
nérale de la France, la tâche eut été importante. La montagne est, en effet, un monde immense, très difficile à parcourir dans tous ses recoins. Nous avons préféré notus limiter au secteur même où nous appelait notre activité: il correspond à la partie de la vallée comprise entre Saint-Julien-de-Maurienne, à l'aval et Bonneval-surArc, à l'amont (4).

Bien que s'inscrivant, entièrement et exclusivement, dans la zone des nappes tectoniques, notre dition parait, at premier abord, ne pas présenter une grande unité. Si l'on se réfère, en effet, aux travaux des géographes ONdE (H.) (1938) et BLANchard (R.) (1945). elle comprend, sans doute, toute lit Haute-Maurienne, sensu stricto, c'est-à-dire toute la partie de la vallée située à l'amont de Modane. mais également une partie de la Moyenne Maurienne. En fait, son unité est surtout d'ordre biologique et phytogéographique: la limite aval de notre dition correspond a la limite amont de pénétration du Hêtre dans la vallée et à la limite aval de l'aire spontanée du Mélèze: notre zone recoupe, d'autre part, ce que les phytosociologues suisses, Braun-Blanourt en particulier (1949), ont appelé le "district du Pin sylvestre » et qui s'étend du sud au nord, depuis la vallée supérieure et moyenne de la Durance et même la région de Tende, jusqu'au Vinschgau tyrolien.

Nous pensons donc que les limites administratives du cantonnement forestier de Modane, qui ont imposé notre choix, se trouvent coincider très heureusement avec celles d'une véritable petite province phytogéographique, étant entendu, tottefois, qu'il y a, au contact des "zones $n$ du mélèze et du hêtre, une zone "mixte * où les denx essences se trouvent représentées, et qui s'étend entre Saint-Jean-de-Maurienne et Saint-Michel-de-Maurienne, soit sur dix kilomètres environ. La a zone $n$ du Hêtre pénètre ainsi dans notre dition - entre Saint-Julien-de-Maurienne et Saint-Michelde-Maurienne très exactement - ce qui ne peut qu'ajouter, évidemment, à l'intérêt de notre étude.

A l'intérieur de cette petite unité, typiquement intra-alpine, nos investigations ont donc porté sur les associations forestières. Il nous a paru nécessairc d'essayer de mener leur étude en même temps que celle du sol qui les supporte, pour tenter de déagger Pinfluence réciproque que sol et végétation cxercent l’un sur l'autre. En même temps, nous avons cherché à obtenir quelques données mésoclimatiques et microclimatiques et à les rattacher aux divers groupements observés. Nous avons essayé, d'autre part, d'apporter quelque clarté aux problèmes suivants: quels peuvent être, aux diverses altitudes et expositions, les climax forestiers et à quels climax du sol correspondent-ils? Quels sont les groupements qui paraissent être éloi-

(4) Pratiquement, la zone forestière s'arrête un peu à l'amont de Bessans Mais cette limite est due, en grande partie, à l'action humaine. 
gnés du climax et quels sont les facteurs qui ont pu freiner - qui peuvent encore freiner - leur évolution progressive? En particulier, quelle est, dans ce domaine, la part de l'influence humaine?

Autre question: à quelles associations forestières déjà décrites peut-on rattacher celles de la Haute-Maurienne? Nous avons pensé, en effet, que l'étude phytosociologique des forêts devait permettre de confirmer les conclusions auxquelles sont arrivés divers chercheurs, concernant les affinités que présente cette vallée avec d'autres régions intra-alpines, et d'éclairer ainsi le problème de l'immigration, en Maurienne, de la flore sylvatique.

Nous avons cherché enfin à rattacher aux diverses associations observées, des structures sylvicoles ou des caractéristiques d'ordre dendrométrique et auxométrique. Il nous a semblé utile, en effet, de ne pas oublier certaines corrélations dont l'importance pratique apparait chaque jour davantage. 


\section{2. - METHODES D'ETUDE DES ASSOCIATIONS}

\section{I.2.1. - Considérations générales.}

L'étude des groupements végétaux, dont la variabilité est grande, doit découler d'un ensemble de déterminations. Comme nous ne cherchions pas à analyser avec une très haute précision la composition quantitative du tapis végétal - ce qui nous eût pratiquement obligé à limiter notre champ d'action - nous n'avons pas fait d'échantillonnage systématique ou au hasard, mais nous avons simplement suivi comme méthorle d'investigation sur le terrain, celle préconisée par Braun-BLAnguet (1951), et nous avons adopté ses échelles, tout au moins en ce qui concerne l'abondance-dominance, soit:

5 - pour les espèces couvrant plus des $3 / 4$ de la surface relevée.

4 - pour les espèces couvrant de $1 / 2$ à $3 / 4$ de la surface relevée,

3 - pour les espèces couvrant de $1 / 4$ à $1 / 2$ de la surface relevée.

2 - pour les espèces couvrant de $1 / 20$ au $1 / 4$ de la surface relevée.

1 - pour les espèces couvrant moins du $1 / 20$ de la surface relevée, mais représentées par des individus assez abondants.

+ - enfin pour les espèces de très faible recouvrement avec peu d'individus.

Pour traduire la sociabilité, par contre - qu'il s'agisse de la sociabilité inhérente à l'espèce ou de la sociabilité écologique [SAUVAGE (Ch.) (1950)], nous avons préféré renoncer aux coefficients de BRAUN-BLANQUET et analyser la microphysionomie des groupements en termes descriptifs ou par des photographies. Cette facon de procéder nous a paru rendre compte de la structure de la végétation d'une façon plus expressive que n'auraient pu le faire des chiffres, dont la signification est moins claire que celle qui s'attache aux coefficients d'abondance-dominance.

Quant à la structure de la végétation arborescente, nous l'avons. sans doute, exprimée en coefficients d'abondance-dominance, mais aussi, grâce à des inventaires complets portant sur un ensemble homogène au point de vue phytosociologique, en courbes de fréquence donnant, par espèce, le nombre d'arbres à l'unité de surface (hec- 
tare), et par catégorie de diamètre (ce diamètre étant pris, sur écorce, à $1,30 \mathrm{~m}$ de hauteur, avec des classes de $5 \mathrm{~cm}$ à partir de $20 \mathrm{~cm}$ ).

Mais une fois les relevés obtenus, leur analyse et leur interprétation n'ont pas été conduites selon les règles mêmes de l'Ecole de Braun-Blanguer. On sait que, dans cette méthode, la comparaison des divers relevés floristiques, c'est-à-dire des divers $\alpha$ individus d'association $n$, fait apparaitre, sans qu'intervienne l'étude préalable du milieu, des noyaux de plantes communes qui permettent de dégager le concept d'association. " La liste des plantes définissant lassociation ne coincide exactement avec aucune des unités végétales étudiées séparément sur le terrain. Mais elle exprime cette réalité, qu'il y a quelque chose de commun entre les unités considérées " [Reynaud-Beauverie (M.A.) (1936)]. Une fois l'association mise en évidence, et caractérisée floristiquement (1), est abordée alors l'étude de sa place dans la systématique phytosociologique, de son écologie, de son aire, de son évolution, etc.

Le but poursuivi dans le présent mémoire étant l'étude des rapports existant entre la végétation et le milietı, nous avons cherché, non seulement à conduire parallelement l'analyse floristique et l'analyse écologique, mais à faire en sorte que cette double analyse s'exprime, aussi clairement que possible, dans les relevés.

Pour ce faire, nous avons été amené à mettre en relief des noyaux de plantes, qui nous ont paru présenter, avec certains facteurs du milieu, des corrélations suffisamment nettes et correspondant à leur optimum de développement. Si donc la juxtaposition des listes d'espèces constitue une des bases de ce travail, l'originalité des groupements végétaux et leurs affinités ont été mises en évidence à l'aide "d'optimales stationnelles n, ou, dans les cas les plus favorables, de "caractéristiques stationnelles o à écologie plus étroite.

Les espèces de méme écologie on d'écologie voisine, qu'il s'agisse de caractéristiques stationnelles - à amplitude écologique étroite ou d'optimales stationnelles - à amplitude plus large, mais à optimum assez localisé — ont été réunies au sein de groupes, dits "groupes écologiques " pour reprendre lexpression de DuviGNEAU (P.) (1946), qui a acquis maintenant droit de cité.

(1) On ne reviendra pas, ici, sur les diverses définitions de l'association qui ont été données par les phytosociologues, et notamment par BraUN-BLANQUet (J.) (1951), Altorge (P.) (1921-22), Molinier (R.) (1935), QUANTIN (A.) (1935), LeMEe (G.) (1937), Jovet (P.) (1949), GuiNochet (M.) (1939), etc. Nous retiendrons, pour notre part, celle d'EMnerger plus rérente (1959): « L'association est un groupement - ou une communauté - naturel de végétaux; elle est floristiquement et statistiquement définie; elle croit dans des conditions écologiques déterminées: les rapports interspécifiques et interindividuels y sont réglés par la concurrence vitale. Flle est lunité de hase đe la végétation, ? 
Les groupes ayant été définis — on reviendra ultérieurement sur ce point - par référence à certaines conditions du milieu assez limitées, que l'on peut appeler facteurs écologiques décisifs, telles que: climat général, tempierature (influencée, toutes choses égales d'ailleurs, par l'altitude), économie de l'eau et aération du sol, état et nature du complexe absorbant du sol et composition et structure de l'humus, on conçoit que certaines espèces se trouvent liées à deux ou plusieurs de ces facteurs et doivent, par conséquent, figurer dans plusicurs groupes. A la limite, une espèce ubiquiste devrait figurer dans tous les groupes, mais une telle manière de faire ne pourrait qualourdir les tahleaux de groupes et masquer, dans une large mesure, les espèces ayant une signification écologique étroite: nous ne l'avons donc pas retenue.

Quant à l'association, elle apparaift comme comprenant, en principe, un groupe indicatewr, disons caractéristique, qui y trouve les meilleures conditions pour son développement et plusieurs fractions d'autres groupes. Son originalité résulte d'ailleurs, tout aussi bien, de la coexistence de ce groupe indicateur et des fractions marginales des autres groupes, fractions narginales qui traduisent, on le verra, les transitions et la variabilité du tapis végétal, dans l'espace comme dans le temps. Certaines associations peuvent ne pas présenter de groupe indicateur bien tranché: dans ce cas, c'est la combinaison toujours originale - des fragments de groupes, qui donne à l'association sat valeur d'unité autonome: il s'agit, le plus souvent, de grotıpements de transition, situés dans des zones de transition.

Cette façon de voir nous parait rejoindre, en fait, celle de nombretix phytosociologues de l'Ecole zuricho-montpellieraine; l'un d'eux - Guinochet (M.) (1955) n'a-t-il pas écrit: "Une espèce caractéristique d'un groupement le caractérise effectivement, mais d'une manière relative, et non absolue, c'est-à-dire en liaison avec (2) les autres caractéristiques et avec les compagnes de haute présence, qui constituent ce qu'on appelle parfois lensemble spérifique (2) normal de ce groupement $n$.

On sait que cette méthode, dite des " groupes écologiques w, est relativement récente et qu'elle a pris naissance à peu près simu1tanément, sous des formes un peu diverses d'ailleurs, en Belgique. en Allemagne et en France. Dans ce dernier pays, où elle connait maintenant un développement particulier avec l'Ecole de Montpellier, sous la direction d'EMrerger, la plupart des chercheurs, avant même que la méthode prít corps, avaient souvent eu le souci d'analyser le tapis végétal à la fois en termes de floristique et en termes d'écologie. Qu'il nous suffise de rappeler, entre autres travaux, ceux de Flahault (Ch.) (1897), d'Allorge (P.) (1921-22), de Leмŕe (G.) (1937), de Jovft (P.) (1949), etc.

(2) Cest nous qui soulignons. 
Ce sont aussi les forestiers qui, à la suite de l'enseignement de Ph. Guinier et de R. Rol, ont, le plus volontiers, mis laccent sur l'intérêt que présentait l'étude de la signification écologique des espèces. Duchaufour, le premier en France, semble-t-il, dans ses "Recherches écologiques sur la chènaie atlantique française $n$, en 1948, a distingué dans ses tableaux de relevés, au sein de chaque association, des groupes d'espèces à écologie affine, véritables indicatrices stationnelles, qui lui ont permis non seulement de caractériser les groupements, mais de traduire les transitions qui permettent de passer - dans l'espace comme dans le temps - d'un groupement à un autre. En 1950, dans ses * Recherches sur l'évolution des sols calcaires en Lorraine $n$, il a montré que dans un groupement donné, celui de la " fruticée mésophile n, pour ne retenir que cet exemple, se rencontraient des espèces relevant de cinq unités écologiques différentes: les caractéristiques de la fruticée, en général, relativement plastiques: celles, plus spécialisées, de la fruticée mésophile: des éléments de fruticée xérophile; d'autres de la forêt de hêtre; des "reliques ", enfin, de la pelouse sur calcaire.

C'est dire que notre travail, tout en faisant appel à une méthode assez récente, se situe, si l'on veut, dans une tradition qui l'est moins.

Bien que le but essentiel du présent mémoire soit de décrire les associations forestières de la Haute-Maurienne, il nous a paru utile, avant d'aborder toute description, d'analyser, au préalable, les raisons qui nous ont fait adopter la méthode des groupes écologiques et de mettre ainsi, en parallèle, cette méthode et celle, plus classique, de Braun-Blanourt. Ce parallèle sera poursuivi aussi loin que possible, et en particulier, lorsque nous dresserons la liste des especes appartenant aux divers groupes écologiques: en face du nom de chacune des espèces retenues pour sa signification écologique, nous indiquerons sa signification dans le système Braun-Blanquet en désignant l'unité plus ou moins compréhensive dont elle est caractéristique dans ce système, chaque fois que possible évidemment, c'est-à-dire chaque fois que l'espèce considérée a été retenue, par les spécialistes, comme caractérisant une unité phytosociologique.

Dans le domaine de la nomenclature, il y a lieu de noter que nous avons suivi les règles préconisées par BAcH (R.). КuocH (R.) et Moor (M.) (1962) et que Rauschert (St.) a analysées en détail (1963).

\section{I.2.1.1. - Continuité du tapis végétal - chaînes et phylums d'associations.}

Qu'elle porte sur les variations régionales d'une même association ou sur des formes de passage que présentent, localement, des associations contiguës, lobservation montre que, malgré certaines 
discontinuités, le tapis végétal, dans son ensemble, présente de nombreuses formes de transition.

Les discontinuités régionales tiennent, en général, à l'existence de barrières isolatrices de grande étendue, qui délimitent alors des régions florales différentes, et à l'isolement insulaire, source d'endémisme; les hêtraies corses, pour ne retenir qu'un exemple, sont, de toutes les hêtraies françaises, les plus originales.

Quant aux discontinutités locales, elles correspondent, le plus souvent, au fait que les facteurs du milieu présentent eux-mêmes des passages sans transition: en Haute-Maurienne où, nous l'avons dit, la roche-mère joue un rỏle certain, on observe fréquemment. dans le trias, des contacts nets entre des quartzites très siliceux et des cargneules, très calcaires; au niveau de tels contacts, le changement de végétation se fait assez brutalement; il en est de même de part et d'autre d'une crète, séparant deux versants d'expositions tranchées, done de climats locaux différents. Certaines associations, d'autre part - celles du bord des eaux, par exemple - s'observent dans des zonations anx limites nettes; PAvILLARD (J.) (1935). entre autres, avait mis l'accent, sur cette discontinuité du tapis végétal, s'opposant à la continutité de variation des facteurs dtı milieu: en loccurrence, diminution progressive de l'humidité du sol quand on s'éloigne des berges d'un étang: en réalité. cette zonation s'explique par la grande variation du facteur humidité entre des points relativement proches, ce qui diminue d'autant la largenr des zones d'interprétation des associations et par l'existence, dans de telles associations, de dominantes exclusives, à amplitude écologique étroite.

Enfin et surtout, l'action humaine s'est exercée de façon très inégale et a contribué nettement à effacer le a flou 0 des contacts pouvant exister dans une végétation entièrement climacique.

D'une façon générale, la végétation présente done des termes de transition qui apparaissent de plus en plus, à mesure que s'amplifient les investigations, et que les aires des chercheurs arrivent à se rejoindre (3).

C'est ainsi que Caricetum firmae de l'étage alpin, ne présente en France et dans la Suisse occiclentale voisine que trois ou quatre espèces caractéristiques: le nombre de ces dernières augmente jusqu'à cinq-six à l'est de la Suisse, à sept dans le Tyrol, à douze dans les Alpes de Carinthie et de Slovénie [Braun-Blanouet (J.) (1954) ]. De même entre le Facelum pracalfino-jurassicum du Centre-Fst de la France et le Fagetum aallictrm du Midi. existent des intermédiaires. dont l'un a été étudié par R. Mor.rnikr et I.

(3) Ellenaekg (H.) (1954), Goodali (D.) (1954), Duvignkaud (P.) (1946), ont insisté sur ce fait. Molınizk (R.) (1935) avait noté: \& A mesure que les travaux se multiplient, les associations sont mieux définies et leurs variatione foristiques indéniables, f'une région à l'autre, sont précisées 3 . 
Ayasse (1955), dans les environs de la Motte du Caire et dont une série a été analysie par Arìnes (J.) (1955). Autre exemple: le Xerobrometum de la région dijonnaise est un des chainons qui relie le Xerobrometum atlantique au Xerobrometum rhénan.

Ces variations géographiques des groupements font que, les conditions écologiques restant pourtant très voisines, il n'y a jamais identité, mais homologie entre des associations appartenant à des territoires floraux tant soit peu différents. D'où la nécessité de ne pas sen tenir aux seuls critères floristiques, mais d'utiliser, en les confrontant avec eux, les critères écologiques.

On aboutit à des constatations du même ordre, si l'on s'attache à l'examen des passages locaux d'une association à une autre. $\mathrm{Ph}$. DuCHAUfoUr (1948) a noté que des séries continues existent entre les chênaies à humus doux et les chênaies acidiphiles. Dans la région subméditerranéenne, particulièrement étudiée, un groupement mixte a été décrit par BLondel. (R.) (1941). Il correspond très exactement à un faciès de transition entre les forèts à Quercus llex (Quercetum ilicis galloprovinciale) et celles à Quercus pubescens (Quercelo-Buxetum). On y observe, en particulier, une augmentation du nombre des espèces du Quercion ilicis vers le sud et le bas de la zone de contact, tandis que les espèces du Quercion pubescentisessiliflorae gagnent en importance vers le nord et le haut. Dans l'arrière pays de Montpellier, des relevés de hètraies pris au contact de la chênaie pubescente ont une prépondérance d'espèces du Quercion, d'autres, au contraire, pris plus hatt, se rapprochent davantage du Fagion [Braun-Blanguet (J.), Roussine (Mme N.), Nègre (R.) (1951)]. En Auvergne, Lemée (G.) (1946) a mis en évidence une série altitudinale, entre un Qucrceto-Carpinetum typicum et un Fagetum gallicum typicum, avec, comme intermédiaires: Querceto Carpinetum montanum et, plus haut, Fagetum tilietosum (4).

MoOr (M.) (1952), de son còté, a insisté sur les passages graduels qui relient les associations les unes aux autres, et montré que, lorsque les pentes sont uniformes et la roche-mère la même, ce serait faire violence à la réalité que de tracer des frontières nettes entre associations.

A l'intérieur d'une mêne association, enfin, peuvent être mises en relief de véritables variations continues: c'est ainsi que BraUNBlanouet (J.). Paldmann (H.) et Bach (R.) (1954), en classant les relevés de 1'Erico-Pinetum dt1 Parc national suisse par ordre d'altitude croissante, ont fait apparaitre une augmentation, avec l'altitude, des espèces des Vaccinio-Picectalia et une diminution concomitante des espèces du Quercion pubescenti-petraeae.

(4) Dans ce domaine également, les exemples pourraient être multipliés. Encore faut-il noter que le réseau des recherches phytosociologiques est, du moins en France, assez lâche. 
Les variations, dans le temps, appellent des observations semblables; les phytosociologues ou les phytogéographes, à la suite de Clements (F.) (1916) et de Giussen (H.) (1926), notamment, se sont presque tous attachés, dans leurs études, à mettre en évidence cette dynamique de la végétation et à montrer comment des phases se succédaient, permettant de lier graduellement les association appartenant au même "phylum ", soit dans une évolution progressive, soit dans une évolution régressive. D'ailleurs, après ce qui a été dit sur les formes de transition dans l'espace, une telle variation dans le temps doit d'autant moins étonner que, pratiquement, le phytosociologue étudie le plus souvent la dynamique des groupements par l'examen, dans l'espace, de leurs variations floristiques et écologiques.

Il faut ajouter, enfin, et ceci nots parait essentiel, que la gradation des variations régionales ou locales, dans l'espace et dans le temps, peut ne pas apparaitre toujours avec netteté, pour les raisons suivantes.

D'une part, sur le terrain, le chercheur fait souvent un choix et évite de faire porter ses investigations sur les intermédiaires, de manière à mettre mieux en relief les caractères originaux des associations qu'il étudie. Son échantillonnage est donc discontinu.

D'autre part, chaque chercheur, souvent selon son tempérament propre, situe à une place plus ou moins élevée dans la hiérarchie, ces formes de transition qui constituent ainsi, soit de véritables unités autonomes, soit de simples variantes géographiques ou écologiques d'unités plus vastes.

Enfin et surtout, le principe mème de classification adopté par 1'Ecole zuricho-montpellieraine — nous reviendrons sur ce point, ultérieurement, car il est capital - qui veut qu'au sein de chaque association ne soient mises en valeur que certaines espèces, en rejetant, dans l'anonymat, de nombreuses compagnes, contribue, par un choix fait dans une seule direction, à masquer les transitions qui s'observent dans la nature. Et si l'on adopte une méthode purement statistique - comme celle de Czekanowski - on constate que, si l'analyse fait apparaitre des noyaux bien tranchés de relevés, ces noyaux sont reliés par des zones de transition, et $\alpha$ qu'il est souvent délicat, selon Gurnochet (M.) (1955), de placer des coupures dans ces suites plus ou moins continues $n$.

Ces caractères du tapis végétal nous paraissent entrainer plusieurs conséquences dont nous avons cherché à tenir compte dans le présent travail: d'abord, celle de donner un intérêt tout particulier à l'étude des groupements de transition. Duchaufour (Ph.) (1948), Duvigneaud (P.),1946) (5), ont montré que la zone d'interpénétra-

(5) Nous avions rédigé le présent chapitre lorsque nous avons pris connaissance de la pénétrante analyse de Duvigneaud (P.) (1946). 
tion des associations permet tout spécialement de saisir les " seuils n Écologiques de certaines espèces, les limites de leur amplitude écologique. Elle oblige ensuite à présenter les résultats sous forme de tableaux synthétiques mettant en évidence les formes de passage d'une association à une autre, soit dans l'espace, soit dans le temps.

L'utilisation de groupes écologiques, qui se relaient d'une association à lautre, qui laissent apparaître, ou subsister, dans telle association des témoins - des pionniers ou des reliques si l'on se réfère au facteur temps - pendant que s'observent, dans cette même association et de façon optimale, donc caractéristique, des espèces d'un autre groupe écologique, nous parait tout particulièrement propre à rendre compte de cette continuité ct de cette variabilité.

Notons, enfin, que cette "évanescence» des groupements, ne nous parait nullement mettre en cause le concept d'association, de mème que l'existence de formes de passage d'un climat à un autre, ou d'un type de sol à un autre, n'altère en rien ces réalités. Elle nous semble simplement donner une importance particulière aux notions d'optimum et d'amplitude écologiques et à la distinction de petites unités (sous-associations, faciès, variantes géographiques), propres à traduire la variabilité du tapis végétal et sa gradualité.

\subsubsection{2. - Critères de différenciation des associations.}

On sait que la "fidélité n de certaines espèces, appelées caractéristiques, c'est-à-dire leur étroite localisation sociologique constitue une notion fondamentale dans le système Braun-Blanquet. En effet, les caractéristiques " permettent d'individualiser floristiquement les associations: elles sont, dans l'ensemble, les meilleurs indicateurs de leur écologie; elles permettent d'apprécier le stade de développement d'une association. Elles permettent des déductions sur la dispersion présente et antérieure d'une association déterminée. De leur présence on peut conclure aux affinités naturelles des associations, ce qui permet la classification de ces associations sur une base floristique. " [Reynaud-Beauverie (M.-A). (1936)].

L'idéal, pour une caractéristique, serait d'avoir une valeur quasiabsolue sur un territoire aussi vaste que possible. Pratiquement mises à part certaines endémiques ou certaines micromorphes dont l'extrême localisation permet surtout de caractériser des formes géographiques d'associations, plutôt que des associations proprement dites - l'observation montre que la plupart des caractéristiques n'ont qu'une valeur très relative, et qu'elles ne sont liées étroitement à une association quu'à l'intérieur d'un territoire limité. Gaussen (H.)

(6) L'intérét de ces petites unités, déjà souligné par Gurnocher (M.) (1938) a śté tout particulièrement analysé par DuvigNeaud (P.) (1946). 
(1954), et plus récemment Ellenberg (H.) (1954), ont noté que, précisément, la notion de caractéristique avait d'autant plus de valeur que la région étudiée était petite.

Cette " relativité " des caractéristiques apparait ainsi, de plus en plus, à mesure que les zones de recherches des spécialistes arrivent à se rejoindre et le méme ELLENBERG (H.) (1954) a pu parler d'une véritable " crise des caractéristiques $n$.

Sans doute, la notion de a caractéristique transgressive " permetelle de pallier, dans une certaine mesure, cette crise, puisqu'une même espèce peut être à la fois caractéristique de deux associations différentes. Mais que cache, en fait, cette notion, a priori antinomique?

On appelle caractéristique transgressive une espèce qui peut être caractéristique de deux associations différentes dans deux territoires différents. Or, si les deux associations en cause sont différentes, ce n'est pas qu'elles soient écologiquement différentes, à quelques nuances près s'entend, c'est qu'elles appartiennent à deux territoires floraux différents - il s'agit donc, pour nous, d'associations homologues - et si l'espèce caractéristique transgressive se retrouve dans chacune d'elles, c'est que, d'une part, son aire englobe les aires des deux associations susdites, et que, d'autre part - ceci est essentiel - elle trouve dans chacune d'elles les conditions de milieu étroitement définies qui lui conviennent.

GuinocheT (M.) (1938), à propos de Leontodon montanum, caractéristique à la fois de l'association Leontidetum montani, de Suisse, et de l'association à Berardia lanuginosa et Brassica repanda des Alpes maritimes, écrit que les divergences qui permettent d'individualiser ces deux groupements tiennent à \& l'existence, dans chacun des deux territoires considérés, d'espèces plus ou moins endémiques (ex: Berardia lanuginosa) n, mais que, "leurs conditions écologiques étant voisines, Leontodon montanum, par exemple, dont l'aire chevauche sur les deux régions envisagées, peut très bien exister dans les deux associations et être liée à chacune d'elles dans leur domaine respectif $n$.

Un autre exemple est celui de la petite Listera cordata, citée par Gaussen (H.) (1954), et au sujet de laquelle Reynaud-Beauverie (1936) a écrit qu' a il n'est donc pas étonnant qu'elle soit caractéristique du Picetum excelsac en Europe centrale, alors qu'elle se trouve dans les bois de Abies alba et mème de Pinus uncinata dans les Pyrénées, de Pseudotsuga à Vancouver, d'Abies balsamea, de Pinus strobus dans les tourbières du Massachusetts ».. car $\alpha$ peu de plantes peuvent se rencontrer sur de grandes aires, tout en restant liées à des conditions de milieu étroitement définies $\mathrm{n}$.

Ces caractéristiques transgressives sont donc, en fait, des caractéristiques stationnelles, d̀ aire vaste, mais à amplitude écologique 
étroite, au moins pour un certain facteur. Leur intérêt nous patrait considérable: elles permettent, en effet, de se rendre compte si des associations floristiquement différentes, parce que appartenant à des territoires floraux différents, sont " homologues " ou non sinon totalement, du moins en ce qui concerne un facteur écologique important ou un groupe de facteurs. Elles s'opposent, si l'on peut dire, aux caractéristiques stationnelles à aire étroite qui sont de véritables endémiques stationnelles, et dont l'intérêt est surtout d'ordre historico-géographique, car elles ne peuvent permettre de définir que des associations "locales $n$.

Ces caractéristiques stationnelles - celles à aire vaste comme celles à aire étroite - méritent done d'être définies non plus d'une façon purement floristique, mais par rapport aux conditions de milieu, toujours assez étroites, auxquelles elles se trouvent liées: réunies au sein des groupes écologiques, elles permettront de caractériser les associations dans une double perspective: floristique et écologique et de mettre en évidence, comme on le verra, les affinités de tous ordres - édaphiques, climatiques... - que présentent entre elles certaines associations.

\section{Le critère de fidélité est-il le seul à retenir?}

Allorge (P.) (1921-22) avait déjà montré que tous les groupements ne sont pas également individualisés par des caractéristiques, et il avait été amené à distinguer des groupements $\alpha$ hautement individualisés $\mathrm{x}$, c'est-à-dire comportant un nombre plus ou moins élevé de caractéristiques, qu'il avait appelées " associations principales $n$, et des groupements présentant, certes, une individualité réelle, mais qui doivent leur originalité non à des caractéristiques, absentes ou représentées seulement par quelques préférentes, mais à un nombre élevé d’espèces constantes. "Malgré la valeur prééminente indéniable, écrivait-il, des caractéristiques pour l'individualisation des groupements, leur absence dans certains d'entre eux n'empêche pas qu'on les considère comme des associations, lorsque la constance de leur cortège floristique est bien établie. BraunBlanguet (J.), Pallmann (H.) et Bach (R.) (1954) n'ont-ils pas caractérisé une variante du Picetum subalpinum vitidietosum, par la rareté des caractéristiques "charakterartenarme Variante o?

GuINochet (M.) (1955), de son côté, étudiant, par la méthode statistique, la signification de Viola cenisia, présente, mais de façon inégale au point de vue constance, dans deux groupements, conclut que sa fréquence étant plus élevée dans l'un d'eux, cette espèce doit alors en ètre considérée comme une des caractéristiques, et il ajoute - c'est nous qui soulignons - : "Peut-être pourra-t-on ou devrat-on d'ailleurs perfectionner la méthode en tenant compte de l'abondance, de la vitalité, etc. ". LEMÉE (G.) (1937), a ainsi appelé ca- 
ractéristiques différentielles d'associations, certaines caractéristiques d'alliance qui se trotwaient ètre plus particulièrement abondantes et vigoureuses dans une ou deux associations de cette alliance. BraunBlanguet (J.). Sissingli (G.), Vlieger (J.) (1939) ont fait de même, par exemple, avec Frica carnea, caractéristique de l'alliance de l'Erico-Pinion et classée conme telle dans les associations Carici-humilis Pinetum, Pinetum austro-alpinum, Rhododendro-hirsuti Mugetum et promue au rang des caractéristiques d'association dans l'Erico-Pinetum et l'Frico Mugetum, on elle manifeste une abondance et une vitalité toutes particulières.

Kuocı (R.) (1954), plus récenment, étudiant les associations forestières suisses comprises dans laire du Sapin blanc, n'a pas craint de considérer comme critère de différenciation de certains groupements la réunion en leur sein d'espèces relativement banales, la combinaison des espèces constantes - combinaison unique acquérant une importance particulière surtout en forêt. I1 rejoignait par là Duchaufour (Ph.) (1948), qui avait noté, à propos de certaines associations de landes: "Molinia coerulca existe aussi bien dans le Schoenctum que dans la lande humide; mais lorsqu'on parle d'une association à Molinia cosrulca et Calluna vulgaris, il ne vient à l'esprit de personne de la confondre avec le Schoenetum w.

On voit donc qu'au critère de fidélité pure, de valeur qualitative, se sont ajoutés, à mesure d'ailleurs que s'élargissait le champ d'étude des chercheurs, des critères de valeur quantitative comme la fréquence, l'abondance, la vitalité d'espèces de fidélité relativement faible. L'utilisation de ces critères, qui sont essentiels, à notre avis. surtout dans le domaine forestier où les espèces de haute fidélité n'existent pratiquement pas, nous parait spécialement efficace lorsque sont mis en évidence des groupes écologiques: la dominance et lexubérance, dans une association donnée, d’une caractéristiquc stationnelle autorisent, en effet, à affirmer que les conditions auxguelles cette espèce se trouve lice, connaissent, dans l'association en cause, un développement particulier, ce qui permet de définir au moins un aspect important de Pécologie de toute lassociation ellemême.

Ceci dit, il faut souligner que le critère abondance-dominance doit être utilisé avec une certaine circonspection surtout lorsgu'on envisage l'ótude des espèces arborescentes. C'est à juste titre, à notre avis, que Braun-Blanguet (J.) (1951), Gurnochet (M.) (1947) à propos des pessières du Jura, Mor.rnier (R.) (1954) en ce qui concerne les forêts de Pin d'Alep, de Pin sylvestre, de Hêtre, ont insisté sur le fait que la seule physionomie des groupements forestiers ne saurait suffire pour définir des associations, et que l'on risquait, en s'appuvant sur elle, de réunir, dans la même unité, des groupements différents ou de séparer des groupements voisins. 
A quoi tiennent, dans ce domaine, les difficultés?

1 faut noter, en premier lieu, que les espéces arborescentes présentent, en général, une certaine plasticité à l'égard de beaucoup de facteurs écologiques, ce qui ne peut, la plupart du temps, les faire considérer comme des caractéristiques stationnelles.

La deuxième difficulté - et elle est majeure - tient au fait que i'homme a exercé une puissante action sur les espèces arborescentes soit directement (plantations par exemple), soit indirectement, en modifiant le jeu de la libre concurrence vitale.

Or, cette action n'est pas toujours très facile à saisir et ce, à notre avis pour deux raisons principales.

Les espèces arborescentes sont sociales et - surtout les espèces résineuses - ont une grosse puissance édificatrice, en ce sens qu'elles modifient sensiblement le milieu originel qu'elles ont connu. Dans certains cas, par conséquent, si l'action humaine continue à jouer consciemment ou non - pour empécher le libre jeu de la concurrence vitale, on risque de voir se créer de véritables associations artificielles, relativement stables, et qui montreront de fortes ressemblances avec des associations naturelles ayant mêmes dominantes, S'observeront dans les deux cas, par exemple, des espèces herbacées liées à un type d'humus: la présence souvent signalée de Pirola secunda, de Goodyera repens dans les peuplements artificiels de Pin sylvestre ou de Listera cordata dans des plantations d'Epicéa (7) constitue quelques exemples de cette " convergence " (8).

La deuxième raison tient au frit qu'une espèce arborescente dominante artificielle dans une association, dans une certaine région, peut être naturellement dominante dans tune association d'une autre région, où la concurrence vitale n'est plus la même, ni le matériel foral. Par exemple, la dominance du Pin sylvestre dans les stations à Chène pubescent ou à Hêtre des Alpes du Sud, traduit une influence humaine; mais en est-il de même au coeur du district du Pin sylvestre, que nous aurons l'occasion d'étudier, d'où le Hêtre et le Chêne pubescent sont complètement et naturellement absents? Très vraisemblablement pas! Le Pin d'Alep, " dominant artificiel " dans les groupements dégradés de Chêne vert n'est-il pas considéré comme faisant " naturellement " partie de l'Oleolentiscetum? [Molinier (R.) (1954)]. Et ne peut-on pas envisager que les mélézeins du Briançonnais ont un certain caractère climacique? Le sapin. essence à écologie pourtant étroite et caractéristique selon

(7) En forêt de Mazan (Arclèche), ơ en forêt du Trévezel (Aigoual), par exemple.

(8) Richard (J.-L.) a, tout récemment, observé dans le Jura qu' \& en climat très froid, comme c'est le cas en forêt des Ecorces, il est imaginable qu'une association secondaire, imitant le Vaccinio-Piccion, puisse se substituer localement et temporairement au Fagion, à labri d'une vieille forêt secondaire très dense d'Epicéa s (1961, p. 144). 
Kuoch (R.) (1954), de l'Abietum albae, a, par contre, une dominance des plus suspectes dans les "sapinières " guitées des Alpes ou du Jura...

Ces quelques exemples montrent qu'il s'agit là d'un des problèmes les plus difficiles de la Phytosociologie forestière, surtout lorsque les investigations se situent à la jointure, si l'on peut dire, de groupements naturels et de groupements plus artificiels, mais présentant la même espèce arborescente.

Il n'était donc pas inutile de l'évoquer.

\section{I.2.1.3. - Associations affines et Systématique phytosociolo- gique.}

Il importe maintenant d'analyser comment la structure en groupes écologiques permet de rendre compte des affinités que présentent entre elles certaines associations, non sans avoir examiné les résultats donnés par la méthode de l'Ecole Zuricho-Montpelliéraine, dans ce même domaine des affinités.

Dans cette dernière méthode, comme on sait, les associations affines sont classies en Alliances, ces dernières en Ordres, les Ordres en Classes, tandis que sont définies des caractéristiques d'Alliances, d'Ordre..., espèces communes à plusieurs associations, mais liées précisément, plus ou moins étroitement, à l'unité compréhensive qu'elles caractérisent. II s'agit d'une classification hiérarchisée.

Ce système, dont le mérite est grand du point de vue même où nous nous plaçons, car il nous a facilité l'étude des groupes écologiques, a été critiqué par certains chercheurs dont Gams (H.) (1933), Goodall (D.) (1954), Ehrendorfer (F.) (1954), Duvigneaud (P.) (1946), et tout récemment NÈGre (R.) (1959), et Susmel (L.) (1959-60). S'il fait apparaitre, en effet, les affinités existant entre associations d'une même unité compréhensive, il risque de masquer pratiquement celles existant entre associations d'alliances, d'ordres différents. Les coupures aux différents niveaux peuvent isoler des associations relativement " voisines " et en rapprocher d'autres, au contraire, plus lointaines: dans une même alliance, par exemple, les associations extrêmes ou " marginales " présentent souvent entre elles moins d'affinités qu'elles n'en ont avec les associations marginales d'une alliance voisine.

C'est ainsi que le Seslerio-Fagetum anthericosum de Moor (alliance Fagion) présente avec l'Erico-Pinetum hyloconietosum de Braun-Blanguet (alliance Erico-Pinion) un coefficient de communauté de $19 \%$. Ce coefficient n'est que de $17 \%$ entre ce même Seslerion-Fagetum et l'Equiseto-Abietetum de Moor, appartenant pourtant tous deux à la même alliance. Par contre, cet Equiseto- 
Abietetum, méso-hygrophile, présente avec le Querco-Carpinetum aretosum d'EtTER (H.) (1943), Alliance Fraxino-Carpinion), un coefficient de communauté de $21,7 \%$.

Sans doute, comme l'a noté Pavillard (J.) (1935), le coefficient de communauté de JACCARD doit-il être interprété avec circonspection, car, " en matière d’affinités dans le domaine biologique, il ne suffit pas de compter, il faut peser. ' Or, les mêmes observations pourraient être faites si l'on mettait dans la balance certaines espèces d'importance sociologique spéciale: par exemple, le QuercoCarpinetum aretosum et le Querco-Carpinetum luzuletosum d'ETTER (H.), tous deux appartenant à l'alliance du Fraxino-Carpinion présentent respectivement sept et trois espèces caractéristiques d'alliance, ce qui correspond à un coefficient de communauté d'alliance. si l'on veut, de $42,8 \%$; mais le Querco-Carpinetum Insuletosum et le Querco-Betuletum - qui, lui, appartient à une autre alliance: le Quercion roboris-sessiliflorae - ont un coefficient de communauté de $77 \%$, et encore ne tenons-nous pas compte du coefficient de présence des caractéristiques d'alliance communes.

Il en est ainsi de beatucoup d'associations " marginales ". Il faut ajouter, enfin, comme l'a souligné EHRENDORFER (F.) (1954), que plus grandes et plus vastes sont les unités, plus profondes sont les lignes de séparation: c'est ainsi que, entre le Loiseleurio-Cetrarietum, l'Erico-Pinetum, le Piceetum montanum et le Genisto-Arctostaphyletum appartenant tous à l'ordre des Vaccinio-Piceetalia, les espèces caractéristiques communes sont trìs rares et les espèces que ces groupement ont en commun ont une importance très subordonnée.

Ces quelques exemples montrent à quel point il est difficile même lorsqu'on s'en tient au seul aspect statique de la végétation d'enserrer dans un réseau simple, la complexité du tapis végétal. I1 en est ainsi, d'ailleurs, à des degrés divers sans doute, pour tous les systèmes de classification.

Dans le cas particulier de la Phytosociologie, la difficulté tient à notre avis, à la nature complexe des groupements qui présentent. en effet, entre eux, des affinités, non pas dans une seule direction, mais dans plusieurs: d'une part, les diverses stations où sont effectués les relevés présentent une micro-hétérogénéité qui n’enlève rien à l'unité du groupement, mais qui se traduit par des micromosaiques floristiques, c'est-à-dire pratiquement par la coexistence de divers grotupes écologiques différents (9). Cette hétérogénéité

(9) Soulignée par Guinocher (M.) (1938) et de nombreux phytosociologues dont NÈGRe (R.) pour la zone aride du Maroc (1959), cette micro-hétérogénéité a fait lobjet - en ce qui concerne le sol - d'une étude statistique de Manil. (G.) et de ses collaborateurs dlans la région du plateau Ardennais (1953). 
peut ètre, en particulier, celle des divers horizons du sol: par exemple, un sol peu profond, reposant sur une roche-mère calcaire, mais décarbonaté en surface, et même désaturé, présentera des espèces acidiphiles qui s'observeront côte à côte, avec des espèces neutrophiles qui petvent trouver dans les horizons inférieurs les conditions auxquelles elles sont normalement liées.

Cette hétérogénéité n'intervient pas seule, d'autre part: l'amplitude écologique des groupes ou des espèces joue également un rôle essentiel: seule une analyse précise du milieu, comme l'examen du comportement des espèces dans leur optimum, doit permettre de faire la part entre la plus ou moins grande plasticité du matériel végétal et 1'hétérogénéité du milieu proprement dit.

Il est facile d'imaginer, en outre, que les affinités deviennent de plus en plus diverses à mesure qu'on s'éloigne d'un groupement typique pour s'atténuer ensuite, lorsque se trouve atteint un autre groupement typique, comme l'a notamment montré Duvigneaud (P.) (1946).

Dans le système de classification de 1'Ecole de Zurich-Montpellier, ces affinités - c'est-à-dire le caractère polydimensionnel de l'association - sont souvent masquées, car ne sont mises en pleine lumière, pour un groupement donné, que les caractéristiques des diverses unités compréhensives auxquelles appartient ledit groupement, alors que certaines espèces intéressantes, caractéristiques par exemple, d'autres unités. sont rejetées dans la foule plus ou moins anonyme des compagnes.

Il arrive toutefois qu'il soit fait état, dans certains tableaux, du caractère composite des associations: les groupes d'espèces mis alors en évidence nous paraissent présenter d'étroites analogies avee les groupes écologiques proprement dits, à ceci près que les premiers restent purement floristiques, alors que les seconds sont rattachés. corrélativement. à un facteur écologique décisif; c'est ainsi que Moor (M.) (1952) dans les tableaux de l'Equiseto-Abietetum, de 1'Abieti-Fagetum elymetosum, et du Fagetum silvaticae allietosum - toutes associations appartenant à l'alliance du Fagion - a mis en relief les espèces caractéristiques des Vaccinio-Piceetalia, BraunBlanquet (J.) (1949), dans le tableau d'association de l'EuphrasioPinctum — rattaché à l'alliance de l'Ononido-Pinion - a détaché, du groupe des compagnes, certaines espèces, caractéristiques du Ouercion pubescenti-sessiliflorae, et d'autres, caractéristiques de l'Erico-Pinion. De même, le tableau du Curvuletum elynetosum. dans l'étude du même auteur sur la végétation alpine des Alpes Françaises (1954), comprend à côté des compagnes, deux grands grounes d'espèces: les caractéristiques des Caricetalia curvulae (acidophiles) et les caractéristiques des Seslerietalia (basophiles). Ces quelques exemples montrent que rien n'interdit d'assouplir la 
classification hiérarchisée, tout en restant dans le domaine purement floristique, et que les analogies entre la méthode floristique et celle des groupes écologiques, sont formément très étroites (10).

Ils confirment les difficultés du classement des associations en raison de leur variabilité; ils montrent, enfin, l'intérêt qu'il y a à utiliser conjointement l'analyse floristique et l'analyse écologique et l'inconvénient que présente - comme l'a souligné fortement Nì̀re (R.) (1959) — d'utiliser le seul critère floristique, quelle que soit l'ampleur de l'unité étudiée.

C'est pourquoi nous n'avons pas cherché à nous enfermer dans un système rigide de classification; nous pensons que la méthode d'analyse des groupements adoptéc dans ce travail, c'est-à-dire la mise en évidence, en leur sein, de groupes d'espèces caractéristiques stationnelles, doit permettre d'éclairer suffisamment les rapports dordre édaphique, climatique, etc. qui les unissent.

\section{I.2.1.4. - Associations végétales et milieu, Examen critique.}

Il reste enfin à s'interroger sur les difficultés que peut soulever l'application d'une telle méthode, dans laquelle, il faut le rappeler, l'analyse écologique ne précède pas l'analyse floristique. mais lui reste parallèle.

Les critiques formulées à l'encontre des méthodes écologiques, en général, peuvent se résumer ainsi.

Le milieu physico-chimique étant extrêmement complexe, et comprenant un grand nombre de facteurs où peuvent apparaitre des interréactions compensatrices on cumulatives, il est très difficile, voire impossible, de le cerner, et il apparait alors comme une notion assez abstraite.

En second lieu, ce milieu n'explique pas tout: le végétal agit sur lui ; et surtout, des facteurs biotiques extrinsèques jouent également des rôles multiples, dont les plus importants ne sont pas forcément les plus apparents (antibiotisme de sécrétion).

L'histoire de la flore enfin, les moyens de dissémination et les aptitudes variées à la compétition des diverses espèces interviennent aussi - et à des degrés divers - pour expliquer la composition qualitative et quantitative des associations.

Que le milieu soit extraordinairement complexe, la chose ne paraît pas douteuse. La plupart des chercheurs - dont Prenant (M.) (1934) a résumé certaines conclusions - qu'ils se soient occupés d'écologie animale ou végétale, l'ont souligné maintes fois. Dans le domaine de la Phytosociologie, Gurnocher (M.) (1955) y a insisté

(10) Il suffit, pour s'en convaincre, de se reporter, par exemple, au travail de Braun-Blanouet (J.) et de Jrnny (H.): * Vegetationsentwicklung und Bodenbildung in der Alpinenstufe der Zentralalpen (Klimaxgebiet des Caricion curvulae) > (1926). 
tout particulièrement. Sans doute, l'utilisation des groupes écologiques reste-t-elle délicate, et mème si l'on donne à la corrélation qui les lie au milieu une valeur relative et non absolue. En effet, certains facteurs présentent entre eux de véritables corrélations internes: l'exemple le plus connu est, en Pédologie, celui des sols peu évolués reposant sur une roche-mère calcaire, qui sont, en général. chatuds et secs : on risque, comme l'a noté Duchaufour (Ph.) (1952), d'appeler calcicoles des espèces n'offrant aucune exigence particulière vis-à-vis des carbonates ou de l'ion calcium, mais qui trouvent sur de tels sols, les conditions de chaleur et de sécheresse qui leur conviennent.

En conséquence, nous n'avons pas cherché à faire une analyse fine, qui relèverait d'ailleurs de la physiologie écologique expérimentale. Nous nous sommes contenté - il s'agit là, si l'on veut, d'une première approximation - de repérer les facteurs simples (11) ou, mieux, les complexes de facteurs dont Pobservation montre qu'ils sont corrélativencut et naturellement liés au développement optimum de certaines espèces. Les relations mises ainsi en évidence ne sont pas forcément causales, mais doivent être considérées comme de simples corrélations, dont l'importance, à la fois théorique et pratique, n'en est pas moins considérable; elles sont, d'autre part. celles observées dans la nature, en ce sens que le milieu naturel peut parfois se montrer plus éloigné du milieı optimum qu'un milien artificiel expérimental (12).

En ce qui concerne le deuxième groupe d'objections, à savoir que le milieu physico-chimique n'explique pas tout, nous noterons ce qui stuit.

En attachant une importance particulière à la pédologie et en envisageant, lors de l'étude d'un sol, non point tel ou tel facteur. mais un complexe de facteurs liés entre enx, nous intégrons, en fait, heaucoup de biologie proprement dite.

$\mathrm{E}_{\mathrm{n}}$ second liet. le fait que le végétal agisse sur le milieu et réciproquement n'enlève rien à l'intérêt de la méthode des groupes écologiques. Bien au contraire: l'étude des associations nous parait grandement facilitée si l'analyse floristique et l'analyse écologique sont menées de front.

Le rôle jouté par certains facteurs biotiques — dont 1'homme au premier chef - est, sans doute, loin d'être négligeable et les fores-

(11) De tels facteurs sont ceux étudiés par les phytosociologues lorsqu'ils déterminent récologie globale des associations: * en matière d'écologie, la seule chose à laquelle nous puissions prétendre est la recherche du ou des facteurs prédominants, đécisifs, pour chaque association, qu'ils soient la cause initiale ou la résultante du complexe écologique correspondant à chacune d'elles ? [GuiNochet (M.) (1938)].

(12) Cf. PREnant (M.) (1934, p. 48). 
tiers - GUIN1ER (Ph.) (1950), ROL (R.) (1945), notamment ont insisté à maintes reprises à son sujet. On a souvent rapproché, en effet, les termes: écologie et physionomie, et montré qu'à une certaine échelle tout au moins, la physionomie, c'est-à-dire pratiquement la dominance de telle ou telle espèce, était incapable de rendre compte des caractères particuliers des groupements, de leur comportement, de leur évolution.. Cette critique, - qui vise surtout les dominances d'origine biotique, et c'est la raison pour laquelle nous en reparlons ici - est parfaitement fondée dans certains cas, mais nous croyons l'avoir montré, pas dans tous. L'essentiel est donc de faire la part entre ce qui relève d'une influence humaine et ce qui n'en dépend pas, ou n'en dépend pas directement.

D'autre part, la méthode dite écologique est loin de ne retenir comme critère que la dominance; nous ne pensons done pas qu'elle aggrave ou qu'elle esquive la difficulté. Le plus souvent, au contraire, l'étude du comportement d'une espèce - considérée isolément - à l'égard du seul milieu physico-chimique, doit faciliter celle de son comportement lorsqu'intervient l'influence humaine.

Quant aux phénomènes d'antibiotisme étudiés maintenant par un grand nombre de chercheurs et mis en lumière notamment par Gưot (L.) (1951-1954) et ses collaborateurs dans les pelouses à Brachypodium pinnatum et Bromus erectus du Nord de la France, ils semblent, sans doute, expliquer à eux seuls, l'absence ou la rareté de certaines espèces dans un groupement végétal déterminé (13).

Cet antibiotisme n'est, au fond, qu'une forme de la concurrence vitale, terme souvent utilisé, mais d'une façon très vague, par les forestiers à propos de certaines espèces arborescentes, qui paraissent se localiser dans des stations où une telle concurrence est faible ou nulle et dont le Pin à crochets est un des meilleurs exemples.

Mais, même dans le cas où cet antibiotisme agit de façon positive (14), c'est-à-dire facilite directement la germination de telle ou telle espèce, il ne met nullement en cause les corrélations qui lient les espèces à certaines stations. S'il est très probable, en effet, que de nombreuses espèces doivent leur élimination de stations leur convenant à la sécrétion d'autres espèces - que l'on doit alors, légitimement, considérer comme mieux adaptées - il n'en reste pas moins que la présence, avec une vitalité normale, d'une espèce au sein d'un groupement, implique qu'elle se trouve en équilibre - au moins provisoire - avec les conditions écologiques (même prises

(13) On trouvera dans Schaeffer et Morenu (1958-59), une liste de travaux ayant plus spécialement trait à l'antibiotisme en forèt.

(14) Il faut noter que l'antibiotisme agit le plus souvent de façon positive, en dernier ressort: en éliminant certaines espèces d'une station donnée, il en favorise d'autres, en effet, dn même coup, qui voient diminuer la concurrence vitale. 
dans le sens étroit d'environnement physico-chimique) de la station et même si cette dernière n'est pas idéalement la meilleure pour elle.

Dans ce domaine encore, nous pensons que l'étude de l'écologie des espèces - ce terme fút-il pris dans son sens le plus étroit ne peut que contribuer à éclairer les relations complexes, d'ordre spécifiquement biologique, qui lient les diverses espèces d'une même association.

Quant à l'aptitude à la compétition et aux moyens de dissémination des espèces - notions qui se rattachent aux précédentes d'ailleurs - ils seraient négligés pour certaines d'entre elles, évidemment, si l'on se référait au seul test de l'abondance-dominance, ce qui n'est pas. D'autre part, pour une même espèce, l'étude de son comportement - variable suivant les stations - doit précisément permettre de déceler, toujours de façon corrélative, son amplitude et son optimum écologique, et de faire ainsi la part, au sein d'un groupement, de ce qui revient, pour cette espéce, aux facteurs intrinsèques définis ci-dessus et à ceux du milieu proprement dit.

Nous pensons, enfin, que l'existence de régions florales qui s'expliquent par l'histoire des vicissitudes de la végétation, ne soulève pas de difficultés particulières, puisque l'on peut toujours mettre en évidence des différentielles géographiques, liées plus ou moins à certaines stations. En tous cas, dans ce domaine encore - où se trouve posé le problème même des aires des espèces - la méthode des groupes écologiques non seulement n'aggrave pas les difficultés, mais risque d'apporter des explications, puisqu'elle confronte, à chaque pas, floristique et écologie.

En résumé, ne cherchant pas à définir d'abord les milieux ou d'abord les associations, nous avons adopté une position moyenne en essayant de mener parallelement l'analyse floristique et lanalyse écologique. Quelles que soient en effet, les formes d'action de la concurrence vitale, la végétation nous parait présenter les liens positifs, d'abord avec le milieu avec lequel elle est obligatoirement en équilibre (15); mème lorsqu'tune part importante du milieu comme 1'humus, par exemple, doit la plus grande partie de son origine à certaines espèces: arbres, éricacées, graminées... il n'est pas d'une spécificité telle que ses saprophytes eux-mêmes apparaissent comme les satellites obligatoires de telle ou telle espèce édificatrice. Le parasitisme lui-mème est, la plupart du temps, lié indirectement au milieu.

Mais d'autre part, ce dernier est d'une telle complexité qu'il est difficile de laborder de front, surtout de façon analytique. Il est

(15) \& L'association traduit, en somme, un état d'équilibre entre la végétation et les conditions du milieu. Elle représente une réalité, mouvante sans doute, car elle varie dans le temps et dans l'espace, dans la mesure où le milieu varie lui-même s [Molinier (R.) (1935)]. 
donc intéressant d'envisager des complexes de facteurs: dans le monde forestier tout particulièrement, la pédologie offre ainsi des ressources extrìmement fécondes. Mais les obstacles s'accumulent vite dès que le climat intervient - le climat du sol y compris, évidemment, dont s'occupe la pédologie - : ce dernier est un facteur fluctuant, où l'intégration du temps exige certains délais, et que les données usuelles fournies par la météorologie n'arrivent pas à définir avec assez de précision. Dans certains cas, l'utilisation de termes phytogéographiques, bien que vagues, s'avère alors indispensable, et ces derniers ne prennent toute leur valeur qu'accolés aux noms de certaines espèces.

Ainsi la floristique doit épauler l'écologie, et réciproquement.

\subsection{2. - Groupes écologiques.}

L'étude des groupes écologiques a été conduite en mème temps que celle des associations dont ils relèvent, mais également par l'examen de certains transects : passages verticaux, ou en long, passages latéraux.

Dans toute la mesure du possible, nous avons suivi des profils le long desquels un seul facteur variait: par exemple, sur un sol carbonaté, à une altitude donnée, nous avons comparé la flore d'un versant sud et celle d'un versant nord; sur une mème roche-mère et sur un même versant, nous avons suivi la chaine des sols et celle de la végétation correspondante, etc.

Nous avons ainsi obtenu des résultats permettant de définir des corrélations de valeur relative, les uns non chiffrés, les autres chiffrés et résultant d'analyses mésoclimatiques, microclimatiques et pédologiques.

Nous avons enfin confronté ces résultats avec ceux obtenus par d'autres chercheurs, qu'ils se soient préoccupés de la synécologie des groupements forestiers, ou qu'ils se soient plus particulièrement penchés sur le comportement de telle ou telle espèce (16).

\subsubsection{1. - Facteurs analysés.}

L'observation nous a montré que la flore sylvatique de notre dition était corrélativement liée aux facteurs écologiques ci-après: climat général ; température (influencée, toutes choses égales d'ailleurs, par l'altitude); économie de l'eau et aération du sol (la première dépendant dans une large mesure de l'exposition); état et

(16) On trouvera dans le numéro d'août 1954 de la revue \& Allgemeine Forst und Jagdzeitung \& 1'analyse des principaux groupes écologiques forestiers de l'Allemagne du Sud tels qu'ils résultent des études de FABER, GArsherg, Hauff, Schlenker, Kreh, Oberdorfer, Schönhar.

Il faut citer aussi les travaux de Z Z.ATNIK (A.) (1957). 
nature du complexe absorbant du sol et composition et structure de Phumus; ces divers facteurs présentant entre eux, d'ailleurs, certaines relations de dépendance: par exemple économie de l'eau et humus,

En ce qui concerne le climat général, nous n'avons pas craint, malgré le sens vague qu'on peut leur reprocher de posséder, d'utiliser certains termes phytogéographiques. Comme l'a noté JovET (P.). (1949), ces qualificatifs ne précisent pas obligatoirement le centre de l'origine des espèces qu'ils définissent, mais ils a donnent une image suffisamment approchée de leur amplitude écologique, ... relativement aux conditions climatiques $n$.

Ils ont été utilisés par certains phytosociologues, dont MeuseL (H.) (1940), et ils nous paraissent intéressants, lorsque s'observent, côte à còte, des espèces appartenant à des éléments floristiques différents: c'est ainsi que, en Haute-Maurienne, les associations de Pin sylvestre ou de Pin à crochets présentent à la fois des espèces dont l'optimum de développement se situe dans la région méditerranéo-montagnarde, et d'autres dont l'optimum se situe dans le sud de l'Europe orientale ou dans les régions steppiques voisines de la Caspienne (espèces sarmatiques de Braun-Branquet).

De même, parmi les espèces mésophiles médio-européennes du cortège des forèts feuillues, certaines ne se rencontrent absolument pas dans notre dition; un contingent, par contre, pénètre dans le monde intra-alpin, au delà de Saint-Julien-de-Maurienne: dans ce groupe, certaines especes ne dépassent presque pas la zone subbriançonnaise, tandis que d'autres s'observent au cour de la HauteMaurienne. Nous avons donc jugé utile de distinguer les unes des autres.

A l'altitude est liée la notion d'étage, ce dernier devant être toujours envisagé, ainsi que l'a montré EMBerger (L.) (1954), d'une façon relative et non absolue, c'est-à-dire dans le contexte d'un climat régional donné. En ce qui concerne cette étude, par conséquent, les noms de divers étages s'entendent accolés au terme générique d' $\alpha$ intra-alpin $n$ qui constitue un type de climat de montagne, froid et sec, et dont les caractéristiques seront analysées plus loin. L'action de laltitude est évidemment fort complexe: elle porte essentiellement sur l'économie de l'eau et la température; l'économie de l'eau fait l'objet de commentaires à part; disons simplement que si les précipitations augmentent avec l'altitude — tout au moins pour les étages sylvatiques qui nous intéressent - l'évaporation, qui apparait comme un phénomène opposé, varie dans le même sens.

En ce qui concerne la température, qui diminue avec l'altitude, nous l'avons, en quelque sorte, intégrée dans les termes qui désignent les étages, cetux-ci étant entendus dans le sens que leur donnent traditionnellement les biogéographes [OzENDA (P.) (1964)]. 
A ces étages ne sont pas liées, d'une façon absolue, des limites altitudinales parce qu'elles varient d'une région à une autre selon le climat général et d'autres facteurs: c'est ainsi que, dans notre dition, typiquement intra-alpine, l'étage montagnard qui est, à l'Envers celui des Sapinières et, à l'Adret, celui des Pineraies de Pin sylvestre, monte plus haut que dans les Préalpes (1 $800 \mathrm{~m}$ contre $1600 \mathrm{~m})$; il en est de même de l'étage subalpin qui atteint $2300 \mathrm{~m}$ environ et où l'on rencontre, dans sa partie inférieure, des pessières, et, dans sa partie supérieure, des pineraies de Pin Cembro (sur sol calcimorphe, des pineraies de Pin à crochets).

Nous avons dû nous contenter de ces termes, sans doute assez vagues, qui impliquent une certaine adaptation des espèces ainsi qualifićes aux conditions thermiques globales qui règnent dans ces divers étages, à chaque exposition, car nous ne possédons malheureusement pas assez de données de températures, et suffisamment étalées dans le temps. Les bioclimatologistes, qui auront évidemment à tenir compte au plus haut point des données de la phytosociologie, devront tout particulièrement s'attacher à serrer de plus près ce problème, en disposant notamment leurs appareils le long de certains transects judicieusement choisis.

Cependant, les mesures mésoclimatiques et microclimatiques (17) que nous avons faites, permettent d'apporter quelques chiffres; de même - et surtout - les données de certains postes météorologiques qui étaient, précisément, sinon alignés sur un même versant, du moins point trop éloignés d'un mème profil, nous ont permis de rattacher aux principaux étages, quelques caractéristiques thermiques.

Le rôle considérable joué par léconomie de leau - du sol et de l'atmosphère - dans la vie des plantes et de leurs groupements n'a pas à être rappelé. Mais il s'agit d'un phénomène complexe, difficile à étudier. Des bioclimatologistes comme Earberger (L.) (19321942-1953-1954), ThorNTHWATte (C.-W.) (1948), des agronomes comme TuRc (L.) (1953), Henin (S.) (1936), des pédologues, des phytosociologues, des hydrauliciens même comme CoUTAGNE (1942), ont cherché, non sans résultats importants, à le cerner et à exprimer ses principales caractéristiques par des valeurs simples, couvrant des facteurs très complexes.

Nous avons pu, pour l'eau du sol, utiliser dans un assez grand nombre d'associations et pour une saison de végétation, un appareil à potentiel capillaire; nous avons complété ses données en calculant pour certains groupements - mais de façon plus fragmentaire - le pouvoir évaporant relatif de l'atmosphère ( $\mathrm{cf}$. ci-après le chapitre sur les méthodes d'analyse climatique). Les résultats

(17) Ces termes seront définis au chapitre I.4, lors de l'analyse des méthodes d'étude des climats. 
obtenus sont venus corroborer ce que montre la simple observation et qui permet de distinguer de façon relative, nous insistons sur ce dernier terme:

- des espèces méso-hygrophiles qui exigent une eau de rétention disponible, suffisamment abondante. Ces espèces, appartenant pour la plupart à la mégaphorbiaie, selon le terme expressif de J. BRIQUET, s'observent essentiellement sur versant Nord, dans les couloirs d'avalanches ou les combes à neige, ou à proximité des torrents qui entretiennent en permanence une atmosphère humide;

- des espèces mésophiles, exigeant un sol frais, c'est-à-dire dont les réserves 'eau restent toujours au-dessus du point de flétrissement, espèces de versants nord, où l'insolation est peu vive et le pouvoir évaporant de l'atmosphère atténué;

- des espéces xérophiles, qui sont adaptées à une dessiccation accentuée du sol et qui s'observent sur les versants sud et ouest, où l'insolation est plus vive et le pouvoir évaporant de l'atmosphère élevé.

Il faut noter ici qu'il existe d'ailleurs entre l'économie de l'eau du sol et la structure de l'humus, dont il va être question, une correspondance étroite, bien mise en évidence par Duchaufour ( $\mathrm{Ph}$.) (1960).

De létat du complexe absorbant (richesse en bases) et de la composition et de la structurc de lhumus, dépendent très étroitement la nutrition minérale et la nutrition azotée des végétaux. Comme il existe une relation étroite, d'une part, entre l'état de ce complexe et la structure de l'humus, et, d'autre part, entre cette structure et l'économie de l'eau du sol, on conçoit que l'humus ait pu être considéré par Duchaufour (Ph.) (1960), notamment comme intégrant tout un ensemble des propriétés du sol.

Cette importance des bases, sinon de l'humus, a toujours été reconnue, et plus particulièrement par les phytosociologues qui se sont occupés comme Braun-Blanguet (J.) (1948) et Guinochet (M.) (1938), de l'étage alpin, puisque le calcium, si l'on peut dire, leur a servi à asseoir les grandes divisions des groupements qu'ils avaient à analyser.

D'une façon très générale, tous les groupements que nous avons étudiés reposaient sur des sols aérés, drainés, un peu hydromorphes parfois, mais jamais formés en conditions permanentes d'anaérobiose (tourbes) ou semi-permanentes (anmoors). Nous avons donc pu distinguer des espẻces assez étroitement adaptées aux divers types d'humus formés en conditions d'aérobiose, définis par DuCHAUFOUR (Ph.) (1957-1960), soit :

1) des espèces calciphiles, correspondant à des humus calciques ou eutrophes, c'est-à-dire présentant une grande richesse en bases et caractérisant les sols calcimorphes. Dans ces sols à mull-mo- 
der calcique, le complexe absorbant est voisin de la saturation, toujours riche en calcium échangeable; l'humification est rapide (en condition d'aération normale), le rapport $\mathrm{C} / \mathrm{N}$ bas (autour de 10), sauf pour les moder calciques; le $\mathrm{pH}$ souvent supérieur à 7 .

2) les espèces subacidiphiles liées soit à un mull type aéré, soit, le plus souvent à un mull faisant le passage vers un moder (mullmoder). Le complexe absorbant de ces humus est partiellement désaturé, avec un rapport $\mathrm{C} / \mathrm{N}$ compris entre 10 et 20 ; la minéralisation est assez rapide et le $\mathrm{pH}$ se situe aux environs de 5 ou de 6 . En forêt résineuse, l'horizon $A_{1}$ est toujours surmonté d'un petit horizon $\mathrm{A}_{0}$ à $\mathrm{C} / \mathrm{N}$ élevé et riche en matière organique.

3) des espèces acidiphiles, qui s'observent sur des sols et des humus oligotrophes, appauvris en bases; l'humus est du type moder: le complexe saturant est encore plus désaturé (taux de 10 à $20 \%$ ), l'humification est plus lente que dans le mull, le rapport $\mathrm{C} / \mathrm{N}$ peu différent par contre, la minéralisation également; mais la cohésion argile-humus est faible, et la structure qui en résulte est du type particulaire. Le $\mathrm{pH}$ est, en général, inférieur à 5 .

4) des especes caracteristiques d'hunus brut (mor): l'humus brut se caractérise par un taux de saturation du complexe absorbant très faible, une minéralisation très lente tout à fait caractéristique, un $\mathrm{pH}$ inférieur à 5 ; le mélange matière organique, matière minérale à peu près nul, le rapport $\mathrm{C} / \mathrm{N}$ étant toujours supérieur à 20 dans l'horizon $\mathrm{A}_{0}$.

\subsubsection{2. - Groupes écologiques résultant de la combinaison des facteurs analysés.}

L'interférence des facteurs analysés - et bien qu'il s'agisse de complexes de facteurs, plutôt que de facteurs simples - donne d'assez nombreuses combinaisons.

Si l'on s'en tient à la seule économie de l'eau et aux types d'humus, on peut dresser le tableau schématique ci-après, abstraction faite des espèces caractéristiques des anmoors et des tourbes, dont nous avons dit qu'elles n'étaient pas présentes dans les forêts de notre dition.

En adoptant un troisième axe de référence (altitudes), on obtiendrait, toujours de manière très schématique, un nombre de groupes multiplié par celui des étages retenus; en se référant au climat général, aux aires, le nombre de groupes serait, encore, à multiplier par celui de ces " paramètres ». 
On risque donc d'arriver à des chiffres considérables, et à un tableau synoptique fort complexe.

\begin{tabular}{|c|c|c|c|}
\hline \multirow{4}{*}{ 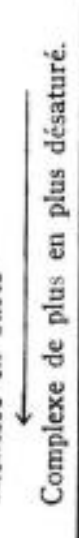 } & $\begin{array}{l}\text { Milieu sec } \\
\text { (Espéces } \\
\text { xérophiles) }\end{array}$ & $\begin{array}{l}\text { Milieu moyennement } \\
\text { humide (Espèces mésoxé- } \\
\text { rophiles à mésophiles) }\end{array}$ & $\begin{array}{l}\text { Milieu humide } \\
\text { (Espèces méso- } \\
\text { hygrophiles) }\end{array}$ \\
\hline & $\begin{array}{l}\text { xéro-calciphiles } \\
\text { (moder et mull } \\
\text { calciques) }\end{array}$ & $\begin{array}{l}\text { Méso-caiciphiles - } \\
\text { (mull calcique) }\end{array}$ & $\begin{array}{l}\text { Méso-hygro-calciphiles } \\
\text { (hydromull calcique) }\end{array}$ \\
\hline & $\begin{array}{l}\text { xéro-subacidiphiles } \\
\text { de mull } \\
\text { (sand-mull) }\end{array}$ & $\begin{array}{l}\text { Mésophiles de mull } \\
\text { forestier - } \\
\text { (mull mésotrophe) }\end{array}$ & $\begin{array}{l}\text { Méso-hygrophiles } \\
\text { d'hydromull } \\
\text { (hydromull mésotrophe) }\end{array}$ \\
\hline & $\begin{array}{l}\text { xéro-acidiphiles } \\
\text { de moder }\end{array}$ & $\begin{array}{l}\text { Mésophiles de moder } \\
\text { forestier }\end{array}$ & $\begin{array}{l}\text { Méso-hygrophiles } \\
\text { d'hydro-moder }\end{array}$ \\
\hline & $\begin{array}{l}\text { xérophiles de mor } \\
\text { (xéromor) }\end{array}$ & Mésophiles de mor & $\begin{array}{l}\text { Méso-hygrophiles } \\
\text { d'hydro-mor }\end{array}$ \\
\hline
\end{tabular}

(1) Les termes d'hydromuil et d'hydromor adoptés par Duchsufour ( $\mathrm{Ph}$.) (1960-1965) sont empruntés à LAFond.

En fait, la complexité est moins grande qu'il ne paraît au premier abord et pour les raisons suivantes.

En premier lieu, toutes les combinaisons écologiques ne s'observent pas en forêt, dans notre dition: la vigueur des pentes, en particulier, fait que toutes les stations sont drainées, à l'exception de quelques micro-stations peut-être, de très faible surface et dont l'importance pratique est absolument négligeable. Ne s'observent donc pas de moders hydromorphes ni d'hydromors, et a fortiori, d'anmoors ou de tourbes, comme il a été déjà dit.

D'autre part, il est relativement rare qu'une espèce se trouve être spécialisée à l'égard de chacun des grands facteurs analysés, et ceci est, dans une certaine mesure, regrettable, car il s'agirait là d'une véritable caractéristique stationnelle. Vaccinium Myrtillus, par exemple, mésophile d'humus brut, possède une vaste amplitude quant à l'altitude et a une aire très vaste; Erica carnea, qui peut être considérée, à bien des égards comme une espèce très spécialisée (aire, type d'humus) s'étend cependant en altitude - du moins en Haute-Maurienne - de l'étage montagnard à l'étage subalpin; Poa Chaixii et Milum effusum, localisés sur les sols à mull forestier et mésophiles, ont une aire vaste et s'observent à des altitudes 
diverses. Les espèces présentent donc presque toujours une certaine amplitude, au moins dans une direction. Ceci est surtout vrai pour les espèces xérophiles qui paraissent, dans l'ensemble, indifférentes au chimisme du sol. Cette observation rejoint celle de Duchaufour $(\mathrm{Ph}$.$) qui, dans un récent article (1960), notait que, « lorsqu'un$ facteur physique important (eau, aération) atteint une valeur extrême - soit par son excès, soit par son insuffisance - il tend à l'emporter sur les facteurs chimiques, qui, en comparaison, n'exercent qu'une influence secondaire sur la végétation $n$. Et de citer l'exemple de Cladonia sylvatica rencontré sur milieu sec, pourvu qu'il ne soit pas calcaire, c'est-à-dire poussant aussi bien sur xéromoder que sur xéromor ou même sur mull sableux (xéromull) et celui de Molinia coerulea, " espèce acidiphile exigeante en eau, mais supportant des phases de dessiccation marquée, et qui se rencontre aussi bien sur hydromoder que sur anmoor et sur tourbe $n$.

Tout ceci explique que, dans le tableau général des groupes écologiques, et pour ne pas l'alourdir, nous n'ayons pas mis en évidence tous les facteurs, mais d'abord: l'économie de l'eau et la nature de l'humus, ce qui permet de délimiter des complexes de groupes, en nombre limité, à l'intérieur desquels peuvent être mis en évidence, si besoin est, les groupes etıx-mêmes - en fonction de l'altitude, tout particulièrement.

Un tableau relativement simplifié peut donc être élaboré puisque, lors de l'étude des associations, il sera facile de faire les distinctions qui s'imposent: par exemple, parmi les mésohygrophiles de mull forestier (hydromtll forestier), certaines espèces ont une aire géographique plus étroite que les autres: Almus viridis, Achillea macrophylla. Aconitum paniculatum, ne s'observent, ni daus les Pyrénées, ni dans le Massif Central; leur aire s'étend sur l'Apennin, les Carpathes et les Balkans, au delà des Alpes (18). Cette spécialisation géographique doit, ou en faire des caractéristiques sensu stricto d'une association relativement étroite, ou, mieux, des différentielles géographiques d'une unité plus vaste, correspondant, si l'on veut, à l' "Adenostylion ".

Par ailleurs, certaines espèces qui ont, on l'a vu, à l'égard d'un facteur déterminé une amplitude étroite, mais à l'égard d'un autre facteur une amplitude plus vaste, figureront dans notre tableau, au besoin dans plusieurs cases: Deschampsia flexuosa par exemple, ne parait pas avoir un optimum nettement localisé en ce qui concerne l'économie de l'eau; elle est, par contre, étroitement liée aux sols à moder: cette spécialisation a une valeur incontestable, et l'espèce doit figurer dans notre tableau, car elle peut être - et doit être - considérée comme une différentielle de grande valeur.

(18) Achillea macrophylla est mème localisće dans les seuls Apennins, en dehors des Alpes. 
A l'inverse, Arctostaphylos Uva-ursi est relativement indifférente au type d'humus, à son $\mathrm{pH}$ etc. Elle se localise, par contre, dans les stations xériques. Comme il s'agit, en outre, d'une orophile (montagnarde à subalpine) à aire circumboréale, elle mérite également de prendre place dans un - ou plusieurs - groupes écologiques (19).

I1 nous parait nécessaire, en effet - et en cela nous partageons tout à fait le point de vue de Duvigneaud (P.) (1946) de réduire le nombre souvent considérable des a compagnes ".

Parmi ces compagnes (20), il en est peu, en définitive, qui soient dénuées d'intérêt, nous l'avons dit à propos de la systématique des associations. Certaines compagnes, en effet, souvent réduites à ce rang dans le système de l'Ecole de Zurich-Montpellier, parce qu'elles ne caractérisent aucune des unités auxquelles appartient l'association où elles figurent, doivent permettre, cependant, de mettre en relief des affinités très intéressantes entre groupements paraissant éloignés les uns des autres, si l'on se réfère à l'ordre ou à la classe dont ils relèvent. C'est ainsi que des espèces déjà citées, comme $P o q$ Chaixii ou Milium effusum, dont l'aire est vaste et qui s'observent dans de nombreuses associations forestières, sont liées à des sols à mull ou mull-moder forestier: leur relative abondance dans le faciès à Calamagrostis villosa du Rhodoreto-Vaccinietum a donc une signification pédologique des plus précieuses.

D'une façon générale, les espèces dont il nous a paru difficile de préciser loptinum, mais qui ont, cependant, une signification écologique assez étroite au moins quant à un facteur important du milieu, figurent dans nos tableat1x, dans plusieurs cases, mais entre parcnthèses.

Notre tableau va donc permettre, dans une certaine mesure, de rendre concret le degrés de spécialisation des espèces, eu égard, tout at moins, aux facteurs retenus. Une espèce liée à la fois à un type de climat, à un étage, à une certaine valeur de l'économie de l'eau et à un type de sol et d'humus, sera une authentique caractéristique stationnelle, et de degré 4 , si l'on osait chiffrer une telle spécialisation: une autre, liée à trois facteurs seulement ou à deux, sera moins caractéristique: elle pourrait être qualifiée de différentielle, et ainsi de suite... Mais, l'on conçoit que l'intérêt d'une différentielle soit atssi considérable — sinon plus — que celui d'une caractéristique authentique, marquée. par ce fait même, par un certain endemisme. Cette différentielle permettra, en effet, de comparer des associations homologues, c'est-d̀-dire à lécologie semblable, alors que

(19) Au lieu de faire figurer ces espèces dans plusieurs groupes, nous les avons réunies, en fait, dans un groupe $d^{\prime}$ \& indifférentes s, plus compréhensif que les autres, par conséquent.

(20) On doit considérer comme telles, les ubiquistes et, provisoirement, les espèces dont l'ćcologie est trop mal connue. 
leur matériel floral peut différer, par ailleurs et pour des raisons historico-géographiques (21). Une caractéristique, sensu-stricto, n'aurait, par contre, qu'une valeur locale et, à la limite, une caractéristique à endémisme élevé, da aire très étroite, finirait par ne plus présenter un gros intérêt, tout au moins dans le domaine de l'écologie comparée.

Nous voudrions, pour clore cette analyse générale, insister sur plusieurs autres points qui nous paraissent essentiels.

1) il ne faudrait pas voir, dans les limites que nous avons tracées, des frontières intangibles: d'abord et surtout, parce que nous connaissons encore mal l'écologie des espèces (seule, une analyse statistique, s'appuyant sur de très nombreuses mesures, permettra de serrer de plus près un tel problème); ensuite, parce que, quoi qu'on fasse, la nature se laissera toujours difficilement enfermer dans les conceptions systématiques de l'esprit humain.

2) il est probable, en second lieu, que le comportement de certaines espèces, tel qu'il nous est apparu, soit en contradiction plus ou moins nette avec celui que ces mêmes espèces présentent dans une autre région, ồ la concurrence vitale, en particulier, est différente: il nous semble que, dans ce domaine, la méthode dite des groupes écologiques, doit alors faciliter, par sa nature même, l'étude des micromorphes, des écotypes ou l'analyse de la compensation de certains facteurs écologiques, tous éléments qui doivent permettre. précisément. d'expliquer les " anomalies " du comportement des espèces en cause.

3) il faut noter, enfin, que la mise en évidence des groupes écologiques et du degré de spécialisation des espèces - même en l'absence de données chiffrées — doit rendre plus aisée l'étude de la structure des associations et éclairer les raisons qui font choisir les caractéristiques, les différentielles, etc.... ce qui ne peut, en définitive, qu'atténuer le caractère trop sotuvent ésotérique des travaux de Phytosociologie.

\section{I.2.2.3, - Tableaux des groupes écologiques.}

En face du nom de chacune des espèces (nomenclature de la Flore de Fournier (P.) (1961), nous avons indiqué sa " signification phytosociologique $n$, dans la classification de l'Ecole de Zurich-Montpellier, c'est-à-dire l'unité, plus ou moins compréhensive dont elle est caractéristique, dans la mesure, évidemment, où elle a été retenue comme telle par les chercheurs de cette Ecole. haut.

(21) On retrouve là, la notion de caractéristique transgressive, analysée plus 
Ne figurent dans les tableaux généraux, que les espèces rencontrées dans les associations décrites. Il est bien certain qu'un plus vaste tableau, englobant plusieurs régions florales et tous les étages dans chaque climat régional - dans la mesure où ils peuvent $y$ exister - présenterait une plus grande symétrie et permettrait de mieux mettre en relief cette extraordinaire complexité qui résulte du recoupement des milieus et des régions florales. En commentant chaque tableau, nous aurons l'occasion de mettre laccent sur ces prolongements.

\section{Groupes écologiques xérophiles.}

Parmi les espèces xérophiles, nous avons cru pouvoir distinguer cinq groupes: le premier est celui des espèces liées à un sol calcimorphe, où l'on peut mettre à part celles qui jouent le róle de pionniers; le second comprend des espèces qui s'observent sans doute sur des sols calcimorphes, mais qui se rencontrent aussi et sans doute de façon optimale, sur des rankers à xéromull, leur présence sur des xéromoders francs étant beaucoup moins affirmée; le troisième et le quatrième englobent les espèces acidiphiles, de moder et de mor; dans le cinquieme enfin, nous avons groupé les espèces qui nous ont vraiment paru indifférentes au chimisme du sol, mais qui trouvent leur optimum dans des conditions fort nettes de xéricité.

Ce dernier groupe contient un assez grand nombre d'especes: ceci s'explique par le fait déjà signalé - mais qui méritera d'être approfondi - à savoir que les propriétés chimiques du substratum s'effacent, en quelque sorte, devant ses propriétés physiques, lorsque celles-ci prennent des valeurs extrêmes. I1 faut dire que les espèces xérophiles sont souvent liées à des sols peu évolués, pauvres en matiere organique, donc particulièrement sensibles aux fluctuations climatiques: or, la matière organique joue un róle important, au départ, dans l'altération des roches-mères et dans l'évolution ultérieure des sols, vers des types à humus eutrophe ou à humus acide oligotrophe. On conçoit qu'en son absence, ou étant donné sa rareté, les propriétés des sols, - il faudrait presque dire des lithosols - soient voisines, quelle que soit la composition chimique des roches-mères en cause et dépendent surtout de caractéristiques physiques (texture et microclimat tout particulièrement). Il est possible, d'ailleurs, que certaines espèces classíes par nous dans ce groupe, telles que: Asperula aristata, Hicracium lanatum, Helianthemum canum, Fumana procumbens..., soient à rattacher au groupe des espèces de sols calcimorphes. En forèt xérophile - où les rankers d̀ moder sont très rares, car les affleurements de quartzites le sont aussi - nous ne les avons pas observées, effecti- 
vement, sur sol silicaté; mais hors forêt, il nous a semblé qu'elles étaient adaptées à divers types de substrats (22).

On observera, d'abord, que les espèces de sols calcimorphes on les indifférentes relèvent d'unités phytosociologiques assez variées. Ceci tient en partie aux espèces qui jouent le rôle de pionniers et se rencontrent dans les associations diverses de " garides n. (Festucetalia vallesiacae); certaines sont des a transgressives on de la classe méditerranéenne et méditerranéo-montagnarde des Ononido-Rosmarinetea. Les espèces de sols plus évolués possèdent aussi des appartenances un peu diverses: ceci montre simplement que la flore des forêts xérophiles et héliophiles présente d'étroites affinités avec celle des pelouses relevant du mème " phylum $»$.

Les espèces de rankers à xéromull, comme celles des rankers à xéromoder, se trouvent, par contre, moins « dispersées " que les précédentes. Les premières sont, soit des espèces assez plastiques de pelouses médio-européennes (de la classe des Festuco-Brometea qui englobe les Festucetalia vallesiacae, liées aux contrées à caractère climatique plutót continental et les Brometalia, ordre plus " atlantique $")$, soit des espèces rencontrées dans les chênaies pubescentes (Quercetalia pubescentis), toutes espèces, comme on le voit, qui sont nettement a transgressives $n$, puisqu'on les observe presque toutes, en Haute-Maurienne, jusque dans l'étage subalpin du Pin à crochets.

Les secondes - liées à un xéromoder - sont, pour la plupart, considérées comme des caractéristiques de pelouses alpines à sol humique silicaté (Caricetalia curvulae; Caricetea curvulae); en forêt, elles se rencontrent dans l'étage subalpin, parfois même dans le montagnard.

Parmi les autres acidiphiles, certaines ont une appartenance vraiment trop a étroite n: Lusula nivea (caractéristique du LuzuloFagion), Deschampsia flexuosa et Veronica officinalis (caractéristiques des Quercetea robori-sessiliflorae) débordent, en effet, très largement, le cadre phytosociologique que leur ont attribué certains chercheurs.

Quant à la rareté des espèces liées à des xéromors, elle tient au fait qu'il y a un peu antinomie entre l'existence d'un humus brut et d'une forte xéricité et que l'aire de répartition d'espèces comme Calluna vulgaris, Leucobryum glaucum, les éloigne de notre dition.

\section{Groupes écologiques méso-xérophiles et mésophiles.}

Les espèces appartenant à ces groupes sont en nombre plus considérable que dans les groupes précédents, et encore faut-il remarquer que certaines sont sociales: Ericacées, Calamagrostis villosa,

(22) La difficulté tient au fait que les affleurement de quartzites sont plus ou moins influencées par l'existence de moraines calcaires non indiquées par la carte géologique. I1 eût donc fallu faire des analyses systématiques de rhizosphère. 
etc. et valent surtout par le nombre d'individus qui les représentent. Ceci s'explique par le fait que le climat même de la forêt, se situe, au point de vue humidité de l'air et du sol, dans la "bande » des mésophiles et des mésoxérophiles et aussi parce que, sur les versants secs et chauds, l'homme a partiellement détruit la forêt pour $y$ installer ses cultures, ce qui a contribué à diminuer l'importance des groupes xérophiles, en forêt.

Comme on peut le constater en se référant aux tableatux, ont été groupées dans un même ensemble les espèces mésophiles et les espèces méso-xérophiles. Il nous a paru, en effet, difficile de faire des coupures dans un domaine où manquent par trop des observations chiffrées. Ce que l'on doit retenir, c'est que les espèces liées aux sols calcinorphes, plus chands et plus filtrants, sont les plus rérophiles, et tout particulièrement celles de de l'étage du Chène pubescent.

Par contre, il nous a paru intéressant de distinguer - au moins dans les deux premiers groupes - les espèces qui pénètrent dans le monde intra-alpin et celles qui n'y pénètrent pas, ou à peine, et de dresser les limites d'aire de ces dernières espéces. Nous y reviendrons lors de l'étude des éléments floristiques de notre dition.

La signification phytosociologique des espèces rencontrées mérite, là aussi, d'ètre analysée:

Les espèces de sols à mull calcique qui ne pénètrent pas au coeur de la Haute-Maurienne relèvent, soit des Quercetalia pubescentis (les plus xérophiles et les plus thermophiles), soit des Fagetalia.

Celles qui pénètrent au cour de notre région, sont des caractéristiques de pelouses alpines basiphiles (Seslerietalia) ou de forêts de Pins basiphiles des Alpes centrales (Erico-Pinion). On notera, à nouveau, l'inconvénient qu'il y a à user de termes purement floristiques, pour définir des réalités floristico-écologiques, car si les espèces des Seslerietalia se rencontrent bien sur pelouses basiphiles, dans les étages alpin et subalpin, elles sont très fréquentes dans les forêts à base de Pin à crochets (étage subalpin) ou même de Pin sylvestre et de Sapin, chaque fois que s'observent des sols calcimorphes.

Parmi les espèces de ce groupe, certaines comme Gymnadenia conopea, Carex glauca, Melica nutans, Cypripedium Calceolus, n'ont jamais été considérées comme des caractéristiques; ce sont des planitio-orophiles, qui se rencontrent, en effet, dans des associations ne paraissant pas présenter entre elles le moindre rapport, d'où leur caractère de compagnes. En fait, elles se retrouvent de façon très nette - du moins, en Haute-Maurienne - dans toutes les associations se développant sur sol carbonaté ou à complexe saturé, et méritent donc d'être réhabilitées à ce titre.

Nous avons, enfin, cru bon de placer dans ce groupe des calciphiles des espèces hémicaprophytes d'humus brut - presque toutes 
des Piroles - mais d'un humus marqué par le calcium. Ces espèces se rencontrent, en effet, dans des associations reposant sur un substratum calcaire (pineraies de Pin sylvestre et de Pin à crochets). On pourrait penser, a priori, qu'elles sont liées au seul humus des Pins. Il n'en est rien, puisque certaines d'entre elles au moins ( $P i$ rola rotundifolia, Goodyera repens) se rencontrent aussi et fréquemment sous Epicea, dans l'Asplenio-Piceetum du Jura et des Préalpes, développé sur lapiaz ou sur éboulis de calcaire dur.

Quoi qu'il en soit, l'écologie de ces espèces n'est pas encore très nette et méritera d'étre précisée.

Les espèces liées au mull forestier présentent une homogénéité de classement dans le système BRAUN-BLANguET plus grande encore que celle des espèces précédentes, puisque toutes relèvent de l'alliance du Fagion (les plus orophiles) ou de l'ordre des Fagetalia; les plus plastiques étant considérées comme des caractéristiques de la classe des Querco-Fagetea.

Cette remarquable correspondance entre l'appartenance de ces diverses caractéristiques à des unités phytosociologiques emboitées les unes dans les autres, et le fait qu'elles sont liées à des sols bruns forestiers, à mull, tient à ce qu'elles s'observent effectivement de façon optimale, dans les associations de forèts feuillues, proches du climax, sinon climaciques, où l'équilibre s'est établi entre la végétation et le sol (Hêtraies climaciques du Fagion, chênaies climaciques du Fraxino-carpinion).

Mais il faut noter que, si certaines d'entre elles ne pénètrent pas dans le domaine intra-alpin, où règnent en maitres les résineux, d'autres, par contre, comme Actaea spicata, Lilium Martagon, Paris quadrifolia, Euphorbia dulcis, etc., se rencontrent au niveau de l'étage montagnard, dans la sapinière à Epicea (Abietetum albae de KuocI (R.) (1954), où les hêtraies n'ont cependant jamais existé. C'est qu'il s'agit de caractéristiques stationnelles, liées à des sols à mull qu'elles retrouvent dans ces forêts, caractérisées, on le verra, par des sols à mull-moder, c'est-à-dire présentant le plus souvent un type d'humus qui se situe à la limite du mull et du moder. Ces espèces, il faut le noter, se localisent plus particulièrement dans les trouées de la futaie jardinée où se produit une minéralisation du mull-moder ou du moder et dans les zones à colluvionnement, favorables à l'existence d'un mull franc.

Les espèces du Fagion qui, par contre, ne s'observent pas en dehors de l'aire du Hêtre, peuvent ètre valablement considérées comme formant avec ce dernier un véritable groupe écologique, tout au moins lorsque les conditions édaphiques correspondent à des sols à mull.

Parmi les acidiphiles, nous avons cru pouvoir distinguer celles qui sont plus précisément liées à des sols à mull-moder, celles qui, 
plus acidiphiles, s'observent de façon optimale sur sols à moder, celles, enfin (Ericacées surtout), des sols à humus brut.

Les unes et les autres appartiennent à l'alliance du Vaccinio-Piceion et à l'ordre des Vaccinio-Piceetalia, à l'exception de Gentiana punctata, Campanula barbata, Avena versicolor, Antennaria dioica qui se rencontrent dans les pelouses alpines et subalpines du Caricion curvulae sur sol humique silicaté (ces dernières espèces, on doit le noter, s'observent surtout dans la sous-association à Calamagrostis villosa de la pineraie de Pin Cembro dont les affinités avec les pelouses du Caricion curvulae sont des plus nettes).

Prenanthes purpurea et surtout Poa Chaixii, bien qu'appartenant à l'alliance du Fagion, nous ont paru - tout au moins dans notre dition - davantage liées à des sols à mull-moder qu'à des sols à mull franc. Poa Chaixii s'observe fréquemment, en effet, dans la sous-association à Calamagostris villosa de la pineraie de Pin Cembro, alors qu'on ne le rencontre nulle part ailleurs. Son abondance dans certaines forêts du Massif Central, sur sol ocre podzolique, semble bien confirmer cette écologie.

Chose curieuse, apparemment: certaines planitio-orophiles, sur lesquelles nous avons déjà appelé l'attention, puisqu'elles figurent dans les groupes des xérophiles: Deschampsia flexuosa et Veronica officinalis sont considérées, par les chercheurs de l'Ecole de BRAuNBLANQUET, comme des caractéristiques de la classe des Quercetea robori-sessiliflorae, classe qui pourrait paraitre, a priori, comme assez éloignée de celle des Vaccinio-Piceetea; en fait, ces classes présentent entre elles des affinitís édaphiques incontestables que mettent bien en évidence les groupes écologiques. De telles affinités existent aussi entre les chênaies acidiphiles et certains types de hêtraies comme le Deschampsieto-Fagetum des Cévennes [BraunBlanqueti J.) (1953)], ou les hêtraies à Silene ruprestis et Polypodium vulgare décrites dans les Pyrénées par Susplugas (J.) (1942). Ces hêtraies acidiphiles arrivent - et la chose ne doit pas paraitre étonnante, après ce que nous venons de dire - à ne plus présenter, ou presque plus, d'espèces caractéristiques du Fagion, et les spécialistes estiment que ces types de hêtraie se rattachent davantage à l'ordre des Quercetalia roboris, chênaies acidiphiles [Braun-Blanguet (J.), Roussine (Mme), NÈgre (R.) (1951, p. 228)] qu'à celui des Fagetalia.

La méthode de classement de Braun-Blanguet - nous aurons l'occasion de revenir sur ce point - aboutit donc, au moins dans ce cas particulier, à une inextricable difficulté, alors que l'utilisation des groupes écologiques permet de mettre pleinement en lumière les affinités qui existent entre hêtraies à mull et chênaies à mull et celles qui existent entre hêtraies à moder et chênaies à moder (affinités édaphiques); comme elle permet, dans le mème étage, de relier les différents types de hêtraies, car le groupe des espèces 
montagnardes liées à un sol à mull, par exemple, se retrouve, bien que forcément tronqué, mais non jamais totalement absent, dans les autres types édaphiques de hêtraies (affinités climatiques).

A noter, toutefois, que, récemment, l'alliance du Fagion a été divisée en trois sous-alliances:

l'Eu-Fagion (hêtraies sur sols à mull), le Cephalanthero-Fagion (hêtraies sur sols à mull calcique) et le Lusulo-Fagion (hêtraies sur sols à moder) [OBERDORFER (E.) (1956)]. Cette façon de faire, qui a évidemment clarifié le problème soulevé ci-dessus, mériterait d'être généralisée, car elle permettrait de faire se rencontrer, sinon se fusionner, les méthodes dites floristiques et celles dites écologiques. Nous reviendrons sur ce point important, dans nos conclusions générales.

Si l'on examine, enfin, les espèces caractéristiques des sols d̀ humus brut (sols ocres podzoliques à mor, sols podzoliques ou podzols), on constate qu'elles sont presque toutes des subalpines, à l'exception de Vaccinium Myrtillus dont l'amplitude à l'égard de l'altitude est vaste: ceci s'explique par le fait, sur lequel nous aurons loccasion de revenir. qu'il y a une étroite corrélation entre les conditions écologiques régnant dans l'étage subalpin et celles qui facilitent la formation d'humus brut; il s'agit d'espèces caractéristiques de l'alliance du Vaccinio-Piccion et, plus précisément, de la sousalliance Rhodoreto-Vaccinion, de l'étage subalpin supérieur.

\section{Groupes écologiques méso-hygrophiles.}

Etant donné l'absence, nous l'avons déjà dit, dans les forêts de la Haute-Maurienne, de sols non aérés, à hydromorphie permanente. ot même semi-permanente, les espèces liées à des anmoors et à des tourbes sont totalement absentes; les hydromoders calciques sont également très rares - la perméabilité des roches-mères carbonatées et la vigueur des pentes expliquent cette rareté - si bien que les méso-hygrophiles lićes à ce type d'humus forment un maigre cortège: d'autant plus que les méso-xérophiles de moder ou de mull calcique, comme Sesleria coerulea, Calamagrostis veria, Carex qlauca, etc, présentent une certaine plasticité, quant à l'économie de leau, et s'observent également dans les parties les plus humides des sols calcimorphes.

Le groupe des méso-hygrophiles des sols à mull est, par contre, bien étoffé. Presquue toutes ses espèces appartiennent à la classe des Betulo-Adenostyletea, et, plus précisément, à l'alliance de 1'Adenostylion. Cette homogénéité s'explique, si l'on se réfère au fait qu'il s'agit d'espèces orophiles liées à un type de climat et à un type de sol colluvial bien définis.

Il nous a semblé utile, enfin, de faire figurer dans un groupe à hydromor. Listera cordata et Vaccinium uliginosum, qui nous paraissent trouver leur optimum sur un tel type d'humus - elles figuraient déjà, mais entre parenthèses, dans le groupe des mésophiles à mor. 


\section{GROUPE DES ESPECES XEROPHILES}

Espèces - Signification écologique

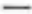

\author{
Unités \\ dont ces espèces \\ sont caractéristiques
}

1. - Espèces liées à des sols calcimorphes.

\section{1. - Espèces pionniers}

Dryas octopetala

Teucrium montan

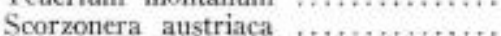

Koeleria Vallesiana ...............

Euphorbia Gerardiana subsp. Loiseuleri..$\ldots \ldots \ldots \ldots \ldots \ldots \ldots \ldots \ldots \ldots$ Laserpitium gallicum ............

\section{Bilyno-Seslerictea}

Thlapeetalia rotundifolii

Ononido-Rosmarinetea

Festucetalia vallesiacae

Festucetalia vallesiacae

Festucetalia vallesiacie

Stipo-centranthetum

\section{2. - Espèces de xéromull colcique on de xéromull-moder calcique.}

Ononis rotundifolia

Astragalus monspessulanus

Globularia Willkommii ...............

Epipactis atropurpurea ..............

Gymnadenia odoratissima $\ldots \ldots \ldots \ldots$

(Polygala Chamaebuxus) ............

Onobrychis montana ...............

Brunella grandiflora $\ldots \ldots \ldots \ldots \ldots$.

Giobularia cordifolia.

Campopthecium lutescens.

Rhytidium rugosum.

Brachytecium glareosum.

Encalypta streptocarpa.

(Tortella tortuosa).
Ononido-Pinion

Ononido-Pinion

Ononido-Rosmarinetea

Ononido-Rosmarinetea

Erico-Pinion

Erico-Pinion

Erico-Pinion

Seslerietala coeruleae

Festuco-Brometea

2. - Espèces lices à des rankers à tínomull.

Polygonatum officinale

Chrysanthemum corymbosum .......

Trifolium rubens .................

Laserpitium latifolium ............

Geranium sanguineum .............

Berberis vulgaris ................

Vincetoxicum officinale ................

Coronilla Emerus ................

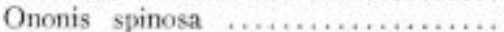

Hippocrepis comosa .............

Brachypodium pinnatum ...........

Centaurea Scabiosa

Gallium Mollugo subsp. corrudifolium.

Pimpinella Saxifraga
Quercetalia pubescentis Quercetalia pubescentis Quercetalia pubescentis Quercetalia pubescentis Quercetalia pubescentis Quercetalia pubescentis Quercetalia pubescentis Quercion pubescentis

Bromion erecti

Bromion erecti

Brometalia

Festuco-Brometea

Festuco-Brometea

Festuco-Brometea 
Espèces - Signification écologique

Anthyllis Vulneraria

Petcedanum Oreoselinum ...........

Carex humilis ....................

Calamagrostis argentea ............

Rosa pimpinellifolia.

Carlina acaulis var. caulescens.

Thuidium abietinum.

\section{Unités \\ dont ces espéces \\ sont caractéristiques}

Festuco-Brometea

Festuco-Brometea

Ononido Rosmarinetea

Thlaspeetea rotundifolii

\section{3. - Espièces liées à des rankers à séromoder.}

Mintuartia laricifolia

Phyteuma betonicifolium ..........

Trifolium alpestre $\ldots \ldots \ldots \ldots \ldots \ldots \ldots$

Silene rupestris ...............

Sempervivum arachnoideum,$\ldots \ldots$.

Sempervivum montanum .........

(Campanula barbata) .............

Centaurea uniflora..$\ldots \ldots \ldots \ldots \ldots$

Veronica fruticans ..............

Iaserpitium Halleri ..............

Antennaria dioica ................

Hieracium Peleterianum ..........

Hieracium picroides.

Silene nutans.

Sedum rupestre subsp. montanum.

(Luzula nivea) .................

(Deschampsia flexuosa) ...........

(Veronica officinalis) ............

Polytrichum piliferum ............

Hedwigia albicans

(Hypnum cupressiforme subsp, ericetorum)

(Polytrichum juniperinum)

(Dicranum scoparium).
Deschampsio-Pinion

Deschampsio-Pinion

Quercetalia pubescentis

Androsetalia

Caricetalia curvulae

Caricetalia curvulae.

Caricetalia curvulae

Caricetalia curvulae

Caricetalia curvulae

Caricetea curvulae

Caricetea curvulae

Caricetea curvulae

\section{Luzulo-Fagion}

Quercetea robori-sessiliflorae

Quercetea robori-sessiliflorae

Cisto-Lavanduletea

Cisto-Lavanduletea

Vaccinium Vitis-Idaea

Vaccinio-Piceetalia

Racomitrium heterostichum.

(Pleurozium Schreberi).

\section{5. - Espèces indifférentes.}

Aretostaphylos Uva-ursi ..........

Potentilla puberula ................

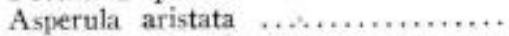

Fumana procumbens ...............

Helianthemum canum ............

Euphorbia Cyparissias

Vaccinio-Piceetalia

Festucetalia vallesiacae

Festucetalia vallesiacae

Ononido-Rosmarinetea

Ononido-Rosmarinetea

Festuco-Brometea 
Espèces - Signification écologique

Potentilla verna

Chrysanthemum leucanthemum subsp montanum,

Hieracium bifidum.

Hieracium lanatum.

Teucrium Chamaedrys.

Carex Halleriana.

Anthericum Liliago.

Laserpitium Siler.

Thymus Serpyllum subsp. Trachselianus.

Aethionema saxatile.

Viola saxatilis.

Dianthus Caryophyllus subsp. silvestris.

Tortula ruralis.

\section{Unités \\ dont ces espèces \\ sont caractéristiques}

Festuco-Brometea

\section{GROUPES DES ESPECES MESO-XEROPHILES \\ ET MESOPHILES}

Fspèces - Signification écologique

\section{Unités}

dont ces espèces

sont caractéristiques

1. - Espèces liées à des sols à mull mu moder calcique.

1.1. - Espices ne pénétrant pas au caru de la Haute-Maurienne.

Cephalanthera rubra

Cephalanthera Xyphophyllum ......

Viola mirabilis

Elymus europaeus

Mercurialis perennis ...............

Orobus vernus .........................

Brachypodium silvaticum
Quercetalia pubescentis

Quercetalia pubescentis

Quercetalia pubescentis

Fagion

Fagetalia

Fagetalia

Querco-Fagetea

\section{2. - Espices sobserzint an caur de la Haute-Maurienus.}

Dryas octopetala

Sesleria coerulea subsp. calcaria ....

Carex ferruginea

Seabiosa lucida ...................

Aster Bellidiastrum

Elyno-Seslerietea

Seslerietalia

Seslerietalia

Seslerietalia

Seslerietalia

Carcluus defloratus .................. 
Espèces - Signification écologique

Phyteuma orbiculare

Valeriana montana

Carex sempervirens .

Sorbus Chamaemespilus ............

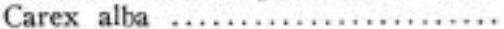

Erica carnea .....................

Coronilla vaginalis $\ldots \ldots \ldots \ldots \ldots \ldots$.

Vicia galloprovincialis ...........

Melampyrum pratense subsp. alpestre.

Polygala Chamaebuxus .............

Cotoneaster tomentosa.......... .

Calamagrostis varia.

Carex glauca.

Gymnadena conopea.

Melica nutans.

Cypripedium Calceolus,

Ctenidium molluscum.

(Tortella tortuosa).

Hémisaprophytes (mor calcicue).

Pirola rotundifolia

Pirola chlorantha $. . . \ldots \ldots \ldots \ldots . . .$.

Goodyera repens
Unités

dont ces espèces

sont caractéristiques

Seslerietalia

Thlaspeetalia

Curvuletalia

Erico-Pinion

Erico-Pinion

Erico-Pinion

Erico-Pinion

Erico-Pinion

Erico-Pinion

Erico-Pinion

Quercetalia pubescentis

\section{2. - Espèces liées à des sols à mull.}

2.1. - Espèces ne pénétrant pas au caur de la Haute-Maurienne.

Festuca silvatica

Epilobium montanum ,...........

Lamium Galeobdolon .............

Melica uniflora

Asperula odorata $\ldots \ldots \ldots \ldots \ldots \ldots$

Sanicula europaea ..............

Bromus asper (subsp. Benekeni) ....

Dentaria pinnata . .............

Carex silvatica ..................

Viburnum Opulus ..............

Hedera Helix

Eurynchium striatum.

Catharinea undulata.
Fagion

Fagion

Fagetalia

Fagetalia

Fagetalia

Fagetalia

Fagetalia

Fagetalia

Fagetalia

Fagetalia

Querco-Fagetea

\section{2. - Especes pentrant au caur de la Haute-Manriene.}

Actaea spicata ................. Fagion

Lilium Martagon ................ Fagion

Paris quadrifolia .................. Fagion

Euphorbia dulcis ................... 
Espèces - Signification écologique

Polystichum Filix-mas

Viola silvestris $\ldots \ldots \ldots \ldots \ldots \ldots \ldots . . . . . .$.

Mycelis muralis

Neottia Nidus avis ................

Moehringia trinervia ...............

Millium effusum ....................

Phyteuma spicatum ................

Carex digitata

Ranunculus Breynimus ............

Lonicera alpigena.

Campanula rhomboidalis.

Clematis alpina.

\section{Unités \\ dont ces espèces sont caractéristiques}

Fagetalia

Fagetalia

Fagetalia

Fagetalia

Fagetalia

Fagetalia

Fagetalia

Querco-Fagetea

Querco-Fagetea

\section{3. - Espéces liées à des sols à mull-moder et à moder.}

3.1. - Espéces de mull-moder.

Poa Chaixii

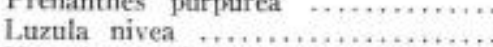

Veronica latifolia ...................

Melampyrum silvaticum ............

Aquilegia atroviolacea ............

Polystichum spinulosum subsp. dilatatum $\ldots \ldots \ldots \ldots \ldots \ldots \ldots \ldots \ldots \ldots \ldots \ldots$

Galium rotundifolium ..............

Rubus saxatilis ....................

Rosa pendulina $\ldots \ldots \ldots \ldots \ldots \ldots . . . . .$.

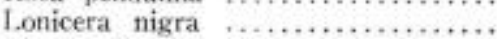

Luzula silvatica subsp. Sieberi .......

Pirola secunda $\ldots \ldots \ldots \ldots \ldots \ldots \ldots$.

Valeriana tripteris.

Oxalis Acetosella.

Dryopteris Linnaeana.

Poa nemoralis.

Brachythecium velutinum.

Mnium cuspidatum.

\section{2. - Lispèces de moder.}

Gentiana punctata

Campanula barbata .....................

Avena versicolor $\ldots . . . \ldots \ldots \ldots \ldots . . .$.

Antemnaria dioica . .........., . . .

Calamagrostis villosa ...............

Saxifraga cuneifolia ..............

Homogyne alpina .................

Pirola minor

Luzula flavescens

(Vaccinium Myrtillus)

\section{Fagion}

Fagion

Luzulo-Fagion

Vaccinio-Piceion

Vaccinio-Piceion

Vaccinio-Piccion

Vaccinio-Piceion

Vaccinio-Piccion (et Fagion)

Vaccinio-Piceetalia

Vaccinio-Piceetalia

Vaccinio-Piceetalia

Vaccinio-Piceetalia

Vaccinio-Picectalia
Caricion curvulae Caricetalia curvulac Caricion curvulae Caricetea curvulae Vaccinio-Piceion Vaccinio-Piceion Vaccinio-Piceetalia Vaccinio-Piceetalia Vaccinio-Picectalia Vaccinio-Piceetalia 
Espèces - Signification écologique

(Vaccinium Vitis-Idaea)

Festuca flavescens - a.s.

Deschampsia flexuosa ...............

Veronica officinalis...$\ldots \ldots \ldots \ldots$.

Maienthemum bifolium,

Polypodium vulgare.

Anthoxantum odoratum.

Brachytecium Starkei.

Isothecium myurum.

Plagiothecium denticulatum.

Mnium spinosum

(Dicranum scoparium).

4. - Espèces liées à des sols à mor.

Rhododendron ferrugineum ........

Lonicera coerulea ................

Vaccinium Myrtillus ..............

(Vaccinium Vitis-Idaea) ............

Empetrum hermaphroditum .........

(Listera cordata) .................

(Vaccinium uliginosum) $\ldots . . . . . . .$.

Peltigera aphtosa $. . . . \ldots \ldots \ldots . . . .$.

Barbilophozia lycopodioides ........

Pleurozium Schreberi.

Depranocladus uncinatus,

Barbilophozia Hatscheri.

Barbilophozia barbata.

Dicranum scoparium.

Cetraria islandica.

\section{Unités}

dont ces espèces

sont caractéristiques

Vaccinio-Piceetalia

(Vaccinio-Piceetalia)

Quercetea robori-sessilifforae

Quercetea robori-sessiliflorac

Vaccinio-Piceion
Vaccinio-Piccion

Vaccinio-Piceion

Vaccinio-Piceetalia

Vaccinio-Piceetalia

Vaccinio-Piceetalia

Vaccinio-Piceion

Vaccinio-Piceetalia

Vaccinio-Piceion

Vaccinio-Piccion

\section{GROUPE DES ESPECES MESOHYGROPHILES}

Espèces - Signification écologique

1. - Espèces de mull et de meder calciques.

Salix retusa,$\ldots \ldots \ldots \ldots \ldots \ldots \ldots$
Salix reticulata $\ldots \ldots \ldots \ldots \ldots \ldots \ldots$

Tofieldia calyculata

(Carex ferruginea) .................

Chrysohypnum chrysophyllum,

\section{Unités \\ dont ces espèces \\ sont caractéristicues}

A rabidion coeruleae Arabidion coeruleae Caricion Davallianae Seslerictalia 
Espèces - Signification écologique

2. - Espèces de mull.

Achillea macrophylla

Sisymbrium tanacetifolium .........

Alnus viridis ....................

Aconitum paniculatum .............

Stellaria nemorum subsp. montana ..

Streptopus amplexifolius ...........

Ranuneulus aconitifolius ............

Cicerbita alpina $. \ldots \ldots \ldots \ldots \ldots . . . . .$.

Myosotis silvatica subsp. alpestris ..

Athyrium alpestre ................

Veratrum album...............

Thalictrum aquilegifolium ..........

Phyteuma Halleri .................

Peucedanum Ostruthium .............

Adenostyles Alliariae ...............

Rumex arifolius .................

Saxifraga rotundifolia .............

Chaerophyllum hirsutum ............

Aconitum vulparia ................

Geranium silvaticum ...............

Polygonatum verticillatum ..........

Mnium punctatum.

Brachythecium reflexum.

(Chrysohypnum stellatum).

\section{Unités \\ dont ces especes \\ sont caractéristiques}

Adenostylion

Adenostylion

Adenostylion

Adenostylion

Adenostylion

Adenostylion

Adenostylion

Adenostylion

Adenostylion

Adenostylion

Adenostylion

Adenostylion

Adenostylion

Adenostyletalia

Adenostyletalia

Adenostyletalia

Adenostyletalia

Adenostyletalia

Adenostyletalia

Betulo-Ádenostyletea

Betulo-Adenostyletea

\section{Espices de mor.}

Listera cordata

Vaccinio-Piceion

Vaccinium uliginosum ............... Vaccinio-Picectalia

\section{I.2.3. - Spectres floristico-écologiques - Cercles d'exposition.}

Dans nos tableaux de relevés, les espèces ne sont done plus classées en caractéristiques d'association, d'alliance, d'ordre, de classe, mais figurent dans des grotupes écologiques qui donnent une image de la structure floristique et écologique du groupement analysé.

Pour donner une image plus condensée de cette structure, nous avons dessiné des spectres "floristico-écologiques " analogues, wutatis mutandis, aux spectres biologiques de RAUNKIER, mais où nous avons tenu compte de l'abondance-dominance des espèces et non de leur seule présence; ce qui traduit mieux la réalité, comme l'on fait remarquer CarLes (J.) (1948) en ce qui concerne les spectres biologiques, et Quezer. (P.) et Rioux (J.-A.) (1950) à propos des spectres dits "zonaux $n$. 
Ces spectres ne concernent sans doute que deux grands " complexes de facteurs $n$ : l'économie de l'eau, de l'air et du sol; la structure et le chimisme de l'humus. Nous avons pensé qu'il était inutile de les surcharger en distinguant les espèces liées à tel ou tel étage, mises d'ailleurs en évidence dans les tableaux analytiques.

Ces spectres, enfin, ont été établis par le calcul du degré de couverture de chaque groupe, exprimé en pour cent du total (on sait que le degré de couverture se calcule ainsi : le chiffre d'abondancedominance 5 , correspond à un degré de $87,5 \%$, moyenne entre 75 et $100 \%$, le chiffre de 4 à $62,5 \%$, etc.).

Enfin, pour traduire de façon synthétique le rôle joué par l'exposition dans la répartition des associations, nous avons dessiné des "cercles d'exposition " comme l'ont fait notamment BRAUN-BLANguet (J.), Palmann (H.) et Bach (R.) (1954), lors de leur étude du Parc national suisse.

Pour calculer le pourcentage de relevés observés à telle ou telle exposition, nous nous sommes référé aux tableaux mêmes d'associations qui nous ont paru offrir, dans ce domaine, un échantillonnage acceptable, étant entendu qu'il eût été plus exact, statistiquement, de s'appuyer sur un échantillonnage plus vaste, obtenu, par exemple, à partir d'un réseau appliqué sur la carte mème des associations.

Il faut noter que les images de répartition des associations en fonction de l'exposition ainsi obtenues, correspondent non aux potentialités des groupements dans ce domaine, mais simplement à leur répartition de fait, en Haute-Maurienne; il est bien certain, en effet, que dans notre dition, toutes les expositions ne sont pas également représentées, ce qui diminue forcément quelque peu l'intérêt d'une telle représentation qui reste cependant, dans l'ensemble, extrêmement suggestive.

On ne doit pas oublier, enfin, que l'exposition prise en soi ne donne qu'une idée partielle d'un phénomène complexe qui sera brièvement analysé: la pente joue un rôle considérable, de même le dessin et la proximité des flancs regardant une exposition donnée. Les géographes ont ainsi pu parler de " faux adrets ", près du fond des talwegs, là où les rayons solaires se trouvent interceptés plus longtemps qu'à altitude plus élevée, par le flanc, tout proche, d'exposition nord.

Il apparait, dans ces conditions, qu'un a cercle d'exposition » gagnerait à ètre établi en fonction, soit de la durée d'ensoleillement, soit, ce qui cernerait de plus près la réalité, en fonction de l'énergie solaire reçue. Mais l'étude de ces paramètres, on le verra, soulève de nombreuses difficultés, plus matérielles que techniques, d'ailleurs. 


\section{I.3. - METHODES D'ETUDE DES SOLS}

Les grandeurs pédologiques n'étant pas strictement les mêmes en tous les points d'un individu d'association, il eût été évidemment intressant de caractériser chaque facteur par une moyenne ou un indice de dispersion (1). Toutefois, étant donné que les unités floristiques étaient assez sensiblement différentes les unes des autres, et qu'il ne s'agissait pas de faire une étude détaillée de l'homogénéité de chaque milieu édaphique, nous n'avons fait, pour chaque association, que quelques relevés pédologiques dans les stations qui nous ont paru les plus typiques. Nous avons, en outre, examiné à l'ceil divers profils, sans faire de prélèvements: cet examen, complété par l'étude de la réaction à l'acide chlorhydrique des divers horirons, nous a permis de nous assurer de l'homogénéité générale du milieu édaphique étudié.

\section{I.3.1. - Méthodes d'analyse utilisées.}

Toutes les analyses ont été faites au Laboratoire de Pédologie de l'Ecole Nationale des Eaux et Forêts.

a) Analyse mócanique. - L'analyse granulométrique adoptée a été celle dite de la méthode internationale décrite par Demolon (G.) (1943), modifiée par Robinson (G.-W.) (1949). On sait que l'on obtient d'une part les cailloux et les graviers (arrêtés par les mailles du tamis à $2 \mathrm{~mm}$ ), d'autre part, la terre fine qui comprend:

les argiles - éléments inférieurs à $2 \mu$,

les limons fins entre $2 \mu$ et $20 \mu$ (ou $0,02 \mathrm{~mm}$ ),

les limons grossiers et les sables fins - entre $20 \mu$ et $0,2 \mathrm{~mm}$, les sables grossiers entre 0,2 et $2 \mathrm{~mm}$.

b) Analyses chimiques. - Le dosage de la matière organique totale, par calcination, n'a été utilisé que pour les humus bruts (horizon $\mathrm{A}_{0}$ ) (2). Pour les sols plus riches en argile, la teneur en

(1) Les travaux de MaxIL (G.) et de ses collaborateurs (1953) montrent qu'un tel résultat, toujours long à obtenir, doit suivre une première analyse; cette dernière est, en général, suffisante si elle est faite dans la station optima: la phytosociologie guide le pédologue et réciproquement.

(2) Dans ce cas, la matic̀re organique est très abondante; il $y$ a très peu de matière fine, donc d'eau de constitution et de carbonates (le poids d'eau évaporée et celui de $\mathrm{CO}_{2}$ perdu par les carbonates lors de la calcination sont donc relativement négligeables). 
matière organique a été déduite du pourcentage de carbone organique par application du coefficient 1,72 .

Le carbone organique a été dosé par la méthode d'ANNe (1945), l'azote total par la méthode de KJELDAHL, l'azote minéral (ammoniacal ou nitrique) par la méthode Drouineau (G.) (1947). Le rapport $\mathrm{C} / \mathrm{N}$ en a été déduit.

Le fer libre a été dosé, pour certains sols lessivés ou podzoliques, par la méthode Deв (B.-C.) (1950) qui utilise des réactifs faibles, tartrate d'ammonium et hydrosulfite de soude, solubilisant le fer à l'état ferreux.

Les carbonates ont été dosés au calcimètre Bernard (mesure du dégagement de $\mathrm{CO}_{2}$ par attaque à $\mathrm{HCl}$ au $1 / 2$ ). Le carbonate de chaux correspond à une première phase de dégagement rapide de $\mathrm{CO}_{2}$, le carbonate de magnésium à une deuxième phase de dégagement lent.

Complexe absorbant: la capacité totale d'échange a été déterminée à $\mathrm{pH} 7$, par percolation lente et progressive d'une solution d'acétate d'ammonium normale.

La somme des bases échangeables a été calculée par la méthode de Bray et Willehtre (1929) ou par la somme au mille équivalent des trois bases fondamentales: $\mathrm{Ca}-\mathrm{Mg}-\mathrm{K}$.

c) Mesure du $p H$.

Le $\mathrm{pH}$ a été mesuré sur le terrain par la méthode colorimétrique, avec référence à une échelle graduée. Les colorants utilisés ont été:

- le bleu de Bromothymol pour les $\mathrm{pH}$ de 6 à 7,6 ,

- le rouge de Méthyle pour les $\mathrm{pH}$ de 4,6 à 7,6 ,

- le vert de Bromocrésol pour les $\mathrm{pH}$ de 3,8 à 5,2 ,

- le bleu de Bromophénol pour les $\mathrm{pH}$ de 3 à 4,5 . et, au laboratoire, à l'aide d'un pHmètre.

\section{I.3.2. - Expression des résultats - Interprétation.}

Les résultats des analyses sont exprimés en pour cent de terre sèche à l'air (à $20^{\circ}$ ). L'humidité indique donc la perte en eau de la terre sèche à l'air, après étuvage de plusieurs heures à l'étuve en $105^{\circ}$.

Le fer libre est exprimé en fer métal pour $100 \mathrm{~g}$.

Enfin, la chaux échangeable est exprimée en milliéquivalent pour $100 \mathrm{~g}$ de terre sèche à l'air.

Certains indices ont été mis en évidence, pour faciliter l'interprétation des résultats.

pour les sols podzoliques et certains sols lessivés, l'indice d'entrainetaux d'argile de l'horizon B

ment de l'argile: $\frac{}{\text { taux d'argile de l'horizon A }}$ permet de distin- 
guer les sols lessivés et les sols podzoliques; le rapport de lessivage taux de fer libre en B

du fer $\longrightarrow$, permet de différencier les sols bruns taux de fer libre en A

des sols bruns lessivés et des sols ocres podzoliques.

D'une façon générale, nous nous sommes moins attachés à l'analyse granulométrique qu'à l'analyse d'éléments qui permettent le mieux de caractériser le type de sol et le type d'humus, c'est-à-dire pour les divers horizons: la présence ou l'absence de carbonates; l'état du complexe absorbant; la teneur en matière organique des horizons humiques et leur $\mathrm{pH}$. Presque tous les sols étudiés appartiennent, en effet, au groupe des rankers de KuBIENA (W.-L.) (1953) ou des sols calcimorphes jeunes: il s'agit donc de sols peu évolués, soumis à une érosion incessante: presque tous les horizons sont riches en cailloux; de ce fait, l'analyse granulométrique ne permet pas d'établir d'efficaces distinctions.

\section{I.3.3. - Description et classification des types de sols étudiés.}

Cette jeunesse des sols n'empèche pas, toutefois, de décrire des types sensiblement différents: la composition de la roche-mère donne à certains d'entre eux des caractères d'autant plus nets - et d'autant plus permanents - que l'érosion intervient, continuellement, pour maintenir ces caractères; la nature des végétaux, le microclimat local jouent un róle très important également et contribuent, par le biais des phénomènes de décarbonatation, de lessivage, ou de podzolisation, a former une gamme de sols relativement variée.

Les sols rencontrés dans les forêts de notre dition peuvent être divisés ainsi.

1) les sols calcimorphes, c'est-a-dire les sols reposant sur des rochesmères calcaires ou gypseuses, de profil $\mathrm{AC}$, à complexe absorbant saturé; il s'agit, par conséquent, de rendzines,

Parmi ces rendzines, il faut distinguer:

a) les rendzines jeunes, très érodées, où l'horizon A est de faible épaisseur, donc pauvres en matière organique.

b) les rendsines brunifiées, décarbonatées dans l'horizon supérieur et passant aux sols bruns calcaires; ce sont des sols également pauvres en matière organique.

c) les sols humiques carbonatés, carbonatés dans les horizons supérieurs, à horizon humifère épais, riches, par conséquent, en matière organique et pauvres en argile (moins de $20 \%$ ). 
d) les sols humiques carbonatés à humus brut qui présentent une couche de mor reposant, soit directement sur la roche-mère calcaire, soit sur un horizon técarbonaté.

2) les sols bruns à mull forestier, comportant peu d'humus (cryptomull), ce dernier étant plus ou moins acide, décarbonatés - au moins dans les horizons supérieurs - et à complexe saturant plus ou moins désaturé. Il s'agit, très généralement, de sols bruns jeunes, développés sur colluvium.

3) les sols à moder et à mor.

Certains types de sols présentent un humus marginal, à la limite du mull et du moder. Ils forment le passage entre les sols à mull et les sols à moder. Ces derniers sont, le plus souvent des rankers à moder, ou des rankers ocres podsoliques, présentant, eux, les deux horizons $A_{2}$ et $B$; ils sont toujours riches en cailloux, même dans l'horizon $A_{2}$, ce qui est un signe incontestable de jeunesse.

On notera que nous n'avons pas observé de sols hydromorphes non drainés, les plus humides étant ceux de l'Alnetum viridis que l'on peut qualifier de sols colluviaux à hydromull.

Le tableau ci-après résume la classification des sols observés, leurs principaux caractères ainsi que leurs aftinités:

\begin{tabular}{|c|c|c|}
\hline Groupes & Types dans le groupe & Types d'humus \\
\hline $\begin{array}{c}\text { Sols } \\
\text { calcimorphes }\end{array}$ & $\begin{array}{l}\text { Rendzine jeune sèche } \\
\text { Sol humique carbonaté } \\
\text { Sol humique carbonaté } \\
\text { à humus brut } \\
\text { Rendzine brunifiée }\end{array}$ & $\begin{array}{l}\text { Moder calcique } \\
\text { Moder ou mull calcique } \\
\text { Mor décalcifié } \\
\text { Mull forestier }\end{array}$ \\
\hline $\begin{array}{c}\text { Sols } \\
\text { bruns d mull }\end{array}$ & $\begin{array}{l}\text { Sol brun jeune } \\
\text { Sol brun calcaire }\end{array}$ & $\begin{array}{l}\text { Mull forestier, parfois } \\
\text { mull-moder } \\
\text { Mull iorestier }\end{array}$ \\
\hline $\begin{array}{c}\text { Sols d } \\
\text { moder ou mor }\end{array}$ & $\begin{array}{l}\text { Ranker à moder } \\
\text { Ranker à mor } \\
\text { Ranker ocre podzolique } \\
\text { Sol podzolique jeune } \\
\text { Podzol. }\end{array}$ & $\begin{array}{l}\text { Moder } \\
\text { Mor } \\
\text { Moder } \\
\text { Mor } \\
\text { Mor }\end{array}$ \\
\hline
\end{tabular}


Les sols humiques carbonatés à humus brut et les sols à mor (rankers, sols podzoliques ou podzols) sont " analogues ", quant à leur horizon supérieur; les sols bruns calcaires et les rendzines le sont, à l'inverse si l'on peut dire, quant à leurs horizons inférieurs. On conçoit mieux, dès lors, combien la phytosociologie a besoin de la pédologie... et réciproquement d'ailleurs. 


\section{I.4. - METHODES D'ETUDE MESOCLIMATIQUE ET MICROCLIMATIQUE}

\section{I.4.1. - Méthodes suivies.}

Il n'est pas inutile, au préalable, d'apporter quelques précisions sur le vocabulaire utilisé, encore que, dans le domaine de l'étude des climats, la terminologie reste assez vague.

Lorsqu'on envisage le climat d'une région de plusieurs centaines de kilometres carrés, on parlera de climat tout court ou, mieux, de macroclimat. Ce macroclimat est donc analysé à partir de postes séparés de plusieurs dizaines de kilomètres: on pourra ainsi définir un macroclimat parisien, un macroclimat breton, etc.

Par contre, il s'agira de mésoclimat ou de climat local, si l'unité naturelle étudiée, s'étend seulement sur un nombre de kilomètres carrés, ne dépassant pas le millier, par exemple. Dorgnon a ainsi analysé le mésoclimat de la forèt de Fontainebleau [dans IABLoKoff (A.-Kh.) (1953)], qui s'étend sur $170 \mathrm{~km}^{2}$ environ.

Lorsqu'on descend dans l'échelle des dimensions, on peut alors légitimement parler de microclimat: ce dernier pouvant correspondre à des volumes très petits, de l'ordre du $\mathrm{cm}^{3}$ ou moins encore, ou à des volumes ou des surfaces sensiblement plus vastes.

Les coupures entre ces différents termes n'ont jamais été définies avec précision et l'on conçoit qu'ils couvrent plusieurs acceptions, puisque l'on embrasse tout le réel climatique avec trois termes seulement.

Dans l'ensemble, comme on le verra au cours de ce travail, et compte tenu des observations ci-dessus, les mesures que nous avons effectuées sont d'ordre microclimatique, alors que les données dont nous nous sommes servi et qui provenaient de postes météorologiques sont d’ordre mésoclimatique.

En matière de microclimatologie, la difficulté tient parfois, comme l'a souligné GurNochet (M.) (1938), à des obstacles matériels. L'idéal eût été de posséder un nombre d'appareils enregistreurs au moins égal au nombre d'associations étudiées et de faire porter les observations simultanément sur tous ces groupements, au cours de Pannée ou, à défaut, au cours d'une saison de végétation. Les résultats obtenus - quoique relatifs - eussent eu une valeur certaine. 
Ne disposant que de deux groupes d'appareils (hygromètre à cheveu, thermometre enregistreur, thermomètre de sol également enregistreurr), nous avons dû̀ nous limiter à des comparaisons portant simultanément sur deux individus d'associations, choisis évidemment parmi les plus typiques.

Pour augmenter lampleur des investigations dans l'espace, nous avons limité à trois semaines environ la durée des observations couplées. D'autre part, at lietı de changer chaque fois les appareils dans les deux " individus " étudiés simultanément, nous avons laissé un groupe d'appareils durant toute la saison de végétation dans un même individu: en l'occurence, il s'est agi d'un individu d'une Pineraie xérophile à Ononis rotındifolia. Astragalus monspessulanus et Carex humilis (Ononido-Pinetum de BraUn-BLANQUET), l'autre groupe étant transporté toutes les trois semaines dans une association différente. Cette façon de procéder nous a permis, pour l'association en cause, de faire une étude microclimatique assez étendue dans le temps.

\section{I.4.2. - Dispositifs techniques et expression des résultats.}

Les appareils mesurant la température de l'air (thermomètres enregistreurs), l'état hygrométrique (hygromètres à cheveux) ont été placés, à des hauteurs convenables, - en général $1.50 \mathrm{~m}$ - dans de petites caisses en bois, ouvertes à leurs deux extrémités, ce qui permettait à l'air de circuler librement, tout en évitant aux appareils d'être atteints directement par les rayons du soleil, car ces deux extrémités étaient protégées par des branchages.

Les appareils de mesure de la température du sol (thermomètres enregistreurs) ont été posés à même le sol et le thermomètre enfoncé à diverses profondeurs, done toujours à l'abri des radiations solaires directes.

Les résultats concernant les températures et les états hygrométriques n'appellent aucun commentaire spécial (1). Mais nous avons cherché, à partir de ces grandeurs, à définir - de facon relatizé s'entend - le porvoir f́vaporant de latnosphère. Nous avons pensé que ce pouvoir évaporant pouvait être traduit par la formule de Dalton $Q=$ K.S. $\frac{\left(\mathrm{F}_{\mathrm{t}}-\mathrm{f}_{\mathrm{t}}\right)}{\mathrm{H}}$ qui concerne la vitesse d'évaporation d'une masse de liquide au repos: S étant la surface libre du liquicle. $\mathrm{K}$ un coefficient lié à la vitesse de l'air. $\mathrm{F}_{t}$ la tension maxima de vapeur d'eau à la température $t$. $f$ sa tension réelle à la même température, $\mathrm{H}$ la pression atmosphérique.

(1) Si ce n'est que les hygromètres à cheveu sont des appareils peu fidèles que nous avons étalonnés le plus souvent possible. 
Nous avons éliminé les coefficients $\mathrm{K}$ et $\mathrm{S}$ étant donné qu'il s'agissait de mesures relatives et que l'influence du vent, en forêt, sur les deux versants de la vallée est certainement comparable, quelle que soit l'altitude (il n'en serait pas de mème au fond du talweg où se localise la brise de vallée et dans l'étage alpin, où ne joue plus la protection des arbres). En choisissant une associationréférence, nous avons pu établir, pour cette association, une courbe du déficit de saturation (égal, par définition à $\mathrm{F}_{\mathrm{t}}-\mathrm{f}_{\mathrm{t}}$ soit $\mathrm{f}-\mathrm{F}_{\mathrm{t}}$ (1 - e), e étant le degré hygrométrique), qui traduisait le pouvoir évaporant de l'air, à $1,50 \mathrm{~m}$ par exemple, si les appareils étaient placés à cette hauteur, pour cette association.

Lorsque la comparaison a porté sur des associations à la même altitude, le facteur $\mathrm{H}$ a été, de ce fait, éliminé : lorsque cette comparaison a porté sur des associations situées à des altitudes différentes, nous avons, pour chacune d'elles, multiplié le déficit de satu$\mathrm{Hr}$

ration le concernant, par le coefficient $\frac{}{\mathrm{H}}, \mathrm{Hr}$ étant la pression

atmosphérique régnant au niveau de l'association-référence, $\mathrm{H}$ celle régnant au niveau de l'association étudiée. Nous avons admis que les variations, dans le temps, de la pression atmosphérique qui sont absolument négligeables lorsqu'elles sont régulières, étaient les mêmes quelle que soit l'altitude, nos comparaisons ayant toujours porté sur des associations voisines dans l'espace.

Sans doute le pouvoir évaporant relatif ainsi défini n'intègre-t-il pas tous les facteurs régissant l'économie de l'eau, de l'air et du sol, mais il nous a paru devoir être retenu pour donner une image des conditions locales auxquelles sont adaptées les espèces qualifiées de xérophiles, mésophiles, méso-hygrophiles, surtout si on le rapproche du régime, également local, des températures de l'air et du sol. 


\section{I.5. - METHODES D'ETUDE AUXOMETRIQUE}

Bien que l'étude des forèts faisant l'objet du présent mémoire soit essentiellement phytosociologique et écologique, il nous a paru intéressant, en utilisant les résultats obtenus lors de l'élaboration des Aménagements, de donner un aperçu de leur structure et de leur production. Il s'agit là, par conséquent, non d'une analyse absolument exhaustive, qui eût exigé la mise en cuvre de moyens utilisés par les Stations de Recherches forestières, mais d'une première approximation assise sur des données assez solides, pour ne pas être infirmée par les recherches plus poussées qui mériteront d'être faites ultérieurement.

On sait que la comparaison des productions de diverses associations forestières doit être conduite avec une certaine circonspection, car ces productions dépendent, en effet, de divers paramètres, rappelés récemment par VINEY (R.) (1955), et dont il importe de dire quelques mots.

Pour une futaie jardinée idéale, et dans des conditions stationnelles données, la production est constante, aux fluctuations près dues aux coupes (pour une parcelle) et à celles dues à la variation des facteurs météorologiques. Pour une futaie équienne, toujours dans des conditions stationnelles données, la production varie sensiblement avec l'âge, et les tables de production montrent que cette production, disons instantanée, passe par un maximum aux environs de 80 ans (1), tandis que la production moyenne depuis l'origine voit son maximum décalé par rapport à celui de la production instantanée.

$\mathrm{Si}$ donc l'on veut comparer la production de deux forêts, il faut évidemment s'adresser soit à des peuplements d'âge connu, soit à des peuplements où s'observent, de façon équilibrée, tous les âges.

Si l'on observe que, dans les montagnes françaises - et la HauteMaurienne ne fait pas exception à la règle - la futaie jardinée idéale, de structure exponentionnelle permanente, se rencontre bien rarement - et ce, pour de multiples raisons dont la principale tient à l'action humaine - on conçoit que la production instantanée soit liée non seulement aux conditions stationnelles, mais aussi aux structures; c'est la raison pour laquelle nous avons toujours accolé

(1) Un tel chiffire n'est donné ici que pour fixer les idées. Il varie, évidemment avec l'essence et les conditions stationnelles. 
la production de chaque type de forêt à sa structure, cette dernière étant traduite par une courbe de fréquence des diamètres, à l'hectare de surface boisée (2). On pourra penser que, dans ces conditions, les chiffres de production obtenus ont une signification assez restreinte; il en serait ainsi, évidemment, s'il s'agissait de peuplements appartenant à une classe d'âge bien délimitée: la production trouvée ne correspondrait qu'à l'une des données d'une table de production inconnue, elle, et qui intéresserait tous les âges.

En fait, sans être idéalement jardinés, les peuplements en cause sauf exceptions dont il sera question ci-après, ont une structure relativement stable, car nos calculs n'ont pas porté sur une parcelle, mais sur plusieurs dont l'équilibre structural est comparable à celui d'une série forestière normale.

Quant aux méthodes suivies, elles diffèrent quelque peu suivant que nous avons eu affaire à des associations couvrant des parcelles entières ou à des associations ne couvrant que des portions de parcelles.

Dans le premier cas, nous avions un inventaire complet des arbres. Nous aurions pu calculer la production par comparaison d'inventaires. Malheureusement, une telle méthode implique que l'on soit sûr des deux inventaires, ce qui n'est pas le cas en Maurienne, où les événements de guerre ont singulièrement perturbé la comptabilité des produits exploités. Nous avons donc adopté la méthode des sondages à la tarière, effectués par groupes d'arbres sur des surfaces non exactement mesurées et selon un quadrillage non strictement systématique (3). A partir des carottes de sondage, nous avons calculé la moyenne des accroissements de vingt ans (la rotation des coupes étant de vingt ans), par catégorie de diamètre; lorsque ces moyennes des $\mathrm{dD}$ se sont avérées être en régression linéaire par rapport aux diamètres, nous avons établi des droites de régression; nous sommes passés aux dV en utilisant une formule simple, sans doute un peu schématique, mais employée fréquemment, notamment par Meyer (H.-A.) $(1942,1952)$ aux Etats-Unis et par Loetsch (F.) (1953) en Allemagne.

Dans le cas où les associations se présentaient sous forme fragmentaire et n'occupaient pas, par conséquent, de parcelles entières

(2) Pour tenir compte dans toute la mesure du possible des vides (rochers, etc...) nous avons rapporté le chiffre de production à la seule surface boisée, appelée par les forestiers: surface régulatrice.

(3) L'idéal eût été de sonder les arbres de placeaux circulaires de surface donnée (5 ares par exemple), selon un quadrillage géométrique. On eût pu, soit par mesure directe, ce qui est la meilleure solution biométrique, soit par référence à un tarif et application d'une formule donnant $\mathrm{dV}$ en fonction de $\mathrm{dD}$, calculer, par placeau la somme des dV de chaque arbre et avoir ainsi un dV correspondant à chaque cercle échantillonné, d'où un calcul de variance et d'erreur probable. Notre méthode est, comme nous l'avons dit, moins rigoureuse car nous avons été dans l'obligation d'utiliser des données obtenues, par des procédés moins stricts, lors de l'élahoration des aménagements. 
(pineraie xérophile sur sol calcimorphe; pineraie acidiphile sur ranker à moder), nous avons délimité sur le terrain des échantillons de surface définie où nous avons sondé tous les arbres. Il est bien entendu que les échantillons ainsi délimités ne représentent qu'un aspect de l'association en cause, mais c'était, et de loin, le plus fréquent. On conçoit dès lors que, dans ce cas particulier, les productions trouvées aient une valeur plus restreinte, d'autant qu'elles se trouvent liées à une structure régulière, les peuplements correspondants étant, assez curieusement d'ailleurs, très équiennes, ce que nous ont confirmé des sondages exécutés à la base des arbres, jusqu'à leur moelle. 
DEUXIËME PARTIE

\author{
Caractères généraux \\ de la Haute-Maurienne
}



Ce qui importe, c'est moins de faire une analyse très fine des caractéristiques du climat et des roches-mères, et des modalités de l'action humaine, que de dégager les traits écologiques d'ensemble qui donnent son originalité à cette partie de la vallée, et qui permettent de prendre une vue générale de l'ambiance climatique et humaine, dans laquelle se sont développées, et se développent, les associations forestières étudiées.

\section{II.1. - LE CLIMAT}

\section{II.1.1. - Les précipitations.}

\section{II.1.1.1. - Les moyennes annuelles.}

Une carte des isohyètes annuelle des Alpes (1) montre que la Maurienne, dans les Alpes du Nord, est vraiment le domaine des précipitations déficientes, et constitue bien ce que ONDE (H.) (1938) a appelé a un ilot de sécheresse $n$. Cette pénurie pluviométrique qui tient $\alpha$ à la présence de barrages successifs tendus sur le trajet des vents humides venus de l'Atlantique $»$, dessine des zones concentriques en forme d'ellipses aplaties.

Les observations de 1889-1910 [SANson (J.) (1945)], donnent pour Valmeinier, à plus de $1500 \mathrm{~m}$ d'altitude: $812 \mathrm{~mm}$-; pour Modane $(1060 \mathrm{~m})$ : $639 \mathrm{~mm}$-; pour Lanslebourg $(1410 \mathrm{~m}): 709$ mm -; pour Bessans (1710 m): $714 \mathrm{~mm}$ - ; pour Bonneval-surArc, enfin $(1790 \mathrm{~m}): 821 \mathrm{~mm}$.

Les observations de 1934-1950, traduites par Balseinte (R.) (1955), dans un profil que nous reproduisons ci-après, donnent des chiffres plus élevés: Saint-Jean-de-Maurienne $(550 \mathrm{~m}): 680 \mathrm{~mm}$; Valmeinier : $854 \mathrm{~mm}$; Le Thyl (1 $360 \mathrm{~m})$ : $952 \mathrm{~mm}$; Saint-Martinla-Porte $(820 \mathrm{~m}): 763 \mathrm{~mm}$; La Praz $(990 \mathrm{~m}): 730 \mathrm{~mm}$; Aussois $(1490 \mathrm{~m})$ : $710 \mathrm{~mm}$; Termignon $(1290 \mathrm{~m})$ : $720 \mathrm{~mm}$; Bessans $(1710 \mathrm{~m}): 820 \mathrm{~mm}$; Bonneval $(1790 \mathrm{~m}): 947 \mathrm{~mm}$ (2).

(1) Celles dressées par Blanchard (R.) ,1956) ou Gaussen (H.) (1951), par exemple.

(2) Les chiffres que nous ont fournis les services de l'E.D.F. pour la période 1949-1958 sont les suivants: Le Thyl : $946 \mathrm{~mm}$; Orelle-Prémont $(830 \mathrm{~m})$ : $719 \mathrm{~mm}$; Avrieux $(1100 \mathrm{~m}): 517 \mathrm{~mm}$; Termignon: $646 \mathrm{~mm}$; Bessans : $850 \mathrm{~mm}$. Le chiffre d'Avrieux nous a été fourni par l'O.N.E.R.A., à Avrieux.

Certaines de ces données sont supérieures à celles de BALSEINTES (période 1934-1950), d'autres inférieures. 
Comme on le voit, c'est entre Saint-Jean-de-Maurienne et Termignon que se situe, au fond du talweg, la zone la plus sèche. Ce " creux pluviométrique " est d'ailleurs caractérisé par la raréfaction du nombre de jours de pluie: 96 à Saint-Jean-de-Maurienne, 81 à la Praz, 83 à Modane (3). On peut méme préciser, si l'on se réfère aux données citées en note, que c'est entre Modane, à la sortie des gorges houillères et Termignon, vers Avrieux sans doute, que doit être fixé le pôle de sécheresse mauriennais (fig. 2).

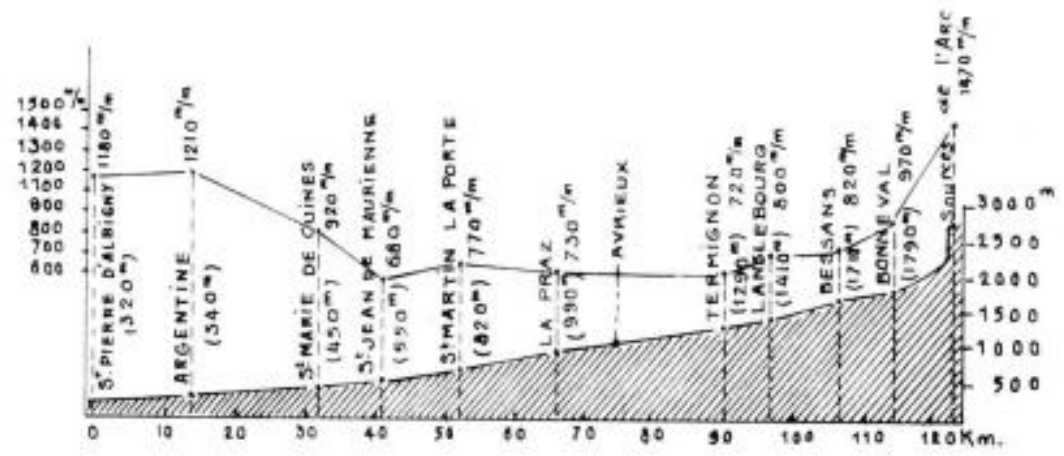

Fig. 2.

Les moyennes de précipitations.

Légende. - Le profil des moyennes annuelles des précipitations, dressé par BALSEINTE pour la période 1934-1950, fait apparaitre un creux pluviométrique entre la Praz et Termignon - le chiffre de Saint-Jean-de-Maurienne étant suspect.

Si l'on se réfère aux chiffres de la décennic 1949-1958, il semble bien que le creux puisse être plus précisément localisé autour d'Avrieux.

Le relèvement qui s'observe après Termignon, et que mettent en évidence, aussi bien la carte détaillée des isohyètes établie par ONDE (H.) (1938), que le profil déjà signalé dressé par BALSEINTE, est dû essentiellement à l'action de la lombarde italienne qui s'est chargée d'humidité sur l'Adriatique.

Les variations avec l'altitude appellent quelques commentaires: ONDE (H.) (1938) avait abouti à deux conclusions intéressantes: d'une part, la très faible valeur, en général, du gradient pluviométrique qui, de $1,15 \mathrm{~mm}$ entre Bessans et Bonneval, dans une zone exceptionnelle très arrosée par les averses padanes, passe à 0,54 $\mathrm{mm}$ à la hauteur de Lanslebourg et tombe jusqu'à $0,06 \mathrm{~mm}$ dans la vallée des Arves, au sud de Saint-Jean-de-Maurienne; d'autre part, son augmentation plus rapide au delà de $2000 \mathrm{~m}$, " à partir d'un niveau où la circulation atmosphérique est déjà beaucoup moins entravée " [ONDE (H.) (1938)].

(3) Cités par Blanchard (R.) (1945). 
En se référant aux résultats donnés par des totalisateurs placés à haute altitude, et en les recoupant avec ceux obtenus par la méthode dite hydrométrique, ONDE trouvait, pour le Haut-Arc, les chiffres suivants: $0,25 \mathrm{~mm}$ de 1000 à $2000 \mathrm{~m}-; 1 \mathrm{~mm}$ de 2000 à $3500 \mathrm{~m}$-. Comme il le notait, les observations de 1934-1937 (3 ans) dans le bassin du Saint-Benoit aboutissaient à des résultats concordants: Aussois $(1490 \mathrm{~m})$ dans l'étage du Pin sylvestre a reçu: $814 \mathrm{~mm}$; Plan d'Amont (2 $100 \mathrm{~m})$, dans l'étage du Pin Cembro: $928 \mathrm{~mm}$ - (gradient: $0,18 \mathrm{~mm}$ ).

On peut se demander, toutefois, si les précipitations en altitude ne sont pas quelque peu sous-estimées: en comparant, mois par mois, les précipitations de deux postes situés sur la même verticale, Orelle $(830 \mathrm{~m})$ et Bissorte $(2120 \mathrm{~m})$. et celles des postes du Car-

\begin{tabular}{|c|c|c|c|c|c|c|c|c|c|c|c|c|c|c|}
\hline \multirow[b]{2}{*}{ 1. } & \multirow[b]{2}{*}{ B10.00rte $(2120 \mathrm{~m})$} & J. & 7. & n. & A. & s. & J. & Jt & A. & 8. & 0. & N. & D. & Totre. \\
\hline & & 63 & 67 & 28 & 48 & 88 & 105 & 61 & 100 & 108 & 70 & 68 & 59 & 865 \\
\hline & Or.116-Primont (830a) & 70 & 81 & 31 & 47 & 53 & 67 & 44 & 71 & 54 & 53 & 77 & 6: & 712 \\
\hline 2) & Carreles $(2020 \mathrm{~m})$ & 84 & 103 & 29 & 48 & 55 & 90 & 49 & 76 & 70 & 68 & 48 & 92 & 822 \\
\hline & Lnecoole (1 $500 \mathrm{~m})$ & 90 & 111 & 27 & 44 & 43 & 78 & 38 & 57 & se & 62 & 50 & 92 & 790 \\
\hline
\end{tabular}

1) Moyennes $1948-1958$ incl.

2) Moyennes 1952-1958 incl.

relet $(2020 \mathrm{~m})$ et d'Aussois $(1500 \mathrm{~m})$, on constate que de novembre à mars les précipitations sont plus fortes à basse altitude qưà haute altitude. II semble done que les quantités de neige pour les postes à altitude élevée pêchent par défaut. Consulté sur ce problème, M. BALSEINTE nous a fait comnaitre que, si l'on se réfère aux bassins versants de l'Isère en amont du Pont du Laisinant $\left(45 \mathrm{~km}^{2}\right)$ - altitude moyenne $2700 \mathrm{~m}$ - et de P'Averole, affluent de 1 Arc $\left(46 \mathrm{~km}^{2}\right)$ - altitude moyenne $2900 \mathrm{~m}$ - on constate que la lane d'eau anuuelle moyenne écotulée par l'Isère pour la période 1951-1956 a été de $1274 \mathrm{~mm}$ et par l'Averole (période 19481956) de $1372 \mathrm{~mm}$. En adoptant des coefficients d'écoulement égaux à l'unité, on aurait donc à $2700 \mathrm{~m}$ (pour la Haute Tarentaise): $1275 \mathrm{~mm}$ et à $2900 \mathrm{~m}$ (pour la Haute-Maurienne): 1370 $\mathrm{mm}$ - ; par extrapolation linéaire - et il est bien difficile d’adopter une autre hypothèse — on aurait $950 \mathrm{~mm}$ de précipitations pour le Carrelet et $1000 \mathrm{~mm}$ pour Bissorte - chiffres supérieurs à ceux précédemment fournis, et qui permettent, par un calcul proportionnel portant sur les seules précipitations hivernales, d'avoir des résultats apparemment plus cohérents.

Quelles que soient d'ailleurs les réserves que l'on puisse faire sur les données concernant les précipitations à haute altitude, un fait 
est acquis: c'est que ces précipitations, vers $2000-2200 \mathrm{~m}-$ restent faibles, puisqu'elles ne dépassent pas 1000 à $1100 \mathrm{~mm}$. Au caur de la zone intra-alpine manriennaise, les étages silvatiques connaissent donc une pénurie cerlaine des précipitations.

\section{II.1.1.2. - Les régimes moyens.}

BÉnÉvent (E.) (1926), dans son étude sur le climat des Alpes françaises, avait divisé ces dernières en quatre grands secteurs d'après le régime de leurs précipitations: le secteur à régime méditerranéen, celui à régime de marge méditerranéenne, celui à régime continental, celui, enfin, à régime à tendance continentale, auquel appartenait la Maurienne. L. " indicatif " saisonnier de ce secteur était le stuivant: Automne - Eté - Printemps - Hiver.

Les récents travaux de BLANCHARD (R.) (1956) montrent que, dans les Alpes du Nord, le régime à tendance continentale est "si profondément perturbé, qu'en fait, il n'existe plus n, et qu' " il s'est donc produit, au cours des quarante dernières années, tune véritable offensive des précipitations hivernales $n$, attribuées à l'adoucissement des températures de cette saison (5).

Si l'on se réfère, en effet, aux tableaux ou aux cartes dressés par Balseinte (R.) (1955), ou par Blanchard (R.) (1956), on s'aperçoit que l'hiver, en Maurienne, se place, actuellement, soit en première, soit en seconde position, ceci pour les années " normales n : il rétrograde souvent, par contre, à la $3^{e}$ place, sinon à la dernière, pour les années de faible pluviosité. L'indicatif n'est donc plus celui d'un régime à tendance continentale: A.E.P.H., mais soit A.H.E.P., typiquement océanique, soit, le plus souvent: H.A.E.P.. régime que BALSEINTE (R.) (1955) propose d'appeler * alpin $n$, " à maximum d'hiver, expliqué par le relief élevé de la masse montagneuse, analogue au régine maritime $»$. C'est. en tout cas, le régime moyen des stations de la Haute-Maurienne, pour la période décennale allant de 1949 ì 1958.

Il faut noter toutefois que Г'utilisation d' " indicatifs a ne doit pas faire oublier qu'ils n'ont pas, en soi, une très grande valeur s'ils ne sont pas accompagnés de "coefficients de pluviosité ", mensuels ou saisonniers, qui, seuls, traduisent la dominance quantitative relative, de telle ou telle saison ou de tel ou tel mois; on se rend compte ainsi, que pour ces dernières années, diverses stations de Haute-Maurienne présentent un "creux " très net en mars, déjà rencontré d'ailleurs par BALseinte pour la période 1939-1950 et

(5) Ce problème a fait l'objet de plusieurs études, dont BALsernte ( $R$.) a rendu compte dans la Revue de Géographie Alpine (1955), Il semble bien que l'augmentation des précipitations hivernales soit due à une amélioration des mesures nivométriques (augmentation fictive, par conséquent) et, en partie, à un changement climatique. 
d'un autre en juillet, lequel coïncide avec un maximum de température (6) (fig. 3).

Il faut noter surtout que ce climat océanique est tout à fait particulier, parce qu'il est sec et que les hivers y sont froids. EMBERGER (L.) (1942), dans son « Projet d'une classification biogéographique

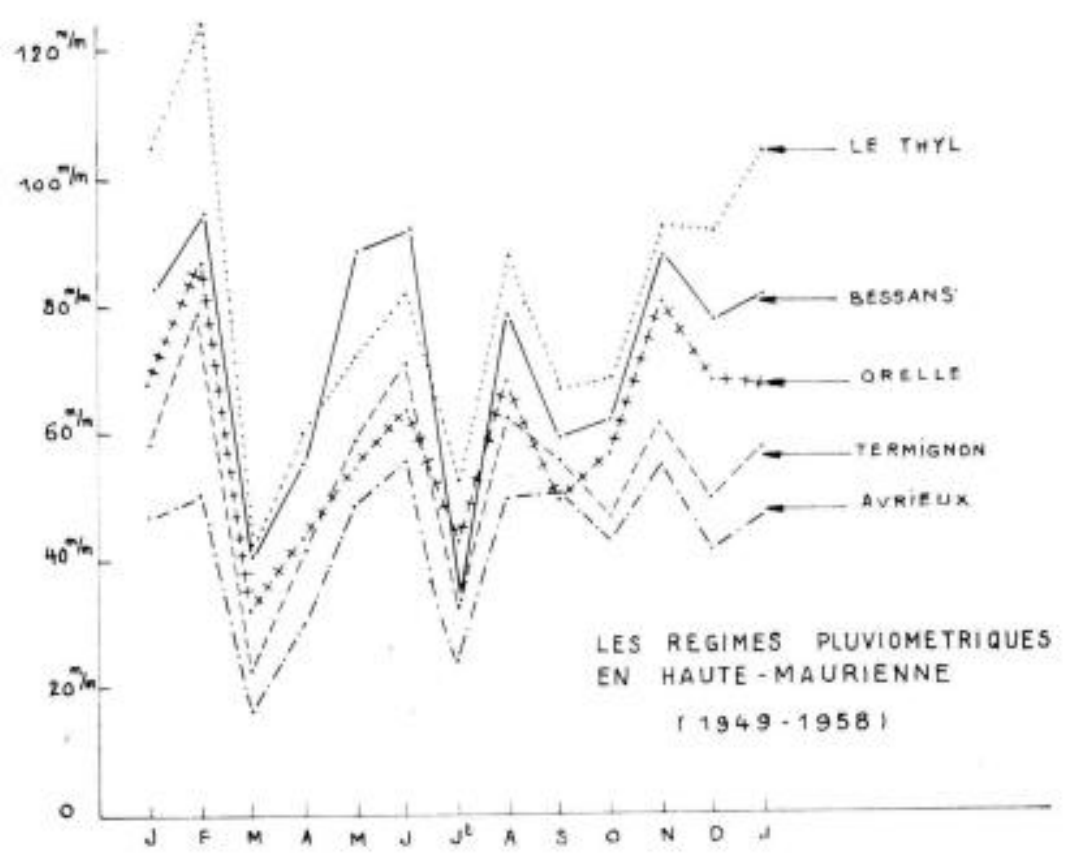

Fig. 3.

Les régines moyens des précipifations.

(1949-1958)

\begin{tabular}{|c|c|c|c|c|c|c|c|c|c|c|c|c|c|c|}
\hline IS øux & Altit. & J & $\boldsymbol{P}$ & x & A & M & $\mathrm{J}$ & $\mathrm{J}^{\mathrm{t}}$ & A & s & 0 & s & D & sot. \\
\hline Lo Thyl & $1380 \mathrm{~m}$ & 105 & 125 & 41 & 60 & 72 & 82 & 53 & 88 & 67 & 70 & 93 & 90 & 946 \\
\hline $\begin{array}{l}\text { Oralle } \\
\text { Pressont }\end{array}$ & $830 n$ & 68 & 87 & 32 & 44 & 5) & 64 & 43 & 69 & 50 & 57 & $\mathrm{~B} 1$ & 69 & 719 \\
\hline Arritoux & $11 \mathrm{CCa}$ & 47 & 50 & 17 & 30 & 49 & 26 & 25 & jo & 51 & 43 & 55 & 42 & 517 \\
\hline Ternienod & $1290 \mathrm{n}$ & 28 & 79 & 23 & 43 & 39 & 71 & 35 & 63 & 26 & 47 & 62 & 50 & 646 \\
\hline Bounans & 17400 & 86 & $\underline{25}$ & 37 & 55 & dy & 92 & 33 & 79 & 29 & 62 & 89 & 78 & 850 \\
\hline
\end{tabular}

(6) Voir, ci-après, le c diagramme ombrothermique s d'Avrieux. A noter que la brièveté de la période d'observations accentue les crochets du profil. 
des climats $»(7)$, s'est d'ailleurs posé la question de savoir si des climats météorologiquement océaniques - mais très froids en hiver - ne devraient pas être rattachés du point de vue bioclimato-

\section{COSFEICIENTS \\ SMISONNIERS}

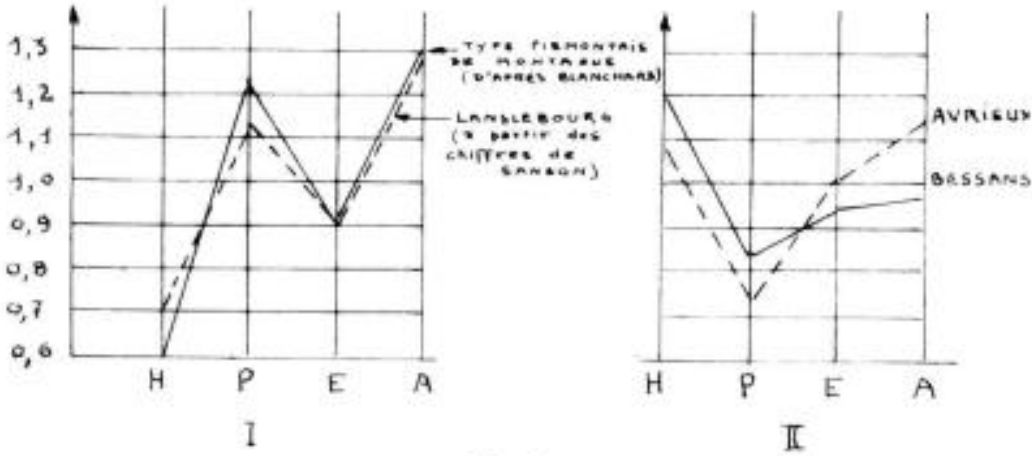

FIG. 4.

Les indices saisonniers.

Légende. - Des chiffres fournis par Sanson pour Lanslebourg (période 1891-1930), il apparaissait que le climat de cette localité s'apparentait de très près au elimat piémontais montagnard, défini par BLANCHAkD (R.) (1956), type A.P.E.H., qui n'est pas sans présenter dse affinités méditerranéennes (I).

Au cours de la décennie 1949-1958, et en l'absence de mesures concernant Lanslebourg, on constate que le régime moyen des précipitations en HauteMaurienne ne correspond plus à ce que BENEVENT avait appelé régime à tendance continentale (indicatif: A.E.P.H.) et que les influences piémontaises et briançonnaises mises en relief par ONDE se sont singulièrement atté nuées (II).

Le régime est devenu ce que BM.SEINTE a appelé * alpin \$ - nous préférerions le terme d" " alpien s - c'est-à-dire \& analogue au régime océanique (A.H.E.P.) mais à maximum d'hiver, expliqué par le relief élevé de la masse montagneuse (H.A.E.P.) *.

\begin{tabular}{|c|c|c|c|c|}
\hline Looslit tos & Printexp $(P)$ & $8+6(z)$ & Au torne (A) & Hiver (B) \\
\hline Le rayl $\ldots \ldots$ & 0,72 & 0,93 & 0,98 & 1,38 \\
\hline ore1le $\ldots .$. & 0,73 & 0,97 & 1,05 & 1,21 \\
\hline Avrioux ..... & 0,74 & 1,01 & 1,16 & 1,09 \\
\hline Terne1gnon .... & 0,77 & 1,04 & 1,16 & 1,09 \\
\hline Bessans $\ldots \ldots$ & 0,25 & 0,95 & 0,99 & 1,22 \\
\hline
\end{tabular}

(7) Oủ il cite, d'ailleurs, Saint-Jean-de-Maurienne et Modane à propos des pluviosités de type océanique. 
logique aux climats continentaux, puisque, dans les deux cas, la saison de végétation est l'été.

Pour répondre à une telle question, il est indispensable de ne pas isoler le régime des précipitations qui ne constitue qu'un aspect du climat, d'autres caractéristiques et de l'accoler, en particulier, à celui des températures. C'est ce qui sera fait ci-après.

Qu'en est-il, depuis 1' " offensive des précipitations hivernales " et de l" "océanisation " du régime des précipitations, des influences briançonnaises et piémontaises que ONDE (H.) (1938) avait mises en relief en Haute-Maurienne, au point de distinguer, dans la zone à tendance continentale de BÉNÉVENT, un " sous-secteur mattriennais " et qui apparaissent si nettement dans le régime des précipitations de Lanslebourg déduit des chiffres donnés par SANSON (J.) (1945) (fig. 4) ?

Il n'est pas très facile de répondre à une telle question, d'autant que le poste de Lanslebourg a cessé de fonctionner, et que celui du Mont-Cenis, marginal en tout état de cause, n'est suivi sérieusement que depuis 1957.

On peut peut-être retenir des courbes des années 1948 à 1959 que les intumescences de mai, juin et de novembre, le creux de juillet - particulièrement nets pour Bessans (fig. 3) traduisent cette influence. Mais elle ne parait plus être celle que ONDE avait analysée, car la tendance à l'égalisation due à l' " invasion hivernale n s'est étendue, comme l'a souligné Blanchard (R.) (1956) sur une partie du Briançonnais.

Il n'est pas sans intérêt de rappeler, enfin, que le climat intraalpin se " continentalise ", lorsque l'on passe de la Maurienne en Valais, puis en Engadine, puis plus à l'Est encore, dans la région de Loeben en Autriche (fig. 5); de toutes les vallées sèches des Alpes, la Maurienne apparait ainsi comme la plus " atlantique n, ce qui n'a d'ailleurs rien d'étonnant.

\section{II.1.1.3. - La variabilité des précipitations.}

Contrairement à ce qu'on aurait pu attendre (8), la Maurienne ne parait pas présenter une plus grande irrégularité annuelle des précipitations que les Préalpes humides (9), alors que dans les

(8) PéguY (Ch.-P.) (1961, p. 198) : « On constate, d'une façon générale, que l'irrégularité des pluies tend à être plus forte dans les pays teintés d'aridité, comme si la variabilité des totaux pluviométriques annuels accompagnait presque inévitablement leur médiocrité. ?

(9) Si l'on se réfère aux travaux de Blanchard (R.) (1956, p. 159), Il faut noter toutefois que BLANCHARD n'a pas adopté rigoureusement la même période de référence pour les diverses stations étudiées, ce qui, dans le cas particulier, peut fausser sensiblement certains résultats (le coefficient absolu d'irrégularité est en effet le rapport entre les précipitations de l'année de pluviosité maximum et celles de lannée de pluviosité minimum). 
Alpes du Sud, par contre, les coefficients d'irrégularité sont très élevés (entre 3 et 4 contre 2 en Maurienne).

Quant à l'irrégularité saisonnière, on notera avec intérêt que le printemps est particulièrement capricieux (coefficient de 8,1 à Aussois pour la décennie 1941-1950), beaucoup plus que l'été (coefficient 2,6 ); d'une année sur l'autre, par conséquent, la germination des graines et le développement des plantules vont se trouver soumises à des chances très inégales.

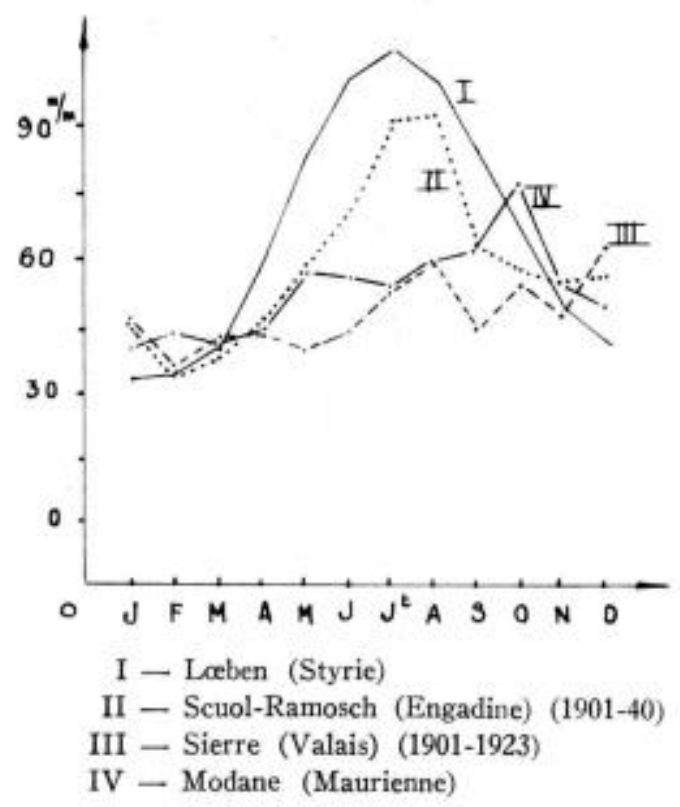

Fig. 5.

Les régimes des précipitations dans diverses régions des Alpes.

Légende. - Quels que soient les chiffres des dernières décennies - et dont on a dit quíils différaient de ceux correspondant au début du siècle le climat de la Haute-Maurienne apparait comme plus océanique que celui du Valais (intumescence en juillet-aoutt) et, a fortiori, que ceux de l'Engadine et de la Styrie qui sont nettement continentaux.

[Chiffres de Loeben d'après Braun-Blanguet (J.) (1961) - de SucolRamosh d'après UtringrR (H.) (1949 - de Sierre d'après Boute (M.) (1950) - de Modane d'après BÉNEvent (E.) (1926).

\section{II.1.1.4. - La neige.}

Les quatre courbes de la figure 6 , établies à partir de chiffres trouvés dans la thèse de ONDE $(H).(1938,414)$, permettent de se faire une idée de l'importance de la neige qui tombe en Haute- 
Maurienne, lorsqu'on remonte la vallée, depuis Saint-Martin-laPorte jusqu'à Bessans.

Quant aux coefficients de nivosité (rapport des chutes nivales exprimées en eau de fusion, aux précipitations totales), ils augmentent à mesure qu'on s'élève le long du talweg, pour atteindre $36,1 \%$ à Bessans.

Hauteurs de neige.

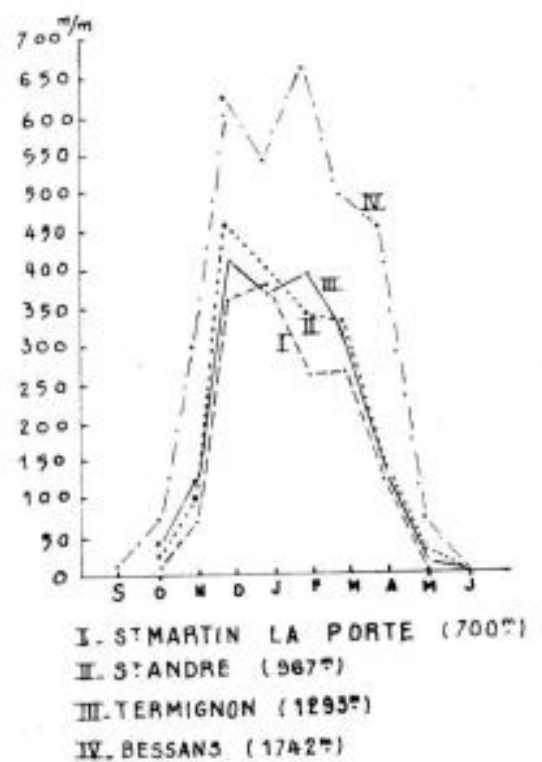

Durées d'enneigement.

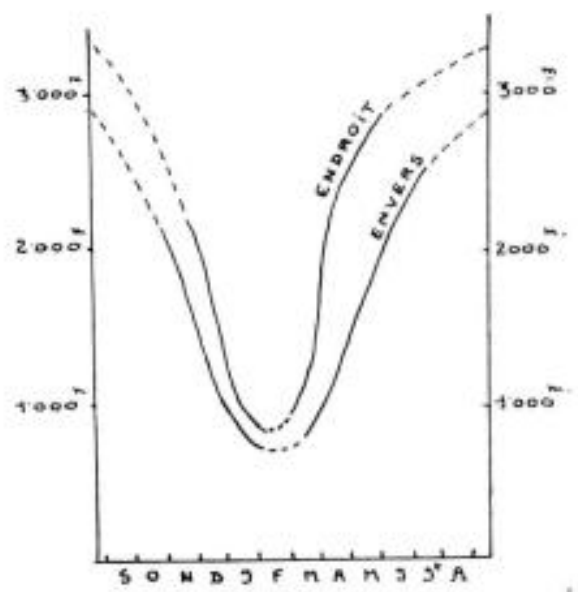

Fig. 6.

L'enneigement.

Légende, - S'il est difficile de mettre en évidence une influence écologique nette du régime proprement dit des précipitations neigeuses, il est, par contre, aisé de voir combien la durée de l'enneigement, selon l'exposition, peut avoir de conséquences, non seulement en hiver (à exposition sud, les risques de gelées hivernales sont plus grands) mais au printemps où le démarrage de la végétation dépend étroitement de l'exposition et de la durée d'enneigement. Dans un couloir à avalanches, où le culot de neige fond tardivement, nous avons vu des amélanchiers fleurir avec un retard de deux mois, par rapport à leurs congénères placés en conditions normales.

La durée d'enneigement est une notion intéressante à retenir, car elle intègre plusieurs facteurs interdépendants: importance des précipitations solides, températures, exposition, pente, durée d'insolation $(10)$.

(10) En particulier, une carte à grande échelle donnant la c chronologie de déneigement $\$$ des diverses associations d'une zone donnée, serait des plus instructives. J.-L. RICHARD (1961) en a donné un bon exemple, as Creux du Van, dans le Jura Suisse. 
Dans les Alpes, elle présente, comme l'a souligné Peguy (Ch.-P.) (1961), une très grande variabilité annuelle; nous avons cependant essayé de dégager des moyennes (11), traduites par deux courbes l'une concernant l'envers et l'autre l'endroit.

Ces courbes montrent la nette différence existant entre les durées d'enneigement des deux versants d'exposition opposée, le déneigement rapide, du bas en haut des versants, de l'Endroit, celui, plus progressif de l'Envers.

On ne saurait passer sous silence, enfin, le rôle des avalanches dans la structure du paysage forestier. Les photos des pages 709-710 montrent que les couloirs d'avalanches intéressent aussi bien, et plus encore peut-être, les versants sud que les versants nord; au delà de Bessans, vers Bonneval, un régime permanent et étendu de coulées de neige empêche pratiquement l'installation de la forêt sur les pentes non occupées par l'homme.

\section{II.1.2. - L'humidité atmosphérique.}

Grâce au centre météorologique d'Avrieux qui comporte un poste de mesures hygrométriques (à thermomètres sec et mouillé), on peut se faire une idée de l'humidité atmosphérique à $9 \mathrm{~h}$ du ma-
.... Bg St Maurice
I. $9 \mathrm{~h},-$ II, $15 \mathrm{~h}$.
Avrieux
I. 9 h. - II. 15 h.

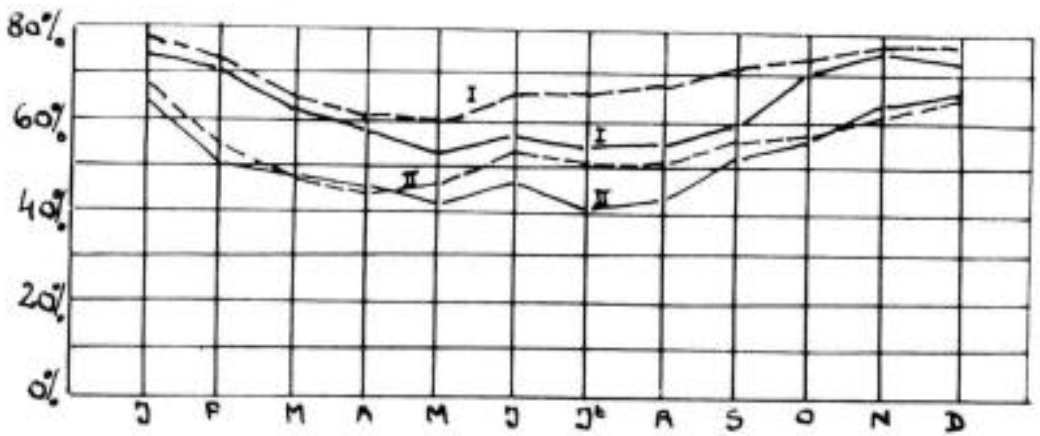

FIG. 7.

L'humidité atmosphérique.

Légende. - L'atmosphère de Bourg-Saint-Maurice, à $860 \mathrm{~m}$ d'altitude en Tarentaise, présente un degré hygrométrique, à 9 heures et à 15 heures, presque constamment plus élevé que celui d'Avrieux, en Maurienne, à $1100 \mathrm{~m}$. Les différences les plus fortes se manifestent au cour de la saison de végétation, c'est-à-dire de mai à septembre.

(11) Grâce aux précieux renseignements fournis par Chakvoz, Chef de district des Eaux et Forêts à Termignon, que nous remercions ici. 
tin et à 15 h régnant au cour de la zone de sécheresse de la Maurienne (12).

On constate qu'en moyenne (pour la période 1958-1963), l'état hygrométrique à $9 \mathrm{~h}$ ne dépasse jamais $80 \%$, ce qui est peu et

\begin{tabular}{|c|c|c|c|c|c|c|c|c|c|c|c|c|c|}
\hline Looal1 t6. & Heures: & $\mathrm{J}$ & 7 & s & $A$ & $x$ & $\mathrm{~J}$ & $\mathrm{~s}^{\mathrm{t}}$ & $\mathrm{A}$ & 31 & 0 & I1 & $D$ \\
\hline \multirow{2}{*}{$\begin{array}{l}\text { SOUHO-St-MAU- } \\
\text { RICE }\end{array}$} & $9 \mathrm{~b}$ & 78,8 & 73,1 & 65,5 & 61,4 & 60,6 & 65,2 & 66,0 & 67,9 & 70,9 & 73,8 & 77,5 & 77,3 \\
\hline & $15 \mathrm{~B}$ & 68,1 & 54,7 & 47,8 & 43,7 & 46,6 & 52,9 & 50,9 & 51,4 & 56,3 & 59,3 & 62,5 & 66,0 \\
\hline \multirow[t]{2}{*}{ AVHIOJX } & $9:$ & 74,3 & 70,8 & 62,9 & 28,7 & 23,1 & 26,9 & 54,6 & 55,4 & 60,1 & 70,9 & 76,9 & 73,6 \\
\hline & $15 \mathrm{~h}$ & 64,4 & 50,1 & 47,9 & 44,7 & 41 , & 46,4 & 40,5 & 41,9 & 51,2 & 57,0 & 64,8 & 67,7 \\
\hline
\end{tabular}

que la moyenne des minimums durant la saison de végétation (de mars à septembre) se situe entre 40 ot $50 \%$.

Ces chiffres sont à rapprocher de ceux de Bourg-Saint-Maurice, en Tarentaise, car ils confirment ce que l'on savait déjà du climat des deux vallées, par l'analyse de la flore et de la pluviosité, à savoir que la Maurienne est plus sèche que la Tarentaise.

\section{II.1.3. - Les températures.}

\section{II.1.3.1, - Les moyennes annuelles.}

La Maurienne n'offre aucune originalité profonde en ce qui concerne les moyennes annuelles. Nous aurons d'ailleurs à les accoler aux moyennes des précipitations et il n'y a pas lieu d'insister.

\section{II.1.3.2. - Les régimes.}

Les courbes des variations mensuelles de températures - vraies ou réduites au niveau de la mer - étudiées en Maurienne par Bénévent (E.) (1926, Onde (H.) (1938) et Blanchard (R.) (1943), montrent que si l'hiver est rude, de printemps y est plus chaud, par exemple, que dans la Combe de Savoie et, en tout cas, que son démarrage y est plus brutal.

Ces deux derniers faits doivent être ainsi traduits: les Moyenne et Haute-Maurienne, par leurs températures des mois de mai, juin, juillet, août - qui constituent la saison utile - rattrapent " le temps perdu " depuis le début du printemps. ONDE (H.) (1938) a montré, en outre, que les poussées thermiques sont plus régulières

(12) Entre $12 \mathrm{~h}$ et $15 \mathrm{~h}$ environ, l'état hygrométrique journalier passe par un minimum assez étalé. La courbe de $15 \mathrm{~h}$ de la fig. 7 correspond donc approximativement à la moyenne des minimums. 
sur les flancs des versants et à haute altitude, qu'au fond de la vallée où les fluctuations de l'insolation irrégularisent quelque peu les courbes thermiques. L'exemple de la Turra, dans l'étage alpin, et celui de Lanslebourg, dans le talweg, sont à retenir à ce point de vue.

A altitudes égales, les températures moyennes mensuelles du monde intra-alpin dépassent, en général, celles des Préalpes et les maximums absolus moyens sont, plus nettement encore, supérieurs à leurs homologues: "ceux de Saint-Jean-de-Maurienne, station plus élevée de $170 \mathrm{~m}$, écrasent ceux de Saint-Laurent-du-Pont, en Chartreuse, de $4,1^{\circ}$ en septembre, de $4,5^{\circ}$ en avril, de $5,5^{\circ}$ en octobre, enfin de $3,65^{\circ}$ pour l'année entière... Entre Termignon et les Contamines - dans le Massif du Mont-Blanc et assises $100 \mathrm{~m}$ plus bas - le contraste demeure prononcé: $2^{\circ}$ à l'avantage du village mauriennais en été, dont $2,6^{\circ}$ en août. " [BLANchaRd (R.) (1943, 245)].

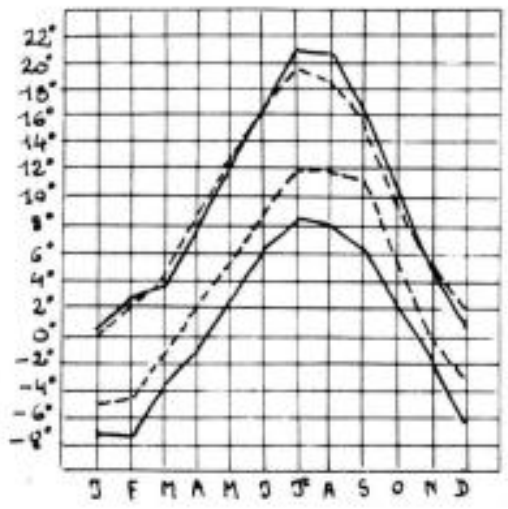

$1891-1930$

Saint-Hilaire-du-Touvet Lanslebourg

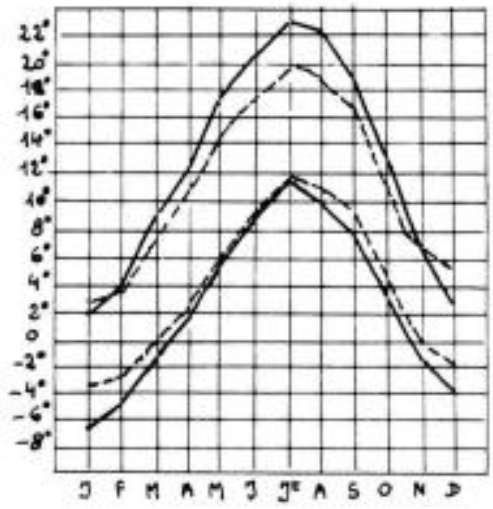

1949-1961

.... Saint-Hilaire-du-Touvet Avrieux

FiG. 8.

Les températiones.

Légende. - Lamplitude des absolus moyens de température (moyenues mensuelles des maximums et des minimums, figurant sur les graphiques cidessus) est sensiblement plus grande dans les Alpes internes (Haute-Maurienne), que dans les Préalpes (Chartreuse).

Si l'on veut bien se rappeler que, dans une région donnce, cette amplitude augmente quand l'altitude baisse. Saint-Hilaire-du-Touvet (à $1130 \mathrm{~m}$ ) malgrè $P$ \& avantage 8 que lui donne son altitude, présente des oscillations de température plus amorties que des stations plus élevées (Lanslebourg: $1400 \mathrm{~m}$ ), mais situées dans des régions toutes différentes (période 1891-1930).

Le phénomène se confirme pour la période 1949-1961 avec deux stations situées à la méme altitude: Avrieux en Haute-Maurienne présent un régime de température nettement plus brutal que Saint-Hilaire-du-Touvet dans les Préalpes. 
Si l'on compare enfin, à altitudes voisines, sinon égales, des régions à pluviosité élevée, comme la Chartreuse (Saint-Hilaire-duTouvet $1127 \mathrm{~m}: 1202 \mathrm{~mm}$ ) à la Haute-Maurienne, sèche et ensoleillée (Lanslebourg $1400 \mathrm{~m}: 704 \mathrm{~mm}$ ), on s'aperçoit que ce sont ces dernières régions, comme il fallait s'y attendre, qui présentent le régime thermique le plus brutal. Cette grande amplitude des absolus moyens de température constitue une des caractéristiques, d notre aris, du climat intra-alpin (fig. 8).

\section{II.1.3.3. - Les gelées.}

11 s'agit là d'un domaine mal exploré, les seules données dont on puisse faire état concernent uniquement ou presque le fond du talweg puisque s'y trouvent rassemblés les appareils d'observation. Selon ONDE (H.) (1938), la période des gelées continues - celles d'hiver - " s'étend sur 5 mois ou plus », à l'amont de Modane; à Bessans, situé, il est vrai, dans une cuvette où l'air froid stagne, " la moyenne des deux années 1934-1936 fait apparaitre un régime des plus rigoureux $n$, de 7 mois pleins commençant dès octobre, le mois de mai ayant connt encore 16 jours de gelée. A l'aval de Modane, on peut admettre que cette période est de 4 mois et, au delà du Pas du Roc, de moins de 3 mois.

Les gelées hors saison, dont la localisation est capricieuse car elle obéit aux facteurs topographiques eux-mêmes très variés, sont assez fréquentes. "De Modane à Lanslevillard, les gelées de juillet sont réputées exceptionnelles (tous les 20 ou 30 ans dit-on à Bramans, mais on parle volontiers de celles de juin (ONDE, ibidem). Ce régime assez sévère des gelées hors saison s'explique, en Mauriense, par la sécheresse de lair qui permet un grand rayonnement nocturne: la Tarentaise, en effet, vallée voisine, mais plus humide, "bénéficie d'un régime de gelées plus uniforme, moins capricieux " et d'une durée de gelée continue sensiblement plus faible (ONDE, ibid.).

\section{II.1.3.4. - L'insolation.}

II n'est pas très facile de donner une vue d'ensemble d'un phénomène qui est essentiellement * local ». L'insolation en un lieu donné dépend, en effet, de nombreux paramètres dont certains communs à plusieurs points - comme la latitude ou l'altitude mais dont la plupart sont vraiment particuliers, comme la position du plan du lieu par rapport au plan horizontal, sa position par rapport au dessin des crêtes voisines qui interceptent plus ou moins longtemps et à tel moment de la journée les rayons solaires, etc. On conçoit, dans ces conditions, que les mesures des durées d'insolation faites par héliographes ou même par hélioramas [PERS (R.) $(1937)]$, si elles sont intéressantes, restent encore insuffisantes: 
elles ne tiennent pas compte des l'incidence des rayons solaires qui varie au cours de la journée et d'une saison à une autre et dont l'importance se traduit par la loi, bien connue, des sinus, ni de l'énergie diffusée par l'atmosphère, alors même que le lieu d'observation est à l'ombre. Seul le solarigraphe, appareil très coûteux et fragile, permettrait de se faire une idée exacte de la quantité d'énergie réellement reçue au sol pendant chaque journée.

Cependant, en l'absence de telles données, les chiffres ci-après permettent de se rendre compte des différences qui peuvent exister en Maurienne entre l'adret et l'envers; ils seront à rappeler lorsque seront étudiées les associations s'observant sur des versants d'expositions opposées et que sera analysé, même fragmentairement, leur microclimat.

A Lanslebourg (latitude $45^{\circ} 20^{\prime}$ ) où le versant exposé au nord a une pente très régulière faisant un angle de $26^{\circ}$ par rapport au plan horizontal et où celui exposé au Sud a une pente moyenne de $31^{\circ}$, comme à Avrieux (latitude $45^{\circ} 13^{\prime}$ ) où ces mêmes pentes

\begin{tabular}{|c|c|c|c|c|c|c|}
\hline \multirow{2}{*}{ Detea } & \multicolumn{3}{|c|}{ LANSLBBOURG } & \multicolumn{3}{|c|}{ A VR IBUX } \\
\hline & Verbant So: & & Vereant Sud & Versant Box & & Vorsant sud \\
\hline 15.1 & $-3^{\circ}$ & (I) & $53^{\circ}$ & $-4^{\circ} 30^{\prime}$ & (I) & $49 \cdot 101$ \\
\hline 15.2 & $3030^{\prime}$ & & $61 \cdot 30^{\prime}$ & $3^{\circ}$ & & $56^{\circ} 40^{\prime}$ \\
\hline 15.3. & $16^{\circ}$ & & $73^{\circ}$ & $14^{\circ} 30^{\prime \prime}$ & & $680^{\circ} 10^{\prime}$ \\
\hline 15. 4 & $28^{\bullet} 30^{\prime}$ & & $85 \bullet 30^{\prime}$ & $27^{\circ}$ & & $70 \cdot 50^{\prime}$ \\
\hline 15. 5 & $37 \cdot 20^{\prime}$ & & $94^{\circ} \cdot 20^{\prime}$ & $35 \cdot 50^{\prime}$ & & $89 \cdot 30^{\prime}$ \\
\hline 15.6 & $41^{\circ} 50^{\prime}$ & & $98 \cdot 50^{\prime}$ & $40^{\circ} 20^{\prime}$ & & $94^{\circ}$ \\
\hline 15. 7 & $40^{\circ} 40^{\prime}$ & & $97^{\circ} 40^{\prime}$ & $39 \cdot 10^{\prime}$ & & $92^{\circ} 50^{\prime}$ \\
\hline 15.8 & $32^{\circ} 50^{\prime}$ & & $89 \cdot 501$ & $31^{\circ} 20^{\prime}$ & & $85^{\circ}$ \\
\hline 15. 9 & $22^{\circ}$ & & $71^{\circ}$ & $20^{\circ} 30^{\prime}$ & & $74^{\circ} 10^{\prime}$ \\
\hline 15.10 & $10^{\circ}$ & & $67^{\circ}$ & $8 \cdot 40^{\prime}$ & & $62^{\circ} 20^{\prime}$ \\
\hline 15.11 & 0.90 & & $27^{\circ} 10^{\prime}$ & $-1^{\circ} 20^{\prime}$ & (I) & $52^{\circ} 20^{\prime}$ \\
\hline 15.12 & $-4^{\circ} 10^{\prime}$ & (I) & $52 \cdot 50 *$ & $-5^{\bullet} 80^{\prime}$ & (I) & $48 \cdot$ \\
\hline
\end{tabular}

(1) Envers à lombre toute la journée.

sont respectivement de $27^{\circ} 30^{\prime}$ et $26^{\circ}$, les angles faits par les rayons solaires à midi, à lenvers et à lendroit, par rapport aux plans des versants montrent à quel point le versant exposé au sud peut ètre favorisé, non seulement en hiver, ce qui a pour conséquence indirecte de diminuer la durée d'enneigement, mais également au printemps, au moment du démarrage de la végétation. 


\section{II.1.4, - Images synthétiques du climat.}

Pour prendre une vue d'ensemble du climat haut-mauriennais, et ce, aussi brièvement que possible après les développements précédents - nous nous sommes servi des données dont nous pouvions disposer: les précipitations et les températures, et nous les avons traduites sous forme de climatogrammes de divers types (courbes ombrothermiques selon BAgnouls (F.) et GAUSSEN (H.) (1953) (13), aires ombrothermiques selon REY (P.) (1960) (14).

Que constatons-nous?

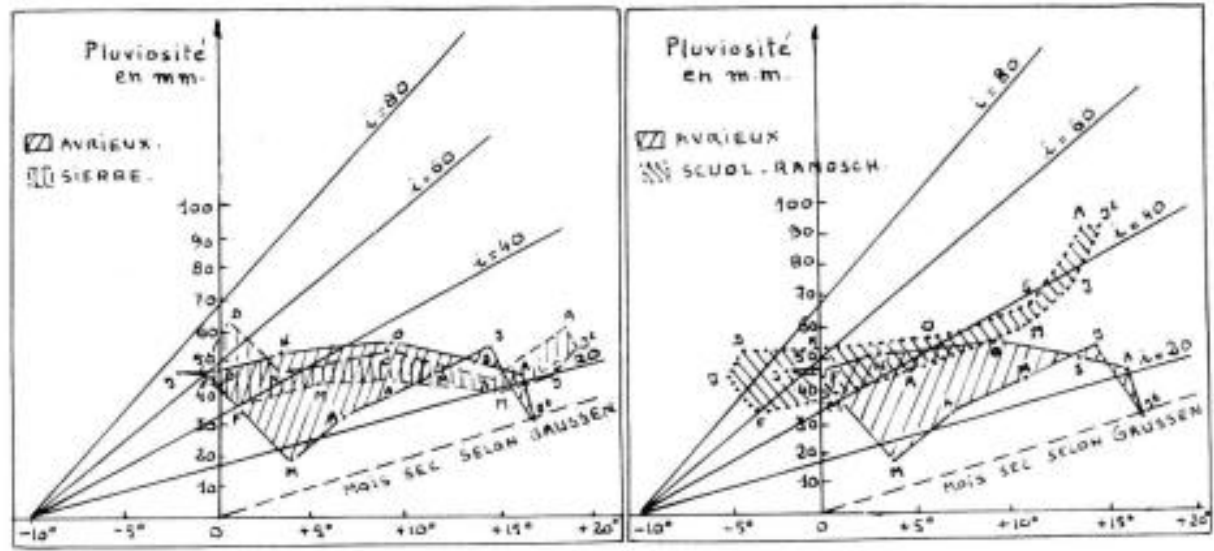

Fic. 9.

Climatogrammes de trois stations typiques des Alpes internes.

Légende:

I. - Sierre, dans le Valais (alt. $552 \mathrm{~m}$ : période 1901-1923).

II. - Scuol-Ramosch, en Engadine (alt. $1237 \mathrm{~m}$ : période 1901-1940).

III. - Avrieux, en Haute-Maurienne (alt. $1150 \mathrm{~m}$; période 1949-1961).

On notera la rigueur des hivers de Setrol-Ramosch, en Engadine, situé seulement $90 \mathrm{~m}$ plus haut qu'Avrieux, et le régime continental des précipitations de cette station, nettement plus marqué que celui des précipitations de Sierre.

(13) On sait que Bagnouls (F.) et Gaussex (H.) (1953) font figurer stir un même graphique les courbes des régimes thermiques et pluviométriques en adoptant le rapport de $2 \mathrm{~mm}$ pour 1 degré: un tel rapport résulte du fait que lauteur définit comme mois sec, un mois recevant une hauteur d'eau (en $\mathrm{mm}$ ) inférieure au double de la température moyenne (en degrés centigrades). On observera que GAsparix avait utilisé un système analogue, le rapport standard étant de 2,6 [dans Cotragse (1942)].

(14) L'intérêt du climatogramme à deux variables (température et précipitations) et de laire ombrothermique, où ces mémes variables apparaissent, c'est qu'ils permettent de tracer \& des réseaux de courbes figurant telle fonction empirique ou expérimentale, liant les précipitations aux températures s et, en particulier, \& les lignes d'égale valeur des indices d'aridité de de MarTONNE \& [Ṕguy (Ch.-P.), (1961)], ou la relation de BMgNouls et Gaussen délimitant la zone des mois secs. 
Qu'il n'y a pas eu, en Haute-Maurienne, de mois sec au sens de Gaussen, durant les périodes d'observation suivies par Sanson pour Lanslebourg, mais que, par contre, pour Avrieux, au creux de l'ilot de sécheresse et pour la période allant de 1949 à 1961 , soit 13 ans, il y a eu, en juillet, une petite période de sécheresse. Cette xéricité d'Avrieux se traduit, en porticulier, par un coefficient de * méditerranéité » d'Emberger (L.) (1943) $\frac{(\mathrm{Pe})}{\mathrm{M}}$ de 5,6 seulement, inférieur par conséquent au seuil de 7 et qui, si le régime des précipitations était méditerranéen, pourrait faire classer Avrieux et le secteur avoisinant, dans la "zone méditerranéenne s d'EmBERGER, au sens phytogéographique du mot.

On notera que si le Valais central et la Hatte-Engadine dont les climats nous paraissent bien représentés par ceux de Sierre et de Scuol-Ramosch (fig. 9), connaissent un climat à tendance continentale (faible pour le Valais, très net pour l'Engadine) celui d'Avrieux leur est tout à fait comparable: les trois climatogrammes se situcnt dans la même zone d'indices d'aridité; mais alors que celui d'Avrieux présente un appendice estival légèrement tourné vers le bas - nous en avons déjà traduit la signification - celui de Sierre et plus encore celui de Scuol-Ramosch ont un appendice estival tourné vers le haut, ce qui est l'image du régime continental de leurs précipitations.

Par contre, la comparaison des climatogrammes d'Avrieux et de Saint-Hilaire-du-Touvet, station préalpine de Chartreuse, à la même altitude, met bien en évidence les différences fondamentales qui existent entre le climat des Préalpes et celui des Alpes internes. Si les deux graphiques ont. à peu près, la mème forme avec toutefois, pour Avrieux, un appendice estival nettement plus marqué, ils se situent dans des "zones d'aridité " tout à fait différentes: les températures sont assez sensiblement plus étalées à Avrieux, ce qui traduit une continentalité plus forte et le décalage des températures au printemps, et ce, au bénéfice de la station intra-alpine, est des plus nets. Ces deux caractéristiques - déjà soulignées sont essenticllement dues à la siccité de latmosphère (fig. 11).

L'aire ombrothermique de la Hatute-Maurienne, que nous avons essayé de tracer. malgré la rareté des documents, comparée à celles obtenues par REV (P.) (1960) pour le Conflent, le Velay, le Vivarais - régions qui nous ont paru assez affines quant au climat montre bien que notre dition se situe, dans l'ensemble, dans une zone à la fois plus froide et plus sèche que le Haut-Conflent, par exemple, pourtant très xériqutue (fig. 12).

Cette sécheresse absolue, qui est à l'origine de la brutalité du climat, laquelle se traduit, on l'a vu, par un régime particulièrement capricieux des gelées, un rapide réchauffement au printemps, est 
bien, en définitive, la caractéristique fondamentale du climat de la Haute-Maurienne. Elle explique - on le verra au cours de ce travail - les affinités que présente la flore de cette vallée avec celle du Valais et de l'Engadine, régions pourtant sensiblement plus continentales. Ainsi se trouvera vérifié le point de vue exprimé par EMrerger (L.) (1954), à savoir qu'un climat météorologiquement

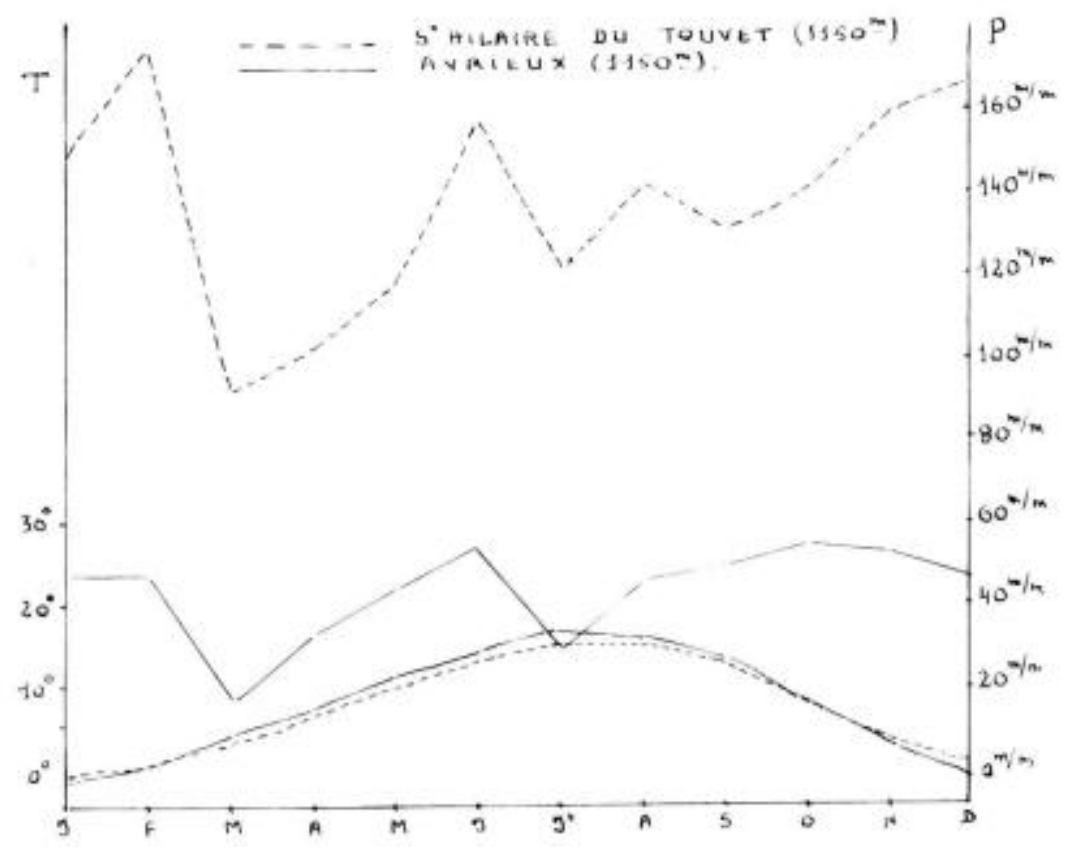

Fig. 10 .

Courbes ombrothermiques.

Léyende, - Les courbes ombrothermiques établies selon les normes de Bagnouls et Gausses, d'Avrieux et de Saint-Hilaire-du-Touvet (en Chartreuse), pour la période 1949-1961, mettent très nettement en relief ce qui différencie climat préalpin (humide et relativement froid de mars à novembre) et climat intra-alpin (sec et relativement chaud de mars à novembre).

\begin{tabular}{|c|c|c|c|c|c|c|c|c|c|c|c|c|c|c|}
\hline Leosist the & & $\mathrm{J}$ & F & $x$ & $A$ & $u$ & 3 & $\mathrm{~J}^{\mathrm{t}}$ & A & s & 0 & I & D & $\begin{array}{l}\text { Totalux } \\
\text { ou noyen }\end{array}$ \\
\hline \multirow[t]{2}{*}{$\begin{array}{l}\text { ATRIEUX } \\
(1 \quad 100 \mathrm{~m})\end{array}$} & \multirow{2}{*}{$\begin{array}{l}P \\
P\end{array}$} & 46,8 & 47,0 & 17,9 & $3 s, 7$ & 46,0 & 54,6 & 30,3 & 46,8 & 20,3 & 53,6 & 23,4 & 47,2 & $527 \mathrm{~mm}$ \\
\hline & & $-2,1$ & $-0,2$ & 3,0 & 7,0 & 31,1 & 14,6 & 16,7 & 16,1 & 13,7 & 8,4 & 2,9 & $-6,3$ & $7.6^{\circ} 0$ \\
\hline \multirow{2}{*}{ 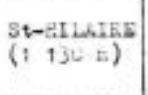 } & \multirow[t]{2}{*}{ p } & is: & 176 & $\$ 2$ & 102 & 118 & 1)â & 122 & 143 & 132 & 142 & 161 & 168 & $1665 \mathrm{~mm}$ \\
\hline & & $-\infty, 5$ & $c, t$ & 3,5 & 6,3 & 10,0 & 13,4 & 15,6 & 12,0 & 13,1 & $B, 1$ & 3,3 & 1,1 & $7,4^{\circ} \mathrm{C}$ \\
\hline
\end{tabular}




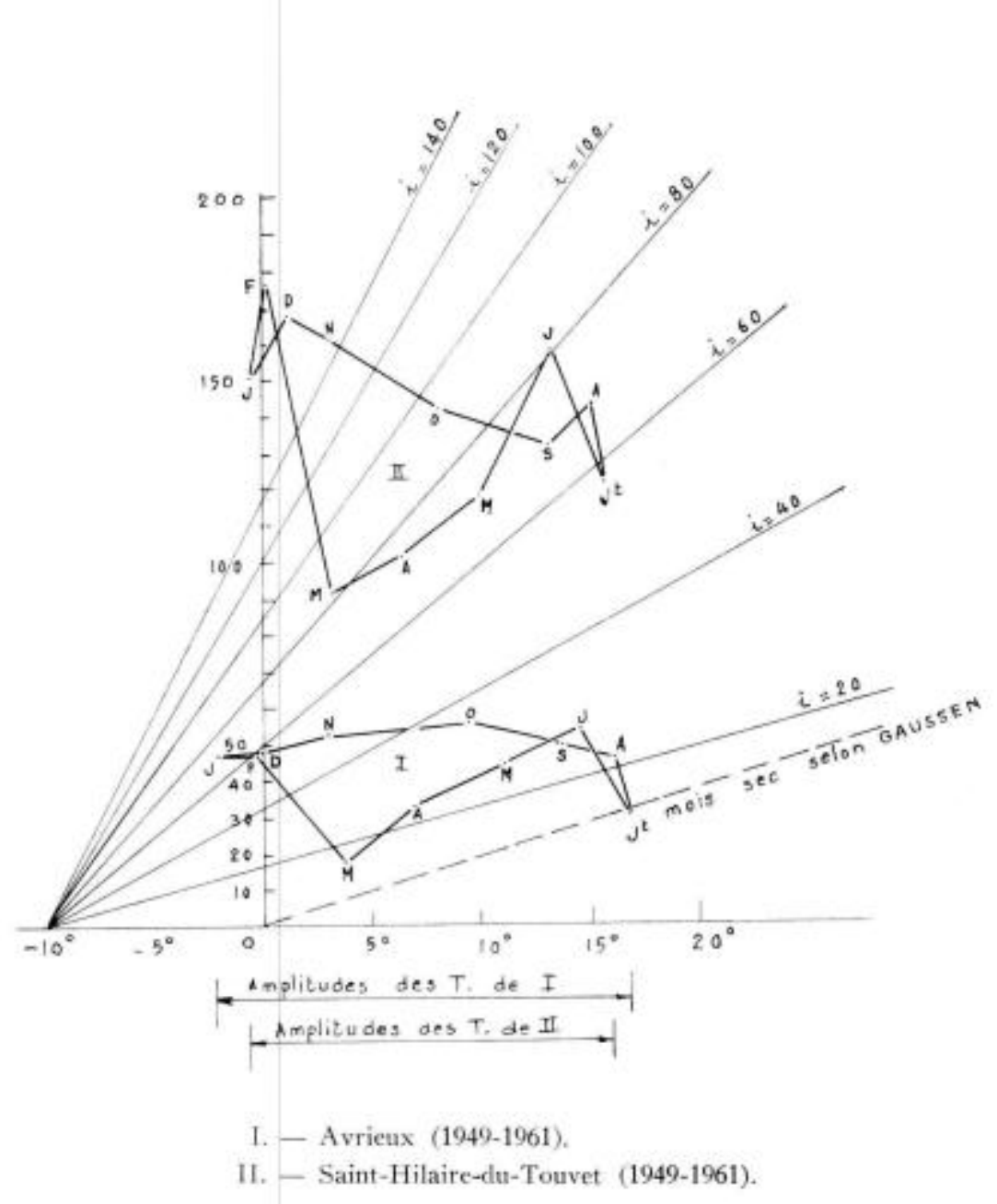

F16. 11.

Climatogrammes d'Avrieux (Haute-Maurienne). it de Saint-Hilairc-du-Touvet (Chartreuse).

Légende. - Si, dans leusemble, les deux climatogrammes préalpin et intraalpin ont la mème forme, ce qui traduit ce que BLANCHARD a appelé \& l'océanisation $>$ du elimat intra-alpin, on remarquera qu'ils se situent dans deux \& zones d'oridité > nettement différentes et que les températures à Avrieux sont constamment plus élevées que celles de Saint-Hilaire-du-Touvet de mars à novembre, et plus basses en hiver.

Les climatogrammes du type ci-dessus traciuisent mieux que les courbes ombrothermiques l'aridité du climat; ces dernières, par contre, mettent mieux en relief les différences de température. 


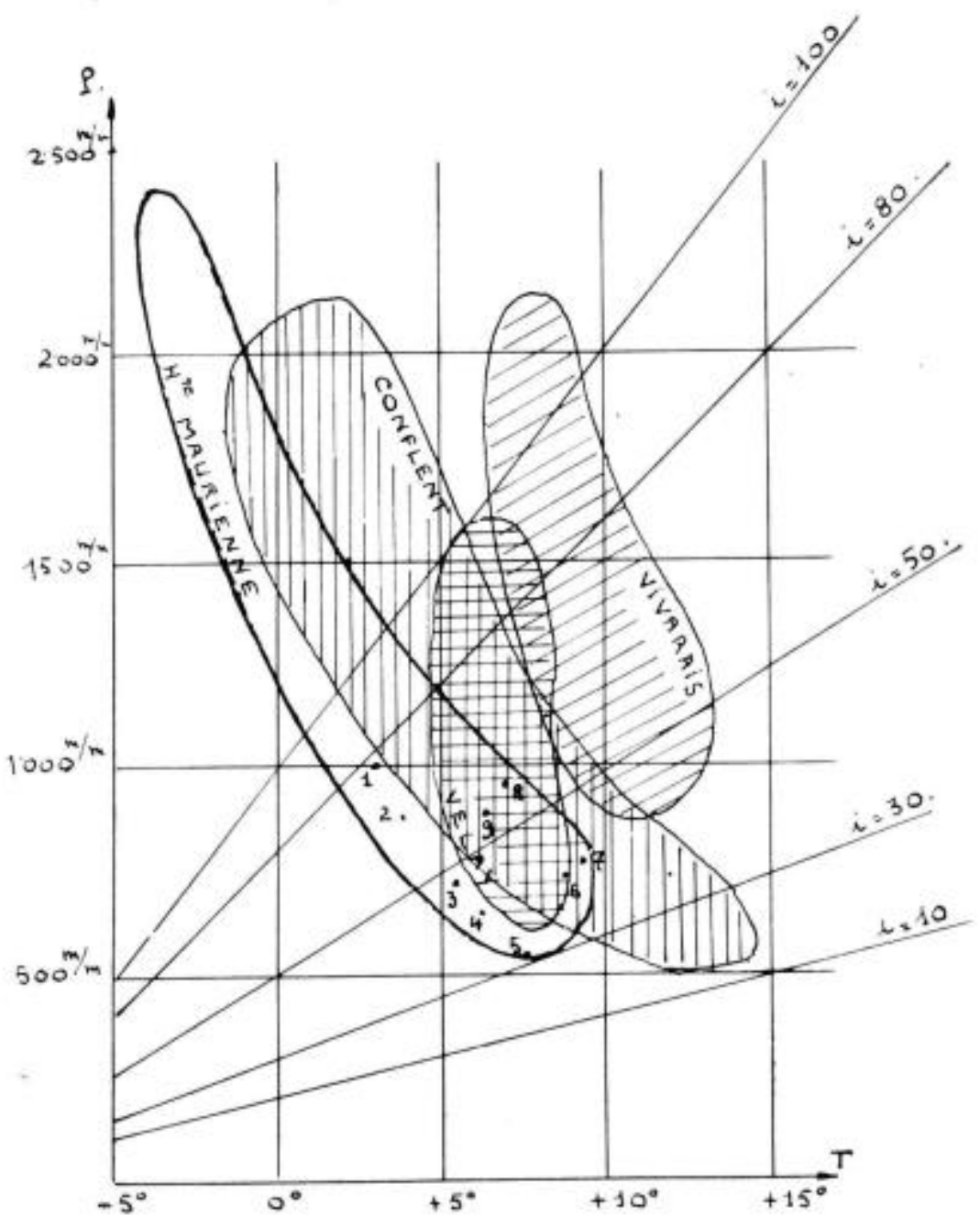

Fig. 12.

Aire ombrothermique de la Haute-Mourienne.

1: Bissorte; 2: Bessans; 3: Lanslebourg; 4: Termignon; 5: Avrieux; 6: Orelle Prémont; 7: Saint-Martin-la-Porte: 8: Le Thyl; 9; Valmeinier.

Légende. - En amont de Modane, la Haute-Maurienne apparait comme plus sèche que le Conflent.

A noter que la régression générale pluviosité/température voit son coefficient angulaire augmenter vers les pluviosité fortes; à haute altitude, en effet, le gradient pluviométrique, selon les travaux de $\mathrm{ONDE}$, augmente sensiblement; si l'on admet, en première approximation, que celui des températures reste à petu près constant, on conçoit que le coefficient angulaire de la régression $\mathrm{P} / \mathrm{T}$ augmente dans les mẻmes proportions, 
océanique - au moins quant au régime de la pluviosité - doit être, phytogéographiquement, rattaché aux climats continentaux, lorsqu'il est sec, et froid et hiver.

On notera, au passage, que les étages forestiers se situent entre les indices d'aridité de 90 et de 30 , ce qui constitue une amplitude étroite, si l'on veut bien tenir compte de l'importance des différences d'altitude, mais qui s'explique par la faiblesse du gradient pluviométrique, đéjà soulignée.

Cette aire ombrothermique, purement géographique, ne prendra d'ailleurs sa véritable signification que comparée aux aires, également ombrothermiques, des grandes essences forestières et à celles des associations telles qu'elles ont pu être déjà étudiées. Nous aurons donc l'occasion de la retrouver, à la fin de ce travail, lorsque nous essaierons de prendre une vue synthétique des facteurs écologiques décisifs qui se trouvent liés corrélativement, sinon causalement, aux groupements forestiers que nous aurons analysés. 


\section{II.2. - LES ROCHES-MERES}

Si l'étude des roches-mères présente le plus grand intérêt, puisque ce sont elles qui expliquent largement la nature des sols, surtout en montagne, où l'érosion joue un rôle de premier plan, il n'y a pas lieu, dans cette vue d'ensemble, de trop s'y attarder, car on les retrouvera dans l'horizon $\mathrm{C}$ des sols, qui est le niveau de la zone de leur altération.

Trois points sont cependant à souligner:

Le premier, déjà exprimé, est que les zones tectoniques et pétrographiques sont, en gros, perpendiculaires à l'axe des vallées et se retrouvent de part et d'autre de chaque versant, à des expositions opposées par conséquent, ce qui doit permettre de faire d'intéressantes comparaisons.

Il faut noter ensuite l'extrême variété des roches-mères et leur relative richesse en calcium. En effet, malgré l'apparente simplicté de la carte géologique, le substratum pétrographique est très « nuancé $n$, comme le montre le récent travail d'ELLENBERGER sur la géologie du pays de Vanoise (1958). Se rencontrent ainsi, et souvent cotte à côte, des roches aussi différentes que des quartzites plus ou moins colorés par divers sels, et des gypses ou des cargneules, les premières typiquement siliceuses et les secondes carbonatées. Mais, dans l'ensemble, mis à part les quartzites que l'on rencontre peu en forêt, sauf dans le synclinal de Chavières et au niveau du premier ombilic glaciaire d'Aussois, les roches-mères totalement siliceuses sont relativement rares. Même les grès micacés du Houiller, à la Praz, près de Modane, s'ils sont riches en quartz $\left(80 \%\right.$ de $\left.\mathrm{Si} \mathrm{O}_{2}\right)$, ne sont pas, pour autant décalcifiés $(1,25$ de $\mathrm{CaO}$ ). Quant aux cargneules, on retiendra qu'un échantillon pris dans le ravin de Saint-Antoine, à l'amont de Modane, a donné les pourcentages suivants: $20,4 \%$ de dolomite ferrifère, $73 \%$ de calcite, 7,6\% d'insolubles aux acides courants (1). On sait aussi que les schistes lustrés, présentent de a minces zones de calcite plus ou moins importantes, à côté d'autres « de quartz et d'un feutrage de mica blanc, chargé d'ilménite et de rutile n.

(1) dans Ellenberger (1958). 
Un troisième point, enfin, est à retenir : c'est l'importance que revêtent, en montagne, les apports divers (éboulis ou moraines). Ces apports, lorsqu'ils sont de faible importance, ne figurent pas, en général sur les cartes géologiques, toujours à petite échelle; par exemple, nous avons souvent rencontré des fragments de dépôts morainiques carbonatés, sur des quartzites, que la carte géologique ne laissait évidemment pas soupconner. Quant aux éboulis, ils ne sont jamais à négliger: une roche-mère donnée est toujours peu

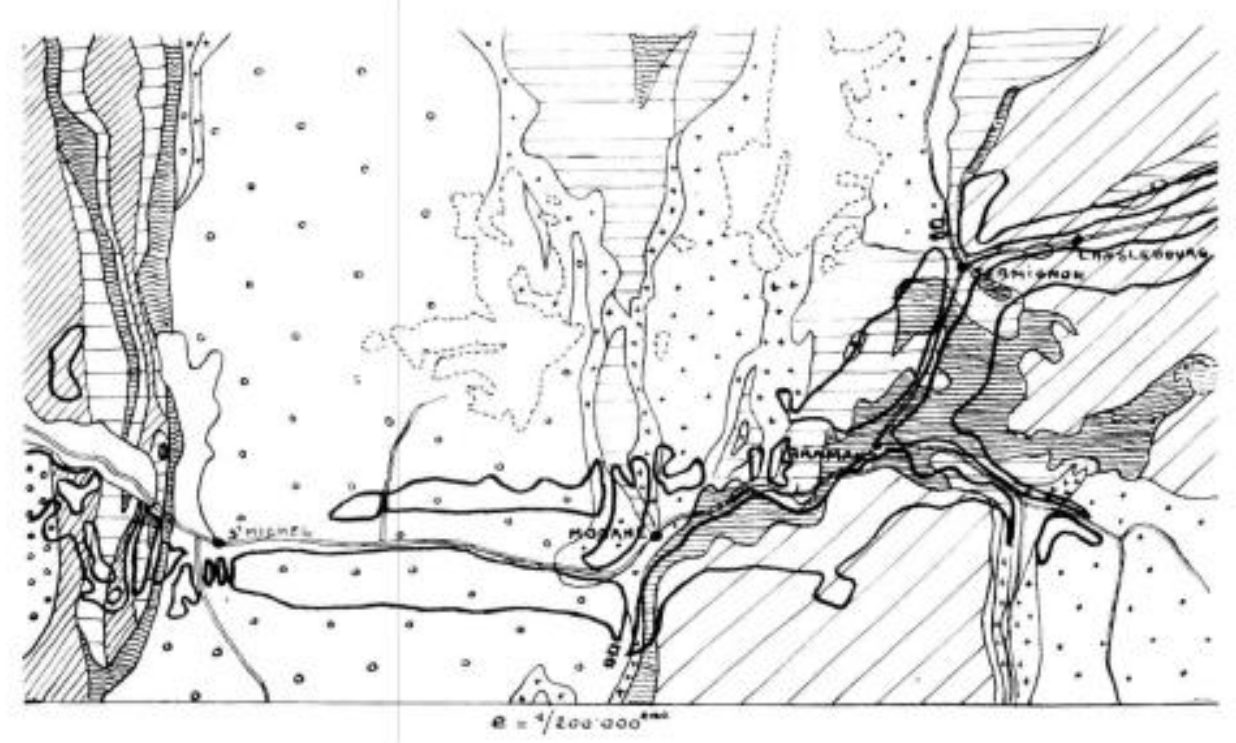

Calcaires francs ou dolomitiques.

Schistes calcaires.

$\square /$ Schistes lustrés.

道国 Gypses et Cargneules.

ح Limites de forèt. $\because$ Grès et Schistes silicatés (Houiller-Flysch).

$\because$ Schistes silicatés métamorphiques.

$\because$ Quartzites.

Alluvions et Moraines.

(.... Glaciers.

FIG. 13.

Les roches-mires.

Légende. - Le schéma géologique ci-dessus met en évidence l'extrème diversité des roches-mères, comme la situation de la vallée par rapport aux grandes zones pétrographiques et qui fait qu'une mème roche-mère occupe les deux flancs d'expositions opposées.

On notera que le \& creux pluviométrique s qui se situe entre Modane et Termignon coincide avec une zone calcaire, de gypse et de cargneules, qui contribuent à accentuer la xéricité des stations correspondantes. 
ou prou " influencée " par celle qui la surmonte, en altitude, alors même qu'aucun éboulis jroprement dit ne s'est produit: il s'agit, si l'on veut, d'un lessivage oblique superficiel.

Le schéma géologique ci-dessus permet de prendre une vue d'ensemble des grands types de roches-mères rencontrées dans la région, et de leur répartition, sans qu'il soit besoin d'autres commentaires. 


\section{II.3. - L'ACTION HUMAINE}

Etudier, en Haute-Maurienne, l'influence humaine dans le domaine forestier, c'est faire tout le procès de la forêt puisque, aussi bien, l'homme, par une action destructrice ou constructive a parfois détruit, parfois recomposé ou, au moins altéré, la physionomie et la composition floristique des forêts primitives.

Les modalités de cette action ont été souvent analysées pour les Alpes Françaises, par les géographes dont Blanciand (R.) (1956), Onde (H.) (1938), Péguy (Ch.-P.) (1947), Vexret (P.) (1944), par les phytogéographes et les forestiers dont Guinier ( $\mathrm{Ph}$.) (1950), Mougrn (P.) (1931), Lenoble (F.) (1923), Fourchy (P.) (1944); nous aurons l'occasion, lors de l'étude des diverses associations, de chercher à déterminer comment cette action se manifeste dans le détail; ce que nous voudrions, dans ce bref chapitre, c'est en prendre une vue d'ensemble, rappelet son ancienneté, décrire son effet le plus brutal et le plus spectaculaire: le défrichement.

\section{II.3.1. - Les témoignages.}

Les textes et les cartes peuvent, dans certains cas, apporter des renseignements intéressants: Movgr (P.) (1931), en a fait état à propos d'un petit massif disparu à Entre-deux-Eaux, sur la commune de Termignon. De tels documents, comme l'a montré FourCHY (P.) (1944), doivent toutefois être interprétés avec une extrême circonspection, car rien n'est plus difficile, en un sens, que de définir une forêt: l'état boisé comporte, en effet, depuis la lande ou la pelouse, toutes les ganmes de densité, alors que les descriptions ne connaissent pratiquement que deux termes: lande et forêt.

La découverte de souches signalée par Mougin (P.) (1931) et par ONDE (H.) (1938), notamment à la Plagnette, à cinq kilomètres à vol d'oiseau de la plus proche forêt, dans la vallée de Valloire, au pied du Galibier, montre que les vallées de la Valloirette et de la Neuvachette ont été largement déboisées par l'homme. Les tourbières de la vallée de Valmeinier sont particulièrement riches en tronc d'arbres (cf. photo) qui, à l'analyse, ont été identifiés comme étant des Pins Cembros. De même, dans les zones dénudées au-dessus du Thyl, peut-on ohserver des souches, indices d'un ancien boisement. 
La toponymie locale, qui a fourni au chanoire Gros (A.) (1935) la matière d'un dictionnaire, enseigne également que beaucoup de noms de lieux comme Sardières, Eterpets près de Valloire, Essarts près de Saint-Julien-de-Maurienne, pour ne citer que ceux-là, tirent leur origine d'anciens défrichements.

Mais c'est, en définitive, l'aspect du manteau forestier et de la flore, qui constituent, à notre avis, les meilleurs des témoignages.

\section{PLANCHE I}

\section{LES TEMOINS DU PASSE}

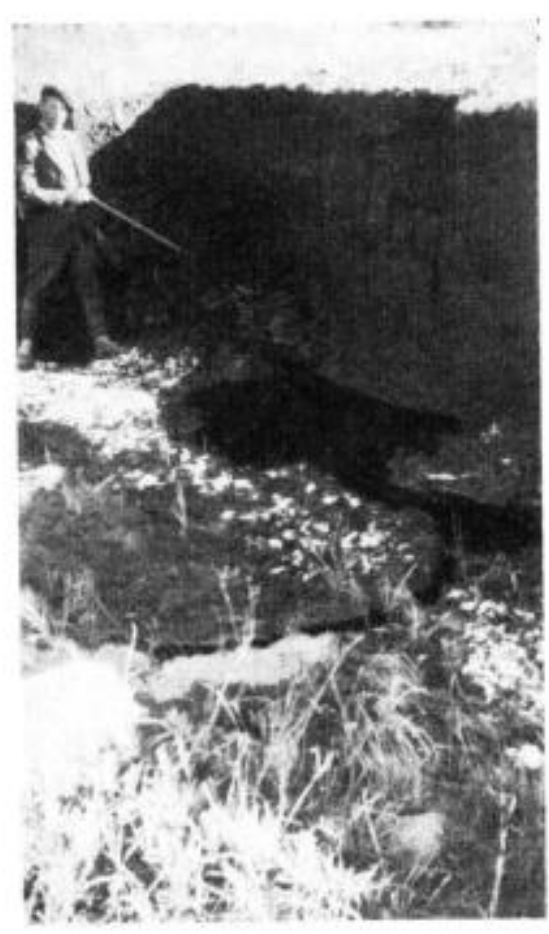

Souche profondément enfouie dans une tourbière.

Vallée de Valmeinier.

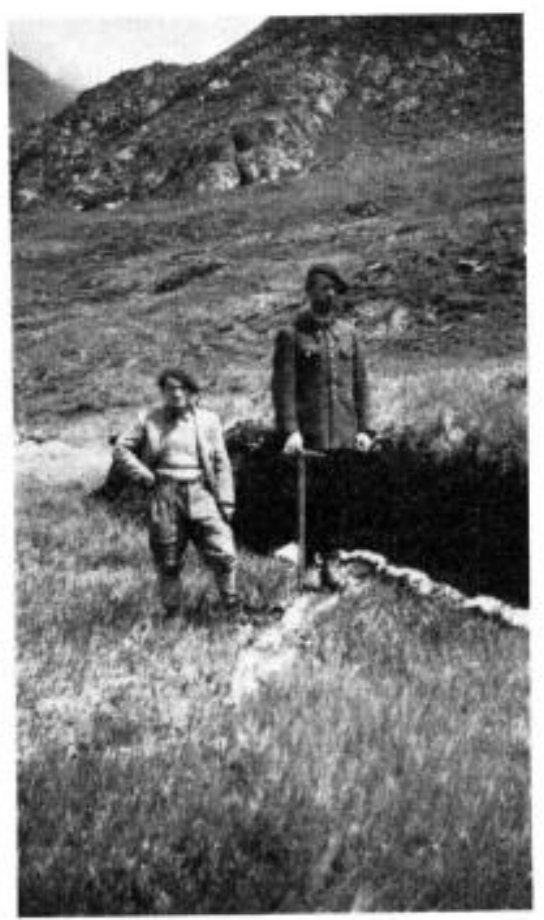

Truncs d'arbres dans une tourbière aux Mottets (région du Galibier)

Ce qu'il $y$ a lieu de souligner, c'est que certains facteurs naturels ont joué et jouent encore un rôle, pour favoriser ou pour inhiber laction humaine et que "la répartition actuelle des massifs forestiers et des zones déboisées n'est pas sans dépendre, dans une large mesure, du milieu physique n [ONDE (H.) (1938)]. 
PLANCHE II

L'OCCUPATION HUMAINE ET SON RECUL ACTUEL

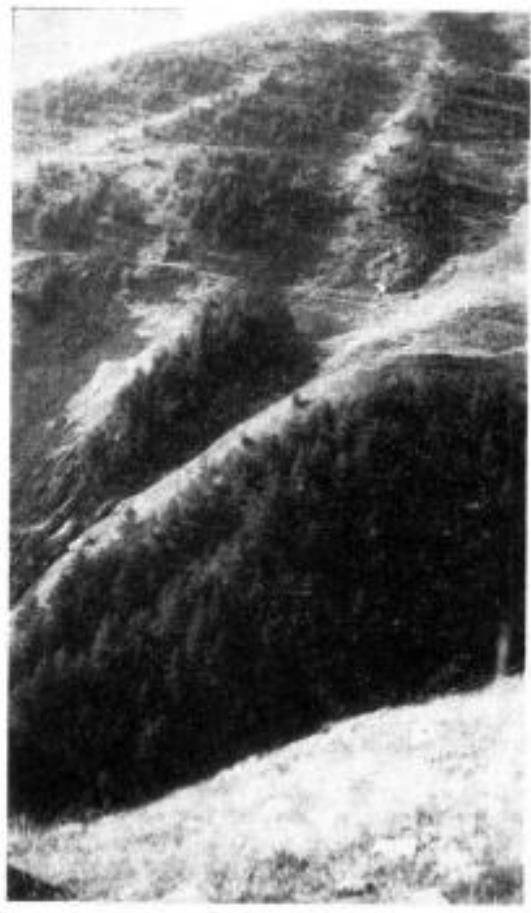

Envahissement des pâturage par le Mélèze (région de Valloires)

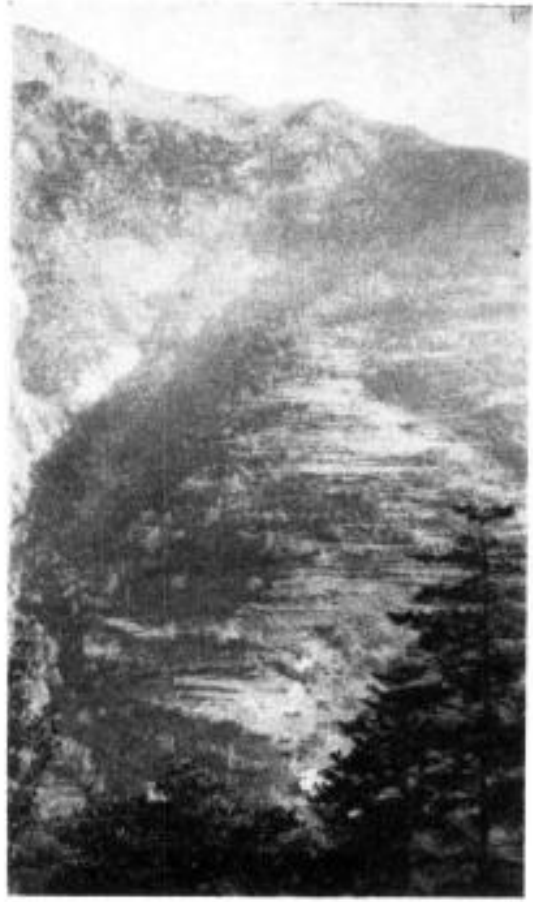

L'adiret dans les gorges houillères Puissante occupation humainc.

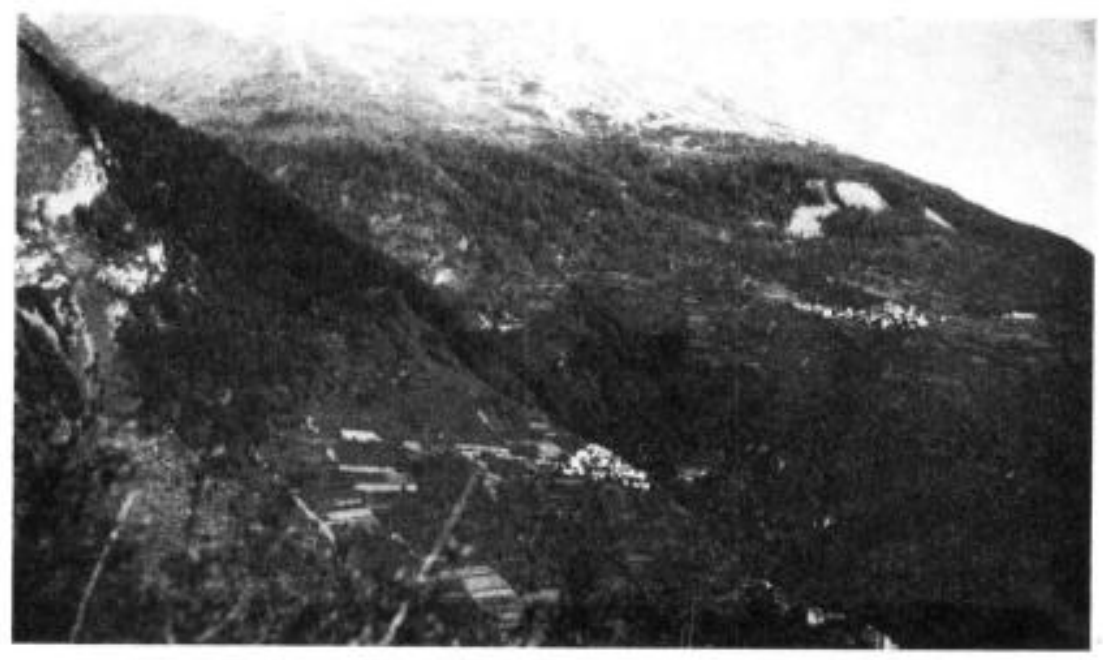

Les grandes clairières de villages sur moraines. Albanne, à droite, et sa ceinture de mélèzeins. Albanette, aे gauche. 


\section{II.3.2. - Les facteurs naturels du défrichement.}

L'exposition, plus exactement la durée de l'insolation hivernale, a fixé l'habitat humain sur les adrets. En Maurienne, orientée d'ouest en est, le phénomène est particulièrement net. Une fois franchie la zone subbriançonnaise au relief vigoureux, l'homme a trouvé le long des gorges houillères, à l'adret - où, probablement par suite d'une action différentielle des glaciers, la pente se trouve être moins forte qu'à l'envers - un terrain de choix pour son habitat, ce qui explique la suppression presque complête de tout étage forestier entre le talweg et 1600 et $1700 \mathrm{~m}$ environ, alors que l'envers est entièrement boisé, troué seulement de clairières de chalets.

Il en est de même de Modane jusqu'à Lanslebourg et au-delà ; il faudrait dire " à peu près de même $n$, car, pour des raisons que l'on va analyser, l'implantation humaine dans ce secteur amont est moins uniforme.

La pente a joué, aussi, un rôle considérable. Toutes les clairières, qu'il s'agisse de clairières de chalets trouant la forêt ou de clairières de villages, comme celles de Montricher, d'Albanne, d'Aussois, de Sardières, correspondent à des replats, ou à des zones de faible pente. A l'inverse, la vigueur du relief, dans les gorges de la Valloirette et même dans les schistes lustrées, a préservé d'importants massifs.

Il est bien certain que le relief, en effet, a vu son action interférer avec celle due à la roche-mère - l'un dépendant de l'autre, dans une large mesure: c'est ainsi que les molles croupes liasiques et houillères de la vallée de la Valloirette ont incontestablement favorisé un aménagement pastoral intensif aux dépens de la forêt; de même, les dépôts morainiques profonds de la région d'Albanne, de Montricher ou d'Aussois. A l'inverse, les cargneules et les gypses de la région de Sollières et de Sardières, par leur sécheresse, ont rebuté l'homme, ce qui explique que le versant sud, dans ce secteur, soit presque entièrement couvert de forêts.

\section{II.3.3. - Ancienneté de l'action humaine - Recul actuel.}

Les nombreux vestiges laissés par l'homme de la préhistoire: pierres à cupules, haches en serpentine de Bessans et de Lanslevillard, objets divers de Pabri sous roche de Loutraz, à Modane, montrent l'ancienneté de l'occupation humaine en Haute-Maurienne et donnent à penser que les pâturages élevés et les grands cols ont été fréquentés dès le retrait des glaciers [ONDE (H.) (1938)]. Cette densité d'occupation se confirme à lâge des métaux: des sépultures de l'âge du bronze et du fer ont été retrouvées à Montdenis, SaintAndré, Saint-Martin-la-Porte. Dès cette époque, les possibilités de 
la vie pastorale, la richesse minière de la vallée et la facilité du passage ont contribué à retenir les hommes dans cette sauvage région.

Ainsi, dès les temps préhistoriques, l'homme en s'installant dans ce pays, a forcément modifié la contenance et la structure des massifs forestiers. Occupant d'abord les grands replats d'altitude, il a fait descendre la lisière supérieure de la forêt, surtout à Montdenis, à Valloire, à Valmeinier. Plus tard, sans doute, les hommes sédentaires du fond de la vallée, ont ouvert des clairières de ci, de là, et aménagé les adrets. Sans entrer dans les détails, on peut dire que cette emprise de l'homme n'a fait que s'accentuer durant l'époque romaine, le Moyen Age, et au-delà. Les données de l'archéologie comme celles de la toponomastique montrent, en effet, que l'ampleur du mouvement démographique n'a cessé de s'accroitre pour atteindre son maximum au XVIIr siècle [ONDE (H.) (1938)].

Depuis, l'homme recule et la forêt avance: les pelouses autrefois fauchées sont progressivement envahies par les Ericacées, puis par les Epicéas et les Sapins (Valmeinier); ailleurs, c'est le Mélèze qui trouve dans les sols bruns à mull de prairies, des conditions d'expansion particulièrement propices (Valloire); ailleurs encore, sur les roches-mères carbonatées (Aussois - Sardières), c'est le Pin sylvestre ou le Pin à crochets, précédés ou suivis par Arctostaphylos Uva-ursi ou Erica carnea; à haute altitude, dans l'étage subalpin supérieur, sur schistes du Houiller ou sur schistes lustrés, c'est le Mélèze et le Pin Cembro qui reprennent possession du terrain, déjà envahi par la lande à Juniperus nana ou Rhododendron ferrugineum. Mais il s'agit là de processus de détail, qui pourront être étudiés dans les pages qui suivent.

$\mathrm{Ce}$ que nous avons voulu marquer, c'est l'importance de l'emprise humaine: cette emprise s'est, sans doute, manifestée de façon inégale suivant les lieux. Elle ne doit cependant jamais être perdue de vue dans une étude phytosociologique et méritait, de ce fait, de prendre place parmi les grands facteurs écologiques. 


\section{II.4. - ELEMENTS FLORISTIQUES DE LA REGION ETUDIEE}

\section{II.4.1. - Considérations générales.}

Bien que l'étude des principaux éléments floristiques de la Haute-Maurienne puisse, légitimement, trouver sa place à la fin de ce travail, il nous parait préférable de prendre, sans plus attendre, une vue d'ensemble de cet aspect important de la biogéographie végétale de cette vallée, ce qui permettra de rendre plus claire l'analyse des groupements forestiers faisant l'objet des prochains chapitres.

On sait que l'on donne le nom de cortège floristique à l'ensemble des espèces qui caractérisent un territoire donné. Comme le fait remarquer OzENDA (P.) (1964 - 73) " on emploie, plus fréquemment d'ailleurs, l'expression « élément floristique », bien qu'elle ait fait l'objet de critiques du fait, qu'en toute rigueur, le mot élément doit désigner quelque chose de simple, et non pas un ensemble collectif $n$. Toutefois, si l'on observe que la flore d'un territoire donné n'est presque jamais totalement composée d'espèces endémiques, mais de plusieurs " éléments ", l'utilisation de ce terme se justifie pleinement, étant entendu qu'il englobe lui-mème des sous-unités.

Dans le cas particulier qui nous occupe, nous n'envisagerons que la flore silvatique. Notre analyse, d'autre part, se situera dans une perspective de Phytosociologie écologique, en ce sens que nous rattacherons les especes aux associations ou aux unités plus vastes qu'elles caractérisent, comme aux conditions écologiques essentielles auxquelles elles sont liées.

Nous insisterons enfin sur les " échelons d'appauvrissement " que présente tel ou tel élément floristique, à mesure que l'on s'enfonce au cceur de notre dition.

Les tableaux des groupes écologiques montrent que la flore silvatique de la Haute-Maurienne comprend plusieurs éléments: un élément médio-européen, mais appauvri au contact de cet ilot de sécheresse que constitue notre vallée, un élément nordique et des 
hautes montagnes de l'Europe, un élément subméditerranéen et méditerraneo-sarmatique, un élément endémique des Alpes occidentales françaises, enfin.

\section{II.4.2. - L'élément médio-européen.}

Mises à part quelques espèces des pelouses sèches médio-européennes, de la classe des Festuco-Brometea ou de l'ordre des Brometalia, comme Brachypodium pinnatum, Euphorbia Cyparissias, Pimpinella Saxifraga, etc. qui jouent, en forêt mauriennaise, un ròle secondaire, l'élément médio-européen est essentiellement représenté par des orophiles silvatiques dont certaines n'atteignent même pas les limites de notre dition, alors que d'autres s'avancent plus ou moins loin, vers le coeur de la haute vallée.

Parmi les caractéristiques des Fagetalia (liées à des sols à mull), nous n'avons jamais rencontré ni Polygonatum multiflorum, ni Asarum europaeum, ni Euphorbia amygdaloìdes, ni Arum maculatum, ni Allium ursinum.

Il faut noter également l'absence de certaines espèces fréquentes dans les hetraies ou dans les hètraies à sapin méridionales: Geranium nodosum, Galium aristatum (1), alors que Trochischantes nodiflorus est assez abondant dans un type de hêtraie à Sapin, dans les gorges de la Valloirette, sur les calcaires de la zone subbriançonnaise où il trouve d'ailleurs sa limite.

N'ont jamais été rencontrées, non plus, les méso-hygrophiles des Erablaies-Frênaies, telles que Adoxa moschatellina, Scilla bifolia, Carex remota, Carex pendula, Impatiens Noli-tangere, Glechoma hederacea, Lysimachia nemorum, etc., ou les espèces de mull-moder calcique des Erablaies sur éboulis calcaire: Lunaria rediviva, Phyllitis Scolopendrium, Asplenium viride.

On doit rappeler, en outre, que si certaines espèces médio-européennes du cortège du Hêtre, caractéristiques de l'Eu-Fagion, se rencontrent jusqu'à la limite, et mème au-delà de la zone calcaire subbriançonnaise, elles n'atteignent pas Modane, sinon Saint-Michel-de-Maurienne. C'est dire qu'elles ne pénètrent pas au coeur de la Haute-Maurienne (2). La figure 14 donne les limites de ces espèces, du moins des plus importantes: on voit que Mercurialis perennis s'arrête avant Montricher, que Festuca silvatica n'atteint pas les gorges de la Valloirette, tandis que Fagus silvatica, Lamium Galeobdolon, Sanicula Europaea, Carex silvatica, Elymus Europaeus, Epilobium montanum pénètrent légèrement dans les gorges houillères; Asperula odorata et Hedera Helix trouvant leur limite un peu plus à l'amont, près du Thyl.

(1) Ce dernier est cependant signalé au Mont-Cenis, sans autre indication par Cariot et St-Lager (1897), mais Perrier de la Bathie (H.) (1918-18) n'en fait nulle mention.

(2) On en trouvera la liste dans les tableaux des groupes écologiques. 
Il est intéressant de noter qu’en Haute-Tarentaise, Festuca silvatica, Asperula odorata, Epilobium montanum se rencontrent, sans ètre abondantes, jusqu'au delà de Sainte-Foye-en-Tarentaise, au cour, par conséquent, de la partie haute de cette vallée.

Parmi les espèces de l'élément médio-européen liées plus précisément aux hêtraies à Sapin ou aux hêtraies à Erable, il en est qui, par contre, pénètrent vraiment dans la zone intra-alpine: on en observe certaines dans la "sapinière vraie $n$, Abietetum albae de Kuoch (R.) (1959), proche par son écologie des hêtraies à Sapin : il s'agit, parmi les espèces du Fagion, de Prenanthes purpurea, Neottia Nidus-avis, Lilium Martagon, Aconitum Vulparia, Actaea spicata (cette dernière très rare), parmi celles des Fagetalia, de Phyteuma spicatum, Viola silvestris, Paris quadrifolia, Milium effusum, Euphorbia dulcis; les autres relèvent de l'alliance Adenostylion et sont très abondantes dans l'Accri-Fagetum de l'étage montagnard supérieur des Préalpes, du Jura ou des Vosges: ce sont les " hattes herbes " qui, en Haute-Maurienne, au lieu d'occuper un sous-étage, se localisent dans des stations très particulières (couloirs à Aune vert), où elles rencontrent les conditions d'humidité qui leur conviennent. Nous aurons l'occasion de les retrouver lors de l'étude de l'Alnetum viridis.

Toutes les espèces de l'élément médio-européen, dont il vient d'être question, sont liées à des sols bruns forestiers à mull.

Parmi les espèces acidiphiles qui se développent sur des sols bruns, plus ou moins lessivés, à moder, les moins orophiles - et probablement les plus " atlantiques n-sont absentes: Hypericum pulchrum, Teucrium Scorodonia, Lathyrus montanus, Carex pilulifera, alors que Veronica officinalis, Deschampsia flexuosa, considérées, à tort, comme caractéristiques de la seule classe des Quercetea robori-sessili-florae — sont présentes, et grimpent jusque dans l'étage stubalpin.

Après avoir analysé l'appauvrissement progressif que présente, dans notre région, l'élément médio-européen, il importe d'appeler lattention sur detıx espèces qui appartiennent à la partie orientale de cet élément et qui jouent un rôle physionomique important dans certaines de nos forêts.

Il s'agit d'abord d'Erica carnea, abondante dans les forêts calciphiles de Pin sylvestre et de Pin à crochets, d'Italie (Val d'Aoste, Alpes dolomitiques...), de Strisse (Valais, Engadine...), d'Autriche (Tyrol, Styric...), de Yougoslavie (Alpes dinariques, vallée de la Drina) (3).

(3) Dans les Alpes dinariques, Erica carnea n'est pas spécialement liće aux Pins, mais plutót à l'Epicéa et au Sapin; dans la vallée de la Drina, Picea omorica, dont l'aire est fort limitée, lui est intimement associé [TREguBov (S.) (1934)]. 
On sait qu'en France, cette espèce est très rare (4), sauf précisément en Haute-Maurienne, où son aire couvre plusieurs kilomètres carrés (cf. fig. 14).

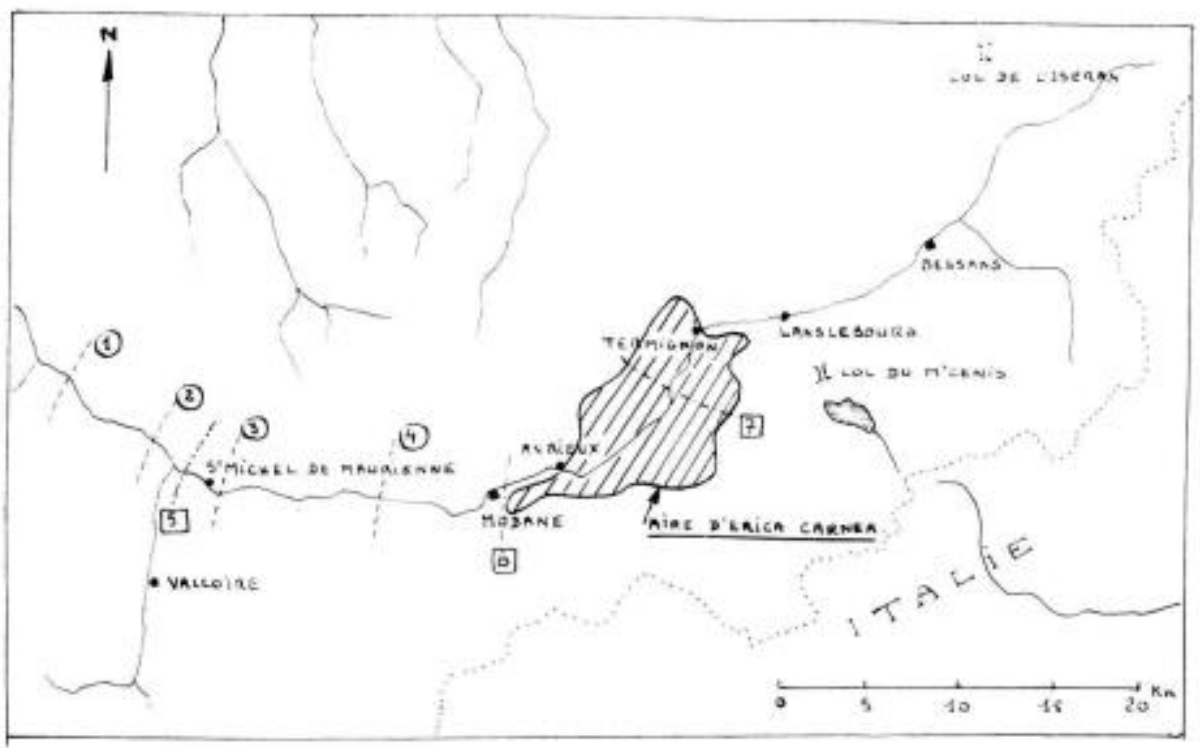

Fig. 14.

Limites de quelques especes importantes.

Légende. - Elément médioeuropéen.

1. - limite de Mercurialis perennis.

2. - limite de Festuca silvatica.

3. - limite de Fagus silvatica, Lamium Galeobdolon, Sanicula Europaea, Carex silvatica, Elymus Europacus, Epilobium montonum.

4. - limite de Hedera Helix et Asperula odorata. Elément subméditerranéen.

5. - limite de Chrysanthemum corymbosum, Campanula persicifolia, Cephalanthera xyphophyllum, Cephalanthera pallens, Viola mirabilis.

6. - limite de Quercus pubescens.

7. - limite de Coronilla Emerus.

Il est évidemment tentant de se demander si son exceptionnelle abondance dans notre vallée - alors qu'elle est très rare ailleurs dans les Alpes occidentales françaises - tient surtout à des facteurs historico-géographiques, ou surtout à des facteurs écologi-

(4) On trouvera dans Perrier de la Bathie (H.) (1917-1928 - T. II, 94) des indications sur les stations de cette espèce en Savoie et Haute-Savoie. En dehors de ces deux départements elle ne s'observe que dans les Alpes maritimes, près du col de Tende. 
ques. Il est difficile de répondre à une telle question car, si l'espèce est bien connue en ce qui concerne ses exigences édaphiques [PALLmanN (H.) (1947) et Braun-Blanguet (J.), Pallalann (H.) et BacH (R.) (1954)], il est actuellement très délicat de cerner les facteurs climatiques auxquels elle est liée, car, dans ce domaine, nous l'avons déjà souligné, les données sont assez rares et incomplètes et l'étude du microclimat des stations à Erica carnea reste à faire.

Toutefois, si l'on se réfère au fait que, dans les Alpes (5) Erica carnea se rencontre dans tous les ilots de sécheresse des vallées intra-alpines, sans pour autant y jouer le ròle d'une espèce xérophile puisqu'elle fuit les versants à exposition plein sud, on conviendra que sa présence en Haute-Maurienne est tout à fait normale. Ce qui l'est moins - mais nous sortons ici, un peu, de notre sujet - c'est son absence totale en Briançonnais, qui connait pourtant un climat assez analogue à celui du Val d'Aoste, ou du Val de Suse, où l'espèce est présente.

La deuxième espèce qui doit retenir notre attention, car son importance n'est pas seulement d'ordre floristique, mais aussi phytosociologique, est Calamagrostis villosa (CHAIX) Mutel.

Cette espèce est restée longtemps méconnue dans les Alpes françaises, puisque, jusqu'à ces dernières années, elle y était considérée comme fort rare et n'était signalée qu'en Tarentaise (où EvRARD et Chermezon (1917-1918) l'avaient découverte), en Vallouise et dans le vallon de Mollières dans les Alpes maritimes. Perrier de la BAтHiE (H.), lui-mème, ne la citait pas dans son catalogue des plantes vasculaires de la Savoie (1917-1928).

On sait, maintenant, que ses stations sont nombreuses dans les Alpes internes de notre pays: elle s'observe, en effet, en Oisans, dans la haute vallée du Vénéon [elle figure, dans un relevé synthétique du Rhodorcto-Empetretum décrit par Nk̀GRE (R.) (1950)], en Haute-Maurienne, où elle est particulièrement abondante [BARTOLI (Ch.) (1955)], dans les vallées du Giffre, de Vallorcine, de l'Arve, du Reposoir [de Leiris (H.) (1961-1965)], dans le Brian-

(5) Dans les Alpes Dinariques, les précipitations sont fortes (avec un régime de type atlantique), en particulier dans le massif du Sneznik, où elles atteignent, annuellement, 2000 à $3000 \mathrm{~mm}$ pour des altitudes inférieures à $1000 \mathrm{~m}$. Mais, selon Wessely, cité par Tregurov (S.) (1941), la « zone climatique du karst se caractérise par des changements de température subits et violents, une température moyenne annuelle basse, des montées de température rapides au printemps; il s'agit donc d'un climat marqué par une * influence danubienne et continentale s incontestable.

On observera que, dans le massif précité, Erica carnea parait jouer un rồle secondaire. Elle se localise dans une association baptisće par HoRvar (I.), Abicto-Calamagrostidetum, plus orécisément, dans la sous-association à épicéa de cette unité qui occupe les versants sud, surtout ceux des trous à gelées [Tregubov (S.) et Divers (1957)]. 
çonnais... Nous l'avons rencontrée en forèts de Bourg-Saint-Maurice, de Pralognan, en Tarentaise, et dans l'étage subalpin de versant nord des vallons de la Madone, du Boreon, de Mollières, dans les Alpes maritimes, où elle joue un rôle très important.

On constate donc que cette espèce, dont l'aire d'après HEGI (G.) (1906-1957) couvre l'Europe centrale et septentrionale (6) et qui donne sa physionomie au tapis herbacé de certains types de pessières et de pineraies de Pin Cembro intra-alpines (7), est presque partout présente dans les Alpes internes françaises.

Sa limite occidentale n'est pas connue avec précision, mais tout porte à croire qu'elle n'atteint pas le massif de Belledonne, puisque nous ne l'avons pas rencontrée dans les pineraies de Pin Cembro de Chamrousse.

Il s'agit donc d'une espèce qui est beaucoup moins localisée qu'Erica carnea, mais qui reste intéressante parce que, dans les Alpes, elle ne descend guère en dessous de l'étage subalpin et qu'elle présente, incontestablement, un caractère d'espèce d'Europe centrale.

\section{II.4.3. - L'élément nordique et des hautes montagnes de l'Europe.}

On sait que de nombreuses espèces du domaine nordique (région euro-sibérienne de la zone holarctique) se retrouvent dans les hautes montagnes de l'Europe, l'analogie entre flore alpine et flore arctique s'expliquant par l'histoire des glaciations quaternaires.

C'est essentiellement dans l'étage subalpin, à la lisière inférieure du domaine des hautes montagnes, que l'on peut rencontrer les espèces communes à ces deux domaines, puisque l'étage alpin échappe à notre analyse et qu'il n'existe pas, dans notre dition, de tourbières boisées, dont on sait qu'elles servent souvent de refuge à des espèces arctiques.

Si l'on se réfère aux relevés des phytosociologues russes, finlandais et scandinaves [Braun-Blanguet (J.), Sissingh (G.) et VlieGER (J.) (1939)], on constate que de nombreuses espèces circumboréales, liées dans le Nord de l'Europe ou même de la Sibérie à la sous-alliance, Piceion septentrionale, se retrouvent dans l'étage subalpin, et même dans l'étage montagnard, de notre haute vallée: il s'agit des Piroles, des Vacciniées, d'Arctostaphyllos Uva-ursi, d'Empetrum hermaphroditum, de Peltigera aphtosa, de Lophosia lycopodioides, etc. Par contre, manquent à notre région: Linnaea borealis - dont on sait l'extrême rareté en France, alors qu'elle

(6) Sans atteindre les pays scandinaves, toutefois.

(7) de Lerris, déjà cité, l'a surtout observée, toutefois, dans les aulnaies vertes. 
est relativement commune dans les Alpes centres - et Trientalis Europaea (8).

On ne doit pas oublier que deux espèces arborescentes ont une aire typiquement nordique bien que leur plasticité, due à l'existence de races écologiques, soit grande et leur permette de prospérer dans des conditions assez éloignées de celles qu'elles connaissent dans le nord de l'Europe; il s'agit ici de Pinus silvestris et surtout de Picea excelsa, cette dernière espèce étant moins thermophile et moins " atlantique " que la première. Ces deux arbres forment, dans notre région, d'importants massifs.

Par contre, les forêts résineuses subalpines de la Haute-Maurienne - et elles partagent ce caractère avec les forêts du même type des hautes montagnes de l'Europe - se distinguent de celles du nord du continent ou de la Sibérie, par la présence de Larix decidua, Pinus Cembro (9), Pinus uncinata, Rhododendron ferrugineum, etc., que l'on peut considérer comme des endémiques de ce domaine et qui jouent done le rôle de différentielles.

\section{II.4.4. - L'élément sub-méditerranéen et méditerranéo-sar- matique.}

Cet élément comprend les espèces qui, selon la nomenclature de Gaussen (H.) (1954) et de Guillaume (H.) (1957), dépassent la limite de f'Olivier et parmi lesquelles Gaussen ( $\mathrm{H}$.) distingue celles qui restent dans la limite du Chêne vert (propéméditerranéennes), celles qui accompagnent l'Alaterne et l'Erable de Montpellier (semiméditerranéennes), celles enfin qui forment les dernières irradiations, et que le Chêne pubescent pourrait servir à caractériser (latéméditerranéennes).

Il est bien certain que, s'il fallait faire un inventaire complet des espèces de cet élément qui atteignent le cour de la Haute-Maurienne. il faudrait ne pas se limiter aux forêts, mais étudier surtout les "garides ", où ces espèces trouvent les meilleures conditions, en général, à leur développement. C'est dire que de par la force des choses, notre analyse se trouve quelque peut tronquée.

Parmi les espèces caractéristiques de l'ordre des Quercetalia pubescentis, certaines ne paraissent pas atteindre les limites aval de notre dition: telles sont Inula hirta, Veronica teucrium, Polygonum dumetorum, etc.; d'autres s'arrêtent à l'entrée des gorges houillères, dans la zone subbriançonnaise: Chrysanthemum corymbosum, Campaurla persicifolia. Cephalanthera xiphophyllum, Cephalanthera

(8) Toutes ces espèces, ou presque, sont liées à des sols à humus brut. Elles trouvent done leur plein développement dans l'étage subalpin où les conditions climatiques sont telles que la minéralisation de l'humus ne s'effectue que très lentement. Il en est de même dans les régions nordiques.

(9) On sait qu'une espèce affine de Pinus cembro: Pinus sibirica (Rupr.) Mayr, a une aire nettement distincte en Sibérie, entre l'Oural et le lac Baikal, a basse altitude [DEBazAC (E.) (1964)]. 
pallens, Viola mirabilis, Cephalanthera rubra (10), etc,; d'autres, par contre, s'enfoncent vraiment au cceur de la zone de sécheresse, telles Viburnum lantana, Amelanchier rotundifolia, Laserpitium latifolium, Trifolium alpestre, Trifolium rubens, Trifolium medium, Geranium sanguineum.

Les caractéristiques de l'alliance Quercion pubescentis, plus spécialisées sans doute, sont plus rares: nous n'avons jamais rencontré, en effet, ni Melittis melissophyllum, ni Sorbus torminalis, ni Bupleurum falcatum, ni Rhus cotinus, etc., alors que Quercus pubescens - avec Acer Monspessulanum, - forme un petit ilot, sur des rochers permiens particulièrement bien exposés, un peu à l'amont de Modane (11). Coronilla Emerus, quand à elle, se rencontre encore, quoique fort rarement, bien au-delà de la limite du Chêne pubescent, dans les bois de Pin sylvestre de Sollières-Sardières (cf. fig. 14).

Si l'on considère, maintenant, les espèces des pelouses maigres des terrains calcaires et marneux du phylum du Chène pubescent, on constate que les plus plastiques - les caractéristiques de la classe des Ononido-Rosmarinetea - sont nombreuses, qui trouvent refuge dans les pineraies xérophiles de notre vallée: il s'agit de Carex humilis, espèce mediterraneo-sarmatique très abondante dans un type de pineraie, l'Ononido-Pinetum, Coronilla minima, Helianthemum canum, Globularia Willkommii, Ononis pusilla, Fumana procumbens, Teucrium montanum. Sont totalement absentes, par contre, les caractéristiques de l'ordre des Ononidetalia striatae, Anthyllis montana et Lavandula vera, en particulier.

A ces espèces, il faut ajouter Carex Halleriana, Astragalus Monspessulanus (12), Ononis rotundifolia.

Il faut enfin noter que certaines subméditerranéennes rencontrćes dans les pineraies de Pin sylvestre du Brianconnais ne s'observent pas dans les pineraies analogues de la Haute-Maurienne: il s'agit d'Onobrychis saxatilis, Astragalus vesicarius. Astragalus purpurens (13).

\section{II.4.5. - L'élément endémique des Alpes occidentales fran- çaises.}

En forêt, cet élément se réduit à une seule espèce, Festuca flavescens, mais qui joue un rôle très important par son abondance

(10) Nous avons cependant trouvé une petite station isolée de Cephanthera rubra, dans une pineraie de Pin sylvestre, au-dessus de Modane.

(11) Jusqu'à ces dernières années, les derniers chénes pubescents observés étaient ceux de la Buffaz, au-dessus de Saint-Michel-de-Mauricnne, à l'entrée des gorges houillères [BARTOLI (Ch.) (1961)].

(12) Ces especes sont surtout abondantes dans des associations relevant de l'ordre des Rosmarinetalia (classe des Ononido-Rosmarinetea).

(13) Elles caractérisent POnobrychideto-Pinetum [Braun-Blanguet (J. (1964)]. 
et sa localisation écologique - versants frais, du montagnard au subalpin, sur sols à moder.

Très répandue dans les forèts des Alpes maritimes internes (nous l'avons observée dans les sapinières et les pessières et cembraies subalpines des vallons de la Madone, de Salese, de Mollières, du Boréon), elle est en Ubaye [LAVAGNe (A.) (1964)], en Queyras [CARIot et SAINT-LAger (1897)], en Oisans [NÈGre (R.) (1950)], en Briançonnais [CADEL (G.) et GiLot (J.-Cl.) (1963)], dans la vallée de Chamonix [CARIot et SAINT-Lager (ibidem)], mais parait curieusement être absente de la Tarentaise: Gensac (P.) (1964), ne la cite pas dans les pessières de la Moyenne-Tarentaise et nous ne l'avons pas rencontrée dans des forêts de la Haute-Tarentaise où elle aurait dû, pourtant, se trouver (pessière subalpine de Malgovert, de Sainte-Foye-en-Tarentaise, etc.).

Quoi qu'il en soit, et bien que sa limite occidentale ne soit pas conntue avec précision, elle parait avoir, dans les Alpes françaises. un caractère intra-alpin, comme Calamagrostis villosa. Mais, à l'inverse de cette dernière espèce, elle est moins localisée en altitude. 

TROISIÈME PARTIE

\section{Les Associations forestières}





\section{III.1. - PREAMBULE}

Pour mieux comprendre la végétation forestière de la HauteMaurienne, et singulièrement celle de ses marges - hêtraies et chênaie pubescente - il nous a paru préférable d'analyser d'abord les associations qui y trouvent leur optimum, c'est-à-dire les associations de résineux purs: pineraies de Pin sylvestre, de Pin à crochets, sapinières, pessières, pineraies de Pin Cembro, en y ajoutant une association de feuillus pratiquement inséparable de celles des résineux: l'aulnaie à Alnus viridis.

Il sera plus facile d'aborder ensuite l'étude des associations marginales, peut-être plus fortement marquées par l'homme et on verra pourquoi, et d'une plus grande complexité: il s'agit de divers types de hêtraies et d'un type de chênaie pubescente. Ces associations qui se situent aux confins aval de notre dition ont évidemment dans le paysage une place relativement réduite et une importance économique pratiquement négligeable. Elles n'en présentent pas moins un intérêt phytogéographique certain. 


\section{III.2. - LES PINERAIES DE PIN SYLVESTRE}

On a pu distinguer, de la vallée de l'Ubaye dans les Basses-Alpes, jusqu'à Leoben, dans la haute vallée de la Mür, en Autriche, toute une zone phyto-géographique qui se trouve coincider avec les parties les plus sèches des vallées intra-alpines et où le Pin sylvestre joue un rôle de premier plan. Cette zone a été précisément appelée la "région du pin " [Braun-Blanguet (J.) et Richard (R.) (1949)].

La Haute-Maurienne est évidemment coupée par cette écharpe et présente diverses associations de cette essence, dont nous allons brièvement définir les grands traits.

Trois faits essentiels s'imposent tout de suite à l'observateur:

- le premier, est que ces associations sont étroitement liées à un étage. On ne les observe pratiquement plus atu-dessus de $1700-$ $1750 \mathrm{~m}$; c'est dire que leur domaine, selon la nomenclature que nous avons adoptée, est l'étage montagnard.

- le second, c'est qu'elles se rencontrent à toutes les expositions, aussi bien, par conséquent, sur versant exposé au nord que sur celui exposé au sud.

- le troisième enfin, c'est qu'elles s'observent sur toutes les roches-mères, donc sur des types de sols assez variés, mais avec une restriction importante, toutefois: à exposition nord, en effet, elles se localisent sur les sols carbonatés reposant eux-mèmes sur des cargneules, des moraines calcaires, etc., laissant la place à la sapinière sur les sols bruns liés aux quartzites, aux schistes lustrés, aux schistes et grès du Houiller; à exposition sud, au contraire, on les rencontre indifféremment sur schistes lustrés, schistes et grès du Houiller, quartzites, encore que sous forme fragmentaire, car l'homme, dans l'étage montagnard sec, a presque totalement détruit la forêt, sauf sur les cargneules et les gypses, pour y installer ses villages et ses cultures.

On peut donc distinguer des associations de sols calcimorphes, qui seront soit xérophiles, soit mésophiles, soit mésoxérophiles et des associations de sols silicimorphes (acidiphiles) qui seront uniquement xérophiles puisque localisées sur versant sec, comme il vient d'être dit. 


\section{III.2.1. - Les pineraies de Pin sylvestre sur sols calcimor- phes.}

III.2.1.1. - La pineraie xérophile à Coronilla minima et Ononis rotundifolia.

1. Organisation sociologique et composition floristique - Faciès.

Ce que nous avons déjà dit lors de l'étude des groupes écologiques xérophiles, nots dispensera de faire ici de longs commentaires.

Dans la strate arborescente, le Pin sylvestre domine de façon absolue; il est quelquefois accompagné du Pin à crochets et de l'Epicéa: ce dernier atteint rarement l'état adulte et se présente toujours sous forme d'individus souffreteux.

Dans les strates arbustives et herbacées, où l'élément a xérophile $n$, pris dans un sens large est pratiquement le seul à être représenté, on peut distinguer plusieurs groupes, dont certains, rappelons-le. ont un caractère " géographique ". II faut noter, en premier lieu, ceux dont les espèces sont plus étroitement liées que les autres à des sols calcimorphes: les xérophiles d'abord, comme Coronilla minima, Ononis rotundifolia, Astragalus Monspessulamus, etc. toutes subméditerranéennes: les mésoxérophiles ensuite, comme Sesleria coerulea, Calanagrostis varia, Melampyrum pratense subsp. alpestre, etc, qui se retrouveront en grande abondance dans les associations affines du versant est ou nord, c'est-à-dire plus mésophiles.

Dans le reste du cortège des xérophiles et dont l'indifférence à l'égard du chimisme du sol est plus grande, il y a lieu de mettre à part les especes sud-etropéennes à affinités "orientales ", comme Polygala Chamacbuxus, Carex humilis, Astragalus Onobrychis et les subméditerranéennes comme Amelanclier rotundifolia, Teucrium Chamaedrys, Calamagrostis argentea, Carex Halleriana, etc.; le reste du cortège comprenant des médio-européennes, dont certaines à affinités boreo-arctiques comme Arctostaphylos Uva-ursi.

C'est, à notre avis, la coexistence de ces deux grands groupes orophile subméditerranécn et sud-oriental, d'une part, et médioeuropéen et boréal d'autre part, qui domne à la pineraie xérophile intra-alpine toute son originalité.

I a présence d'autres groupes qui jouent le róle de groupes différentiels permet de distinguer trois sous-associations:

- tout d'abord, une sous-association riche en espèces pionniers à Hieracium lanatum. Asperula aristata, Fumana procumbens, etc. qui s'observent sur les sols les moins évolués, les plus squelettiques, les moins riches, par conséquent, en matière organique. Cette sousassociation correspond à la phase initiale de lassociation. 
- une sous-association type, correspondant à la phase optimale de l'association, d'où ont disparu les espèces pionniers et où, corrélativement, sont quelque peu représentées des mésoxérophiles de sols calcimorphes.

- une sous-association, enfin, à Fagus silvatica, localisée dans la zone subbriançonnaise, aux confins de l'aire du Hêtre et sur laquelle nous aurons l'occasion de revenir lors de l'étude des hêtraies.

Les espèces différentielles en sont: Coronilla Emerus, Cephalanthera rubra, Chrysanthemum corymbosum, Acer opulifolium, Fagus silvatica... toutes espèces qui s'observent dans les chênaies pubescentes ou dans certaines hêtraies thermophiles proches de ces chênaies. On notera au passage que, dans cette unité, les mésoxérophiles et mésophiles de mull ou de moder calcique sont mieux représentées et jouent ainsi, dans une certaine mesure, le rỏle de différentielles.

Il faut mettre l'accent enfin - qu'il s'agisse de l'une ou de l'autre de ces trois unités - sur la rareté des mousses qui ne s'observent que dans des microstations privilégiées: le pied des arbres, en particulier.

Si l’on se réfère au tableau général d'association — en s'en tenant au seul groupement type - ou au spectre floristico-écologique qui en est l'image (fig. 32), on constate que lorganisation sociologique de la pineraie à Coronilla minima et Ononis rotundifolia est assez complexe, malgré les apparences. Se trouvent, en effet, réunies sur la même station, des espèces dont les aires possèdent des centres de gravité assez divers: l'étude précise de leur écologie - qui reste à faire - devra donc, là plus qu'ailleurs, se référer tout particulièrement at microclimat des sols où on les observe; elle permettra, il faut l'espérer, d'accoler des termes plus précis aux termes géographiques ou phytogéographiques que nous avons dû utiliser faute de mieux et de donner ainsi, de l'organisation sociologique de cette pineraie une vue plus fine.

La structure est uniforme. La strate arborescente est le plus souvent régulière (cf. ci-après: caractéristiques sylvicoles). Arctostaphylos Uva-ursi est presque toujours présente: elle forme soit des taches isolées entre lesquelles s'observent, par petits groupes, Astragalus Monspessulanus, Ononis rotundifolia, Carex humilis... soit une nappe quasi continue d'où surgissent ces mêmes espèces dont la tendance, même dans ce cas là, est de se localiser dans de petits " vides $n$.

La strate arbustive est assez lâche, et mis à part les trotiées de régénération riches en jeunes Pins sylvestres, elle comprend essentiellement l'Amelanchier et le Genévrier à moitié prostré (forme intermedia). 
PLANCHE IV

Les pineraies de Pin sylvestre xérophiles.

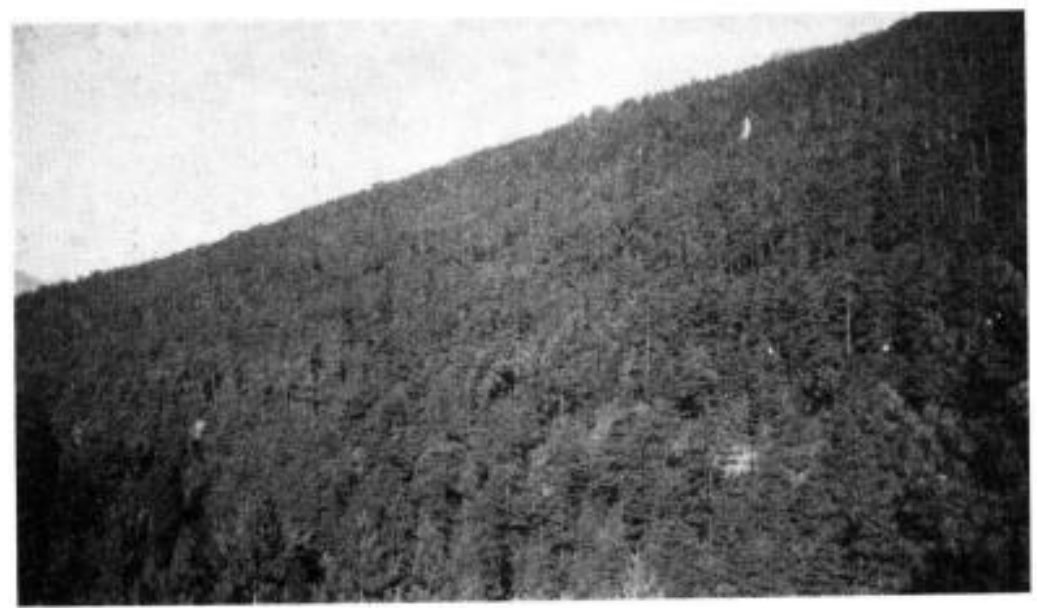

La pineraie xérophile à Ononis rotundifolia et Coronilla minina. Forêt d'Aussois - Vue d'ensemble.

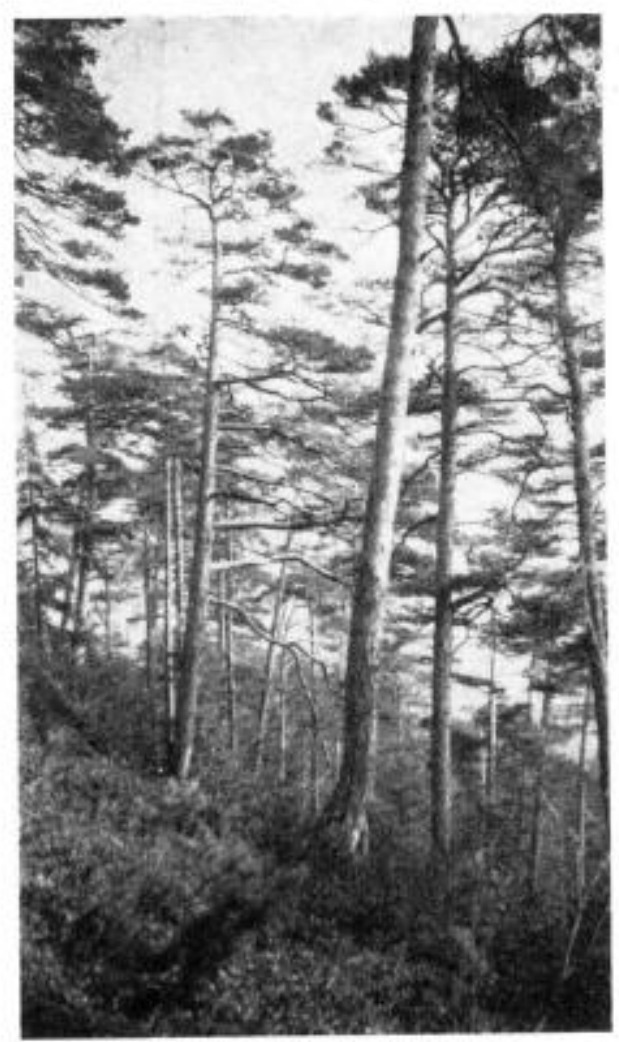

La pineraie xérophile. Vue de détail. 


\section{$2^{\circ}$ Identification - Dénomination,}

A la suite du dernier travail de Braun-Blanguet (J.) sur la végétation xérophile des vallées intra-alpines (1961), travail qui avait été précédé d'analyses plus fragmentaires (1959), on peut distinguer dans la " région du Pin " quatre associations principales xérophiles de Pin sylvestre, reposant sur sols calcimorphes; ces groupements, dont il faut dire tout de suite qu'ils sont très proches les uns des autres, se caractérisent, chacun, par un cortège de différentielles géographiques qui va s'affaiblissant, lorsque l'on passe du Briançonnais, à la Maurienne, au Valais, puis à la Styrie; c'est ainsi que dans l'Onobrychideto-Pinetum du Briançonnais se rencontrent des méditerranéo-montagnardes assez strictes, comme Onobrychis saxatilis, Daphne Cneorum subsp. Verloti, Astragalus purpureus, Astragalus austriacus qui disparaissent plus au nord, en Maurienne ou dans le Valais; là, un deuxième groupe composé d'espèces à amplitude écologique plus large: Coronilla minima, Odontites viscosa, Ononis pusilla, et qui s'observait dans la précédente association, permet de distinguer une deuxième association: l'Odondito-Pinetum; tandis que I'Ononido-Pinetum - troisième association - se différencie surtout négativement, par l'absence des espèces précédemment citées et, positivement, par la présence d'un dernier reliquat de méditerranéo-montagnardes, dont Astragalus Monspessulanus, Ononis rotundifolia; quant à l'Astragalo-Pinetum de la Styrie, il doit son originalité à deux caractéristiques territoriales: Astragalus vesicarius subsp. venostanum et Oxytropis Halleri subsp. velutinum.

Où situer, dans cet ensemble, les pineraies xérophiles et neutrophiles de la Haute-Maurienne? Elles ne relèvent pas, indiscutablement, des deux groupements " extrêmes $n$ : 1'OnobryclidetoPinctum briançonnais et l'Astragalo-Pinetum autrichien; par contre, elles doivent être placées entre lOdondito-Pinetum et lononido-Pinetum du Valais. Sans entrer dans des discussions byzantines sur un tel problème de classification. on doit constater que, par Coronilla minima, souvent présente, nos pineraies diffèrent quelque peu de l'Ononido-Pinctum; elles ne peuvent, par contre, être totalement issimilées à l'Odondito-Pinctum, dont elles sont pourtant très proches, car Odondites viscosa reste extrêmement rare (1).

Il r'est pas inutile de rappeler ici que les pineraies en cause ont été réunies par Braux-Blaxqurt dans une alliance spéciale, l'Ono-

(1) La présence de cette espèce en Maurienne que Huguesin prétendait avoir trouvée à Lanslebourg, était mise en doute par PERrier de la Bathif (H.) (1917-1928) qui ne l'avait jamais rencontrée. Nous l'avons observée sur le plateau de Sardières, sur gypse, dans un peuplement rabougri de Pin à crochets, et, en un point de la pineraic de Sollières-Sardières, où, localement, elle était abondante. BRAEx-Bt.ANoUET (J.) T'a également rencontrée au Verney. près de Bramans (1961). 
nido-Pinion, apparenté d'abord à l'ordre méditerranéo-montagnard des Ononidetalia striatae (1949). Ce dernier classement présentait l'inconvénient de masquer les affinités existant entre ces associations et celles rattachées à lalliance de l'Erico-Pinion, et qui relèvent des Vaccinio-Piceetalia. Récemment, Braun-Blanguet a donc modifié son classement primitif pour apparenter l'OnonidoPinion aux Vaccinio-Piceefalia (1959). Mais ce classement lui-même n'est pas satisfaisant, puisqu'il fait entrer des associations neutrophiles, qu'elles soient xérophiles (Ononido-Pinion) ou mésophiles (Erico-Pinion), dans un ordre (Vaccinio-Picectalia) et une classe (Vaccinio-Picetea) qui comportent essentiellement des associations acidiphiles.

HoRvat (I.) (1959) a supprimé cet inconvénient en créant une nouvelle classe, celle des Erico-Pinetea plus homogène, par conséquent, que l'ancienne classe des Vaccinio-Piceefea, laquelle subsiste évidemment en gagnant, à son tour, de 1'homogénéité.

On notera que cette division est tout à fait comparable, bien qu'il s'agisse d'unités plus vastes, à celle déjà signalée et qui concernait l'alliance du Fagion. Nous aurons d'ailleurs l'occasion, in fine, de revenir sur ce problème capital de la nomenclature et de la classification.

\section{$3^{\circ}$ Répartition - Ecolog̣ie.}

a) Répartition générale.

Qu'il s'agisse de la sous-association vraiment intra-alpine ou de celle qui, dans la zone subbriançonnaise jouxte la hêtraie thermophile et xérophile, le cercle d'exposition montre leur étroite localisation quant à Texposition: dès qu'une inflexion secondaire du terrain permet à des conditions moins xériques de se manifester. les espèces les plus xérophiles disparaissent pour laisser la place à des mésoxérophiles comme Erica carnea, etc. (fig, 30).

Dans la zone subbriançonnaise, la pineraie xérophile passe donc, latéralement, à une hètraie, ot même à une sapinière vraie: plus en amont - les gorges houillères franchies, - elle passe, dans les mêmes conditions, à une pineraie mésophile on à une sapinière vraie, tandis qu'en altitude, vers $1750 \mathrm{~m} .1800 \mathrm{~m}$, au maximum, elle laisse la place, dans l'étage subalpin. à des peuplements homologues de Pin à crochets.

\section{b) Climat local et microclimat.}

Bien que le poste météorologique d'Avrieux se trouve situé à la limite inférieure de lassociation, ses données déjà analysées permettent de se faire une idée assez exacte du climat local régnant au bas de la pineraie sèche en Haute-Maurienne. En rapprochant les deux courbes de précipitations d'Avrienx (1 $100 \mathrm{~m}$ ) et d'Aussois $(1500 \mathrm{~m})$ situés à la mème exposition et l'un aut-dessus de l'autre, 
et en extrapolant linéairement les données jusqu'à $1750 \mathrm{~m}$, limite de l'étage du Pin sylvestre, on peut donner une assez bonne image des conditions climatiques tout au moins celles concernant les précipitations, régnant dans l'étage montagnard sec (fig. 17).

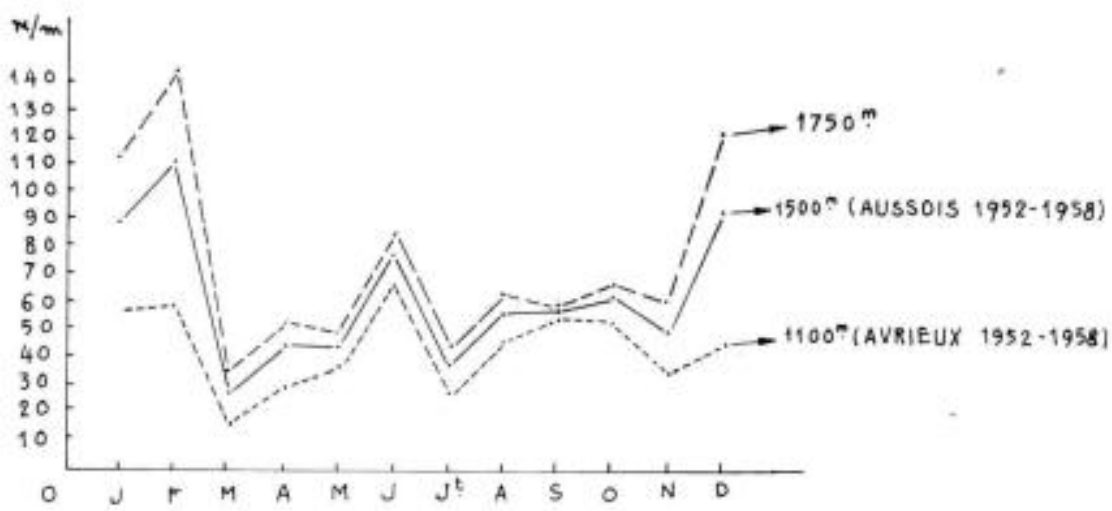

Fic. 17.

Les pineraies de Pin sylvestre - Misoclimat.

Légende. - A exposition sud, ou sud-est, pour la période 1952-1958, le régime des précipitations dans l'étage du Pin sylvestre est représenté par les courbes ci-dessus, les moyennes annuelles étant les suivantes: Avrieux: 525 mm; Aussois: $741 \mathrm{~mm}$; à $1750 \mathrm{~m}$ (par extrapolation linéaire): $891 \mathrm{~mm}$.

En ce qui concerne le microclimat, plusieurs observations importantes s'imposent. Si l'on compare un individu de pineraie sèche (Ononido-Pinetum) à un individu de pineraie mésophile (EricoPinetum), le premier à altitude de $1600 \mathrm{~m}$, le second à $1300 \mathrm{~m}$, on constate que les moyennes de température de l'air dans l'EricoPinetum sont un peu plus élevées que dans r'Ononido-Pinetum, comme on pouvait s'y attendre, puisque l'altitude plus basse joue en faveur, si l'on peut dire, de l'association mésophile; par contre, et ceci nous parait tout à fait remarquable, le sol $-\grave{a}-2 \mathrm{~cm}-$ s'avère être, en moyenne, plus chaud, à $1600 \mathrm{~m}$ dans l'OnonidoPinetum qu'à $1300 \mathrm{~m}$ dans lérico-Pinetum. Et si l'on considère les maximums, pour le sol, il est facile de se rendre compte qu'ils sont plus éloignés l'un de l'autre que ne le sont entre elles les valeurs moyennes (fig. 18).

De telles caractéristiques apparaissent encore mieux sur les graphiques concernant un individu de pineraie sèche et un de sapinière, car, dans ce cas, les deux individus se trouvaient situés, à très peu près, à la même altitude (fig. 19).

Autre fait à noter: si la température de l'air - à $1,50 \mathrm{~m}$ - présente, dans la pineraie sèche, des oscillations fortes, ces oscilla- 
Températures de l'air

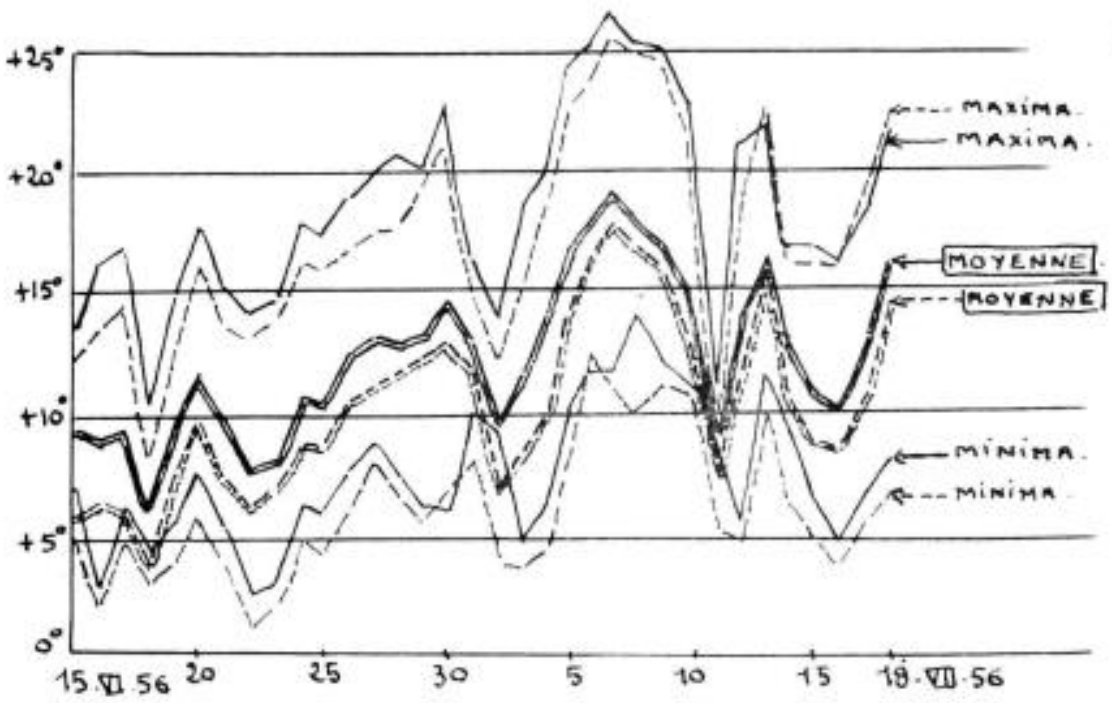

Températures du sol.

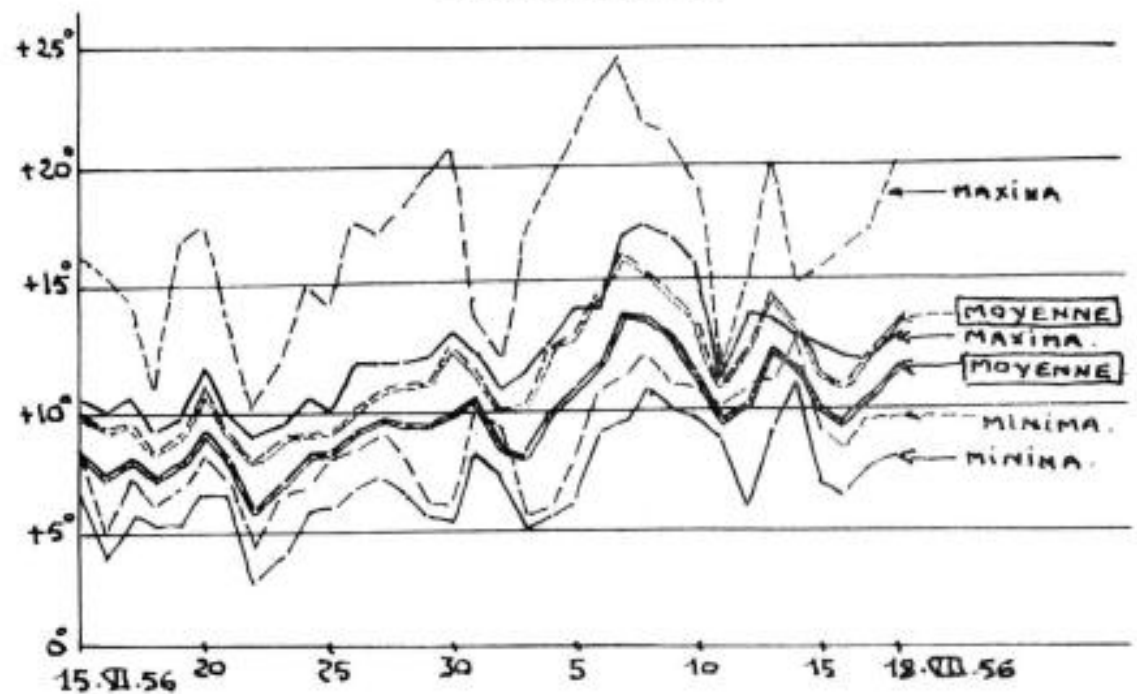

Oninido-Pinetum (1 $600 \mathrm{~m}$ ).

Erico-Pinetum (1 $300 \mathrm{~m})$.

Fig. 18.

Les pineraies de Pin svlocstre - Microclinat.

Légende. - Les courbes ci-dessus, dessinées à partir de données obtentes dans deux individus de pineraies, l'un xérophile, Tautre mésophile, mais à des allitudes diffirentes, montrent que la température de l'air, à $1,50 \mathrm{~m}$, est plus élevée à $1300 \mathrm{~m}$ à exposition nord, cửà $1600 \mathrm{~m}$ म̀ exposition sud.

Par contre, les courbes qui concernent les temperatures du sol à $-0,02 \mathrm{~m}$, font apparaitre le phénomène inverse. La supériorité thermique de l'OnonidoPinetum par rapport à PErico-Pinetum se traduit done an niveatu du sol, alors méme que l'altitude le défavorise. 
Températures du sol (Maximums et minimums)
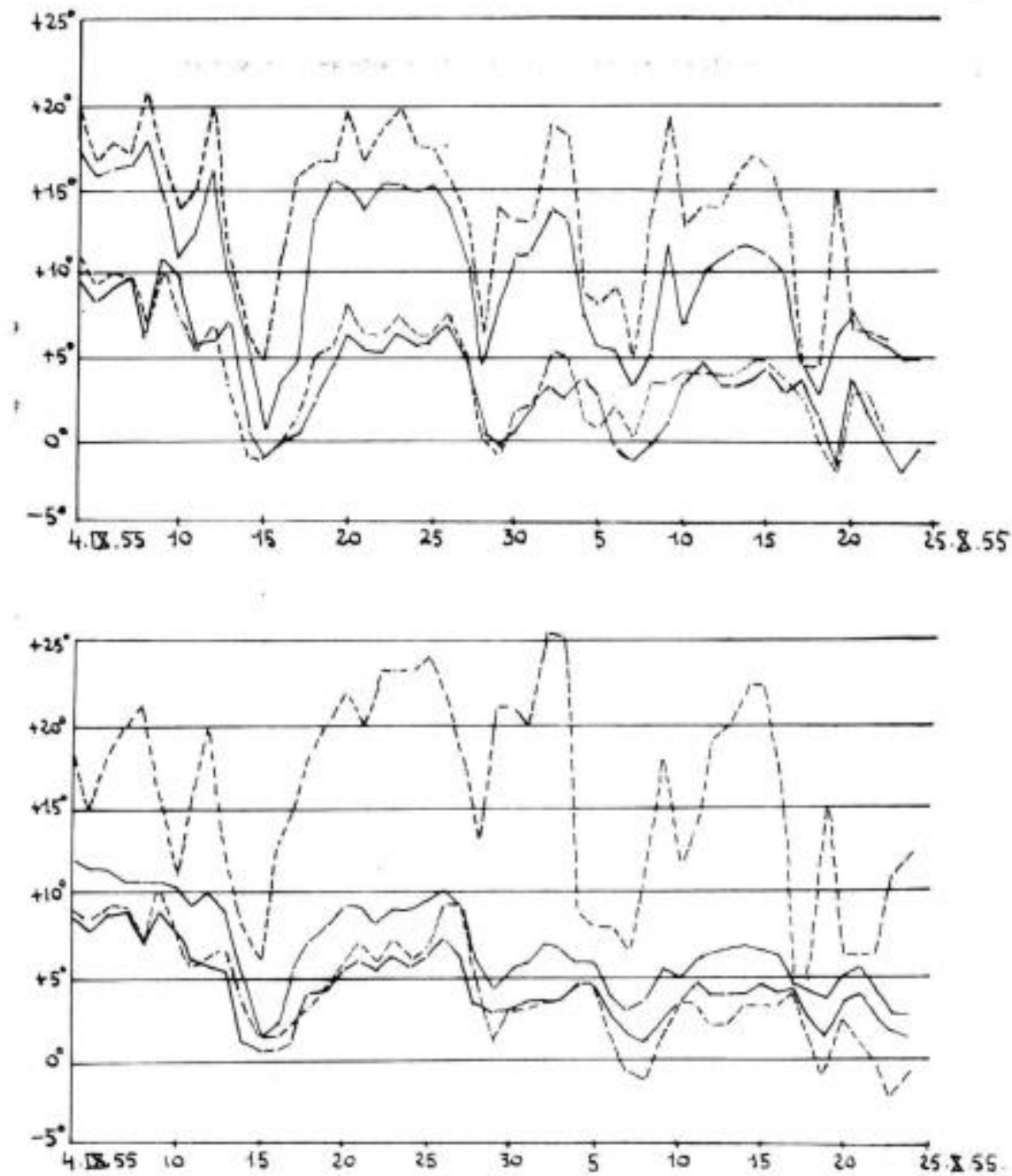

-... Ononido-Pinetum (1600 m).

Abietetum albae $(1600 \mathrm{~m})$.

F16. 19.

Legende. - Si les températures de l'air (à $1,50 \mathrm{~m}$ ) - maximums et minimums - des deux individus d'associations en catse (Ononido-Pinefum et Abietełsm albae), bien que situće à la méme altitude, ne diffèrent que peu, celles du sol $(\mathrm{a}-0,02 \mathrm{~m})$ sont, par contre très dissemblables.

On notera que les températures convergent, les jours pluvieux (15-9-1955 17-10-1955). 
tions sont comparables à celles de la température de l'air de la pineraie mésophile, et même à celles de la température de l'air de la sapinière; par contre, la température du sol - à $-2 \mathrm{~cm}-$ dans la pineraie sèche, présente par rapport à celle des deux autres associations, une originalité certaine: le climat thermique du sol $(\mathrm{a}-2 \mathrm{~cm}$ ) de la pineraie sèche est très nettement plus brutal que celui de la pineraie mésophile et plus fortement encore que celui de la sapinière. De telle sorte que, si l'on mettait en parallèle les températures de l'air des trois associations en cause, à même altitude s'entend, il y aurait des différences sans doute, mais elles seraient relativement faibles; par contre, les températures du sol (à $-2 \mathrm{~cm})$ présenteraient entre elles des différences fortes et des plus significatives.

Qu'il s'agisse de régime thermique moyen ou des oscillations journalières de la température, le microclimat du sol — dans ses premiers centimètres - de la pineraie sèche, présente des caractères propres qui nous paraissent constituer l'un des traits les plus saillants de toute l'écologie de l'association, et l'on jugera vite de leur importance, si l'on se réfère au fait que c'est au niveau du sol - et dans ses premiers centimètres - que se produisent les phénomènes capitaux de la germination.

Ces résultats viennent confirmer ceux obtenus en Suisse, tout au moins sur l'essentiel, car les conditions d'observation n'ont pas été les mèmes dans les deux cas. Dans le Parc national suisse, en effet, les associations comparées ne sont pas identiques aux nôtres: il s'agit plutöt de vicariants subalpins: d'une part le Pineto-Caricetum humilis, d'autre part le Mugeto-Ericetum (on dirait, maintenant, selon la nomenclature nouvelle, Carici humilis Pinetum et Erico-Mugetum); les températures du sol ont été prises à la surface; les individus choisis se trouvaient à la même altitude: $1900 \mathrm{~m}$. Comme on le voit sur le tableau ci-après, les différences de température au niveau du sol - que nous avons mises en évidence entre les deux associations, sont beaucoup plus fortes que les différences de température de l'air, à $1,50 \mathrm{~m}$.

\begin{tabular}{|c|c|c|c|c|c|c|}
\hline \multirow[b]{3}{*}{ 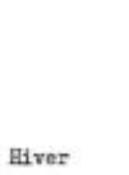 } & \multicolumn{3}{|c|}{$\begin{array}{l}\text { Tespérature moyene de } \\
\text { I'nir }\end{array}$} & \multicolumn{3}{|c|}{$\begin{array}{l}\text { Tenperature moyenne au } \\
\text { nivesu du sol }(0 \mathrm{~cm})\end{array}$} \\
\hline & Pinerate & Nugetaie & $\Delta t$ & Pinerale & Nugetste & $\Delta t^{\prime}$ \\
\hline & $2,2^{\circ}$ & $-0,6^{\circ}$ & $2, e^{\circ}$ & 110 & $-0,3^{0}$ & $11,3^{\circ}$ \\
\hline Printeaps & $10,5^{\circ}$ & $9,9^{\circ}$ & $0,6^{\circ}$ & $23^{\circ}$ & $16,5^{\circ}$ & $6,5^{\circ}$ \\
\hline Eté & $16,7^{\circ}$ & $15,1^{\circ}$ & $1,6^{\circ}$ & $31,3^{\circ}$ & $23,4^{\circ}$ & $7,9^{\circ}$ \\
\hline Automne & $15,6^{\circ}$ & $13,5^{\circ}$ & $2,2^{6}$ & $26,88^{\circ}$ & $17,7^{\circ}$ & $9.1^{\circ}$ \\
\hline
\end{tabular}

Si l'on s'éloigne de la surface du sol, le phénomène s'inverse par contre. 
La chute de température est des plus nettes à $-15 \mathrm{~cm}$ et ses variations deviennent comparables à celles de la température des horizons superficiels des associations mésophiles (fig. 20).

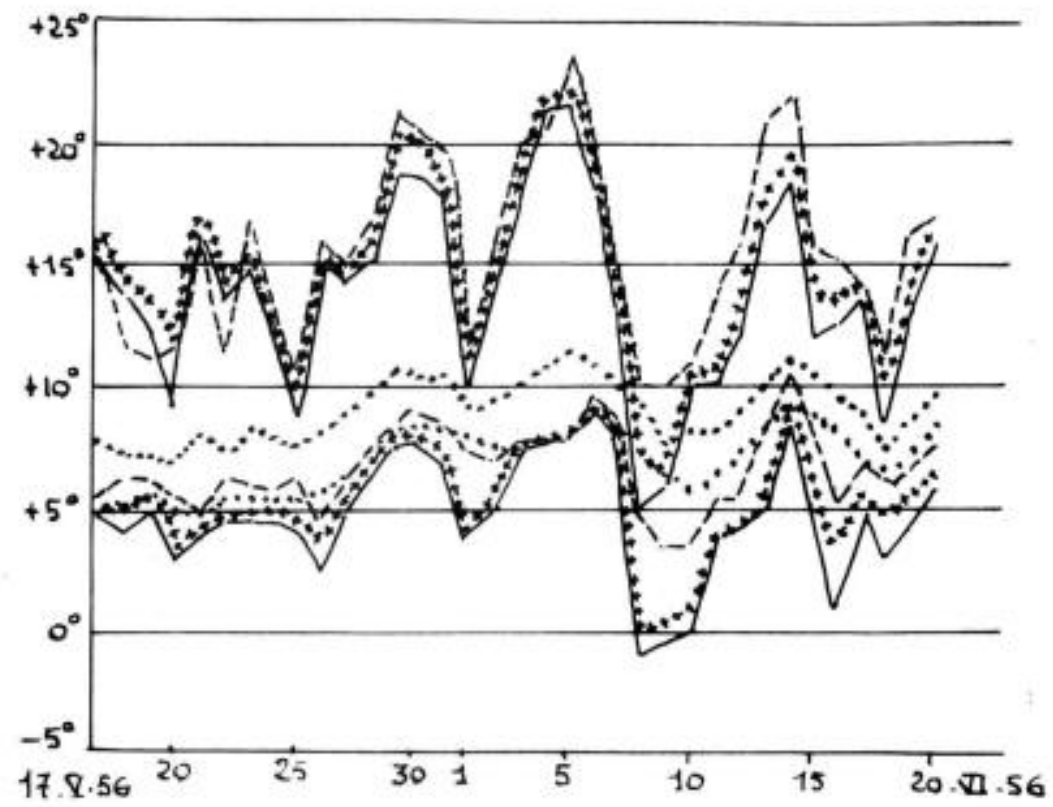

-. . Température du sol à $-0,02 \mathrm{~m}$ (max. et min).

$\ldots$. Température du sol à $-0,15 \mathrm{~m}$ (maxi. et min.).

- Température de 1'air à $+1,50 \mathrm{~m}$ (bax, et min.).

+++ Température de l'air à $+0,20 \mathrm{~m}$ (max. et $\min$.).

Fig. 20.

Les pineraies de Pin sylvestre xérophiles - Microclimat.

Légende. - Bien qu'effectuée sur un laps de temps un peu court pour que l'on puisse en tirer des conclusions fermes, la mesure des températures à différents niveaux, dans un individu de pineraie de Pin sylvestre à Ononis rotundifolia et Coronilla minima, montre que la température du sol à $-0,02 \mathrm{~m}$, est, dans l'ensemble, plus élevée que celle de l'air, même à $+0,20 \mathrm{~m}$, mais qu'elle s'abaisse sensiblement, en présentant de faibles oscillations journalières, dès que l'on atteint et dépasse une certaine profondeur $(-0,15 \mathrm{~m})$.

Bien que nous n'ayons pu dresser les profils thermiques complets des associations comparées, il apparait très nettement cependant, que l'Ononido-Pinetrem possède, par rapport à l'Erico-Pinetum et l'Abietetum albae un profil thermique différent de ceux de ces dernières associations, et ceci tient essentiellement aux régimes thermiques qui règnent près de la surface du sol, dans les trois groupements en cause. 

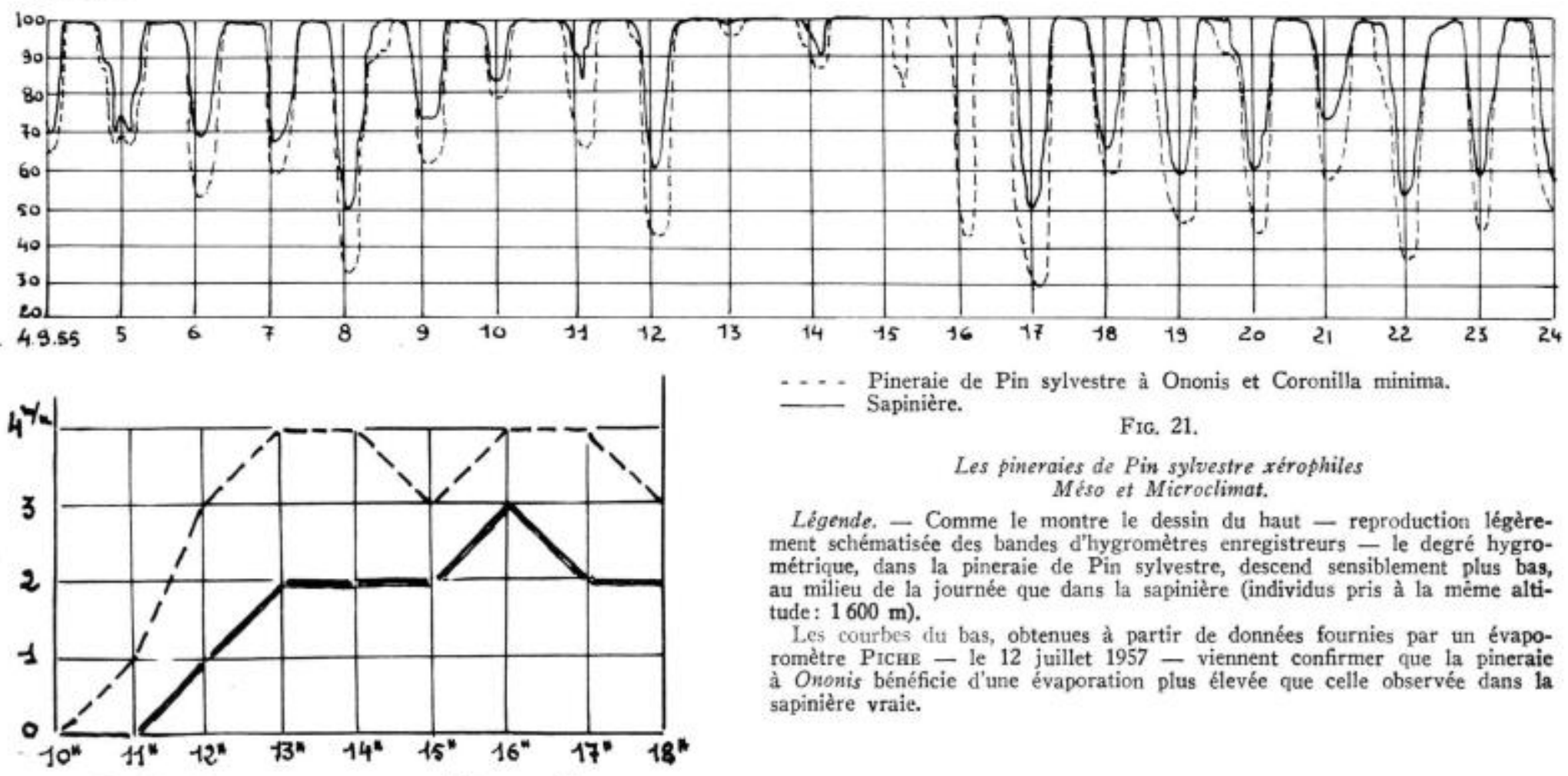

-.. Pineraie de Pin sylvestre à Ononis et Coronilla minima.

- Sapinière.

Fic. 21.

Les pineraies de Pin sylvestre xérophiles Méso et Microclimat.

Légende. - Comme le montre le dessin du haut - reproduction légèrement schématisée des bandes d'hygromètres enregistreurs - le degré hygrométrique, dans la pineraie de Pin sylvestre, descend sensiblement plus bas, au milieu de la journée que dans la sapinière (individus pris à la mẻme altitude: $1600 \mathrm{~m}$ ).

Les courbes du bas, obtenues à partir de données fournies par un évaporomètre PicHE - le 12 juillet 1957 - viennent confirmer que la pineraie à Ononis bénéficie d'une évaporation plus élevée que celle observée dans la sapinière vraie. 
De telles différences dans les régimes thermiques s'expliquent évidemment par l'exposition, la pente, etc.; la sécheresse de l'air et celle du sol dont il sera question ci-après, jouent certainement un rôle de premier plan: sur nos courbes, en effet, on constate une convergence des températures des jours de pluie ou simplement couverts. Cette sécheresse - que nous avons traduite par le déficit
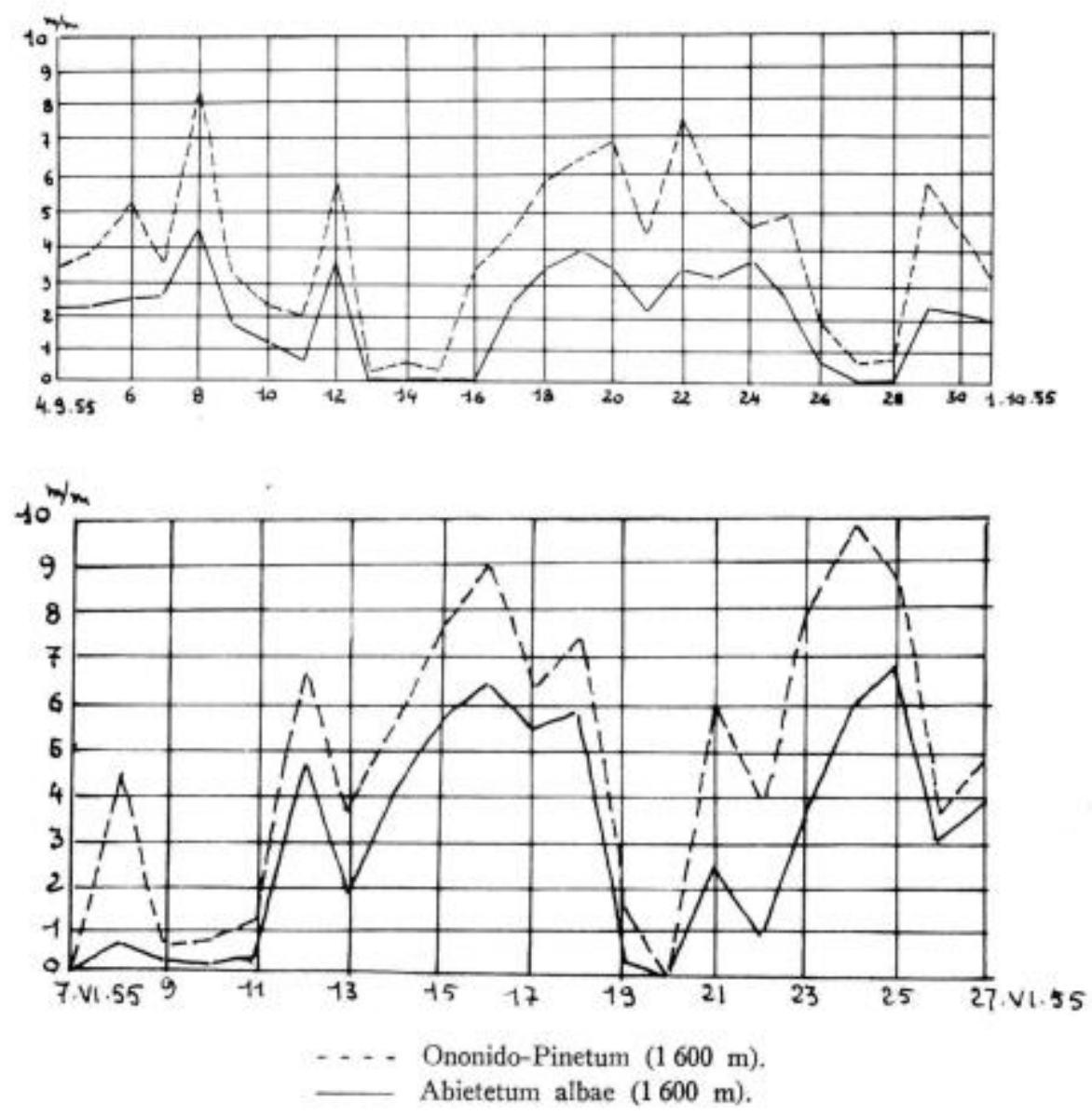

Fig. 22.

Les pineraies de Pin sylvestre xérophiles Méso et Microclimat.

Légende. - Comme on le voit sur ces deux schémas, résultant d'expériences faites à des époques différentes, le pouvoir évaporant de l'atmosphère, à 1,50 $\mathrm{m}$, entre 8 et 16 heures, est toujours nettement plus élevée dans l'Onowido$P$ inetum que dans l'Abietetum albae, sauf, évidemment, les jours couverts et, a fortiori, les jours de pluie, où se manifeste une convergence. 
de saturation à $1,5 \mathrm{~m}$ - montre que ha pineraie connait un régime plus xérique que la sapinière ou la rhodoraie à Pin Cembro (fig. $21-22-23)$.

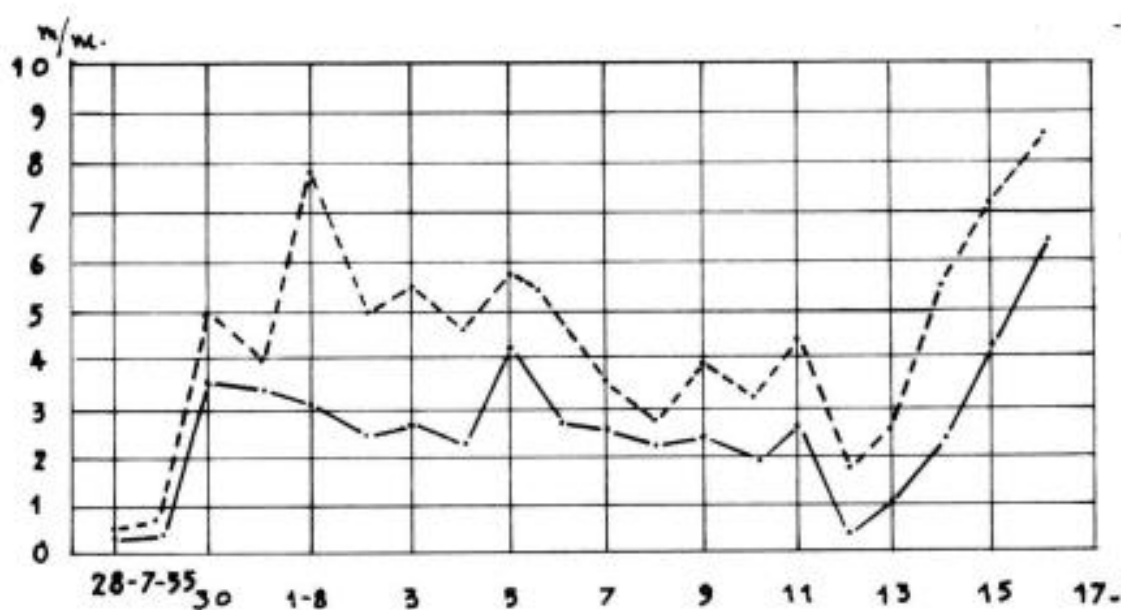

_... Ononido-Pinetum

FIG, 23.

Ligende. - Bien que la rhodoraie à Pin Cembro soit située à une altitude plus élevée $(1950 \mathrm{~m})$ - ce qui la favorise dans le domaine de l'évaporation - la pineraie de Pin sylvestre à Ononis rotundifolia (altitude $1240 \mathrm{~m}$ ) présente, tout de méme, un pouvoir évaporant, à $1,50 \mathrm{~m}$ (entre 8 et $16 \mathrm{~h}$.), plus élevé et qui est dù à des conditions thermiques nettement plus favorables.

\section{c) Sols.}

Il ressort de la morphologie des profils et des analyses, que les sols de l'Ononido-Pinetrm sont. soit des xirorendsines dolomitiques, en général (sol $\mathrm{n}^{\circ} 1$ ), soit des xérorendzines dolomitiques brunifées, mais à complexe saturé ot proche de la saturation ( (fig. 24).

La texture est limono-argileuse; 1'humus est un mull-moder calcique très riche en matière organique; on peut penser que la pente empéche, par érosion, la formation d'un humus brut; tout indique une vie biologique peu active: la forte accumulation de matière organique - $52,3 \%$ dans le sol $1,33,2 \%$ dans le sol $2-$ le $\mathrm{C} / \mathrm{N}$ élevé $\left(33,8\right.$ dans le sol $n^{\circ} 1,26,3$ dans le sol $\left.n^{\circ} 2\right)$, la structure peu agglomérée, la décarbonatation souvent accentuée des horizons supérieurs. Il faut vraisemblablement attribuer à l'cxcès d'insolation ot de dessication cette évolution des sols et la légère tendance à lacidification en surface que l'on y observe. Nous verrons que dans 
des associations un peu plus mésophiles — sur même roche-mère les conditions écologiques permettent une meilleure vie biologique, ce qui entraine une structure en grumeaux et une carbonatation qui s'affirme jusqu'à la surface.

Ononido-Pinetum

Types de sols

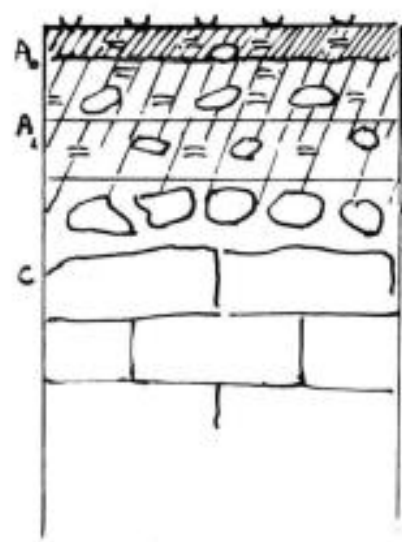

Rendzine jeune à mull-moder calcique.

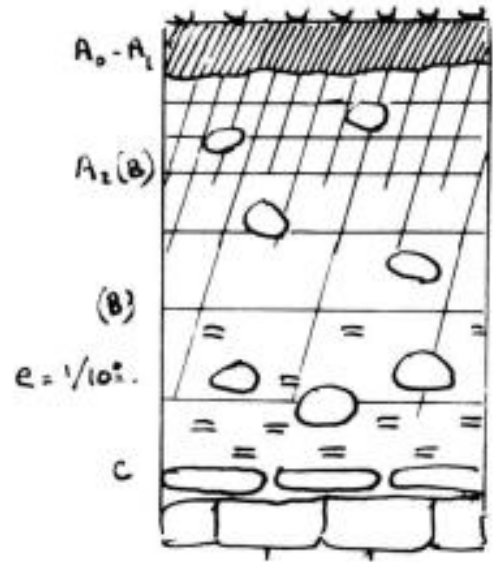

Rendzine brunifiée à mull-moder calcique.

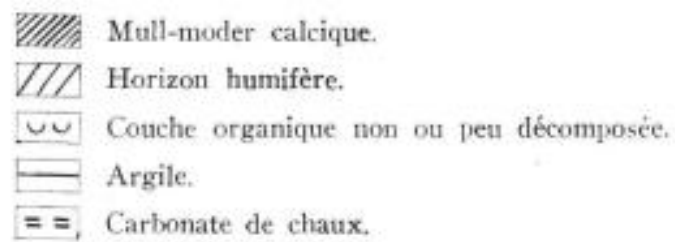

Description morphologique (fig. 24).

- Profil $n^{\circ} 1$.

$$
A_{0}(0-3 \mathrm{~cm})
$$

- horizon de mull-moder calcique de structure à grains grossiers, brun noirâtre.

- squelette constitué de morceaux de cargneule, correspondant à $30 \%$ du volume total.

- racines nombreuses.

$$
A_{1}-C(4-15 \mathrm{~cm})
$$

- passage brutal à la roche-mère - horizon de couleur brun ocracée; structure particulaire à gros grains - squelette formant $80 \%$ du volume total. 
- Analyses.

Sol $n^{\circ} 1$ - Forît de Modane.

Plle 5 - Exp. S. - Altitude: $1460 \mathrm{~m}$ - Pente: $40 \%$ - Roche-mère : cargneule.

Relevé floristique $\mathrm{n}^{\circ} 11$

\begin{tabular}{|c|c|c|c|c|c|c|c|}
\hline Horizona & $\begin{array}{l}\text { Pror. de } \\
\text { prélìven. }\end{array}$ & pH & $\begin{array}{l}\text { Matiòre } \\
\text { organiquo }\end{array}$ & c & K & $\mathrm{c} / \mathrm{s}$ & $\begin{array}{c}\cos \mathrm{Ca} \& \mathrm{~N} \\
\text { total }\end{array}$ \\
\hline$\Lambda_{0}$ & $-3 \mathrm{ce}$ & 8,2 & 52,3 & 30,7 & 0,91 & 33,8 & 10,6 \\
\hline$\Lambda_{1}-c$ & $-15 \mathrm{ca}$ & 8,5 & 8,6 & & & & 52,6 \\
\hline
\end{tabular}

Sol $n^{\circ} 2$ - Forèt a'Aussois

2 série - Plle I - Exp. S. - Altitude: $1620 \mathrm{~m}$ - Pente $40 \%$ Roche-mère: cargneule.

\begin{tabular}{|c|c|c|c|c|c|c|c|c|c|}
\hline $\begin{array}{l}\text { Pro- } \\
\text { foo- } \\
\text { deur }\end{array}$ & Horizons & $\begin{array}{l}\text { Prof. de } \\
\text { prélèv. }\end{array}$ & Canilloux & $\mathrm{pH}$ & Argile & $\begin{array}{c}\text { Linons } \\
\text { fins }\end{array}$ & $\begin{array}{l}\text { Linons } \\
\text { gross. }\end{array}$ & $\begin{array}{c}\text { Sebles } \\
\text { finn }\end{array}$ & $\begin{array}{l}\text { Sables } \\
\text { groas. }\end{array}$ \\
\hline \multirow{3}{*}{$-5 \mathrm{~cm}$} & $\Lambda_{0}-\Lambda_{1}$ & aurface & & & & & \multirow{3}{*}{$\begin{array}{l}15,9 \\
18,4\end{array}$} & \multirow{3}{*}{$\begin{array}{l}18,1 \\
14,3\end{array}$} & \multirow{3}{*}{$\begin{array}{r}6,6 \\
16,7\end{array}$} \\
\hline & $\boldsymbol{z}_{2}-(\mathrm{B})$ & $-10 \mathrm{~cm}$ & 14 & 7,4 & 21,2 & 30,1 & & & \\
\hline & (B) & $-25 \mathrm{ca}$ & 24 & 7,7 & 15,8 & 30,5 & & & \\
\hline \multirow[t]{2}{*}{$-40 \mathrm{cs}$} & (B) $\mathrm{C}$ & $-45 \mathrm{~cm}$ & 51 & 7,9 & 7,7 & 15,5 & 17,6 & 37,4 & 19,3 \\
\hline & Horizone & $\begin{array}{l}\text { matiere } \\
\text { orgnn. }\end{array}$ & C & S & $c / s$ & $\begin{array}{l}\mathrm{CO}_{3} \mathrm{Cs}_{8} \\
\text { total }\end{array}$ & $\begin{array}{l}\infty_{3} \mathrm{Cn} \\
\text { actif }\end{array}$ & \multicolumn{2}{|c|}{ Fer 2ibre } \\
\hline & $A_{0}-A_{1}$ & 33,2 & 16,6 & 0,63 & 26,3 & \multirow[b]{2}{*}{ traces } & & \\
\hline & $A_{2}(B)$ & 5,6 & 3,23 & & & & & \multicolumn{2}{|c|}{1,62} \\
\hline & (B) & 2,5 & 1,43 & & & 5,3 & 2,1 & \multicolumn{2}{|c|}{1,62} \\
\hline & (B) $\mathrm{C}$ & 1,7 & 0,87 & & & 42,2 & 6,7 & \multicolumn{2}{|c|}{1,16} \\
\hline & Horizons & $\stackrel{\mathrm{K}}{\text { Echang. }}$ & écha & & $\begin{array}{c}\mathrm{Kg} \\
\text { Échang. }\end{array}$ & s & s. & \multicolumn{2}{|l|}{$S / T$} \\
\hline & $\begin{array}{l}A_{0}-A_{1} \\
A_{2}(B) \\
(B) \\
\text { (B) C }\end{array}$ & 0,70 & 54 & & 4,35 & 59,05 & 53,7 & \multicolumn{2}{|l|}{$\begin{array}{c}\text { Baturé } \\
n \\
n \\
n\end{array}$} \\
\hline
\end{tabular}


- cargneule triasique.

- Profil $n^{\circ} 2$.

$A_{0}-A_{1}(0-5 \mathrm{~cm})$

- horizon de mull-moder calcique brun noirâtre - structure sub-grumeleuse, à grains grossiers - limite inférieure nette, très peu de cailloux ( $5 \%$ Inviron), racines nombreuses.

$$
A_{2}-(6-10 \mathrm{~cm})
$$

- couleur brun ocracée foncée - structure à grains grossiers.

- squelette constitué de morceaux de cargneule ( $10 \%$ du volume total).

- racines moins nombreuses.

$$
\text { B }(11-45 \mathrm{~cm})
$$

- mème structure - couleur plus claire.

- squelette correspondant à $20 \%$ du volume total.

\section{C}

- cargneule triasique.

\section{- Etat hydrique.}

Nous avons cherché, en outre, à traduire l'état hydrique du sol de l'Ononido-Pinetum, à l'aide d'un pédohygromètre et, en opérant, comme pour l'air, par comparaison.

Bien que les mesures n'aient porté que sur une seule saison de végétation (10 mai - 10 novembre 1957) et qu'elles aient été un peu interrompues, en juillet (2), elles nous paraissent pleines de signification (fig. 25).

On constate d'abord, et ce caractère s'est trouvé renforcé en 1957 à la suite d'un été particulièrement humide, que c'est surtout la sécheresse de fin de saison qui offre le plus gros intérêt: « en effet, après la fonte des neiges, le sol emmagasine une certaine réserve d'eau en profondeur (plus ou moins importante, suivant les stations) et cette réserve alimente ensuite, par remontée capillaire lente, les horizons de surface, en compensant plus ou moins les pertes par évaporation; jusqu'à ce que survienne une sorte de rupture d'équilibre caractéristique, qui est marquée par une augmentation très rapide du $\mathrm{pF} \gg(3)$.

(2) à la suite de la crue dévastatrice de lArc qui a coupé certaines voies de communication.

(3) Duchaufour, in litteris. 
Fig. 25.

Les pineraies de $P$ in sylvestre xérophiles Microclimat du sol.

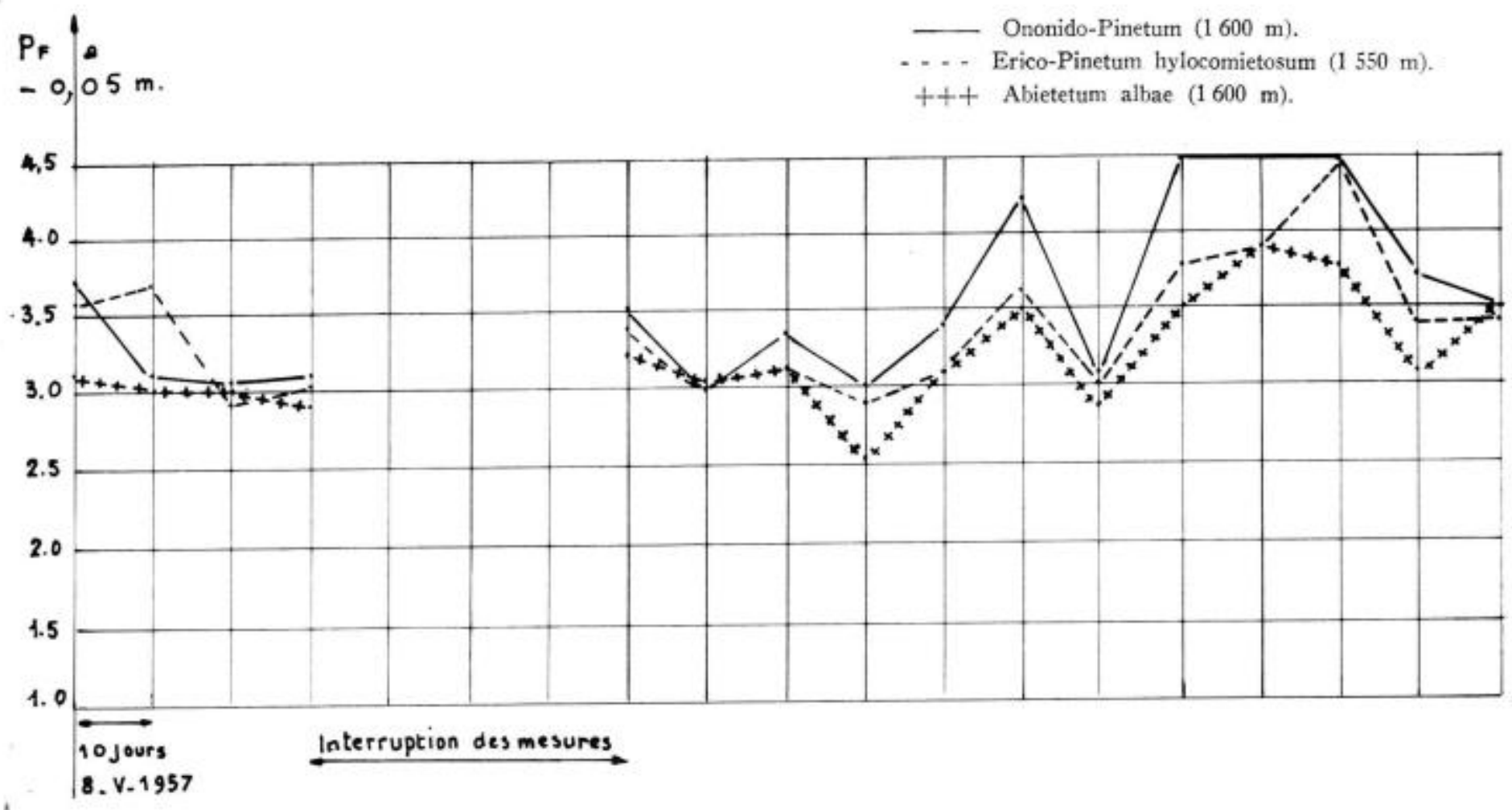


PF i

$-0,25 ?$

4.5

45

3.5

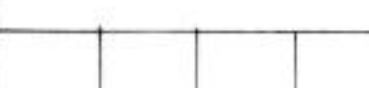

Ononido-Pinetum (1600 m).

-. - Erico-Pinetum hylocomietosum (1 $550 \mathrm{~m})$.

+++ Abietetum albae (1600 m).

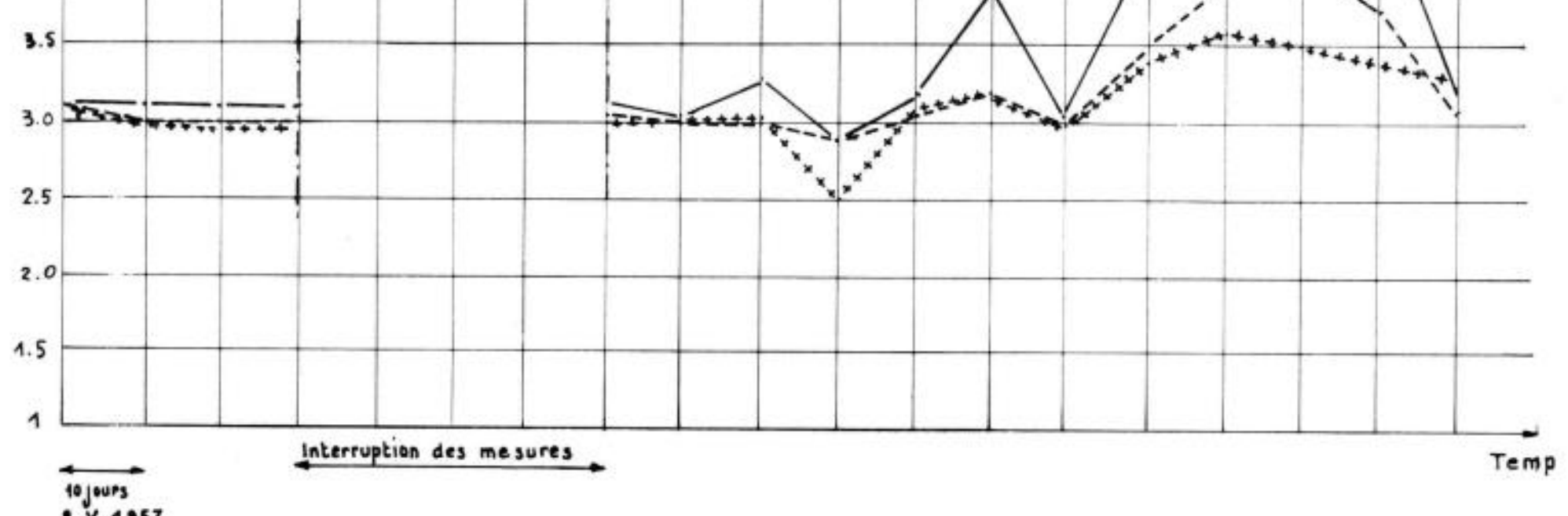

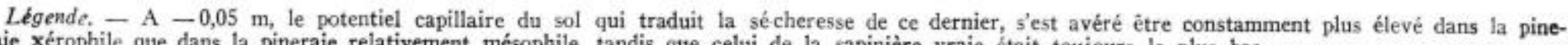
raie xérophile que dans la pineraie relativement mésophile, tandis que celui de la sapinière vraie était toujours le plus bas.

Ces résultats viennent corroborer ceux obtenus à partir des mesures du pouvoir évaporant de l'air.

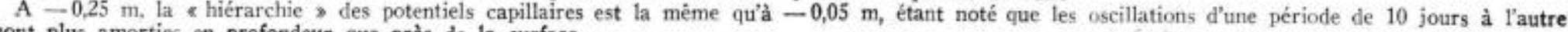
sont plus amorties en profondeur que près de la surface. 
On constate, d'autre part, qu'en valeur absolue - et qu'il s'agisse du $\mathrm{pF}$ de surface $(-5 \mathrm{~cm}$ ) ou de celui régnant en profondeur ( $-25 \mathrm{~cm})$, l'Ononido-Pinetum présente une sécheresse du sol constamment supérieure à celles de l'Erico-Pinetum et de l'Abietetum albae, associations qui seront analysées plus loin. Cette sécheresse chiffrée se conjugue avec celle de l'air et justifie le terme de xérophile, accolé aux espèces qui se trouvent localisées dans ce type de pineraie et, par contre-coup, celui de mésophile accolé aux espèces rencontrées dans des associations plus fraiches.

Chose importante à souligner et qui vient ajouter ses effets à la brutalité du régime thermique du sol déjà analysé, les oscillations du $\mathrm{pF}$ sont sensiblement plus accentuées dans la pineraie xérophile que dans celle à Erica carnea et mousses ou que dans la sapinière vraie et ceci, aussi bien à $-25 \mathrm{~cm}$ qu'à $-5 \mathrm{~cm}$. Le régime hydrique du sol, comme on pouvait s'y attendre, est donc brutal, lui aussi.

\subsubsection{2. - La pineraie mésoxérophile à Erica carnea et Carex humilis.}

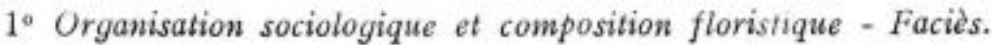

Il s'agit ici d'une association se situant à mi-chemin entre la pineraie xérophile à Ononis rotundifolia et Astragalus Monpessulanus et la pineraie mésophile à Erica carnea et Hylocomies. Si nous avons cru devoir en faire une unité autonome, c'est qu'elle couvre des dizaines et des dizaines d'hectares dans des conditions écologiques - et tout particulièrement d'exposition — qui se situent entre celles que connaissent les deux pineraies " extrêmes $n$.

Le tableau d'association montre que les groupes de xérophiles perdent de leur importance: les xérophiles subméditerranéennes subsistent, dans leur ensemble, mais avec un coefficient d'abondance-dominance sensiblement atténué; il en est de même des xérophiles médio-européennes.

Par contre, les mésophiles de sols calcimorphes sont très nettement mieux représentés: certaines d'ailleurs étaient totalement absentes de la pineraie xérophile comme Erica carnea, Carex alba... Apparaissent de même, certaines espèces liées à des sols de mullmoder comme Melampyrum silvaticum, Lusula silvatica subsp. Sieberi; les mousses, enfin (Rhytidiadelphus triquetrus tout particulièrement), sont sensiblement plus abondantes.

Le spectre floristico-écologique de ce groupement - que l'on rapprochera de celui précédemment défini (fig. 31) traduit bien le caractère $\alpha$ mixte $»$ de la composition floristique. Est à retenir tout particulièrement la proportion relative d'Arctostaphylos Uva ursi et d'Erica carnea, qui se trouve ètre intermédiaire entre les proportions observées dans les deux associations extrêmes (fig. 26 et 31). 
PLANCHE $V$

Pineraie de Pin syluestre méso-xérophile.
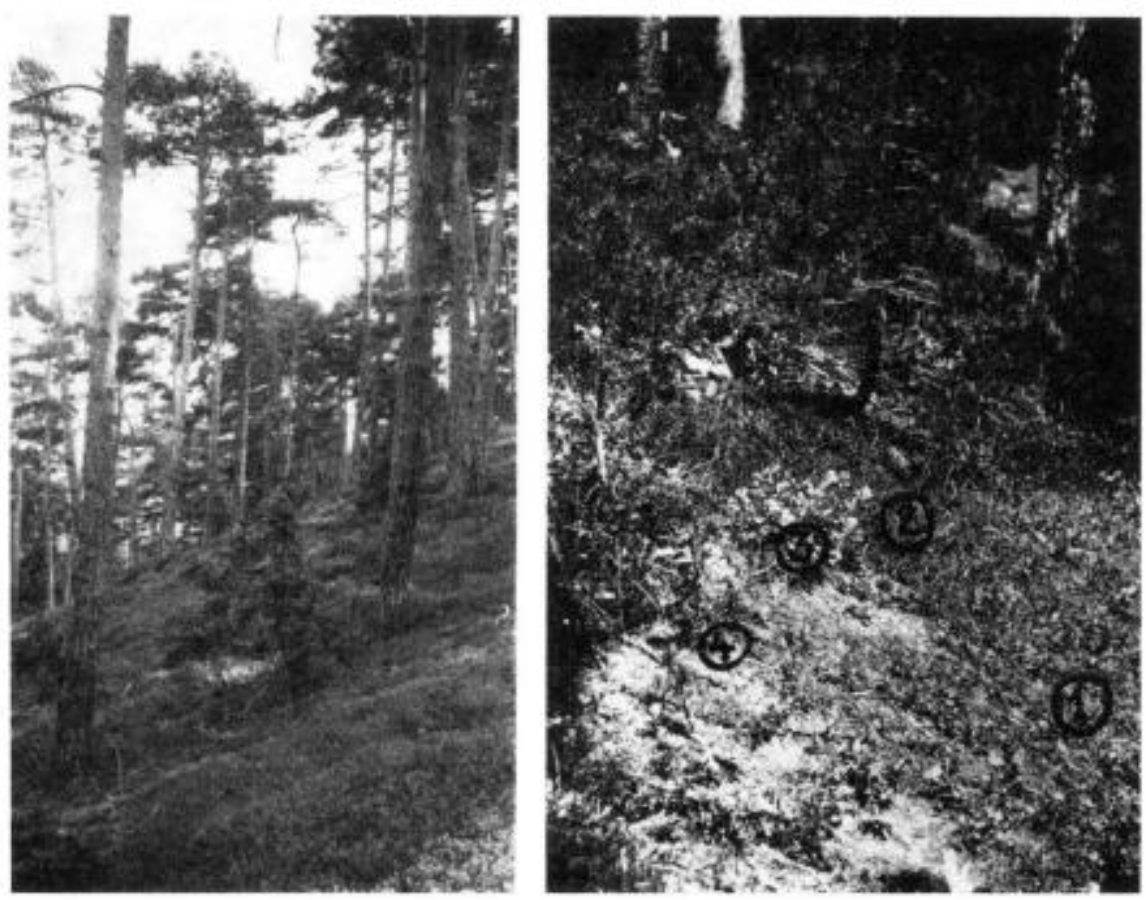

A gauche: Pineraie méso-xérophile à Erica carnea et Carex humilis. Vue d'ensemble. Forèt de Sollières-Sardières.

A droite: Cette photo montre la physionomie de détail de la pineraie de Pin sylvestre à Erica carnea et Carex humilis, qui, à exposition * intermédiaire >. fait le passage entre la pineraie franchement xérophile à Ononis rotundifolia et Coronilla minima et la pineraic relativement mésophile à Erica carnea et Hylocomies,

Arctostaphylos Uva ursi (1) et Erica carnea (2) forment des ilots qui, en général, ne se mélent pas. Sur des plages nues, se rencontrent les espèces les plus xérophiles: Ononis rotundifolia (3), Carex humilis (4), Astragaltus Monspessulanus (non représenté). Les mousses sont presque totalement absentes. 
Quant à la structuré, elle est également caractéristique; au-dessous d'une strate arborescente plus ou moins régulière et d'une strate arbustive en général peu fournie, la végétation s'étend en trois taches principales: Erica carnea forme ses propres plages et se trouve rarement mêlée vraiment à Arctostaphylos Uva ursi; cette dernière s'étend également en taches à peu près pures, à côté d'Erica carnea; les vides entre ces deux espèces sont occupés par Carex humilis, Epipactis atrorubens, etc, et, d'une façon générale, par les espèces les plus xérophiles (planche V).

Quant à Vaccinium Vitis Idaea, elle se trouve surtout localisée au pied des arbres ou sur les emplacements d'anciennes souches - là où une couche $A_{0}$ d'humus brut peut se former et se maintenir.

\section{$2^{\circ}$ Identification - Dénomination.}

Parmi les groupements intra-alpins de Pin sylvestre déjà étudiés et qui participent à la fois de types extrêmes: xérophile et mésophile, il y a lieu d'en retenir deux: d'une part la sous-association à Peucedanum oreoselinum de lErico-Pinetum, d'autre part le Carici humilis Pinetum [Braun-Blanguet (J.), Pallaann (H.), BACH (R.) (1954)].

Le premier appartient à l'étage montagnard - et même à la base de l'étage montagnard, le second à l'étage subalpin - très exactement, le subalpin inférieur. Comme il y a, entre ces deux unités, de très étroites affinités, il n'est évidemment pas très facile de savoir quelle est la plus proche de notre association mixte. La très grande abondance d'Erica carnea dans la première (presque toujours de coefficient 5), fait que notre groupement se rapproche davantage du Carici humilis Pinetum, sans cependant lui être identique, évidemment: en effet, certaines espèces suisses sont absentes dans notre dition, comme Laserpitium Gaudinii ou y sont d'une rareté extrême, comme Daphne striata, Viola pinnata. D'autres comme Saponaria ocymoides, Campanula rapunculoides sont plus fréquentes dans l'association suisse, mais sans être toutefois absentes de la nôtre.

Il nous semble que ce groupement pourait être dénommé, au moins de façon provisoire: Ononido-Pinetum ericetosum.

\section{$3^{\circ}$ Répartition - Ecologie.}

S'observant entre 1200 et $1800 \mathrm{~m}$ au maximum (4), notre association occupe donc l'étage montagnard; elle est relayée plus haut - c'est-à-dire dans l'étage subalpin - par une association vicariante de Pin à crochets; le cercle d'exposition montre qu'elle laisse

(4) Cette altitude inféricure à $1200 \mathrm{~m}$ s'explique par le fait que, lorsque les conditions écologiques permettent l'installation de l'association - au delà d'Avrieux - , le fond du talweg atteint cette altitude. 
la place, latéralement, soit à l'Ononido-Pinetum, lorsque les conditions deviennent plus xériques (exposition plein sud ou sol vraiment superficiel), soit à l'Erico-Pinetum, lorsque, au contraire, les conditions écologiques deviennent plus humides et plus fraiches (exposition nord) (fig. 31).

Bien que nous n'ayons pas fait de mesures microclimatiques dans cette association, on peut penser que, toutes choses égales d'ailleurs, elle connait des conditions climatiques intermédiaires entre celles des pineraies que nous avons déjà qualifiées d'extrêmes.

Les sols. - Il résulte de nos observations que l'on a affaire à des rendzines, parfois légèrement brunifiées, mais qui présentent, dans l'ensemble, des conditions biologiques meilleures que celles rencontrées dans 1'Ononido-Pinetum. Dans l'exemple ci-après, qui correspond à une rendzine évoluant vers un sol brun, sous un horizon $A_{0}$ de très faible épaisseur - et dont la présence s'explique par la pente qui est de $15 \%$ seulement - l'horizon $A_{1}$ possède un $\mathrm{C} / \mathrm{N}$ sensiblement plus bas $(18,4)$ que celui rencontré précédemment $(26,4)$; d'autre part, la décarbonatation est moins accentuée, puisque $\mathrm{CO}_{3} \mathrm{Ca}$ s'observe dès la surface - à l'état de traces, il est vrai.

$$
\begin{gathered}
\text { Forêt de Sollières-Sardières - } 2^{*} \text { série - Parcelle } 0 \\
\text { Exp. S-SE - Alt. : } 1460 \mathrm{~m}-\text { Pente : } 25 \% \\
\text { Roche-mère: cargnetules et maraines calcaires } \\
\text { Relevé floristique } \mathrm{n}^{\circ} 9
\end{gathered}
$$

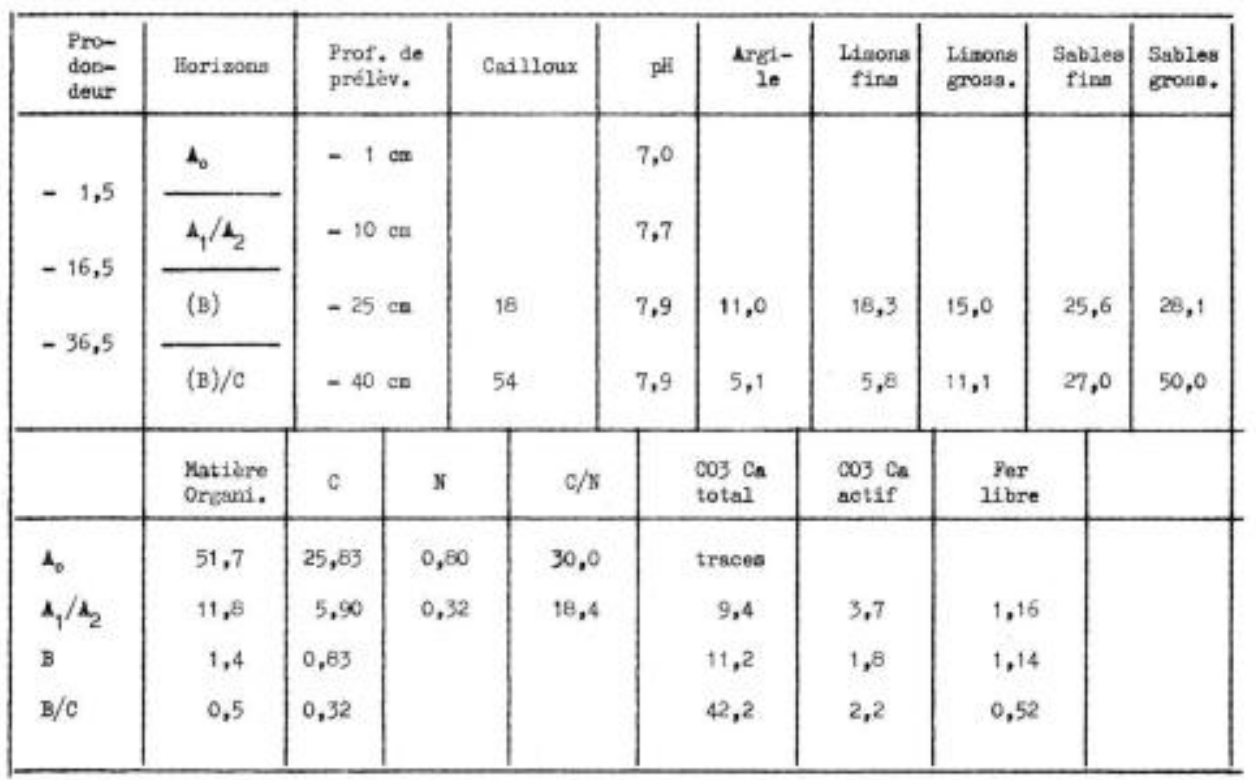




\section{I.2.1.3. - La pineraie mésophile à Erica carnea el Hylo- comies.}

\section{$1^{\circ}$ Organisation sociologique et composition floristique.}

Du point de vue floristico-écologique, cette association se trouve caractérisée à la fois par labsence à peu près totale de certaines xérophiles et par la présence, en grande abondance, de mésophiles de sols calcimorphes, dont Erica carnea.

En effet, les xérophiles subméditerranéennes comme Astragalus Monspessulanus, Ononis rotundifolia, etc. disparaissent presque complètement; seul subsiste avec une certaine constance: Amelanchier rotundifolia; les xérophiles médio-européennes - plus plastiques à l'égard de la séchcresse que les précédentes - sont encore représentées, mais avec une constance, et surtout une abondancedominance, nettement plus faibles que celles qui étaient les leurs dans l'Ononido-Pinetum.

Par contre, les mésophiles des sols calcimorphes forment un bon noyau: en tête, Erica carnea, espèce d'Europe Centrale, typiquement intra-alpine, qui trouve dans une telle pineraie - et dans son vicariant subalpin à Pin à crochets - un développement considérable; puis Melampyrum pratense subsp. alpestre, Carex alba, Sesleria coerulea, Valeriana montana, Carex glanca, Calanagrostis varia, etc.

On notera aussi la relative abondance d'espèces que l'on retrouvera dans la sapinière, comme Melampyrum silvaticum, Luzula silvatica subsp. Sieberi, et qui, bien que certainement plastiques à l'égard du chimisme du sol, trotvent leur optimum sur des sols de mull-moder forestier.

La strate muscinale, enfin, est très vigoureuse et comprend des espèces pour la plupart indifférentes, comme Rhytidiadelphus triquetrus et Hylocomium proliferum (syn, H. splendens).

Une petite observation est à faire concernant la composition floristique: Vaccinium Vitis idaea, caractéristique d'humus brut, relativement xérophile, et Hypnum Schreberi, caractéristique d'humus brut également, mais moins orophile, s'observent sotvent dans l'association. D'une façon générale, elles se rencontrent au pied des arbres ou sur lemplacement de souches - totalement recouvertes - là où l'acidification est la plus forte.

Le spectre floristico-écologique - qui traduit de façon imagée l'organisation sociologique de l'association - permet de bien saisir les différences essentielles qui la séparent des associations affines de Pin sylvestre (fig. 31). Là encore, la proportion relative d'Erica carnea et d'Arctostaphylos Uva ursi constitue un indice intéressant (fig. 26).

La structure n'est plus aussi complexe que celle des associations précédemment étudiées: elle est du type continu, du moins dans son ensemble: Erica carnea en effet, forme une nappe dense, pres- 
que sans trous, avec les Hylocomies; en émergent quelques arbustes comme Amelanchier rotundifolia, Cotoneaster tomentosa et, évidemment, les arbres, c'est-à-dire d'abord les Pins sylvestres en nette majorité, puis quelques Epicéas dont la forme et la vigueur sont supérieures à celles des Epicéas de l'Ononido-Pinetum, enfin de

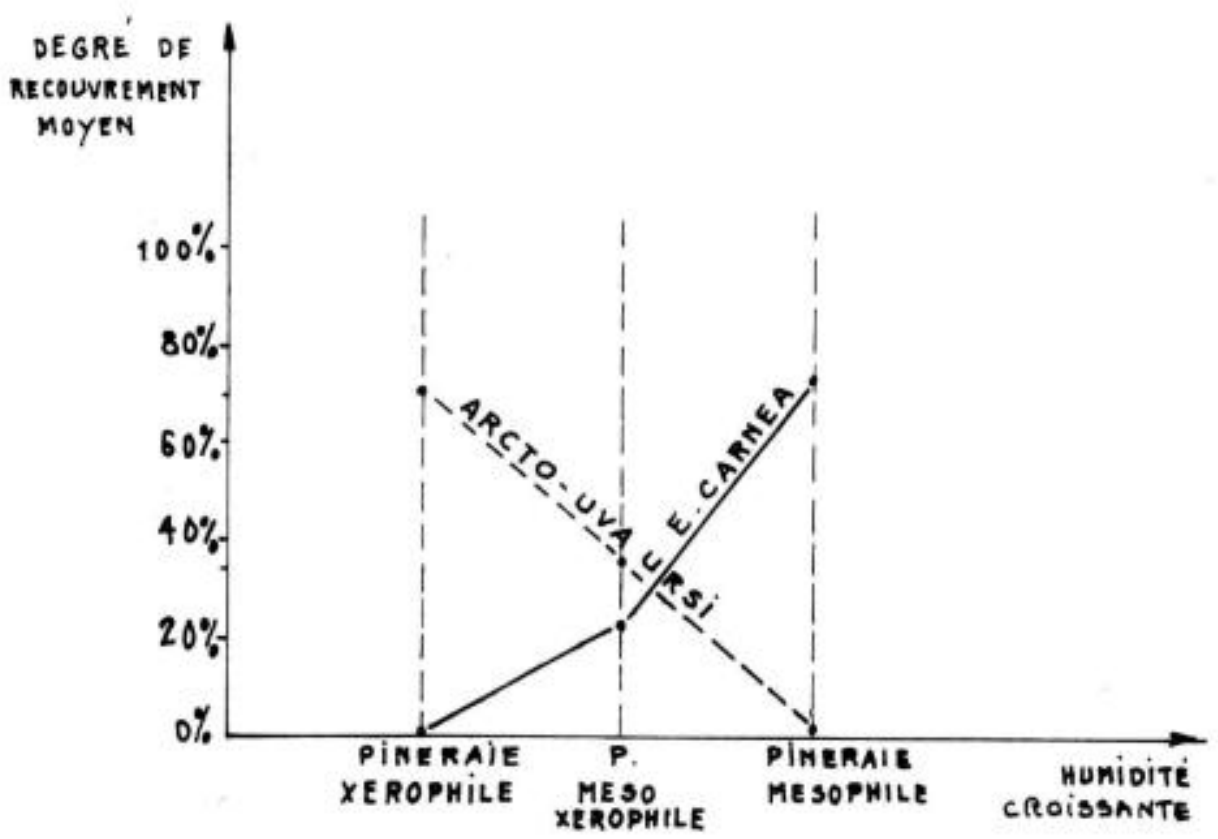

Fig. 26.

Légende. - Le schéma ci-dessus obtenu à partir de nombreux televés, montre nettement que la Busserolle est sensiblement plus xérophile que la Bruyère carnée, la première connaissant une exubérance marquée à exposition sud, la seconde à exposition nord.

rares Sapins dont la présence est tout de même à relever: il s'agit le plus souvent de jeunes individus qui appartiennent pour la plupart encore à la strate arbustive.

\section{Identification - Dénomination.}

C'est, évidemment à l'Erico-Pinetum hylocomietosum décrit en Suisse, pour la première fois, et où on le rencontre dans toutes les vallées intra-alpines (Rhin, Inn, Rhône) que doit ètre identifié notre groupement (ancienne appellation: Pincto-Ericetum hylocomietosum Br. Bl, 1939 - Braun-Blanguet (J.), Sissingh (G.) et Vlieger (D.) (1939). 


\section{Répartition - Ecologie.}

Apparaissant un peu au-delà de Modane et coincidant avec une partie de l'aire d'Erica carnca (5), la pineraie à Erica carnea et Hylocomies obéit à une triple localisation: son étage est l'étage montagnard; elle s'observe uniquement sur sol calcimorphe, et ce, à exposition fraiche: nord ou est. Et encore, doit-on apporter quelques nuances: sur roche-mère gypseuse, lorsque l'érosion rajeunit sans cesse le sol, le Pin sylvestre, dans son étage, est totalement remplacé par le $\mathrm{Pin}$ à crochets. Si donc l'on se rappelle que, sur versant nord, la nappe des schistes lustrés descend presque jusqu'au fond du talweg, ne laissant aux gypses et aux calcaires que le golfe qui s'épanotit entre Bramans et Termignon; si l'on tient compte de l'importance du gypse dans ce golfe, et du fait qu'il est

Erico-Pinetum

Types de sols

Profil n* 1

$$
\mathrm{e}=1 / 15^{\circ}
$$

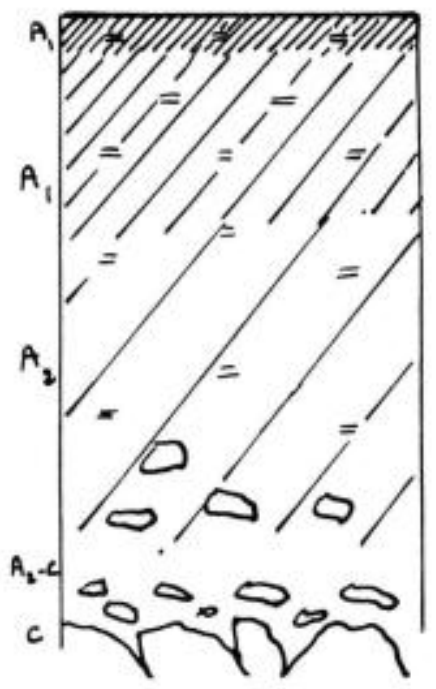

Sol humique carbonaté

\section{Profil $n^{\circ} 2$}

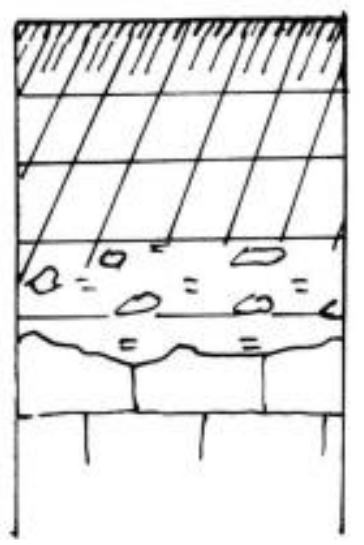

Rendzine brunifiée.

$$
\begin{aligned}
& \text { WII/A Horizon humifère. } \\
& ==\text { Argile. } \\
& =\text { Carbonate de chaux. }
\end{aligned}
$$

(5) Erica carnea se développe aussi, dans l'étage subalpin, comme on le verra, où elle s'associe au Pin à crochets. 


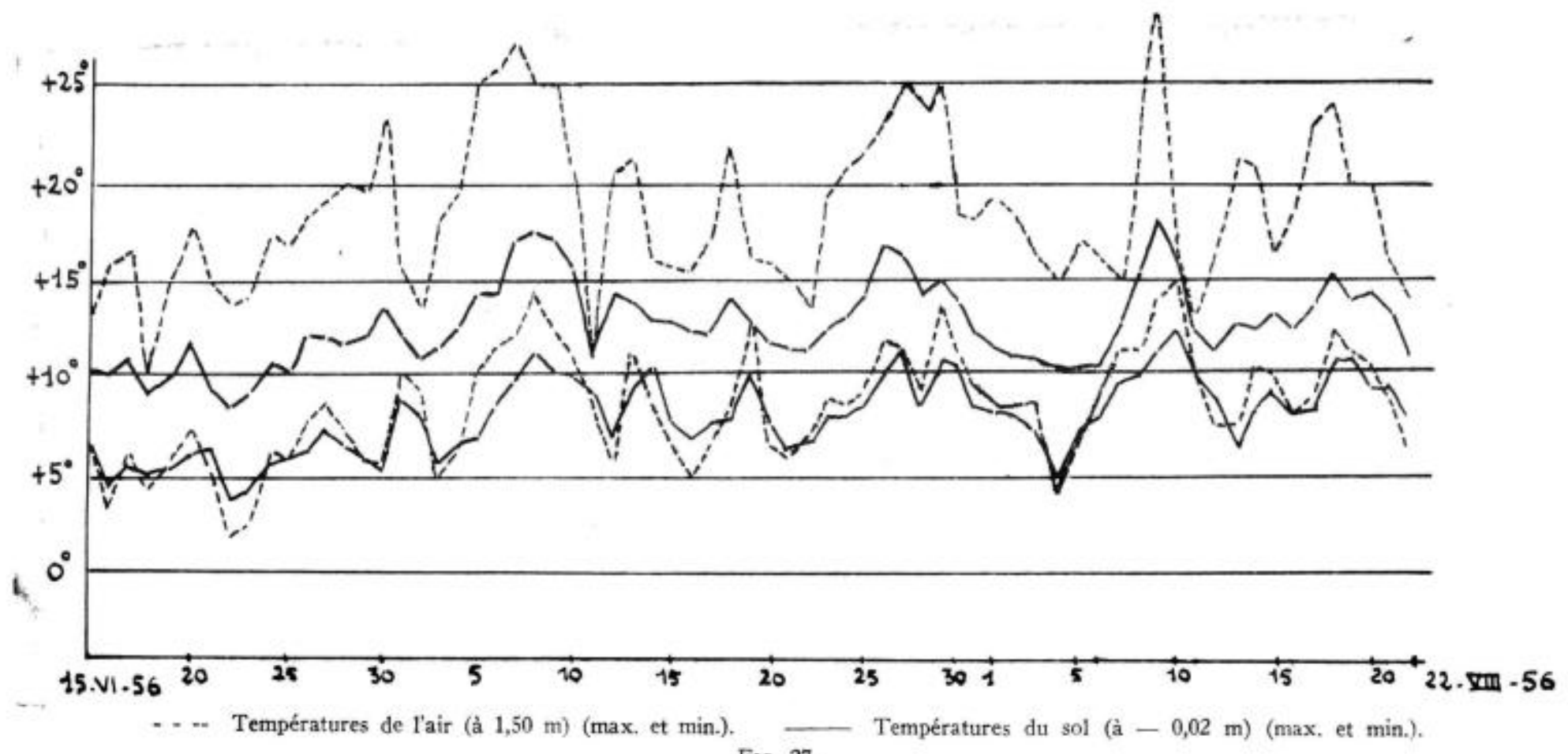

FiG. 27.

Les pineraies de Pin sylvestre mésophiles - Microclimat

Légende. - Alors que, dans 1'Ononido-Pinétum, les températures de l'air -à $1,50 \mathrm{~m}$ - et surtout celles du sol - à $-0,2 \mathrm{~m}-$ connaissent de fortes oscillations (fig. 19), on constate que, dans l'Erico-Pinetum (fig. ci-dessus) ces oscillations sont plus amorties, surtout celles de la température du sol. 
partiellement recoupé par l'étage subalpin où règne le Pin à crochets qu'il s'agisse de gypse ou non, on concevra qu'en définitive, les peuplements de Pins sylvestres à Erica carnea et Hylocomies soient relativement rares et, en tout cas, beaucoup moins étendus que les peuplements mésoxérophiles où voisinent Erica carnea, Astragalus Monspessulanus, Carex humilis.

L'analyse microclimatique que nous avons faite au sujet de l'Ononido-Pinetum et où nous avions comparé un individu de cette association et un de l'Erico-Pinetum, nous dispensera de longs développements. Nous avons vu que ce qui caractérisait le microclimat de l'Erico-Pinetum se situait au niveau du sol: ce dernier apparait, pendant la belle saison tout au moins, comme sensiblement plus froid que l'air qui le surmonte et ses oscillations thermiques journalières sont nettement plus amorties que celles de lair (fig. 27).

Les sols. - Dans 1'Erico-Pinetum, les sols, d'après nos observations, sont dans l'ensemble des rendzines, dont certaines ont une tendance plus nette encore que dans les groupements précédents à évoluer vers des sols bruns.

Un exemple de sol typiquentent carbonaté est fourni par un individu d'Erico-Pinetum en forêt de Villarodin-Bourget, tout à fait particulier, puisque développé sur gypse. C'est la grande profondeur du sol (près de $1 \mathrm{~m}$ ) qui explique, à notre avis, la disparition du Pin à crochets sur une telle roche-mère (dès que le gypse affleure, dans l'ensemble du groupement, le Pin à crochets apparait aussitôt).

II s'agit d'un sol humique carbonaté dont nous donnons ci-après la description morphologique et une analyse sommaire.

Description morphologique (fig, 28).

- Profil $n^{0} 1$.

$A_{1}$ surface $(0-5 \mathrm{~cm})$

- horizon de mull calcique, très noir, structure à grumeaux, arrondis de 1 à $4 \mathrm{~mm}$. Feutrage dense de racines.

$A_{1}$ profond $(6-20 \mathrm{~cm})$

- horizon gris - structure à grains grossiers. Lacis de racines beancoup moins dense.

$$
\left.\mathrm{A}_{2}-\mathrm{C} \text { (vers } 90 \mathrm{~cm}\right)
$$

- horizon blanchâtre, couleur du gypse - Pas de structure nette - squelette ahondant ( $80 \%$ du volume). 
Forêt de Villarodin-Bourget

$1^{\text {re }}$ série - Exp. N - Alt. $1300 \mathrm{~m}$ - Roche-mère: gypse - Pente $50 \%$ Relevé floristique $\mathrm{n}^{\circ} 19$

\begin{tabular}{|c|c|c|c|c|c|c|c|c|c|}
\hline $\begin{array}{l}\text { Prof. de } \\
\text { préliov. }\end{array}$ & Horiwone & $\mathrm{pH}$ & $\begin{array}{l}\text { Kat. } \\
\text { organ. }\end{array}$ & c & N & $c / v$ & $00^{3} \mathrm{Ca}$ & $c^{3}$ ue $_{6}$ & $\begin{array}{l}\mathbf{x}^{7} \mathrm{Cs}_{e} \\
\text { ankydre }\end{array}$ \\
\hline$-5 \mathrm{du}$ & $h_{1}$ & 7,8 & 25,6 & 15,06 & 0,70 & 21,5 & 6,6 & 6 & 0 \\
\hline-20 on & $\mathrm{A}_{1}$ & B & 6,5 & 3,87 & 0,21 & 18,4 & 21,4 & 12 & 0 \\
\hline-35 on & $h_{2}$ & 8,2 & 5,1 & 3,01 & & & 19,4 & 14,2 & Traees \\
\hline-90 on & $b_{2}-c$ & $B$ & & & & & 9,4 & 6,3 & $40(1)$ \\
\hline
\end{tabular}

(1) gypse: environ $50 \%$.

En forét de Sollières-Sardières, sur moraine calcaire et sur plus faible pente, l'évolution vers un sol brun, par contre, est des plus nettes, comme le montre, en particulier, la décarbonation de la partie supérieure du profil - jusqu'à $15 \mathrm{~cm}$ - dont l'analyse sommaire est donnée ci-après.

- horizon très mince, humifère, brun noirâtre à petits grumeaux de 1 à 2 $\mathrm{mm}$. Feutrage très dense de racines d'Erica carnea.

- structure à petits grumeaux argilohumiques - horizon de couleur brune - texture limono-argileuse.

(B) (non analysé)

- petits fragments de calcaires et de schistes noirs triasiques - couleur brune.

Forêt de Sollières-Sardières

$2^{*}$ séric - Plle S - Exp. N - Alt. $1450 \mathrm{~m}$ - Pente $20 \%$

Relevé floristique $n^{*} 20$

\begin{tabular}{|c|c|c|c|c|c|c|c|c|c|}
\hline $\begin{array}{l}\text { Pro- } \\
\text { Pon:- } \\
\text { deur }\end{array}$ & Hori $20 \mathrm{ng}$ & $\begin{array}{l}\text { Prof. de } \\
\text { préliven. }\end{array}$ & pH & C & $\begin{array}{l}\text { zat. } \\
\text { organ. }\end{array}$ & $n$ & $c / 4$ & $\begin{array}{l}\mathrm{C}_{\mathrm{B}}+\mathrm{H} \\
\text { Éhang. }\end{array}$ & $\mathrm{Co}^{3} \mathrm{Cs}$ \\
\hline$-3 \mathrm{en}$ & & - $2 \mathrm{~cm}$ & 6,6 & 13,6 & 23,2 & 0,56 & 24,4 & 31,2 & o \\
\hline$-20 \mathrm{cn}$ & $A_{2}-(B)$ & $-15 \mathrm{~cm}$ & 6,9 & & 5,1 & & & 17 & 0 \\
\hline
\end{tabular}

(1) en milliéquivalents pour $100 \mathrm{~g}$. 


\section{- Etat hydrique.}

Les courbes, déjà commentées, montrent que l'état hydrique du sol de l'Erico-Pinetum, s'il se situe entre ceux de l'Ononido-Pinetum et de l'Abietctum albae se rapproche davantage de l'état hydrique de cette dernière association; ceci ne nous parait pas étonnant, si l'on se réfère au fait que, dans les deux cas, les expositions sont identiques; la différence s'explique, à notre avis, par des différences de granulométrie; d'après ce que nous avons pu constater, le sol de l'Erico-Pinetum est toujours plus riche en cailloux, donc mieux drainé et un peu plus chaud, ce qui entraine une évaporation plus intense.

\section{III.2.2. - Les pineraies de Pin sylvestre acidiphiles.}

Sur roche-mère silicatée et sur versant nord, la pineraie est pratiquement absente et se trouve remplacée par la sapinière.

Il n'en est pas de même sur versant sud, où elle a dû occuper une place importante dans toute la partie supérieure de la vallée, c'est-à-dire à partir de St-Michel-de-Maurienne. Mais l'influence humaine a été telle qu'il n'en subsiste plus que quelques lambeaux le long des gorges Houillères, et que le seul peuplement important s'observe dans le synclinal géologique de Chavières, sur quartsites triasiques très superficiels, ce qui donne d'ailleurs à ce peuplement une composition floristique assez originale.

Cette localisation étroite explique que les peuplements de Pin sylvestre acidiphiles soient moins variés que leurs homologues de sols calcimorphes, et que l'on ne doive parler que d'une association en distinguant au besoin des sous-unités.

\section{$1^{\circ}$ Organisation sociologique et composition floristique.}

Sur le tableau d'association, est mis en évidence un premier groupe d'espèces acidiplililes de sol à moder, qui ne figurait pas dans les tableaux des autres pineraies, et qui peut d'ailleurs être scindé en trois parties: le noyau principal est constitué par des espèces telles que: Deschampsia flexuosa, Phyteuma betonicifolitm, Hieracium picroides, Veronica officinalis, etc. Quant aux deux autres noyaux, ils permettent de différencier des sous-associations:

- le premier, avec Minuartia laricifolia et Sempervivum arachnoideum, correspond au groupement le plus xérophile, sur ranker à moder,

- le second s'observe sur un sol plus profond et dans des conditions moins xériques. Il comprend essentiellement des caractéristiques d'humus brut, dont Vaccinium Vitis idaea, qui joue le principal rôle et Vaccinium Myrtillus. 
PLANCHE VI

Le Deschampsio-Pinetum
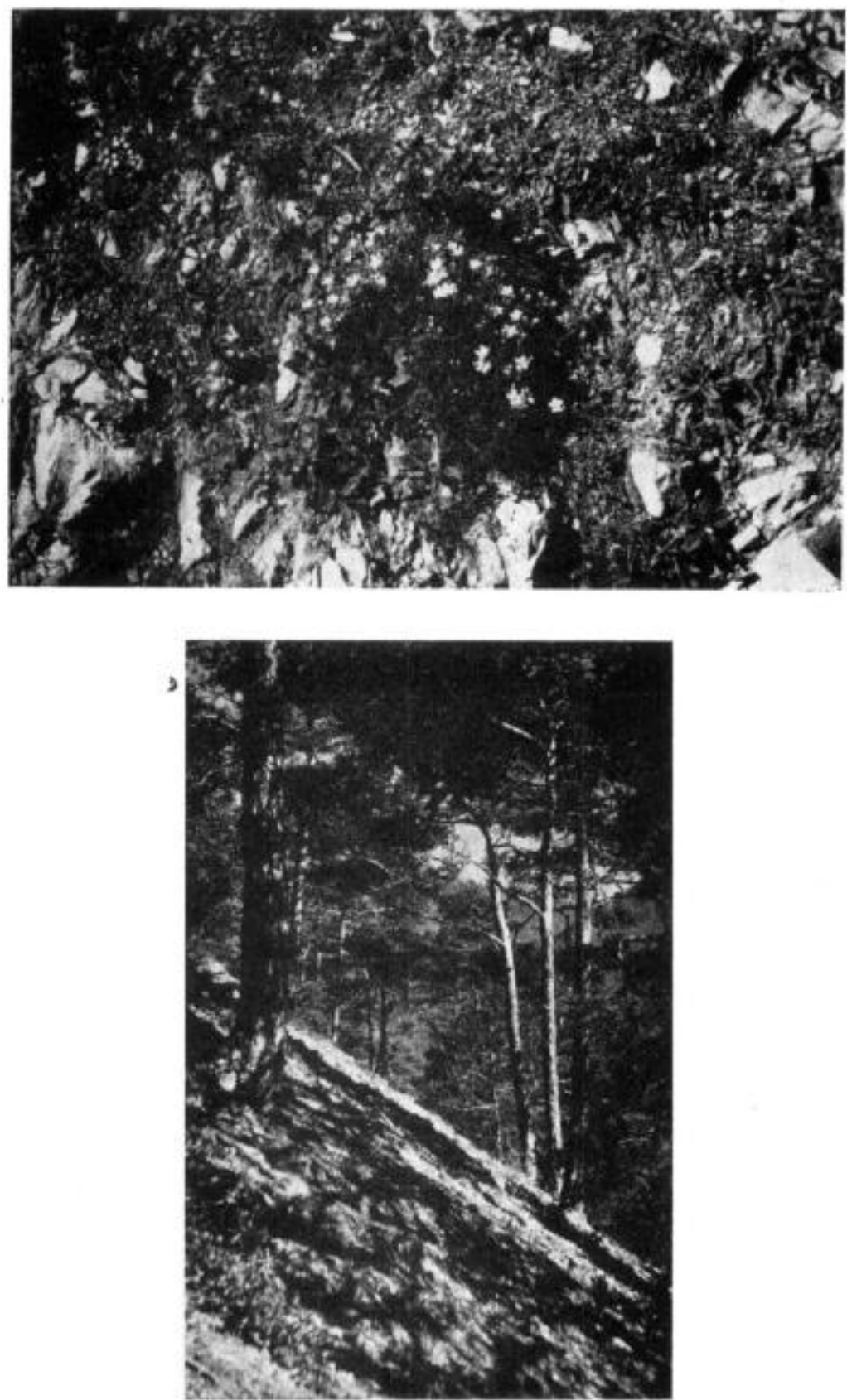

Légende, - Sur quartzites, la pineraie xéro-calciphile laisse la place ì une pineraie à Deschampsia fletuxuosa et Minuartia laricifolia (Deschampsia-Pinetum de Braun-Blanguet).

La couverture végétale est très faible (moins de $40 \%$ ) car la pente est très forte.

L'ombre portée des fûts et des feuillages des Pins empéche quelque peu de voir les touffes de la Canche flexueuse.

Sur la vue de détail du haut, apparait nettement une belle touffe de Minuartia laricifolia; en haut, à gauche, Sempervirus arachnoideum; par places. Hieracium Peleterianum. 
11 est intéressant de noter, dans ce groupe des acidiphiles, Galium rotundifolium qui, en Haute-Maurienne, est extrêmement rare. Nous ne l'avons, en effet, jamais rencontré dans les associations mésophiles, qu'il s'agisse de la sapinière ou de la pessière subalpine, mais nous l'avons quelquefois observé - deux ou trois fois peutêtre - dans les parties les plus fraiches de la pessière subalpine à Vaccinium Vitis idaca. Il est donc curieux d'en trouver quelques petites taches dans cette pineraie, alors qu'il s'agit d'une espèce plutôt mésophile, qui trouve son plein développement dans certaines sapinières (6).

A ce groupe des acidiphiles qui est évidemment le groupe caractéristique de l'association se joint un vaste ensemble de xérophiles assez indifférentes au chimisme du sol, et oủ il nous a paru utile de distinguer les subméditerranéennes d'une part, et les médioeuropéennes, moins thermophiles, de l'autre.

Le spectre floristico-écologique traduit, une fois de plus, l'organisation sociologique de l'association qui se trouve rappeler, par bien des côtés, l'Ononido-Pinetum, mais qui en diffère toutefois, par son noyau d'espèces acidiphiles (fig. 31).

La structure des strates herbacée et arbustive est assez diffuse; le degré de couverture est, dans l'ensemble, faible et les taches d'Arctostaphylos Uva ursi toujours disjointes, entre les touffes de Deschampsia flexuosa.

\section{2॰ Identification - Dénomination.}

Jusqu'au très récent travail de BRAUn-BLAnqueT, déjà signalé (1961), ancune association de Pin sylvestre parmi celles décrites dans les Alpes, ne semblait s'identifier à la nôtre. Arcuinger (L.) (1952) avait bien analysé des pineraies acidiphiles, mais il s'agissait, soit d'associations plus ou moins transitoires, proches des chênaies dégradées (Querco-Betuletum) et riches en espèces des landes médio-européennes, soit d'associations de Pins de marais proches de l'aulnaie glutineuse ( ( Pinetum silvestris paludosum turfosum "). C'était finalement lassociation décrite par Braun-Blanguft sous le nom provisoire de Picectum subalpinum pinetosum (1954) qui paraissait la plus proche de celle qui nous occupe par labondance de Vaccinium Vitis idaea, et la présence d'autres espèces acidiphiles telles: Deschampsia flexuosa, Laserpitium Halleri... Depuis, BRAUNBLANQUET a donné une brève description (1961) d'une pineraie appelée par luti Deschampsio-Pinetum et observée dans la région de Briançon, en France, de Salbertrand et de Fenestrelle en Italie

(6) Galium rotundifolium, aux marges de son aire, se localise sur moder: au centre de son aire, il parait plus plastique, d'après ce que nous avons pu nbserver dans le Vercors et le Trièves. 
et qui, comme le montre le relevé ci-après pris au Villard-Laté, entre Briançon et le Monetier, est tout à fait comparable aux nótres.

\author{
5. Pinus silvestris \\ 1. Deschampsia flexuoso \\ 1. Minuartia laricifolia \\ + Trifolium alpestre \\ + Antennaria dioica \\ + Juniperus communis
}

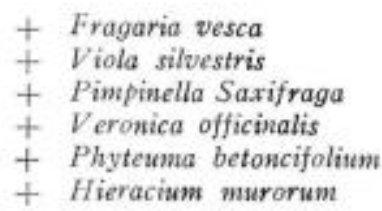

\section{$3^{\circ}$ Répartition - Ecologie.}

$\mathrm{La}$ pineraie acidiphile que l'on observe en Haute-Maurienne dans l'étage montagnard jusqu'à la limite du subalpin, sur versant sud et sur roches-mères pauvres en bases - grès et schistes du Houiller, schistes métamorphiques, quartzites du Trias - se trouve, de ce fait, localisée le long des gorges houillères, entre St-Michel-deMaurienne et Modane, et un peu au-delà, jusqu'à la cascade StBenoit. Plus à l'amont, en effet, ce sont les cargneules, les gypses, les calcaires, puis les schistes lustrés - eux-mèmes plus ou moins carbonatés - qui relaient les roches vraiment silicatées.

Or, si l'homme n'a pu coloniser que très partiellement la zone des cargneules et des calcaires, au relief souvent heurté et particulièrement peu propice à la culture, et a, de ce fait, permis à la forêt de sol calcimorphe de se maintenir sur d'assez grandes surfaces, il a, par contre, occupé presque totalement l'étage montagnard, de l" "Endroit ", non seulement le long des gorges houilleres, mais au-delà, autour du village du Bourget, où les quartzites triasiques se trouvent très souvent recouverts de placages morainiques. On conçoit dès lors que la pineraie acidiphile soit très peu représentée et s'observe, au bas de la pessière subalpine sèche, dans les rares endroits, où le relief, les éboulis ont empéché tout défrichement. Il est tout de même une exception à cet état de fait, on l'a vu: dans le synclinal géologique de Chavière, au droit de Modane, des quartzites très redressés sont occupés par la forêt presque jusqu'au talweg: ils portent la pineraie à Minuaria laricifolia et Sempervivum arachnoideum.

Nous n'avons pu, pour des raisons déjà dites, étudier le microclimat de la pineraie acidiphile. On doit penser qu'il n'est pas très éloigné de celui des autres pineraies situées dans des conditions d'exposition analogues.

Les sols, par contre, présentent des caractères propres. L'analyse du ranker, portant la pineraie à Minuartia laricifolia, montre qu'il s'agit d'un sol sans carbonates, à $\mathrm{pH}$ particulièrement bas, et où la quantité de calcium échangeable est beaucoup plus faible que celle des sols précédemment étudiés. L'humus est du type moder, à structure particulaire, riche en grains de quartz nus, et dont le rapport $\mathrm{C} / \mathrm{N}: 19,7$, est comparable à celui d'un mull (fig. 29). 
Description morphologique (fig. 29).

$$
A_{1}(0-4 \mathrm{~cm})
$$

- Moder brun noirattre, de structure particulaire avec très nombreux grains de quartz nus. Transition nette avec l'horizon (C).

$$
\text { (C) }(5-8 \mathrm{~cm})
$$

- Roche-mère en décomposition. Squelette représentant $80 \%$ du volume total; le reste de texture sableuse cotleur blanchâtre.

- Roche-mère: quartzite triasique.

Deschampsio-Pinetum

Type de sol

$$
\mathrm{e}=1 / 10^{*}
$$

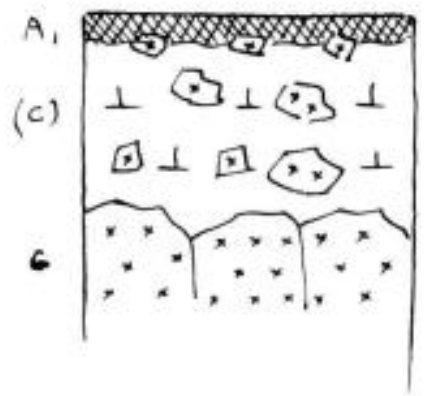

Hunus de moxler.

11 Roche-mére en cours d'altération.

$x x$ Roche-mère siliceuse non altérée.

Sol - Forêt de Modane

\begin{tabular}{|c|c|c|c|c|c|c|c|c|c|}
\hline $\begin{array}{l}\text { Prom } \\
\text { form } \\
\text { deur }\end{array}$ & $\begin{array}{l}\text { Bor1- } \\
\text { zons }\end{array}$ & $\begin{array}{l}\text { Prof. do } \\
\text { próliv. }\end{array}$ & pH & $\begin{array}{l}\text { Mat. } \\
\text { org. }\end{array}$ & $c$ & $\mathrm{~N}$ & $c / s$ & $\begin{array}{l}\infty_{3} \mathrm{C}_{\mathrm{a}} \\
\text { tolal }\end{array}$ & $\begin{array}{l}\mathrm{Ca}^{+\mathrm{H}} \\
\text { fehang. }\end{array}$ \\
\hline \multirow{3}{*}{$\begin{array}{l}-4 \mathrm{~cm} \\
-10 \mathrm{~cm}\end{array}$} & $A_{1}$ & \multirow{4}{*}{$\begin{array}{l}-1 \mathrm{~cm} \\
-8 \mathrm{~cm} \\
-15 \mathrm{~cm}\end{array}$} & \multirow{4}{*}{$\begin{array}{l}5,2 \\
4,1 \\
5,4\end{array}$} & \multirow[t]{4}{*}{9,4} & \multirow[t]{4}{*}{5,5} & \multirow[t]{4}{*}{0,26} & \multirow[t]{4}{*}{19,7} & 0 & 8,6 \\
\hline & (c) & & & & & & & 0 & 2,6 \\
\hline & c & & & & & & & 0 & 1,4 \\
\hline$-200 \mathrm{~cm}$ & & & & & & & & & \\
\hline
\end{tabular}

Plle 4 - Exp. SW - Alt.: $1300 \mathrm{~m}$ - Roche-mère: quartzites

Relevé floristique $\mathrm{n}^{\circ} 1$ 


\section{III.2.3. - Vue d'ensemble sur les pineraies de Pin sylvestre.}

\section{III.2.3.1. - Affinités phytosociologiques.}

De toutes les associations forestières, les pineraies de Pin sylvestre - parce que spécifiquement intra-alpines - sont celles, sans doute, qui permettent le mieux de saisir loriginalité des diverses vallées sèches des Alpes, comme les affinités qui existent entre elles. De ce point de vue, on pent conclure des analyses précédentes, que la Haute-Maurienne est, en définitive, plus proche du Valais que du Briançonnais, ce qui vient confirmer les données des régimes pluviométriques: c'est tout près de l'Ononido-Pinetum et de l'Odonditi-Pinetum Valaisans que se situent les pineraies xérophiles de notre dition, qui diffèrent quelque peu, par contre, de l'Onobrychideto-Pinetum briançonnais et de 1'Astragalo-Pinetum autrichien. Cette très proche parenté subsiste, si l'on considère les pineraies plus mésophiles (7); dans leur domaine, en effet, il est une espèce

\section{Les pineraies de Pin sylvestre}

Cercles d'exposition

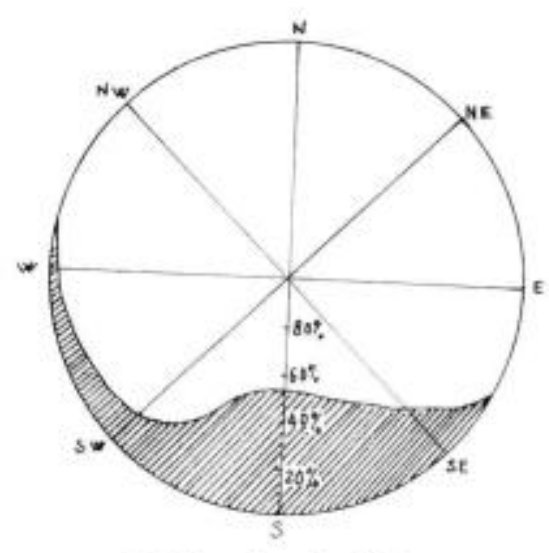

I. Pineraie xérophile.

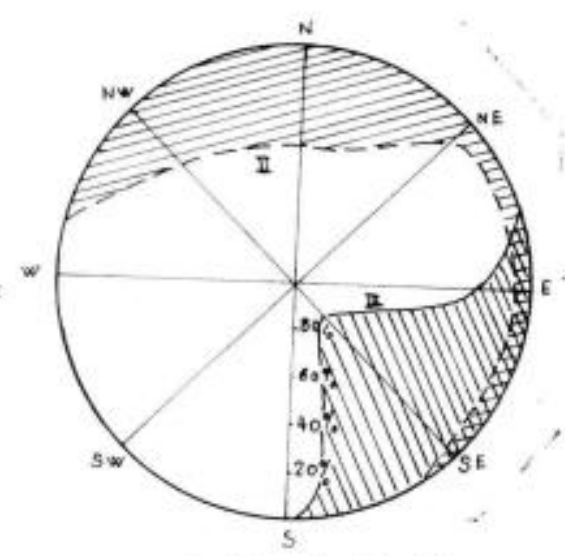

I. Pineraie xérophile.

Fig. 30 .

... II. Pineraie mésoxérophile. III. Pineraie méso-érophile.

Légende, - Bien que les cercles d'exposition doivent être interprétés avec une certaine circonspection pour des raisons déjà dites, on constate qu'ils rendent parfaitement compte des conditions écologiques régnant dans chacune des associations en cause.

On les rapprochera des spectres floristico-écologiques correspondants.

I. - Pineraie xérophile à Ononis rotundifolia et Coronilla minima.

II. - Pineraie mésoxérophile à Erica carnea sans mousses.

III. - Pineraie mésophile à Erica carnea et mousses.

(7) celles du Briançonnais ne sont pas encore bien connues; elles ne comportent pas, en tout état de cause, l'espèce sociale qui donne leur physionomie aux pineraies de la Maurienne, Erica carnea. 
Les pineraies de $P$ in sylvestre

Spectres floristico-écologiques

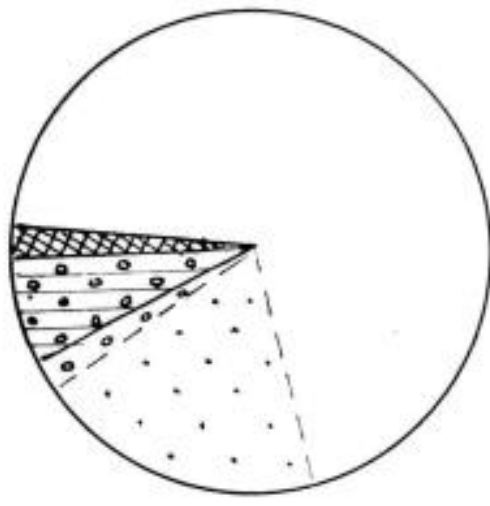

I. Ononido-Pinetum silvestris. (Relevés 7 à 14)

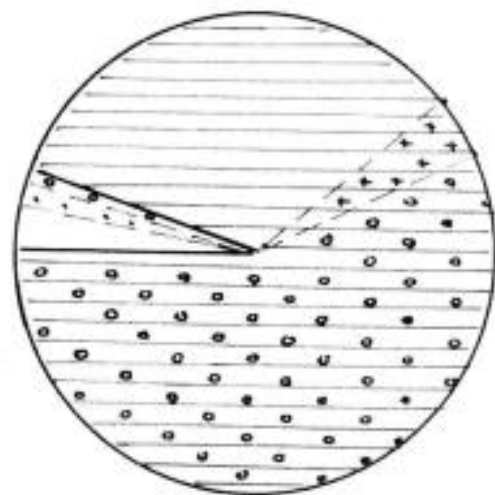

III. Erico-Pinetum silvestris hylocemietosum. (Relevés 13 à 19)

Xérophiles indifférentes.

... Xérophiles de mull.

-o0 xérophiles d'humus calcique.

-- Xérophiles de moder ou de mor.

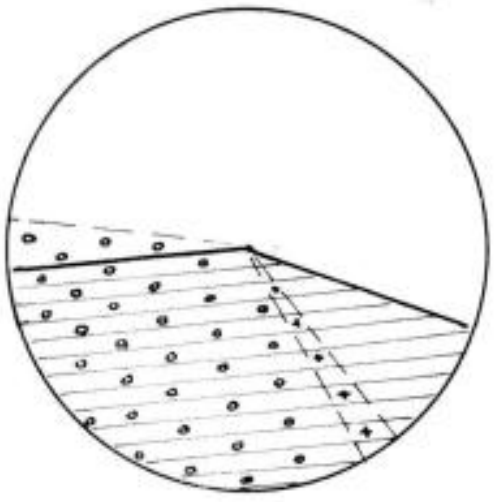

II. Ononido-Pinuetum silvestris ericetosum.

(Relevés 6 à 12)

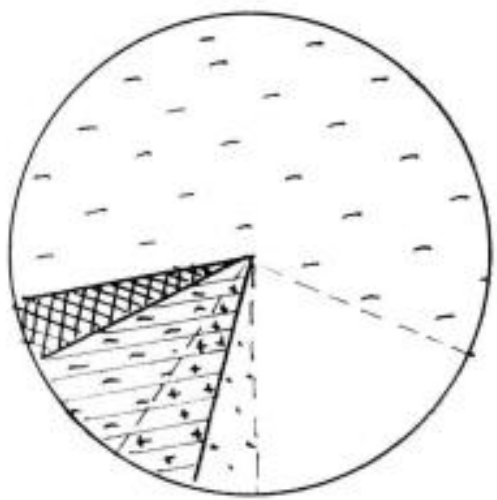

IV. Deschampsio-Pinetum silvestris. (Relevés 1 à 9)

4- Mésophiles de mull-moder.

$\frac{20}{4}$ Mésophiles de mull calcique.

-- Mésophiles de moder ou de mor.

Diverses.

Mésophiles (ou M..X.) indifférentes.

Fic. 31 .

Les spectres montrent l'envahissement progressif des mésophiles et dess méso-xérophiles, lorsque lon passe de la pineraie à Ononis rotundifolia et Coronilla minima à la pineraie à Erica carnea sans Mousses puis à la pineraie relativement mésophile à Ericar carnea et Mousses. 
qui joue un ròle tout à fait remarquable: Erica carnea. Très répandue dans les vallées intra-alpines de l'Europe centrale, en Suisse, en Autriche, en Italie du Nord, en Yougoslavie, cette espèce, très rare dans les Alpes occidentales françaises, ne s'observe en grande abondance qu'en Haute-Maurienne.

Ces affinités phytosociologiques que les pineraies permettent de souligner ne concernent pas, comme on le verra, ces set1s groupements; nous aurons l'occasion, tout au long de cette étude, de les mettre en relief pour d'autres associations et nous essaierons, in fine, de les expliquer à la fois par la similitude des conditions écologiques régnant en Haute-Maurienne et dans les autres vallées sèches de l'Europe centrale et par les facilités de communication que notre vallée a offertes aux courants floraux venus de l'est.

\section{III.2.3.2, - Evolution - Climax.}

Un fait essentiel est à retenir: à exposition nord, lErico-Pinetum est spécialisé édaphiquement (sols calcimorphes), alors qu'à exposition sud, les pineraies s'observent sur toutes roches-mères avec un contingent d'espèces non spécialisées et un contingent d'espèces spécialisées (calci-neutrophiles sur sols calcimorphes; acidiphiles sur sols silicatés).

Les questions qui se posent sont donc les suivantes: à exposition fraiche l'Erico-Pinetum, d'une part; à exposition sèche l'OnonidoPinetum et son vicariant acidiphile le Deschampsio-Pinetum, d'autre part, sont-elles, ou non, des associations climaciques?

Il nous est apparu que, lorsque le sol se décarbonate et se désature en calcium dans les horizons supérieurs, à exposition nord, et de calcimorphe devient sol brun, c'est l'Abietetum albae [sapinière vraie de Kuoch (R.) (1954) qui sera analysée plus loin] qui apparait. On rencontre des peuplements où le Pin sylvestre a disparu avant même qu'Erica carnea et son cortège l'aient fait: c'est dire qu'entre l'Erico-Pinetum et la sapinière neutrophile à Carex alba. se rencontrent d'incontestables intermédiaires et qui paraissent bien se situer dans une série génétique.

Mais il est bien certain que cette évolution est d'autant plus difficile que l'érosion est plus forte: cette dernière dépend de la composition et de la dureté de la roche-mère comme de la vigueur du relief. Sur cargneule et sur moraine, roches-mères tendres, Yinstallation est certainement très difficile et l'Erico-Pinetum constitue une association spécialisée permanente: sur calcaire franc, l'évolution parait plus aisée, comme on l'observe en forêt de Sollières-Envers.

A noter que dans la haute vallée du Rhin et en Basse-Engadine. [Braun-Blanguet (J.), Pallaman (H.) et Bach (R.) (1954)] 
après une étude de détail des phases initiales de l'Erico-Pinetum, indiquent que l'association évolue climaciquement vers une pessière vraie montagnarde (Piceetum montanum) typiquement intra-alpine, d'où se trouve exclu le sapin, par suite de conditions climatiques très sèches et très continentales.

Le problème du climax à exposition sud est sans doute plus délicat, car le long des gorges houillères, où l'on aurait pu analyser le passage entre hêtraies et pineraies, l'action humaine s'est fait sentir avec une vigueur déjà soulignée. On saisit toutefois ce passage, avec une grande netteté, en forêt d'Albanne, dans les gorges de la Valloirette. Sur le flane de ces gorges à exposition générale tournée vers l'est, on constate qu'aux expositions secondaires les plus fraiches, le Hêtre domine, avec un cortège qui sera analysé plus loin, alors qu'aux expositions sud, le Pin sylvestre le remplace presque totalement, avec un cortège quelque peu différent (Ononido-Pinetum fagetosum) et ce, alors que laction humaine s'est exercée de toute évidence, de la même facon de part et d'autre de telles petites arêtes secondaires (8).

On peut donc penser qu'entre St-Michel-de-Maurienne et Modane, le bas de l'étage montagnard était occupé par la chênaic pubescente qui arrivait à disparaitre, par laminage dû à l'élévation du fond de la vallée; au-dessus, la hêtraie devait céder peu à peu la place aux pineraies, dont les premières enclaves s'observent, on vient de le voir, à l'aval des gorges houillères, dans la vallée de la Valloirette. On ne voit d'ailleurs pas en dehors des pineraies de Pin sylvestre - quelles associations pourraient tenir leur place - puisque ni la hêtraie ni la chênaie pubescente n'ont atteint la Haute-Maurienne, sensu stricto.

Sans doute, 1'Abietefum albae arrive-t-il - sous sa forme la plus xérique - à occuper certains versants tournés vers le sud-est. A Attssois, en particulier, il est en contact presque direct avec l'Ononido-Pinetum. Mais sa localisation dans les ravins ou sur le plateau au sol profond et à l'enneigement prolongé donne à penser qu'aux versants " plein sud " et même sur sol profond, il ne peut s'adapter an climat brutal précédemment analysé.

C'est pourquoi nous pensons que la pincraic sérophile - acidiphile ou neutrophile - constitue un climax, plus ou moins homogène évidemment, si l'on observe qu'en montagne l'influence de la roche-mère se manifeste toujours peu ou prou (9). Nous reviendrons sur ce dernier point d'ailleurs in fine.

(8) Dans les parties les plus abruptes, cette action peut ètre même consiđérée comme négligeable.

(9) Le point de vue exprimé par Richard (J.-L.) (1961) à propos des associations jurassiennes est à rappeler ici, à savoir que e l'influence de la roche-mère se fait sentir jusquau stade final d'une série évolutive $\$$. 


\section{III.2.3.3. - Structures sylvicoles - Productions.}

D'une façon générale, la structure, c'est-à-dire la répartition des tiges dans l'espace de l'une ou l'autre des pineraies de Pin sylvestre

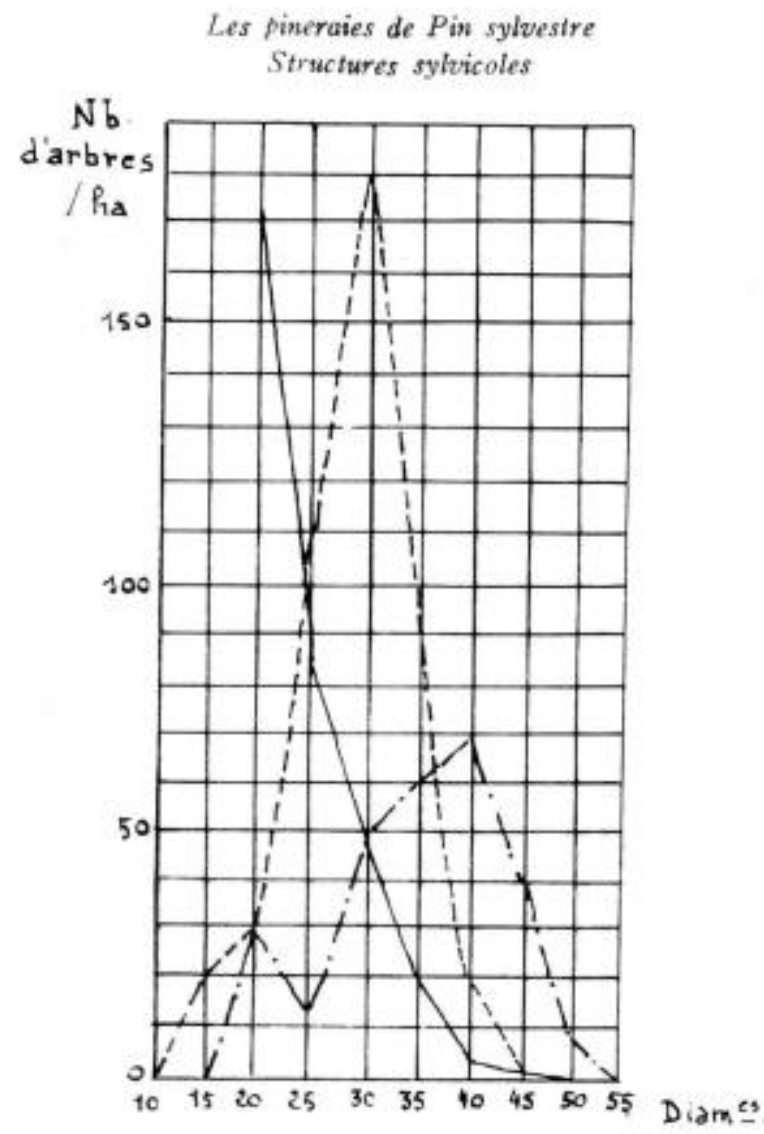

Pineraie mésoxérophile.

sur 97,7 ha ,Sollières. $2^{\circ}$ Série, Plles L.O.P.Q.T.).

.... Pineraie mésophile.

(Sollières 2" Sćrie. Plle $\mathrm{S}$ pie.

-.. Pineraie xérophile.

(Aussois. $1^{\text {r* }}$ Série. Plle $\mathrm{H}$ pie.

FIG. 32.

Légende. - Les structures des deux derniers types de pineraie montrent une grande régularité; mais il s'agit, il faut le noter, de structures obtenues à partir d'un échantillonnage restreint, alors que celle des la pineraie mésoxérophile résulte de comptages effectués sur près de 100 hectares. 
apparait comme régulière. On peut penser que cette structure est due à l'action humaine, soit que l'exploitation ait été faite par coupes à blanc, à tire et aire, soit que l'envahissement de certains terrains pastoraux ait été brutal; on ne doit cependant pas négliger le rôle joué par le tempérament, même du Pin sylvestre, essence de lumière.

Cette structure se traduit par des courbes de type gaussien, plus ou moins aplaties selon l'âge des peuplements et selon, aussi, leur vitesse d'accroissement pour un âge donné (10) (fig. 33).

En ce qui concerne les productions - que l'on se gardera bien de mettre en parallèle pour des raisons déjà dites - on retiendra surtout qu'elles sont de très faible importance: sur sol calcimorphe, l'Ononido-Pinetum échantillonné, ayant à peu près 230 ans, produit actuellement (moyenne des 20 dernières années) $1,36 \mathrm{~m}^{3} /$ hectare et par an (tarif algan $\mathrm{n}^{\circ} 5$ ), la pineraie " mixte " à Erica carnea et Carex humilis (d'âges mêlés, produit $1,59 \mathrm{~m}^{3}$ ) (tarif algan $\mathrm{n}^{\circ} 6$ ), l'Erico-Pinetum a 130 ans environ, $2,35 \mathrm{~m}^{3}$ (même tarif). Sur ranker initial, le Deschampsio-Pinetum produit $1.54 \mathrm{~m}^{3}$ (tarif algan $\mathrm{n}^{\circ} 3$ ), l'âge de l'échantillon retenu étant d'environ 175 ans.

(10) Fait exception à la règle la pineraie mésoxérophile qui présente une structure de futaie jardinée. Ceci tient essentiellement au fait que l'échantillonnage a porté sur des parcelles entières: la structure régulière par bouquets se trouve ainsi un peu masquée. 


\section{III.3. - LES PINERAIES DE PIN A CROCHETS}

En Haute-Maurienne, les pineraies de Pin à crochets occupent une surface assez considérable, et leur localisation obéit à des conditions écologiques étroites, dont il importe de définir tout de suite les grands traits.

En effet, bien que le Pin à crochets s'avère adapté, en France, aux substrats les plus sévères - il se localise pratiquement, dans notre dition, sur les seuls sols calcimorphes, laissant notamment la place à l'Epicéa, au Pin sylvestre, ot au Mélèze, sur les éboulis de quartzite les plus stériles.

Dans son étage - qui, on le verra, est essenticllement létagc subalpin - le Pin à crochets devrait donc se rencontrer sur tous les sols calcimorphes. Mais ici une nuance s'impose: dans la zone subbriançonnaise, pourtant riche en calcaires et méme en gypse, le Pin à crochets est relativement rare et se réfugie pratiquement sur quelques arêtes difficilement accessibles du massif des Encombres: ceci tient au fait que l'étage subalpin de cette zone est, en fait, très peu étendu. que le relief $y$ est particulièrement vigoureux - d'où un régime d'éboulis et d'avalanches très important - et que, partout où les pentes ne sont pas trop accentuées, elles sont occupées par des pâturages: les sols calcimorphes de l'étage montagnard, eux, sont recouverts par des groupements de Chène pubescent, de Hêtre, de Pin sylvestre ou de Sapin. Dans la zone de Vanoise, par contre, très riche en calcaires, cargneules, gypses et qui se développe à l'amont de lat nappe Houillère, les forêts de Pin à crochets couvrent de vastes étendues dans un étage subalpin luimème de grande surface. Elles laissent la place, dans l'étage montagnard, aux pineraies de Pin sylvestre. c'est-à-dire au-dessous de $1750-1800 \mathrm{~m}$, à une exception près toutefois déjà soulignée et qui est importante: sur gypse, en effet, là où l'érosion empêche tout approfondissement et toute évolution du sol, le Pin à crochets prend - et totalement - la place du Pin sylvestre dans Pétage montagnard lui-même; il s'agit donc d'une spécialisation au deuxième degré, en quelque sorte. Dans cet étage, les associations qu'il forme sont tout à fait semblables à celles du Pin sylvestre, car il constitue. en fait, la seule espèce différentielle. Toutefois, étant donné qu'il 


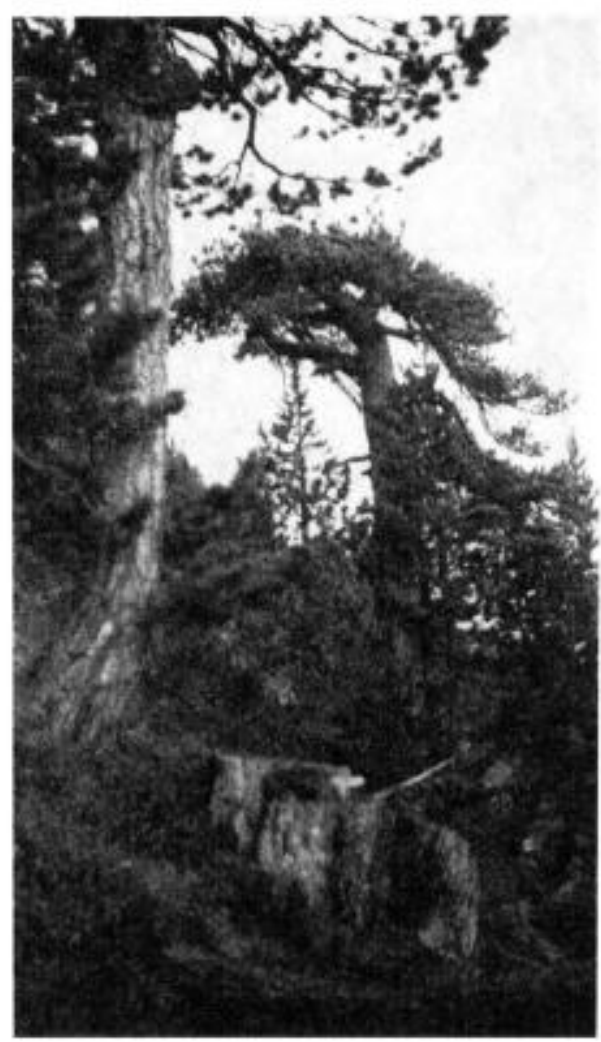

Vers $2300 \mathrm{~m}$ d'altitude, formes tourmentées des arbres de lisière... 
dominc de façon absolue - et naturellement - il nous a paru difficile de faire de ces groupements des sous-unités d'associations de Pin sylvestre... d'où le P'in sylvestre serait complètement absent, èt il nous a semblé préférable de les étudier en même temps que les associations subalpines de Pins à crochets, dont elles diffèrent très peu d'ailleurs.

Il faut noter que les pineraies de Pin it crochets présentent avec les pineraies de Pin sylvestre, un parallélisme très étroit, à cette différence près qu'il n'existe pas, dans notre dition, de pineraies de Pin à crochets acidiphiles.

Une telle parenté nous dispensera de faire une analyse poussée des organisations sociologiques.

Il n'est pas inutile de rappeler que le Pin à crochets qui nous occupe correspond au Pinus montana subsp, uncinata de Ramon, espèce à forme érigéc des Alpes Françaises et des Pyrénées - comme des autres montagnes françaises, d'ailleurs, et dont les caractères ont été rappelés par Guinier (Ph.) et Pourter (J.) (1951). Cet arbre peut prendre des formes prostrées dans les couloirs d'avalanches ou sur certaines crêtes battues par les vents: mais il s'agit alors de véritables accomodats et non de génotypes, comme GuINIER et POURTET en ont observé, par exemple, à l'Alpe du Buffalora, au nord de l'Ofenpass, en Suisse (1).

\section{III.3.1. - Les pineraies de Pin à crochets xérophiles.}

$1^{\circ}$ Organisation sociologique et composition floristique - Faciès.

Comme dans l'Ononido-Pinetum, les groupes écologiques xérophiles dominent de façon absolue, et ce sont les mêmes, du moins dans létage montagnard, sur gypse: les calciphiles dont les subméditerranéennes, les indifférentes médio-européennes et circumboréales, où Arctostaphylos Uva-ursi joue le premier róle, etc. Les relevés 1 et 2 correspondent à un faciès pionnier, particulièrement sec, avec Hieracium lanatum, Viola rupestris, Scorsonera austriaca. Astragalus sempervirens, etc.

Il est curieux de noter que les relevés de l'étage subalpin ne diffèrent pas tellement de ceux de l'étage montagnard. Les oro-subméditerranéennes tendent à disparaître complètement toutefois, tandis qu'apparaissent d'autres espèces subalpines jouant le róle de différentielles comme Carex semperzirens, Senecio Doronicum, Minuartia Villarsii. La flore de cette sous-association stbalpine n'est évidemment pas sans rappeler celle de l'association à Sesleria coerulea et Avena montana qui la relaie dans Pétage alpin.

(1) On regrettera que les phytosociologues suisses aient appelé Mugetum toutes les associations de Pins à crochets, alor's qu'il s'agit le plus souvent dans le Jura tout particulièrement - non du Pinus Mugho, mais du Pinus montana subsp. uncinata. 
PLANCHE VIII

Les pineraies de $P$ in à crochels

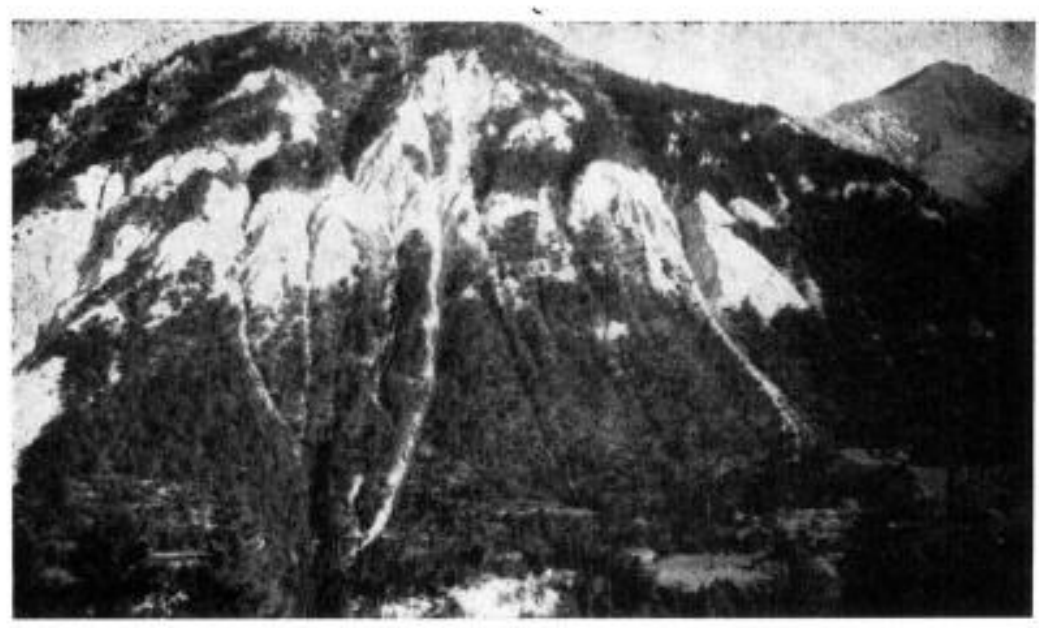

La pincraie xérophile sur gypse - Vallée de Bramans Vuc générale.

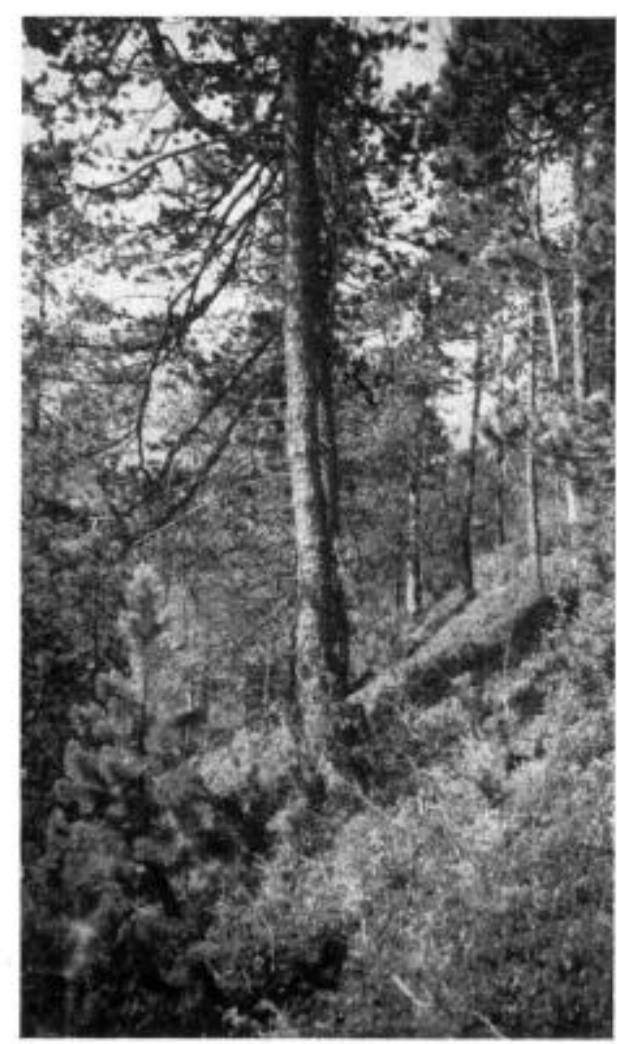

La pineraie de Pin à crochets xérophile sur rendzine gypseuse Vue de détail. 
Le spectre floristico-écologique rappelle de très près celui de l'Ononido-Pinetum (fig. 35).

La structure, tout au moins celle de la strate arborescente, est assez typique: alors que, dans $\mathrm{J} O$ Ononido-Pinetum, les Pins sylvestres atteignent d'assez fortes dimensions et forment alors des peuplements peu denses, les Pins à crochets, dans l'association en cause, sont souvent très denses et de très petites dimensions, ce qui est en relation directe avec l'extrème pauvreté de la station. La structure des strates arbustive et herbacée est, soit du type mosaique avec de nombreux vides et des taches d'Arctostaphylos Uva-ursi, soit, le plus souvent, du type "tapis continu ", la Busserole couvrant complètement le sol.

\section{Identification - Dénomination.}

Parmi les groupements de Pin à crochets décrits à ce jour notamment en Suisse - les plus xérophiles paraissent être la variante à Pinus mugo du Carici humilis Pinetum et la sous-association à Carex humilis de l'Erico-Mugetum. De ces deux groupements, très proches l'un de l'autre, d'ailleurs, le plus xérophile est le premier où se trouvent présentés des espèces, comme Saponaria ocymoides, Teucrium montanum, Globularia cordifolia, etc, et doit être rapproché de la forme subalpine de notre association, sans conteste possible. Mises à part les différences tenant aux aires des espèces et qui expliquent l'absence dans l'association de Hatte-Maurienne de Laserpitium Gaudinii, absent en France, de Daphne striata ou de Viola pinnata, extrêmement rares en Savoie, le nombre d'espèces communes est des plus significatifs, en effet. Par contre, la sousassociation " montagnarde », particulièrement riche en oro-méditerranéennes, ne parait pas avoir été décrite: il faut dire qu'elle s'explique, dans nos Alpes, par l'abondance des gypses qui paraissent beaucoup plus rares en Suisse (2).

\section{3o Répartition - Ecologie.}

En l'absence de données microclimatiques, il y a lieu d'insister sur l'étroite localisation de cette association que l'on observe sur les seuls versants exposés en plein Midi et sur sols calcimorphes. Une telle exposition et de tels types de sols ne se rencontrent, dans la vallée principale de l'Arc, qu'au-dessus de Lanslevillard, là où une fenêtre de calcaires triasiques émerge de la nappe des schistes lustrés; des conditions tout aussi xériques se trouvent également remplies, dans certains vallons secondaires, et tout particulièrement dans la vallée d'Ambin, où les formations de gypse prennent un dévéloppement considérable. C'est dans cette vallée que, sur gypse, et dans l'étage montagnard, c'est-à-dire entre 1300 et $1700 \mathrm{~m}$ en-

(2) S'il fallait lui donner un nom, ce serait celui d'Ononido-Mugetum, plus exactement d'Ononido-Pinetum wincinatae. 
viron, la pineraie de Pin à crochets à Coronilla minima et Ononis rotundifolia, variante sur gypse à Pins à crochets de l'Ononido-Pinetum, connait un développement particulier.

Quelles altitudes peut atteindre la pineraie xérophile de Pin à crochets? Le plus souvent, elle est limitée par les formes de terrain qui s'arrondissent au sommet des versants abrupts des torrents, avant que ne soit atteint l'étage alpin, et sont, de ce fait, occupés par l'homme; mais il n'en est pas toujours ainsi, par exemple, audessus de Lanslevillard, où la forèt - malgré un régime sévère d'avalanches qui lui donne un aspect misérable - atteint $2.300 \mathrm{~m}$ à pet près.

Les sols s'apparentent tout à fait à ceux de l'Ononido-Pinetum. Il s'agit soit de rendzines jeunes - sur gypse tout particulièrement. dans l'étage montagnard - (sol $\left.\mathrm{n}^{\circ} 1\right)$ - soit de rendsines un peut décarbonatées on surface ( sol $^{\circ} 2$ ), dont une analyse sommaire est donnée ci-après:

\section{Sol $n^{*} 1$ - Forét de Bramans}

Exp.: Sud - Altitude $1500 \mathrm{~m}$ - Roche-mère: Gypse

Canton de la Villette - Relevé floristique $\mathrm{n}^{\circ} 1$

\begin{tabular}{|c|c|c|c|c|c|c|}
\hline $\begin{array}{l}\text { Profor- } \\
\text { deur }\end{array}$ & Horizans & $\begin{array}{l}\text { Profond. de } \\
\text { prélèvenent }\end{array}$ & pH & $\begin{array}{c}\text { Matière } \\
\text { organique }\end{array}$ & $\begin{array}{l}\infty^{3} \mathrm{cs}_{\mathrm{s}} \\
\text { netif }\end{array}$ & $\begin{array}{c}\mathrm{Cn}^{+4} \\
\text { Gebangeable }\end{array}$ \\
\hline \multirow{3}{*}{$30 \mathrm{~cm}$} & $A_{1}$ & $-2 a n$ & 7,9 & 35,2 & 1 & Sature \\
\hline & $\mathbf{A}_{1}$ & $-20 \mathrm{cs}$ & 8,5 & 5,4 & 11 & Saturé \\
\hline & $4_{1} / 0$ & $-40 \mathrm{~cm}$ & 8,5 & 4,2 & 12,3 & Sature \\
\hline
\end{tabular}

Sol $n^{\circ} 2$ - Forìt de Termignon

Exp. Sud - 8ltitude: $1900 \mathrm{~m}$ - Roche-mère: Moraine calcaire.

Relevé floristique $\mathrm{n}^{\circ} 10$

\begin{tabular}{|c|c|c|c|c|c|c|c|c|c|}
\hline Profond- & Borizons & $\begin{array}{l}\text { Profond. } \\
\text { de preilav. }\end{array}$ & $\mathrm{pH}$ & Argile & Limon & $\begin{array}{c}\text { Sablee } \\
\text { fins }\end{array}$ & $\begin{array}{l}\text { Snbles } \\
\text { grosa. }\end{array}$ & $\begin{array}{l}\text { Matière } \\
\text { organ. }\end{array}$ & $\infty^{3} \mathrm{Ca}$ \\
\hline \multirow{2}{*}{$40 \mathrm{~cm}$} & $\Lambda_{1}$ & $-5 \mathrm{cos}$ & 7,4 & & & & & 40,2 & Traces \\
\hline & $\mathbf{A}_{1} / \mathrm{c}$ & $-45 c m$ & 7,4 & 19 & 30 & 23,9 & 11,9 & 11,1 & 9,1 \\
\hline
\end{tabular}




\section{III.3.2. - Les pineraies de Pin à crochets méso-xérophiles.}

$1^{\circ}$ Organisation sociologique et composition floristique.

Le tableau d'association met bien en évidence le caractère de ce groupement, véritable intermédiaire entre le groupement xérophile et le groupement mésophile dont il sera question ci-après. Il présente, de ce fait. les plus étroites affinités avec l'association mésorérophile de Pin sylvestre, dont nous avons fait, également, une unité autonome.

Mésophiles et xérophiles se trouvent à peu près représentées dans la même proportion: tout particulièrement, le rapport Erica carnea/Arctostaphylos Uva-ursi est sensiblement égal à 1 .

Trois faciès principaux peuvent ètre distingués:

- un faciès montagnard, sur gypse, où l'Epicéa est à peu près complètement absent, et où les espèces xérophiles subméditerranéennes s'observent en plus grande abondance que dans les autres faciès, qu'il s'agisse de xérophiles neutrophiles ou d'indifférentes. Ces espèces, dans cet étage et sur les sols particulièrement secs, reposant sur gypse, trouvent les conditions thermiques et xériques qui leur conviennent, plus facilement que dans l'étage subalpin et sur des sols plus évolués, à granulométrie plus fine.

- un faciès subalpin inférieur, entre $1750 \mathrm{~m}$ et $2000 \mathrm{~m}$, où l'Epicéa est presque toujours présent. On notera toutefois qu'il reste en seconde position par rapport au Pin à crochets, alors que dans l'association mésophile à Erica carnea et Hylocomies, il arrive à égalité avec ce dernier. Les mésophiles — ou mésoxérophiles notamment Sesleria coerulea sont plus abondantes que dans le faciès précédent, à l'inverse des xérophiles.

- un faciès subalpin supérieur, à partir de $2000 \mathrm{~m}$ où l'Epicéa disparait, laissant la place au seul Pin à crochets. Les méditerranéomontagnardes sont absentes tandis que Carex sempervirens, Dryas octopetala, qui sont essentiellement stbalpines dans notre dition. jouent le rôle de différentielles.

Le spectre floristico-écologique, comme la structure, rappellent tout à fait ceux de la pineraie homologue de Pin sylvestre: Erica carnea et Arctostaphylos Uva-ursi forment des taches plus ou moins contiguës: les places vides étant occupées par Carex humilis, Ononis rotundifolia, Astragalus Monspessulanus... et dans l'étage subalpin par Carex sempervirens et surtout Dryas octopetala (fig. 35).

\section{Identification - Dénomination.}

Si l'on se réfère aux divers relevés phytosociologiques de pineraies de $\mathrm{P}$ in à crochets qui ont été effectués, notamment en Suisse, il apparait que notre groupement présente les plus étroites affinités avec la sous-association de l'Erico-Mugetum dite à Carex humilis 
PLANCHE IX

Les pineraies de Pin à crochets

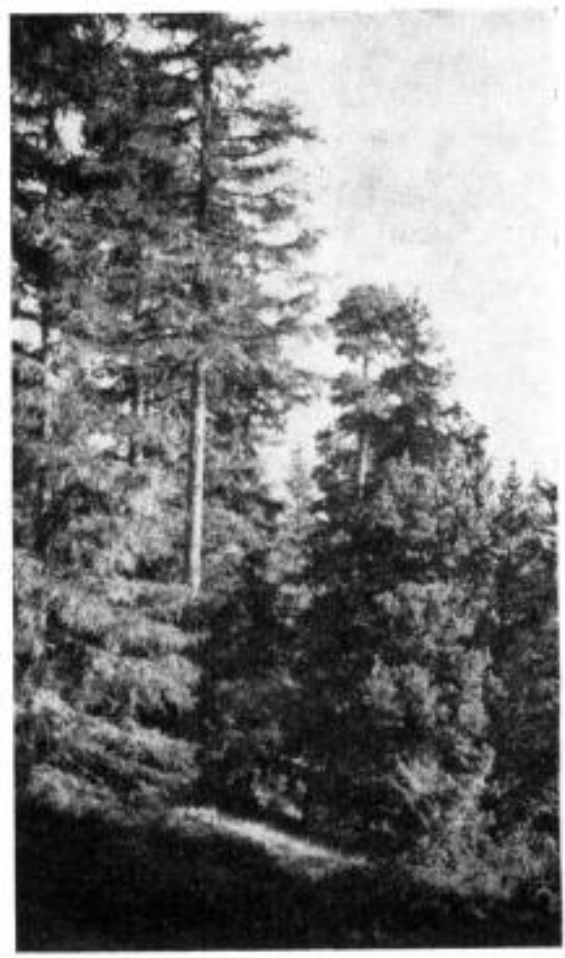

Pineraie mésoxérophile à Erica carnea et Carex humilis. Etage subalpin inférieur $(1900 \mathrm{~m})$ - Forét de Sollières-Sardières. (Noter la présence de l'Epicéa.)

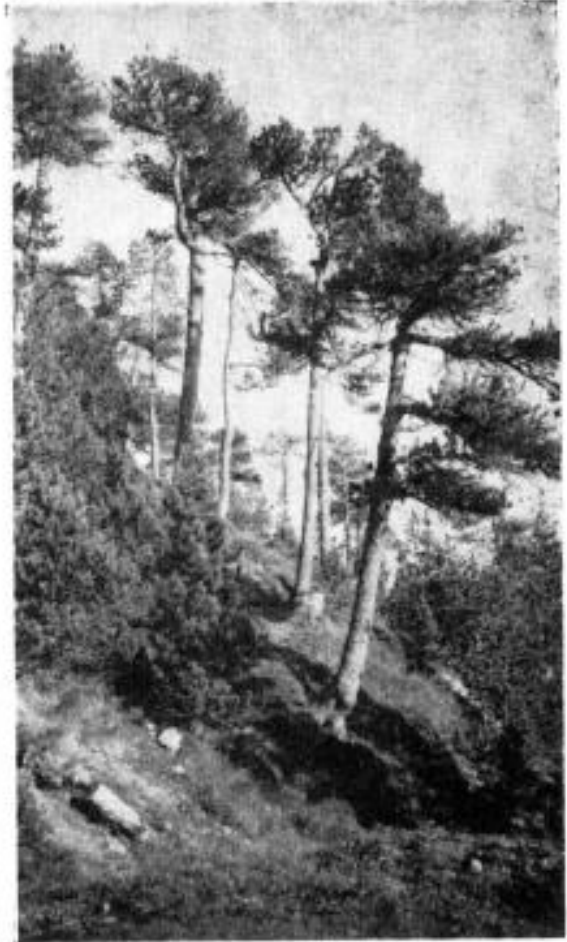

Pineraic mésoxérophile à Erica carnea et Carex humilis. Etage subalpin supéricur $(2200 \mathrm{~m})$ - Forèt de Sollières-Sardières.

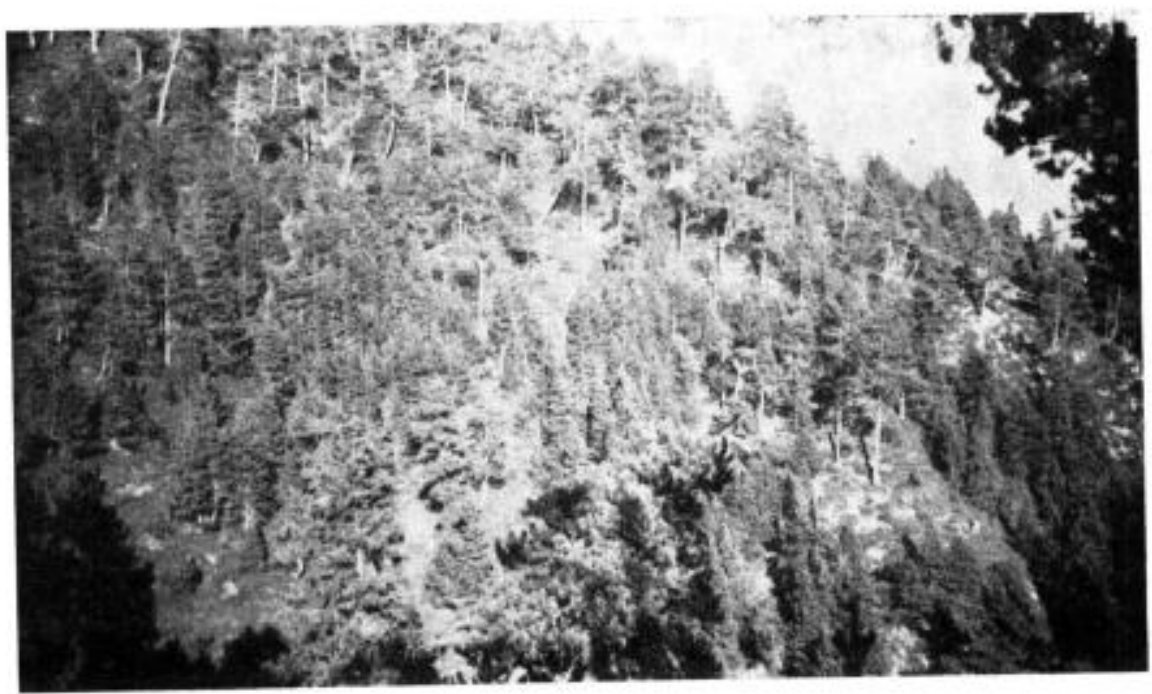

Pineraie mésoxérophile. Etage subalpin supérieur (2200 m) Vue gencesale. 
(Erico-Mugetum caricetosum humilis), encore que les relevés suisses nous paraissent correspondre à des individus un peu plus mésophiles que les nôtres, plus proches, par conséquent, de la sousassociation à Hylocomies.

\section{Répartition - Ecologie.}

En l'absence de données microclimatiques, il y a lieu de retenir la très remarquable localisation de cette association aux expositions " intermédiaircs $n$. Comme les roches-mères carbonatées s'observent, dans la partie de la vallée où PArc prend une direction SWNE, les expositions se trouvent ètre en majorité nord-ouest et sudest, ce qui explique que la pineraie mésoxérophile de Pin à crochets - comme son homologue à Pin sylvestre - couvre des surfaces étendues en Haute-Maurienne, lassociation xérophile se localisant sur les versants sud, secondaires et la mésophile sur les versants nord, secondaires.

Les sols, tous de type calcimorphe, présentent toutefois des nuances.

Certains sont à assimiler aux sols humiques carbonatés proprement dits : sols $n^{\circ} 1$ et 2 . Il s'agit de sols à $\mathrm{pH}$ élevé dans presque tout le profil, riches en matière organique, à l'humus structuré en grumeaux et carbonatés jusque dans l'horizon de surface, parvres en argile.

Sol $n^{\circ} I$ - Forêt $d^{r}$ Anssois

Fxp. SE - Altitude : $2200 \mathrm{~m}$ - Roche-mère: cargneule

Relevé floristique $\mathrm{n}^{\circ} 13$

\begin{tabular}{|c|c|c|c|c|c|c|c|c|}
\hline Dorizons & $\begin{array}{l}\text { Profond. } \\
\text { de prol. }\end{array}$ & pH & Argile & Linora & $\begin{array}{c}\text { Sables } \\
\text { fins }\end{array}$ & $\begin{array}{l}\text { asbles } \\
\text { Gross. }\end{array}$ & $\begin{array}{l}\text { Nat. } \\
\text { org. }\end{array}$ & , $\mathrm{CO}_{3} \mathrm{Ca}$ \\
\hline 4 & $-2 \mathrm{~cm}$ & 7,6 & & & & & 34 & 25,3 \\
\hline$A_{1} / C$ & $-15 \mathrm{cs}$ & 7,6 & 5,5 & 11 & 54 & 17,7 & 9,4 & 63,7 \\
\hline
\end{tabular}

Sol $n^{*} 2$ - Forêt d'Aussois

Exp. W - Altitude: $1850 \mathrm{~m}$ - Roche-mère: cargneule (grouins)

Relevé floristique $\mathrm{n}^{\circ} 12$

\begin{tabular}{|c|c|c|c|c|c|c|c|c|c|}
\hline Borizons & $\begin{array}{l}\text { Profond. } \\
\text { de pról. }\end{array}$ & Cailloux & pHi & $\begin{array}{l}\text { Mat. } \\
\text { org. }\end{array}$ & c & s & $\mathrm{c} / \mathrm{s}$ & $\begin{array}{l}\cos ^{3} c_{a} \\
\text { total }\end{array}$ & $\begin{array}{l}\omega^{3} c_{0} \\
\operatorname{actif}\end{array}$ \\
\hline 4 & $-2 \mathrm{~cm}$ & & 7.4 & 36.9 & 18.45 & 0.83 & 22.2 & 4.3 & 5.0 \\
\hline$\Lambda_{1}$ & $-10 \mathrm{~cm}$ & 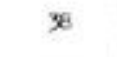 & 7.7 & 19.6 & 9.78 & 0.50 & 19.6 & 22.4 & 4.4 \\
\hline$A_{1} / C$ & $-40 \mathrm{ct}$ & 36 & 8.0 & 3.8 & 2.21 & & & 30.0 & 2.7 \\
\hline
\end{tabular}


D'autres - sols $\mathrm{n}^{\circ} 3$ et 4 - présentent un début de décarbonatation dans 1'horizon de surface, un abaissement corrélatif de $\mathrm{pH}$ et sont à assimiler à des sols humiques carbonatés passant au type d humus brut. La teneur en matière organique est particulièrement élevée.

Sol $n^{\circ} 2$ - For êt d"Aussois

Exp. W - Altitude: $1900 \mathrm{~m}$ - Roche-mère: cargneule

Relevé floristique $\mathrm{n}^{\circ} 11$

\begin{tabular}{|c|c|c|c|c|c|c|c|c|c|}
\hline Borizons & $\begin{array}{l}\text { Frofond. } \\
\text { de prél. }\end{array}$ & pdi & Argd le & Limong & $\begin{array}{c}\text { Sables } \\
\text { find }\end{array}$ & $\begin{array}{l}\text { asbles } \\
\text { groas. }\end{array}$ & $\begin{array}{l}\text { Nat. } \\
\text { org. }\end{array}$ & $\infty_{3} C_{n}$ & $\mathrm{H}_{2} \mathrm{O}$ \\
\hline$A_{0} / A_{1}$ & $-5 \mathrm{~cm}$ & 6 & & & & & 68 & tracea & \\
\hline 4 & $-20 \mathrm{~cm}$ & 7,2 & 14,5 & 27 & 20,2 & 9,5 & 14,2 & trpoes & 4,6 \\
\hline$A_{1} / c$ & $-50 \mathrm{~cm}$ & 7,6 & 10 & 29 & 31,4 & 20,5 & 6,7 & 37 & 2,4 \\
\hline
\end{tabular}

Sol $n^{\circ} 4$ - Forêt de Bramans

Exp. NW - Altitude: $1750 \mathrm{~m}$ - Roche-mère: Calcaire triasique

Relevé floristique $\mathrm{n}^{\circ} 6$

\begin{tabular}{|c|c|c|c|c|c|c|c|c|c|}
\hline Barisons & $\begin{array}{l}\text { Profond. } \\
\text { de prél. }\end{array}$ & $\mathrm{pH}$ & Argile & Ltrong & $\begin{array}{c}\text { Sable } \\
\text { fin }\end{array}$ & $\begin{array}{l}\text { Sable } \\
\text { gross. }\end{array}$ & $\begin{array}{l}\text { Nat. } \\
\text { org. }\end{array}$ & $\infty_{3} \mathrm{Ce}$ & $\mathrm{B}_{2} \mathrm{O}$ \\
\hline$A_{0} / A_{1}$ & $-5 \mathrm{cn}$ & 6,8 & 8 & 18,5 & traces & traces & 62,9 & 0 & 11,5 \\
\hline$A_{1} / c$ & $-15 \mathrm{~cm}$ & 7,6 & 12,5 & 19 & 45,6 & 2 & 15,5 & 57 & 4,4 \\
\hline
\end{tabular}

D'autres, enfin - qui correspondent en général à un faciès riche en Brachypodium pinnatum - sont des sols bruns calcaires ou des rendzines brunifiées, décarbonatés à plus grande profondeur et présentant un taux de matière organique relativement faible, structurée en petits grumeaux ( $\left(\mathrm{sol} \mathrm{n}^{\circ} 5\right.$ ).

Sol $n^{\circ} 5-$ Forêt $d^{\prime}$ Aussois

Exp. SE - Altitude: $1900 \mathrm{~m}$ - Roche-mère: moraine calcaire

Relevé floristique $\mathrm{n}^{\circ} 10$

\begin{tabular}{|c|c|c|c|c|c|c|c|c|c|c|}
\hline Pror. & Horizoas & $\begin{array}{l}\text { Profond.| } \\
\text { de pré1. }\end{array}$ & $\mathrm{pH}$ & Argile & Limon & $\begin{array}{c}\text { Sabie } \\
\mathrm{fin}\end{array}$ & $\begin{array}{l}\text { Sable } \\
\text { grass. }\end{array}$ & $\begin{array}{l}\text { Nat. } \\
\text { org. }\end{array}$ & $\mathrm{CO}_{3} \cdot \mathrm{Ca}$ & $\mathrm{H}_{2} \mathrm{O}$ \\
\hline$-50 n$ & $A_{1}$ & $-3 \mathrm{~cm}$ & 6,4 & 14 & 19 & 35,4 & 5,7 & 20,7 & 0 & 5,2 \\
\hline $20 \mathrm{~cm}$ & $h_{2}$ (B) & $-10 \mathrm{~cm}$ & 7 & 18 & 23 & 43,2 & 9,5 & 4,4 & 0,9 & 1,9 \\
\hline
\end{tabular}


Une telle diversité s'explique par la multiplicité des conditions écologiques locales: sur roche-mère dure, riche en carbonate de chaux, sur faible pente, un sol humique carbonaté aura tendance à évoluer vers le type à humus brut, tandis qu'une moraine calcaire plus ou moins riche en éléments silicatés, évoluera, dans les mêmes conditions, vers une sol brun calcaire. Sur forte pente, la décarbonatation se fera plus difficilement. Mais, dans l'ensemble, cette diversité reste de faible ampleur et n'atteint que les couches supérieures du sol, de telle sorte qu'elle ne retentit pratiquement pas sur la végétation.

On va voir qu'il n'en est plus de même avec les pineraies situées en conditions plus froides et plus humides.

\section{III.3.3. - Les pineraies de Pin à crochets mésophiles.}

Les groupements mésophiles de Pin à crochets se présentent sous deux physionomies nettement différentes, encore que leur écologie soit très voisine, sinon identique. Dans un type, Erica carnea domine de façon absolue - comme dans l'Erico-Pinetum; dans l'autre, son puissant tapis est remplacé par les touffes très abondantes et très vigoureuses de Carex ferruginea.

Cette curieuse vicariance de deux espèces qui paraissent s'exclure mutuellement s'explique, parce que l'aire d'Erica carnea ne recouvre pas toute la zone calcaire de la Vanoise, mais s'arrête un peu à l'amont de Modane. Or, dans la région de Modane, à exposition général nord, s'observent encore des calcaires, des cargneules et même des gypses, qui se trouvent le plus souvent - à la suite des caprices de l'érosion - exposés secondairement à l'ouest, et qui portent, par conséquent, des pineraies xérophiles de Pin sylvestre, mais dont certains se rencontrent à exposition plein nord, dans l'étage subalpin, et au-dessus d'une zone de roches-mères silicatées (quartzites, houiller métamorphique), portant une sapinière vraie. Ce sont ces calcaires, cargneules et gypses qui servent de substrat à la pineraie de Pin à crochets, à Carex ferruginea.

Il parait utile d'analyser à part - quoique très brièvement ce type un peu particulier de pineraie.

\section{III.3.3.1. - Pineraie de Pin à crochets à Erica carnea et Hy- locomies.}

$1^{\circ}$ Organisation sociologique et composition floristique - Faciès. xérophiles, tout particulièrement des oro-subméditerranéennes qui

Ce qui caractérise cette association c'est, d'une part, la rareté des cont pratiquement absentes, d'autre part l'abondance et la constance des mésophiles et mésoxérophiles, parmi lesquelles les calci-neutrophiles jouent un grand róle avec, en tête, comme pour les pine- 
PLANCHE X

Les pineraies de Pin à crochets

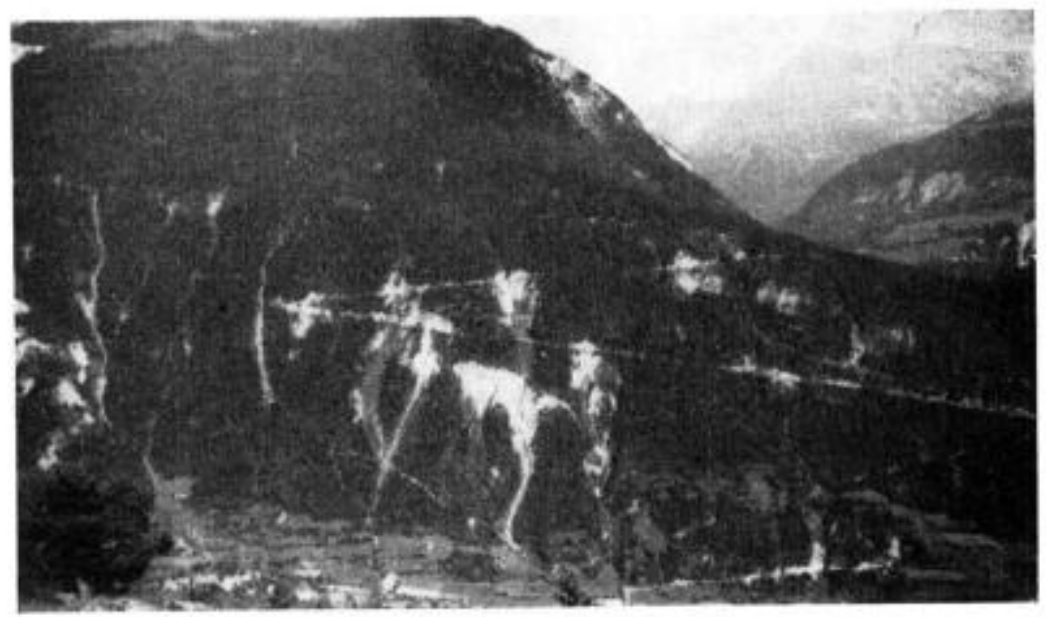

La pineraie de Pin à crochets mésophile sur rendzine gypseuse (étage montagnard) - Forèt de Bramans. 
raies homologues de Pin sylvestre: Erica carnea accompagnés d'autres espèces sud-européennes.

On notera que, dans l'étage montagnard, où le Pin à crochets repose sur gypse, les espèces du mull-moder sont relativement rares: ceci tient à la grande solubilité de la roche-mère qui, en donnant de fortes pentes, freine l'évolution des sols et empêche, en particulier, la décarbonatation des horizons supérieurs. Dans l'étage subalpin, où les calcaires et les cargneules sont plus abondants et où le climat est plus froid, le phénomène de décarbonatation peut se produire plus facilement et se traduit par une plus grande abondance de l'Epicéa, et des espèces de mull-moder qui jouent le róle de différentielles.

Il est intéressant d'observer, à ce sujet, que certains relevés correspondent à des stades intermédiaires: dans l'étage montagnard supérieur, aux confins de l'étage subalpin, tout un contingent d'espèces appartient aux associations mésophiles de sols bruns plus ou moins évolués: Lusula nivea, Prenanthes purpurea, Veronica urticifolia, Lusula Sieberi, etc. dont certaines (Veronica urticifolia, Luzula nivea) sont, en général, localisées dans la sapinière; plus haut, s'ajoutent à ce contingent des espèces plus spécialement subalpines comme Calamagrostis villosa, Homogyne alpina, localisées, elles, soit dans la pessière subalpine, soit dans les groupements de Pin Cembro; plus haut encore, c'est Rhododendron ferrugineum, Lonicera coerulea, qui apparaissent et qui forment un faciès pouvant être rattaché aussi bien aux associations de Pin à crochets qu'à celles de Pin Cembro (3).

D'une façon générale en tout cas - et le fait a déjà été souligné par Braun-Blanguet (J.), Pallmann (H.) et Bach (R.) dans leur étude sur le Parc National Suisse (1954) - la pineraie mésophile de $P$ in à crochets s'avère être plus riche en acidiphiles que son homologue de Pin sylvestre. Ceci tient à la plus grande rigueur du climat, plus humide et plus froid - qui donne, dans l'étage subalpin, beaucoup plus facilement qu'à altitude plus basse, des sols humiques carbonatés à humus brut ou des sols brunifiés.

Le spectre floristico-écologique - qui est à rapprocher de celui de la pineraie mésophile homologue à Pin sylvestre - traduit très clairement ce fait (fig. 35).

Ouant d̀ la structure, elle est tout à fait comparable à celle de l'Erico-Pinetum: tapis à peu près continu d'Erica carnea, d'où émergent les autres espèces, et sous lequel s'étend une strate muscinale toujours vigoureuse.

2० Identification - Dénomination.

C'est évidemment à l'Erico-Mugetum décrit plusieurs fois en Suisse, que doit être identifié notre groupement, très exactement

(3) Nous en avons fait une sous-association de la rhodoraie à Pin Cembro. 
à la sous-association Hylocomietosum; les deux groupements coïncident à peu près parfaitement, sauf l'absence dans le groupement mauriennais de quelques espèces n'atteignant pas notre dition: Rhododendron hirsutum, Crepis alpestris subsp. silvatica, Daphne striata, Laserpitium Gaudinii [Braun-Blanguet (J.), Sissingh (G.), VlieGER (J.) (1939)].

\section{$3^{\circ}$ Répartition - Ecologie.}

La localisation de la pineraie mésophile de Pin à crochets quant à l'exposition, tout au moins, est tout à fait semblable à celle de la pineraie de Pin sylvestre homologue: le cercle d'exposition le montre d'ailleurs (fig. 34).

En altitude et compte tenu, encore une fois, des peuplements sur gypse, elle s'étage entre $1300 \mathrm{~m}$ et $2200 \mathrm{~m}$; mais au sommet, elle a tendance à perdre son autonomie et à prendre la forme de rhodoraie mixte à Pin Cembro et Erica carnea.

Ses sols sont tous du type calcinorphe, mais présentent une évolution plus ou moins profonde déjà signalée lors de l'étude des groupes écologiques.

Sur roches-mères riches en carbonates et panvres en argile, il s'agit de sols humiques carbonatés vrais ou dégradés à humus brut ;

Erico-Mugetum - Types de sols

$\mathrm{e}=1 / 15^{\circ}$

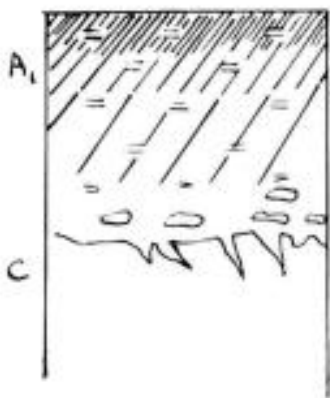

Profil $\mathrm{n}^{\circ} 1$.

Sol humique carbonaté.

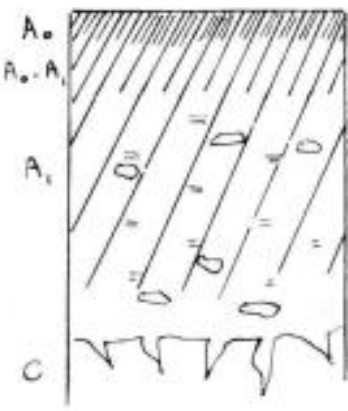

Profil $\mathrm{n}^{\circ} 2$

Sol humique décarbonaté en surface.

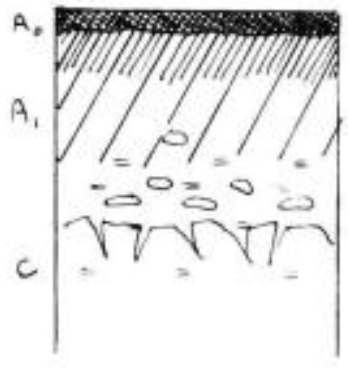

Profil $\mathrm{n}^{\circ} 3$.

Sol humique calcique décarbonaté acidifié.

Horizon humifère acidifié.

$==$ Carbonate de chaux.

Mull-moder calcique.

FIG, 33.

Sols humiques carbonatés des pineraies de Pin à crochets. 
dans ce dernier cas, l'acidité de l'horizon de surface, comme l'absence de carbonate qu'il présente, correspond moins à une décarbonatation qu'à une accumulation d'humus brut que traduit bien la teneur en matière organique et qui est due aux conditions climatiques, particulièrement froides et relativement humides.

Sur roches-mères plus pauvres en carbonates, comme les moraines, l'évolution est un peu différente: au départ, si l'on peut dire, le sol est plus proche des rendzines que des sols humiques carbonatés proprement dits; il se produit une décarbonatation dans les horizons de surface, la rendzine se brunifie et passe au sol brun plus ou moins lessivé à mull-moder. Une telle évolution explique, comme dans le premier cas, l'existence de sub-acidiphiles, mais il s'agira plutót d'espèces caractéristiques de mull-moder que de mor.

Nous donnons, ci-après, des exemples de sols humiques carbonatés plus ou moins décarbonatés et d'autres, correspondant à des sols bruns rendziniformes. On notera, dans les deux cas, l'absence de carbonates dans les horizons supérieurs et l'abaissement du $\mathrm{pH}$ dans ces mêmes horizons; mais alors que la teneur en matière organique est forte sur sol humique carbonaté, elle est relativement faible sur les sols bruns.

\section{Description morphologique.}

- Profil $n^{\circ} 1$.

$A_{1}$ supérieur $(0-2 \mathrm{~cm})$

- horizon humifère de mull calcique, structure grenue - couleur noire feutrage dense de racines d'Erica carnea.

$A_{1}$ profond $(3-30 \mathrm{~cm})$

- horizon humifère, structure grenue - couleur gris-noirâtre progressivement dégradée - feutrage de racines moins dense.

$$
A_{1}-(31-40 \mathrm{~cm})
$$

- horizon très peu humifère - structure subparticulaire (grains de gypse) - couleur gris-blanchâtre - texture sablo-limoneuse.

- Profil $n^{\circ} 2$.

$$
A_{0} \text { (surface) }(0-3 \mathrm{~cm})
$$

- horizon très humifère, de mull-moder calcique - structure grenue couleur très noire. 


$$
A_{0}-A_{1} \text { (profond) }
$$

$(4-20 \mathrm{~cm})$

- horizon très humifère, de mull-moder calcique; structure en grumeaux de 2 à $3 \mathrm{~mm}$ - feutrage dense de racines d'Erica carnea.

$$
A_{1}(21-60 \mathrm{~cm})
$$

- horizon légèrement humifère, structure subparticulaire (grains de gypse — couleur gris-blanchâtre - texture sablo-limoneuse.

\section{C}

- Gypse à structure subparticulaire, meuble - couleur blanchâtre.

- Profil $n^{\circ} 3$.

$$
A_{0}(0-4 \mathrm{~cm})
$$

- horizon de mor calcique, structure particulaire avec parties un peu fibreuses - couleur très noire avec passage brutal à l'horizon $\mathrm{A}_{1}$.

$$
A_{1}(5-20 \mathrm{~cm})
$$

- horizon humifère, de mull-moder calcique, structure en grains, couleur gris-blanchâtre - texture sablo-limoneuse.

\section{C}

- gypse de structure subparticulaire, meuble, de couleur blanchâtre.

- Analyses.

\begin{tabular}{|c|c|c|c|c|c|c|c|c|}
\hline Borizone & $\begin{array}{l}\text { Profond. } \\
\text { prólèv. }\end{array}$ & $\mathrm{pH}$ & $\begin{array}{l}\text { Kat. } \\
\text { org. }\end{array}$ & c & s & $\mathrm{c} / \mathrm{s}$ & $\cos \mathrm{cm}$ & $\cos \mathrm{N}_{8}$ \\
\hline$A_{1}$ & $-2 \mathrm{~cm}$ & 8,2 & 25 & 14,75 & 0,63 & 23,4 & 6,4 & 16,4 \\
\hline 4 & $-10 \mathrm{~cm}$ & 8,3 & 11,3 & 6,66 & 0,31 & 21,4 & 12,0 & 29,0 \\
\hline$A_{1}-A_{2}$ & $-30 \mathrm{~cm}$ & 8,4 & 3,4 & 2,02 & & & 14,4 & 28,0 \\
\hline & $-40 \mathrm{ch}$ & 8,4 & 2,1 & 1,23 & & & 12,8 & 37,2 \\
\hline
\end{tabular}

Sol $n^{\circ} 1$ - Forêt d'Avrieux

Altitude : $1500 \mathrm{~m}$ - Exp. Nord - Pente: $50 \%$ - Roche-mère: Gypse.

Relevé floristique $\mathrm{n}^{\bullet} 17$ 
Sol $n^{\circ} 2$ - Forêt de Bramans.

Plle 8 - Altitude : $1300 \mathrm{~m}$ - Exp. Nord - Pente: $100 \%$ - Roche-mère: Gypse

Relevé floristique $\mathrm{n}^{\circ} 17$

\begin{tabular}{|c|c|c|c|c|c|c|c|c|c|c|c|}
\hline $\begin{array}{l}\text { Hori- } \\
\text { sons }\end{array}$ & $\begin{array}{l}\text { Prof. de } \\
\text { prólèv. }\end{array}$ & $\mathrm{pH}$ & $\begin{array}{c}\text { Ar- } \\
\text { gilo }\end{array}$ & $\begin{array}{c}\text { Linons } \\
\text { fins }\end{array}$ & $\begin{array}{l}\text { Linonas } \\
\text { grogs. }\end{array}$ & $\begin{array}{l}\text { Sables } \\
\text { fing }\end{array}$ & $\begin{array}{l}\text { Sables } \\
\text { gross. }\end{array}$ & $\begin{array}{l}\text { Yeat. } \\
\text { org. }\end{array}$ & $c$ & $\mathbb{N}$ & $c / \mathrm{s}$ \\
\hline $\begin{array}{c}\Lambda_{0} \\
\Lambda_{0}-\Lambda_{1} \\
\Lambda_{1} \\
c\end{array}$ & $\begin{array}{l}-3 \mathrm{cn} \\
-15 \mathrm{~cm} \\
-55 \mathrm{~cm} \\
-65 \mathrm{~cm}\end{array}$ & $\begin{array}{l}7,1 \\
7,3 \\
7,9 \\
8,1\end{array}$ & $\begin{array}{r}20,5 \\
8,9\end{array}$ & $\begin{array}{l}24,8 \\
17,8\end{array}$ & $\begin{array}{l}19,0 \\
23,3\end{array}$ & $\begin{array}{l}19,0 \\
28,2\end{array}$ & $\begin{array}{r}8,6 \\
20,2\end{array}$ & $\begin{array}{r}\frac{60,5}{50,2} \\
5,6 \\
0,8\end{array}$ & $\begin{array}{l}30,26 \\
25,09 \\
3,23 \\
0,46\end{array}$ & $\begin{array}{l}0,85 \\
0,76 \\
0,22\end{array}$ & $\begin{array}{l}35,6 \\
33,0 \\
14,7\end{array}$ \\
\hline & \multicolumn{3}{|c|}{$\infty 0^{3} \mathrm{Ca}_{\mathrm{B}}$ total } & \multicolumn{2}{|c|}{$\cos ^{3} \mathrm{Ca}$ actle } & Fer libre & \multicolumn{5}{|c|}{\begin{tabular}{|l|l|l}
$\mathrm{Cs}^{+4}$ Gehangeable & $\mathrm{K}^{+4}$ fehangeable
\end{tabular}} \\
\hline $\begin{array}{l}1 \\
2 \\
3 \\
4\end{array}$ & & \multicolumn{2}{|l|}{$\begin{array}{c}\text { néant } \\
5,5\end{array}$} & 8,5 & $\begin{array}{l}5 \\
9 \\
4\end{array}$ & $\begin{array}{l}0,54 \\
0,21\end{array}$ & \multicolumn{2}{|c|}{103,12} & \multicolumn{3}{|c|}{0,93} \\
\hline
\end{tabular}

Sol $n^{0} 3$ - Forêt de Bramans.

Altitude : $1450 \mathrm{~m}$ - Exp. Est - Roche-mère: Gypse - Pente : $10 \%$.

Relevé floristique $\mathrm{n}^{\circ} 20$

\begin{tabular}{|c|c|c|c|c|c|c|c|c|}
\hline Hort zone & $\begin{array}{l}\text { profond. } \\
\text { đe prél. }\end{array}$ & $\mathrm{p}$ सI & c & $\begin{array}{l}\text { Mat. } \\
\text { org. }\end{array}$ & $\infty \mathrm{Ca}_{\mathrm{s}}$ & $\cos N_{8}$ & $\begin{array}{l}\mathrm{Ca} \text { setif } \\
\%\end{array}$ & $\begin{array}{c}\mathrm{Ca} \\
\text { échang. }\end{array}$ \\
\hline 10 & $-2 \mathrm{~cm}$ & 4.8 & 49,32 & 㔭, 8 & pisant & & & 44,2 \\
\hline$\Lambda_{1}$ & $-8 \mathrm{~cm}$ & 6,2 & 3.99 & 6,7 & traces & & & 21,5 \\
\hline $4 / c$ & $-20 \mathrm{ca}$ & 7,8 & 1,04 & 1,7 & 4,8 & 5 & 21,2 & \\
\hline C & $-40 \mathrm{~cm}$ & 7,9 & 0,92 & 1,5 & 24,6 & 18,1 & 67,5 & \\
\hline
\end{tabular}

Sols de rendsines passant a des sols bruns.

Sol $n^{\circ} 4$ - Forêt de Termignon

Altitude: $1900 \mathrm{~m}$ - Exp. N - Roche-mère: moraine calcaire Relevé floristique $\mathrm{n}^{\circ}$

\begin{tabular}{|c|c|c|c|c|c|c|c|c|c|c|}
\hline Horizons & $\begin{array}{l}\text { Profond. } \\
\text { te prél. }\end{array}$ & $\mathrm{pHI}$ & Argale & Limong & $\begin{array}{l}\text { Sable } \\
\text { fin }\end{array}$ & $\begin{array}{l}\text { Sable } \\
\text { Erosa. }\end{array}$ & $\begin{array}{l}\text { Mnt. } \\
\text { org. }\end{array}$ & Per & $\infty^{3} \mathrm{Ca}$ & $\mathrm{H}^{2} \mathrm{O}$ \\
\hline$A_{1}$ & $-5 \mathrm{an}$ & 5.4 & 3 & 29 & 37,5 & 12,2 & 13.5 & 1,3 & 0 & 4,5 \\
\hline (B) & $-30 \mathrm{~cm}$ & 6,4 & 10 & 23 & 43,5 & 20,7 & 1,2 & 1,7 & 0 & 1,6 \\
\hline (B) $/ \mathrm{C}$ & $-40 \mathrm{~cm}$ & 6,8 & 8 & 23 & 40,7 & 25,1 & 1,4 & 2,0 & 0 & 1,8 \\
\hline
\end{tabular}


Sol $n^{\circ} 5$ - Forêt de Termignon

Altitude: $1950 \mathrm{~m}$ - Exp. $\mathrm{N}$ - Roche-mère: moraine calcaire

Relevé floristique $\mathrm{n}^{\circ}$

\begin{tabular}{|c|c|c|c|c|c|c|c|c|c|c|}
\hline Borizons & $\begin{array}{l}\text { Profond: } \\
\text { de príl. }\end{array}$ & $\mathrm{pH}^{\mathrm{H}}$ & Angile & Lisouns & $\begin{array}{c}\text { Sable } \\
\text { fins }\end{array}$ & $\begin{array}{l}\text { Sable } \\
\text { Eross. }\end{array}$ & $\begin{array}{l}\text { Mat. } \\
\text { org. }\end{array}$ & Fer & $\infty_{3} C_{\Delta}$ & $\mathrm{H}_{2} \mathrm{O}$ \\
\hline$A_{1}$ & $-4 \mathrm{~cm}$ & $\underline{6,6}$ & & & & & 28.7 & & 0 & \\
\hline (B) & $-10 \mathrm{~cm}$ & 7,0 & 19,5 & 34,0 & 28,0 & 8,2 & 6,3 & 2,6 & 0 & 4,0 \\
\hline,$^{(\mathrm{B}) / \mathrm{c}}$ & $-25 \mathrm{~cm}$ & 7,4 & 14,0 & 32,5 & 36,6 & 10,7 & 3,5 & 2,5 & 9,2 & 2,7 \\
\hline
\end{tabular}

III.3.3.2. - Pineraie de Pin à crochets à Carex ferruginea.

$1^{\circ}$ Organisation sociologique et composition floristique.

Si l'on se réfère au tableat d'association, il est facile de se convaincre qu'il s'agit en fait d'un groupement très proche du précédent, puisque les mêmes groupes écologiques s'y observent et, à très peu près, avec la même importance. La physionomie de la strate herbacée ou sous-arbustier y est cependant différente: les touffes du Carex ferruginea, qui ne sont pas contiguës et qui laissent entre elles des vides occupés partiellement par les autres espèces dont Sesleria coerulea, Carex alba, Valeriana montana, Polygala chamaebuxus, etc., ont un tout autre aspect, en effet, que les touffes d'Erica carnea.

\section{Identification - Dénomination.}

Il est tout à fait naturel de rapprocher notre groupement de la sous-association à Carex ferruginea de l'Erico-Mugetum observée dans le Parc national suisse par Braun-Blanouet (Erico-Mugefum caricetosum ferrugineae). Il faut cependant noter que le groupement mauriennais ne comprend absolument pas Erica carnea et qu'il est moins hygrophile que son homologue suisse, où se rencontrent Saxifraga aisoïdes, Parnassia palustris, Bartsia alpina, Pedicularis verticillata [Braun-Blanguet (J.), Pallmann (H.), BACH (R.) (1954)].

\section{$3^{\circ}$ Répartition - Ecologie.}

Ce qui a été dit de la position respective des pineraies à Erica carnea et de celles à Carex ferruginea, nous dispensera d'insister sur la répartition de ces dernières dont la place, dans le paysage, est d'ailleurs assez restreinte.

Leur écologie étant tout à fait semblable à celle de l'Erico-Mugetum à Hyloconies, leur microclimat doit être aussi humide que celui de l'Erico-Pinetum, mais plus froid; les sols où nous les avons rencontrées étaient des rendzines, le plus souvent colluviales. 


\section{III.3.4. - Vue d'ensemble sur les pineraies de Pin à crochets.}

\section{III.3.4.1. - Affinités phytosociologiques.}

La très proche parenté qui existe entre pineraies de Pin à crochets et pineraies de Pin sylvestre, et que les tableaux d'association comme les commentaires qui les accompagnaient ont bien mises en relief, entraine comme corollaire que ce qui a été dit concernant les unes peut être répété, lorsqu'il s'agit des autres.

Les affinités climatiques entre la Haute-Maurienne et certaines vallées des Alpes centrales, dont le Valais, mises en évidence par l'analyse des pineraies de Pin sylvestre se trouvent donc confirmées par celle des pineraies de Pin à crochets.

\section{III.3.4.2. - Evolution - Climax.}

La localisation, à exposition sud comme à exposition nord, des pineraies de Pin à crochets sur les seuls sols calcimorphes met en relief une spécialisation plus poussée que celle des pineraies de Pin sylvestre.

A exposition nord, nous pensons que l'Erico-Mugetum doit peu à peu laisser la place, à mesure que le sol se décarbonate et se décalcifie, dans l'étage montagnard, sur gypse, à l'Abietetum albae; dans l'étage subalpin inférieur à Piceetum subalpinum mésophile,

\section{Les pineraies de Pin à crochets}

Cercles d'exposition

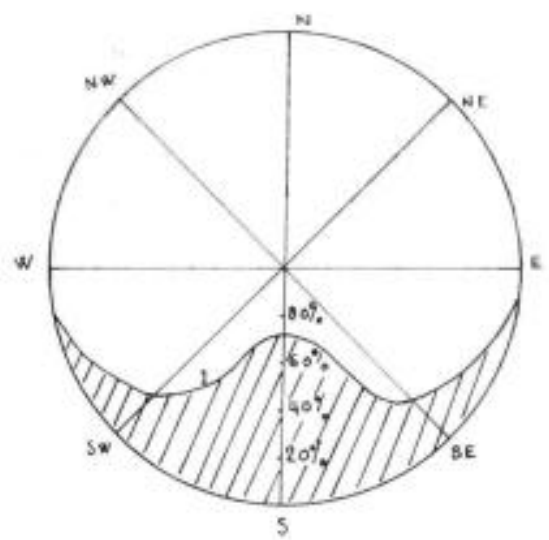

I. Pineraie xérophile.

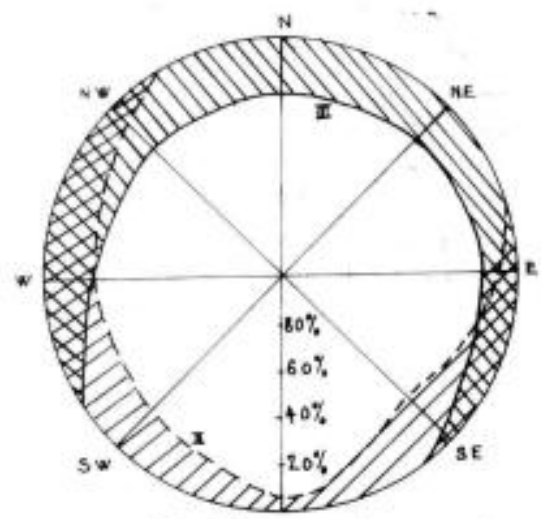

... II. Pineraie mésoxérophile.

Fig. 34.

Légende. - Les remarques faites à propos des cercles d'exposition des pineraies de Pin sylvestre sont évidemment pleinement applicables à ceux des pineraies de $\mathrm{P}$ in à crochets. 
Les pineraies de $P$ in à crochets

Spectres floristico-écologiques

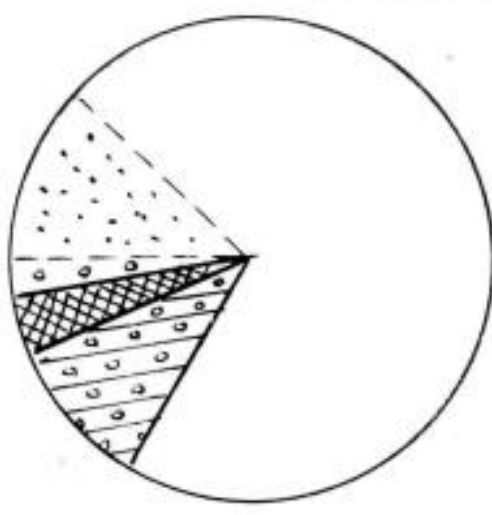

I. Ononido-Pinetum uncinatae (Relevés 6 à 15)

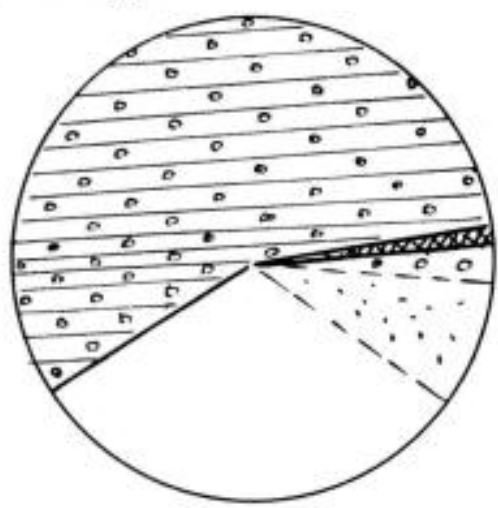

II. Ononido-Pinuetum unicinatae ericetosum

(Relevés 1 à 13)

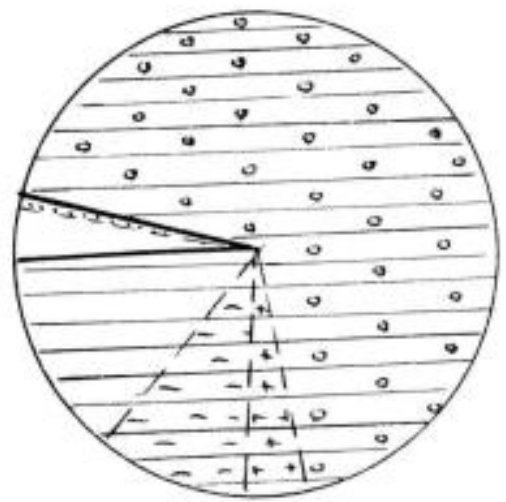

III. Erico-Pinetum uncinatae hylocomietosum.

(Relevés 14 à 25)

Xérophiles indifférentes.

○ O Xérophiles de mull calcique.

... Xérophiles de mull.

Mésophiles (ou M..X.) indifférentes.
वंब Mésophiles d'humus calcique.

Mésophiles de mull/moder.

- Mésophiles de moder ou de mor.

Diverses.

\section{FIG. 35}

Légende. - Les spectres des pineraies de Pin à crochets sont tout à fait comparables à ceux des pineraies de $P$ in sylvestre.

On notera toutefois que dans la pineraie de $P$ in à crochets relativement mésophile (à Erica carnea et Hylocomies) l'abondance-dominance des acidiphiles est sensiblement plus élevée que celle observée dans l'association homologue de Pin sylvestre, l'acidification plus poussée des sols dans l'étage subalpin s'expliquant par les conditions climatiques sévères qui règent dans cet étage. 
dans l'étage subalpin supérieur, à la rhodoraie à Pin Cembro. On a vu que certains relevés montraient nettement cette évolution. Mais il s'agit là, est-il besoin de le dire, d'un processus lent, parce que constamment freiné par l'érosion. Cette dernière joue un ròle primordial sur les roches tendres, gypses en premier lieu et cargneules, de telle sorte que, sur ces roches-mères, les pineraies de Pin à crochets doivent être considérées comme des associations spécialisées permanentes.

On peut penser qu'à exposition tournée vers le sud, un processus de même ordre se manifeste.

Dans l'étage montagnard, sur gypse par conséquent, tout porte à croire que le Pin sylvestre doive peu à peu remplacer le Pin à crochets, au fur et à mesure que le sol s'approfondit, même sans décarbonatation; mais ici encore, la friabilité et la solubilité de la roche-mère sont telles, qu'il y a lieu de parler d'association spécialisée permanente; sur la rive droite du torrent d'Ambin, où on rencontre les seuls peuplements xérophiles de Pin à crochets sur gypse, nous n'avons pu saisir cette évolution, car la pente est particulièrement forte et l'érosion considérable.

Dans l'étage subalpin inférieur, sur calcaire plus dur, c'est vers une pessière sèche que doit conduire l'évolution; pessière qui serait certainement marquée par la nature de la roche-mère et qui serait, par conséquent, moins acidiphile que celle rencontrée sur Houiller. Les indices d'une telle évolution apparaissent au canton de la Travestaz, à Termignon (relevés 6 à 9).

Dans l'étage subalpin supéricur, c'est probablement vers une juniperaie à Pin Cembro - qui sera décrite dans un prochain chapitre - que tendrait la pineraie de Pin à crochets xérophile. Mais cette dernière est très peu représentée, car à la limite de l'étage alpin, l'homme a colonisé les adrets; les rares individus que l'on rencontre se trouvent sur le trajet d'avalanches - puissant outil d'érosion - ou au pied d'une falaise de cargneule, source inépuisable de carbonate - de telle sorte que, là aussi, le terme de permanent s'impose également.

\section{III.3.4.3. - Structures sylvicoles - Productions.}

Ce qui caractérise les pineraies de Pin à crochets dans le domaine des structures, ce sont les faibles diamètres liés à la fois à la pauvreté du substrat et à la rigueur du climat (pineraies subalpines), soit au seul substrat (pineraies montagnardes sur gypse).

On observe donc, soit des courbes à forme exponentielle, mais très peu " étalées " vers les gros diamètres (courbe $\mathrm{n}^{\circ} 1$, donnant la structure d'une pineraie mésoxérophile à Erica carnea et Carex humilis dans l'étage stbalpin à Sollières-Sardières), soit des courbes en cloche, montrant que le nombre d'arbres est considérable avec de faibles diamètres, ce qui traduit la stérilité du sol 
Les pineraies de Pin à crochets Structures sylvicoles

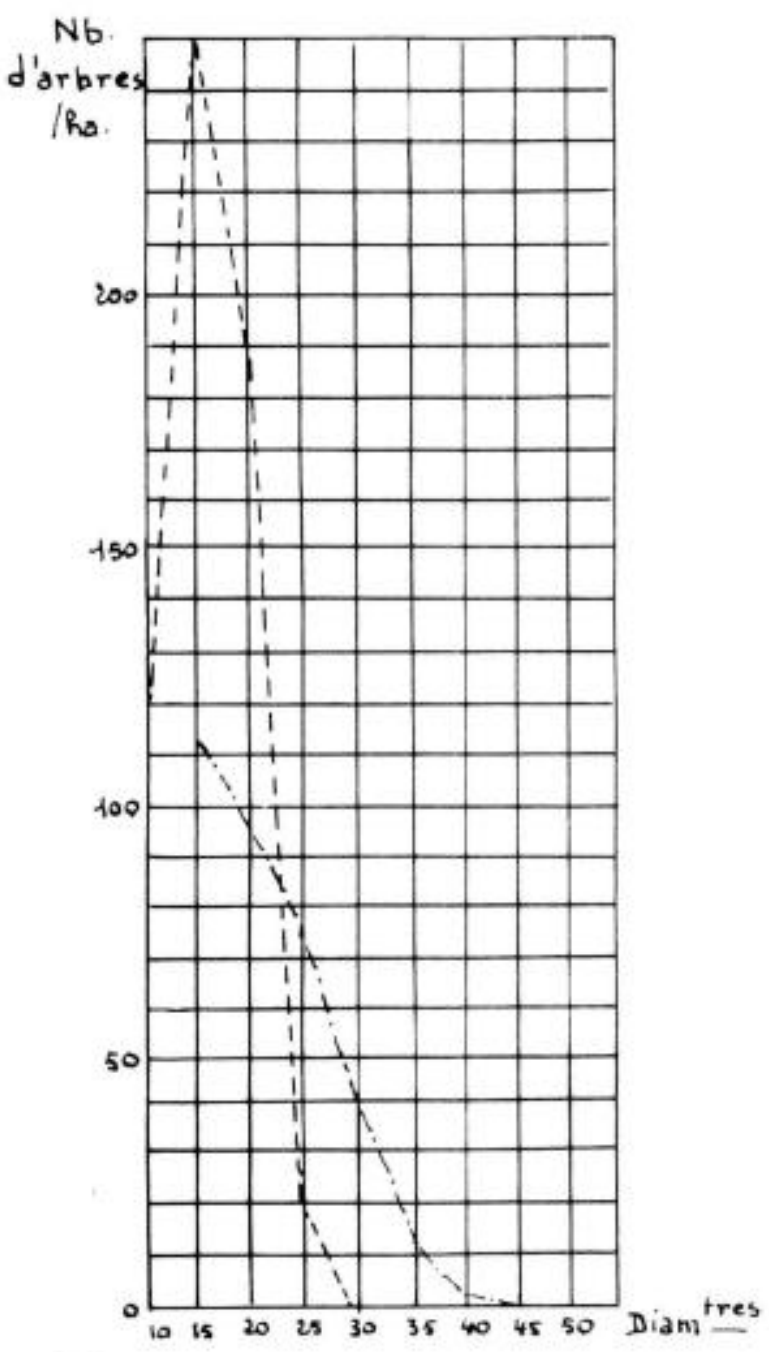

Pineraie xérophile (Bramans).

Pineraic méso-xérophlic subalpine. Sollières, $3^{\circ}$ Séric - 45,8 ha).

Fic, 36.

Légende. - Ce que l'on doit retenir du schéma ci-dessus - surtout si on le rapproche du schéma analogue concernant les pineraies de Pin sylvestre c'est la faiblesse des diamètres.

Cette faiblesse est particulièrement nette dans la pineraie xérophile de Bramans située en plein versant sud et sur gypse. 
(courbe $\mathrm{n}^{\circ} 2$ donnant la structure d'une pineraie xérophile, à Astragalus Monspessulanus et Ononis rotundifolia, dans l'étage montagnard, sur gypse, à Bramans).

L'on ne doit donc pas s'étonner que les productions apparaissent comme désiroires: $0,75 \mathrm{~m}^{3}$ par hectare et par an, pour le premier peuplement (tarif Algan $\mathrm{n}^{\circ} 3-41 \mathrm{~m}^{3}$ à l'hectare), et $0,73 \mathrm{~m}^{3}$ seulement pour le second (tarif Algan $\mathrm{n}^{0} 2-47 \mathrm{~m}^{3}$ à l'hectare). 


\section{III.4. - LES SAPINIERES}

\section{III.4.1. - Analyse phytosociologique et écologique.}

$\mathrm{Si}$, dans le domaine montagnard subatlantique, Hêtre et Sapin sont le plus souvent compagnons fidèles, dans le domaine intraalpin, le Sapin continue à prospérer et à former des massifs étendus, alors que le Hêtre est totalement - et naturellement - absent. Là, on observe une sapinière à Epicéa, mais sans Hêtre.

Bien qu'elle couvre de grandes surfaces, puisqu'elle s'étend, sur versand nord, de Lanslevillard à Montricher, à l'extrémité aval de notre dition (1), la sapinière, dans son ensemble, présente une physionomie très constante et des variations de composition floristique assez faibles, ce qui n'est pas le cas, comme nous l'avons vu, des pineraies de Pin sylvestre, de Pin à crochets ou de Pin Cembro. Aussi nous a-t-il paru rationnel de ne distinguer qu'une seule unité, comportant un noyau important d'espèces communes, et de mettre simplement en évidence diverses sous-associations, dont l'importance écologique n'est d'ailleurs pas négligeable.

1. Organisation sociologique et composition floristique - Sousassociations et faciès.

Le tableau général d'association fait apparaitre un noyau d'espèces communes, et deux groupes principaux de différentielles, dont il sera question ci-après.

Dans le noyau de base, le Sapin et l'Epicéa, dans la strate arborescente, sont à peu près les seules espèces à être représentées, le Sapin possédant presque toujours une abondance-dominance supérieure à celle de l'Epicéa (2).

Dans les autres strates, doivent être d'abord distinguées des espèces comme Melampyrum silvaticum, Lusula nivea, Veronica urticifolia, Prenanthes purpurea, Lusula Sieberi et Hieracium murorum, qui sont les plus abondantes et les plus constantes. Il s'agit d'espèces mésophiles liées à des sols à mull-moder, plus ou moins lessivés, mais présentant à l'égard du chimisme du sol une certaine

(1) Mises à part les zones à Pin à crochets ou à Pin sylvestre de Solliêres et de Bramans sur gypse et cargneule.

(2) Le Mélèze dont la présence est due à l'action humaine s'observe aussi, parfois, dans cet étage. 


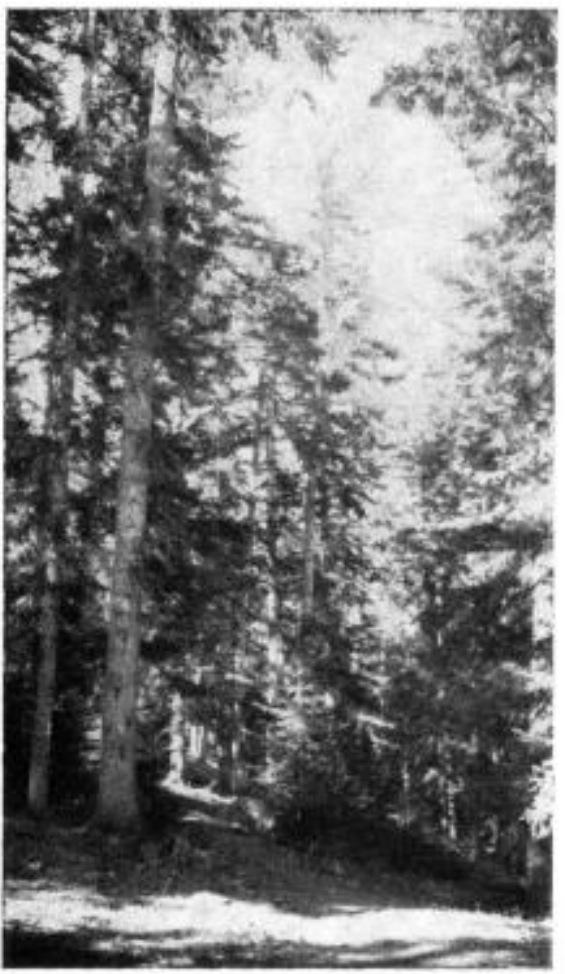

1

Sapinière d'Aussois.

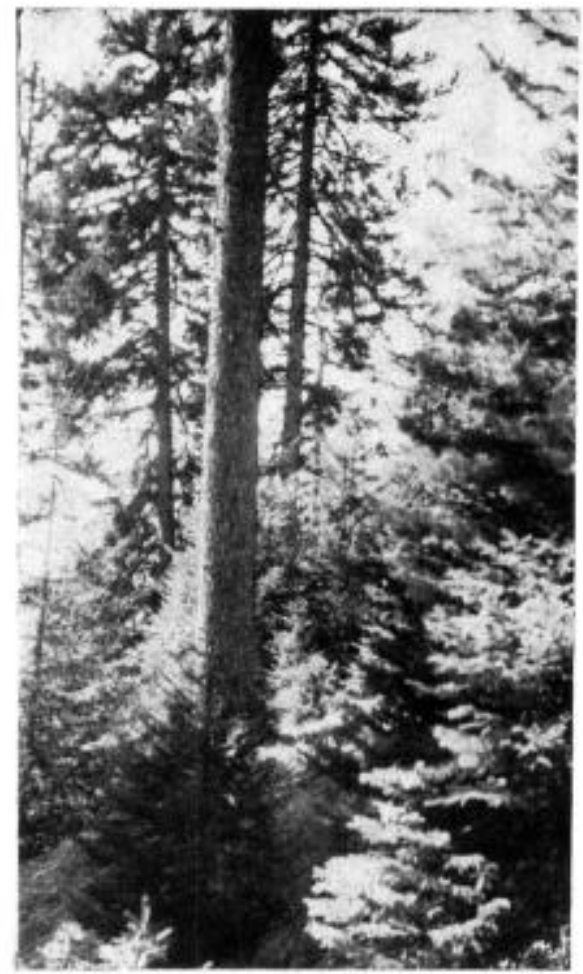

2

Trouées de régénération

en forêt de Termignon. 
plasticité. Elles atteignent l'optimum de leur développement dans l'étage montagnard, ce qui ne veut pas dire qu'on ne les observe pas dans l'étage subalpin: les relevés faits dans la pessière subalpine et dans la rhodoraie à Pin Cembro le montrent; Veronica urticifolia, cependant, est, à ce point de vue, la moins plastique et peut être considérée comme une bonne caractéristique de ce type de sapinière.

Les autres espèces du noyau: Clematis alpina, Lonicera nigra, Rosa alpina, nous paraissent présenter une plus grande plasticité, bien que leur écologie rappelle celle des précédentes.

Sont également assez constantes, Vaccinium Myrtillus, et surtout une endémique des Alpes occidentales: Festuca flavescens; ces deux dernières espèces possèdent, dans le faciès à Saxifraga cuneifolia, dont il sera question ci-après, une abondante différentielle qu'il importe, dès à présent, de souligner.

On notera, avec intérêt, l'absence ou l'extrême rareté d'espèces, pourtant toujours présentes, dans les sapinières à hêtre (AbietiFagetum) comme Actaea spicata, Lamium Galeobdolon, Melica uniflora, Asperula odorata, Sanicula europaea, Dentaria pinnata, Carex silvatica, etc. sur lesquelles nous avons déjà appelé l'attention.

Au groupe précédemment défini et qui forme le noyau de base, viennent s'agglutiner, selon les conditions écologiques, d'autres groupes qui permettent de distinguer des faciès.

Stur roche-mère carbonatée - et l'on verra que, dans les exemples pris, le sol est, lui aussi, quelque peu carbonaté - on rencontre des espèces liées précisément à des sols calcimorphes: Sesleria coerulea, Carex alba, Calamagrostis varia, Aster Bellidiastrum, etc. Ces espèces se rencontrent seules, le plus souvent, mais il arrive que parfois, s'ajoutent au cortège, soit Erica carnea, soit Carex ferruginea (relevés 28-29). On notera qu'il s'agit ici de relevés pris au contact de l'Erico-Pinetum, ou de la pineraie de Pins à crochets à Carex ferruginea, et qui se présentent comme des termes de transition entre ces associations et la sapinière. Ce qu'il y a d'assez remarquable, c'est que, dans de tels individus, le Sapin - de forme médiocre d'ailleurs - est nettement dominant.

Sur roche-mère presque totalement silicatée (schistes et surtout grès du Houiller), les espèces de sols calcimorphes disparaissent très nettement, tandis que font leur apparition des espèces liées à des sols de moder typiques: Saxifraga cuneifolia, Deschampsia flexuosa; Festuca flavescens, qui était très peu abondant dans le faciès précédent, prend une grande importance, déjà soulignée, d'ailleurs; de même les espèces liées de façon optimale à des sols de mor: Vaccinium Myrtillus et Vaccinium Vitis idaea.

Sur roche-mère mixte (schistes lustrés) c'est-à-dire à la fois silicatée et carbonatée, ni le groupe des espèces de moder calcique, ni celui des espèces de moder acide ne sont vraiment représentés. 
Seules figurent les espèces du noyau principal, dont nous avons déjà dit qu'elles nous paraissent liées à des sols lessivés, à mull-moder. tout en présentant une plasticité certaine à l'égard du type d'humus et de la richesse en bases.

Deux autres faciès sont à signaler, qui n'ont - quant à la surface occupée - presque aucune importance, mais qui méritent cependant d'être brièvement analysés:

- une faciès méso-hygrophile, qui correspond à de petites cuvettes où la neige séjourne plus longtemps qu'ailleurs, et un faciès d̀ Festuca silvatica (cette dernière, toujours rare et localisée), au contact des hêtraies de la bordure aval de notre dition.

La structure est, pour lia strate herbacée, du type " diffus »: les espèces sociales, ne forment pas đe nappe étendue, ni même de taches; il s'agit de minuscules bouquets: Melampyrum silvaticum est souvent très dominant, mais comme il s'agit d'une espèce annuelle assez grêle, le sol n'est pas complètement couvert. Emergent de ce tapis lâche, Veronica uricicifolia. Hieracium murorum, et les autres espèces du cortège.

Dans le faciès à Saxifraga ctmeifolia, - que l'on observe dans les gorges houillères où la pente est exceptionnellement forte se rencontrent de nombreux éhoulis, et c'est le plus souvent sur les rochers que se localise, parmi les mousses, dont Isothecium viviparum, le Saxifrage à feuilles en coin.

Enfin, dominant les strates herbacées et muscinales le Sapin et l'Epicéa, dont les structures sylvicoles seront analysées à leur place.

\section{Identification - Dénomination.}

On sait que, dans la classification de Braun-Blanouet, la sapinière proprement dite n'existe pas et que dans les relevés correspondant à des forêts résineuses de la classe des Vaccinio-Piceetalia, Abies alba fait toujours figure d'espèce compagne.

La chose s'explique: Abies alba, en effet, s'observe - et souvent avec une grande abondance-dominance - à la fois dans certains types de forêts mixtes résineuses et feuillues relevant de lalliance du Fagion (Abieti-Fageta divers) et dans d'autres types relevant de l'alliance du Vaccinio-Piceion. Cette appartenance à deux unités quii se trouvent elles-mêmes être incluses dans des unités plus vastes n'ayant apparemment pas de rapports (Ouerco-Fagetea, d'une part et Vaccinio-Piceefea, de l'autre) a abouti à ne donner au sapin aucune valeur sociologique, ni même écologique et à appeler Piceeta des sapinières naturelles, à Epicéa sans doute, mais sans Hêtre et très riches en Sapin. La chose en soi ne serait peut-être pas très grave, si elle n'aboutissait, en fait, à sous-estimer le rôle naturel du Sapin dans certaines de ces associations ou à dénommer Piceeta, des associations où, de toute évidence, l'Epicéa n'a jamais existé 
naturellement, comme dans les Pyrénées (3). A l'inverse, il faut le reconnaitre, les forestiers ont eu tendance à appeler sapinières des peuplements riches en Sapin sans doute, mais du fait de l'homme et qui sont, au vrai, des hêtraies à Sapin (Abieti-Fageta des phytosociologues).

Ces inconvénients - qui tiennent essentiellement au mode de classification adopté - n'ont pas échappé à certains chercheurs: c'est ainsi que Kuoch (R.) (1954) a appelé Abietetum albae des sapinières qui ne sont pas des Abieti-Fageta et baptisées par d'at1tres: "Piceeta \#. Cet Abietetum albae et ses diverses formes doivent donc s'identifier avec le Piceetum normale de Beger ( $\mathrm{H}$.) (1922). le Piceetum montanum galietosum et le Piceetum transalpinum de Braun-Blanquet (J.), avec le Piceetum abieto-festucetosum, enfin, de Braun-Blanouet (J.) également. Kuoch a distingué une sous-association à Melampyrum silvaticum, avec deux faciès: l'un à Carex alba, l'autre à Saxifraga cuncifolia; une sous-association à Festuca silvatica et Elymus europoeus (très proche de l'AbietiFagetum); une sous-association enfin à Isothecium viviparum. A côté de cet Abietetum albae, ont été décrits un Myrtillo-Abietetum (sur le plateau suisse), un Equiseto-Abietetum, enfin un AdenostyloAbictetum et un Rhododendro-Abietetum.

Si certains de nos relevés (faciès méso-hygrophile - relevés $n^{\circ} 13$ à 18) peuvent être rattachés à l'Adenostylo-Abietetum (sous-association prenanthetosum), l'ensemble présente les plus grandes affinités avec l'Abietetum albae melampyretosum proprement dit, avec ses deux faciès à Carex alba et Saxifraga cuneifolia. A noter que notre faciès intermédiaire n'a pas été expressément mis en évidence par KuOcH, bien que certains relevés classés dans son faciès à Carex alba soient particulièrement pauvres en différentielles de ce faciès et puissent ainsi être considérés comme se rattachant à notre sapinière sur schistes lustrés.

De petites nuances - et qui méritent d'ètre signalées - font que 1 'Abietetum albae de la Haute-Maurienne, diffère quelque peu de celui défini par Kuoch: Coronilla Emerus, Galium rotundifolium. Asperula odorata, Festuca silvatica, Elymus europaeus sont assez souvent présentes dans l'association stiisse, alors que dans la sapinière de Haute-Maurienne, elles sont absentes; de même Carex silvatica, Euphorbia amygdaloïdes, Ranunculus lanuginosus qui jotient dans 1'Abietetum albae melampyretosum de KuOCH, il est vrai, un rôle des plus secondaires. Mais ces différences sont les seules et elles s'expliquent surtout par le fait que les relevés de $\mathrm{Kuoch}$ ont été pris sur une vaste surface géographique, et souvent en contact étroit avec les sous-associations plus mésophiles

(3) Braun-Blanguet (J.), Roussine (Mme), Nìgre (R.) (1951 - 267). 
qui font le passage avec 1'Abieli-Fagetum (sous-association festucetosum et elymetosum) alors que les nottres ont été concentrés sur la seule Haute-Maurienne.

Une dernière question se pose: le faciès à Festuca silvatica que nous avons distingué doit-il être simplement intégré à la sous-association Abietetum albae melampyretostsm ou peut-il ètre rapproché de la sous-association Abietctum albac festucetosum? Il s'agit ici, sans doute, d'une nuance secondaire, d'autant plus que les deux sous-associations en cause sont elles-mèmes très proches l'une de lautre, la seconde étant cependant plus hygrophile avec Aconitum vulparia, Dentaria digitata, Ramucults aconitifolius, Gentiana asclepiadea, etc, Etant donné que, dans notre faciès, Festuca silvafica est loin d'ètre abondante, an contraire de ce qui se passe dans la sous-association suisse et que les especes citées plus haut n'y figurent pas, il parait plus rationnel de ne point distinguer de sousunité. Mais il n'était tout de mẻme pas inutile de signaler ce petit faciès, d'autant plus qu'il se trouve au contact de la zone du Hêtre.

\section{$3^{\circ}$ Répartition - Ecologie.}

a) Répartition - Dans son étude sur l'Abietetum albae, KuocH (H.) (1954), indique que cette sapinière colonise toutes les expositions et s'étend, entre 1200 et $1700 \mathrm{~m}$ d'altitude, aux expositions ensoleillées et entre 1000 et $1500 \mathrm{~m}$ - aux expositions ombra-
gées.

En Maurienne, la localisation de la sapinière quant à l'exposition est plus étroite; elle fuit l'exposition sud et se plait à exposition nord. Quant aux expositions intermédiaires, il est assez curieux de constater que, dans la zone Flysch-Subbrianconnaise, c'est à exposition ouest qu'elle s'observe, le versant d'exposition est étant occupé par une hètraie thermophile plus ou moins riche en Epicéa et en Pin sylvestre. A l'amont de notre dition, par contre, au-delà des gorges houillères, c'est l'inverse qui se produit: la sapinière domine à exposition est, les pineraies à exposition ouest. Une telle symétrie dont l'axe se situterait at milieu des gorges houillères et perpendiculairement à la ligne de talweg, parait s'expliquer par la direction des vents pluvieux. A l'aval, les vents venus de l'Atlantique et qui ont franchi le rideau Sept-I aux - Lauzières viennent buter contre la zone subbriançonnaise allongée du nord au sud; à l'amont, c'est le régime de la Lombarde qui règne et qui vient
de l'est.

Comment expliquer, maintenant, cette absence de la sapinière haut-mauriennaise aux " adrets w? Nous verrions volontiers l'explication dans le régime des pluies: en Haute-Maurienne, les influences piémontaises ou même méditerranéennes déjà soulignées, font que l'été présente un certain "creux " pluviométrique; dans les Alpes intermédiaires suisses (en moyen Valais et au nord des 
Grisons), les influences continentales font de la saison chaude une saison relativement humide. Dans le premier cas, la sapinière fuyant la sécheresse relative de l'été se localiserait assez strictement aux ubacs - ce qu'elle fait beaucoup plus encore, dans le Briançonnais où le régime est plus franchement méditerranéen — alors que plus à l'est, la relative humidité de la saison chaude lui permettrait une plus large extension (4).

On pourrait aussi penser qu'à exposition sud, les défrichements de l'homme ont éliminé la sapinière ou ont provoqué son remplacement par des pineraies qui ne seraient donc pas climaciques. Une telle explication n'est pas satisfaisante. Que l'action humaine ait accentué la xéricité des versants sud, la chose est probable; mais comment expliquer que, sur les inflexions secondaires - et souvent de très faible étendue - du versant général stıd, apparaisse la sapinière dès que l'exposition regarde l'est et le nord-est (5), et que, à l'inverse, elle disparaisse sur un versant général nord dès qu'une inflexion secondaire regarde vers l'ouest ou le sud-ouest? Il est extrêmement peu probable que la pression de l'homme ait été plus forte sur de telles surfaces qui sont souvent minuscules.

En altitude, la sapinière s'étend de $950 \mathrm{~m}$ environ à $1750 \mathrm{~m}$; son altitude de base est à peu près celle du fond du talweg (elle s'élève donc avec ce dernier), compte tenu du fait qu'il existe toujours une frange semi-forestière, semi-agricole, et d'épaisseur variable, vers le fond de la vallée. Ses limites supérieures ne sont pas faciles à tracer, - autrement que conventionnellement - car le passage avec la pessière subalpine se fait très progressivement et les différences floristiques entre les deux associations ne sont pas marquées. Elle parait donc s'arrêter un peu à l'amont de Lanslebourg, sans que son étage soit à proprement "laminé n; certains indices (abondance de Veronica urticifolia, Lusula nivea et de jeunes semis de Sapin, etc.) montrent que plus à l'amont, en forêt de Lanslevillard où l'Epicéa domine nettement, la sapinière doit, vers le bas de la forêt s'entend, c'est-à-dire en dessous de $1700 \mathrm{~m}$ environ, retrouver une place perdue du fait de l'homme.

b) Climat - Cette localisation sur versant nord, donne au climat de la sapinière ses caractéristiques essentielles. Lors de l'étude des pineraies - et plus particulièrement de l'Ononido-Pinetum - nous avons déjà eu l'occasion de mettre en parallèle certains paramètres de ce microclimat et ceux du microclimat des pineraies.

(4) Cette explication rejoint celle donnée par Carles (J.) (1953) à propos de la répartition du Hêtre et du Sapin au nord et au sud du Mézenc, sur la bordure orientale du Massif Central. \& Le Sapin s'accommode fort mal de cette sécheresse estivale et tend à disparaitre dans la mesure où elle se fait plus sentir, à moins qu'un ubac particulièrement frais ne lui permette de franchir ce mois sans dommage,

(5) Comme sur les flancs du Fort du Sappey, près de Modane, à l'amont des gorges houillères. 
Il s'agit d'un microclimat relativement mésophile, aux oscillations thermiques un peu amorties par rapport à celles de la pineraie et où - caractéristique essentielle - le microclimat du sol s'avère être sensiblement plus froid que celui de lair (6).

On notera aussi que le pouvoir évaporant de l'atmosphère reste au-dessous de celui de la pineraie, ce que corrobore l'étude compa-

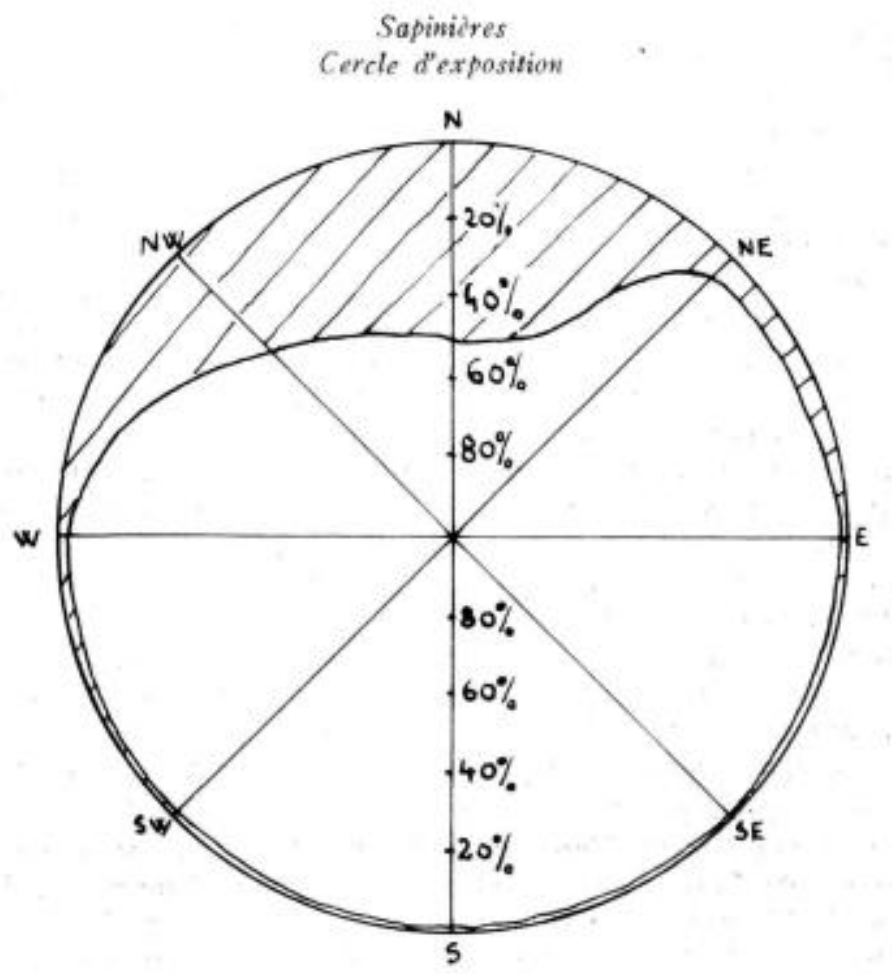

F1G, 37.

Légende. - Quel que soit le type de sapinière, cette dernière se localise très nettement sur les versants exposés au nord.

Les rares sapinières sur versants exposés au sud se trouvent, soit situées sur terrain plat ou presque plat, en prolongement d'une sapinière s'étendant sur un versant secondaire exposé au nord (sapinière d'Aussois), soit situées sur un $>$ faux adret 3 , au fond d'un talweg encaissé (sapinière de la Travestaz, à Termignon).

rative - fort brève - de l'évaporation directe, mesurée à l'évaporomètre Piche.

S'il fallait donner un aperçu du climat général de cette sapinière, les relevés déjà traduits en courbes des postes météorologiques de

(6) Tout au moins durant la saison de végétation et aux périodes où nous avons fait nos mesures. 
Lanslebourg $(1400 \mathrm{~m})$ (période 1891-1930) et de ceux d'Orelle $(830 \mathrm{~m})$ et de Termignon $(1300 \mathrm{~m})$ (période 1949-1958), tous postes situés dans la zone d'altitude de la sapinière, seraient à retenir.

c) Sols - Chimisme - Etat hydrique.

Comme pour les autres associations, les sols sont peu profonds, peu évolués. Ce qui les caractérise, par rapport à ceux des pineraies de Pin sylvestre neutrophiles ou des pineraies de Pin à crochets, c'est qu'il ne s'agit plus - sauf dans le faciès à Carex alba - de sols calcimorphes, c'est-à-dire carbonatés ou à complexe absorbant saturé, mais de sols bruns plus ou moins lessivés, soit à mull, soit à mull-moder; ils diffèrent donc également des sols de la rhodoraie à Pin Cembro ou de la pessière subalpine, qui sont des sols podzoliques, plus ou moins évolués, à humus brut (mor).

Sur roches-mères calcaires et dans le faciès à Carex alba et Calamagrostis varia (relevés $\mathrm{n}^{\circ} 28$ à 36 ), les sols - comme on pouvait s'y attendre - restent encore du type calcimorphe, mais montrent le plus souvent une évolution nette vers un sol brun (sols $\mathrm{n}^{\circ} 1$ et $\mathrm{n}^{\circ} 2$ ).

Sol ñ 1 - Forêt d'Aussois.

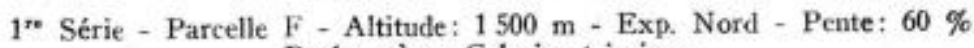
Roche-mère: Calcaire triasique.

\begin{tabular}{|c|c|c|c|c|c|c|c|}
\hline Hori zons & $\begin{array}{l}\text { Profond. } \\
\text { de prélà. }\end{array}$ & pii & engile & $\begin{array}{l}\text { Limone } \\
\text { fins }\end{array}$ & $\begin{array}{l}\text { Linona } \\
\text { grosaiers }\end{array}$ & $\begin{array}{l}\text { Sables } \\
\text { finn }\end{array}$ & $\begin{array}{l}\text { Sablen } \\
\text { growiers }\end{array}$ \\
\hline (B) $/ c^{k}$ & $\begin{array}{l}-2 \mathrm{en} \\
-20 \mathrm{ar}\end{array}$ & $\begin{array}{l}7,2 \\
7,7\end{array}$ & 17,2 & 30,2 & 12,8 & 12,7 & 20,5 \\
\hline Hor12ons & $\begin{array}{l}\text { Ratière } \\
\text { orean. }\end{array}$ & c & F & $c / \hbar$ & $\begin{array}{l}\mathrm{Co}^{3} \mathrm{Ca}_{\mathrm{a}} \\
\text { total }\end{array}$ & $\begin{array}{c}\text { Fer } \\
21 \text { bre }\end{array}$ & \\
\hline$A_{1}$ & 19,5 & $9,7 B$ & $c, 48$ & 20,4 & 1,9 & 1,28 & \\
\hline (B) $/ \mathrm{C}$ & 4,0 & 2,31 & & & 9,1 & 1,97 & \\
\hline
\end{tabular}

Sol n'2 - Forèt d'Aussois.

$1^{\text {rt }}$ série - Parcelle H - Altitude: $1600 \mathrm{~m}$ - Exp. SE - Pente : $10 \%$ Roche-mère: Calcaire triasique.

\begin{tabular}{|c|c|c|c|c|c|c|c|c|}
\hline Hori 20n8 & $\begin{array}{l}\text { Profond. } \\
\text { de prélev }\end{array}$ & pa & Argilo & Limons & $\begin{array}{c}\text { Snbles } \\
\text { In nas }\end{array}$ & $\begin{array}{c}\text { Sables } \\
\text { erooniers }\end{array}$ & $\begin{array}{l}\text { Mat. } \\
\text { organ. }\end{array}$ & $\mathrm{co}^{3} \mathrm{ca}$ \\
\hline$A_{1}$ & $-5 \mathrm{cs}$ & 7,4 & 17,5 & 20,5 & 30,5 & 5,3 & 23,3 & traces \\
\hline (B) & -15 ox & 6,6 & 23,0 & 22,5 & 37,9 & 11,3 & 4,3 & 0,9 \\
\hline (B) $/ \mathrm{c}$ & $-35 \mathrm{~cm}$ & 7,6 & 16,0 & 22,5 & 33,3 & 25,1 & 2,4 & 23,0 \\
\hline
\end{tabular}


Ils sont très semblables à ceux rencontrés dans certains individus de l'Erico-Pinetum ou de l'Erico-Mugetum et où s'observaient à cóté d'Erica carnea, de nombreuses espèces stubacidiphiles comme Melampyrum silvaticum, Lusula silvatica subsp. Sieberi, Prenanthes purpurea, Veronica urticifolia, etc.

Les différences floristiques entre pineraic à Erica carnea sous sa forme la plus acidiphile et sapinière vraie à Carex alba - tout particulièrement l'abondance d'Erica carnea dans le premier cas et son absence dans l'autre - ne peuvent donc s'expliquer par le chimisme du sol, mais probablement par l'action humaine qui, en ouvrant de vastes trouées dans tne sapinière fermée, sur sol calcimorphe, a favorisé le Pin sylvestre et son cortège d'espèces héliophiles, dont Erica carnea.

Sur roches-mères mixtes (schistes lustrés), et dans le faciès " moyen " précédemment défini, les sols ne sont plus calcimorphes, mais du type brun, plus on moins lessivés, à mull ou même à mullmoder.

L'exemple ci-après, pris en forêt de Termignon, et qui correspond à un sol lessivé peu évolué, montre que sous l'influence d'un humus à $\mathrm{C} / \mathrm{N}$ élevé, le lessivage commence à intervenir (taux de fer croissant avec la profondeur) alors que l'altération superficielle est encore en cours (taux d'argile décroissant avec la profondeur, libération importante et fixation de $\mathrm{Ca}$ dans les horizons humiques).

\section{Forêt de Termignon.}

Parcelle 8 - Altitude: $1550 \mathrm{~m}$ - Exp. Nord - Pente : $30 \%$

Roche-mère: Schistes lustré5.

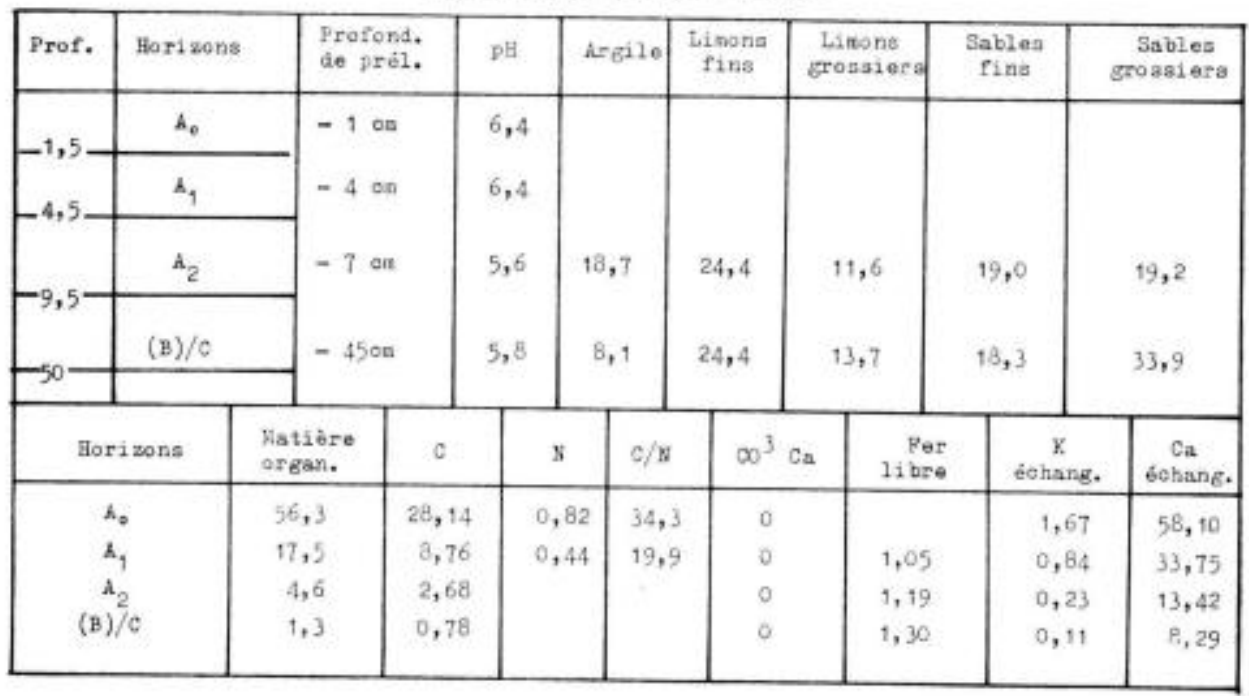


Sur roches-mères très silicatées - schistes et surtout grès du Houiller, - mais dont nous avons vu lors de l'étude géologique qu'elles n'étaient pas dépourvues de calcium, les sols sont très voisins de ceux précédemment étudiés.

L'exemple ci-après, pris en forêt des Fourneaux, sur grès du Houiller, le montre: la couche $\mathrm{A}_{0}$ est nette, mais peu épaisse. Son $\mathrm{C} / \mathrm{N}$ est élevé, mais pas celui de $\mathrm{A}_{1}$. Le taux de fer augmente légèrement en profondeur marquant un début d'entrainement, tandis que la libération d'une quantité de $\mathrm{Ca}$ beaucoup plus importante en surface qu'en profondeur indique une altération encore incomplète des horizons profonds comme une concentration de cet élément dans l'horizon humique.

On a affaire, comme dans le cas précédent, à un sol brun lessivé, peu évolué, mais développé sur éboulis à assez gros blocs.

\section{Forêt de Fournécux.}

Parcelle E - Altitude: $1400 \mathrm{~m}$ - Exp.: Uord - Pente: $20 \%$ Roche-mère: Grès du Houiller.

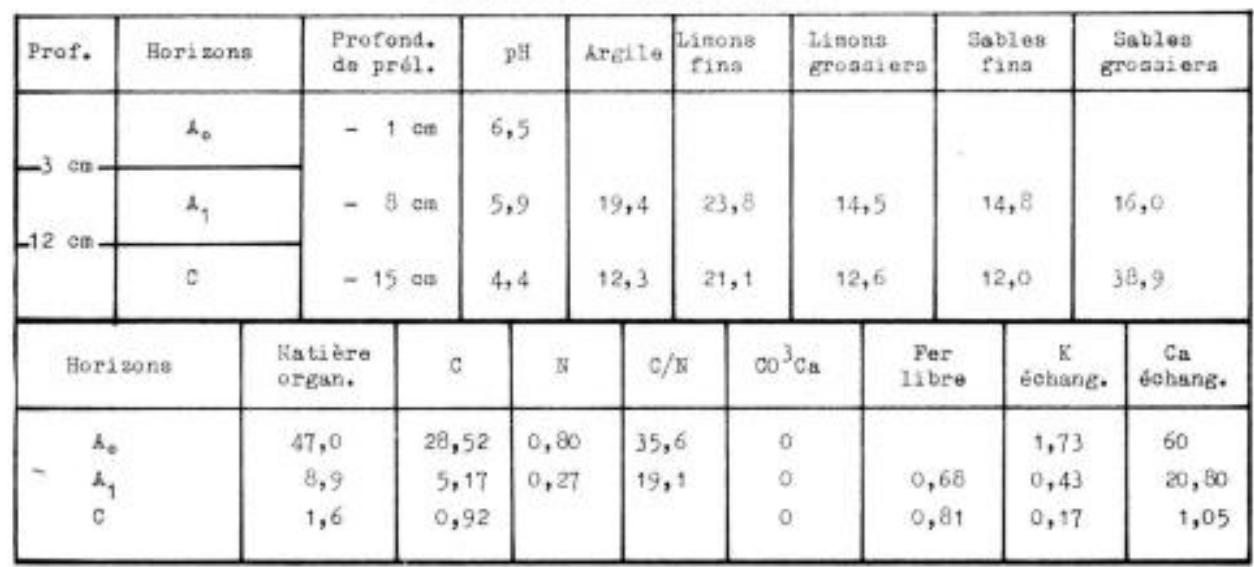

Ce qui explique dans ce faciès la présence de Saxifraga cuneifolia et l'abondance de Festuca flavescens, localisées, le plus souvent, sur les blocs ou près des blocs, sur un $\mathrm{A}_{\sigma}$ formé par les mousses, où Isothecium viviparum joue le premier róle, ce sont les caractéristiques de l'horizon $\mathrm{C}$, au niveau de la zone d'altération de la roche-mère: le $\mathrm{pH}$ est sensiblement plus bas que dans le cas précédent comme la teneur en calcium échangeable.

En ce qui concerne l'état hydrique, il faut rappeler les courbes déjà analysées lors de l'étude de l'Erico-Pinetum et de l'OnonidoPinetum. Le $\mathrm{pF}$ de l'Abietetum albae - à $-5 \mathrm{~cm}$ comme à $25 \mathrm{~cm}$ - toujours un peu inférieur à celui de l'Erico-Pinetum et beaucoup plus encore à celui de l'Ononido-Pinetum, montre que, des trois associations, l'Abietetum albae est la plus mésophile. 


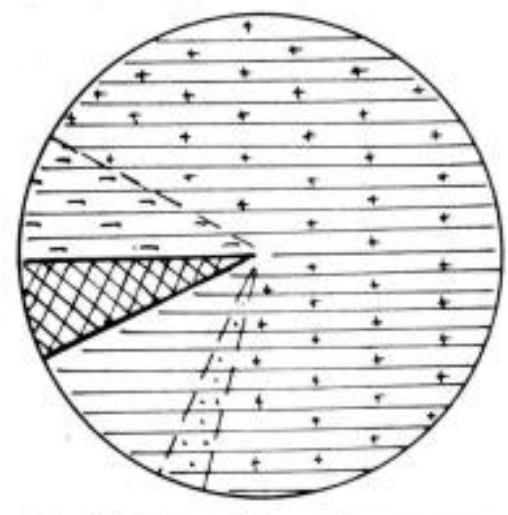

I. Abietetum albae melampyretosum typicum (Relevés 1 à 12).

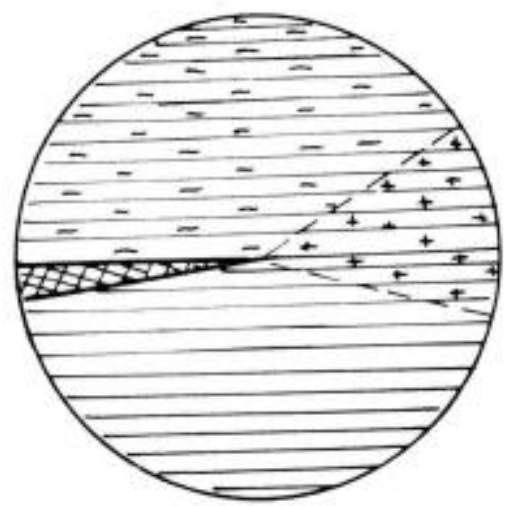

II. Abietetum albae melampyretosum à S, cuneifolia (Relevés 19 à 27).

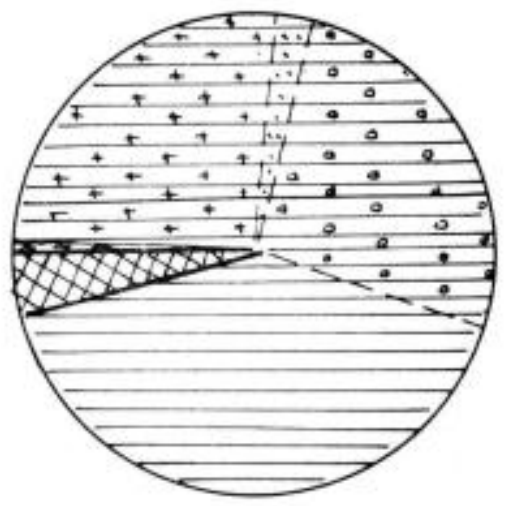

JII. Abietetum albae melampyretosum à C, alba.

(Relevés 28 à 35 ).

=- Mésophiles de moder et de mor.

$+{ }_{+}+$Mésophiles de mull-moder.

$\because$ Mésophiles de mull.
[ㅇ. Mésophiles de mull calcique.

$\square$ Mésophiles indifférentes (mousses). monerses.

Fic. 38.

\section{LES SAPINIERES}

Spectres floristico-écologiques.

Légende. - Sur schistes lustrés, les espèces liées à un mull-moder dominent alors que sur grès du Houiller, les espèces de moder franc prennent la première place; sur sols bruns calcaires, les espèces liées à un humus calcique jouent un rôle non négligeable.

A noter, dans les deux derniers types ,l'importance physionomique des mousses. 


\section{III.4.2. - Vue d'ensemble sur les sapinières.}

\subsubsection{1. - Affinités phytosociologiques.}

La sapinière vraie à Mélampyre silvatique (Abietetum albae melampyretosum) de KuOcH, qui se rencontre, en Suisse, dans les parties moyennes du Valais et de l'Engadine (Zwischenalpen) est donc une association typiquement intra-alpine, encore qu'elle soit remplacée dans les zones les plus continentales (Kontinentale Hochalpen) par une pessière de montagne (Piceetum montanum melicetosum), d'où le Sapin est absent.

Sa présence en Haute-Maurienne vient donc confirmer à nouveau les affinités climatiques qui existent entre celle vallée et d'autres, situées plus au coeur de l'Europe Centrale.

Mais, alors que dans les Alpes occidentales françaises, la Maurienne est la seule à posséder, sur une vaste étendue, des associations comme 1'Erico-Pinetum, ou l'Erico-Mugetum (7), on rencontre dans la plupart des autres vallées internes, l'Abietetum albae, en Haute-Tarentaise, en Briançonnais, en Ubaye et dans les Alpes maritimes.

\section{III.4.2.2. - Evolution - Climax.}

On a vu, à propos des pineraies de Pin sylvestre et de celles de Pin à crochets sur gypse, que la sapinière vraie correspondait certainement, dans l'étage montagnard et sur versant frais, à une association climacique, laissant la place sur les versants les plus secs et les plus chauds, à la pineraie xérophile de Pin sylvestre.

Il s'agit, si l'on veut, d'un climax correspondant à des conditions pédologiques moyennes, alors qu'à la même exposition et dans le même étage, les associations spécialisées édaphiquement de Pin sylvestre ou de Pin à crochets peuvent être considérées - étant donné leur permanence sur laquelle nous avons insisté - comme des « climax spéciaux $n$; en montagne, en effet, et en dehors de toute intervention humaine, la végétation forestière, à une exposition donnée dans un étage, ne pouvait pas, à cause des phénomènes d'érosion, ne pas présenter de nombreuses " nuances pédologiques ", analogues, en définitive, à celles que nous lui connaissons, l'influence humaine se faisant sentir.

\section{III.4.2.3. - Structures forestières - Productions.}

D'une façon générale, les peuplements de l'Abietetum albae présentent une structure régulière, surtout dans les grandes forêts de Lanslebourg et de Termignon (forêt d'Arc). Cette structure se traduit évidemment par une courbe d'allure gaussienne (ex.: parcelle 14 , de la forêt de Lanslebourg).

(2) Si l'on considère ces associations au sens strict, c'est-à-dire caractérisées par la présence d'Erica carnea, 


\section{Nombre \\ al'hect tare}

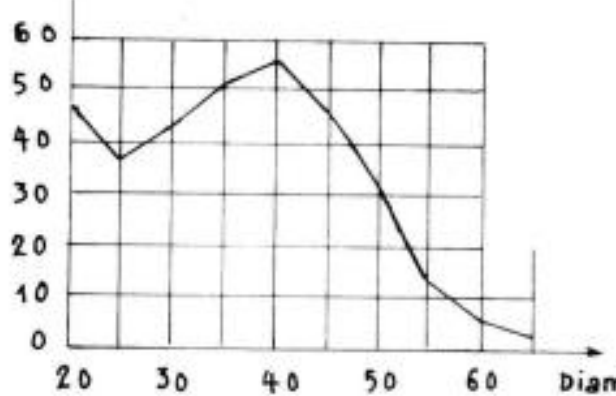

Termignon - Parcelle 25

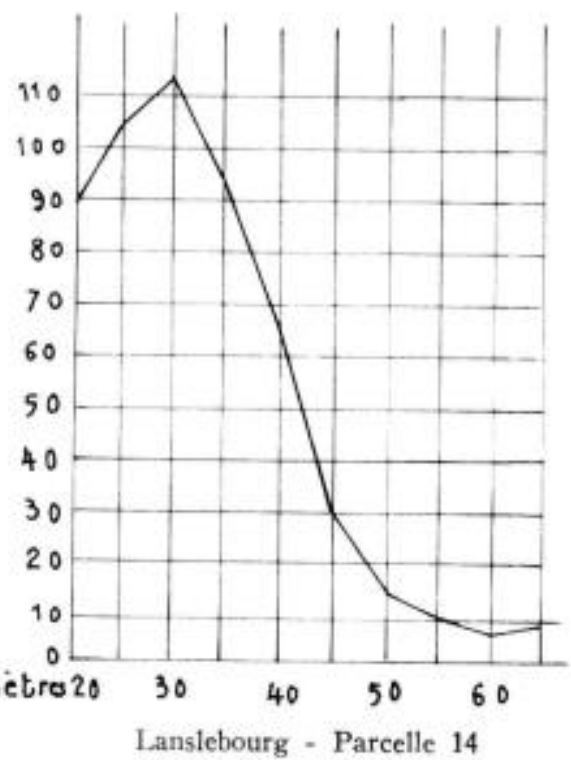

Lanslebourg - Parcelle 14

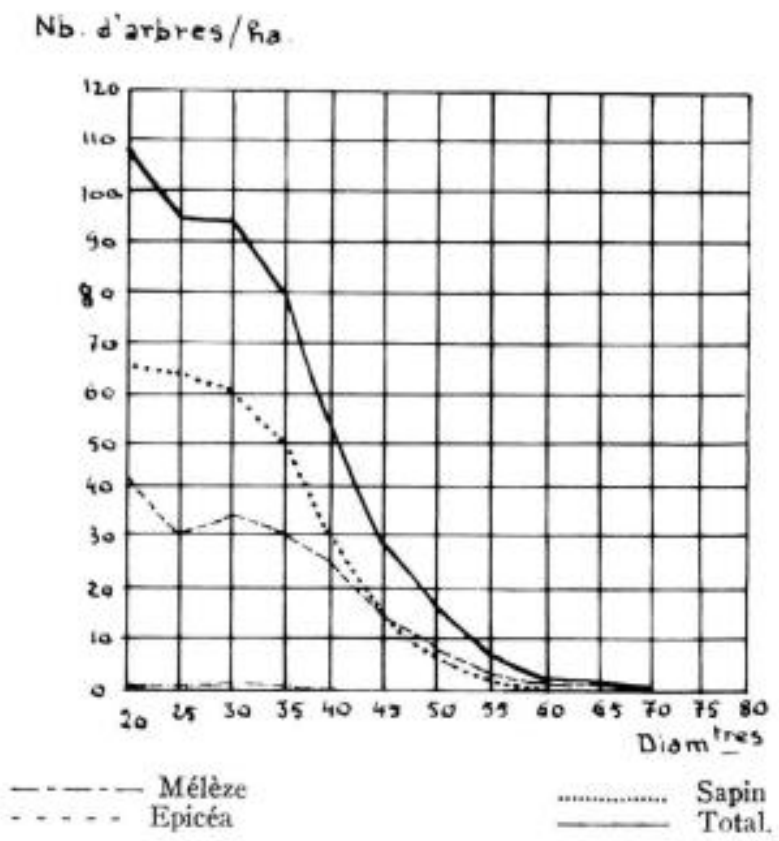

FiG. 39.

Sapinières

Structures sylvicoles 
Le but poursuivi par le forestier étant d'obtenir une structure jardinée, on conçoit que des trouées plus ou moins vastes soient assises partout oì se manifeste une régénération vigoureuse. Cet effort se traduira - et se traduit parfois, dès maintenant - par un relèvement, sur les courbes, du nombre des arbres de petit diamètre (ex.: parcelle 25 , de la forêts de Termignon).

L'échantillonnage de 103 hectares en forêt de Lanslebourg montre que la production de la sapinière vraie est relativement élevée: $4,8 \mathrm{~m}^{3} / \mathrm{ha} /$ an (tarif Algan $\mathrm{n}^{\circ} 8$ - volume à l'hectare: $380 \mathrm{~m}^{3}$ ).

Légende. - Que l'on considère la structure de parcelles envisagées comme des unités autonomes ou celle d'un ensemble de parcelles (fig. 39), les sapinières de la Haute-Maurienne apparaissement comme très peu chargées en gros bois $(0,70 \mathrm{~m}$ de diamètre et au-delà), mais présentent, par contre, une proportion excessive de bois moyens $(0,30$ à $0,40 \mathrm{~m}$ de diamètre).

Une telle structure résulte d'une action humaine assez intense qui, avant le rattachement de la Savoie à la France, a éliminé systématiquement tous les gros bois en favorisant indirectement une régénération puissante, puis du róle protecteur des forestiers qui ont laissé se développer et s'enrichir une futaie déséquilibrée au départ.

II appartient maintenant à ces derniers, en augmentant les possibilités, de passer d'une phase d'enrichissement à une phase de régénération, mais non d'une façon massive, de manière à atteindre le plus tôt possible, une structure jardinée équilibrée et qui se traduira par une courbe exponentielle.

$\mathrm{La}$ courbe générale montre, en outre, le ròle respectif que jouent la sapinière sur schistes lustrés, les deux essences: sapin et épicéa. On notera que cette dernière espèce est mieux représentée dans les gros diamètres, ce qui traduit, à notre avis, une influence humaine ancienne.

A la suite de coupes abusives, signalées ci-dessus, l'épicéa s'est reproduit plus puissamment que le sapin; ce dernier, par contre, s'est, par la suite, beaucoup plus régénéré que son congénère. On doit penser qu'un équilibre s'établira entre les deux essences, sans qu'il y ait lieu de parler d'alternance; le sapin dominera constamment l'épicéa, dans une proportion difficile à fixer et variable selon les stations, sans que l'épicéa, d'ailleurs, disparaisse. Les bouquets mixtes de régénération sont d'ailleurs, actuellement, très fréguent et traduisent cet équilibre. 


\section{III.5. - LES PESSIERES}

Si l'on faisait le bilan de l'importance de chaque essence, dans les forêts de la Haute-Maurienne, l'Epicéa [Picea excelsa (Lamk) Link] arriverait certainement en tête. C'est en effet, la seule espèce arborescente à être représentée à peu près dans toutes les associations, et si elle est rare dans la rhodoraie à Pin Cembro et dans la plupart des pineraies de Pin sylvestre, elle est constante et souvent abondante dans la sapinière et dans certains faciès des pineraies de Pin à crochets. En outre, elle est dominante absolue dans des associations auxquelles elle doit donner son nom: les pessières.

Contrairement à ce qui se passe dans certains secteurs des Préalpes de Savoie ou de Haute-Savoie, où l'Epicéa fait figure d'envahisseur et arrive à dominer dans un étage, qui est celui du Hêtre ou du Sapin et du Hêtre, les pessières de la Haute-Maurienne sont localisées dans l'étage subalpin.

Ces pessières colonisent toutes les expositions, mais il est bien certain que celles de versant sud présentent par rapport à celles de versant nord des différences notables qui font que doivent être distinguées des pessières d" "adret "ot xérophiles et des pessières d' " envers ", mésophiles ou mésohygrophiles. Entre les deux extrêmes, on peut rencontrer des types de transition, assez rares d'ailleurs étant donné que les grands versants d'expositions " intermédiaires " sont faits de roches-mères carbonatées et sont occupés essentiellement par des pineraies de Pin sylvestre et de Pin à crochets, comme on l'a vu.

\section{III.5.1. - Les pessières xérophiles.}

$1^{\circ}$ Organisation sociologique et Composition floristique Sous-associations.

Comme le montrent certains relevés du tableau général (relevés $\mathrm{n}^{\circ} 1$ à 6 ), existe, sur schistes lustrés, à l'amont de Termignon, une pessière xérophile à flore relativement pauvre.

Dans l'ensemble, les peuplements correspondant à ce type sont assez jeunes ( 80 ans environ), souvent très denses et de structure très régulière. Encore peu évolués, ils se sont développés brutalement sur d'anciennes friches à moutons, plus ou moins envahies 


\section{LES PESSIERES}

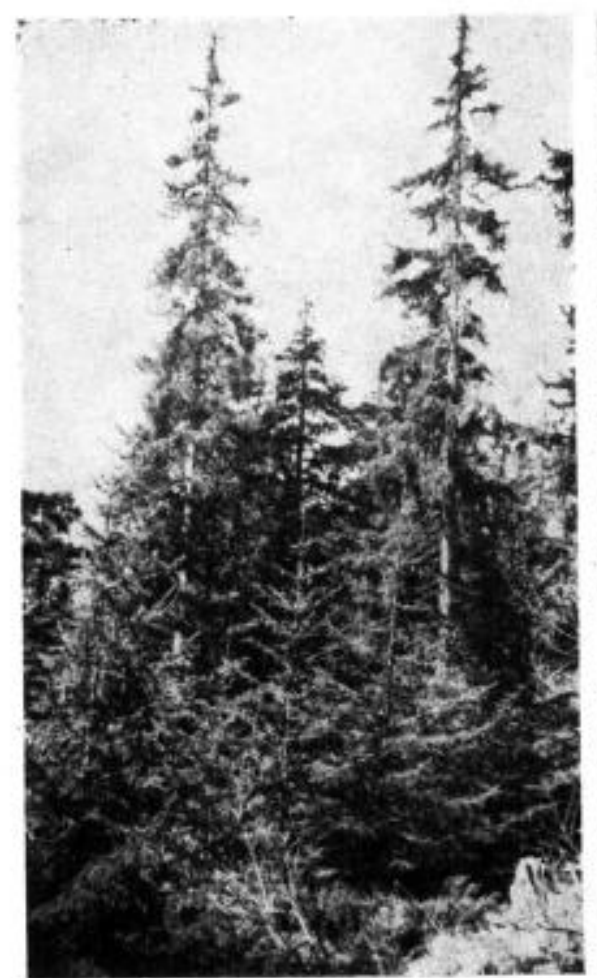

Pessière subalpine xérophile à $V a c$ cinium Vitis-idaea sur schistes du Houiller.

Une trouée de régénération. Forêt de Saint-André

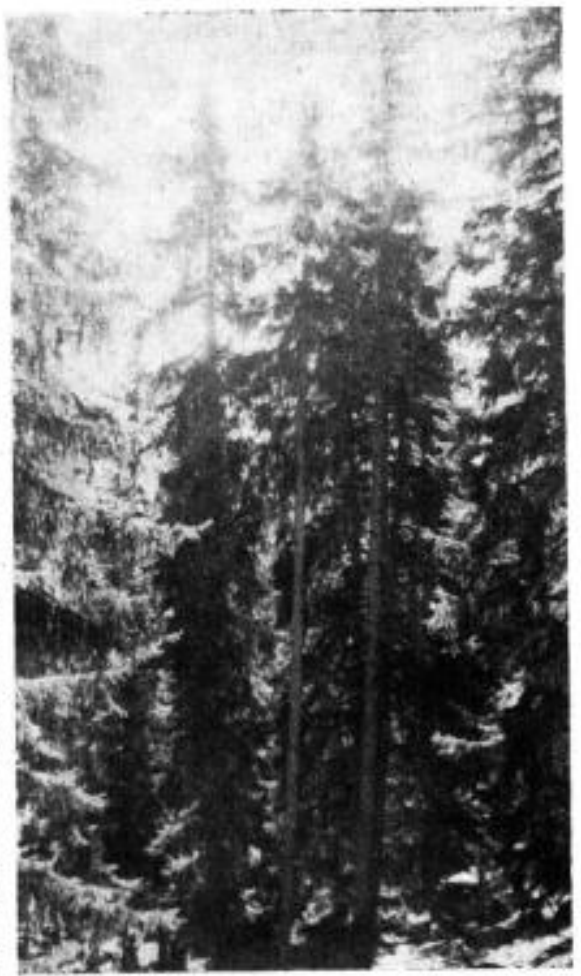

Pessière subalpin xérophile à $V a c-$ cinium Vitis-idaea sur schistes du Hotiller.

Forêt de Saint-André Un beau peuplement. 
par la busserole: leur densité est telle qu'elle a étouffé toute végétation ou presque et il faudra attendre, pour les mieux étudier, qu'ils aient vieilli et se soient irrégularisés.

Dans les rares endroits, où le tapis végétal arbustif et herbacé subsiste et se développe, on constate que sa flore rappelle assez bien celle de la pineraie subalpine de Pin à crochets, sur sol calcimorphe; mais ici, la richesse en éléments silicatés de la rochemère élimine le Pin à crochets au profit de l'Epicéa.

Sur grès et schistes du Houiller et sur quartzites triasiques, entre Saint-Michel-de-Maurienne et le Bourget, et contrairement à ce que l'on observe sur schistes lustrés, un autre type de pessière xérophile subalpine présente tous les signes d'un peuplement installé là depuis les temps les plus reculés: il s'agit d'un groupement acidiphile (relevés $\mathrm{n}^{\circ} 7$ à 15 ).

Dans la strate arborescente, il y a dominance presque absolue de l'Epicéa; seuls le Pin Cembro, vers le haut de l'étage, et le Pin sylvestre, vers le bas, se mélangent à lui, mais en restant toujours très subordonnés; le Mélèze est totalement absent.

Dans les autres strates, les groupes écologiques les mieux représentés sont ceux des xérophiles et des mésoxérophiles acidiphiles: on retrouve dans ce type de pessière certaines xéro-acidiphiles rencontrées dans la pineraie de Pin sylvestre sur ranker à moder, telles Phyteuma betonicifolium, Silene rupestris, etc; s'y ajoutent des subalpines comme Laserpitium Halleri. Dans le groupe des mésoxérophies, est à retenir la dominance de Vaccinium Vitis-idaea, qui donne aux strates inférieures leur physionomie, alors que Vaccinium Myrtillus est très nettement stubordonnée.

Se joignent aux groupes des acidiphiles, un cortège, assez maigre d'ailleurs, de xérophiles indifférentes quant au type d'humus et quelques mésophiles ou mẻme mésohygrophiles de mull, dont la présence - toujours discrète - s'explique par l'hétérogénéité du milieu: il existe, en effet, dans la pessière xérophile, des microstations abritées où règnent des conditions rappelant celles du versant général opposé.

Comme on peut s'y attendre, le spectre floristico-écologique de la pessière sur sol subcalcimorphe rappelle celui de la pineraie de Pin à crochets, tandis que le spectre de la pessière acidiphile rappelle à la fois ceux de la pineraie de Pin sylvestre et de la pineraie de Pin Cembro acidiphiles.

\section{Identification - Dénomination.}

Il serait évidemment prématuré de vouloir donner un nom au premier des deux groupements analysés - bien qu'il ne paraisse pas avoir été déjà décrit - car il s'agit d'une association en pleine évolution, comme il a été dit et qui méritera d'être revue ultérieurement. 
La seconde sous-association, acidiphile, est à rapprocher, par contre, du Piceetum subalpinum vaccinietosum Vitis-idaeae décrit par Braun-Blanguet (J.), Palmann (H.) et Bach (R.), en Engadine (1954). Si l'on met en parallèle notre tableau et celui des auteurs suisses, on constate tout de suite qu'il s'agit, dans les deux cas, d'une pessière subalpine sèche, floristiquement assez pauvre, où Vaccinium Vitis-idaea domine, alors que Vaccinium Myrtillus joue un rôle secondaire.

A noter toutefois que le groupement de la Haute-Maurienne apparait dans l'ensemble comme plus xérophile que son homologue suisse, non seulement par rapport à la variante à Peltigere aphtosa et Hylocomies la plus mésophile, mais également par rapport à la variante qui se caractérise, selon BraUN-BLANQUET lui-même, par la rareté des caractéristiques (Charakterartenarme variante). Certaines espèces mésophiles, comme Veronica urticifolia, Luzula silvatica subsp. Sieberi, Calamagrostis villosa, qui figurent avec une certaine constance dans les relevés suisses, ne se rencontrent dans les nôtres qu'avec une constance sensiblement plus faible. Il en est de même de Larix decidua, dont nous avons souligné l'absence dans notre association, mais qui se montre très fréquent dans l'association suisse, quoique avec une abondance-dominance faible, puisqu'elle n'atteint que rarement, deux.

\section{$3^{\circ}$ Répartition - Ecologie.}

Les pessières subalpines sèches - qu'il s'agisse de la pessière à Carex humilis et Polygala chamaebuxus ou de celle à Vaccinium Vitis-idaea, - se localisent aux expositions sud, la première sur schistes lustrés, la seconde sur grès et schistes du Houiller.

Cette dernière s'étage, en altitude, entre $1800 \mathrm{~m}$ et $2000 \mathrm{~m}$ environ; vers le bas, elle passe à la pineraie xérophile acidiphile déjà analysée et vers le haut, elle est relayée par la pineraie de Pin Cembro à Juniperus nana.

Sur schistes lustrés, l'occupation humaine dans l'étage subalpin supérieur, et même dans la partie haute de l'étage subalpin inférieur, fait que la pessière s'arrête plus bas que dans les gorges houillères et qu'elle se trouve en contact soit avec des pelouses pâturées ou fauchées, soit avec une lande à base de busserolle, toutes formations qui occupent, selon toute vraisemblance, le sousétage du Pin Cembro. Vers le bas, la pessière passe à la pineraie xérophile de Pin sylvestre à Ononis rotundifolia.

Latéralement, les pessières xérophiles ne sont en contact avec aucun groupement forestier: à l'amont, en effet, au-delà de Lanslevillard, l'occupation humaine (chalets d'alpage) et un régime sévère d'avalanches ont éliminé complètement la forêt sur tout le versant sud; il en est de même à l'aval, après Orelle, autour des divers hameaux du Thyl, mais où seule a pratiquement joué l'action humaine. 
En l'absence de mesures microclimatiques de l'air et du sol, on retiendra la pluviosité au fort du Sappey, situé sur un verrou accolé au versant général sud, au-dessus de Modane, à $1750 \mathrm{~m}$ d'altitude, c'est-à-dire au niveau de la pessière xérophile. Alors que, pendant la même période d'observations la pluviosité à Modane - au pied de la pineraie de Pin sylvestre - a été de $641 \mathrm{~mm}$, elle a été au Sappey de $734 \mathrm{~mm}$. Le microclimat de la pessière xérophile comme son climat local - doivent donc rappeler ceux de la pineraie de Pin sylvestre xérophile, mais être plus froid, et plus humide, donc moins brutal.

En ce qui concerne les sols, nous n'avons retenu que les profils de ceux de la pessière acidiphile: les sols de la pessière sur schistes lustrés sont, en effet, très peu évolués et correspondent à des sols bruns jeunes de pelouse, surmontés, sous les peuplements les plus denses, d'une petite couche de $\mathrm{A}_{0}$.

Les sols de la pessière à airelle rouge montrent, par contre, une évolution plus marquée: ils sont tous de type podzolique, mais à horizons " ramassés ". Il s'agit soit de sols ocres podzoliques jeu-

\section{Pessière subalpine sérophile}

Sol $\mathrm{n}^{\circ} 1$

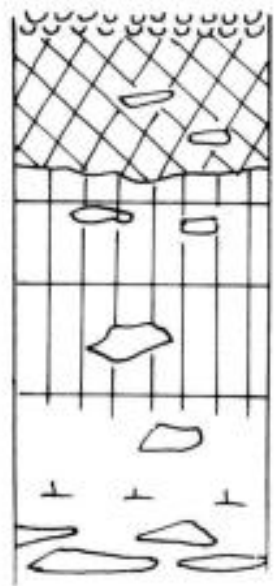

Sol ocre podzolique.

$$
e=1 / 10 \quad \text { Sol } n^{\circ} 2
$$

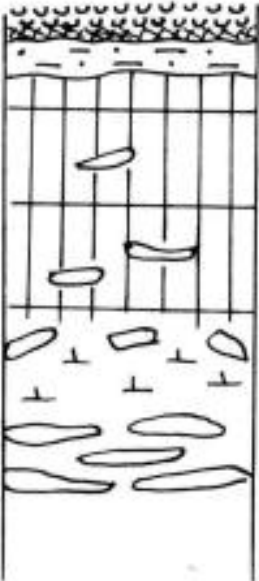

Sol podzolique.

verves: Humus brut.

MXX Moder.

Argile.

[.- Horizon cendreux lessivé.

WIII Fer ferrique (rouille).

$\perp \perp$ Zone d'altération.

Fic. 40 , 
nes $\left(\mathrm{sol} \mathrm{n}^{\circ} 1\right.$ - relevé floristique $\mathrm{n}^{\circ} 3$ ), à horizon $\mathrm{A}_{2}$ pratiquement inexistant, soit de sols podzoliques jeunes un peu plus évolués, à horizon $\mathrm{A}_{2}$ net, mais de faible épaisseur (sol $\mathrm{n}^{\circ} 2$ - relevé floristique $n^{\circ} 7$ ).

\section{Description morphologique (fig. 40)}

\section{Sol $n^{\circ} 1$.}

$A_{0}(0-3 \mathrm{~cm})$ - horizon humifère noirâtre - structure fibreuse - feutrage de racines d'airelle rouge.

$A_{1}(4-12 \mathrm{~cm})$ - horizon humifère, brun-gris - texture limoneuse - structure particulaire - racines d'Epicéa.

B $(13-40 \mathrm{~cm})$ - horizon rouille brunâtre - texture limoneuse nombreux fragments de schistes (30\% du volume).

Sol $n^{\circ} 2$.

Les horizons $A_{0}, A_{1}, B_{1}$ sont tout à fait semblables à ceux du sol précédent.

Mais un horizon $\mathrm{A}_{2}$, cendreux, gris, de texture limono-sableuse et de structure particulaire s'observe, en un mince liséré, sous l'horizon humifère.

Analyse sommaire

Sol 1 - Relevé floristique $\mathrm{n}^{\circ} 19$ - sol ocre podzolique

\begin{tabular}{|c|c|c|c|c|c|c|c|}
\hline Jori zons & Bpaisseur & $p^{H}$ & $c$ & Mat.ors. & $\cos ^{3} \mathrm{Cs}$ & $\underset{\text { deh. }}{\mathrm{Ca}}$ & res \\
\hline 4. & 3 ox & 4,4 & 45,34 & 77 & IX) & 26,5 & \\
\hline$\cdot h_{1}$ & $12 \mathrm{~cm}$ & 4,1 & 3,22 & 5,4 & Sesunt & 4,3 & 0,98 \\
\hline b & $40 \mathrm{~cm}$ & 4,3 & 0,28 & 1,6 & verent & 2,1 & 1,01 \\
\hline
\end{tabular}

Sol 2 - Relevé floristique $n^{\circ} 20$ - sol ocre podzolique

\begin{tabular}{|c|c|c|c|c|c|c|c|}
\hline Horisons & Epaissour & pI & c & Nat, org. & $\mathrm{CO}^{3} \mathrm{Oa}$ & $\begin{array}{c}\mathrm{Ca} \\
\text { Cck. }\end{array}$ & Por \\
\hline \multirow{4}{*}{ 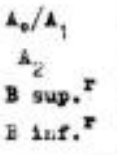 } & \multirow{4}{*}{$\begin{array}{l}2 \mathrm{~cm} \\
2 \mathrm{~cm} \\
30 \mathrm{~cm}\end{array}$} & 4 & 40,76 & 69,3 & Nennt & 3,5 & \\
\hline & & 3,7 & 2,46 & 4,1 & Jéunt & 2,3 & 0,72 \\
\hline & & 4,2 & 1,47 & 2,3 & Heant & 1,1 & 1,12 \\
\hline & & 4,2 & 1,62 & 2,7 & Neunt & 1,2 & 1,84 \\
\hline
\end{tabular}

Alors que la pineraie acidiphile de Pin sylvestre repose sur un ranker à moder, on notera qu'à altitude plus élevée, et bien que l'essence soit moins podzolisante, on a affaire à un sol podzolique ou ocre podzolique - à nor. Ceci nous parait tenir à plusieurs 
Potentiel capillaire à $-0,05 \mathrm{~m}$

\section{Ononido-Pinetum}

Piceetum subalpinum vaccinietosum vitis-idaeae.

++++ Abietetum albae.

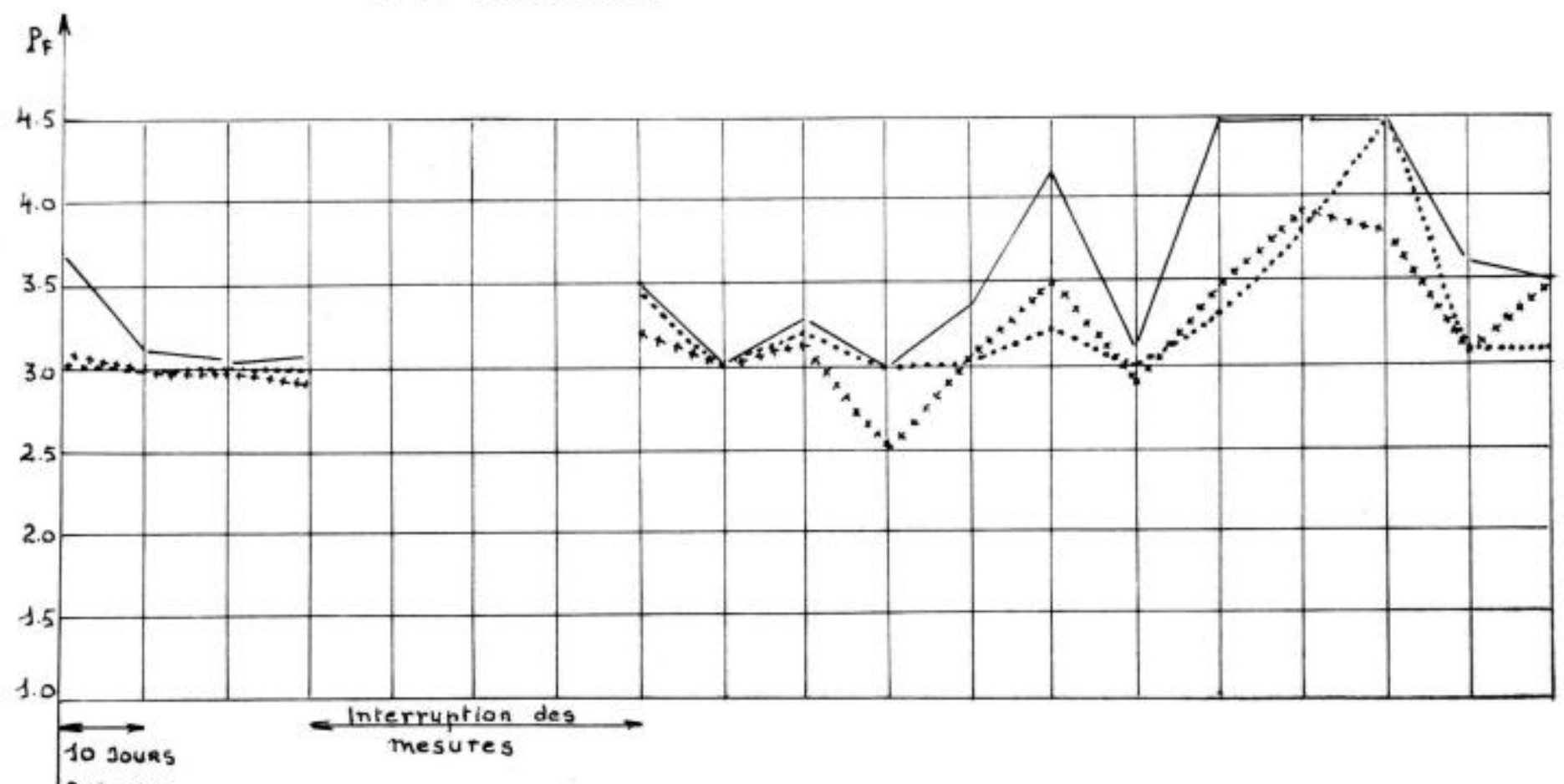




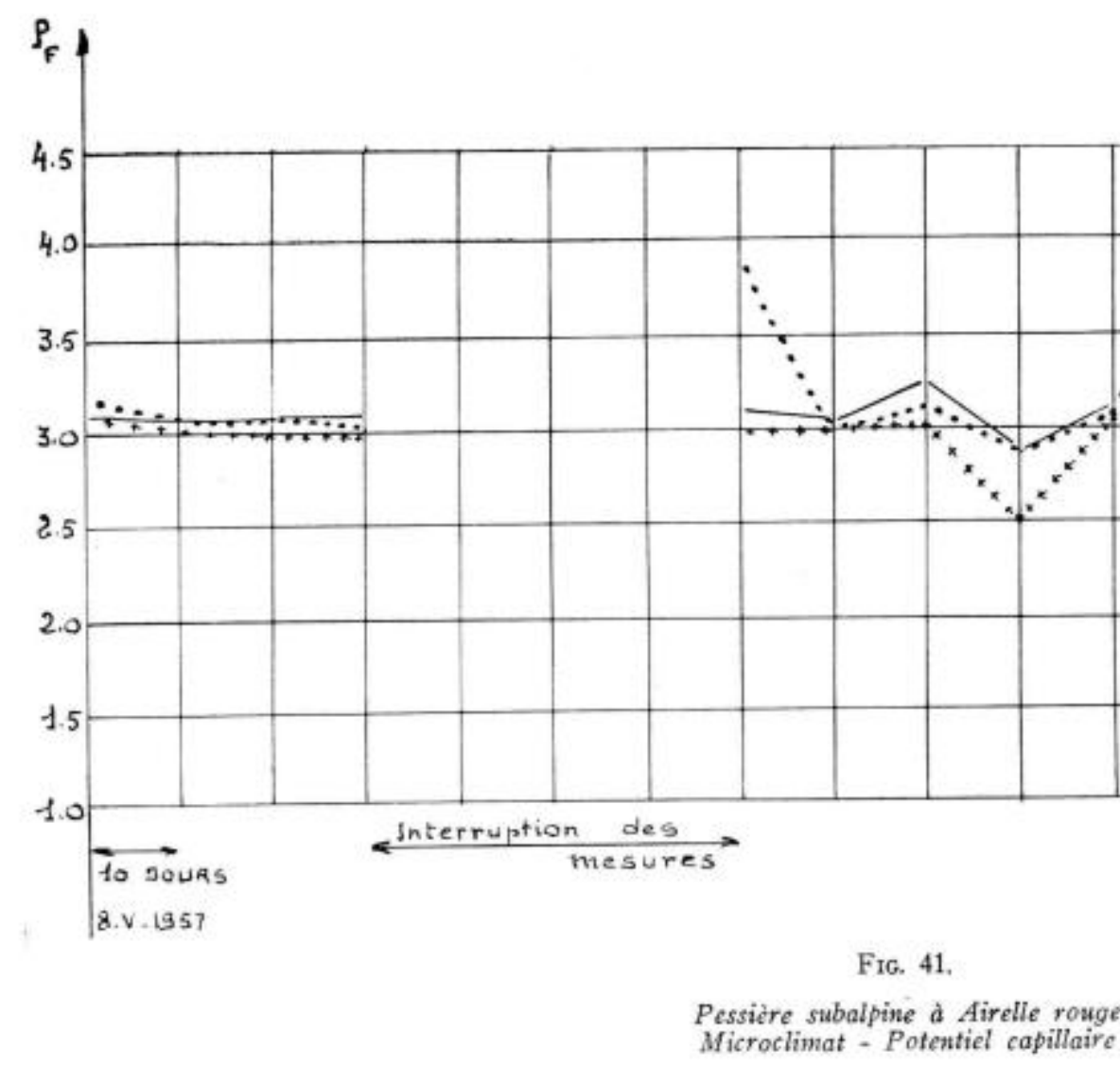




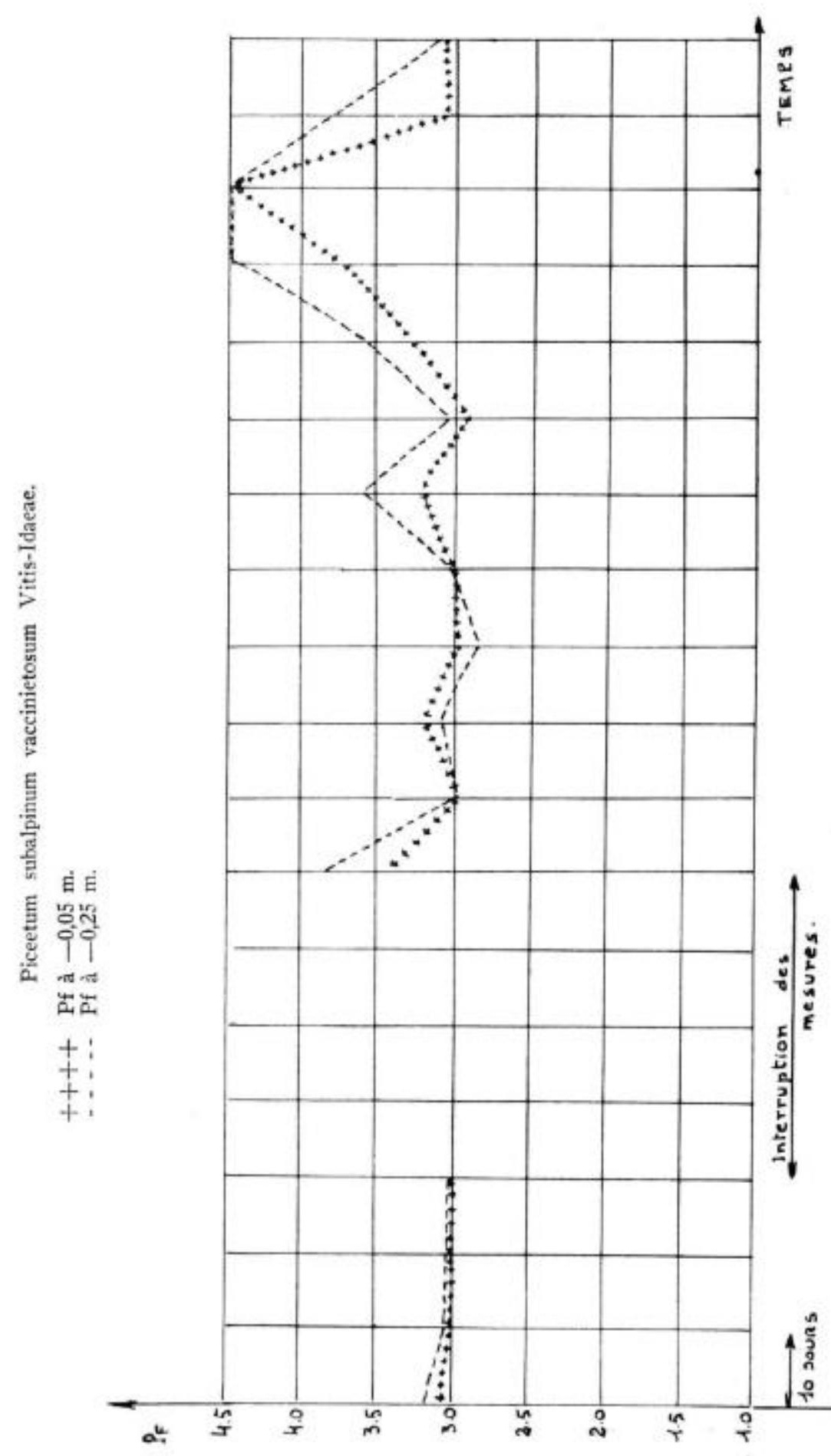




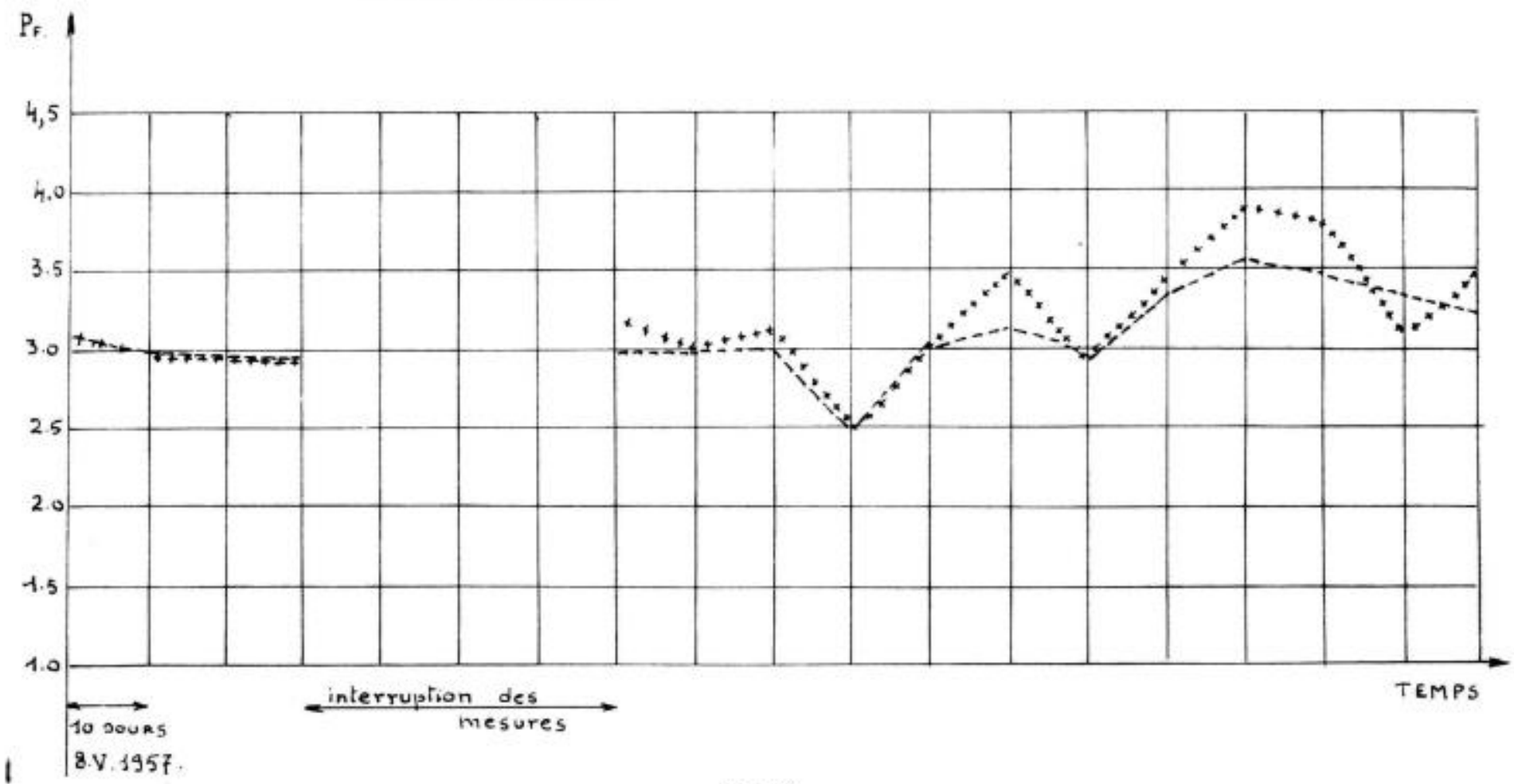

Fig. 42.

Pessière subalpine à Airelle rouge

Potentiel capillaire à $-0,05$ et $-0,25$

Comparaison avec celui de l'Abietetum albae. 
raisons: d'une façon générale, les pentes où se rencontre la pineraie, sont sensiblement plus fortes que celles où s'observe la pessière: l'érosion y joue donc un plus grand rôle: l'humidité et le froid sont, en outre, plus élevés au niveau de cette dernière: l'humus doit donc s'y décomposer plus lentement; enfin, et surtout sans doute, la pessière est nettement plus riche en Ericacées, dont le róle dans la podzolisation - et toutes choses égales d'ailleurs - nous parait primordial.

Mais, on observera que les sols restent peu évolués: un tel caractère s'explique sans doute par l'érosion, mais aussi par la sécheresse du mésoclimat qui est elle-même à l'origine du faible développement des Ericacées acidifiantes.

L'étude du potentiel capillaire à $-0,25 \mathrm{~m}$ de profondeur montre que le sol de la pessière subalpine à Airelle rouge est moins sec, comme on pouvait s'y attendre, que celui de la pineraie de Pin sylvestre à Ononis rotundifolia, et qu'il est sensiblement plus sec que celui de la sapinière vraie. Près de la surface, par contre, à - $0,05 \mathrm{~m}$, le phénomène est moins net, bien que dans l'ensemble, la même hiérarchie s'observe. Si l'on se réfère aux données correspondant aux observations $n^{\circ} 8$ à 14 , il semble, en effet, que, près de la surface, l'humus brut de la pessière subalpine et le moder qui lui succède en profondeur, jotent plus que le mull de la sapinière, un róle de "volant hydrique s (fig. 41).

Ce rôle apparait plus nettement - étant entendu qu'il s'agit là d'un fait qui appellera, pour être confirmé, de nombreuses mesures - si l'on met en parallèle, pour chacune des deux associations, les courbes du pF à $-0,05 \mathrm{~m}$ et à $-0,25 \mathrm{~m}$. On constate que le sol de la pessière subalpine est plus sec en profondeur qu'en surface, alors que celui de la sapinière est plus sec en surface qu'en profondeur. Ces résultats - très fragmentaires - viendraient confirmer l'une des conclusions de Rousseau (L.-Z.) obtenues à l'occasion de l'étude de l'influence du type d'humus sur le développement des plantules de sapin (1959), à savoir que le mull est plus perméable que le moder, le complexe colloïdal argilo-humique dans ce type d'humus étant peu hygrophile (fig. 42).

\section{III.5.2. - Les pessières mésophiles et mésohygrophiles.}

$1^{\circ}$ Organisation sociologique et composition floristique - Sousassociations.

Pour " situer » d'une façon générale les pessières mésophiles ou mésohygrophiles, il importe de les comparer à celle de versant sud, mais aussi aux sapinières à Epicéa de l'étage montagnard, sur versant nord, qu'elles relaient dans l'étage subalpin. 
Le tableau général des relevés dans les pessières fait apparaitre trois faits essentiels:

les groupes écologiques xérophiles - qui englobent les espèces xéro-acidiphiles, les xéro-calciphiles et les indifférentes - bien représentés dans les deux types de pessière xérophile, disparaissent totalement dans les pessières mésophiles,

le groupe des mésophiles, par contre, est heaucoup plus étoffé dans les pessières fraîches que dans les autres: si certaines espèces sont présentes dans les det1x groupes d'associations, certaines, comme Sarifraga cuncifolia, Festuca flavescens, sont localisées dans l'association mésophile, tandis que d'autres y connaissent une plus grande constance et une plus grande abondance, telles Calamagrostis villosa, Homogyne alpina, Vaccinitrm Myrtillus: A l'inverse, on observera le recul de Vaccinium Vitis-idaea dont la dominance n'est supérieure à celle de Vaccinium Myrtillus que dans des relevés de transition (relevés $n^{\circ} 17$ et 18 ) pris, il faut le noter, sur un versant secondaire tourné vers l'est, du versant général sud. Le groupe des mésohygrophiles, enfin, est très nettement plus étoffé, comme on pouvait s'y attendre, surtout dans la sous-association à Adenostyles Alliariae et Cicerbita alpina.

Par rapport à la sapinière vraie (Abietetum albae), les différences sont moins nettes et cela se conçoit si l'on veut bien retenir que le passage d'une association à l'autre, sur un même versant, se fait selon des transitions insensibles, alors que d'un versant à l'autre, et dans le même étage, les transitions sont plus brutales.

Des différences n'en existent pas moins et elles méritent d'être soulignées:

Tout d'abord apparaissent dans la pessière subalpine, un certain nombre d'espèces subalpines ou mème alpines qui ne se rencontrent pratiquement pas dans la sapinière, telles Soldanella alpina, Ajuga pyramidalis et surtout Calamagrostis villosa, Rhododendron ferrugineum (ces deux dernières espèces qui sont assez rares dans l'étage subalpin inférieur, prennent tout leur développement dans le sousétage du Pin Cembro). A l'inverse, certaines espèces rencontrées dans 1'Abietetum albae, comme Carex digitata, Phvterma spicatum tendent à disparaitre dans létage subalpin, tandis que d'autres, comme Veronica urticifolia, Lusula nivea, subsistent mais avec une abondance-dominance sensiblement affaiblie.

Quoi qu'il en soit de ces différences, positives ou négatives, on retrouve dans la pessière subalpine fraiche des sous-associations analogues à celles de la sapinière à Epicéa; il s'agit donc, véritablement, d'unités vicariantes subalpines, proches de celles observées dans l'étage montagnard.

Une sous-association type sur schistes lustrés, d'abord riche en mésophiles de mull-moder, où Calamagrostis villosa est bien représentée. 
PLANCHE XIII

LES PESSIERES

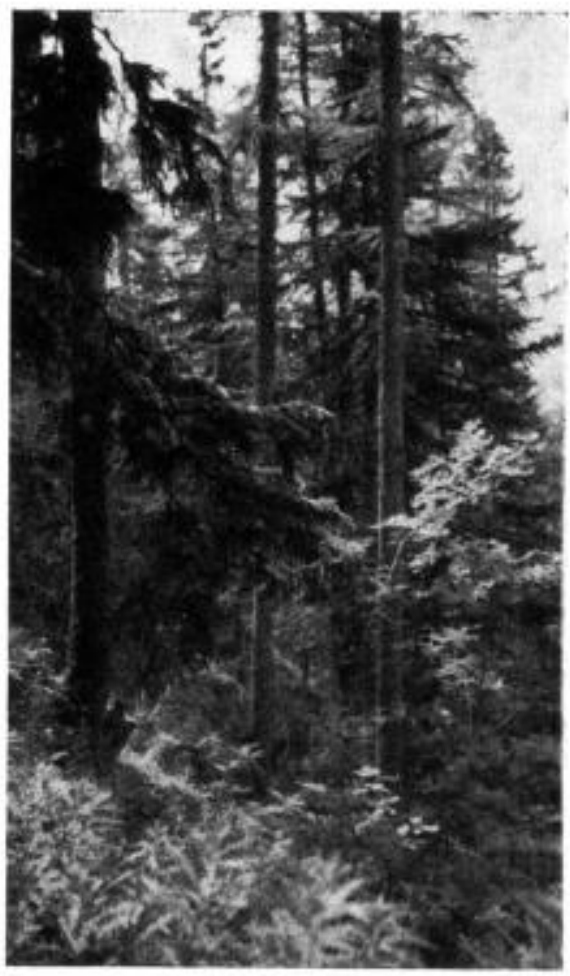

Pessière subalpine mésophile avec trouée à Epilobe au premier plan.

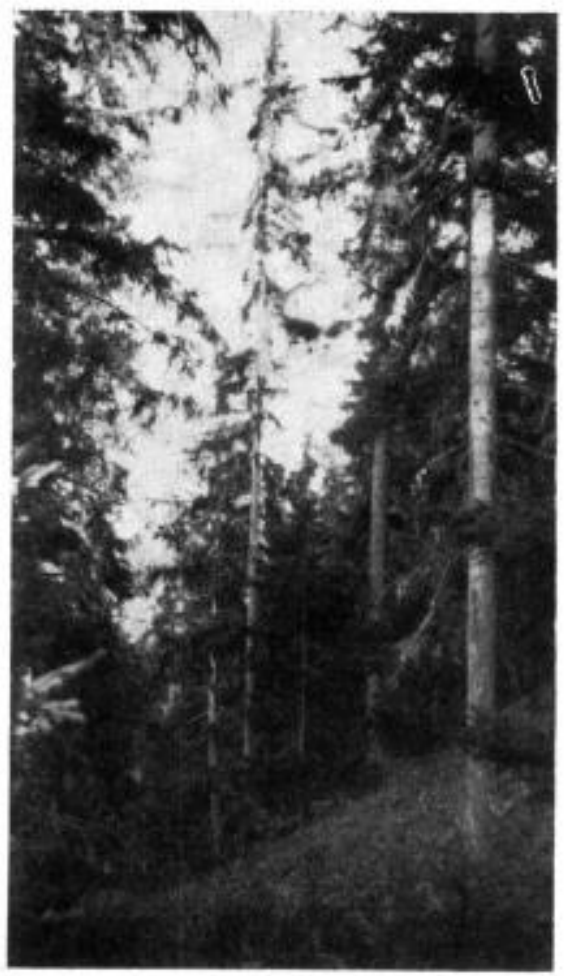

Pessière subalpine mésophile. Structure jardinée. Forêt de Lanslebourg. 
Une sous-association à Saxifraga Cuneifolia qui est presque totalement localisée dans les gorges houillères et qui se caractérise par une grande richesse en mésophiles de moder.

Une sous-association mésohygrophile, at contact de l'Alnetum viridis, riche en espèces de 1'Adenostylion. Dans ce dernier groupement et bien que, dans l'ensemble la végétation donne l'impression d'un tout homogène, on se trouve à la limite d'une répartition en mosaique. Les éléments liés à un humus brut ou à un moder (Festuca flavescens, Vaccinium Myrtillus) se localisent le plus souvent, en effet, près des troncs et sur les souches ou autour d'elles, où ils trouvent des conditions pédologiques quii leur conviennent particulièrement. On constate, d'autre part, que certaines petites dépressions de quelques mètres carrés seulement sont exclusivement occupées par Adenostyles Alliariae et Cicerbita alpina; dans ces dépressions, à peine prononcées pourtant, mais où la neige subsiste plus longtemps qu'ailleurs, la régénération de l'Epicéa s'avère particulièrement difficile. On pent donc les assimiler dans une certaine mesure - et bien qu'elles soient minuscules - à celles observées dans certaines forêts du Haut-Jura ou de la Chartreuse où le dessin de la mosaique est infiniment plus net et où vivent côte à còte, dans des milieux édaphiques très différents. 1'Asplenio-Piceetum et le Ciccrbito-Adenostyletum.

\section{$2^{\circ}$ Dénomination - Identification.}

Nous avons vu que la pessière subalpine acidiphile de versant sud, s'identifiait avec le Piceetum subalpinum vaccinitosum Vitisidaege de Braun-Blanguet (J.). Palmann (H.) et Bach (R.) (1954). L'homologue de ce Piccetum sur versant nord (8) est le Piccetum subalpinum myrtilletosum décrit en Suisse, en Autriche et en Italie par divers auteurs. Notre groupement peut-il être rapporté à cette pessière?

I1 est de fait que Braun-Blanguet a ainsi appelé un individu dont le relevé a été pris au-dessus de Lanslebourg, en Haute-Maurienne, sur schistes lustrés (1961). Ce relevé dont nous donnons ci-après le détail, s'intègre parfaitement dans notre groupement (9).

Caractéristiques de lassociation:
2.3. Festuca flavescens
+ Lonicera vigra
+ Lusula Lutulina (syn. L. fla- vescens)

Caractéristiques d'alliance (Vaccinio-Piccion):
4.1. Picea excelsa
2.1. Melampyrum silvaticum
+ Lusula silvatica.
+ Veronica latifolia

(8) Mise à part la forme mésohygrophile qui, malgré sa structure, peut ètre rapportée à un Adenostylo-Piceetum.

(9) Le premier chiffre correspond à l'abondance-dominance; le second à la sociabilité. 
Caractéristiques d'ordre (Vaccinio-Piceetalia):

2.1. Hieracium murorom

+2. Vaccintion Myrtillus

+ Pirola secunda

+ Rubus saxatilis

\section{Compagnes:}

1.1 Larix decidua

+ Hepatica nobilis $(=H$. triloba)

+ Sorbus Aucuparia

+ Phyteuma Halleri

+ Hieracium prenanthoides

(+) Ranuncalus montanus

$2.3 / 2$ Rhytidiadelphus triquetris

+2. Hylocomium proliferum
1.1. Homogync alpina

+2. Vaccinium Vitis-idaea

+ Rosa pendulina $(=R$. alpina $)$

Si l'on rapproche, d'autre part. notre tableatu de celui établi assez récemment en Suisse par Braun-Blanouet (J.), Pallmann (H.) et BACH (R.) (1954), on constate que l'on retrotive dans les deux cas bon nombre d'espèces communes appartenant soit à l'alliance du Vaccinio-Piceion comme: Melampyrum silvaticum, Calamagrostis villosa, Lusula silvatica ssp. Sieberi... soit à l'ordre des VaccinioPiceetalia, et même des espèces appartenant à l'une ou l'autre de ces deux unités et considérées comme caractéristiques de l'association, telles Lonicera nigra ou Pirola uniflora.

Cependant - et c'est là où nous voulions en venir - notre groupement diffère tout de mẻme du Piccetum subalpinum myrtilletosum type, par l'absence totale de certaines espèces considérées d'ailleurs - en plus de celles déja citées - comme caractéristiques de l'association à savoir: Lycopodium annotinum, Listera cordata, Ptilium crista-castrensis et par le développement relativement faible de $V$ acrinium Myrtillus.

Or, ces espèces ont une signification écologique des plus nettes: ce sont des orophiles étroitement liées à un humus brut assez riche en eau, proche de l'hydromor. Leur absence dans notre groupement tient au fait que les sols de la pessière subalpine mésophile de notre dition ne sont pas des sols à humus brut vraiment caractérisé et épais, mais, soit des sols à mull-moder ou à moder, soit des sols à mor de faible épaisseur, très peu évolués, très jeunes.

Pour expliquer ce caractère, il parait difficile, a priori, d'incriminer le climat, puisque la pessière subalpine type se rencontre au oceur du monde intra-alpin, en Suisse, notamment, encore que l'on puisse observer que le climat intra-alpin suisse est plus froid et plus continental que celui de la Haute-Maurienne. Il faut noter. à ce propos, que nous avons rencontré, en Haute-Tarentaise, des pessières subalpines nettement caractérisées, avec notamment, Lycopodium annotinum, Ptilium crista-castrensis (forêt de Malgovert, aut-dessus de Bourg-Saint-Maurice, forêt d'Issertan, au-dessus de Pralognan), mais que, par contre, dans les Alpes maritimes, dans 
les vallons de la Madone, du Boréon, de Molière, les pessières subalpines mésophiles nous ont paru très proches de celles de la HauteMaurienne: nous n'y avons jamais observé Listera cordata, Lycopodium annotinum, Ptilium crista-castrensis. Il n'est donc pas impossible d'imaginer qu'il existe ane forme sud-occidentale de la pessière subalpine mésophile appauvrie en espèces liées à l'hydromor. De prochaines prospections effectuées dans les Alpes du sud, pourront nous éclairer sur ce point.

Il ne faudrait pas, toutefois, oublier l'influence humaine. Il ne parait pas douteux, en effet, que la pessière subalpine du versant nord de notre dition a subi cette influence. Le pâturage des bovins s'exerce encore assez fréquemment à son niveau - sur schistes lustrés, tout particulièrement, où les formes du relief sont arrondies; il semble surtout que ce soit dans l'étage subalpin inférieur que l'industrie du charbon de bois se soit manifestée avec le plus de vigueur, la vidange de grosses billes de bois s'avérant longue et difficile. D'anciennes places à charbon s'observent, en effet, quelquefois dans cet étage, alors que nous n'en avons jamais rencontré dans celui de la sapinière. Cette action humaine explique, en outre, à notre avis, la grande abondance du mélèze, la faible dominance de Vaccinium Myrtillus et la jeunesse générale de la forêt, à ce niveau.

\section{$3^{\circ}$ Répartition - Ecologie.}

La pessière mésophile ou mésohygrophile s'étend donc aux expositions fraîches entre $1700-1750 \mathrm{~m}$ et $1950 \mathrm{~m}$ environ. Vers le haut, elle passe aux peuplements de Pin Cembro (rhodoraie ou calamagrostidaie à Pin Cembro) et vers le bas, à la sapinière à Epicéa (Abietetum albae).

Son climat se situe évidemment entre celui de ces deux associations sans qu'il soit possible, en l'absence de poste météorologique situé à son niveau, d'en donner une image exacte.

Les sols sur sclistes lustrés, il s'agit de sols bruns jeunes, légèrement lessivés à mull passant au moder, rappelant, par conséquent, ceux de la sapinière sur même roche-mère.

Une analyse sommaire d'un sol de ce type est donnée ci-après:

\begin{tabular}{|c|c|c|c|c|c|c|}
\hline Hori zone & $\begin{array}{l}\text { epais- } \\
\text { soura }\end{array}$ & $\mathrm{pH}$ & $\begin{array}{l}\text { Vistiore } \\
\text { orgar. }\end{array}$ & $\mathrm{Co}^{3} \mathrm{Ca}$ & $\begin{array}{l}\text { Cn éonan- } \\
\text { ooubl. }\end{array}$ & For \\
\hline$A_{1}$ & $50 x$ & 5,4 & 26,1 & $v$ & 26,6 & \\
\hline$h_{2}$ & $15 \mathrm{om}$ & 5 & 4 & 0 & 5,0 & 2,0 \\
\hline (B) & 20 on & 4,7 & 2,3 & c & 1,1 & 2,13 \\
\hline
\end{tabular}

sur grès ou schistes du houiller (sous-association à Saxifraga cuneifolia), l'humus est un moder passant au mor, tandis que le sol lui- 
même appartient au type ocre-podzolique (sol 1 et 2 ) soit mème au type podzolique jeune (sol 3) présentant un horizon cendreux mais d'épaisseur très faible (moins de $2 \mathrm{~cm}$ ).

Sol $n^{*} 1$ - Relevé floristique $\mathrm{r}^{*} 18$ - Sol ocre podzolique

\begin{tabular}{|c|c|c|c|c|c|c|c|c|c|c|}
\hline $\begin{array}{l}\text { Hort- } \\
\text { zons }\end{array}$ & $\begin{array}{l}\text { Byals- } \\
\text { seur }\end{array}$ & $\mathrm{P}^{\mathrm{H}}$ & $\begin{array}{l}\text { Not. } \\
\text { orean. } \\
\text { orean. }\end{array}$ & $00^{3} \mathrm{Cat}$ & Fer & hreslo & tion & $\begin{array}{l}\text { Sables } \\
\text { fins }\end{array}$ & $\begin{array}{l}\text { Sables } \\
\text { gaoss. }\end{array}$ & $\mathrm{H}^{2} \mathrm{O}$ \\
\hline ho/A1 & 500 & 4,8 & 54,6 & 0 & & & & & & \\
\hline B1 $(-10$ & )$_{40 \mathrm{en}}$ & 4,6 & 6,6 & 0 & 1,6 & 18 & 27 & 24,1 & 21,4 & 2,9 \\
\hline$B 2(-40)$ & & 5,0 & 2,9 & o & 1,6 & 16 & 20.5 & 27,9 & 21,3 & 2,4 \\
\hline
\end{tabular}

Sol $n^{*} 2$ - Relevé floristique $n^{*} 19$ - Sol ocre podzolique

\begin{tabular}{|c|c|c|c|c|c|c|c|c|c|c|}
\hline $\begin{array}{l}\text { Eori- } \\
\text { gons }\end{array}$ & $\begin{array}{l}E_{p a z a-} \\
\text { sour }\end{array}$ & pH & $\begin{array}{l}\text { Mat. } \\
\text { oxern. }\end{array}$ & $\mathrm{Co}^{3} \mathrm{Cs}$ & Por & hirgile & Linon & $\begin{array}{l}\text { solus } \\
\text { fins }\end{array}$ & $\begin{array}{l}\text { Sables } \\
\text { broses. }\end{array}$ & $\mathrm{H}^{2} \mathrm{C}$ \\
\hline $10 / 11$ & 4 on & 4,5 & 20,7 & c & 1,12 & 16,5 & 19,5 & 28,8 & 12,5 & 2 \\
\hline B1 $(-10)$ & $\lambda_{30}$ & 4,2 & 4 & 0 & 1,42 & 17 & 26,5 & $3>, y$ & 15,6 & 1 \\
\hline $\mathrm{B} 2(-25)$ & 30 os & 4,6 & 2,2 & o & 1,64 & 15 & 25 & 39,7 & 17,1 & 1 \\
\hline
\end{tabular}

Sol $n^{\circ} 3$ - Relevé floristique $n^{\circ} 21$ - Sol podzolique jeune

\begin{tabular}{|c|c|c|c|c|c|c|c|c|c|c|}
\hline $\begin{array}{l}\text { Hori- } \\
\text { sone }\end{array}$ & $\begin{array}{l}\text { Epain- } \\
\text { seur }\end{array}$ & $p E$ & $\begin{array}{l}\text { hat. } \\
\text { orban. }\end{array}$ & $\mathrm{Co}^{3} \mathrm{Ca}$ & Por & Argile & Lrwor: & $\begin{array}{l}\text { seblea } \\
\text { spins }\end{array}$ & $\begin{array}{l}\text { Sebles } \\
\text { sross. }\end{array}$ & $\mathrm{H}^{2} \mathrm{O}$ \\
\hline Ao $/$ Al $_{1}$ & 3 oif & 4,4 & 65,2 & $c$ & & & & & & \\
\hline A2 & 2 ou & 4,2 & 10,2 & 4 & $0, y$ & 17,5 & 29 & 24,6 & 15,3 & 3,4 \\
\hline B & 30 as & 4,5 & 2,2 & 0 & 1,4 & 16 & 29,5 & 28,7 & 29,2 & 2,4 \\
\hline
\end{tabular}

\section{III.5.3. - Vue d'ensemble sur les pessières.}

III.5.3.1. - Affinités phytosociologiques.

Ce qui a été déjà dit au sujet des deux types extrêmes de pessière subalpine rencontrés en Haute-Maurienne, nous dispensera de nouveaux commentaires.

On notera simplement que la pessière de notre dition diffère, des pessières de l'Europe centrale par la présence de Festuca flavescens qui n'est d'ailleurs pas liée à l'étage subalpin, puisqu'elle s'observe aussi, mais avec une moins grande abondance, dans l'étage montagnard; il n'existe pas, en outre, dans notre dition, de sousassociation à Sphaignes (Piceetum subalpinum sphagnetosum) ni de pessière à Sphaignes, sensu stricto (Sphagno-Piceetum). 


\section{LES PESSIERES}

Spectres floristico-ćcologiques

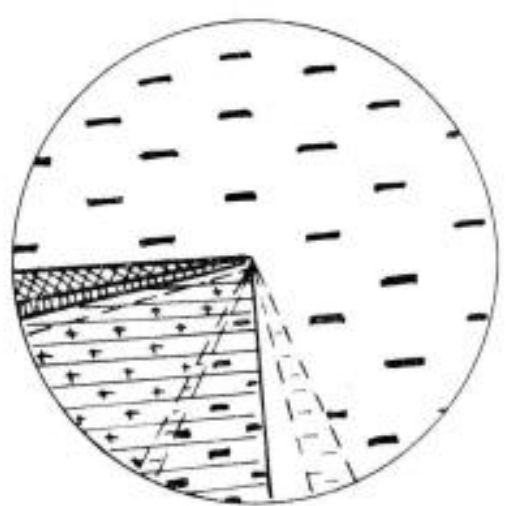

I. Picectum subalpinum vaccinetosum Vitis-idaeae (Relevés 1 à 11)

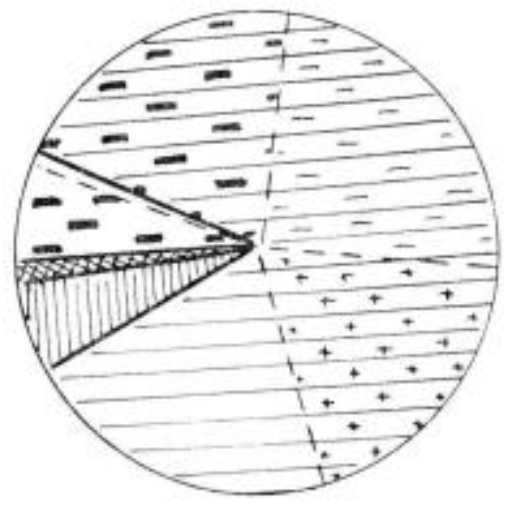

II. Piceetum subalpinum myrtilletosum (Relevés 16 à 25)

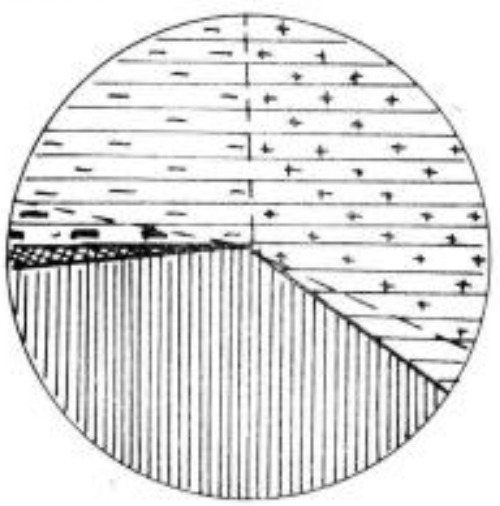

III. Piccetum subalpinum adenostyletosum. (Relevés 26 à 35)

- - Xérophiles de mor

[- - Xérophiles de moder Autres xérophiles

- Mésophiles de mor

- $=$ Mésophiles de moder

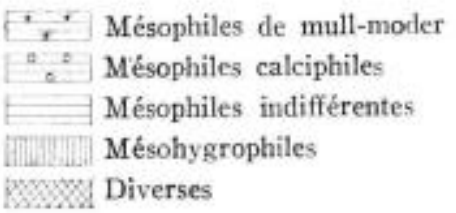

FIG. 43.

Légende. - Le spectre de la pessière xérophile se situe bien entre celui de la pineraie de Pin sylvestre à Deschampsia flexuosa et celui de la pineraie de $\mathrm{P}$ in Cembro homologue; de méme, celui de la pessière mésophile à Vaccinium Myrtillus est intermédiaire entre celui de la sapinière et celui de la rhodoraie à Pin Cembro. 


\section{Les Pessières}

Structures sylvicoles

I. Pessière mésophile

Nb.darbres/Ra.

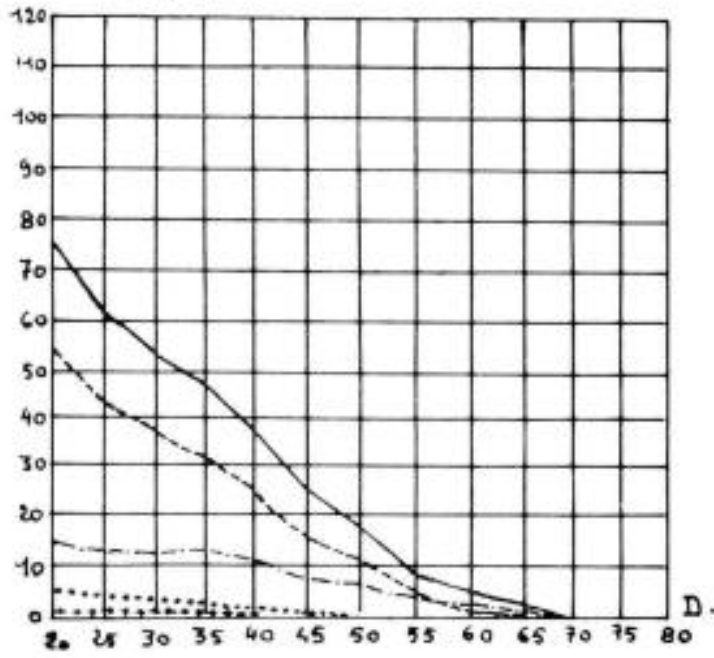

...... Epicéa

-... Mélèze

….......... Pin Cembro

\section{Pessière xérophile}

$\mathrm{Nb}$ d'arbres/ha

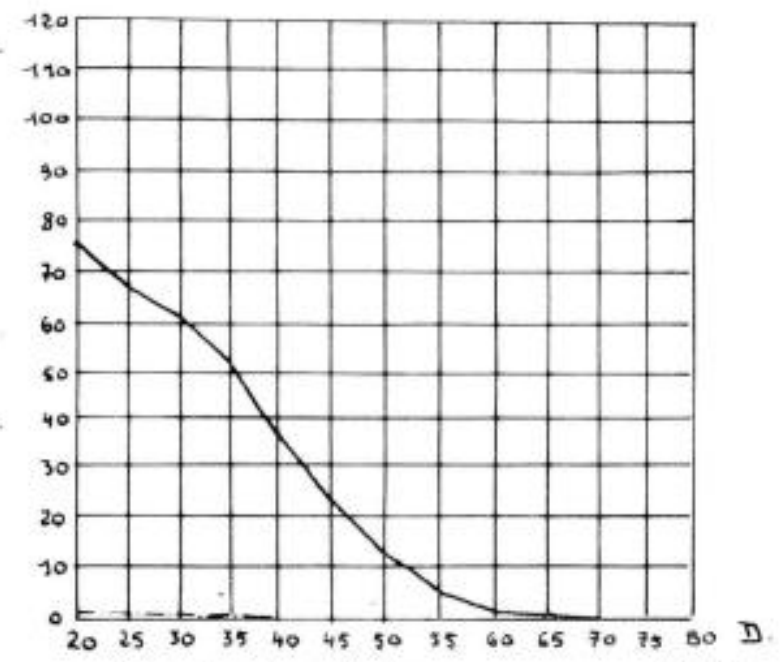

-- - Pins sylvestre et Cembro

Epicéa.

Fig. 44. 


\section{III.5.3.2. - Evolution - Climax.}

Nous retrouvons, à propos des pessières, un problème général et c'est la raison pour laquelle nous l'envisagerons, dans son ensemble, à la fin de ce travail : c'est celui de l'influence qu'exerce en montagne, où l'érosion joue un rôle considérable, la nature de la rochemère, influence qui amène à se poser la question suivante, dans le cas particulier qui nous occupe: existe-t-il ou non, à chaque exposition, un seul climax dans l'étage subalpin inférieur?

Les tableaux de relevés montrent qu'il y a une convergence certaine entre pessière sur schistes lustrés et pessière sur grès du Houiller - pour ne retenir que les pessières mésophiles. Mais, il faut noter que des différences subsistent: présence de Saxifraga cuneifolia et plus grande abondance de Festuca flavescens sur les sols à moder dérivant du Houiller.

Seule, une analyse statistique permettrait de dire, si ces différences sont plus faibles que celles observées dans l'étage montagnard; elles sont en tout cas plus fortes que celles que l'on pourra retenir dans l'étage subalpin supérieur, domaine du Pin Cembro où l'on ne peut pratiquement pas faire de distinction de types en fonction de la roche-mêre - sauf sur gypse, où l'érosion a beau jeu d'amenuiser la couche d'humus brut en formation.

Il semble donc bien que plus l'altitude est élevée, dans le domaine forestier, plus grande est l'uniformité des stations.

\section{III.5.3.3. - Structures forestières - Production.}

Les schémas 1 et 2 mettent très clairement en évidence les structures globales des deux grands types de Pessière: mésophile à Vaccinium Myrtillus et xérophile à Vaccinium Vitis-idaea.

Ligende. - Les structures globales des deux pessières subalpines (I. - Pessière mésophile - 195 ha; II. - Pessière xérophile - 135 ha) sont tout à fait comparables, étant noté toutefois que la pessière xérophile est un peu moins riche en gros bois.

Par contre, la pessière mésophile apparait comme sensiblement plus riche en espèces arborescentes que son homologue de versant sud; l'importance du mélèze est à retenir et elle traduit, dans l'ensemble, une influence humaine marquée.

La structure de ces deux grands types de pessière présente un certain déséquilibre (excès de bois de $30-35$; déficit de bois de 20 ) qu'il appartient au forestier de résorber sans plus attendre. 


\section{III.6. - LES PINERAIES DE PIN CEMBRO}

En Haute-Maurienne, les peuplements de Pin Cembro sont largement représentés, principalement sur les versants frais où on les observe sur toutes les roches-mères, tout au moins là où la pente ou la nature du terrain (éboulis), a empêché l'occupation humaine: de plus en plus cependant, à la suite du recul de l'homme, ils tendent à coloniser tout l'étage subalpin supérieur (saut, évidemment, les stations spécialisées), ce qui se traduit par l'existence d'un certain nombre de peuplements encore jeunes, peu évolués, particulièrement riches en Mélèzes.

Sur versants sud, par contre, ils sont rares et se localisent pratiquement sur les ćboulis dut Houiller ou sur les quartzites du synclinal de Chavières, non tutilisés par l'homme; sur schistes lustrés, aux formes plus arrondies et à la fertilité plus grande, l'influence humaine les a éliminés, et depuis fort longtemps selon toute vraisemblance.

Alors que sur versant nord - ou est - les espèces mésophiles dominent - comme Rhododendron ferrugineum et Calamagrostis rillosa - sur versants sud, ces espèces sont exceptionnellement représentées; il en est de méme de Vaccinium Myrtillus qui cède le pas à Vaccinitm Vitis-idaea, tandis que Juniperus communis stubsp. nana et intermedia et Arclostaphylos Uva ursi sont presque toujours présents. Et ces différences ne sont pas les seules, comme on le verra ci-après.

Elles sont telles, en tout cas, qu'elles permettent de distinguer des pineraies de Cembro mésophiles et d'autres relativement xírophiles.

\section{III.6.1. - Les pineraies de Pin Cembro mésophiles.}

Toutes ces pineraies ont un contingent d'espèces communes dont les plus importantes sont: Rhododendron ferrugineum, Lonicera cocrulea, Vaccinium Myrtillus, Vaccinium Vitis-idaea, Calamagrostis villosa... On peut cependant y distinguer des sous-associations et des faciès caractérisés par des espèces différentielles proprement dites ou par des "abondances-dominances différentielles " de certaines espèces, présentes partout, mais de façon très inégale. 


\section{PLANCHE XIV}

\section{Les pineraies de Pin Cembro}

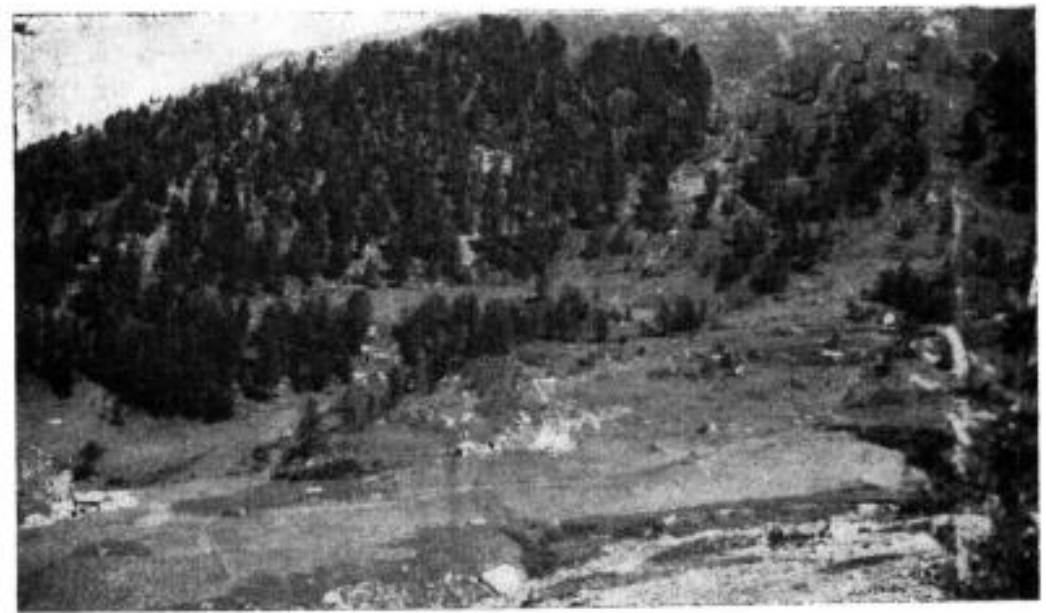

Pineraic de Pin Cembro sur schistes lustrés Vallon de Bramanette

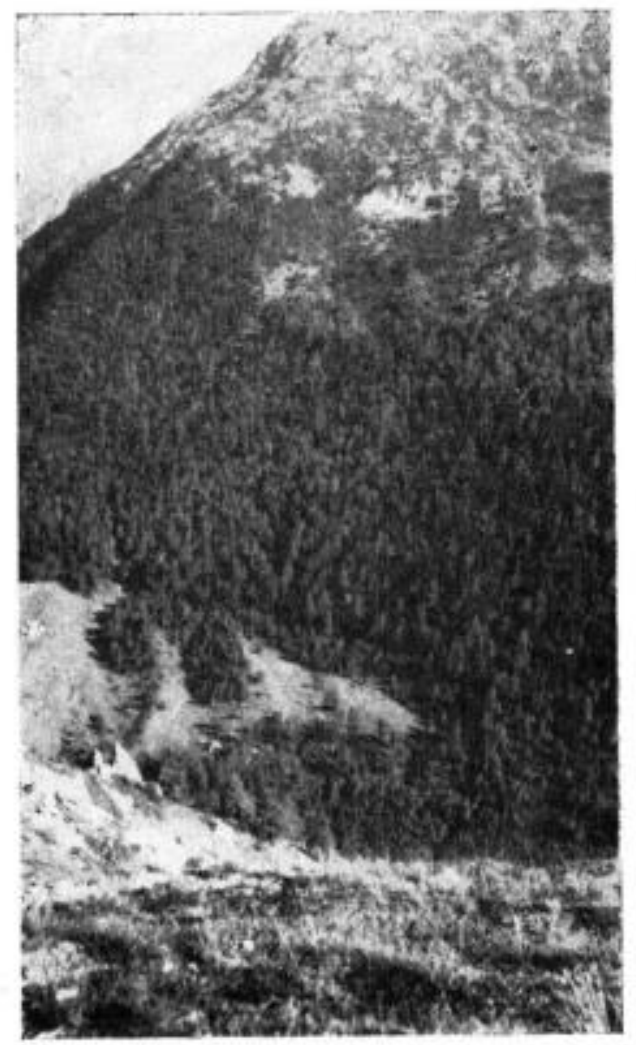

Pineraie de Pin Cembro sur Permo-houiller métamorphique. Massif d'Ambin - Vallon d'Etache. 
Il nous semble que trois types principas $x$ peuvent ètre mis en évidence:

- dans le premier, labondance du Rhododendron ferrugineum donne au groupement sa physionomie. Il est parfois dominant (faciès typique); parfois il laisse la première place à Vaccinium $M y$ tillus (faciès à Vaccinium Myrtillus); quelquefois enfin, il se présente par taches très disjointes entre lesquelles s'observent des espèces prairiales qui jouent alors le rôle de différentielles (faciès pâturé).

- dans le second, c'est Calomagrostis villosa, en nappe quasi continue - d'où émergent quelques rares bouquets de Rhododendron, - qui domine. Il se trouve d'ailleurs accompagné d'espèces prairiales, lui aussi.

- dans le troisic̀me, enfin, c'est Pinus montana subsp. uncinata et des espèces de mull ou de moder calciques qui jouent le ròle de différentielles.

Nous analyserons successivement ces trois sous-associations et ces faciès.

III.6.1.1. - La pineraie de Pin Cembro à Rhododendron ferrugineum.

\section{Organisation sociologique et composition floristique.}

On notera tout de suite que les deux faciès: celui à Rhododendron ferrugineum et celui à Vaccinium Myrtillus présentent la même organisation sociologique et la même composition floristique. Nous les étudierons done sans faire de distinction.

II y a lieu, sans tarder, de mettre laccent sur la constance et la dominance, dans la strate arborescente, du Pin Cembro qui constitue une excellente caractéristique; à ses côtés, Lari.x decidua est nettement moins bien représenté, sauf dans les peuplements jeunes: il en sera reparlé à propos de la dynamique de l'association; quant à Picea excelsa, il est toujours relégué en troisième position et ce, d'autant plus que l'on se rapproche de l'étage alpin.

Les groupes écologiques les mieux représentés sont ceux des espèces mésophiles à humus brut et des mésophiles à moder.

Parmi les premières, Rhododendron ferrugineum et Lonicera coerulea nous paraissent être les meilleures caractéristiques (10) parce que localisées dans l'étage subalpin. Ces espèces ont, en outre, un haut degré de constance. A leur côté, Vaccinium Myrtilltus est également très constant, son ahondance-dominance dans certains relevés ponvant même permettre de distinguer un faciès; il en est

(10) avec Pinus. Cembro, par conséquent, qui doit ĉtre considéré également comme caractéristique des sols à humus brut. 
de mème de Vaccinium Vitis idaca, toujours subordonné toutefois. Par contre, Empetrum hermaphroditum et Vaccinium uliginosum s'observent beaucoup plus rarement; ils n'atteignent leur optimum, dans notre dition, quau-dessus de la forêt de Pin Cembro, là où la lande prend nettement le pas sur la forêt proprement dite [Empetreto-Vaccinietum (11)].

Parmi les espèces de sols à moder, ou à mull-moder, sont à retenir Lusula silvatica subsp. Sieberi (12), Festuca flavescens et surtout Calamagrostis villosa qui se localise dans l'étage subalpin.

Parmi les Cryptogames, Barbilophozia lycopodioides, Barbilophosia barbata et Brachythecium Starkei, etc. sont à mettre à part parce que orophiles - ; les autres forment un groupe assez compact avec, notamment, Dicranum scoparium et Pleurozium Schreberi (13).

Mises à part les mésophiles indifférentes, surtout représentées par des Mousses d'ailleurs — dont Rhytidiadelphus triquetrus notre groupement comprend encore quelques mésohygrophiles dont Sorbus aucuparia et pas d'espèces de sols à mull, sauf celles qui, comme Geranium silvaticum paraissent avoir à l'égard de l'humus, une plasticité plus grande que celle de leurs compagnes.

Si l'on se réfère au tableau général d'association ou aux spectres floristico-écologiques qui en sont l'image, on constate que l'organisation sociologique de la pineraie de Pin Cembro à Rhododendron ferrugineum - ou à Vaccinium Myrtillus - est plus simple que celle des sous-associations voisines en ce sens que le nombre de groupes écologiques y est plus réduit. Ceci tient essentiellement au fait - nous aurons l'occasion de revenir sur ce point - que les conditions de milieu y sont moins diversifiées, moins complexes. Nous verrons, lors de l'étude de l'écologie, qu'elles ne sont cependant pas uniformes, et c'est ce qui explique, en particulier, que, à còté du groupe des mésophiles des sols à mor, se trouve bien représenté un groupe voisin, mais déjà différent, celui des mésophiles de sols à moder.

Cette légère hérérogénéité se retrouve, à peu près semblable à elle-même, dans tous les relevés; il en résulte, pour l'ensemble de la sous-assocation, une homogénéité générale certaine, que traduit

(11) Cette association, qui confine à l'étage alpin et constitue un groupement de transition entre les peluoses de cet étage et la rhodoraie à Pin Cembro, ne sera pas étudiée ici.

(12) Lusula silvatica subsp. Sieberi est considérée par Braun-Blanouet comme une caractéristique de l'association. It nous semble qu'elle ne doive pas ètre retenue comme telle, car elle s'observe avec une constance et une abondance-dominance comparables aussi bien dans la pessière subalpine que dans la sapinière vraie.

(13) Pleurosium Schreberi, considérée en plaine comme localisée sur les humus bruts, nous parait, en montagne - tout at moins dans notre dition comme plus plastique à l'égard du type d'humus. 


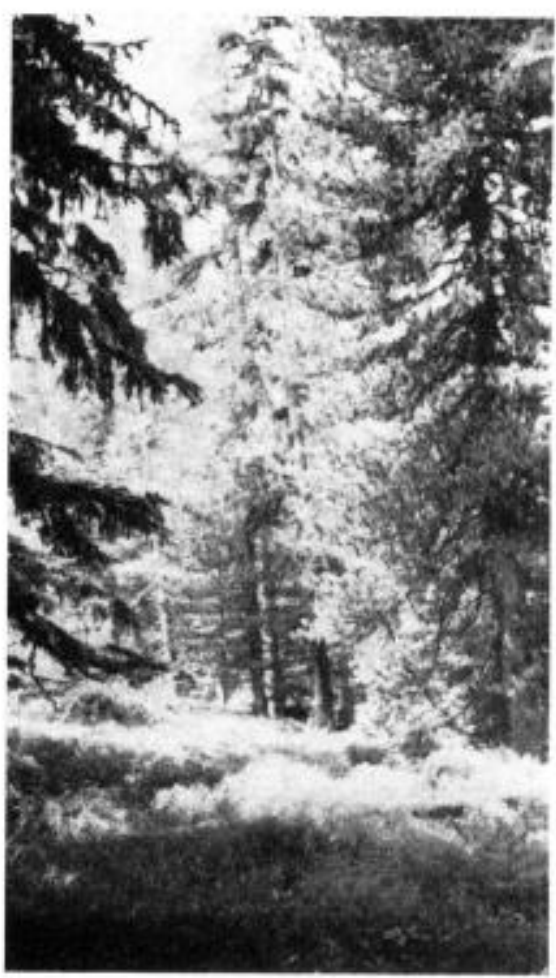

Forét du Thyl (Grès du Houtiller)

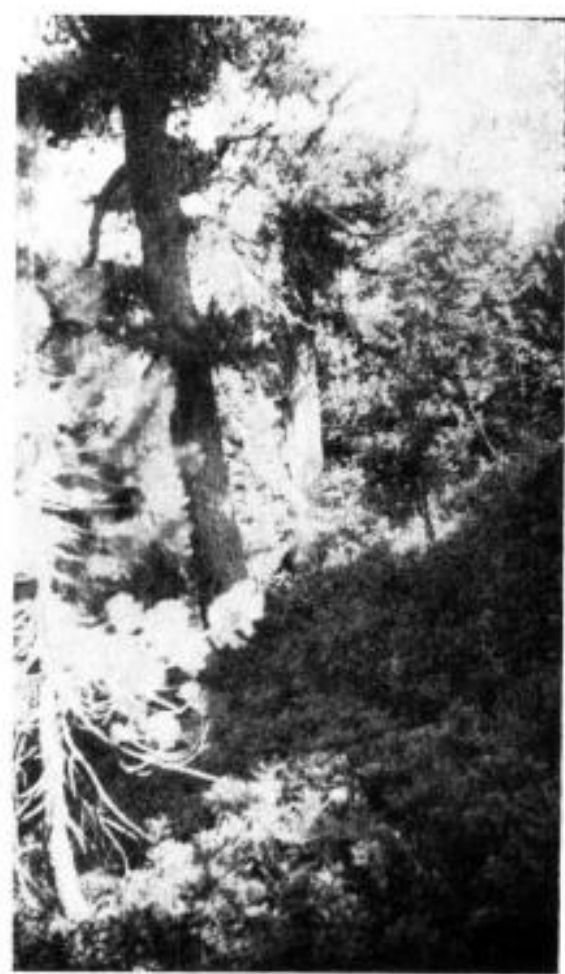

Forèt de Bramans (Schistes phylliteux d'Ambin). 
d'ailleurs le diagramme de présence: sur un total de 60 espèces environ, en effet, on en compte 27 , soit $45 \%$ dont le degré de présence est égal ou supérieur à $40 \%$.

La structure de la pineraie de Pin Cembro à Rhododendron ferrugineum - dont le spectre biologique donne, dans une certaine mesure, une image globale, - a subi, est-il besoin de le dire, l'influence humaine. Et tout particulièrement celle de la strate arborescente, que nous analyserons plus en détail, ultérieurement, lors de l'étude des caractéristiques sylvicoles: il s'agit, le plus souvent, d'une futaie claire, d'aspect régulier, ot̀ la régénération est làche et s'effectue pied par pied et non par masse; les courbes donnant le nombre d'arbres à l'hectare, par essence et par catégories de diamètres permettent de se faire une idée de cette structure. En dehors des jeunes arbres de moins de $0,10 \mathrm{~m}$ de diamètre (mesuré à $1,30 \mathrm{~m}$ ), Sorbus aucuparia constitue, pratiquement à lui seul, toute la sousstrate arborescente.

La strate arbustive est, par contre, particulièrement puissante: les nanophanérophytes, comme Rhododendron ferrugineum, Lonicera coerulea, Vaccinium Myrtillus, Rosa alpina, dominent une sous-strate de chamaephytes représentées essentiellement par Vaccinium Vitis idaea et où s'observe, notamment, Melampyrum silvaticum. Dans cette strate arbustive, les nanophanérophytes ne sont pas, en général, confusément mèlées, - surtout Rhododendron ferrugineum et Vaccinium Myrtillus -: elles forment des taches contiguës, plus ou moins étendues. Il en est de même des graminées - surtout représentées par Calamagrostis villosa - qui constituent des plages assez pures entre celles des Ericacées.

Vient enfin, la strate muscinale, dont l'importance varie beancoup d'un relevé à l'autre.

\section{2。 Identification - Dénomination.}

Si l'on se réfère au tableau général d'association, il est facile de se convaincre, à la lecture de la liste des espèces, que notre sousassociation s'identifie de façon très étroite avec le Rhodoreto-Vaccinietum. Cembretosum décrit en Engadine par Braun-Blanquet et étudié, depuis lors, dans toutes les régions de la Suisse ou de l'Autriche où l'on peut le rencontrer.

Il est intéressant de noter, si l'on se reporte à un tableau complet comme celui figurant dans l'étude sur le Parc national suisse (14) que certaines espèces, observées dans ce pays, ne figurent pas dans nos relevés. Il s'agit d'abord de Linnaea borealis, qui n'est d'ailleurs pas spécifiquement subalpine, et dont les dernières stations dans les Alpes occidentales s'observent dans la vallée de la Tarentaise

(14) Braun-Blanouft (J.), Pallamann (H.), Bach (R.) (1954). 
- au nord de la Maurienne, et dans le massif des Bauges (15). I1 s'agit ensuite de Trientalis europaea, rare en Suisse d'ailleurs, et signalé en Savoie au-dessus de Crest-Volant, dans la Tarentaise également; ces deux espèces, liées à des sols à humus brut, sont considérées par BRAUN-BLANQUET comme caractéristiques de la rhodoraie à Pin Cembro. Le reste du cortège coïncide, par contre - mis à part Rhododendron intermedium - de façon tout à fait remarquable avec son homologue suisse.

\section{3" Répartition - Ecologie.}

\section{Répartition générale}

La pineraie de Pin Cembro à Rhododendron trouve son optimum aux expositions les plus fraiches, nord et est; à l'ouest, elle s'affaiblit pour passer, aux expositions sud, à une association plus xérophile qui sera étudiée ci-après.

En altitude, elle s'étage de $1950 \mathrm{~m}$ environ à $2200 \mathrm{~m}$ environ laissant la place, plus haut, à l'Empetreto-Vaccinietum, au Loiseleurieto-cetrarietum, ou à des associations subalpino-alpines, marquées par le pâturage; là on observe encore quelques Mélèzes ou Pins cembros, petits par la taille, mais non par l'âge. Sur les crêtes où l'enneigement est moindre et la chaleur estivale plus forte, la forêt atteint une altitude maxima, très vraisemblablement " natırelle ", car on peut penser que, dans de telles stations, l'influence humaine a été et reste négligeable. Alors peut être atteinte l'altitude de $2300 \mathrm{~m}$ ! ; les derniers " arbres n - qui n'atteignent pas plus d'un mètre de haut - pouvant s'échelonner dans des groupements de transition avec ceux de l'étage alpin, jusqu'à $2400 \mathrm{~m}$ et même au-delà (16).

Aux altitudes inférieures, elle passe, plus ou moins progressivement, à la pessière subalpine.

\section{Climat}

Le climat général est évidemment subalpin et intra-alpin. Grâce aux postes météorologiques installés par E.D.F. à Plan d'Aval, au-dessus d'Aussois, à $2020 \mathrm{~m}$ et à Bissorte, dans les gorges houillères, à $2120 \mathrm{~m}$, qui se trouvent placés att niveat de la pineraie de Pin Cembro et à son exposition, on peut s'en faire une idée, encore que les renseignements obtenus ne concernent qu'un petit nombre d'années et paraissent sujets à caution, quant à l'importance des précipitations hivernales, comme on 1'a vu.

De 1949 à 1955 inclus, la moyenne annuelle des températures a été pour Bissorte de $2,9^{\circ}$ et pour Avrieux — situé à $1100 \mathrm{~m}$ et

(15) Perrizr de la Bathie (1917-1928), donne des indications détaillées à ce sujet. Fournier (1961) indique Linnaed borealis dans le Briançonnais.

(16) Sur une créte montant à laiguille de Scolette, nous avons rencontré le dernier Pin Cembro, à l'abri, à $2480 \mathrm{~m}$. 
que nous prendrons pour terme de comparaison - de $7,3^{\circ}$. Si l'on retient, en première approximation le chiffre de $0,44^{\circ}$ comme gradient thermométrique annuel, on voit que l'isotherme de $2^{\circ}$ qui correspond, selon OzEnDA (P.) (1954), à la limite de la forêt dans les Alpes du Sud et les Pyrénées se place à $2300 \mathrm{~m}$ environ au centre de notre dition, chiffre qui parait en effet très acceptable. Si l'on utilise maintenant l'isotherme de juillet (on sait que la limite nord des forêts dans l'Europe septentrionale suit sensiblement l'isotherme $10^{\circ}$ du mois de juillet et que, dans les Alpes suisses, la température moyenne de juillet au niveau de la limite des forêts oscille

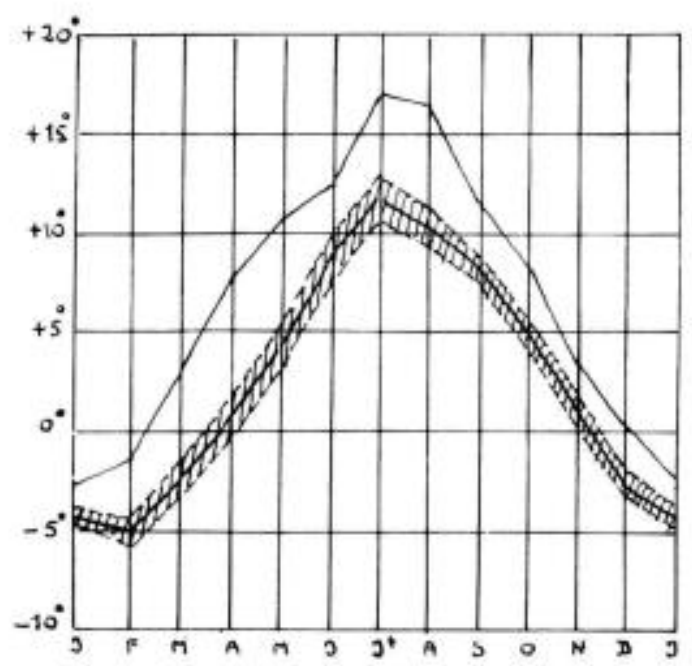

I. Avrieux. Zone du Pin sylvestre.

II. Bissorte. Zone du Pin Cembro. (1949-1955)

QIIIA Etage du Pin Cembro.

FIG. 45 .

Légende, - Régime des températures au nivean de la pineraie de Pin Cembro. Les courbes ci-dessus permettent de se faire une idée du régime moyen des températures ayant régné de 1949 à 1955 dans l'étage mésophile du Pin Cembro (Bissorte: $2120 \mathrm{~m}$ d'altitude) et dans celui, xérophile, du Pin sylvestre (Avrieux : $1100 \mathrm{~m}$ ).

On voit que les différences de température ont été les plus fortes au printemps, c'est-à-dire de mars à mai inclus, comme on pouvait s'y attendre, puisque c'est à ce moment-là que la pineraie de Pin sylvestre est déneigée, alors que celle de Pin Cembro conserve encore sa couveture de neige.

A partir du mois d'août, au contraire, la descente des températures à Avrieux est plus rapide qu'à Bissorte.

L'altitude joue donc le rôle de \& volant thermique $»$, tout at moins en ce qui concerne la température de l'air. 
entre $10,7^{\circ}$ et $7,8^{\circ}$ [OzENDA (1954) 301]), on constate, en utilisant cette fois-ci le gradient de juillet, soit $0,54^{\circ}$, que l'altitude de 2300 $\mathrm{m}$ correspond à un isotherme de $10,6^{\circ}$ qui se situe bien dans les limites définies ci-dessus.

En retenant toujours l'hypothèse que les températures moyennes, pour chaque mois, varient linéairement avec l'altitude, on peut tracer la " zone thermométrique $»-$ et son régime - qui correspond à l'étage occupé par la rhodoraie à Pin Cembro (fig. 45).

Si l'on rapproche les courbes de température de Bissorte (2 120 $\mathrm{m})$ de celles de $\mathrm{S}$. Charl $(1815 \mathrm{~m})$ et de Buffalora $(1977 \mathrm{~m})$, deux stations de Haute-Engadine, situées au niveau de la ceinture des peuplements de Pin Cembro, on constate que ces dernières jouissent d'un climat sensiblement plus froid, surtout en hiver et que la montée des températures au printemps y est plus brutale. Ce fait semble s'expliquer par le caractère plus continental du climat, caractère sur lequel nous avons déjà appelé l'attention (fig. 46).

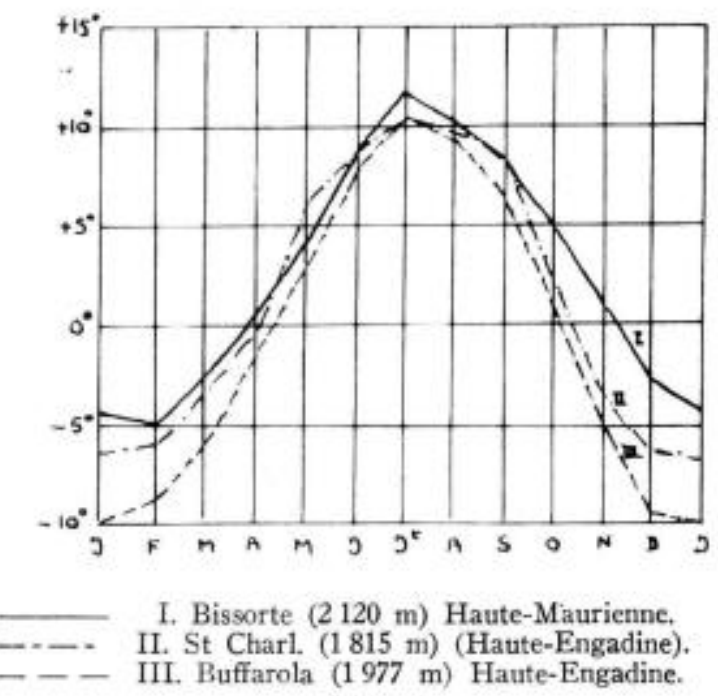

FIG. 46.

Nous avons pu, d'autre part, faire une brève analyse microclimatique qui, bien que portant sur un seul individu d'association, ne nous parait pas dénuée d'intérêt.

Du 28 juillet au 16 août 1955 , nous avons donc comparé un individu de l'Ononido-Pinetum (parcelle 4 de la forêt de Modane altitude: $1240 \mathrm{~m}$, exposition sud) à un individu du RhodoretoVaccinietum cembretosum (parcelle 11 de la forêt de Bramans altitude: $1950 \mathrm{~m}$, exposition nw) (fig. 47). 


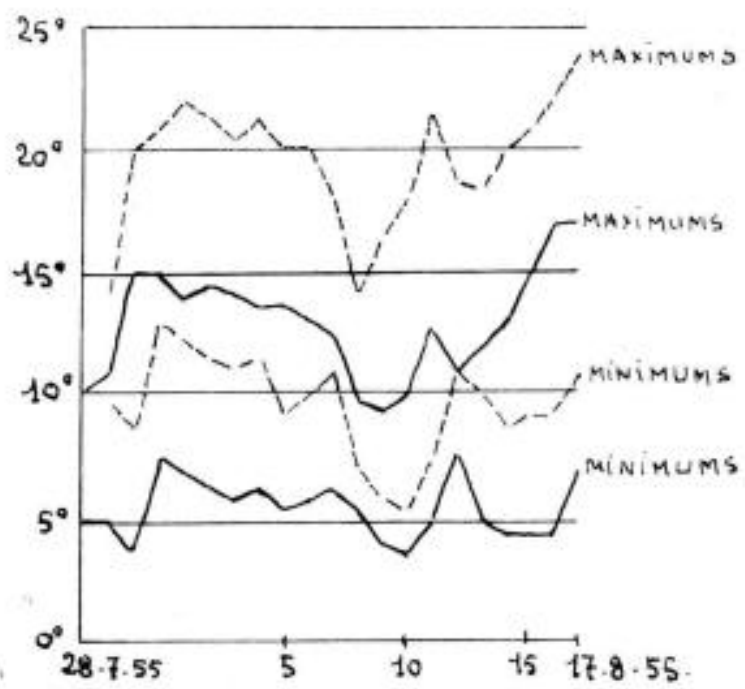

Amplitude des températures extrêmes.

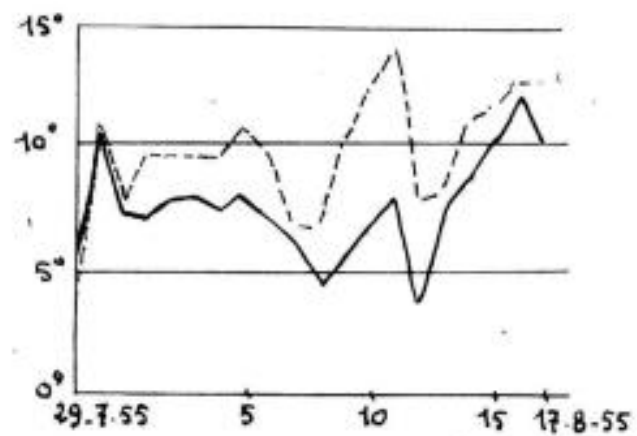

Différences entre les températures extrêmes.

... Ononido-Pinetum (1 $240 \mathrm{~m})$.

Rhodoreto-Vaccinietum cembretosum (1950 m).

FIG. 47. 
On constate, d'abord, que la température moyenne de l'air, à $1,50 \mathrm{~m}$, durant cette période, est sensiblement plus faible dans la pineraie de $\mathrm{P}$ in Cembro que dans celle de Pin sylvestre $\left(8,4^{\circ}\right.$ contre $\left.13,8^{\circ}\right)$. Cette différence n'a rien d'absolu et se fut trouvée atténuée si l'individu de la pineraie de Pin sylvestre avait été choisi à une altitude plus forte, vers la limite supérieure de ses possibilités d'existence. On peut penser qu'elle n'eut pas disparu, loin de là : en effet, en comparant un Pineto caricetum humilis - association qui présente avec l'Ononido-Pinctum d'étroites affinités - situé à $1910 \mathrm{~m}$, à exposition sud, et un Rhodoreto-V accinietum cembretosum situé à $1850 \mathrm{~m}$ à exposition nord, les Suisses ont observé des différences encore très nettes de températures de l'air (prises à $1,50 \mathrm{~m}$ au-dessus du sol) : du 20 juin au 31 juillet $1941: 16,7^{\circ}$ pour la première association contre $14,2^{\circ}$ pour la seconde; et du 31 juillet 1941 au 10 octobre 1941, 15,6 $6^{\circ}$ contre 11,4 $4^{\circ}$ [BraunBlanguet (J.), Pallmann (H.) et Bach (R.) (1954)].

Les amplitudes journalières de la température de l'air sont, comme on pouvait s'y attendre, beaucoup plus fortes dans la pineraie de Pin sylvestre, qui jouit done d'un climat plus brutal.

La température du sol à $-2 \mathrm{~cm}$ est restée toujours inférieure, en moyenne, à celle de l'air (pour la période considérée: $\mathrm{t}$ air (à

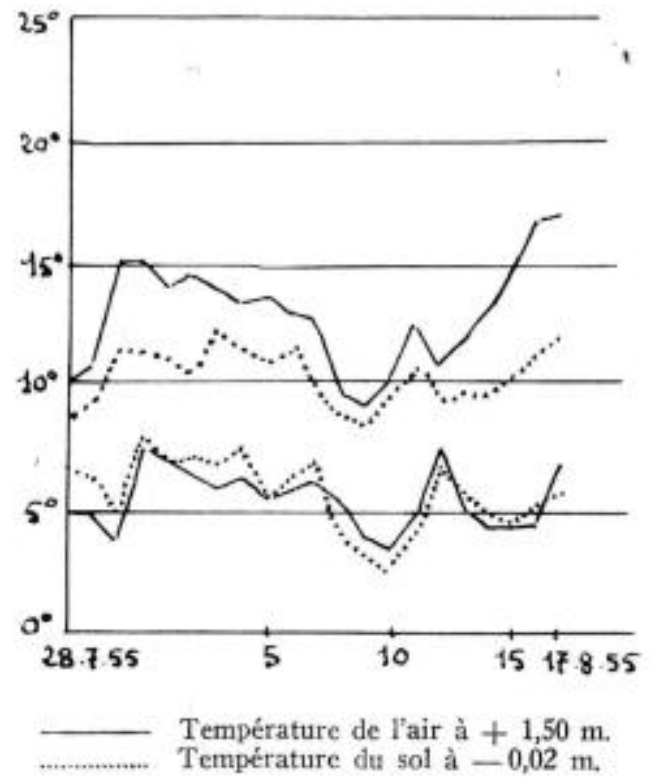

FIG. 48.

Légende. - Températures de l'air et du sol (maximums et minimums) dans une pineraie de Pin Cembro. 
$1,50 \mathrm{~m}): 8,4^{\circ} ; \mathrm{t}$ sol $(\mathrm{a}-2 \mathrm{~cm}): 5,9^{\circ}$, et l'amplitude de ses variations journalières a toujours été inférieure à celle de la température de l'air (fig. 48). Ces résultats concordent avec ceux obtenus dans le Parc national suisse, où la température du sol a été prise plus bas, il faut le noter $(-10 \mathrm{~cm})$. En effet, dans une rhodoraie à Pin Cembro, à $1850 \mathrm{~m}$ d'altitude, la température de l'air à $1,50 \mathrm{~m}$ a été, du 20 juin au 31 juillet 1941 , de $14,2^{\circ}$ en moyenne et celle du sol de $9,6^{\circ}$. Si l'on se reporte aux résultats obtenus par les Suisses, on constate que les différences de températures de l'air et du sol s'atténtuent en hiver, et on peut penser que le maximum de cette différence doit se situer en juillet-août.

Ce qu'il nous parait essentiel de retenir, c'est que le microclimat - de l'air et des horizons superficiels du sol - de la pineraie de Pin Cembro est peu brutal. Il rappelle tout à fait, à ce point de vue, celui de la sapinière vraie ou de la pessière subalpine de versant nord, tout en se montrant plus froid.

Les courbes de déficit de saturation montrent, enfin, qu'il est sensiblement plus humide que celui de la pineraie à Ononis rotundifolia (fig. 23).

\section{Sols}

La pineraie de Pin Cembro à Rhododendron se développe sur toutes les roches-mères présentes, dans l'étage subalpin, en HauteMaurienne. Sur calcaire ou sur gypse, elle s'observe - mais de facon très fragmentaire, nous verrons pourquoi - sous sa forme à Pinus uncinata, que nous étudierons plus loin. Sur schistes lustrés, elle parait, a priori, moins développée que sur les roches métamorphiques du permo-houiller (massif d'Ambin) on sur les grès et les schistes du Houiller. Ce problème, envisagé pour l'ensemble des Alpes suisses - a préoccupé Furrer (E.) (1955): l'examen de la répartition du Pin Cembro sur différentes roches-mères avait d'abord fait conclure - à Klebelsberg (R.) en particulier (1952) - que cet arbre fuyait les schistes lustrés. Il n'en est rien: les schistes lustrés, de composition chimique relativement riche, donnent de bons sols de pelouse, et ce fait a contribué au défrichement des forêts qu'ils portaient, d'autant plus qu'aux confins de l'étage alpin existe souvent un replat glaciaire, où les schistes lustrés, qui se délitent facilement, ont donné des formes arrondies sans gros éboulis. C'est donc sur les schistes lustrés que la forêt de Pin Cembro a le plus souffert de l'action humaine et c'est sur eux, depuis le recul de l'homme que s'observent ses formes les plus jeunes.

La pineraie de Pin Cembro à Rhododendron est, dans l'ensemble, développée sur des sols à humus brut. Mais il ne faudrait pas s'imaginer, toutefois, que le sol est d'un type uniforme ou même que la couche d'humus brut forme un tapis continu, de même épaisseur et sans " trous ». En fait, on rencontre cette pineraie dans des zones 
à forte pente, souvent riches en gros blocs, si bien que l'érosion y est extrêmement diversifiée: il ne faut pas oublier, non plus, que ces forêts bien que difficiles d'accès, continuent à faire l'objet d'exploitations et que l'enlèvement et le trûnage des arbres ajoutent à l'érosion naturelle très différenciée, une érosion "artificielle " non négligeable. Et ceci explique, en particulier. l'existence de " plages n de graminées (Calanagyrostis villosa, Poa Choixii, etc.) dont l'optimum se situe sur des sols à moder - ou à moder-mull et dont nous avons pu constater souvent qu'elles se localisaient aux endroits mème où avaient été exploités des arbres et où s'était donc produit une minéralisation de Thumus. II ne faut pas oublier, enfin, que l'horizon humifere comprend detr couches principales: tune couche $\mathrm{A}_{0}$, d'épaisseur variable on l'a vu, et une couche $\mathrm{A}_{1}$ qui correspond, en fait, à un moder. On comprend mieux, dès lors, l'organisation sociologique de l'association, surtout si l'on tient compte de l'amplitude que peuvent présenter, à l'égard du type d'humus, des espèces affines.

Dans cette mosaique - òt domincut très largement des sols d̀ humus brut - on va done rencontrer des rankers d'érosion et des sols lessivés à moder, toujours localisés; des rankers ì humus brut. c'est-à-dire des sols très jeunes, ne présentant ni horizon $\mathrm{A}_{2}, \mathrm{ni}$ horizon $B$, les couches $A_{0}$ et $\backslash$, reposant directement sur la rochemère en décomposition : enfin et surtout, des sols podzoliques et des podzols, ces derniers s'observant sur les replats où l'érosion ne peut se manifester que difficilement. Tous ces sols ont un caractère commun qui est leur jeunesse : ce caractère, dî à l'érosion, se traduit par une faible épaisseur de tous les horizons et par leur grande richesse en fragments de la roche-mère, fragments qui apparaissent jusque dans les horizons humifères.

Nous donnons ci-après les analyses sommaires de cinq sols et les descriptions de profil de trois d'entre eux.

Le premier est un sol brun lessivé à moder. sur schistes lustrés: les trois suivants, des sols podzoliquues ferrugineux développés sur schistes lustrés, grès du Houiller et le dernier, enfin, un podzol sur grès métamorphique du Permo-Houiller.

Description morphologique - Fig. 49.

Sol $n^{\circ} 1$ - sol lessivé à moder (sur schistes lustrés).

$\mathrm{A}_{0}-(0-1 \mathrm{~cm})$ - litière de feuilles morte.

$A_{1}-(2-13 \mathrm{~cm})$ - horizon humifère, noirâtre - structure particulaire, avec quelques petits agrégats - limite inférieure bien tranchée.

A. - (14 - $43 \mathrm{~cm})$ - couleur gris beige, texture limono-argileuse - structure partiellement détruite, granulaire. Squelette

B - $(44-75 \mathrm{~cm})$ abondant (30\% du volume).

B - $(44-75 \mathrm{~cm})$ - couleur grise (schistes lustrés), texture limoneuse, structure granulaire. Des taches rouilles au niveau des fragments de roche-Squelette abondant $(60 \%$ du volume). 
Sol $n^{*} 2-$ sol podzolique ferruginew (sur grès du Houiller).

$A_{0}-(0-2 \mathrm{~cm})$ - litière de feuilles mortes (rhododendron) - structure fibreuse.

$\mathrm{A}_{1}-(3-12 \mathrm{~cm})$ - horizon humifère noir-brunattre - structure particulaire, avec grains de quartz nus. Limite inférieure assez bien tranchée.

$A_{2}$ - $(13-17 \mathrm{~cm})$ - beige clair, texture limono-sableuse, structure particulaire. Squelette assez abondant ( $20 \%$ du volume).

B - $(18-70 \mathrm{~cm})$ - beige foncé, texture limoneuse - structure grantlaire - squelette abonlant ( $50 \%$ du volume).

Sol n" 3-podzol (sur grès métamorphique dn Permo-Houiller).
$A_{6}-(0-10 \mathrm{~cm})$ - litière de feuilles mortes, de 2 à 3 me d'épaisseur. surmontant une couche de fermentation, de struc-
A - ture fibreuse.
$A_{1}-(11-20 \mathrm{~cm})$ - horizon humifère de structure particulaire, avec grains de guartz nus et contenant de menus frag- ments de schistes sériciteux (10\% du volume).

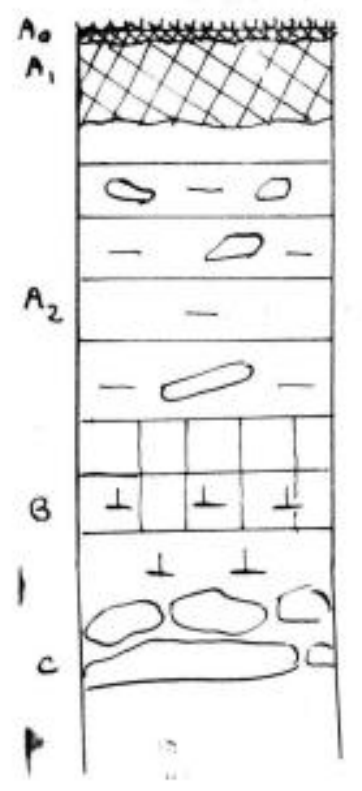

Foovu Humus brut Moder

- Horizon lessivé … Horizon cendrenx

$$
c=1 / 10^{\circ}
$$
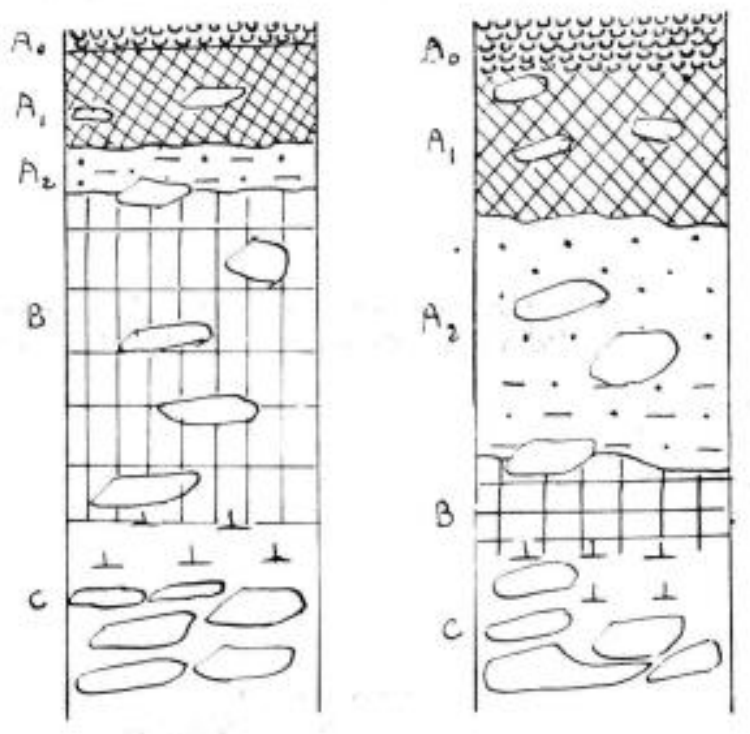

Fic. 49 , 
$\mathrm{A}_{2}-(21-45 \mathrm{~cm})$ - horizon cendreux, à structure particulaire; squelette assez important (15\% du volume).

B-C - $(46-60 \mathrm{~cm})$ - texture limoneuse, couleur ocre foncé, structure granulaire - importants fragments de roche-mère (40 à $50 \%$ du volume total).

Analyses.

1. Sol brun, trìs légèrement lessivé à moder.

Forêt communale d'Avrieux - Canton de la Montonnaz - Parcelle D.

Pente $50 \%$ - Exp. N - Alt.: $2000 \mathrm{~m}$ - Roche-mère: Schistes lustrés

\begin{tabular}{|c|c|c|c|c|c|c|c|c|c|c|c|}
\hline $\begin{array}{l}\text { Bori- } \\
\text { zons }\end{array}$ & $\begin{array}{l}\text { Tpais- } \\
\text { genur }\end{array}$ & $\begin{array}{l}\text { Ordonnéø } \\
\text { du pról. }\end{array}$ & pH & Arg. & Ins, & Sablo & H.O & Yat. 0 . & Cailloux & Fer. & deaervations \\
\hline Al & $12 \mathrm{~cm}$ & $-5 \mathrm{~cm}$ & 5,8 & & & & & & & & \\
\hline$A 2$ & $30 \mathrm{~cm}$ & $-30 \mathrm{ar}$ & 5,8 & I4 & 25 & IB & 1,4 & 2,7 & 73,3 & 1,02 & $\begin{array}{l}\text { Indice } d^{r} \text { ent. } \\
\text { du for : }\end{array}$ \\
\hline $\mathrm{B} / \mathrm{C}$ & $40 \mathrm{~cm}$ & $-50 \mathrm{~cm}$ & 5,7 & 5,5 & 16 & 42 & 0,6 & 0,7 & 71,3 & 1,45 & 1,1 \\
\hline
\end{tabular}

$2^{*}$ Sols podzoliques jeunes.

a) Forêt communale d'Avricux - Canton de la Montonnaz - Parcelle E.

Pente $30 \%$ - Exp. N - Alt: $2100 \mathrm{~m}$ - Roche-mère: Schistes lustrés.

\begin{tabular}{|c|c|c|c|c|c|c|c|c|c|c|c|}
\hline $\begin{array}{l}\text { Bort- } \\
\text { gons }\end{array}$ & $\begin{array}{l}\text { spols- } \\
\text { seur }\end{array}$ & $\begin{array}{l}\text { Profond. } \\
\text { du prél. }\end{array}$ & $\mathrm{pH}$ & Arg. & Lis. & Sable & $\mathrm{a} 2 \mathrm{O}$ & Ist. 0. & Casiloux & Fer & Obeervations \\
\hline $\mathrm{Ao} / \mathrm{Al}$ & 15 or & $-10 \mathrm{ca}$ & 4 & & & & & & & & \\
\hline $\mathrm{A2}$ & $7 \mathrm{~cm}$ & -18 ca & 4,9 & 14 & 29,5 & 15 & 1.7 & 8,2 & 44,4 & 0,40 & $\begin{array}{l}\text { Inlice d'ent. } \\
\text { du fer : }\end{array}$ \\
\hline$B / C$ & 30 ou & $-35 \mathrm{ct}$ & 5,2 & 10 & 27,5 & 26,5 & 1 & 1,9 & 56,8 & 1,42 & 3,8 \\
\hline
\end{tabular}

b) Forêt communale du Thyl - Série unique - Parcelle H.

Relevé floristique $\mathrm{n}^{\circ} 2$ - Roche-mère: grès du Houiller.

\begin{tabular}{|c|c|c|c|c|c|c|c|c|c|c|c|}
\hline $\begin{array}{l}\text { Jori- } \\
\text { zonn }\end{array}$ & $\begin{array}{l}\text { Bpais- } \\
\text { sear }\end{array}$ & $\begin{array}{l}\text { Profond. } \\
\text { du prél. }\end{array}$ & pHe & Arg. & Lin. & 3.P. & s.c. & 120 & Nint. 0. & Per & Ubservatfana \\
\hline$A 0 / 21$ & $12 \mathrm{~cm}$ & -60 & 3,8 & & & & & & 65,4 & & \multirow{3}{*}{$\begin{array}{c}\text { Indice d'ent. } \\
\text { du fer } t \\
1,7\end{array}$} \\
\hline$\$ 2$ & $4 \mathrm{ces}$ & $-15 \mathrm{~cm}$ & 3,8 & 12,5 & 32 & 30,2 & 19,7 & 2,2 & 3,4 & 0,8 & \\
\hline $\mathrm{B} / \mathrm{C}$ & 40 on & $-40 \mathrm{~cm}$ & 4,4 & 10,5 & 21 & 28,7 & 35,8 & 1,8 & 2,2 & 1,4 & \\
\hline
\end{tabular}

c) Forét communale d'Orelle - $1^{\text {re }}$ série - Parcelle n

Relevé floristique $\mathrm{n}^{\circ} 3$

Pente $30 \%$ - Exp. N - Alt. $2020 \mathrm{~m}$ - Roche-mère: grès houiller.

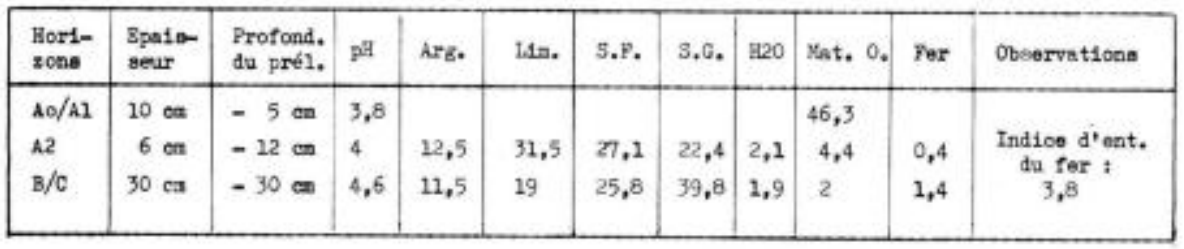


3* Podsol jeunc,

Forit communale de Bramans - Canton d'Ambin - Parcelle II.

Pente $30 \%$ - Exp. N-NW - Alt. : $1920 \mathrm{~m}$ - Roche-mère: éboulis de permohouiller (grès) - Relevé floristique $n^{\prime \prime} 7$.

\begin{tabular}{|c|c|c|c|c|c|c|c|c|c|c|}
\hline $\begin{array}{l}\text { Hor1- } \\
\text { 2ong }\end{array}$ & $\begin{array}{l}\text { Epois- } \\
\text { gear }\end{array}$ & $\begin{array}{l}\text { Profond. } \\
\text { du surel. }\end{array}$ & $\mathrm{pH}$ & $\mathrm{Arg}$. & Linon & Sablo & $h 20$ & Mat, organ. & fer & Oboervationa \\
\hline$A_{0} / h 1$ & $20 \mathrm{~cm}$ & $-10 \mathrm{c}$ & 3,8 & & & & & & & \multirow{3}{*}{$\begin{array}{c}\text { Indiee a'entr. } \\
\text { du for t } \\
4,1\end{array}$} \\
\hline$\$ 2$ & $25 \mathrm{~cm}$ & $-25 \mathrm{~cm}$ & 5,2 & 7 & 23 & 32 & 0,4 & 1,4 & 0,75 & \\
\hline $\mathrm{B} / \mathrm{C}$ & 1200 & $-50 \mathrm{ct}$ & 5,4 & 6 & 15.5 & 34 & 0,7 & 1,2 & 1,02 & \\
\hline
\end{tabular}

Alors que le sol lessivé présente des horizons peu différenciés et un indice d'entrainement du fer de 1,4 seulement, les sols podzoliques et le podzol ont des horizons nets; ils se différencient entre eux, par les caractères suivants: les sols podzoliques ont un horizon $A_{0} / A_{1}$, moins épais que celui du podzol; il en est de même de l'horizon $\mathrm{A}_{2}$. L'indice d'entrainement du fer $y$ est respectivement de 1,7 à 3,8 , alors qu'il atteint 4 dans le podzol. On notera que, pour tous ces sols, le taux d'argile n'augmente pas avec la profondeur. Ce fait, déjà mis en évidence par Duchaufour ( $\mathrm{Ph}$.) s'explique par un phénomène de lessivage oblique s'exerçant le long des pentes. Malgré l'érosion - qui explique l'abondance des fragments de la roche-mère jusque dans les horizons humifères - la forte accumulation d'humus brut a puissamment contribué à podzoliser un sol jeune, par altération directe.

L'accumulation de lhumus brut s'explique, elle, par le climat froid de l'étage subalpin et la présence d'Ericacées particulièrement développées. Cet humus, on le sait, est sensiblement plus riche en azote, en phosphore et en bases que l'humus brut des sols podzoliques ou des podzols atlantiques. On observera, en outre, que, contrairement à ce qui se passe dans les sols homologues atlantiques, l'indice d'entrainement du fer y est faible: ceci s'explique par la libération de ce métal (altération) dans les horizons supérieurs qui en contiennent encore des réserves (fragments de rochemère).

\section{$4^{\circ}$ Faciès.}

Le faciès à myrtille, on l'a vu, correspond à une variante qui diffère très peu de la rhodoraie-type, si ce n'est par la dominance absolue de Vaccinium Myrtillus. Par contre, le faciès que nous appelons " faciès pâturé " présente une organisation floristicosociologique qui appelle une analyse spéciale.

Comme le montrent le tableau d'association - et les spectres correspondants - ce facìs possède une organisation sociologique relativement complexe: en effet, à côté des groupes d'espèces liées à 
des sols à humus brut - dont l'importance diminue - ou à des sols à moder dont l'importance augmente légèrement - s'observe un groupe d'espèces mésophiles, liées à des sols à mull ou à mullmoder. Parmi ces espèces, il en est de relativement ubiquistes, telles Poa Chaixii, Millium effusum, Poa nemoralis...; il en est d'autres, au contraire, qui appartiennent plus spécialement aux prairies à Trisetum flavescens des étages montagnard supérieur et subalpin: Tristeum flavescens, Phleum alpinum, Poa alpina, Campanula rhomboüdalis..., d'autres enfin, plus hygrophiles: Geranium silvaticum, Phyteuma Halleri, font la liaison avec le groupe des mésohygrophiles à mull qui est, dans ce faciès, sensiblement plus étoffé et joue, avec le précédent, un rôle différenciateur.

La complexité de cette organisation - qui se retrouve dans tous les relevés - n'empêche nullement le faciès de présenter, dans son ensemble, une homogénéité certaine.

La structure des strates arbustive et herbacée est du type mosaique: les taches de Rhododendron et de Myrtille laissant entre elles la place à une pelouse, plus ou moins pâturée; les arbres se localisant, soit dans les Ericacées, soit dans les Genévriers, où ils se trouvent protégés de la dent du bétail. Il faut noter ici, que le pâturage étant en régression, on observe souvent une régénération assez abondante du Mélèze dans les places laissées "vides „ par les Rhododendrons ou les Myrtilles.

Les sols, dans une large mesure, reflètent la végétation: sous les taches d'Ericacées, la podzolisation rappelle celle des sols de la rhodoraie type; ailleurs, il s'agit de sols bruns à mull-moder (17) dont le $\mathrm{pH}$ ne descend pas au-dessous de 5 , et où l'on n'observe pas de lessivage du fer. Nous en donnons ci-après deux exemples (18).

\begin{tabular}{|c|c|c|c|c|c|c|c|c|c|c|}
\hline $\begin{array}{l}\text { Hori- } \\
\text { zons }\end{array}$ & $\begin{array}{l}\text { Epais- } \\
\text { seur }\end{array}$ & $\begin{array}{l}\text { Profond. } \\
\text { du prsi. }\end{array}$ & $\mathrm{pH}$ & Arg. & Linon & Sables & b20 & Vlat. org. & fer & Observations \\
\hline $\begin{array}{l}\mathrm{A} 0 / \mathrm{hI} \\
\mathrm{A2} \\
(\mathrm{B})\end{array}$ & $\begin{array}{l}3 \text { en } \\
8 \text { on } \\
?\end{array}$ & $\begin{array}{l}-2 \mathrm{en} \\
-7 \mathrm{~cm} \\
-17 \mathrm{~cm}\end{array}$ & $\begin{array}{l}5,5 \\
6,2 \\
5,8\end{array}$ & $\begin{array}{l}12,5 \\
11\end{array}$ & $\begin{array}{l}35.5 \\
38\end{array}$ & $\begin{array}{r}9 \\
11\end{array}$ & $\begin{array}{l}0,8 \\
0,8\end{array}$ & $\begin{array}{l}0,5 \\
0,2\end{array}$ & $\begin{array}{l}0,84 \\
0,74\end{array}$ & $\begin{array}{l}\text { Indice d'entret. } \\
\text { du fer : } 1\end{array}$ \\
\hline $\begin{array}{c}\mathrm{AO} / \mathrm{AL} \\
\mathrm{A2}(\mathrm{B})\end{array}$ & $\begin{array}{l}3 \mathrm{en} \\
20 \mathrm{en}\end{array}$ & $\begin{array}{l}-2 \mathrm{ca} \\
-15 \mathrm{ca}\end{array}$ & $\begin{array}{l}5,3 \\
5,3\end{array}$ & $\begin{array}{l}11,5 \\
10,5\end{array}$ & $\begin{array}{l}16,5 \\
15\end{array}$ & $\begin{array}{l}17 \\
33,5\end{array}$ & $\begin{array}{l}1,8 \\
1,3\end{array}$ & $\begin{array}{l}7,6 \\
4,1\end{array}$ & $\begin{array}{l}0,56 \\
0,52\end{array}$ & \\
\hline
\end{tabular}

(17) Ces sols présentent quelquefois une couche $A_{0}$ de 2 ou $3 \mathrm{~cm}$ d'épaisseur, au maximum.

(18) $1^{\text {er }}$ sol: forêt communale de Bramans - Canton d'Amblin - Parcelle 11 Pente $40 \%$ - Exp. NW - Alt. $1890 \mathrm{~m}$.

2 sol: forèt communale de Villarodin-Bourget - Canton de la Roche - Parcelle 12 - Pente $40 \%$ - Exp. NW - Alt. $1900 \mathrm{~m}$. 


\section{III.6.1.2. - La pineraie de Pin Cembro à Calamagrostis vil- losa,}

$1^{\circ}$ Organisation sociologique et composition floristique.

Alors que Calamagrostis villosa joue, dans la pineraie à Rhododendron, un rôle relativement secondaire, cette Graminée arrive, dans certains cas, à dominer de façon absolue et à donner à la forêt de Pin Cembro et de Mélèze, unc physionomie particulic̀re.

Un simple coup d'œil jeté sur le tableat général montre que son organisation sociologique et sa composition floristique rappellent de très près celles du faciès pâturé de la Rhodoraie. Il existe d'ailleurs entre ces deux groupements tous les intermédiaires, d'autant qu'un pâturage extensif s'exerce souvent dans la Calamagrostidaie. Mais la dominance de Calantagrostis villosa fait que le groupe écologique des espèces liées à des sols à moder est nettement prédominant sur les autres et c'est, aut fond, ce qui nous a conduit à séparer cette sous-association du groupement affine de la Rhodoraie, étudié précédemment.

Comme dans la Rhodoraie, la strate arborescente présente une structure régulière dans l'ensenble, et la densité des arbres y est faible. On notera, au passage, que le Mélẻze y est bien représenté et qu'il est, le plus souvent, plus abondant que le Pin Cembro luiméme. Ce qui frappe le plus - dans la strate arbustive - c'est, sinon l'absence, du moins, lextrême rareté de la régénération des arbres, dont nous reparlerons d'ailleurs. II semble qu'il faille en rechercher la cause dans la concurrence mécanique et physiologique créée par Calamagrostis villosa qui forme dans l'horizon A un lacis de racines et de radicelles extrêmement dense. Les semis de Mélèze et de Pin Cembro se localisent dans les rares endroits où Calamagrostis villosa a reculé devant une végétation de pelouse à base de Geranium silvaticum, Alchemilla vulgaris, Ranunculus geraniifolius, etc. En creusant des banquettes - qui doivent être éventuellement rafraichies, car la Graminée s'avère rapidement envahissante - des semis s'installent, qui ne connaissent plus la concurrence pour l'eau, en particulier, provoquée par le Calamagrostis.

En dehors de petites tâches de Rhododendron, les Ericacées sont faiblement représentées, sans jamais être absentes: sous la strate des Graminées, en effet, Vaccinium Myrtillus se rencontre toujours, mais le plus souvent sous la forme de petits plants chétifs, et ne formant pas de touffes. Il est accompagné de Mousses, rarement très abondantes.

\section{$2^{\circ}$ Identification - Dénomination.}

C'est évidemment à la sous-association "Calamagrostidetosum " du Rhodoreto-Vaccinictum que doit ètre assimilé notre groupement. Et si l'on se réfère au relevé synthétique de cette sous-association figurant au Prodrome déjà cité, on constate que les différentielles 
indiquées par Braun-Blanquet: Calamagrostis villosa, Veratum album, Ranunculus montanus ( $R$. geraniifolius), Geranium silvaticum, Poa Chaixii, Rosa pendulina, Pencedanum Ostruthium, Rubus Idaeus, Lusula nemorosa var. Erythranthema, correspondent, à très peu près, aux groupes des espèces liées à des sols à mull ou à mull-moder - qu'elles soient mésophiles ou mésohygrophiles qui nous sont apparues comme constituant les groupes différenciateurs.

\section{Répartition - Ecologie.}

En Suisse, le groupement à Calamagrostis villosa est considéré comme plus xérophile et plus thermophile que la Rhodoraie proprement dite et comme ne dépassant qu'exceptionnellement l'altitude de $2000 \mathrm{~m}$. En est-il de même en Haute-Maurienne? En l'absence de mesures climatiques, il est évidemment difficile de répondre à une telle question. Tout ce que l'on peut dire, c'est que la Rhodoraie parait mieux adaptée aux gros éboulis, aux crêtes rocheuses et que, de ce fait, c'est bien à elle, en général, qui colonise les dernières stations susceptibles d'être occupées par la forêt de Pin Cembro. On peut conjecturer en outre, que la plus grande abondance de Mélèze, au feuillage léger, entraine comme corollaire, un climat du sol plus sec que celui de la Rhodoraie.

Ce sol, en tout cas, présente des caractéristiques différentes de celles appartenant aux sols de la Rhodoraie, L'analyse ci-après et le profil correspondant montrent qu'il s'agit d'un sol lessivé, faiblement podzolique (indice d'entrainement: 3): à moder, mais à moder faisant le passage avec un mull. Dans le feutrage très dense des racines de Graminées, en effet, l'humus apparait sous forme de petits grumeaux, mais peu consistants et présentant des grains de quartz, libres. Le rapport $\mathrm{C} / \mathrm{N}(19.3)$ montre que la minéralisation et l'humification sont assez rapides; le rapport S/T élevé, indique, lui, une grande richesse en bases qui rappelle celle d'un mull.

L'influence de l'altitude, des résineux - et, dans une certaine mesure des Ericacées - se trouve donc sensiblement contrebalancée par celle des Graminées dont le rôle s'avère considérable. Il faut ajouter que la plus grande luminosité régnant au niveau du sol, facilite encore la minéralisation de la matière organique.

De telles caractéristiques expliquent l'organisation sociologique et la composition floristique du groupement où dominent nettement des espèces liées à des sols à moder ou à des sols à mull, le type d'humus, pourtant ici très uniforme, permettant aux unes et aux autres, grâce à son caractère marginal, de prospérer (19).

(19) et compte tenu, aussi, de lamplitude écologique des espèces présentes, évidemment. 


\begin{tabular}{|c|c|c|c|c|c|c|c|c|c|c|c|c|}
\hline $\begin{array}{l}\text { Hori- } \\
\text { zons }\end{array}$ & $\begin{array}{l}\text { Speis- } \\
\text { seur }\end{array}$ & $\begin{array}{l}\text { Cad1- } \\
\text { loux }\end{array}$ & $\mathrm{pH}$ & Arg. & $\begin{array}{l}\text { Ifinotis } \\
\text { Pina }\end{array}$ & $\begin{array}{l}\text { Linong } \\
\text { gros }\end{array}$ & $\begin{array}{c}\text { Sables } \\
\text { flas }\end{array}$ & $\begin{array}{l}\text { Siables } \\
\text { gros }\end{array}$ & Mate ors. & 0 & n & $\mathrm{c} / \mathrm{s}$ \\
\hline$A 2$ & $6 \mathrm{~cm}$ & & 4,9 & & & & & & 17,0 & 8,49 & 0,44 & 19,3 \\
\hline 12 & $20 \mathrm{~cm}$ & 14 & 5,2 & 5,0 & 23,4 & 14,4 & 34,2 & 21,6 & 1,0 & 0,60 & & \\
\hline B & $40 \mathrm{~cm}$ & 17 & (b) & 6,2 & 20,3 & 14,4 & 36,4 & 21,1 & 0,9 & 0,55 & & \\
\hline \multirow{2}{*}{$\begin{array}{l}\text { Horl- } \\
\text { tons }\end{array}$} & \multicolumn{6}{|c|}{ Couplexe absorbant (a. val, p. 5 ) } & & & \multirow{2}{*}{\multicolumn{4}{|c|}{ Doservstiona }} \\
\hline & $\begin{array}{c}2 \\
\text { ih } 7\end{array}$ & Ca & K & $\mathrm{Mg}$ & $s$ & $S / T$ & $\mathrm{Cos} \mathrm{Ca}$ & $\begin{array}{c}\text { Per } \\
\text { Litren }\end{array}$ & & & & \\
\hline Al & 23,5 & 10,17 & 0,85 & 2,46 & 13,48 & 57,4 & 0 & 0,52 & \multirow{3}{*}{\multicolumn{4}{|c|}{$\begin{array}{l}\text { Indice d'entr, du for } t \\
3,1\end{array}$}} \\
\hline A2 & 7,6 & 2,94 & $0, \infty 8$ & 0,59 & 3,61 & 47.5 & 0 & 0,42 & & & & \\
\hline B & 6,7 & 2,18 & 0,07 & 0,50 & 2,75 & 41,0 & 0 & 1,30 & & & & \\
\hline
\end{tabular}

\section{III.6.1.3. - La pineraie de Pin Cembro à Rhododendron fer- rugineum et Erica carnea.}

$1^{\circ}$ Organisation sociologique et composition floristique.

Comme le montre le tableau général d'association, cette petite unité se caractérise par la présence de Pinus montana subsp. uncinata et d'espèces liées à des sols calcimorphes (moder calcique tout particulièrement) comme Valeriana montana, Sorbus Chamaemespilus, Erica carnea, Aster Bellidiastrum, Calamagrostis varia, Phyteuma orbiculare, Carex ferruginea... qui forment, comme on le voit sur le tableau, un véritable groupe différentiel. Les espèces liées à des sols à humus brut sont également bien représentées, ainsi d'ailleurs que les espèces de moder, alors que, par contre, les mésophiles et les mésohygrophiles de mull, sont pratiquement absentes. L'ensemble se trouve complété par des mésophiles indifférentes, où les Mousses dominent nettement.

Ce groupement possède un spectre floristico-écologique qui n'est pas sans rappeler, malgré les apparences, celui de la Rhodoraie proprement dite, mais où la moitié environ de "l'espace " occupé par les espèces liées au mor se trouverait couvert par des espèces liées au moder calcique. Nous verrons que la pédologie permet d'expliquer une telle organisation.

La structure est, dans son ensemble, semblable à celle de la Rhodoraie, les plages de Graminées étant toutefois moins importantes. Nous avons observé toutefois, un " individu " pâturé de structure un peu différente où de petits ilots de pelouse riches en calciphiles se trouvaient intégrés dans une Rhodoraie reposant sur roche-mère calcaire.

(b) On notera que le $\mathrm{pH}$ augmente avec la profondeur. Ceci tient au fait, que dans l'horizon humifère, le nombre d'ions $\mathrm{h}+$ dissociés est plus élevé (acides humiques). 


\section{Identification - Dénomination.}

Dans le Parc national suisse, a été distinguée dans la Rhodoraie à Vacciniées (Rhodoreto-Vaccinietum) une sous-association à Pins à crochets: Rhodoreto-Vaccinietum Mugetosum qui se distingue, en fait, très peu du Rhodoreto-Vaccinietum Cembretosum, si ce n'est par la plus grande constance et abondance de Lichens du genre Cladonia et d'espèces d'humus brut, s'observant dans les Alpes internes tout au moins, aux confins des étages alpin et subalpin, comme Empetrum hermaphroditum et Vaccinium uliginosum. La présence de ces espèces s'explique par le fait que la sous-association à Pin à crochets pousse à haute altitude, les relevés de BraunBLANQUET ont été pris en majorité entre 2100 et $2300 \mathrm{~m}$. Quant aux faibles différences floristiques existant entre les deux unités, elle est due au puissant développement d'une couche d'humus brut qui fait " converger " les flores, grâce à des horizons superficiels " analogues " bien que dans le Rhodoreto-Vaccinietum Cembretosum les roches-mères soient presque toujours siliceuses et dans le Rhodoreto-Vaccinietum Mugetosum presque toujours calcaires.

Il existe toutefois des différentielles de sols calcimorphes, non mises en évidence d'ailleurs, par Braun-Blanguet, mais elles sont plutôt rares, et nous avons dit pourquoi; il s'agit d'Erica Carnea, de Sorbus Chamaemespilus, et de Rhododendron intermedium.

Notre groupement de Haute-Maurienne - qui ne se présente qu'exceptionnellement par masse - se distingue de son homologue suisse, d'une part par l'absence des Lichens du genre Cladonia, d'Empetrum hermaphroditum, de Vaccinium uliginosum, d'autre part, par la présence, en plus grande abondance, d'espèces calciphiles. Ceci s'explique par le fait qu'il ne s'observe pas à haute altitude (il se trouve limité, dans la zone calcaire et gypseuse, par un replat occupé par l'homme) et que, l'érosion ou le pâturage aidant, il est relativement proche de l'association de Pin à crochets (Erico-Mugetum) qui précède la pineraie de Pin Cembro à Rhododendron, dans une même série dynamique.

\section{Répartition - Ecologie.}

Il se trouve que, dans notre dition et sur versant frais, les roches carbonatées sont surtout représentées par des gypses - très sensibles à l'érosion - ou par des calcaires triasiques, mais formant des falaises abruptes, au-dessus desquelles l'homme a installé des pâturages. Ceci explique que la pineraie de Pin Cembro à Rhododendron ferrugineum et Erica carnea soit très rare et ne se présente que sous une forme plus ou moins fragmentaire. C'est, en définitive, sur les lèvres plein nord de certains couloirs très enneigés qu'elle se localise pratiquement, entre Termignon et Bramans.

Climat - Si donc son climat général ne peut différer de celui de la Rhodoraie proprement dite, son microclimat - dont nous 
n'avons fait aucune mesure - est peut-êttre, toutes choses égales d'ailleurs, un peu plus froid.

Sol - Les sols, par contre, présentent des caractères propres sommairement analysés ci-après:

$1^{\text {er }}$ exemple - Forêt communale de Bramans - Canton du Jeu - Parcelle 7.

Exp. NW $(350$ g) p. $100 \%$ - Alt. $1920 \mathrm{~m}$ - Roche-mère: Cargneules.

Relevé floristique $n^{\circ} 28$.

\begin{tabular}{|c|c|c|c|c|c|c|c|c|c|}
\hline $\begin{array}{l}\text { Aort- } \\
\text { zons }\end{array}$ & Ipnisseur & $\begin{array}{l}\text { Profond. } \\
\text { da pri1. }\end{array}$ & pat & iat. Crg. & c. & $x$. & $c / s$ & $\begin{array}{l}\text { Cis }+ \text {. } \\
\text { sohurng. }\end{array}$ & $\begin{array}{r}\mathrm{Co}_{0} \mathrm{CB}_{8} \\
\text { nettif }\end{array}$ \\
\hline Ao & $7 \mathrm{~cm}$ & $-4 \mathrm{~cm}$ & 5,2 & 53,3 & 31,36 & 1,26 & 25 & 41 & 0 \\
\hline A1 & & $-12 \mathrm{~cm}$ & 7,5 & 8,9 & & & & 28,2 & 0 \\
\hline Al & $30 \mathrm{con}$ & $-25 \mathrm{~cm}$ & 8,2 & 0,8 & & & & (sat) & 0,6 \\
\hline
\end{tabular}

20 exemple - Forét communale de Sollières - Sardières - $1^{\text {te }}$ série - Parcelle U.

Exp. N (Og.) p. $70 \%$ - Alt. $2000 \mathrm{~m}$ - Roche-mère: Moraine calcaire et cargneules - Relevé floristique $\mathrm{n}^{\circ} 29$.

\begin{tabular}{|c|c|c|c|c|c|c|c|c|c|}
\hline $\begin{array}{l}\text { Hor1- } \\
\text { zons }\end{array}$ & Bpasiaseur & $\begin{array}{l}\text { Profond. } \\
\text { du príl. }\end{array}$ & $\mathrm{pH}$ & Kat. Org. & c. & x. & $c / s$ & $\begin{array}{l}\mathrm{Cn}+ \\
\text { éaharas. }\end{array}$ & $\begin{array}{l}\text { Cos cs } \\
\text { actif }\end{array}$ \\
\hline do & $10 \mathrm{~cm}$ & $-4 \mathrm{~cm}$ & 4,6 & 65,8 & 38,74 & 1,4 & 27,7 & 22,6 & 0 \\
\hline 11 & $18 \mathrm{~cm}$ & -12 on & 6,5 & 4,3 & & & & 13,2 & 0 \\
\hline $11 / 0$ & $30 \mathrm{~cm}$ & $-20 \mathrm{cr}$ & $\theta, 1$ & 1,6 & & & & (sat) & 0,6 \\
\hline
\end{tabular}

Description d'wn profil (sol $n^{\circ} 2$ ) - fig. 50.

$\mathrm{A}_{0}-(0-10 \mathrm{~cm})$ - humus très noir, consistance un peu e grasse s.

$\mathrm{A}_{1}-(11-36 \mathrm{~cm})-$ humus brun-ocracé, à grumeux de $3 \mathrm{~mm}$. Squelette de plus en plus abondant avec la profondeur (fragments de cargneule).

C - $\quad$ - cargneule ocre clair, un peu fissurée.

$$
\mathrm{e}=1 / 10^{\circ}
$$

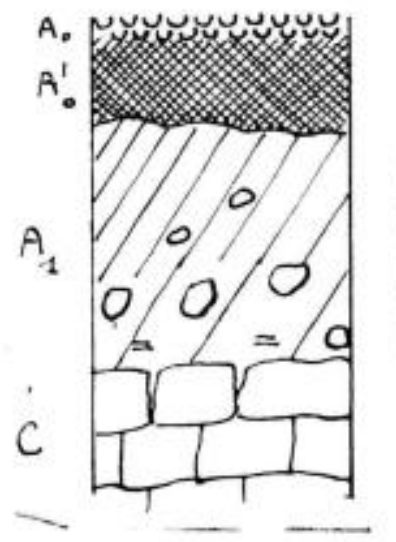

zusury Couche organique

ZzW Humus peu actif

(/7 Humus grumeleux

- Carbonate de chaux

I Roche-mère calcaire

Fig. 50. 
On voit qu'il s'agit de sols calcimorphes à humus brut.

L'humus, en $\mathrm{A}_{0}$, a exactement la morphologie d'un " Mor »; bien qu'acide, il est cependant assez riche en calcium échangeable (surtout celui du $1^{\text {er }}$ sol); le rapport $\mathrm{C} / \mathrm{N}$ est plus faible que dans les Mors a atlantiques $n$ ou il dépasse souvent 30 .

L'humus du $1^{\text {er }}$ sol, où la teneur en Ca est de 41 , doit ètre considéré comme un "Tangel nou mor calcique; celui du second passe au mor vrai.

Alors que l'horizon supérieur est acide, on passe assez vite en profondeur à un $\mathrm{pH}$ supérieur à 8 , et à une saturation en calcium échangeable.

On conçoit que, sur de tels sols, coexistent - au moins provisoirement - deux groupes écologiques, qui paraissent au premier abord $\alpha$ antinomiques $n$. Sur l'humus brut, vont se trouver les espèces adaptées à un tel substrat; c'est-à-dire très frugales, en ce qui concerne l'azote; mais, comme il s'agit d'un humus assez riche en calcium échangeable et qu'au demeurant, les couches inférieures offrent une composition et une structure de moder calcique, s'observeront également les espèces calciphiles.

Il faut ajouter que l'érosion, toujours très diversifiée - (il faudrait parler de micro-érosion) joue un rôle non négligeable et ce, d'autant plus que la roche-mère est friable. La couche d'humus brut a donc une épaisseur très variable d'un point à un autre, si bien que la couche carbonatée peut être très facilement atteinte par les espèces calciphiles. Sur gypse, tout particulièrement, on arrive ainsi à observer, et dans un mélange assez confus, des espèces liées au mor et des espèces pionniers calciphiles comme Dryas octopetala,

\section{III.6.2. - Les pineraies de Pin Cembro xérophiles.}

Ces peuplements xérophiles de Pin Cembro qui s'étendent audessus de la pessière subalpine sèche à Vaccinium Vitis idaea, présentant des caractères propres qui doivent les faire distinguer, à notre avis, des peuplements mésophiles, étant entendu qu'il existe entre eux des termes de transition.

1. Organisation sociologique et composition floristique - Faciess.

Ce qui frappe le plus, dans le tableau d'association, c'est la présence de deux groupes écologiques, non rencontrés dans l'association mésophile.

Le premier est relativement spécialisé, puisqu'il est formé de xérophiles liées à des sols à moder: Ces espèces, toutes orophiles, se rencontrent, soit dans les pelouses acidiphiles sèches du bas de l'étage alpin (associations du Festucion variae dépendant des Caricetalia curvulae), soit dans les landes acidiphiles sèches du haut de l'étage subalpin (Junipereto-Aretostaphyletum, en particulier), le 
second est noins spécialisé, puisqu'il comprend des espèces xérophiles sans doute, mais paraissant indifférentes au chimisme du sol. Ces espèces sont également bien représentées dans le JuniperetoArectostaphyletum, association principale de l'alliance du Juniperion nanae. Nots aurons d'ailleurs l'occasion de commenter ce fait, lors de l'étude de l'identification de notre groupement.

Les groupes des mésophiles et des mésoxérophiles de sols à mor ou à moder présentent, par rapport à ceux de la Rhodoraie, une composition sensiblement différente, att moins quantativement. Rhododendron ferrugineum devient rare et est pratiquement remplacé par Juniperus communis subsp. intermedia et nana; Vaccinium Vitis idaea joue un rôle plus important que Vaccinitum Myrtillus, alors que c'était l'inverse dans la Rhodoraie (sauf dans le relevé 1 qui correspond à un faciès de transition). Quant aux mésophiles proprement dites, comme Calanagrostis villosa, Melampyrum silvaticum, Homogyne alpina, elles se sont très sensiblement raréfiées.

Parmi les mésohygrophiles, seules, subsistent les plus plastiques: Chaerophyllum Villarsii, Peucedanum Ostruthium... Aucune trace, en particulier, des espèces de I'Alnetum Viridis qui s'observaient parfois dans la Rhodoraie.

Dans la strate arborescente, deux faits notables sont à relever: d'une part, l'absence presque absolue de Larix decidua, d'autre part, la relative abondance de Picea excelsa qui, sur versant sud, monte plus haut que sur versant nord. On sait que la répugnance du Mélèze à coloniser les versants sud n'est pas spéciale à la Haute-Maurienne. Fourchy (P.) (1952) a pu montrer qu'il en était ainsi dans toute la zone intra-alpine, du moins, la moins arrosée.

\section{Identification - Dénomination.}

Si lon cherche à repérer, parmi les associations déjà décrites. celles qui présentent le plus d'affinités avec notre groupement, on s'arrête vite sur le Junipereto-Arctostaphyletum (21). Les deux groupements possèdent en commun: Arctostaphylos Uva ursi, Juniperus communis subsp. nana, Cotoneaster integerrina qui sont considérées, entre autres, comme caractéristiques par BRAUN-BLANQUET. Vaccinium Vitis idaea, Vaccinium Myrtillus, Anthoxantum odoratum, Deschampsia flexuosa... pour ne citer que les plus abondantes et les plus constantes. Sont, en outre, à citer, les xérophiles acidiphiles ci-après: Phyteuma betonicifolium. Silene rupestris, Laserpitum Halleri, Veronica fruticane.

A ces ressemblances positives, doivent s'ajouter les affinités négatives, en quelque sorte: dans les deux groupements, les mésophiles sont rares et tout particulièrement Rhododendron ferrugineum. Calamagrostis villosa. Homonyne alpina, etc.

(21) Braun-Blanouet (J.), Sissingh (G.) et Vtimger (J.) 1939 et BraunBlanouet (J.), Pallmann (H.) et Bach (R.) 1954. 
A quoi tiennent les différences? Il y a d'abord celles qui s'expliquent par l'histoire des flores: dans notre association, sont ainsi absentes des espèces propres à l'Europe Centrale, comme Senecio abrotanifolius, Sempervirum $W$ ulfeni. Il en est d'autres - et les plus importantes - qui s'expliquent par l'écologie: le JuniperetoArctostaphyletum est sensiblement plus riche en espèces héliophiles: Arnica montana, Anemone alpina, Nardus stricta, Trifolium alpinum, Gentiana campestris, etc. indiquant assez que ce groupement est relativement proche des associations prairiales du bas de l'étage alpin. Notre association, par contre, bien que fragmentaire, se situerait plus près du climax, semble-t-il. Et ce fait est confirmé par l'importance du Pin Cembro: dans la lande à Busserole et à Genévrier, il est sans doute presque partout présent, mais il ne forme pas de forêt à proprement parler, puisqu'il s'observe en individus clairsemés ou en bouquets isolés: dans notre groupement, au contraire, la strate arborescente a une densité comparable à celle de la pineraie de Pin Cembro à Rhododendron ferrugineum.

Si donc, il fallait donner un nom à cette unité, en s'inspirant des règles suivies par Braun-Blanguet, il serait assez logique de l'appeler: "Junipereto-Arctostaphyletum cembretosum ". Mais nous pensons qu'il est plus sage, avant de fixer toute étiquette, d'attendre une étude plus approfondie, axée sur sa place parmi les autres associations de l'étage.

\section{$3^{\circ}$ Répartition - Ecologie - Faciès.}

On n'observe de tels peuplements, dans notre dition, ni sur rochemère calcaire, ni sur schistes lustrés, mais uniquement sur les schistes et grès du Houiller et sur les quartzites triasiques.

Sur roche-mère calcaire, l'étage subalpin supérieur, aux expositions sud, est, en effet, occupé par l'association à Pinus uncinata et Arctostaphylos Uva ursi ou Erica carnea; il s'agit d'un groupement neutrophile: la rigueur des pentes, la sécheresse et l'évaporation constituant autant de facteurs propres à freiner la formation de sols calcimorphes à humus brut. Sur schistes lustrés, par contre, il semble que l'évolution naturelle devrait, plus facilement, conduire à une Juniperaie à Pin Cembro; mais l'action humaine a été considérable et, si elle a existé, la forêt de Pin Cembro n'a pas laissé de traces.

Par contre, sur les éboulis de schistes ou de grès houillers, ou, sur les quartzites du synclinal de Chavières où l'homme ne pouvait manifestement s'installer, la forêt de Pin Cembro a pu subsister, quoique de façon fragmentaire. Elle se localise donc au niveau d'arêtes peu accusées et monte ainsi jusqu'à plus de $2300 \mathrm{~m}$. Vers le bas, elle passe progressivement, vers $2000 \mathrm{~m}$, à la pessière subalpine à Vaccinium Vitis idaea.

Elle présente un faciès particulier, fort localisé, mais digne de retenir l'attention. Sur quartzites à forte pente, elle repose en effet, 
sur un ranker à moder, particulièrement peu évolué et où s'observent en abondance Phyteuma betonicifolium et Minuartia laricifolia.

\section{III.6.3. - Vue d'ensemble sur les pineraies de Pin Cembro.}

\section{III.6.3.1. - Affinités phytosociologiques.}

Les pineraies de Pin Cembro, à Rhododendron ferrugineum ou à Calamagrostis villosa sont typiquement intra-alpines, comme les associations précédemment décrites; clles s'étendent, en effet [Braun-Blanguet (J.), Sissingh (G.) et Vlieger (J.) (1939)], de la Styrie, en Autriche, aux Alpes maritimes, dans les chaines internes.

Ce que nous avons dit concernant les pineraies de Pin sylvestre ou de Pin à crochets, les sapinières et les pessières, à savoir que

Les pineraies de Pin Cembro

Cercles d'exposition

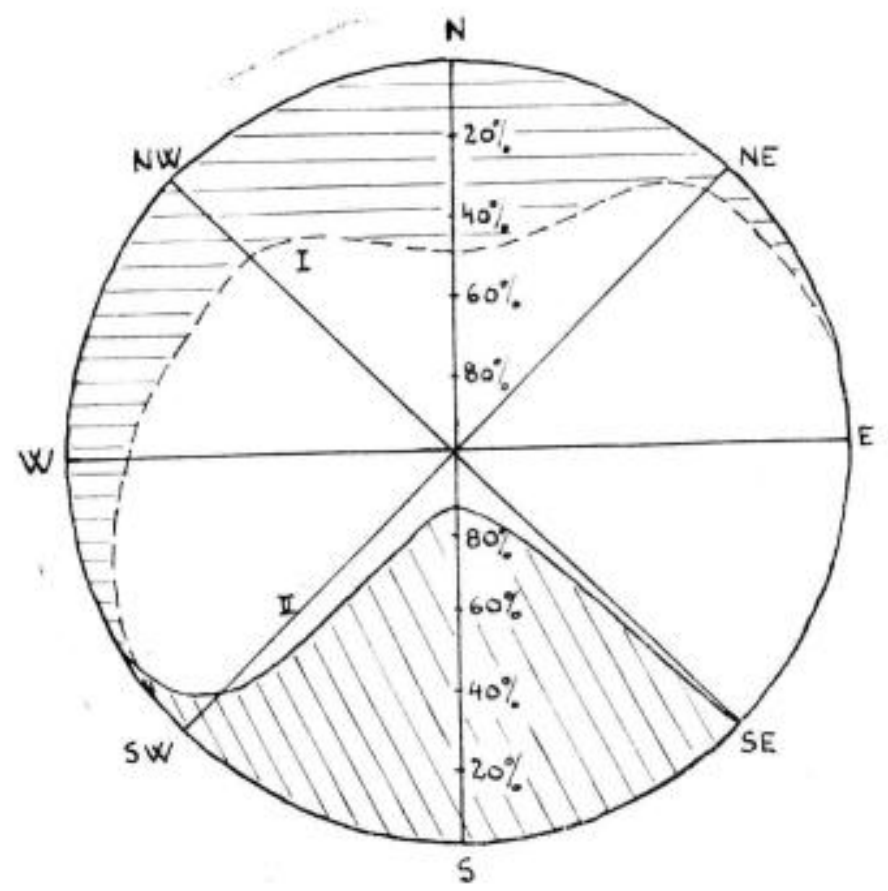

I .... Rhodoraic à Pin Cembro.

11 Juniperaic à Pin Cembro.

Fig. 51. 


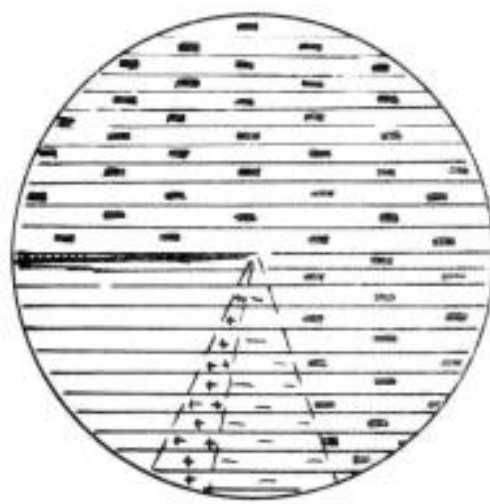

I. Rhodoreto-vaccienetum cembretosum (Relevés 1 à 11).

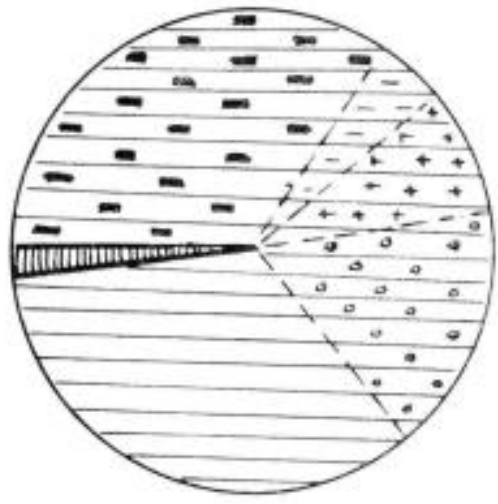

III. Rhodoreto-vaccietum ericetosum. (Relevés 26 à 29)

- - Mésophiles de mor $=$ Mésophiles de moder ++ Mésophiles de mull-moder $\because \because$ Mésophiles de mull $\because$ Mésophiles d'humus calcique Mésophiles indifférentes

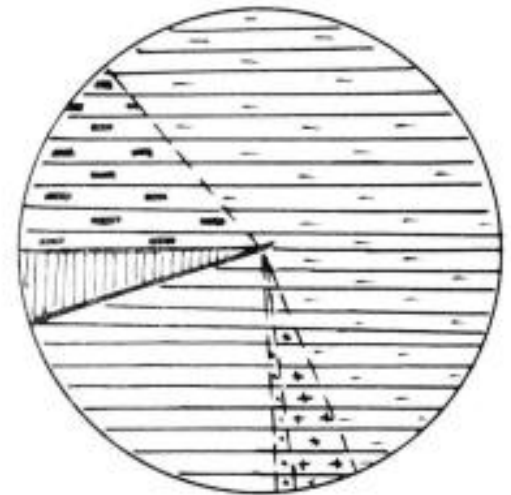

II. Rhodoreto-vaccinieto calamagrostidetosum (Relevés 18 à 25).

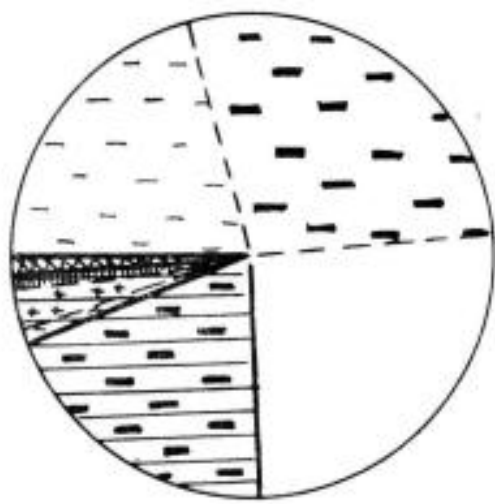

IV. Junipereto-vaccinetietum cembretosum (Relevés 1 à 8 ).

\section{Mésohygrophiles}

--- Xérophiles de mor (V. Vidaea)

$==$ Xérophiles de moder

Autres xérophiles

Diverses

Fic. 52. 
leur présence en Haute-Maurienne met bien en évidence les affinités floristiques et écologiques que cette vallée présente avec d'autres vallées intra-alpines où l'on rencontre ces mêmes groupements, s'applique donc parfaitement aux pineraies de Pin Cembro.

Il $\mathrm{y}$ a lieu de noter, toutefois, que ces dernières peuvent s'observer en dehors du monde intra-alpin proprement dit, puisqu'elles ont essaimé jusqu'à Chamrousse, sur le rebord occidental des massifs cristallins externes. Mais il s'agit, ici, d'un groupement un peu appauvri floristiquement et où manque, en particulier, Calamagrostis villosa.

Il faut signaler aussi que la Rhodoraie à Vacciniées, sans arbres (Rhodoreto-Vaccinictum extra-silvaticum) a une aire d'extension plus vaste que celle du Rhodoreto-Vaccinietum cembretosum, bien que, toujours selon les auteurs cités ci-dessus, elle manque totalement déjà dans le Dévoluy et ne soit pas représentée, du moins à leur connaissance, dans le Queyras ou dans l'Ubaye.

Les deux types extrêmes se partagent les expositions opposées, les peuplements "xérophiles " de Pin Cembro sont beaucoup plus étroitement localisés que leurs homologues mésophiles.

On retiendra que les expositions "intermédiaires ", entre Avrieux et Termignon, où la vallée de l'Arc prend une direction SW-NE, sont essentiellement occupées par des peuplements de Pin à crochets (zone des cargneules et des gypses). De ce fait, les potentialités du Pin Cembro quant à son aptitude à occuper les expositions les plus diverses ne peuvent totalement se manifester.

Dans la pineraie de Pin Cembro à Rhododendron ferrugineum, sur sol podzolique ou podzol, les espèces liées au mor dominent: dans la pineraie à Calanagrostis villosa, ce sont celles liées au moder qui dominent; enfin, dans le type mixte, sur rendzine à humus brut, il $y$ a partage entre des acidiphiles franches et des basiphiles.

Sur versant sud, les espèces xérophiles dominent nettement, dans la pineraie de Pin Cembro à Airelle rouge et Genévrier.

Il n'est pas inutile, enfin, de rappeler que le long des chânes préalpiennes et dans le Jura, une association affine vient relayer, en quelque sorte, la pineraie de Pin Cembro à Rhododendron: il s'agit du Lycopodio-Mugetum décrit récemment de façon complète par Richard (J.-L.) dans le Jura (1961). On rencontre ce groupement dans l'étage subalpin, sur lapiaz, ou en enclaves subalpines dans l'étage montagnard, sur éboulis à microclimat du sol particulièrement froid. $\mathrm{Si}$, dans cette association, le Pin à crochets remplace le Pin Cembro, les mêmes Ericacées se retrouvent: Rhododendron ferrugineum, Vaccinium Myrtillus, Vaccinium Vitis idaea, Vaccinium uhiginosum, Empetrum hermaphroditum, et sur un sol 
" analogue $n$ à celui de la pineraie de Pin Cembro ì Rhododendron, c'est-à-dire à humus brut.

\section{III.6.3.2. - Evolution - Climax.}

Tous les auteurs qui, comme Braun-Blanguet (J.), Pallmann (H.), BAcH (R.) (1954), ont analysé la pineraie de Pin Cembro à Rhododendron ou qui, comme AUER (Ch.) (1947), LUDI (W.) (1945), Duchaufour (Ph.) (1952) ont étudié les groupements, riches en Mélèzes de l'étage subalpin supérieur, se sont accordés pour reconnaitre dans cette pineraie, un groupement final, climacique. L'analogie des sols, au sens de Pallmann, qu'ils reposent sur roche-mère silicatée ou sur roche-mère carbonatée constitue évidemment un puissant argument en faveur de ce point de vue.

Ce qu'il est intéressant de rappeler, c'est que, comme l'a souligné Duchaufour dans son récent ouvrage de Pédologie (1965), l'on se trouve en présence de podzols climatiques qui « s'opposent aux podzols de plaine $"$; ces derniers résultent, en effet, d'une action régressive et sont appauvris en éléments minéraux nutritifs, à la suite d'un lessivage préalable prolongé " alors que les premiers sont souvent plus riches au point de vue chimique, en particulier parce que leurs horizons supérieurs contiennent de nombreux fragments de roche-mère en cours d'altération, précieuses réserves en éléments minéraux $n$.

Mais - la chose est à souligner - à l'inverse de ce que l'on observe dans le domaine atlantique de plaine, la régression est caractérisée par un " brunissement $»$ des sols et la disparition ou la quasi-disparition des horizons typiques des podzols. C'est ce qui se passe dans la sous-association à Calamagrostis villosa dont l'origine s'explique à notre avis, par l'incendie ou, le pâturage (22), et où les sols rencontrés sont, on l'a vu, faiblement podzoliques, avec un horizon humifère à $\mathrm{C} / \mathrm{N}$ particulièrement bas.

\section{III.6.2.3, - Structures sylvicoles - Production.}

Ce qui caractérise la structure sylvicole de la pineraie de Pin Cembro, est le faible nombre d'individus rencontrés à l'hectare, surtout dans les petits diamètres. Il s'agit d'une futaie claire, où l'on n'observe pas, contrairement à ce qui se passe dans l'étage montagnard (sapinière) ou même dans l'étage subalpin inférieur (pessière subalpine), de fourrés de jeunes plants; ces derniers sont isolés les uns des autres: le forestier dit que la régénération se fait pied par pied.

(22) En forêt d'Aletsch, nous avons pu voir un envahissement du Calamagrostis villosa, après incendie. 
La courbe donnant le nombre d'arbres à l'hectare par catégorie de diamètre traduit bien ce phénomène: elle est presque parallèle à l'axe des abscisses.

Quant à la production, elle est évidemment faible, comme on pouvait s'y attendre. 85,9 ha échantillonnés, ont donné le chiffre de $1,8 \mathrm{~m}^{3}$ par ha/an (tarif Algan $\mathrm{n}^{\circ} 6$ ).

Pineraie de Pin Cembro à Rhododendron.

Siructure sylvicole.

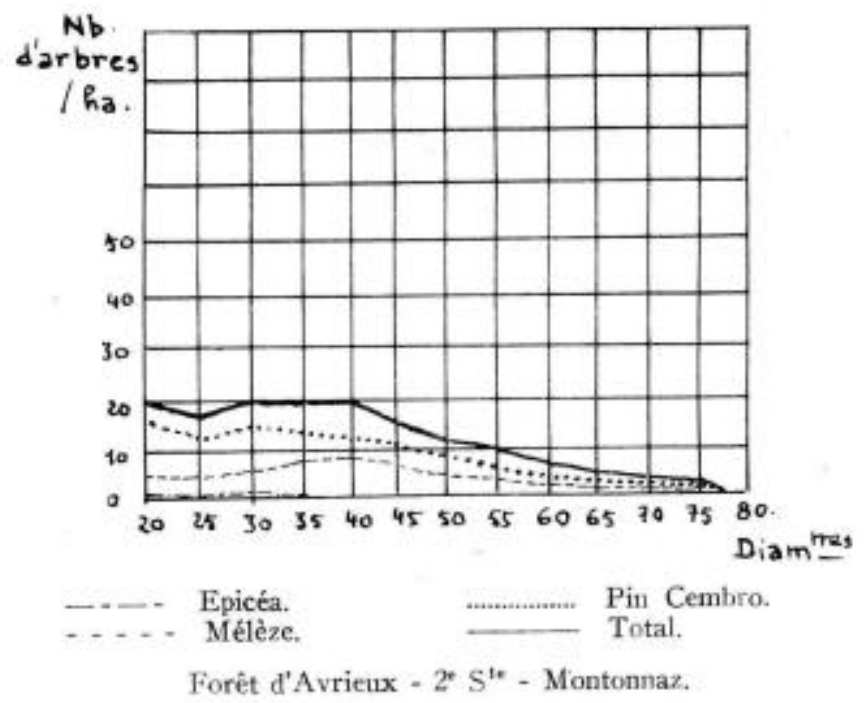

F1G. 53. 


\section{III.7. - L'AULNAIE VERTE (Alnetum viridis)}

\section{III.7.1. - Analyse phytosociologique et écologique.}

De tous les groupements étudiés jusqu'à présent, le groupement à Alnus viridis est celui qui peut paraitre le moins forestier, au sens étroit du mot. Et il en est bien ainsi, si l'on observe que les arbres y sont rares et toujours disséminés.

Mais il nous a semblé, cependant, présenter un réel intérêt et même forestier - : il appartient à un étage silvatique, le subalpin; c'est, dans notre dition, le seul groupement de feuillus de cet étage; il fait corps avec la forêt proprement dite, dans presque tous les cas; il présente, enfin, les plus étroites affinités avec certains faciès de la forêt résineuse, toujours dans notre dition, et, en dehors d'elle, avec des forêts mixtes de l'étage montagnard supérieur.

\section{$1^{\circ}$ Organisation sociologique et composition floristique.}

Le tableau d'association est d'une grande simplicité, en ce sens que le nombre de groupes écologiques est faible et que l'un d'eux - celui des mésohygrophiles des sols colluviaux à mull - domine nettement.

Dans le groupe des mésohygrophiles à mull, il $\mathrm{y}$ a lieu de distinguer certaines espèces qui jouent le rôle de différentielles géographiques et même - quoique à un moindre degré - altitudinales; elles permettent d'individualiser le groupement à Alnus viridis par rapport à d'autres groupements affines des Pyrénées ou du Massif Central: il s'agit d'Alnus viridis, Achillea macrophylla, Aconitum paniculatum, Sisymbrium tanacetifolium qui sont des orophiles sud-européennes et, les trois premières même, des alpinobalkaniques. Ces espéces peuvent, à ce titre, être considérées comme les caractéristiques du groupement.

Les autres espèces du groupe écologique, dont la tête de file est Adenostyles Alliariae ont une aire soit circumboréale, soit médioeuropéenne et ont, quant à l'altitude, une amplitude plus vaste. Il ne nous a pas paru utile de faire de distinctions, à l'intérieur de ce sous-groupe, encore que certaines espèces soient considérées par Braun-Blanguet (J.) (1950), comme caractéristiques d'Alliance (Adenostylion), et d'autres d'Ordre (Adenostyletalia) (23).

(23) $\mathrm{Cf}$, à ce sujet, le tableau général des groupes écologiques, 


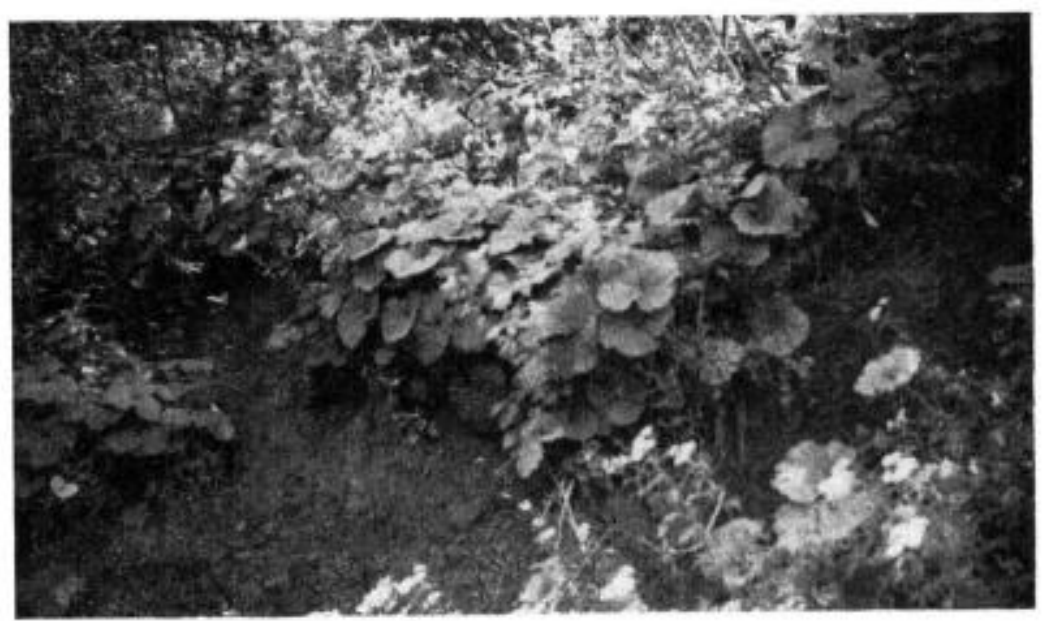

Dans un couloir, en forèt de Lanslebourg.

Sur cette photo, on distingue, outre Alnus viridis, Adenostyles Alliariae, Achillea macrophylla.

Le sol colluvial à mull apparait aussi, nettement. 
A côté de ce groupe, compact et homogène, doit être distingué un fragment de groupe qui comprend des espèces, à notre avis, moins hygrophiles, mais qui, comme les précédentes, s'observent essentiellement sur des sols à mull, à structure aérée. Ces espèces qui se rencontrent, sans doute, en forêt, trouvent leur optimum dans les prairies engraissées de l'étage subalpin ou du montagnard supérieur, à Trisetum flavescens: il s'agit de Geranium silvaticum, et, mieux encore, de Veratrum album, Campanula rhomboidalis, Trollius europaeus, etc. Ce qu'il est intéressant de noter, tout de suite, c'est que ces espèces trouvent donc, dans le sol de l'Aulnaie verte. une structure et une nourriture minérale et azotée comparables à celles des sols de prairies out on les observe si fréquemment et avec une telle abondance.

Nous avons cru devoir mettre à part trois espèces: Viola biflora, Soldanella alpina, Sorbus aucuparia dont l'écologie mériterait d'être précisée; elles sont liées à une grande humidité de l'atmosphère et du sol, mais présentent, par contre, à l'égard de son chimisme, une certaine plasticité. Sorbus aucuparia, en particulier, s'observe quoique avec une vitalité moindre - sur des sols à mor tourbeux dans les associations d'Epicéa à Sphaignes (Sphagno-Piceetum) ou de Pin à crochets à Sphaignes (Sphagno-Mugetum, des Suisses) (24), comme sur les sols podzoliques on les podzols de la pineraie de Pin Cembro à Rhododendron.

Ont été rassemblées, in fine, des espèces assez plastiques - mais mésophiles - et qui, d'une façon générale, trouvent leur optimum sur des sols acides à moder: Oxalis acetosella, très "ubiquiste " quant à l'altitude, Homogyne alpina, etc. Ce sont des espèces échappées des associations voisines, at contact étroit desquelles se trouve l'Aulnaie verte. Parmi elles, des espèces comme Aspidium lonchitis. Valeriana tripteris - à un moindre degré Gentiana punctata trouvent dans le sol de l'Aulnaie verte, la texture grossière qui leur convient et que l'on retrouve dans les éboulis plus ou moins nus où elles se complaisent.

Dans chacun des groupes, nous avons mis à part les Mousses; a fortiori celles qui, quoique mésophiles, paraissent indifférentes att type d'humus.

Le spectre floristico-écologique n'appelle aucun commentaire spécial puisqu'il n'est que la traduction imagée du tableau d'association lui-même.

La structure est pluristrate: quelques arbres se rencontrent parfois (Larix decidus - Picea excelsa), mais toujours très disséminés. Sous les Aunes verts et les Sorbiers des oiseleurs dont les tiges sont fortement courbées vers laval sous l'effet de la reptation des neiges, s'observent une sous-strate arbustive, très lâche, avec surtout Lonicera nigra, et la strate de la megaphorbiaie proprement

(24) De telles associations ne s'ohservent pas en Haute-Maurienne. 
dite qui comprend les grandes espèces: Cicerbita alpina, Adenostyles Alliariae et, sous elles, les petites espèces: Viola biflora, Myosotis silvatica, etc. Quant à̀ la strate muscinale, elle n'est représentée que par quelques individus, très rares dans l'ensemble.

Le spectre biologique montre précisément cette rareté des Bryophytes et l'importance à peu près égale des Phanérophytes et der Hémicryptophytes.

\section{L'Aulnaic verte}

Spectres biologique et floristico-écologique.

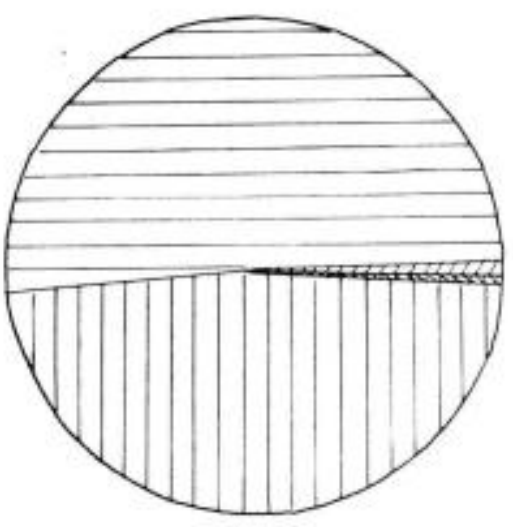

[II] Phanérophytes.

$\square$ Hémi cryptophytes.

QZZZGéophytes.

X...X. Bryophytes.

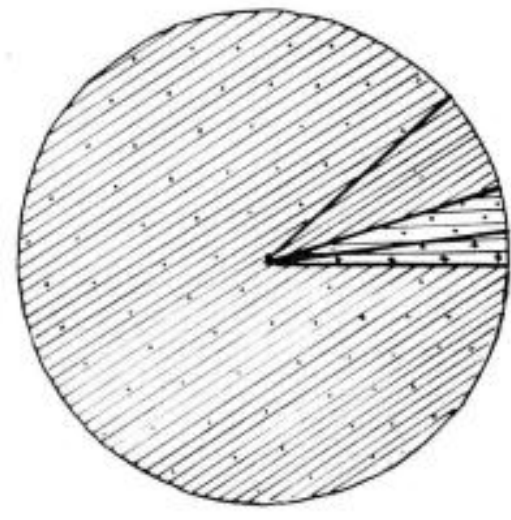

321.7. Méso-hygrophiles de mull.

DU Méso-hygrophiles indifférentes.

Pésophiles de mull.

$+\frac{3}{+}+1$ Mésophiles acidiphiles.

FIG. 54.

Légende. - On notera la dominance écrasante des mésohygrophiles - et singulièrement de celles de mull - ce qui s'explique par les conditions très particulières régnant dans une association hautement spécialisée (sol colluvial à mull, en atmosphère humide, en versant exposé att Nord).

II y a équilibre entre Phanérophytes et Hémicryptophytes, tandis que les Bryophytes sont très rares.

\section{Identification - Dénomination.}

Notre groupement s'identifie facilement avec l'Alnetum viridis décrit en Suisse (25), ou dans les Alpes Françaises, notamment par

(25) On trouvera dans Braun-Blanguet: e Ubersicht der Pflanzen gesselschaften Rätiens in Vegetatio - Vol. II (1949) - Fasc. 2-3 - p. 216, la liste chronologique des auteurs de langue allemande ayant écrit sur cette association, 
Heim (R.), dans le massif du Combeynot (1922) (26), par GurnoCHET (M.), dans le bassin du Giffre (1939), par QUEzEL (P.), dans les Alpes maritimes (1950), par Chasx (G.), aux abords du Lautaret.

Il faut noter ici que nous n'avons pu distinguer aucune sousassociation, alors que Braun-Branguet (J.) (1949) en Suisse, dans les Alpes rhétiques, en a distingué trois : une à Salix Waldsteiniana (sous-espèce orientale du Salix arbuscula), une autre à Salix helvetica, la dernière, enfin, à Salix appendiculata. Ceci tient probablement au fait que notre dition est relativement peu étendue et que nous nous sommes volontairement limité à l'étude des individus d'associations proches des forêts. On peut penser que les taches d'aune vert des vallées déboisées de la Neuvachette ou de la Valloirette recèlent des groupements légèrement différents, plus riches en saules en particulier (27).

\section{$3^{\circ}$ Répartition - Ecologie.}

Dans l'ensemble, l'Aulnaie verte se localise aux expositions fraiches, sur des sols silico-alumineux, dans l'étage suthalpin. Ceci peut paraitre, a priori, en contradiction avec ce que dit Fourchy (P.) de l'aune vert, dans l'Oisans (1951): "On le rencontre partout, à toutes expositions et toutes altitudes; depuis la plaine de l'Oisans $(700 \mathrm{~m})$ jusqu'au col du Lautaret $(2100 \mathrm{~m})$. n

En fat, comme le note le même auteur: " à exposition chande, il se réfugie sur les sols argileux, au voisinage des ravins, où il existe, malgré tout, un peu d"humidité n. En Haute-Maurienne. en tout cas, et compte tenu du fait que l'on ne doit pas confondre Aulne vert et Aulnaie verte (ta présence de quelques pieds d'Aune vert constituant la forme la plus fragmentaire de l'association), sa localisation sur les versants nord ou est, secondairement sur les versants ouest, (à l'aval surtout, où ces versants reçivent les vents pluvieux), comme son absence sur les versants sud, nous paraissent des plus caractéristiques.

L'Alnetum viridis fuit de même les roches-mères calcaires, pauvres en argile, même à exposition plein nord, et tout particulièrement les cargneules, les gypses, on les calcaires francs. Les couloirs d'avalanches, sur de telles roches, sont donc couverts d'Erica carnea et de Pin à crochets rabougris; leur végétation n'est donc pas très différente de celles des forêts voisines. Si le couloir - comme il arrive parfois dans le gypse - est particulièrement profond et

(26) Dans l'étude de HerM, qui date de 1922, les listes de plantes sont données sans coefficients d'abondance-dominance; il ne s'agit pas à la lettre d'une étude phytosociologique.

(27) Comme une excursion rapide a pu nous le montrer. 
étroit, la végétation rappelle celle du Salicetum herbaceae - reticulatae de l'étage alpin, sans que jamais apparaisse l'Aulnaie verte; Salix reticulata, Salix herbacea, Dryas octopetala, Bartsia alpina, Tofieldia calyculata, Pirola rotundifolia, Saxifraga aizoides, Polygonum viviparum, voisinent alors avec Rhododendron ferrugineum qui, lui, se localise sut la lèvre nord du couloir, sur une couche d'humus brut.

Quant à sa localisation en altitude, nous partageons tout à fait l'opinion de FAvarger (Cl.) $(1958,120)$ qui « s'il s'agissait de caractériser l'étage subalpin autrement que par des forêts " l'appellerait volontiers "étage de laulne vert ". Dans cet étage, en HauteMaurienne, il occupe, par vastes nappes, les versants très abrupts et humides que la forêt ne peut coloniser, par suite d'un régime de glissements de neige permanent ou les cirques glaciaires, pleins de moraines gorgées d'eau presque en toutes saisons. Il se développe aussi dans les couloirs d'avalanches, au contact de la pineraie de Pin Cembro et de la pessière subalpine: le long de ces couloirs, qui vont souvent s'encaissant entre des parois rochetses, il descend jusque dans l'étage de la sapinière, mais en perdant une partie de son cortège floristique et en n'occupant plus qu'une surface minime et fort fragmentée, ce qui explique que laune vert puisse s'observer assez bas, en altitude, mais toujours dans des stations très particulières.

Cette triple localisation: exposition fraîche, roche-mère silicoalumineuse, étage subalpin, permet de se faire une idée de l'écologie générale de l'association. Si son climat général est celui de la pessière subalpine ou de la pineraie à Pin Cembro, son climat local - et singulièrement celui du sol et celui régnant sous la strate des Aulnes verts - possède certainement des caractéristiques propres: degré hygrométrique élevé tout particulièrement. Nous n'avons, malheureusement, effectué aucune mesure à ce sujet.

Le sol présente aussi, par rapport à ceux des forêts voisines, des caractères originanx.

Nous avons fait deux prélèvements, sur schistes lustrés seulement, dont les résultats sont condensés dans le tableau ci-après.

\begin{tabular}{|c|c|c|c|c|}
\hline \multicolumn{5}{|c|}{ ler prollevenent - (relevó floriatique $\mathrm{n}^{\circ} 4$ ) } \\
\hline Bor1s. & PQ & Mat. Org. & $\cos ^{3} \mathrm{cs}$ & $\mathrm{Cs}_{\mathrm{s}}$ \\
\hline$A_{1}$ & 6,5 & 6,4 & 0 & 10,7 \\
\hline$h_{1} \mathrm{C}$ & 6,6 & 5,7 & 0 & 11,2 \\
\hline
\end{tabular}




\begin{tabular}{|c|c|c|c|c|c|c|c|c|c|}
\hline \multicolumn{10}{|c|}{ 2ذ̀ne prélèvenent - (relevé flaristique a $a^{0} 5$ ) } \\
\hline Horiz. & pill & Arg. & tinon 7 & Inon G & Sables $?$ & \multicolumn{2}{|c|}{ Bablen C } & Mat. Crg. & For libre \\
\hline$h_{1}$ & 5,7 & 11,3 & 17,7 & 9,7 & 14,9 & \multicolumn{2}{|l|}{40,3} & 4.8 & \\
\hline$A_{1} c$ & 6,3 & 4,0 & 18,1 & 15,7 & 14,0 & \multicolumn{2}{|l|}{45,1} & 4,8 & 1.22 \\
\hline \multirow[b]{2}{*}{ Boriz. } & \multicolumn{5}{|c|}{ Complexe abaorbant a. val. p. $100 \mathrm{~B}}$. & \multicolumn{3}{|c|}{ Hat. org. if } & \multirow{2}{*}{$\operatorname{co}^{3} \mathrm{Cs}_{\mathrm{B}}$} \\
\hline & $a^{2}$ pif 7 & $\begin{array}{c}\mathrm{g} \\
\text { éch. }\end{array}$ & $\begin{array}{l}\text { Ca } \\
\text { ech. }\end{array}$ & 3 & $\begin{array}{l}5 / 2 \\
\times \mathrm{pH} 7\end{array}$ & $c$ & N & $\mathrm{c} / \mathrm{h}$ & \\
\hline$h_{1}$ & 13,2 & 0,19 & 10,92 & 12,09 & 91,6 & $2, \pi 7$ & 0.29 & 2,5 & 0 \\
\hline$A_{1} C$ & 10,6 & 0,07 & 10,62 & 10,94 & saturó & 2,77 & 0,22 & 12,6 & 0 \\
\hline
\end{tabular}

Le profil du sol est le suivant:

$A_{1}(0-20 \mathrm{~cm})$ - Mull de structure grumeleuse à gros grumeaux, peu humifère. Des cailloux, de toutes dimensions, dès l'horizon supéricur - Nombreuses racines,

$A_{1} C(>25 \mathrm{~cm})$ - Horizon s'enrichissant en éléments d'arène, de structure à grumeaux plus fins; cailloux plus nombreux - Moins de racines.

Les analyses, comme la description meme du profil, montrent qu'il s'agit d'un sol à horizons non différenciés: c'est un ranker colluvial d̀ mull actif.

La texture est assez caractéristique: pauvreté en argile et forte richesse en sables grossiers. Alors que les deux horizons ont, dans leur terre fine, la même composition - ou une composition très voisine - la texture, par contre, diffère, l'horizon supérieur étant plus riche en éléments altérés.

I a matière organique peut ne pas paraitre très abondante (moins de $5 \%$ ): mais il s'agit d'une matière riche en azote minéralisé $\left(\mathrm{C} / \mathrm{N}\right.$ particulièrement bas, surtout en $\left.\mathrm{A}_{1}\right)$; indice de mull actif. D'autre part, par suite du colluvionnement qui remanie sans cesse le sol, on l'observe en quantités égales en surface comme à $25 \mathrm{~cm}$ de profondeur. Le sol contient donc, en fait, beaucoup d'éléments azotés directement assimilables, et c'est là son caractère fondamental.

La capacité d'échange est très élevée.

\section{$4^{\circ}$ Evolution.}

Dans quelle mesure PAulnaie verte constitue-t-elle une association permanente? La réponse doit être nuancée.

Si, comme nous l'avons vu, quelques rares Mélèzes et Epicéas arrivent à s'installer et à se maintenir dans les couloirs à Aulne 
vert, il ne semble pas que la forêt puisse vraiment se substituer à l'Aulnaie lorsque les conditions sont vraiment spéciales et se maintiennent telles: zones ou couloirs avalancheux, à forte pente, à grande humidité. L'Alnetum viridis apparaît alors vraiment comme une association permanente, spécialisée.

Mais l'on conçoit, dès lors, qu'un changement dans le régime des avalanches ou dans celui des eaux, ptisse perturber l'association et la faire avancer ou reculer. La consolidation du colluvium qui résulte d'un tel changement de régime hydrique permet alors à la forèt de s'installer: on peut penser que la pessière subalpine à hautes herbes précédemment décrite, constitue une phase dans la série qui va de l'aulnaie verte à la pessière subalpine mésophile, dans l'étage subalpin inférieur.

Fourchy, dans son étude sur l'Oisans, signale aussi l'envahissement par l'Aulne vert, des prairies de fauche et des pâturages abandonnés par l'homme; ce phénomène en Haute-Maurienne n'est pas très net; il mériterait, en tout cas, d'ètre étudié de près, ne serait-ce que pour voir à partir de quelles associations prairiales il s'amorce. II faut noter ici que, dans la région qui nous occupe, l'homme n'a cessé d'exercer une certaine " pression pastorale " , jusqu'à ces dernières années; d'autre part, le climat général est propice atux associations xérophiles ou mésophiles, qu'elles soient prairiales ou forestières si bien que le recul de l'homme dans l'étage subalpin se manifeste par l'avancée de la lande à Erica carnea ou par celle de la lande à Rhododendron ferrugineum.

\section{III.7.2. - Vue d'ensemble sur P'Aulnaie verte.}

Ce qu'il y a lieu d'étudier, in fine, au moins brièvement, ce sont d'une part, les affinités que présente l'Alnetum viridis, non seulement avec les autres associations à hates herbes classées à juste titre par BraUn-BL.ANQUET dans la même alliance, I'Adenostylion, mais aussi avec des associations forestières appartenant au Fagion; ce sont, d'autre part, les difficultés que rencontre la forêt résineuse à s'installer dans de telles associations, alors que le climat général se montre favorable.

Le rapprochement des relevés effectués dans l'Aulnaie verte avec ceux faits dans les associations à hautes herbes (association à Adenostyles Alliariae, de Luguer (A.), dans le Massif Central (1926), Adenostyleto-Cicerbitetum, de Braun-BlanQuet (J.), dans les Alpes rhétiques (1949), notamment, montre à l'évidence qu'il s'agit là d'associations vraiment voisines, à tel point que GurNocher (M.) (1939) pense qu'il « sera certainement nécessaire de les réunir en un groupement unique $n$.

Ce qu'il est plus intéressant de noter, ce sont les affinités qui lient 1'Alnetum viridis (et, d'une façon plus générale, les mégaphorbiaies) et certaines associations feuillues tel 1'Aceri-Fagetum, 
association climax, dans le Jura, de Pétage montagnard supérieur. Les relevés effectués par MooR (M.) (1952) et par Kuoch (R.) (1954), dans ce groupement - tout particulièrement dans la sousassociation à Cirsium oleraceum - font apparaitre une affinité floristique des plus remarquables avec 1'Alnelum viridis. Et ceci, bien que cette profonde parenté soit, dans une certaine mesure. masquée par l'appartenance de l'Aceri-Fagetum à l'alliance du Fagion. En effet, si des espèces comme Rumex arifolius, Cicerbita alpina, Ranunculus aconitifolius, Veratrum album. Thalictrum aquilegifolium, Campanula rhomboïdalis sont mises en évidence dans les relevés de Moor. les deux premières en tant que caractéristiques de l'association, les autres en tant que différentielles de sous-associations, d'autres espèces néso-hygrophiles de mull, sont reléguées dans le groupe anonyme des compagnes: il s'agit essentiellement d'Adenostyles Alliariae, Senecio Fuchsii et nemorensis, Melandrium diurnum, Saxifraga rotundifolia. Stellaria nemorum, Mvosotis silvatica. Ces espèces ont pourtant un grand intérêt de diagnose: elles se présentent, en effet, dans lassociation en cause (Aceri-Fagetum) avec un degré de présence et une ahondance-dominance sensiblement supérieurs à ceux qu'elles possèdent dans les autres associations de l'Alliance.

A cette affinité floristique, correspond une affinité ćcologique. Dans l'Aceri-Fagctum, le sol est à structure aérée. à gros grumeaux, à texture assez grossière: l'humus est du type mull, à forte activité biologique; la teneur en matière organique étant d'environ $5 \%$ et diminuant lentement avec la profondeur: tous caractères, on le voit, semblables à ceux des sols de l'Aulnaie verte.

Quant aux climats, ils présentent aussi des caractères voisins. encore que nous ne possédions pas, malheuretusement, de chiffres précis à leur sujet: dans l'Aulnaie verte règne une forte humidité: mais elle n'est pas celle du climat général: elle appartient à des stations spécialisées; dans la hêtraie à Erablc. cette humidité est "climacique $n$, pourrait-on dire: elle correspond au climat général de l'étage, humide, de " tempérament " océanique. C'est ici qu'il y a lieu de rappeler la très intéressante remarque de FAvarger (Cl.) (1958): "Rien n'illustre mieux, à notre avis, la différence de climat entre les étages subalpin et montagnard que de voir les groupements à hautes herbes s'associer à une forêt climacique dans l'étage montagnard, alors qu'au subalpin n - et. nous ajouterons personnellement: a fortiori au subalpin intraalpin - "elles constituent dans des stations particulièrement fraiches, des associations spécialisées, déterminées par le sol et le climat local $n$.

Quant au deuxième problème, nous ne ferons que l'effleurer, car il appellerait à lui tout seul, des études fines de physiologie écologique qui sortiraient du cadre de ce travail. Comment expliquer que la forêt résineuse connaisse les plus grandes difficultés à s'ins- 
taller dans la mégaphorbiaie et n'y arrive, en définitive, que très rarement?

En ce qui concerne l'Aulnaie verte, on peut évoquer la concurrence de l'Aulne vert, l'ombre qu'il crée, et surtout le colluvionnement auquel il est parfaitement adapté, au contraire du Mélèze et de l'Epicéa. Ces raisons sont peut-être bonnes; elles ne paraissent pas être les seules. On observe quelquefois, en Haute-Maurienne, tout particulièrement en forêt d'Arc - et ceci aussi bien dans l'étage subalpin que dans l'étage montagnard supérieur - de légères dépressions où prospèrent les " hautes herbes n. Ces dépressions se "reboisent " très difficilement.

Des observations semblables ont été faites - il y a longtemps déjà - dans les Préalpes et le Jura, où l'humidité générale est particulièrement favorable à la mégaphorbiaie.

Récemment, Richard (J.-L.) (1960), fait obscrver que dans l'étage montagnard supérieur du Jura a les épicéas isolés qu'on trouve parfois dans la mégaphorbiaie, croissent toujours sur des ilots rocheux avec humus brut et myrtilles qui émergent de la terre argileuse. Il s'agit donc d'une mosaique de deux stations très différentes. Il serait done inutile de vouloir à tout prix régénérer l'Epicéa dans la mégaphorbiaie elle-mène, qui-est probablement une station non forestiere $n$.

Ce phénomène n'a pas échappé aux forestiers français: à MATHEY (A.) (1911), dans la série de Génietux en Grande Chartreuse et, plus récemment, à LAchaussíz (E.) (1947) dans la forêt du Massacre, dans le Haut Jura. Ce dernier, après avoir montré que ni l'accumulation de neige, ni l'intervention de l'Herpotrichia nigra, ne peuvent expliquer de façon satisfaisinte le phénomène observé, on conclut que c'est dans le sol qu'il faut rechercher l'explication: dans les sols de la mégaphorbiaie, à pH stubacide, à mull, doivent se développer des champignons responsables de la fonte des semis. Ainsi ces sols riches, humides, à taux de saturation élevé, considérés autrefois comme les meilleur's sols forestiers, atussi bien pour les résineux que pour les feuillus, s'avèreraient, en définitive, très défavorables à la régénération des résineux, à leur implantation. Et ce qui se passe dans 1'Alnetum viridis - où le colluvionnement joue tout de même un róle particulier, selon toute vraisemblance confirmerait les observations faites dans la mégaphorbiaie proprement dite. Il n'était donc pas intitile d'évoquer à son sujet ce problème forestier. 


\section{III.8. - HETRAIES ET CHENAIE PUBESCENTE}

\section{III.8.1. - Les hêtraies.}

On sait que les hêtraies ne pénètrent pas - tout au moins dans les Alpes occidentales françaises - au cour du monde intra-alpin, d'où la sécheresse semble les exclure.

Ce fait a été souligné pour les principales vallées des Alpes, par de nombreux phytogéographes, notamment par FlaHAULT, pour l'Ubaye (1897), par BRAUN-BLANQUET pour la Haute-Durance (1922), par Ph. Guinier pour la Haute-Tarentaise (1950), par P. FourchY pour l'Oisans (1951); la Haute-Maurienne ne fait pas exception à la régle comme l'ont noté Beauverd (1911), et, plus près de nous, C. Guinet et R. de Vilmorin (1961).

C'est dire que les hêtraies que nous avons analysées sont des groupements finaux, situés dans tue sone de contact, où l'on rencontre, dans l'étage montagnard, de nombreux éléments des pineraies de Pin sylvestre ou de la sapinière vraie, qui vont relayer les hêtraies à l'amont de la vallée. Leur étude, par ce fait même, en est rendue plus difficile.

On les observe, en outre, dans un domaine qui a subi tout particulièrement l'influence humaine: les placages glaciaires et les faibles pentes, propices à l'installation de l'homme, dominent, en effet, autour de Saint-Martin-la-Porte, de Saint-Martin d'Arc et d'Albanne, de telle sorte que les hêtraies se présentent souvent comme des lambeaux at milieu d'un paysage de bocage. Paraissent faire exception à la règle, les deux flancs des gorges de la Valloirette et la rive droite du torrent de Saint-Martin-la-Porte, qui sont, dans l'ensemble, très escarpés. Mais l'on doit noter que même là, l'action des populations et celle du forestier se sont conjuguées (28), pour transformer la physionomie des peuplements originels en éliminant, aussi systématiquement que possible, le Hêtre, excellent bois de feu et concurrent sérieux pour les résineux. Ce fait accentue, lui aussi, les difficultés de l'analyse.

Cette dernière, pour être complète et plus claire, il faut le souligner, aurait gagné à porter également sur les hêtraies de la moyenne Maurienne, ce qui eût permis de mieux saisir l'appauvrissement

(28) On doit $y$ ajouter les ravages causés par deux incendies importants et par les fumées de l'usine de Calypso. 
floristique progressif des hêtraies préalpines lorsqu'elles s'enfoncent dans l'intérieur des vallées; mais il fallait bien fixer des limites à notre travail. Les relevés que nous avons pris nous paraissent cependant suffisants, pour donner une bonne image de ces hêtraies finales, au contact de la zone intra-alpine.

\section{Organisation sociologique et composition floristique.}

Si l'on envisage le tableau général des relevés, on constate tout d'abord que les hêtraies "intra-alpines " sont caractérisées sous leurs formes les plus xérophiles, par la présente très constante, de Polygala Chamaebuxus, élément des pineraies qui n'existe pas dans les hêtraies, même les plus xérophiles, du Jura ou des Préalpes. Cette espèce constitue donc une excellente différentielle géographique.

Il faut noter, en second lieu - et ceci ne doit pas étonner dans un groupement marginal - labsence de nombreuses espèces du Fagion (espèces mésophiles de l'étage montagnard liées à un sol à mull), comme Dentaria pinnata, Polystichum lobatum, Dentaria digitata, Festuca silvatica, on d'espèces des Fagetalia (espèces mésophiles liées au mème type de sol que les précédentes, mais plus plastiques quant à l'altitude) comme Laminm Galeobdolon, Polygonatum multiflorum, Carex silvatica, Euphorbia amygdalö̈des, Asarum europaeum, Arum maculatum, Alliwm ursinum, alors que certaines autres espèces, caractéristiques des mêmes unités dans le système Braun-Blanguet, subsistent encore (Sanicula europaea, Asperula odorata, Bromus Benekeni, Elymus Europaeus).

Si lon entre dans le détail, on constate que deux groupements peuvent être distingués:

- le premier, nettement xérophile et thermophile est riche en Pin sylvestre et en Céphalanthères;

- dans le second, plus mésophile, se rencontrent les espèces du Fagion et des Fagetalia dont il vient d'être question, qui étaient absentes du premier tandis que disparaissent les xérophiles.

Le premier groupement, à Céphalanthères, se présente sous trois aspects quelque peu différents.

En contact très étroit avec les premières enclaves des pineraies, à exposition plein sud, on observe une sous-association ultra-marginale à Carex humilis et Astragalus monspessulanus où le groupe des xéro-calciphiles est particulièrement bien représenté. Ce type ne diffère presque pas de l'Ononido-Pinetum que nous avons distingué à Chrysanthemum corymbosum et Céphalanthères, si ce n'est que le Hêtre $y$ atteint l'âge adulte et reste en sous-étage de façon clairsemée. 


\section{PLANCHE XVII}

\section{LES HETRAIES}

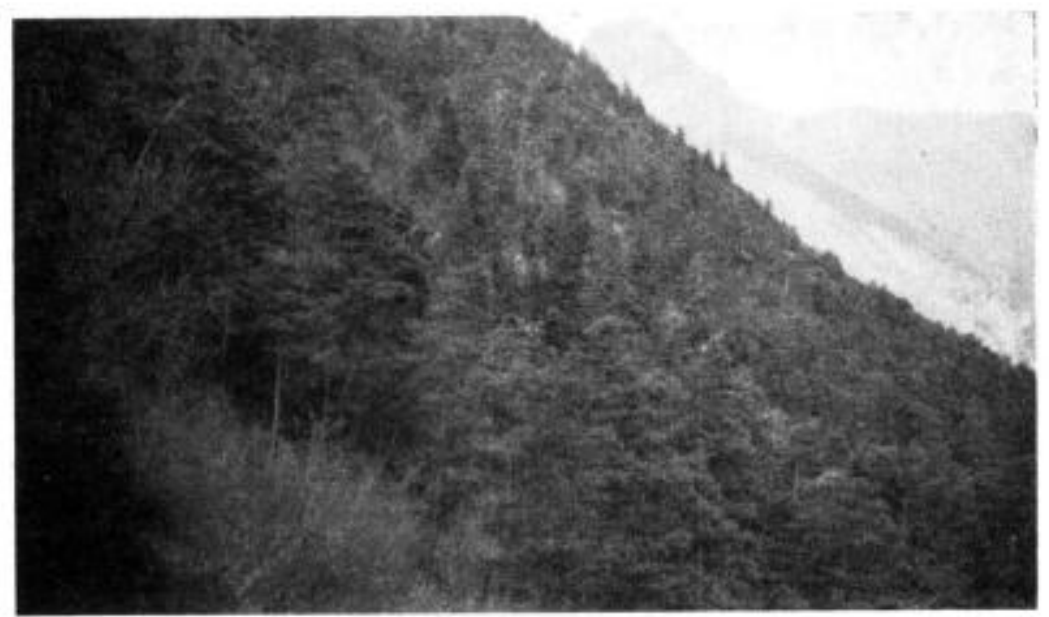

Le hêtraie ì Cephalanthères et Polygala chamacbuxus riche en Pins sylvestres. - Vallée de la Valoirette, - Vue générale.

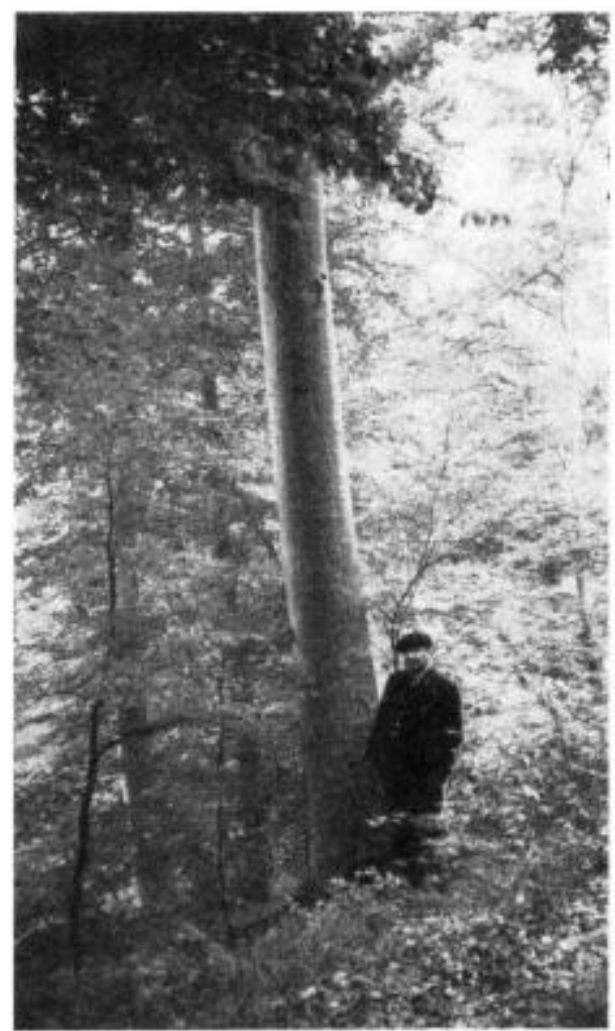

La hêtraie à Asperula odorata et Elymus Europaeus. (Saint-Martin-la-Porte) 
Dans des conditions un peu moins xériques, apparait le groupe des méso-xérophiles calciphiles, avec Sesleria coerulea comme tête de file qui donne aux strates inférieures leur physionomie. Le Pin sylvestre est le plus souvent dominant.

Le troisième groupement - toujours xérophile et se différenciant des hêtraies préalpines par Polygala chamaebruxus - est le moins spécialisé et le moins marginal. Il correspond, à notre avis. au type moyen, de l'association à Céphalanthères.

La deuxième association se différencie de la précédente par l'absence totale de xérophiles, la diminution très sensible des mésoxérophiles - tout particulièrement des Céphalanthères et de Polygala Chanaebuxus - et l'apparition d'une fraction du groupe des espèces mésophiles de mull liées au hêtre: le aroupe à Asperula odorata et Elymus Europaeus. On notera que le groupe à Euphorbia dulcis et Phyteuma spicatum qui reste présent dans l'Abietetum albae et qui est déjà représenté dans la hêtraie à Céphalanthères est ici très étoffé.

Deux faciès peuvent ètre distingués dont l'un riche en sapin; la dominance de ce dernier, toujours guité, est certainement artificielle et due à l'action du forestier qui a porté son effort sur la destruction du Hêtre considéré comme une essence gênante, pour l'essence résineuse; on notera la présence, mais peti constante, de Trochischantes nodiflorus et la relative ahondance de Lusula nivea.

\section{2。Dénomination - Identification.}

a) la hêtraie à Céphalanthères et à Polygala Chamaebruxus.

Parmi les hêtraies les plus thermophiles et les plus xérophiles décrites à ce jour, quatre doivent retenir notre attention: la hêtraie à Buis, proche de la chênaie pubescente à Buis 「Buxo-Fagetum de Braun-Blanouet (J.) et Susplugas (J.) (1937) 1 subméditerranéenne, la hêtraie à laiches [Carici-Fagetum de Moor (M.) (1952)] du Jura, la hêtraie à Seslerie [Seslerio-Fagetum de Moor (M.) (1952)] du Jura également et la hêtraie à Polygala Chamaebuxus [Fagetum silvaticae polygaletosum de KuocH (R.) (1954)] des Alpes intermédiaires.

Ces quatre associations possèdent d'ailleurs en commun un contingent d'espèces que l'on retrouve dans les chênaies pubescentes dont les Céphalanthères, Coronilla Emerus, etc. en dehors de certaines espèces caractéristiques du Fagion et des Fagetalia.

Si l'on examine avec attention la composition floristique de ces quatre groupements, on se convainc aisément que notre association à Céphalanthères et Polygala Chamaebuxus sous sa forme la moins spécialisée, et la moins extrême, doit être rapportée au Fagetum silvaticae polygaletosum de КUосн, bien qu'elle soit encore plus 
pauvre que ce groupement en éléments du Fagion et des Fagetalia et qu'elle soit plus proche que lui des pineraies de Pin sylvestre.

On notera, par contre, que notre sous-association à Sesleria corulea et Carex alba doit être considérée comme une forme $\alpha$ intraalpine " du Seslerio-Fagetum anthericosum jurassien dont elle est proche (29) mais dont elle diffère par la présence de Polygala Chamaebuxus et l'absence d'un certain nombre d'espèces comme $\mathrm{Mer}$ curialis perennis, Polygonatum multiflorum, Euphorbia anygdalö̈des.

\section{b) Ia hêtraie à Asperula odorata ef Elvunus europaeus.}

Cette association ne peut pas être rapportée de façon stricte à un groupement déjà défini. Elle nous parait se situer entre un Fageium silvaticae typicum appauvri et le Fagetum silvaticae polygaletosum, encore que l'on puisse valablement le rattacher à ce dernier si l'on se réfère à sa pauvreté en espèces du Fagion et des Fagetalia.

\section{c) la hêtraie à Sapin et à Trochischantes nodiflorus.}

Bien que Trochischantes nodiflorus ne soit pas très constant, sa présence est à souligner car l'on sait qu'il constitue un élément important de la flore de certaines hêtraies à Sapin et même de sapinières dans les Alpes du sud (30). Le faciès à Trochischantes est donc bien à mettre un peu à part (encore qu'il soit très proche du précédent), car il pourra probablement être rattaché à certains groupements semblables des Alpes du sud, lorsque ces derniers auront été analysés.

\section{Répartition - Ecologie.}

Répartition: Beauverd (G.) (1911), avait déjà signalé que la zone de pénétration du Hêtre en Maurienne s'étendait, en remontant la vallée, " jusqu'à Montdenis, avec des bosquets d'avant-poste à $1600 \mathrm{~m}$, sous le rocher des Encombres, entre Saint-Julien et Saint-Martin-la-Porte n. De Vilmorin (R.) et Guinet (C.) (1961) ont observé également que le Hêtre était absent de la haute-vallée et que a sa dernière station, la plus en amont, se trouvait sur la rive droite, presque en face de Saint-Michel-de-Maurienne n. De fait, nous n'avons nous-mème trouvé aucune station à l'amont de Saint-Michel. Le Hêtre s'arrête donc bien à cette dernière localité.

(29) Comme elle est très proche de l'Erico-Pinetum, abstraction faite d'Erica carnea.

(30) La forêt de Boscodon, an pied du Grand Morgon, près d'Embrun, est en particulier très riche en Trochischantes, à un point tel, que BRnUNBlanguer, dans sa récente description de la flore des vallées intra-alpines (1961) a pu la désigner sous le nom de e Trochisceto-Abietetum ?. Ce type se rencontre d'ailleurs dans toutes les Alpes du Sud. 
Comment se répartissent, entre Saint-Julien-de-Maurienne limite de notre dition - et Saint-Michel, les divers types de hêtraie que nous avons distingués?

Les plus xérophiles s'observent sous Albanne, sur la rive gauche, tournée vers l'est du torrent de la Valloirette et à l'adret, dans le massif des Encombres, au-dessus de l'étage du Chêne pubescent. C'est sur ces deux flancs, assez escarpés que l'on rencontre la hêtraie à Céphalanthères et Polygala Chamaebuxus et les formes de passage vers la pineraie xérophile; elle s'étage dans les gorges entre $1000 \mathrm{~m}$ et $1650 \mathrm{~m}$ et à l'adret entre 1200 et $1600 \mathrm{~m}$.

La hêtraie relativement mésophile et qui, quoique appauvrie n'est pas sans rappeler la hêtraie typique préalpine, occupe, par contre, par lambeaux, les expositions nord et ouest, à partir de $700 \mathrm{~m}$.

\section{Climat - microclimat.}

Les données météorologiques fournies par le poste du Thyl permettent de se faire une idée du climat général régnant dans la zone finale des hêtraies. On a vu que, par rapport au climat typiquement

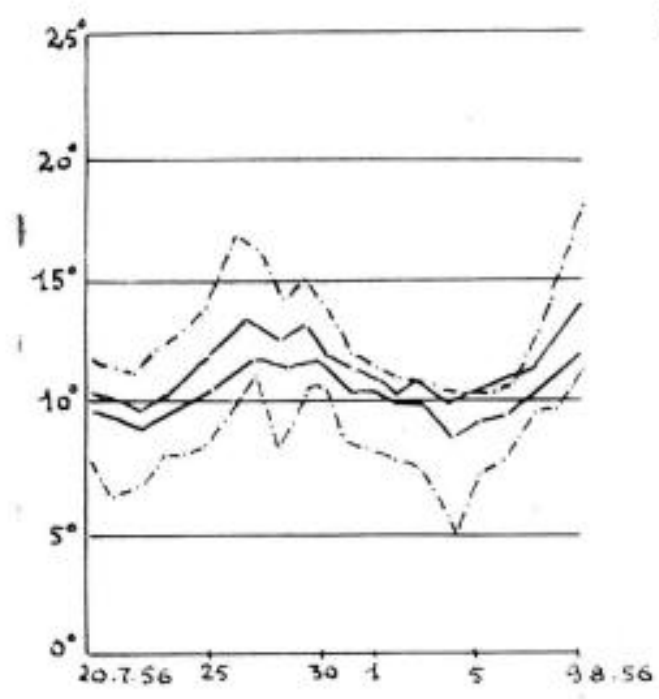

Températures du $501(-0,02 \mathrm{~m})$.

--. Erico-Pinetum (1 $300 \mathrm{~m})$.

Fagetum (1 $300 \mathrm{~m})$.

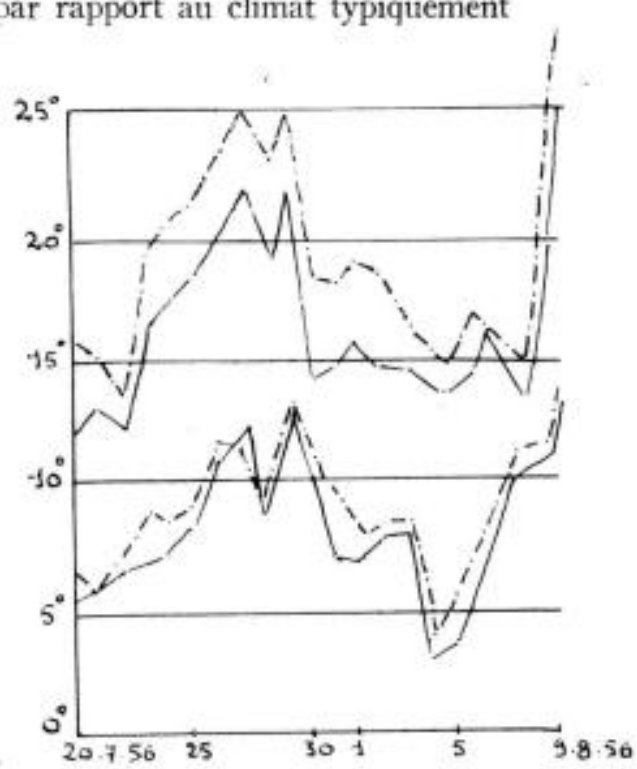

Températures de l'air $(+1,50 \mathrm{~m})$.

-. - Erico-Pinetum. Fagetum.

FiG. 55.

Climats de la hêtraie à Asperula odorata et Elymus Evropaeus et de l'Erico-Pinetum hylocomietosum. 


\section{- hrgaone}

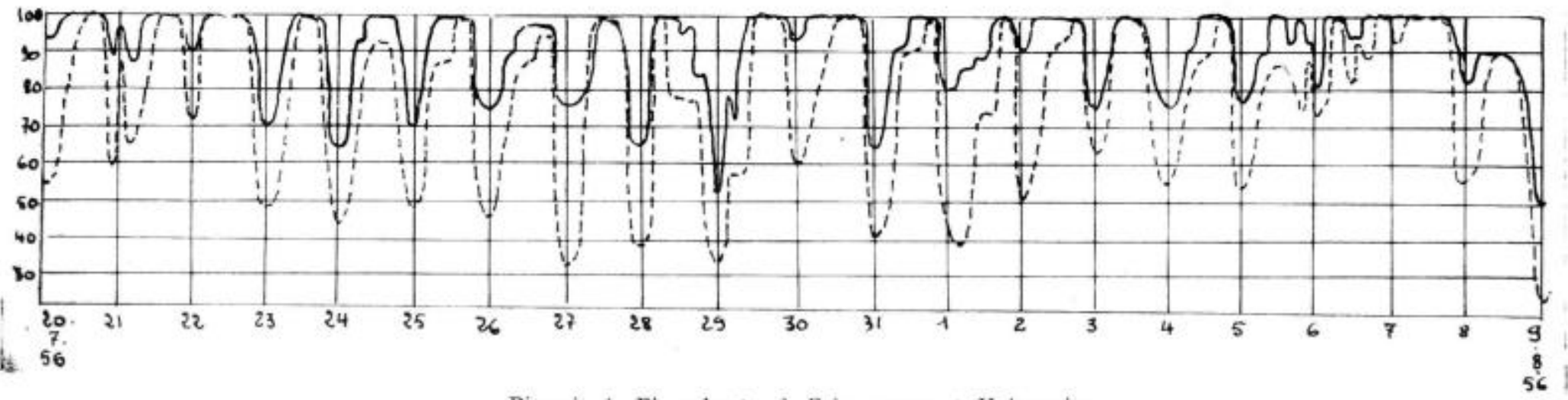

Hetraie it Asperule.

Fig. 56 .

Légende, - Le dessin ci-dessus - reproduction légèrement schématisée d'une bande d'hygromètre enregistreur - montre que le degré hygromé trique à $1,50 \mathrm{~m}$ dans la pineraie à Erica carnea et Hylocomies, au coeur de la zone sèche de la Haute-Maurienne, est descendu en milieu de journée, sensiblement plus bas que dans la Hétraie fraiche à Aspersila odorata, située dans une zone plus arrosée (région de Saint-Martin-la-Porte).

I1 est évident que si, dans ce phénomène, le climat général joue un rỏle primordial, on ne doit pas oublier que la structure méme de la forẻt contribue à accentuer les différences (le feuillage dense du Hêtre créant un microclimat plus humide que celui rencontré sous le feuillage plus clair du Pin sylvestre). 
intra-alpin d'Avrieux, celui du Thyl apparait comme sensiblement plus humide.

Nous aurons d'ailleurs l'occasion, dans la conclusion de ce travail, de revenir sur ce point, en situant les hêtraies et les autres associations forestières de la Haute-Maurienne dans un système de référence ombrothermique.

Les quelques chiffres relatifs au climat que nous avons pu recueillir et qui concernent un individı de hẻtraie à Asperule comparé à un individu d'Erico-Pinetum situé à la même altitude et à la même exposition, montrent que le climat de la hêtraie, aussi bien celui du sol que celui de l'air, est moins brutal que celui observé dans l'Erico-Pinetum, lequel l'était encore beaucoup moins, comme on l'a déjà $\mathrm{vu}$, que celui de l'Ononido-Pinetum. Ce régime, relativement amorti du climat de la hêtraie, tient certainement au degré hygrométrique et à la pluviosité qui, plus forts que dans les pineraies, jouent le rôle de " volant thermique " (fig. 53).

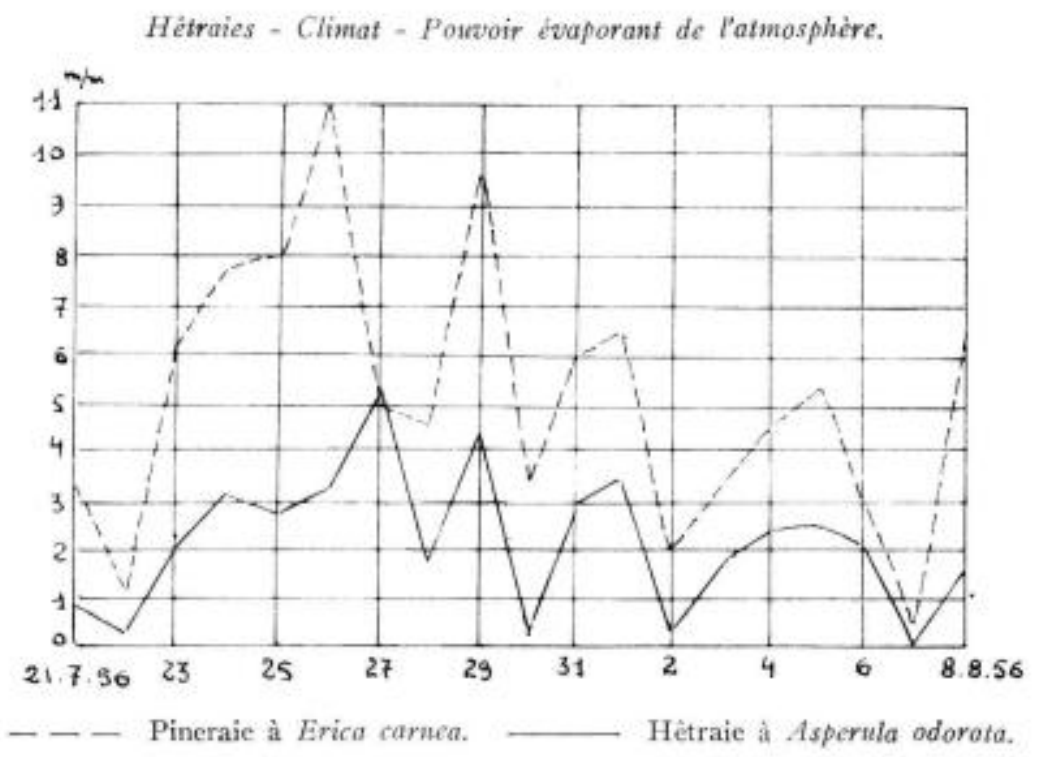

Fic. 57.

Légende, - Fin juillet et début août 1956, le pouvoir évaporant de l'atmoshère à $1,50 \mathrm{~m}$ (entre $8 \mathrm{~h}$ et $16 \mathrm{~h}$ ) s'est avéré étre sensiblement plus élevé dans la Pineraie à Erica carnea et Hylocomies (à $1300 \mathrm{~m}$ ) que dans la Hêtraie à Asperule et Orge d'Europe (à $1300 \mathrm{~m}$ également), située dans une zone déjà plus arrosée (région de Saint-Martin-la-Porte).

Sol - Chimisme - Etat hydrique.

L'examen des profils de sol, comme leur analyse montrant que nos hêtraies reposent, soit sur des sols calcimorphes, plus ou moins 
PF o

Les hêtraies - Potentiel capillaire d $-0,05 \mathrm{~m}$ et $-0,25 \mathrm{~m}$.

$-0,05^{m}$

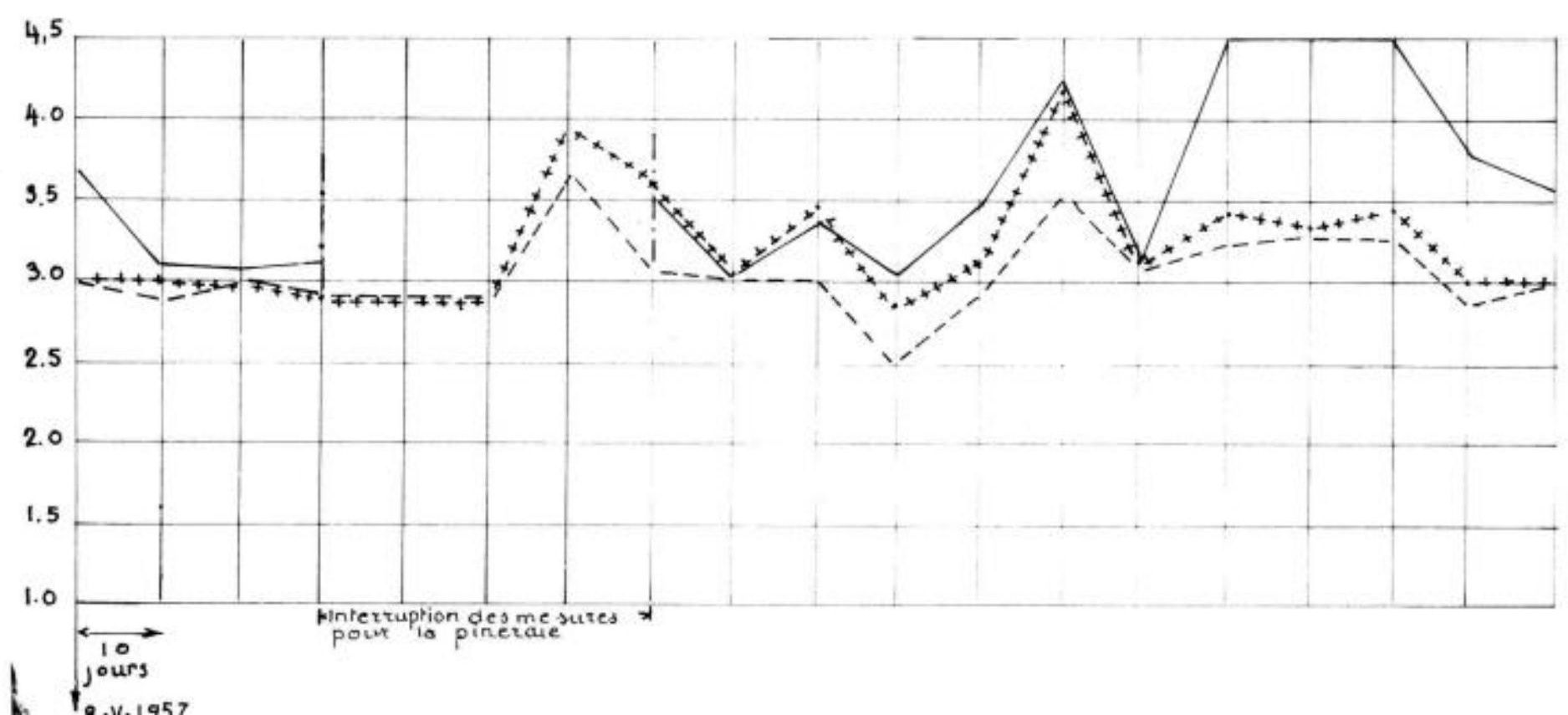




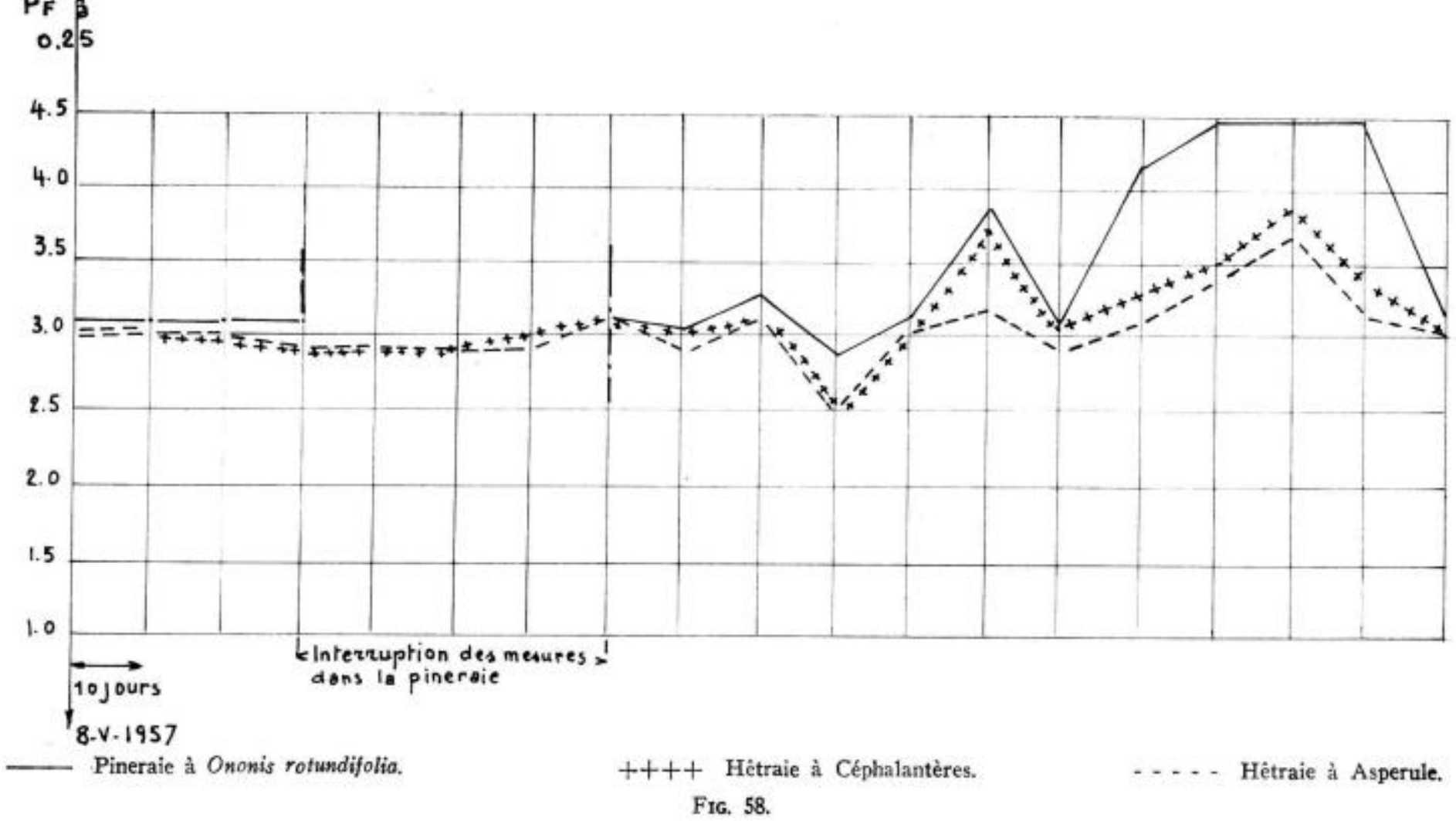


décarbonatés, selon le degré de pente, soit sur des sols bruns à mull. Tous ces sols, est-il besoin de le dire, sont jeunes car soumis à l'action incessante de l'érosion.

D'une façon générale, et comme on peut s'y attendre en se référant aux groupes écologiques, les sols de la hêtraie à Céphalanthères et Polygala Chamaebuxus sous ses diverses formes, sont des rendzines de pente dont certaines un peu brunifiées sur les replats.

Exemple de rendzine colluviale; sol $n^{\circ} 1$ (relevé floristique $n^{\circ} 12$ ).

\begin{tabular}{|l|c|c|c|c|c|}
\hline Hor1 zons & pH & 0 & Mat.0rg. & $\infty^{3} \mathrm{Ca}$ & $\begin{array}{c}\text { Ca } \\
\text { Johangesble }\end{array}$ \\
\hline$A_{1}(-2$ On $)$ & 6,6 & 11,27 & 19,1 & 0,9 & 43 \\
$A_{1}(-1000)$ & 7,4 & 2,18 & 3,7 & 0,4 & 28 \\
\hline
\end{tabular}

Exemple de rendzine brunifiće sur replat: sol $n^{\circ} 2$ (relevé floristique $n^{\circ} 14$ ).

\begin{tabular}{|c|c|c|c|c|c|c|c|}
\hline Hori wons & $\mathrm{pH}$ & c & $\begin{array}{l}\text { Mat. } \\
\text { organ. }\end{array}$ & $\mathrm{Co}^{3} \mathrm{Ca}$ & $\begin{array}{c}C_{A} \\
\text { Colares. }\end{array}$ & $\mathrm{N}$ & $c / s$ \\
\hline A, & 6,9 & 6,7 & 11,3 & Nosent & 30,0 & 0,42 & 15,9 \\
\hline$A_{2} / B$ & 6,2 & & 3,7 & 2,5 & 17,0 & & \\
\hline
\end{tabular}

Dans les hêtraies les plus mésophiles, à Asperule odorante et Sanicle d'Europe les sols sont, dans lensemble, plus décarbonatés, ce qui est en plein accord avec l'organisation sociologique puisque, dans ce type de hêtraies dominent des groupes écologiques d'espèces liées à des sols à mull.

Nous donnons ici, par ordre de décarbonatation croissante, trois exemples de types de sol pris dans ces hêtraies.

Sol $\mathrm{n}^{\circ} 3$ (relevé floristique $\mathrm{n}^{\circ}$ 18)

Rendsine brunifiée, analogue au sol $\mathrm{n}^{\circ} 2$.

\begin{tabular}{|c|c|c|c|c|c|}
\hline Hor1 zone & $\mathrm{pH}$ & c & Nat. organ. & $\operatorname{co}^{3} \mathrm{~cm}$ & C. tobang. \\
\hline$A_{1}$ & 6,7 & 23,7 & 40,4 & Neant & 74,5 \\
\hline B & 6,8 & 6,45 & 10,9 & 2,8 & \\
\hline
\end{tabular}


Sol $n^{*} 4$ (relevé floristique $n^{*} 19$ ).

Sol urun à mull, pas très éloigné d'une rendzine brunifiće.

\begin{tabular}{|c|c|c|c|c|c|}
\hline Horizons & pH & 0 & Nat. organ. & $\infty^{3} \mathrm{Ca}$ & Co bohang. \\
\hline$A_{1}$ & 6,8 & 12,29 & 20,8 & Traces & 64 \\
$\mathrm{~A}_{2} /(B)$ & 6,7 & 6,21 & 10,5 & Traoes & 36 \\
\hline
\end{tabular}

Sol $\mathrm{n}^{\circ} 5$ (relevé floristique $\mathrm{n}^{\circ} 21$ ).

Sol brun à mull.

\begin{tabular}{|c|c|c|c|c|c|c|c|}
\hline Hor1eona & $\mathrm{pll}$ & c & $\begin{array}{l}\text { Mat. } \\
\text { orgati. }\end{array}$ & $\infty^{3} \mathrm{ces}$ & $\begin{array}{c}\mathrm{Cs} \\
\text { colinng. }\end{array}$ & $s$ & $c / s$ \\
\hline$\Lambda_{1}$ & 6,9 & 10,76 & 98,3 & yent & 34,5 & 0,84 & 12,8 \\
\hline$A_{2}$ & 7 & & 5,1 & Moant & 18,8 & & \\
\hline
\end{tabular}

L'étude de l'énergie de rétention de l'ean par le sol, traduite par le potentiel capillaire, montre que le sol de la hêtraie à Céphalanthères et Polygala chamaeburus a constamment présenté, pendant toute la durée de l'expérience déjà signalée, un potentiel capillaire, aussi bien près de la surface $(-5 \mathrm{~cm})$ qu'en profondeur $(-25$ $\mathrm{cm})$ plus élevé que celui de la hêtraie à Asperule, c'est-à-dire qu'il s'est avéré être toujours plus sec, ce à quoi on pouvait s'attendre.

En mettant en parallèle les courbes relatives aux hêtraies et celles concernant l'Ononido-Pinetum, on constate que le sol de la pineraie est sensiblement plus sec que celui des deux hêtraies (fig. 56).

\section{III.8.2. - La chênaie pubescente.}

En Haute-Maurienne, comme dans les autres vallées " internes » des Alpes françaises et même suisses, elle Chêne pubescent pénètre plus profondément que le Hêtre dans le monde intra-alpin (31).

Malgré cela, dans le territoire que nous avons étudié, il ne s'agit presque plus de chênaie pubescente proprement dite - sauf aux abords de Saint-Julien-de-Maturienne - mais plutôt d'individus plus ou moins groupés, car l'homme installé au bas des adrets a

(31) Il en est bien ainsi dans le Valais [Braun-Bt.anourt (J.) et Richard (R.) (1949)], en Tarentaise [Tessier (M.L.) (1907), GuINIER (Ph.) (1950)], dans la haute vallée de la Durance [Humbert (H.) (1951)], dans le Queytas [Flahault (Ch.), (1897)], etc... 
fortement perturbé l'association originelle (traitement en taillis à courte révolution - pacage des chèvres) et l'a même fait disparaitrc le long des gorges houillères.

Aussi ne nous a-t-il pas été possible d'établir, pour ce groupement, un tableau phytosociologique valable.

\section{$1^{\circ}$ Organisation sociologique - Composition floristique.}

Nous définirons la chẻnaie pubescente finale de la Haute-Maurienne (région de Saint-Julien-de-Maturienne) en la comparant à la hêtraie à Céphalanthères et Polygala Chanaebuxus dont elle est évidemment très proche.

On notera dans la chênaie, la dominance de Quercus pubescens et l'existence, comme différentielles strictes, de Cornus mas, Acer Monspessulanum, Colutea arborescens, Cerasus Mahaleb, en même temps que l'absence à peu près totale des rares espèces du Fagion que lon rencontrait encore dans la hetraie xérophile (Lilium Martagon, Neottia nidus avis).

On y rencontre aussi beaucoup plus que dans la hêtraie, des éléments de fruticaie, comme Coronilla Emerus, Rhamnus alpina, Acer Opalus, Viburnum lantana, Cornus sanguinea, Amelanchier ovalis, Cotoneaster tomentosa, qui donnent d'ailleurs it cette chênaie sa physionomie; $s^{\prime} y$ ajoutent d'autres espèces, caractéristiques comme les précédentes dans le système Braun-Blanguet du Quercion pubescenti-sessiliflorae ou des Quercetalia pubescentis, telles Helleborus fatidus, Digitalis lutea, Daphne Laureola, Geranium sanguineum, Campanula persicifolia (32).

La structure de notre chênaie, fortement marquée par l'homme, comme il a été dit, apparait souvent comme une micromosaĩue. où des taches de taillis dense alternent avec de minuscules clairières, dans lesquelles se cantonnent les espèces de "garides n, telles $\mathrm{Al}$ lium sphaerocephalum, Pimpinella saxifraga, Lactuca perennis, Stachys rectus, Fumana procumbens, Globularia Willkommi, Origamum vulgare, Helianthemum canum, Dianthus caryophyllus subsp. silvestris, etc.

Il n'est pas sans intérêt, enfin, de signaler l'absence totale d'espèces, telles que Cytisus sessilifolius, Lonicera etrusca, Inula spiraeifolia, Melittis mellissophylum, sans oublier Buxus sempervirens dont nous reparlerons, et qui jouent un rôle important dans les chênaies pubescentes méridionales.

(32) On notera que certaines de ces espèces (Amelanchier ovalis, Cotonéaster tomentosa) dépassent assez largement le domaine de la chẻnaie ou de la hétraie xérophile, et se retrouvent dans la pineraie de $\mathrm{P}$ in à crochets (étage subalpin). 
PLANCHE XVIII

LA CHENAIE PUBESCENTE

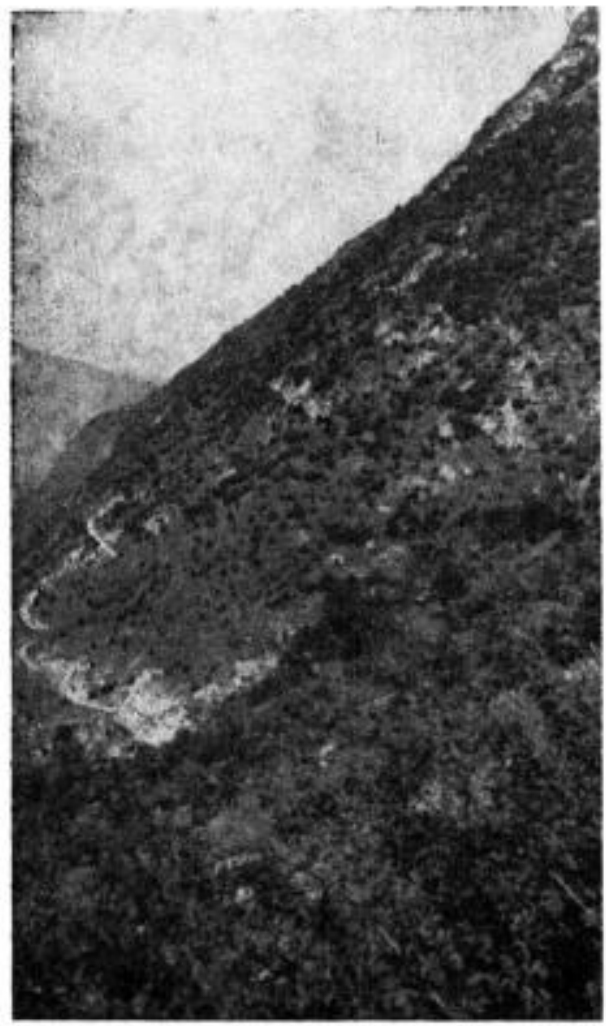

Ladret de Saint-Julien-de-Maurienne domaine de la Chênaie pubescente, dégradée surtout vers le bas. 


\section{2" Identification.}

On sait que les phytosociologues ont distingué en France et en Suisse trois types de chênaie pubescente: le Buxo-Quercetum qui borde, vers le nord, dans la région méditerranéenne, les forêts de chène vert et qui joue un grand róle dans les Préalpes du sud (Baronnies et Diois notamment); ce groupement s'infiltre d'ailleurs, le long de stations privilégiées, dans les Préalpes du nord et atteint le Jura méridional [QUANrrn (A.) (1935)] — le LithospermoQuercetum, moins méditerranéen et moins thermophile [QUANTrN (A.) (1935) - LEMÉE (G.) (1946)], enfin le Campanuleto-Quercetum pubescentis décrit récemment dans le Valais par BRAUNBlanquet (J.) (1959) qui est très proche du précédent dont il se distingue essentiellement par la présence de Campanula bononiensis.

Ces trois groupements ont d'ailleurs en commun un grand nombre d'espèces et ils diffèrent surtout par leur physionomie, le buis par sa dominance ou son absence jouant un grand rôle dans ce domaine.

Il n'est pas très facile de rattacher de façon stricte notre groupement à l'une des trois associations signalées ci-dessus, ćtant donné son appaurrissement floristique. On peut toutefois noter qu'il diffère sensiblement du Buso-Quercetum, car le buis qui ne pénètre absolument pas dans la vallée de la Maurienne, en est totalement absent, comme d'autres espreces méridionales déjà signalées.

Il se rapproche beaucoup plus dit Campanulcto-Quercetum pubescensis, encore que nous nayons jamais rencontré Campamula bononiensis, ni Lithospermum purpureo-cocruleum d'ailleurs (33).

\section{$3^{\circ}$ Répartition.}

Dans la région de Saint-Julien-de-Maurienne-Montricher, la chênaie occupe, soit les adrets (de la zone des cultures au sommet des cônes de déjection, jusqu'à $1200 \mathrm{~m}$ environ où elle est relayée par la hêtraie), soit, sur versant général nord, les versants secondaires exposés à l'est et au sud-est jusqu'à $1000 \mathrm{~m}$ environ.

Elle disparait un peu plus à lamont vers Saint-Martin-la-Porte. où l'homme a installé ses cultures et ses prairies, jusqu'à une assez forte altitude. On retrouve quelques chênes plus ou moins groupés au milieu d'un cortège prairial xérophile, près du hameau de la Buffaz à l'adret, au-dessus de Saint-Michel-de-Maurienne. Cette station était considérée comme la dernière de l'espèce, jusqu'à ces dernières années [Guinet (C.) et de Vilmorin (R.) (1961)].

(33) En fait, le Lithospernum purpureo-coeruleum ne figure pas dans les stations très sèches à Quercus pubescens. Moor (M.) (1962) cité par RıCHaRd (J.L.) (1965), propose de nommer e Coronillo-Quercetum > la véritable chènaic pubescente des pentes rocheuses les plus sèches, où l'on rencontre Quercus pubescens, Acer Opalus, Coronilla coronata, etc. 
Il n'en est rien. Si le long des gorges houillères, à l'adret, où l'homme est installé jusqu'au talweg, on ne trouve pas trace de chêne pubescent, on peut observer sous le fort du Replaton, audessus de Modane, soit à une douzaine de kilomètres à vol d'oiseatu de la station de la Buffaz, une fruticaie bien développée, sans chêne pubescent sans doute, mais assez riche en éléments de la chênaie avec notamment Acer Monspessulanum, Lathyrus latifolitrs, Cerasus Mahaleb, Coronilla Emerus, Amelanchier rotundifolia, Viburmum lantana, Cormus sanquinea, etc. [BArtoli (C.) (1961)]. Plus à l'amont encore, à $1300 \mathrm{~m}$ d'altitude et à l'adret, dans des rochers de verrucano, nous avons pu trouver, cette fois, des chênes pubescents, qui sont vraiment les derniers représentants de l'espèce dans la vallée: Acer Monspessulanum n'était pas absent. On notera, sur l'éboulis que dominent les roches, la présence de Carlina acanthifolia, qui atteint également ici sa limite amont dans la vallée.

\section{Pineraies de Pin svivestre ct chênaic pubescente: le problème du climax.}

Etudiant, dans la région de Sierre, dans le Valais (forêt de Finges), les rapports existant entre l'Odondito-Pinetum et la chênaie à Quercus pubescens, Braun-Blanguet (J.) (1949) concluait que " abandonnés par l'homme, les bois de Pin sylvestre perdraient du terrain aux dépens du Chêne pubescent qui, sur les sols évolués, formerait l'association climatique terminale, tandis que le Pin dans l'association de l'Euphrasieto-Pinetum (Syn. - Odondito-Pinetum) serait refoulé sur les sols pauvres, très secs et peu profonds n.

Fn dautres termes, "la Pineraie pure, grotipement permanent des sols peu évolués, ne constitue pas le climax climatique, comme certains auteurs lont supposé (GAms - Bull. Soc. Murithienne, 44. 1927) $)$.

Bien que nous ayons déjà assez longuement développé notre point de vue sur la question de savoir si les pineraies xérophiles de la Haute-Maurienne constituaient ou non le climax, il nous parait utile de revenir brièvement sur ce point à la suite des observations formulées par Braun-BLANQUET.

Il faut noter, tout d'abord, que l'Odondito-Pinetum de la forêt de Finges se situe à assez basse altitude (entre 560 et $940 \mathrm{~m}$ ) et qu'il se trouve être assez riche en espèces que nous n'avons jamais rencontrées dans nos pineraies, à savoir Quercus pubescens, Ligustrum vulgare, Prunus Mahaleb, Campanula bononiensis, Bupleurum falcatum, Peucedanum Cervaria. II est donc parfaitement concevable qu'à ces altitudes - qui sont celles de l'étage du Chéne puhescent - ce dernier ait reculé du fait de l'homme et laissé la place à un pineraie localisée primitivement sur des sols très secs et peu profonds. 
Mais, nous pensons qu'en Maurienne, au-dessus de l'étage du Chêne pubescent, qui se prolonge assez loin dans la vallée et qui se termine en biseau, à mesure que s'élève le fond du talweg, les hêtraies xérophiles, dont nous avons analysé les groupements finaux, étaient progressivement remplacées par des pineraies xérophiles de Pin sylvestre, climaciques (34).

Il n'est pas possible de supposer, en effet, que ces pineraies qui jouxtent l'étage subalpin et s'étendent bien au-delà des derniers restes de la chênaie pubescente se soient substituées à cette dernière, ni même à la hêtraie dont les éléments marginaux ne dépassent pas, on l'a vu, Saint-Michel-de-Maurienne.

Sans doute, chênaie pubescente et hêtraie ont-elles subi l'influence humaine, nous l'avons dit; mais dans certaines limites seulement.

On doit supposer enfin, comme le suggère d'ailleurs BRAUNBLANQUET, que des enclaves de pineraie - association spécialisée sur sols très superficiels et très secs - pouvaient s'observer dans l'étage même de la Chênaie pubescente intra-alpine, rappelant de près les pineraies climaciques de l'étage montagnard et montagnard supérieur et que, à l'inverse en quelque sorte, des enclaves thermophiles de létage submontagnard ou collinéen pouvaient se rencontrer dans l'étage montagnard.

(34) $\mathrm{Ce}$ passage, dont nous avons vu des exemples dans les gorges de la Valloirette, nous échappe eependant en grande partie, car il devait se situer. à notre avis, le long des gorges houillères où thomme s'est installé an fond du talweg jusqu'au bas de l'étage subalpin. 
QUATRIÈME PARTIE

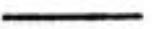

\section{Conclusions}



Nous voudrions, au terme de cette étude, prendre une vue d'ensemble des facteurs écologiques décisifs qui permettent d'expliquer la végétation forestière de la Haute-Maurienne, et en déduire quelques données concernant le problème du climax; rassembler, dans un esprit de synthèse, les réflexions que nous ont suggérées la nomenclature et la systématique phytosociologique de l'Ecole de $\mathrm{Zu}-$ rich-Montpellier, et montrer que les critères de différenciation et de classification des unités phytosociologiques utilisés par cette Ecole peuvent être améliorés et se rapprocher de ceux adoptés par les tenants de la méthode des groupes écologiques; donner, enfin, un bref aperçu des problèmes pratiques qui se posent au sylviculteur.

\section{IV.1. - LES FACTEURS ECOLOGIQUES DECISIFS}

Tout au long de ce travail, nous avons eu l'occasion de montrer que les facteurs écologiques essentiels qui interviennent dans le déterminisme de la composition floristique et de l'organisation sociologique de chaque groupement étaient le climat, la nature de la roche-mère et du sol, le relief, la végétation, l'homme enfin.

\section{IV.1.1. - Le climat.}

Il s'agit, évidemment, nous avons insisté sur ce point, non seulement du climat de l'air, mais plus encore peut-être de celui du sol (température et potentiel capillaire liés à ce climat).

On doit penser que ce sont des facteurs climatiques et non édaphiques ou biotiques qui permettent en Maurienne, comme dans d'autres vallées intra-alpines de France ou de Suisse, de distinguer deux petites provinces biogéographiques: l'une, côté Préalpes, où subsistent le Hêtre et son cortège, l'autre, côté Alpes internes, d'où le Hêtre est absent, et où se développent pineraies xérophiles et Mélèze.

Il apparait, en effet, que le Hêtre n'est pas exclu de la HauteMaurienne par le chimisme des roches-mères ou des sols, puisqu'il présente, dans ce domaine, comme l'ont rappelé récemment DuCHAUFOUR et BONNEAU (1960), une remarquable plasticité; il ne l'a pas été non plus par l'homme, qui l'a sans doute fait un peu reculer, mais qui n'aurait pu le détruire, dans toutes les forêts de versant nord, dans une zone altitudinale lui convenant. 


\section{L'aire ombrothermique de la Haute-Maurienne et celles de trois espèces forestières: \\ Hêtre, Sapin, Chềne pubescent.}

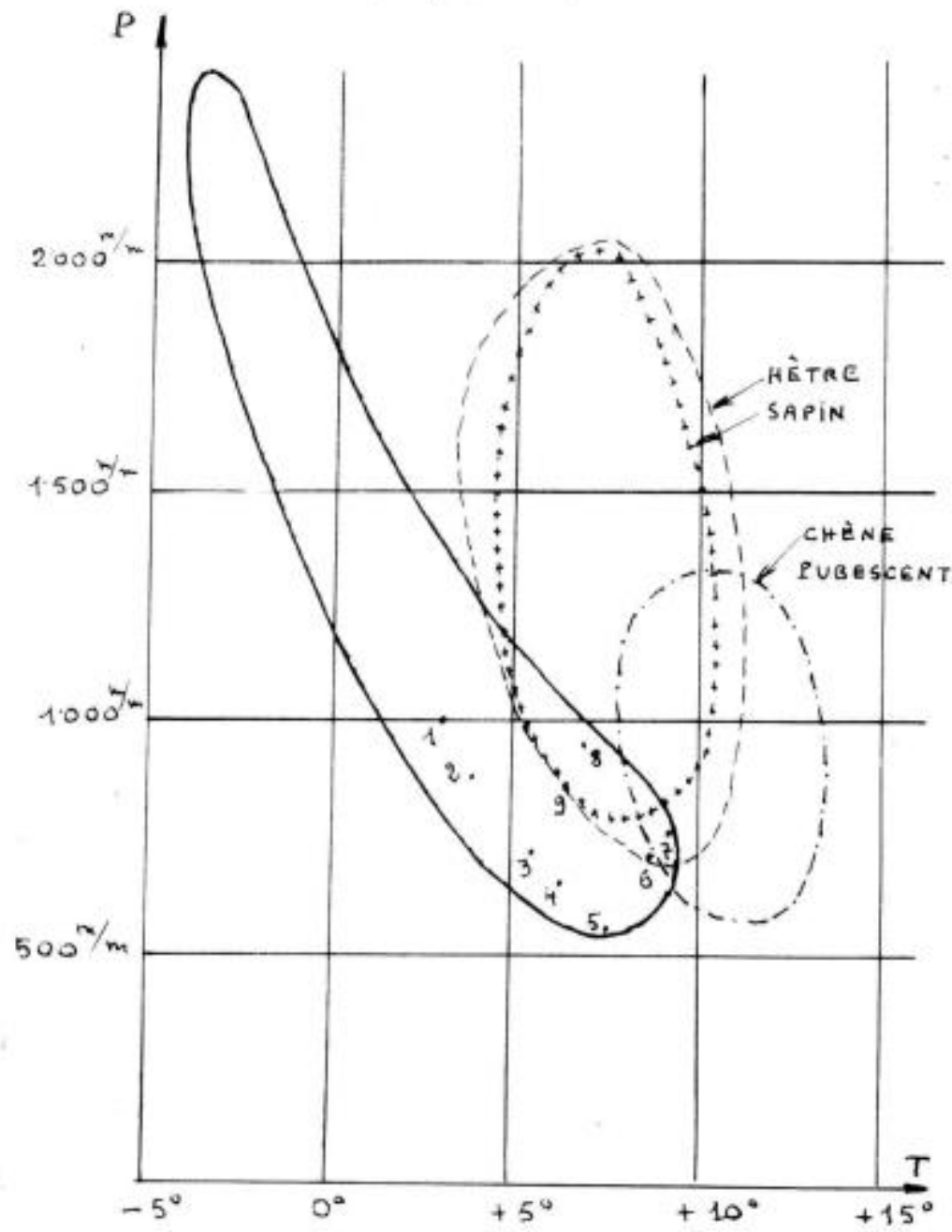

Fir. 59.

Légende. - L'aire réelle du Hètre en Maurienne s'arrète effectivement at niveau de Saint-Michel-de-Maurienne-Valmeinier; l'aire ombrothermique du Hêtre, selon REy, coincide donc remarquablement avec ce que l'on peut observer dans notre région: l'aire du Chéne pubescent rend bien compte des faits également.

Par contre, les sapinières intra-alpines (Abietetum albae de Kuoch) qui se situent jusquaux points 3 et 4 débordent nettement l'aire de REY, qui correspond à un échantillonnage subatlantique, un peu restreint par conséquent.

Les numéros du schéma correspondent aux localités suivantes:

1: Bissorte - 2: Bessans - 3: Lanslebourg - 4: Termignon - 5: Avrieux 6: Orelle - 7: Saint-Martin-la-Porte - 8: Le Thyl - 9: Valmeinier. 
Il est évidemment très difficile de trouver le ou les facteurs climatiques qui, vis-à-vis du Hêtre sont des facteurs limitants: la sécheresse a été souvent invoquée, par CHrist (H.) (1879), par Gaussen (H.) (1926), par Guinier (Ph.) (1950); le régime des gelées tardives également [IssLer (E.) (1931)].

Un tel problème au demeurant - pour la solution duquel il devra être fait appel à des données météorologiques nombreuses et variées - ne peut être abordé que pour l'ensemble des Alpes.

$\mathrm{Ce}$ que nous retiendrons donc, simplement, ici, en n'oubliant point qu'il ne s'agit que de schémas, c'est que l'airc ombrothermique du Hêtre, telle que l'a dessinée REY (P.) (1960) (1) pour le Velay, le Vivarais, le Front Nord Pyrénéen, le Vallespir, le Conflent, la moyenne vallée du Rhône et les Alpes-Maritimes, ce qui constitue déjà un échantillonnage assez étalé (2), se trouve située dans une zone plus chaude et plus arrosée que l'aire ombro-thermique de la Haute-Maurienne. Cette aire du Hêtre recoupe toutefois celle de notre vallée, et dans la région où, précisément, on observe les dernières hêtraies, vers le Thyl et Saint-Martin-la-Porte (3). Si l'on s'en tient donc aux deux seuls facteurs, pluviosité et tèmpérature de l'air, on voit que le Hêtre, d'après le graphique de REY, supporte bien le froid à condition qu'il soit très humide et, dans une certaine mesure, la sécheresse, mais à condition qu'il fasse chaud (fig. 59).

La tentation est grande, évidemment, de considérer, pour la comparer à la précédente, l'aire ombrothermique du Sapin qui s'enfonce profondément dans la zone intra-alpine, ce que ne fait pas le Hêtre. On constate que l'aire ombrothermique du Sapin, telle qu'elle est dessinée par REx, se trouve être englobée par celle du Hêtre, ce qui, schématiquement, nous parait bien rendre compte qu'en montagne subatlantique le Hêtre s'élève plus hatt que le Sapin (étage montagnard supérieur très arrosé et froid) et descend plus bas que lui (étage montagnard inférieur, relativement sec et chaud).

Mais le graphique de Rey ne rend pas compte, toutefois, de l'existence des sapinières de la Haute-Maurienne - entre autres qui connaissent des pluviosités inférieures ou au moins égales à $650 / 700 \mathrm{~mm}$. Ces sapinières montrent que le Sapin — plus que le Hètre - peut supporter un certain froid sec.

(1) Nous avons, en fait, enveloppé par une seule courbe les différentes courbes du Hétre dessinées par REY et qui correspondaicnt aux diverses régions étudiées par lui. Nous avons agi de méme en ce qui concerne les aires du Sapin et du Chẻne pubescent.

(2) Encore que l'on puisse regretter qu'il ne s'étende pas aux Vosges, au Jura, aux Préalpes du nord et du sud.

(3) On observera que la zone d'interférence dépasse, en fait, la zone des hêtraies de Haute-Maurienne. On peut donc conjecturer ce que nous avons déjà souligné, que le Hêtre a dû quelque peu reculer du fait de l'homme; à noter que le Hêtre dans le Velay connaît sur ses marges une température moyenne de $5^{\circ} 2$, pour une pluviosité de $1000 \mathrm{~mm}$ seulement. Mais il est incontestable que la sapinière vraie de Hante-Matsrienne est adaptée à un froid plus sec. 
Quant à l'aire ombrothermique du Chêne pubescent - dont l'échantillonnage a la même ampleur que les deux précédentes elle recoupe celle de la Haute-Maurienne dans la zone la plus chaude (celle de Saint-Julien-de-Maurienne) mais non tout à fait la plus sèche, ce qui, toujours schématiquement, correspond bien aux faits.

A lintérieur de la sone intra-alpine, c'est encore le climat il faudrait parler de climats locaux et de microclimats - qui, par le jeu de l'altitude, joue un rôle déterminant dans la constitution des étages aux diverses expositions: à exposition nord, la pessière subalpine apparait ainsi comme plus froide et plus arrosée que la sapinière, tandis que la pineraie de Pin Cembro l'est plus, ellemème, que la pessière subalpine; à exposition sud, c'est la pessière subalpine sèche (4) qui se trouve étre plus froide et plus arrosée que la pineraie de Pin sylvestre, la juniperaie à Pin Cembro l'étant, à son tour, plus que la pessière.

Mais, ce qu'il faut rappeler ici, ćest le rôle prépondérant joút par le clinat du sol, dans les premiers centimètres de celui-ci. On a vu combien le potentiel capillaire à $-25 \mathrm{~cm}$ et à $-5 \mathrm{~cm}$ et la température vers la surface, pouvaient différer, dans le même étage général, d'une exposition à l'autre et expliquer qu'à exposition tournée vers le sud, une pineraie de Pin sylvestre très xérophile et adaptée à un climat aux variations brutales, puisse prospérer, alors que sur le versant d'en face, se rencontre une sapinière relativement mésophile, au climat du sol sensiblement différent.

Le climat n'a d'ailleurs pas seulement qu'un rôle direct: on a vu, lors de l'étude des pineraies xérophiles de Pin sylvestre, en particulier, que l'excès d'insolation et de dessiccation pouvait permettre d'expliquer l'évolution relativement poussée des sols et la légère tendance à l'acidification que l'on y observe, car la vie biologique $y$ est très peu active.

Sur le versant nord, et à haute altitude, dans l'étage subalpin, les basses températures jouent un rôle analogue: dans le sol de la rhodoraie à Pin Cembro, l'activité biologique, par suite du froid, est également très faible, et les Ericacées aidant, une importante accumulation d'humus se produit, qui est à l'origine des phénomènes de podzolisation déjà décrits.

\section{IV.1.2. - La nature des roches-mères et des sols.}

Ce que l'on doit d'abord souligner, c'est le caractère de jeunesse que présentent tous les sols; ce caractère est dû à l'érosion, car les replats en forêt sont pratiquement inexistants ou de surface minime.

(4) ou la pineraic de Pin à crochets, sur roche-mère carbonatée. 
Cette jeunesse se traduit par l'existence de fragments de rochemère jusque dans les horizons de surface, et ceci, comme on l'a noté, même dans les sols podzoliques de la rhodoraie à Pin Cembro, qui peuvent être considérés, a priori, comme les plus évolués. Ces sols podzoliques, comme l'a souligné Duchaufour ( $\mathrm{Ph}$.) (1960) * sont souvent plus riches au point de vue chimique que les podzols atlantiques. L'humus, qui contient d'importantes réserves de calcium, de phosphore et d'azote, assure aux résineux une nutrition satisfaisante, tout en maintenant un milieu acide favorable aux mycorhizes $x$.

Il découle de laction incessante de l'érosion que la nature de la rochc-mère influence directement et continûment la pédogénèse et, par contre-coup, conditionne étroitement la composition floristique des associations. C'est ainsi que les vastes peuplements de Pin à crochets qui s'étendent entre Bramans et Termignon, sur versant nord, s'expliquent directement par l'existence du gypse extravasé au contact de la nappe des schistes lustrés.

Les différences floristiques sont d'autant plus nettes qu'elles correspondent à des différences marquées du chimisme des rochesmères. Un exemple est particulièrement à retenir à cet égard: on a vu que, dans le synclinal de Chavières, à exposition sud, on observait côte à côte deux associations xérophiles de Pin sylvestre, très dissemblables: d'une part, l'Ononido-Pinetum sur rendzine superficielle reposant elle-même sur cargneules triasiques; d'autre part. le Deschampsio-Pinctum, sur ranker à moder, reposant sur quartzites également triasiques.

Dans la plupart des cas, toutefois, les différences floristiques dues au chimisme du sol et de la roche-mère sont moins marquées: ceci tient au fait que les roches-mères présentent entre elles des caractères moins extrêmes et que les phénomènes de décarbonatation des horizons superficiels affectant les sols reposant sur roches-mères carbonatées tendent à les rendre "analogues " à ceux reposant sur des roches-mères plus silicatées. L'ensemble de 1'Abictetum albae est à rappeler, à ce sujet: les sols sur schistes lustrés - ces derniers, bien que contenant de petits filonets de calcite, sont déjà peut carbonatés - et ceux sur grès ou schistes du Houiller ne diffèrent pas sensiblement, comme il a déjà été dit, et si l'on peut distinguer sur ces derniers un faciès à Saxifraga cuncifolia, ceci tient essentiellement au fait que cette espèce pousse sur les éboulis même de grès, sur la petite couche de moder produite par les mousses dont Isothecium viviparum.

\section{IV.1.3. - Le relief.}

Des deux processus essentiels, qui sont sous la dépendance directe du relief: érosion, c'est-à-dire décapage du sol dans son ensemble 
et lessivage oblique, c'est-à-dire " entrainement le long de la pente, dans la masse du profil, des éléments colloïdaux ou solubles $n$, le premier l'emporte nettement en importance, en Haute-Maurienne, où les pentes en forêt sont toujours très fortes (de 40 à $100 \%$ et au-delà), les replats ayant été colonisés par l'homme.

Outre qu'elle tend à rajeunir constamment les sols, comme on l'a vu, l'érosion contribue à la formation d'éboulis dont le rôle, dans le paysage forestier n'est pas négligeable. Eboulis à gros éléments, par exemple, dans le Permo-hotiller métamorphique, où se complait la rhodoraie à Pin Cembro; éboulis à éléments moyens dans les gorges houillères, en particulier au niveau de la pessière subalpine xérophile; éboulis plus fins, mais plus localisés, un petı partout, ailleurs, donnant naissance à des rendzines ou à des rankers colluviaux.

Mais, ce qu'il faut souligner, c'est que le long d'une même pente. il n'y a pas, au sommet, une rendzine initiale, si l'on est sur calcaire ou un ranker d'érosion, si l'on a affaire à une roche-mère silicatée, puis, plus bas, une rendzine colluviale ou un ranker colluvial; les séquences - si séquences il y a - dessinent un véritable puzzle. car le dessin des falaises ou des affleurements rocheux est lui-même fort complexe; d'autre part, les colluviums, qu'ils soient calcaires ou non, restent soumis à une intense érosion et restent donc de faible profondeur; les différences qu'ils présentent avec les stations d'érosion, proprement dites, sont donc le plus souvent négligeables, du moins en forêt.

Les seules séquences à retenir sont celles qui correspondent aux étages; elles sont dues directement at climat, mais, indirectement. au relief lui-même.

\section{IV.1.4. - La végétation.}

La végétation forestière agit sur le microclimat: de ce point de vue, on doit penser que, dans les pineraies xérophiles, le couvert léger des Pins contribue à donner à leur microclimat, la brutalité que nous lui connaissons, alors que le couvert des Hêtres et celui des Sapins joue un rôle inverse dans les hêtraies ou dans la sapinière.

La végétation agit aussi par l'humus qu'elle produit: la matière organique, comme l'a noté Duchaufour (Ph.) (1960), est en effet "l'intermédiaire entre le monde vivant et le monde minéral, par lequel le premier peut modifier les propriétés du second, dans un sens déterminé ’. A cet égard, on doit retenir que la dominance absolue des résineux, dans nos associations, explique l'existence d'un humus dont les caractères ne sont jamais ceux d'un mull franc, mais ou d'un moder ou d'un mull-moder; ce n'est vraiment que sous les Aulnes verts que l'on rencontre un vrai mull. On doit souligner aussi, car il est remarquable, le rôle respectif que jouent les Gra- 
minées ou les Ericacées, espèces sociales par excellence. Dans l'étage du Pin Cembro, à exposition fraiche, l'abondance du Rhododendron, de Vaccinium Myrtillus, de Vaccinium Vitis idaea, fait se créer un humus brut - les conditions climatiques aidant - alors que, toutes choses égales d'ailleurs, la dominance de Calamagrostis villosa associé à quelques autres Graminées plus rares dont Poa Chaixii, ou mème Milium effusum, aboutit à une brunification des sols, 1'humus produit par ces graminées étant peu acide, riche en calcium, et de décomposition rapide.

\section{IV.1.5. - L'homme.}

L'action humaine dont nous avons déjà analysé l'aspect le plus spectaculaire, à savoir la déforestation laquelle s'est exercée surtout sur les adrets en supprimant partie des chênaies pubescentes et des hêtraies et, dans l'étage subalpin sec, partie des pineraies de Pin Cembro, s'est en outre manifestée, on l'a vu, dans tous les étages, pour modifier la structure sylvicole des peuplements et aussi, mais dans une mesure moindre, à notre avis, leur composition floristique,

Dans l'étage du Pin Cembro, le faciès à Calamagrostis villosa et ce que nous avons appelé le faciès pâturé de la rhodoraie, lui doivent leur extension.

Dans l'étage de la pessière subalpine mésophile, on peut penser que le pâturage et les coupes assez brutales destinées à alimenter l'industrie du charbon de bois, ont contribué - l'érosion aidant à freiner l'évolution des sols et à empêcher que ne s'installe une couche d'humus brut propice à des espèces telles que Listera cordata. Lycopodium annotinum, etc,

Dans l'étage de la sapiniẻre, par contre, cette action est peut-étre moins nette: elle a pu cependant, sur roche-mère calcaire, favoriser l'extension des pineraies calciphiles à Erica carnea et Hylocomies, aux dépens de la sapinière à Carex alba.

Dans toute la zone des gypses et des cargneules, où l'on observe les associations spécialisées de Pin sylvestre ou de Pin à crochets. nous ne pensons pas que l'homme ait. pu vraiment jouer un rôle modificateur, car le relief est particulièrement vigoureux et les arbres de faible valeur.

Dans tous les étages de versant frais enfin, l'homme a créé mais il s'agit en fait de petites surfaces, sauf autour d'Albanne et de Montricher - des mélézeins, véritables prés-bois riches en espèces prairiales, qui évoluent, selon les étages, avec le recul de l'homme, soit vers la thodoraie à Pin Cembro, soit vers la pessière subalpine, soit vers la sapinière. Ces mélézeins, qui n'ont qu'une très faible importance, n'ont pas été analysés ici, car leur étude, malgré l'intérêt qu'elle présentait du point de vue de la dynamique de la végétation, nous eût entrainé trop loin. 


\section{IV.1.6. - Vue d'ensemble.}

Le tableau synoptique et les photos des pages suivantes donnent une vue d'ensemble des groupements décrits et ce, en fonction des grands facteurs écologiques.

On se reportera à la carte au $1 / 50000^{\circ}$ pour les situer géographiquement.

Le tableau général appelle quelques commentaires: nous avons défini les associations ou leurs sous-unités, en utilisant des termes français et des termes latins.

Lorsque les associations avaient déjà un nom, nous l'avons adopté soit totalement, par exemple: "Abietetum albae melampyretosum $n$, soit en le modifiant ou en le complétant conformément aux règles fixées par BACH (R.), Kuoch (R.), Moor (M.) (1962) et par Rauschert (St.) (1963).

Ces règles voulant qu'un groupement végétal soit dénommé en utilisant les noms des plantes représentatives, nous avons abandonné les termes de Rhodoreto-Vaccinictum cembretosum, ou de Rhodoreto-Vaccinietum calamagrostidetosum (que nous avions conservés, dans le corps du texte, pour éviter toute confusion) et leur avons substitué ceux de Rhododendro-Pinetum Cembrae et de Calamagrostio villosae Pinetum Cembrae.

Nous avons, en outre, renoncé au terme de Mugetum, pour des raisons déjà dites, et avons adopté celui de Pinetum uncinatae, ce qui nous obligeait, par contre-coup, à parler de Pinetum silvestris. C'est ainsi que l'Erico-Mugetum est devent l'Erico-Pinetum uncinatae et l'Erico-Pinetum, sensu stricto, l'Erico-Pinetum silvestris.

Il est des cas plus "épineux w: dans la " famille " des pineraies xérophiles sur sol calcimorphe, on trouve, dans l'étage montagnard, l'Ononido-Pinetum silvestris, dans l'étage stubalpin inférieur, un groupement que l'on doit appeler: Ononido-Pinetum uncinatae, à Picea excelsa, et, dans l'étage subalpin supérieur enfin, un groupement qui se situe bien dans la même séquence, mais qui, ne possédant plus d'Ononis et singulièrement d'Ononis rotundifolia, ne peut plus être raisonnablement appelé Ononido-Pinetum uncinatae; si donc, on retient le nom d'une autre espèce, la dénomination obtenue va masquer les affinités très étroites qui existent entre ce groupement et ceux des étages inférieurs. Ce qu'il faudrait, par conséquent, c'est dénommer tous ces groupements affines à l'aide d'une espèce s'observant dans tous les étages et liées à la fois à l'exposition sud et aux sols calcimorphes: Carex humilis, par exemple et, ensuite, les différencier par une espèce plus spécialisée, tout at1 moins quant à l'étage.

Dans le cas particulier évoqué, on aurait la " séquence " ci-après: Carici humilis - Pinetum silvestris ononidetosum rotundifoliae, $\mathrm{Ca}$ rici humilis - Pinetum uncinatae piceetosum excelsae, Carici humilis - Pinetum uncinatae caricetosum sempervirentis. 
Tableau général des associations forestières de la Haute-Masrienne (moins les hêtraies et chênaie pubescente, marginales)

\begin{tabular}{|c|c|c|}
\hline Btagea & \multicolumn{2}{|c|}{$\begin{array}{l}\text { Roohes mères earbonstiea (enreneules, gypoe, ealealre) } \\
\qquad 301 \text { a i mull-qoder onleique }\end{array}$} \\
\hline & Bxposition Sud & Exposition Jord \\
\hline $\begin{array}{l}\text { Btage } \\
\text { montagrord }\end{array}$ & 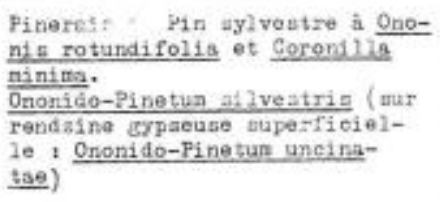 & 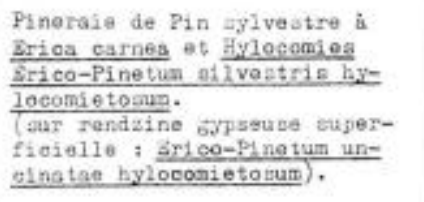 \\
\hline $\begin{array}{l}\text { Rtage } \\
\text { gubsipin } \\
\text { inforiteur }\end{array}$ & 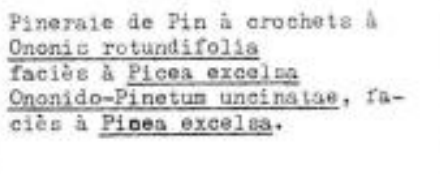 & 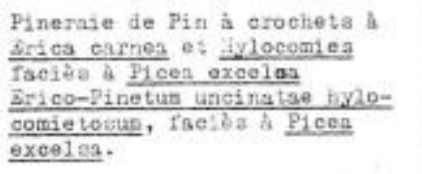 \\
\hline $\begin{array}{l}\text { Btage } \\
\text { aubsilpin } \\
\text { augérieir }\end{array}$ & 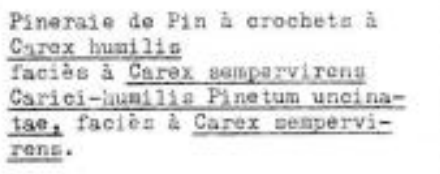 & 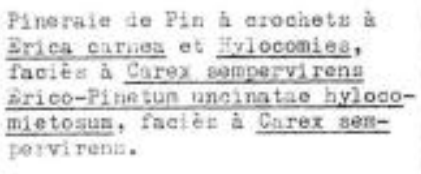 \\
\hline
\end{tabular}

\begin{tabular}{|c|c|c|}
\hline Btage a & 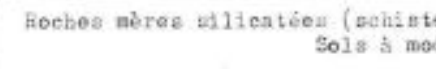 & $\begin{array}{l}\text { ot itrès du Houlller, qunrtaites } \\
\text { et } \mathrm{h} \text { nor }\end{array}$ \\
\hline \multirow[b]{2}{*}{$\begin{array}{l}\text { Btase } \\
\text { nontagnnad }\end{array}$} & Bxposition sud & Jxporition Sord \\
\hline & $\begin{array}{l}\text { Pineraie de Pin aylvestre b } \\
\text { Deachanpsia flexuosa ot lijnuar- } \\
\text { tia 2aricifolis } \\
\text { Deschampaie-P1netur aldveutris }\end{array}$ & 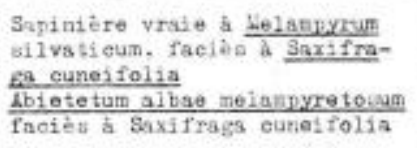 \\
\hline $\begin{array}{l}\text { stage } \\
\text { gubalpin } \\
\text { inferrieur }\end{array}$ & 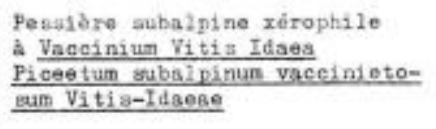 & 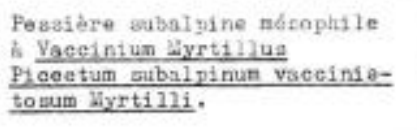 \\
\hline $\begin{array}{l}\text { Btage } \\
\text { subsipin } \\
\text { supériour }\end{array}$ & 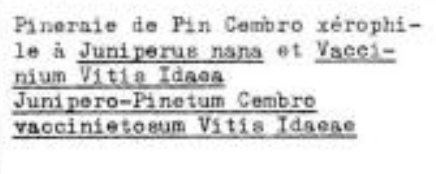 & 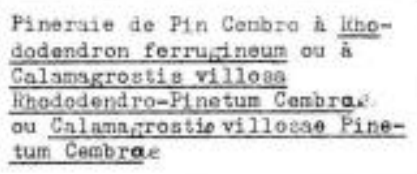 \\
\hline
\end{tabular}




\begin{tabular}{|c|c|c|}
\hline \multirow[t]{2}{*}{ Btages } & \multicolumn{2}{|c|}{$\begin{array}{c}\text { Roches bòrea "mixtes" (aebiates lustrís) } \\
\text { joli i muli-noder ot mor }\end{array}$} \\
\hline & Expoeition Sud & Bxpost tion llord \\
\hline $\begin{array}{l}\text { Btsge } \\
\text { montagnird }\end{array}$ & 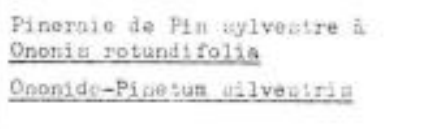 & 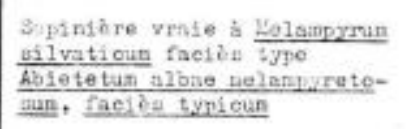 \\
\hline $\begin{array}{l}\text { Btage } \\
\text { eubainin } \\
\text { inforiaur }\end{array}$ & 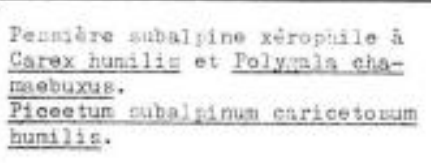 & 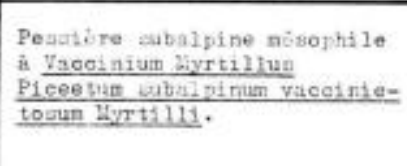 \\
\hline $\begin{array}{l}\text { 3tsage } \\
\text { subaipin } \\
\text { aupórieur }\end{array}$ & $\begin{array}{l}\text { Souv-étree totalement occupé } \\
\text { phr l hoane }\end{array}$ & 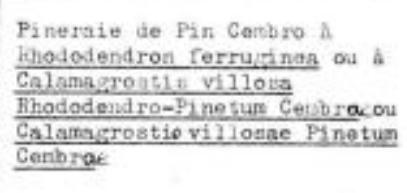 \\
\hline
\end{tabular}

Cette solution, qui nous parait la plus rationnelle, obligerait à remanier une nomenclature déjà acquise (Ononido-Pinetum); nous l'avons donc abandonnée, et avons, très provisoirement, baptisée l'association du subalpin supérieur, comme il est dit ci-dessus.

Il y a lieu de noter que nous n'avons pas fait figurer sur le tableau synoptique et, pour éviter de l'alourdir, les associations " intermédiaires $n$ et singulièrement celles des sols calcimorphes dont nous avons cependant souligné l'importance géographique.

A exposition sud-est ou nord-ouest, on rencontre donc, dans l'étage montagnard, une association où l'on trouve à la fois des espèces xérophiles et des espèces mésophiles. Pour marquer ce double caractère, il y aurait lieu de la dénommer: Ononido-Pinetum silvestris ericetosum; dans l'étage subalpin inférieur, ce serait l'Ononido-Pinetum uncinatae ericetosum et dans le supérieur, enfin. le Carici-humilis Pinetum uncinatac ericetosum.

Inutile d'ajouter que toutes ces dénominations ont, dans notre esprit, une valeur toute provisoire; nous avons eu simplement l'intention d'obéir aux règles récentes, en évitant d'ajouter à la confusion qui règne dans ce domaine. Ces dénominations sont celles qui figurent au tableau synthétique de relevés, à la fin de ce travail. 


\section{PLANCHE XIX}

\section{Les association forestieres \\ et le jeu des facteurs fologiques décisifs.}

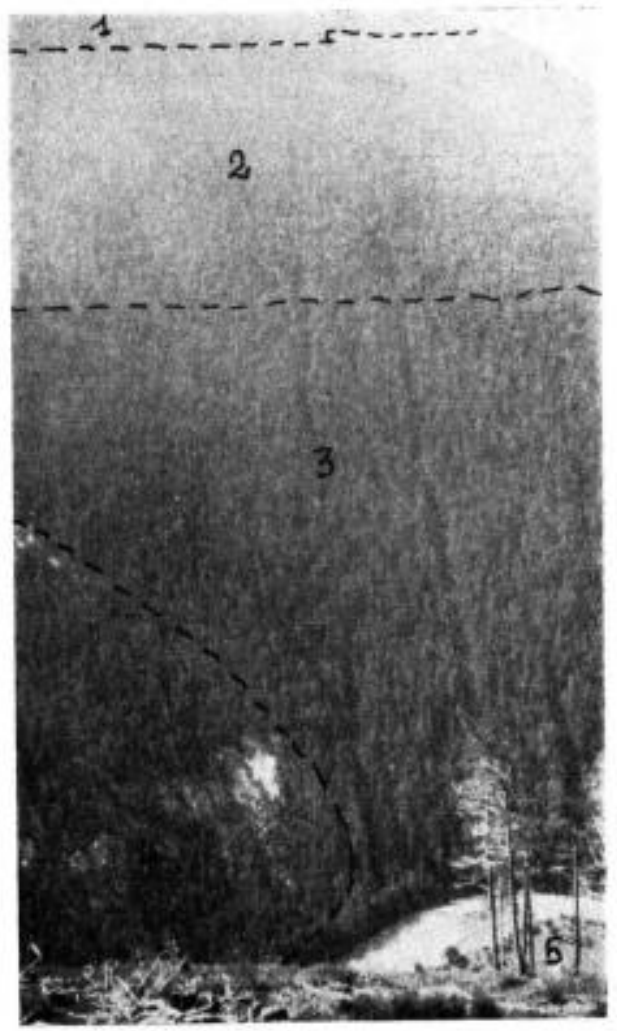

Légende. - Lorsque la roche-mère occupe tout un versant lui-méme peu ondulé, les associations se succèdent de haut en bas, sans solution de continuité et de façon simple: ainsi en forét d'Arc, entre Lanslebourg et Termignon, sur schistes lustrés, s'étagent une sapinière (3), une pessière subalpine (2), une pineraie de Pin Cembro (1).

A l'adret, une pineraie de Pin sylvestre à Ononis rotundifolia incendiée (5). Une lentille de gypse, toutefois, associée aux schistes lustrés, vient rompre l'ordonnance des étages et porte une pineraie de Pin à crochets à Erica carnea et Hylocomies (4). 


\section{PLANCHE XX}

\section{Les association forestières}

et le jeu des facteurs écologiques décisifs.

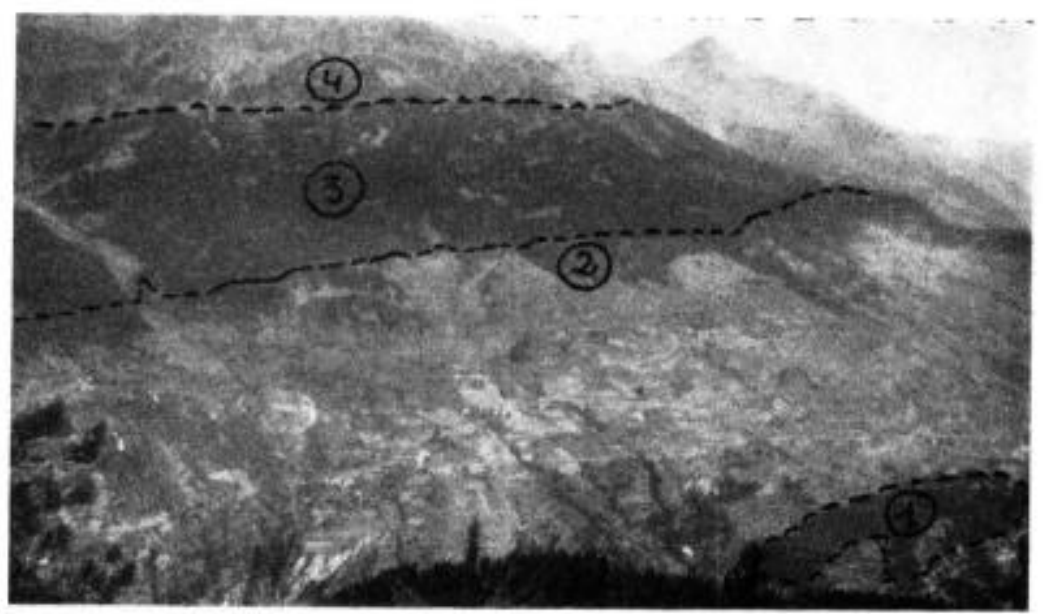

Légende. - Sur grès et schistes du Howiller, à l'adret, l'homme a à peu près totalement occupé l'étage montagnard où subsistent quelques ilots forestiers [Deschampsio-Pinetum (1)].

Dans le bas du manteau forestier, à la limite des étages montagnard et subalpin, l'Epicéa se méle au Pin sylvestre (2); puis s'étend la pessière subalpine xérophile à Vaccinium Vitis-Idaea (3), dans 1'étage subalpin supérieur, on observe des lambeaux d'une pineraie de Pin Cembro xérophile à Vaccinium Vitis-Idaea, Arctostaphylos Uva-ursi, Juniperus nana (4). 


\section{PLANCHE XXI}

Les associations forestières

et le jeu des facteurs écologiques décisifs.

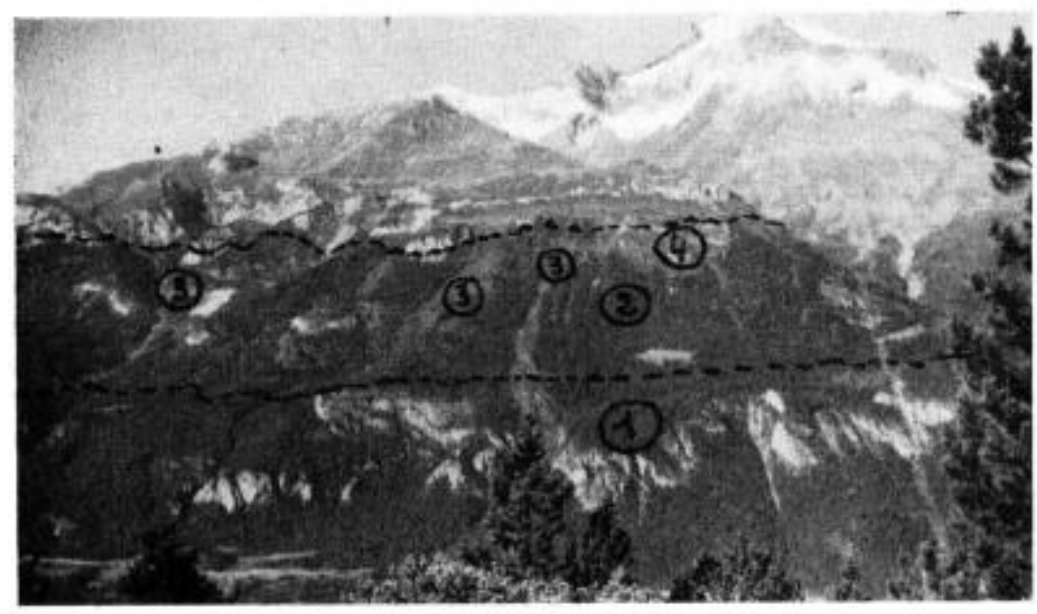

Légende. - La zone des cargneules de la Vanoise (sols calcimorphes) est le domaine, dans l'étage montagnard, du Pin sylvestre [Ononido-Pinetum silvestris; Erico-Pinetum sylvestris (1)] et, dans l'étage subalpin, du Pin à crochets [Ononido-Pinetum uncinatae; Erico-Pinetum uncinatae (2)].

On notera l'abondance des couloirs d'avalanches (3). En (4), partie incendiee. 


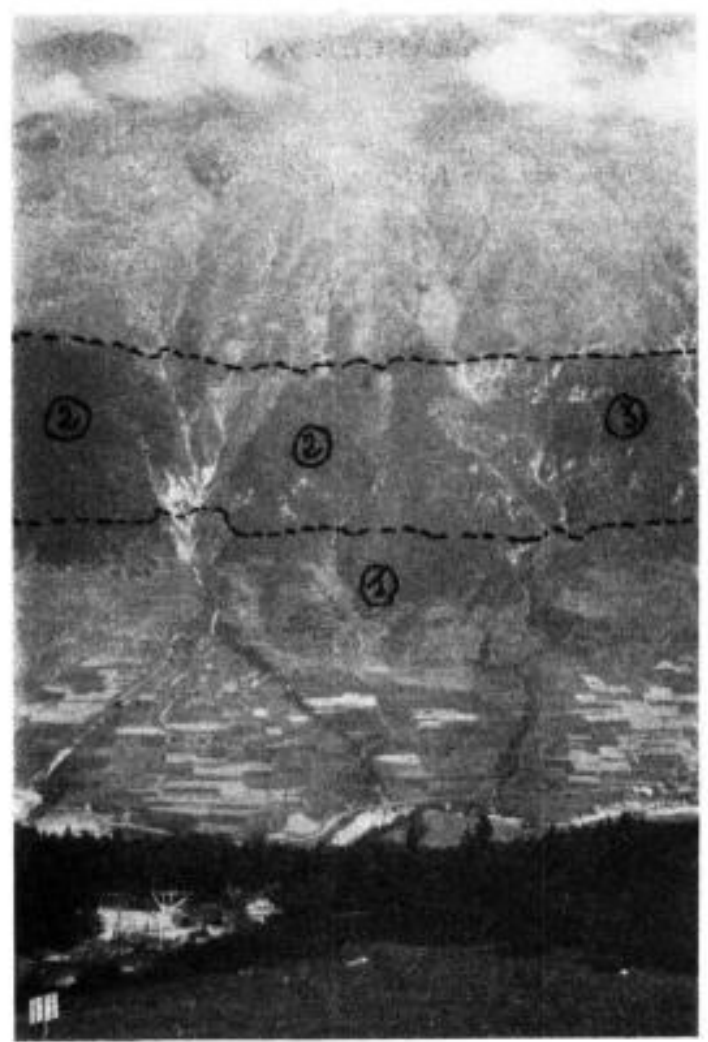

Légende. - L'adret, entre Lansicbourg et Lanslevillard a été fortement occupé par l'homme dans la plus grande partie de l'étage montagnard (jusqu'à $1550-1600 \mathrm{~m}$ ) et dans le subalpin supérieur (à partir de $2000-2050 \mathrm{~m}$ ).

On observe toutefois, entre 1600 et $1800 \mathrm{~m}$, l'Ononido-Pinetum (1) et audessus, la pessière subalpine sèche (2). Cette dernière laisse la place, à droite de la photo, à un Ononido-Mugetsum (3) qui se situe très exactement sur les cargneules et calcaires triasiques d'une fenêtre s'ouvrant au milieu des schistes lustrés.

Le dessin de cette fenètre est tel que les cargneules et calcaires se retrouvent encore au centre de la photo, mais dans le seul étage montagnard où s'observe l'Ononido-Pinetum (1), lequel s'étend donc à la fois sur calcaires triasiques et sur schistes lustrés. 


\section{PLANCHE XXIII}

\section{Les associations forestières}

et le jeu des facteurs écologiques décisits.

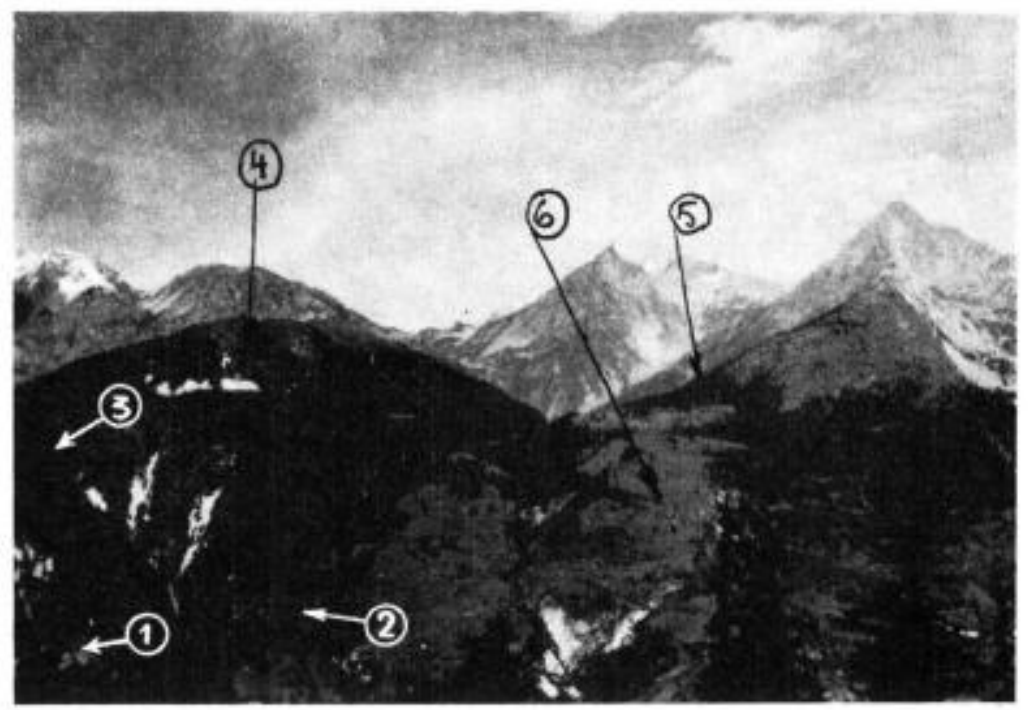

Légende. - Sur versant sud, toujours au niveau de Modane, la répartition des associations forestières est moins complexe.

On notera toutefois, sur cargneules (1) une pineraie xéro-calciphile à Ononis rotundifolia jouxtant une pineraie xéro-acidiphile à Deschampsia flexuosa et Minuartia laricifolia (2).

Cette pineraie, sur quartzites, s'élève assez haut; lui succèdent une pessière subalpine sèche à Airelle rouge (3), puis une pineraie de Pin Cembro relativement xérophile (4), tandis qu'à exposition secondaire nord, sur Permohouiller métamorphique (masquée sur la photo), s'observe une magnifique rhodoraie à Pin Cembro (5).

L'action humaine, qui ne marque pas de recul sensible, s'est exercée dans les zones les moins en pente (6). 


\section{PLANCHE XXIV}

Les associations forestières

et te jeu des facteurs écologiques décisifs.

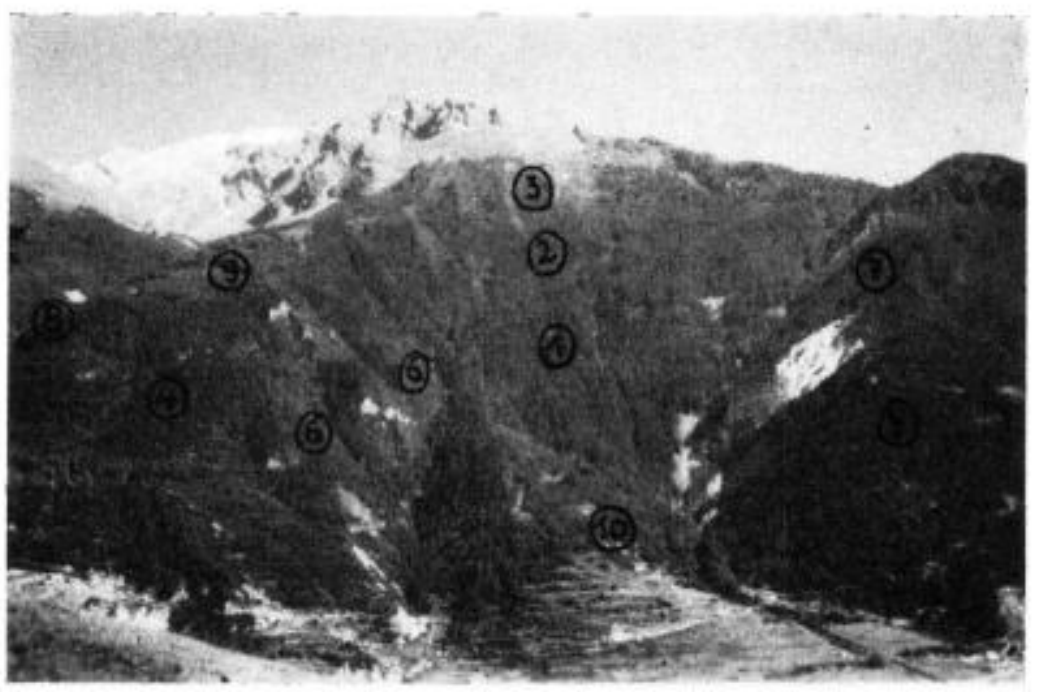

Légende. - Si, le plus souvent, les facteurs écologiques décisifs ne jouent pas tous ensemble (en forêt d'Are, à l'envers, les grands étages de végétation se succèdent sans solution de continuité sur une méme roche-mère, les schistes lustrés), il n'en est pas toujours ainsi, ce qui rend, évidemment plus complexe le tapis forestier.

C'est ainsi qu'en forêt de Modane, à exposition générale nord, sur schistes lustrés, et sur éboulis en provenant, se succèdent du bas en haut: sapinière vraie (1), pessière subalpine (2), pineraie de Pin Cembro à Rhododendron (3).

Mais une zone fort complexe de calcaires, cargneules, gypse, occupe partie du bas du versant, ce qui permet à une sapinière vraie à Carex alba de se développer (4) tandis que vers l'ouest, sur le houiller métamorphique repose une sapinière vraie à Saxifraga cuneifolia (5).

A exposition secondaire ouest, plus chatade et sur cargneules, s'observe, fragmentée, une pineraie sèche à Ononis rotundifolia (6), tandis qu'à l'ouest, toujours sur cargneules, mais à exposition fraiche et dans létage subalpin, on rencontre une pineraie de Pin à crochets à Cares ferruginea (7), sans Erica carnea dont l'aire s'arréte au niveau du ruisseau de Saint-Antoine (8).

L'action humaine, enfin, est venue elle-méme interférer: sur le replat (9), des pelouses autrefois páturées sont actuellement envahies par un mélézein qui, situé à la limite des étages montagnard et subalpin, évoluera, vers le bas, vers une sapinière et plus haut, vers une pessière subalpine.

Cette action s'est exercée aussi au bas des versants à exposition ouest (10) où la pineraie, très jeune, se trouve coupée par des pelouses riches de Brachypodium pinnatum, ou une fruticaie à base de Berberis vulgaris. 


\section{IV.2. - LE PROBLEME DU CLIMAX}

Lors de l'étude de chaque association et une fois analysée l'action humaine, nous nous sommes posé la question de savoir s'il s'agissait d'une association climacique, c'est-à-dire dépendant essentiellement du climat on d'une association spécialisée, c'est-à-dire liée plus étroitement aux conditions locales de station et plus particulièrement à celles résultant de la composition de la roche-mère.

Ce faisant, il nous est apparu que, dans l'ensemble, sous l'action incessante de l'érosion, due elle-même à un relief presque toujours vigoureux " la nature de la roche-mère influence directement et continûment la pédogénèse, et, par contre-coup, conditionne étroitement la composition floristique des associations $x$.

On peut se demander, dans ces conditions - et la question a été posée par divers auteurs, dont ETTER (H.), pour les forêts de la bordure austro-orientale du Plateau suisse (1947), par ELLENBerg (H.), pour les associations de l'étape alpin (1953), par NorRFALise (A.), pour les forêts belges (1954), par Richard (J.-L.), pour les associations forestières du Jura (1961) — si, en HauteMaurienne, comme le plus souvent en montagne, l'influence de la station et de la roche-mère ne l'emporte pas sur celle du climat dans l'orientation générale de l'évolution des sols. En d'autres termes, comme l'a développé Favarger (Cl.) (1960), dans un article de synthèse, on peut se demander, s'il n'y aurait pas lieu de distinguer, dans chaque étage, divers climax liés à la roche-mère et à l'exposition (Klimaxschwarm ou essaim de climax de ETTER), c'est-àdire, en définitive, de faire se rapprocher la notion d'association climacique et celle d'association spécialisée.

Dans cette perspective, une association climacique, dans une région donnée, ne devrait sa qualificition qu'à son importance territoriale, plus grande que celle d'autres associations, dites alors spécialisées permanentes mais pourrait, dans une autre région, où son importance serait minetre, jouer, par contre, à son tour, le rôle d'association spécialisée.

Par exemple, alors que 1'Abieti-Fagetum - surtout sous sa forme festucetosum - couvre de grandes étendues dans les Préalpes et surtout dans le Jura, at niveat de l'étage montagnard moyen. il est très localisé dans les Basses-Vosges gréseuses, où il ne s'observe - sur sol brun à mull - que sur les affleurements toujours de faible surface de grès intermédiaires; dans cette dernière 
région, domine en effet le grès vosgien, presque exclusivement quartzeux, très pauvre en argile (moins de $5 \%$ ) qui porte soit des sapinières à mousses et Vaccinium Myrtillus, à exposition fraiche (Myrtillo-Abietetum), soit des pineraies de Pin sylvestre à Calluno à exposition sèche, toutes associations que l'on pourrait parfaitement qualifier de climaciques, l'Abieti-Fagetum faisant alors figure, par rapport à elles, d'association spécialisée.

Qu'en est-il en Haute-Maurienne?

Notre étude a montré qu'il existe sans doute de grandes associations d'étage, plus ou moins proches du climax: à exposition nord. sapinières dans létage montagnard, pessière subalpine mésophile dans le subalpin inférieur, pineraie de Pin Cembro à Rhododendron dans le subalpin supérieur; à exposition sud, pineraie xérophile de Pin sylvestre dans le montagnard, pessière subalpine xérophile dans le subalpin inférieur, pineraie de Pin Cembro à Genévrier dans le subalpin supérieur et que des phénomènes de " convergence ") tout à fait remarquables ne sont pas niables: la rhodoraie à Pin Cembro sur grès du Houiller ne présente pratiquement pas de différences avec celle développée sur schistes lustrés et arrive à se rencontrer sur cargneules ou même gypse, quoique sous une forme un peu spéciale (rhodoraie à Pin Cembro, à Pin à crochets et Erica carnea), les horizons supérieurs des sols (dans les deux premiers cas: sols podzoliques ou podzols, dans le dernier, sol humique carbonaté à humus brut) étant " analogues $x$ au sens de Pallamann. Une même convergence se rencontre plus bas. dans la pessière subalpine et dans la sapinic̀re.

Mais, malgré ces convergences, les grandes associations ne se présentent pas sous un type uniforme, de telle sorte qu'il n'est pas facile, en définitive, de distinguer ce que représente vraiment l'association " moyenne " climacique.

On rencontre presque toujours des sous-associations ou des variantes dont les compositions floristiques sont liées aux propriétés des horizons minéraux ou de la roche-mère: par exemple, la sapinière sur schistes lustrés n'est pas tout à fait la même que celle sur roche-mère carbonatée (sapinière à Carex alba) ni que celle sur roche-mère plus silicatée (sapinière à Saxifraga cuneifolia). Ces variantes ou sous associations passent, d'ailleurs, et souvent par des intermédiaires très nets, d̀ des associations spécialisées: exemple: on rencontre, quoique rarement, il est vrai, des sapinières à Erica carnea ou Carex ferruginea, qui relient la sapinière à Carex $a l b a$ à la pineraie de $\mathrm{P}$ in sylvestre ou de $\mathrm{Pin}$ à crochets mésophile. à Erica carnea et Hylocomies on à Carex ferruginea qui. elles, sont des associations spécialisées.

Dans l'étage montagnard sec, la pineraie de Pin sylvestre se présente sous deux formes extrêmes: le Deschampsio-Pinetum et 1'Ononido-Pinetum. II est vraisemblable que lassociation la plus proche du climax est celle que l'on rencontre sur schistes lustrés, 
encore qu'elle présente beaucoup d'affinités avec l'Ononido-Pinetum calciphile; mais, là aussi, on l'a vu, il existe des intermédiaire: une variante du Deschampsio-Pinetum, à Carex humilis et Polygaba Chamaebuxus qui, influencée à la fois par la silice des quartzites et le calcaire des moraines, constitue un terme de transition vers l'Ononido-Pinetum.

Il est donc incontestable qu'il n'existe pas une uniformité des associations climatiques et que des formes de passage peuvent, en outre, être observées entre les diverses "variantes subclimaciques " et les associations spécialisées proprement dites. 


\section{IV.3. - LE PROBLEME DE LA NOMENCLATURE ET DE LA CLASSIFICATION DES UNITES PHYTOSOCIOLOGIQUES}

A la suite de l'étude critique que nous avons conduite, dans li première partie de ce travail, des méthodes de classification phytosociologique adoptées par l'Ecole de Braun-Bunnourt, et de l'analyse que nous avons faite des groupes écologiques, nots sommes arrivé à deux résultats apparemment antinomiques: nous avons montré, d'une part, que la classification hiérarchisée de BraunBlanguer prêtait le flanc ì d'assez nombreuses critiques, mais, d'autre part, que les notions d'alliance, d'ordre, de classe avaient une signification écologique, permettant de les justifier.

Le moment est done venu de prendre whe one d'ensemble de ce problème complexe de typologie, dont l'intérêt est considérable, car c'est celui même, pour une large part, des fondements de la phytosociologie.

\section{IV.3.1. - Les grands types possibles de classification hiérar- chisée.}

Pour éclairer un tel problème, il ne nous parait pas inutile d'analyser d'abord - et en restant volontairement dans le seul domaine forestier - les grands types possibles de classification hiérarchisée.

On peut, tout d'abord, faite des rapprochements " horizontaux $n$, c'est-à-dire grouper ensemble les associations forestières d'un mème étage, puis, en grimpant dans la hiérarchie, juxtaposer les groupements de deux étages contigus, et ainsi de suite...

Un tel type de classement - qui se situe surtout dans une perspective dynamique, peut être qualifié de climatique.

Il est possible aussi, de faire des rapprochements "verticaux " et de grouper ensemble, en tunités compréhensives, des associations de divers étages, mais qui présentent entre elles des affinités dues aux caractères du sol: ce type de classement est donc édaphique. On réunira ainsi toutes les associations forestières acidiphiles, toutes les associations subacidiphiles, etc... 
C'est dans cet esprit que Dorng (H.) (1962) a créé la classe des Querco-Picetea, qui s'articule ainsi :

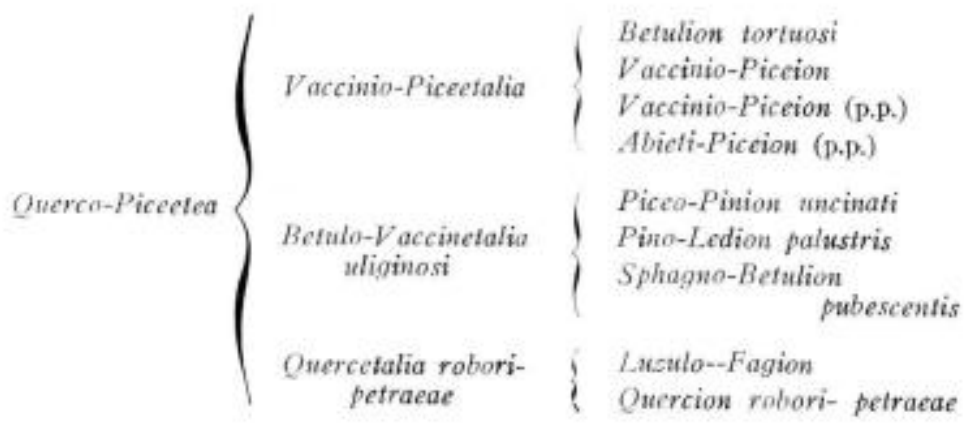

De même, sa classe des Querco-Fagetea parait ne comprendre que les associations liées à des sols à mull (mésophiles et mésohygrophiles), à l'exclusion des associations thermo-neutrophiles des Quercetalia pubescentis, et, bien entendu, des associations acidiphiles.

On peut, enfin, adopter une classification hiérarchisée mixte, où l'on groupe d'abord les associations en fonction de critères édaphiques, en s'en tenant à deux étages contigus, par exemple, et en rassemblant ces groupements édaphiques en une unité compréhensive de type "climatique $"$, correspondant, par conséquent, à deux étages, et ainsi de suite.

C'est ce qu'a fait récenment Soo (R.) (1964), pour certaines forêts hongroises: il a conservé la classe des Querco-Fagetea de Braun-Blanguet, mais a créé, à l'intérieur de cette classe, un ordre nouveau, les Pino-Quercetalia qui groupe toutes les associations acidiphiles de feuillus ou mixtes, et ou l'on rencontre, par conséquent, à la fois les chênaies acidiphiles (Castaneo-Quercion de Soo) et les hétraies acidiphiles (Deschampsio-Fagion), qui, jusqu'à présent, comme on l'a déjà souligné, étaient séparées.

Ces brèves considérations vont nous permettre de mieux comprendre la classification adoptée par l'Ecole de Zurich-Montpellier.

\section{IV.3.2. - Le système de classification de l'Ecole de Zurich- Montpellier.}

Les défauts que présente ce système sont de trois ordres:

a) Critères d'une hiérarchie - Foisonnenent des associations.

On doit d'abord constater que les chercheurs se réclamant de r'Ecole de Zurich-Montpellier ont eu trop souvent tendance à donner une place élevée, dans la hiérarchie, à des unités $\alpha$ locales $n$, 
caractérisées par des endémiques; ils ont ainsi créé de nouvelles associations, là où il n'aurait fallu retenir que des « variantes géographiques " . Ce foisonnement des associations, alors même que ces dernières se trouvent réunies dans la même unité, contribue à masquer les affinités d'ordre écologique souvent extrêmement étroites qui relient certaines d'entre elles et à faire croire que tous les groupements appartenant à la mème unité sont également « distants " les uns des autres, ce qui n'est pas, dans la plupart des cas.

Le tempérament de chaque chercheur, la plus ou moins grande ampleur du domaine qu'il explorait, l'ignorance dans laquelle il était parfois des travaux de ses collègues, ont ainsi abouti $\dot{a}$ faire se créer des unités ayant même rang dans la hiérarchie, mais qui, en fait, ne sont pas également compréhensives.

b) Critères d'une hiérarchie. La signification des caractéristiques des diverses unités compréhensives.

Lorsque l'on analyse la signification écologique des caractéristiques de telle ou telle unité et, singulièrement, de telle ou telle alliance, on constate, nous l'avons vu, que l'amplitude écologique de ces espèces est assez ctroite: les espèces du Fagion sont des espèces mésophiles montagnardes liées à des sols à mull; celles du Vaccinio-Piceion sont des mésophiles liées dans les étages montagnard et subalpin à des sols à moder et à mor; celles de l'Adenostylion sont, dans ces mêmes étages, des mésohygrophiles liées à des sols colluviaux à mull; celles du Quercion robori-petraeae sont des planitiaires mésophiles liées à des sols à moder ou même à mor ; etc.

Les données de l'écologie - et singulièrement de la pédologie dont nous ne cesserons de dire limportance fondamentale - montrent done que, malgré leur signification écologique profonde, les notions d'alliance, d'ordre, etc. sont restées critiquables et critiquées, parce que l'erreur a été d'englober dans ces unités, dont les espèces caractéristiques correspondent d̀ une écologie étroite, mais souvent ignorée, des sous-unités dont la plupart des espèces ne présentaient que très partiellement lamplitude écologique des caractéristiques de l’unité compréhensive.

Toute association possédant une certaine hétérogénéité floristique, due essentiellement à l'amplitude écologique plus ou moins grande des espèces qui la composent et à une relative hétérogénéité de son milieu - nous avons déjà insisté sur ces deux points - il était assez facile, lorsqu'il s'agissait de procéder à une classification hiérarchisée, de ne retenir, pour une association donnée, que les espèces caractéristiques de l'unité compréhensive dans laquelle on voulait l'inclure, alors que, souvent, ces espèces ne jouaient qu'un rôle secondaire, dans l'association en cause. 
Ainsi donc, par le fait de s'en être tenu à la seule floristique, de ne pas avoir analysé l'écologie des "caractéristiques ” des diverses unités, d'avoir, par esprit de système, il faut le dire, choisi et retenu, dans une association donnée, les seules espèces qui facilitaient sa classification, et surtout peut-être, d'avoir négligé et combattu même la notion de "caractéristique stationnelle " pour ne retenir que celle de "caractéristique floristique $n$, les chercheurs de lEcole de Zurich-Montpellier ont souvent abouti a des impasses.

Ils se sont ainsi heurtés à la difficulté de classer dans l'alliance du Fagion - appellation trop vaste, et nous avons dit pourquoi les hêtraies acidiphiles, où se trouvent très largement représentées des espèces de moder et de mor qui s'observent effectivement dans les chênaies acidiphiles, alors que les caractéristiques du Fagion y sont très rares, et ont envisagé de les inclure dans l'alliance du Quercion robori-petraeae, en les considérant comme des formes d'altitude de ces chênaies, ce qui n'était tout de mème pas très satisfaisant; ils ont rencontré des difficultés analogues avec les pineraies intra-alpines neutrophiles (alliances de l'Ononido-Pinion et du Erico-Pinion), incluses dans un ordre qui comprend, pour une très large part, des associations acidiphiles (Vaccinio-Piceetalia), etc.

Le fait d'utiliser un terme floristique a abouti, en outre, à certaines ambiguités: n'est-il pas un pet choquant de classer dans le Fagion des associations, où le hẻtre manque ou est très faiblement représenté - naturellement et non par le fait de l'homme - comme le Myrtillo-Abietetum, 1'Equiseto-Abietetum, 1'Abietetum albae, 1'Adenostylo-Abietetum? Dans le mẻme ordre d'idées, n'est-il pas anormal d'appeler "Piceetum n une association des Pyrénées d'où l'Epicéa est exclu? (5).

Ces anomalies, dont les exemples pourraient être multipliés, sont à l'origine, à notre avis, de l'incompréhension, sinon de l'hostilité, dont ont fạit preuve maints esprits à l'égard du système BraunBlanquet, dont le mérite est grand, cependant, nous l'avons déjà souligné, mais qui peut être amendé et qui ne cesse de l'être d'ailleurs.

Peu à peu, en effet, et à mesure que se sont développées les études écologiques, les chercheurs de cette Ecole ont été amenés à créer de nouvelles unités plus homogènes, en subdivisant les unités originelles trop vastes. Ainsi, on l'a vu, l'alliance du Fagion a été divisée en trois sous-alliances, la classe des Vaccinio-Piceetea a perdu les pineraies neutrophiles, etc.

Dans l'ensemble cependant, et malgré ces mises au point, une certaine " hétérogénéité générale " subsiste encore dans ce système de classification.

(5) Braun-Blanquet (J.), Roussine Mme), Nżcre (R.) 1951. 
c) Hétérogénéité générale de la classification de l'Ecole de Zurich-Montpellier.

Au niveau de l'Alliance, d'abord on constate que certaines de ces unités sont, soit du type climatique, soit du type édaphique, soit du type mixte, sans que des justifications objectives permettent d'expliquer l'appartenance de telle ou telle unité à tel ou tel type de classification.

L'alliance du Fagion, par exemple, comprend toutes les associations de l'étage montagnard, c'est-à-dire aussi bien les groupements subacidiphiles à mull de la sous-alliance Asperulo-Fagion (ou EuFagion) (Fagetun silvaticae, Abieti-Fagetum, Aceri-Fagetum, Equiseto-Abietetum) que les groupements neutrophiles de la sous-alliance Cephalanthero-Fagion (Carici-Fagetum, Seslerio-Fagetum, etc.), ou que les groupements acidiphiles de la sous-alliance LusuloFagion (Melampyro-Fagetum, Lusulo-Fagetum, Deschampsio-Fagetum, etc.). Le classement est ici de type climatique.

Par contre, l'alliance du Quercion robori-petraeae ne comprend que les chênaies acidiphiles (classement édaphique); de même celle du Vaccinio-Piceion ne concerne que les associations acidiphiles de résineux de l'étage subalpin, celle de l'Erico-Pinion les seules associations neutrophiles résineuses des étages montagnard et subalpin.

Cette hétérogénéité se retrouve forcément dans les unités supérieures:

L'ordre des Fagetalia silvaticae englobe les associations de trois étages: planitiaire, collinéen et montagnard, avec toutes les associations de l'étage montagnard (alliance du Fagion), mais avec une partie seulement des associations des étages planitiaire et collinéen (alliance du Fraxino-Carpinion), puisque le Quercion robori-petraeae est rattaché à l'ordre des Quercetalia robori-petraeae.

Si l'on ajoute que la plupart des associations thermophiles de l'étage submontagnard forment l'alliance du Quercion pubescentipetraeae incluse dans l'ordre des Quercetalia pubescentis et se trouvent ainsi rassemblées en des unités plutôt " climatiques " qu' " édaphiques » (elles sont donc séparées d'associations neutrophiles avec lesquelles elles présentent de très étroites aftinités, celles du Cephalanthero-Fagion), on saisira mieux le caractère quelque peu incohérent que présente la classification de Braun-BLANouET, tout au moins en ce qui concerne les forêts.

Ce caractère, il faut le souligner, tient beaucoup au fait que la classification originelle qui était incomplète de par la force des choses a été modifiée, le plus souvent améliorée, mais toujours de façon partielle, au fur et à mesure que se développaient les travaux des spécialistes.

\section{IV.3.3. - Classifications hiérarchisées et non hiérarchisées.}

Si l'on devait peser les mérites respectifs des deux types de classement que nous avons distingués, on pourrait penser, en premier 
lieu, que le classement édaphique présente une plus grande a amplitude $n$ que l'autre, en ce sens que le nombre d'associations liées à un type d'humus donné, à travers tous les étages, est probablement plus grand que celui des associations spécialisées édaphiquement dans le même étage, ce qui devrait permettre de distinguer dans le premier cas plus d'unités compréhensives que dans le second.

Seule une analyse de détail qui dépasse l'objet du présent travail permettrait de se faire une idée sur ce sujet, étant noté que des critères véritablement objectifs manquent pour déterminer dans les deux cas, des unités de même valeur (6).

Pour juger, en second lieu, de la « cohérence $»$ de chacun des classements, il faudrait étudier, avec des coefficients de communauté ad hoc (en tenant compte, non seulement de la présence des espèces, mais également de leur abondance-dominance) les " degrés d'affinité » existant entre des unités analogues des deux systèmes. Nous essaierons, dans un prochain travail, d'éclairer un tel problème.

Quels que soient les résultats obtenus quant à cette " cohérence n, on pourra toujours faire observer que le classement édaphique est, a priori, plus artificiel que le classement climatique; ce dernier, en effet, se situe dans une perspective dynamique et facilite la mise en évidence de " séquences génétiques $»$.

Ce à quoi, certains pourront rétorquer qu'en montagne, la notion de climax unique par étage doit être singulièrement nuancée, - on l'a souligné - et doit plutót laisser la place désormais à la notion d'essaim de climax.

Mais quels que soient, en définitive, les mérites de l'un ou l'autre classement, ils ont tous deux le défaut d'être hiérarchisés et de masquer, par conséquent, ce fait important, que les associations - sauf celles qui sont hautement spécialisées - sont polydimensionnelles, c'est-à-dire composées de groupes écologiques divers.

C'est la raison pour laquelle il nous a paru préférable, dans le tableau général des associations forestières de la Haute-Maurienne, de faire apparaitre ces divers groupes; l'on peut, dès lors, saisir les affinités de tous ordres qui existent entre les divers groupements, et en déduire des schémas qui traduisent ces affinités.

\section{IV.3.4 - Le problème de la nomenclature.}

Cette question des affinités serait éclairée, pensons-nous, si la nomenclature adoptée en Phytosociologie en tenait compte davantage.

(6) C'est, en définitive, l'absence de critères objectifs dans le choix des unités, qui est à l'origine de bien des difficultés: une alliance comme le Quercion robori-patraeae ne se situe pas au même niveau que son homologue le Fraxino-Carpinion, lequel est moins compréhensif que le Fagion. 
Pour ce faire, on pourrait désigner les Alliances par tin terme Horistique: celui de l'espèce arborescente caractéristique, dans unc région et à une exposition donnces d'un étage, ou liée étroitement à certaines conditions stationnelles spéciales.

Par exemple, toutes les associations de Pin à crochets formeraient l'alliance du Pinion uncinatac, celles du Quercus sessiliflora, celle du Quercion sessiliflorac, celles du Fagus silvatica, le Fagion silvaticae, etc, étant entendu que la détermination des associations ne saurait s'appuyer sur le seul critère de la dominance des espèces arborescentes, mais devrait faire appel à une étude floristique et écologique de détail, permettant de fixer avec précision la place du groupenent et de faire la part de Paction humaine dans la dominance de tel ou tel arbre.

Cette façon de dénommer les Alliances qui est, dans la plupart des cas, déjà adoptée par 1'Ecole de Zurich-Montpellier, mais qui devrait l'étre d'une façon plus systématique, permettrait de faire se rapprocher la nomenclature proposée de celle de Gsussen oit les " séries » sont désignées par le nom d'une espèce arborescente, dans tous les étages forestiers s'entend.

Ceci fait, il serait évidemment indispensable de distinguer, à l'intérieur de ces unités, relativement vastes, des "sous-unités édaphiques n. Dans ce dernier domaine, on peut être tenté d'utiliser encore des termes floristiques, mais le choix s'avère difficile, car l'espèce retenue doit se retrouver dans tous les étages d'une région considérée, tout en étant étroitement liée à un setil type d'humus.

Pour les groupements acidiphiles, Deschampsio flexuosa parait a priori une très bonne espèce, puisque, tout en trouvant son optimum sur les moders, elle reste très fréquente et abondante sur les mors et se rencontre de la Chênaie à la Pineraie de Pin Cembro. On parlerait ainsi de Deschampsio-Quercion scssiliflorae, de Deschampsio-Fagion, de Deschampsio-Pinion uncinatae, de Deschampsio-Pinion Silvestris, ce qui marquerait bien les affinités existant entre les associations relevant de ces diverses unités.

Pour les groupements neutrophiles, le probleme est plus délicat. Erica carnea peut passer, a priori, pour une bonne espèce, encore qu'elle soit une orophile et s'observe surtout dans les groupements de résineux; mais elle présente le défaut majeur d'avoir une aire extrêmement restreinte en France (de ce point de vue, le terme d'Erico-Pinctea n'a vraiment valeur d'image qu'en Europe Centrale). Calamagrostis varia serait à lui préférer, avant Sesleria coerulea, bien qu'elle soit aussi une orophile.

Ces exemples nous paraissent suffisants, pour montrer, qu'en tout état de cause, l'utilisation d'un terme floristique présente le grave inconvénient de n'être vraiment suggestif, donc utilisable, que dans les limites de l'aire de l'espèce qu'il désigne. Si la chose est acceptable pour les grandes espèces arborescentes, elle ne l'est plus lorsque l'on utilise deux termes floristiques, car 1" "aire de ce binôme " 
correspond à la partie commune des aires des deux espèces en cause, partie commune, qui risque, dans certains cas, d'être un peu trop restreinte.

C'est la raison pour laquelle nous pensons qu'il est tout de même préférable d'utiliser des termes écologiques - ce qui, d'ailleurs, a parfois été déjà fait, mais trop rarement - car ils permettent d'exprimer de nombreuses nuances et de façon explicite, tout en mettant en relief - et sans limitation d'aire - les analogies.

Pour reprendre nos exemples, on parlera de Neutro-Pinion uncinatae (à mull ou moder calcique), d'Acidi-Pinion uncinatae (à moder ou à mor), de Subacidi-Quercion sessiliflorae (à mull forestier), etc.

A ces préfixes, correspondant à des caractères édapho-chimiques, pourront en être adjoints d'autres, définissant des caractères climatiques, plus précisément liés à l'économie de l'eau: par exemple, dans le Neutro-Fagion, on pourra distinguer un Xéro-neutro-Fagion, un Méso-neutro-Fagion, etc.

Si l'on retient sept types d'humus (mull à moder calciques; mull forestier; moder; mor; anmoor ; mor tourbeux acide; mor tourbeux calcique) et quatre " échelles " d'économie de l'eau (xéro; méso; méso-hygro; hygro) on obtient, pour une alliance, telle que nous l'avons définie ci-dessus, vingt-huit combinaisons. En fait, toutes ne se rencontrent pas dans la nature, car il y a antinomie entre certaines d'entre elles (les humus formés en milieu saturé d'eau ne peuvent être xériques) ou entre telle espèce arborescente et tel type d'humus (les chênaies, les hêtraies ne se rencontrent pas sur tourbière; les pineraies de Pin Cembro ou même les pessières ne s'observent pas sur mull calcique, etc.). Leur nombre reste cependant tel, qu'il permet $d^{\prime}$ " abaisser $"$ les sous-unités, ainsi définies, presque au niveau des associations et des sous-associations. Deux exemples - où nous n'avons pas cherché à être exhaustif - suffiront pour éclairer notre pensée: le Xéro-neutro-Fagion comprendrait les associations ciaprès: Seslerio-Fagetum anthericetosum, Buxo-Fagetum sous-association; le Méso-neutro-Fagion comprendrait le Carici-Fagetum carisetosum albae, le Seslerio-Fagetum hylocomietosum, le Buxo-Fagetum hylocomictosum, le Tilio-Fagetum, le Taxo-Fagetum...

Une telle nomenclature aurait le mérite, nous semble-t-il, de mettre en relief à la fois les « affinités d'étage \# - ou de sousétage - par le nom même de l'espèce arborescente servant de désinence, et celles liées au sol et au microclimat. Elle correspondrait à une réalité, puisque, dans toutes les sous-alliances commençant par le même préfixe, se rencontreraient les espèces du même groupe écologique, ou, du moins, la plupart d'entre elles. 


\section{IV.4. - PROBLEMES SYLVICOLES}

On sait que trois problèmes principaux se posent à l'aménagiste et au sylviculteur dans toute forêt: celui de la structure - c'est-àdire de la répartition des tiges dans l'espace - qui doit ètre telle, que, sur une surface donnée dite "série n, la production soit à peu près constante ou tende à ètre constante:

- celui de la régénération, qui, dans toute la mesure du possible doit être naturelle, être répartie dans l'espace et dans le temps en fonction de la structure et assurer la pérennité du massif, sans àcoups,

- celui, enfin, de la substitution d'essences qui se pose lorsque les produits de la forêt naturelle sont médiocrement intéressants du point de vue économique.

En Haute-Maurienne, le forestier cherche à obtenir une structure jardinée, c'est-à-dire celle où l'équilibre entre les diverses classes d'âge est obtenu sur une surface réduite - en général, celle de la parcelle de gestion, soit 15 à 20 hectares, - ce qui permet et implique d'avoir une régénération continue ou à peu près continue dans le temps et discontinue dans l'espace. Cette structure s'impose en grande partie, non seulement à cause des fortes pentes, où l'érosion doit être évitée au maximum, mais aussi parce que l'on observe du bas en haut d'un versant des associations diverses, formant parfois même un véritable puzzle, qu'il ne serait pas possible de traiter par grands ensembles, comme cela se fait, lorsque l'on adopte un aménagement de futaie régulière.

Cette structure se prète d'autre part à tous les assouplissements possibles et permet de tenir compte au maximum des nuances du milieu, du tempérament des essences, en un mot, de l'existence de telle ou telle association: les dimensions d'exploitabilité, l'ampleur des trouées de régénération dépendront ainsi étroitement de l'association rencontrée. Si dans les pineraies de Pin sylvestre - surtout dans l'Ononido-Pinetum - les trouées doivent être vastes (diamètre de $50 \mathrm{~m}$ par exemple), elles seront plus petites dans la sapinière vraie, où pourra même être pratiqué un jardinage " pied par pied n : dans la rhodoraie à Pin Cembro, où la régénération est sporadique et jamais par tâches importantes, le martelage sera a ponctuel n. 
Il est évident que, pour maitriser toutes ces structures diverses. le forestier doit pouvoir compter sur une régénération suffisante, lui permettant de régénérer une unité de gestion (parcelle) dans le laps de temps correspondant aux divers âges d'exploitabilité.

On peut dire que, dans l'ensemble, toutes les associations de la Haute-Maurienne, qui sont en équilibre avec le milieu, ne posent pas de problèmes, quant à la régénération des essences arborescentes, à l'exception d'une seule: l'association du Pin Cembro à Calamagrostis villosa. Nous avons déjà dit ce qu'il fallait en penser et les remèdes à apporter, lors de l'étude de ce groupement.

Peut-on améliorer le rendement des forêts de notre haute vallée en introduisant des essences exotiques? Nous pensons que non, car il se trouve qu'elles sont, dans l'ensemble, composées presque uniquement de résineux dont on sait l'importance économique et que, admirablement adaptées à des conditions écologiques parfois sévères, elles risqueraient de souffrir de toute introduction d'élément étranger.

On peut cependant retenir que des essais de plantation de Picea omorica, essence calciphile poussant avec Erica carnea, dans une portion très restreinte du territoire de l'Europe méridionale ITREGUBOV (S.) (1934)], pourraient être tentés dans les pineraies de Pin sylvestre et de Pin à crochets, à Erica carnea.

Dans les hêtraies méso-xérophiles dont l'importance est d'ailleurs minime, devraient réussir les Sapins méditerranéens (Abies Nordmanniana notamment) et le Pin noir d'Autriche. Ces mêmes essences, avec le Cèdre de l'Atlas pourraient aussi être utilisées dans l'étage du Chêne pubescent.

Le problème majeur qui se pose, en définitive, au forestier dans notre dition et qui est apparu lors de l'étude de chaque association est celui de P'équilibre des structures. Lorsque l'on envisage chaque parcelle ou chaque série, il est facile de se convaincre que la répartition des tiges par classe de diamètre ne correspond pas à une exponentielle - qui traduirait cet équilibre - mais à une courbe beaucoup trop convexe. Il y a partout insuffisance de jeunes bois et excès de bois moyens. C'est donc la régénération et la place qu'il convient de lui laisser qui doit appeler toute l'attention du forestier.

Dans ce domaine, la connaissance des associations et des conditions écologiques qui leur sont liées apparait comme absolument indispensable. 


\section{RESUME GENERAL}

$1^{\circ}$ Dans une première partie et pour nous en tenir à l'essentiel. nous avons montré que toutes les associations végétales - sauf peut-être celles hautement spécialisées - ont des affinités dans plusieurs directions.

Ce " caractère polydimensionnel " des groupements, tient essentiellement d'une part et surtout, à la plasticité des diverses espèces végétales quii les composent, c'est-à-dire à l'amplitude qu'elles présentent vis-à-vis de tel ou tel facteur du milieu, d'autre part à la relative hétérogénéité de ce dernier.

$2^{\circ}$ Pour traduire un tel caractère, il nous a paru essentiel de faire appel à la notion de groupe écologique, la coexistence de divers groupes ou portions de groupes dans une même association permettant à la fois de caractériser cette dernière et de mettre en évidence toutes les nuances du milieu dans lequel elle se développe et ce dans une double perspective de statique et de dynamique végétale.

$3^{\circ}$ Ce faisant, nous devions renoncer à la classification classique en alliances, en ordres et en classes de Braun-Blanouet, car cette classification, comme nous l'avons montré, masque précisément le caractère "polydimensionnel " des groupements en ne mettant en évidence que les espèces appartenant aux diverses unités compréhensives dans lesquelles se trouve placée une association donnée et en rejetant dans la foule plus ou moins anonyme des compagnes, des espèces qui sont pourtant caractéristiques d'autres unités et dont l'intérêt écologique ne doit donc pas être négligé.

$4^{\circ}$ Nous avons cependant cru utile de rattacher dans toute la mesure du possible, les divers groupes définis par rapport à certains grands facteurs écologiques décisifs, aux unités du système BraunBlanquet. Nous avons ainsi pu montrer que, derrière les termes purement floristiques de ce système - en particulier ceux désignant les alliances - se cachait une réalité écologique profonde. Cette correspondance a été forcément limitée aux espèces rencontrées dans notre territoire d'étude - tout au moins à la plupart d'entre elles.

$5^{\circ}$ Après avoir défini les grands traits écologiques qui caractérisent la Haute-Maurienne, nous avons analysé, et en termes à la 
fois floristiques et écologiques, les associations forestières de cette région, y compris celles - hêtraies et chênaie pubescente - qui font le passage entre le monde préalpin et le monde intra-alpin.

$6^{\circ} \mathrm{Il}$ s'est avéré que les groupes écologiques que nous avions préalablement définis se sont articulés dans les associations décrites. de façon parfaitement cohérente, c'est-à-dire qu'il nous ont permis. chaque fois, d'expliquer les nuances du milieu dans lequel se développent les dites associations.

$7^{\circ}$ Ces nuances du milieu ont été analysées - bien que parfois de façon fragmentaire, et nous avons dit pourquoi - à l'aide d'analyses pédologiques et climatiques de l'air et du sol. Il est ainsi apparu que le micro-climat du sol - traduit en température et en potentiel capillaire - surtout dans ses premiers centimètres, avait une importance capitale, ce qui ne peut faire négliger pour autant l'influence de son chimisme.

Cette analyse est, en outre, venue justifier la définition écologique des divers groupes que nous avions retenus.

$8^{\circ}$ Tout au long de notre travail, nous avons pu montrer les remarquables affinités que présente la Haute-Maurienne avec d'autres vallées internes des Alpes Centrales, notamment le Valais et même l'Engadine.

La grande similitude - pour ne pas dire l'identité de composition floristique des diverses associations forestières de ces vallées distantes pourtant de plusieurs dizaines de kilomètres et séparées entre elles par de puissants barrages montagneux, vient renforcer. à notre avis, la notion même d'association végétale, critiquée par certains; elle montre, en effet, que l'association correspond bien à une réalité incontestable, mais évidemment complexe et variable comme toute chose vivante et ayant une histoire.

9. Nous avons donné quelques chiffres concernant les productions des principales associations étudiées sans alourdir le texte des données proprement dendrométriques qui nous ont permis de les obtenir (résultats des sondages à la tarière de PrESSLER - calcul des droites de régression donnant les accroissements en diamètre en fonction de ce dernier), car ce problème sortait du cadre de notre étude.

$10^{\circ}$ En conclusion, nous avons pris une vue d'ensemble des grands facteurs écologiques qui permettent " d'expliquer " les associations décrites, ce qui nous a permis, au passage, de développer quelques brèves idées générales sur la notion de climax, en montagne.

Nous avons ensuite abordé, dans un esprit de synthèse, l'importante question de la classification des unités phytosociologiques et mis en relief les raisons pour lesquelles le système de classifi- 
cation adopté par l'Ecole de Zurich-Montpellier reste critiquable, bien que les notions de classe, d'ordre et surtout d'alliance correspondent à une réalité écologique. Nous avons esquissé une solution à ce problème en nous en tenant volontairement à quelques grandes lignes.

Nous avons enfin effleuré, de façon très condensée, les problèmes sylvicoles qui se posent au forestier en Haute-Maurienne, problèmes au demeurant assez faciles à résoudre, étant donné que les forêts, presque toutes composées uniquement de résineux, présentent, de ce fait, un intérêt économique indéniable, encore que l'on puisse regretter leur faible croissance. Mais dans ce dernier domaine, la Nature est fort contraignante et les progrès à envisager dans un avenir pas trop lointain résulteront directement de l'amélioration même des structures sylvicoles, actuellement un peu déséquilibrées. 


\section{BIBLIOGRAPHIE}

Atchele (D.) et Schweglek (H.W.), - 1956 - Unsere Moos- und Farnpflanzen.

Stuttgart. 181 p.

Archinger (E.). - 1952 - Die Rotbuchenwälder als Waldentwicklungstypen. Angezuandte Pflansensoziologic.

Veröffentlichungen des Instituts für Angewandte Pflanzensoziologie des Landes Kärnten. 5, 106 p. Vienne.

Aıcringer (E.). - 1952 - Fichtenwälder und Fichtenforste als Waldentwicklungstypen.

Angewandte Pflanzensoziologie. 7, 178 p. Vienne.

AICHINGer (E.). - 1952 - Rotfóhrenwälder als Waldentwicklungstypen. Angewandte Pflansensosiologic. 6, 68 p. Vienne.

AlLioni (C.). - 1785 - Flora pedemontana. Torino.

Allorge (P.). - 1921-1922 - Les associations végétales du Vexin français. Revue gén. de bot. - Thèse. Paris.

ANNE. - 1945 - Dosage rapide du carbone organique des sols. Ann. Agr., 2: 161-172.

AReNes (J.). -1955 - A propos de quelques groupments végétaux relictuels de la Provence et de la Corse.

Mémoires de la Soc. de Biogéographie. Nlle Série, 2 : 29-49.

Augr (Ch.). - 1947 - Untersuchungen uber die natürliche Verjügung der Lärche in Arven - Lärchenwald des Oberengadins.

Mitteilungen der Schweiserischen Anstalt für das forstliche Versuchswesen, 21 (1): 7-140. Zürich,

Bлсн (R.), Kuoch (R.), Iherg (R.), - 1954 - Wälder der Schweizen Alpen im Verbreitungsgebiet der Weisstanne, Entscheidende Standortsfactoren und Böden.

Mitt. der Schweis. für das forstl. Versuchswesen, 30: 261-314. Zürich.

Васн (R.), Киосн (R.), Moor (M.). - 1962 - Die Nomenklatur der Pflanzengesellschaften. Mitt. flor. sos. Arbeitsgem. N.F, 9: 301-308.

Bagnouls (F.) et GaUssen (H.). - 1953 - Saison sèche et indice xérothermique.

Bull. Soc, Hist. Nat. Toulouse, 88: 193-239. Toulouse.

Balsemste (R.). - 1955 - La pluviosité en Savoie.

Rev. Géog. alpine, 43 : 299-355.

Bartol-I (C.). - 1954 - Sur les peuplements de pins à crochets de la HauteMaurienne.

8 Congrès Int. Bot. Section 13, 45-48.

BлктоLi (C.). - 1955 - Note sur le Calamagrostis villosa (Chaix) Mutel, en Haute-Maurienne.

Monde des Plantes. 316.

Bartoli (C.). - 1961 - Aperçu d'ensemble sur les Groupements forestiers de la Haute-Maurienne.

87* session extraordinaire en Savoie.

Bull, Soc, bot. Fr, $108: 70-79$. 
Bartol. (C.). - 1962 - Première note sur les Associations forestięres du Massii de la Grande-Chartreuse.

Annales E.N.E.F, et Stat. Rech, et Exp., 19, fasc. 3: 328-372.

Bartoli (C.). - 1962 - Chronique botanique. A propos d'une thèse récente sur les foréts acidophiles du Jura.

Rev. For. Fr., n" 2: 134-140.

Benuverd (G.). - 1911 - Herborisation de la Société Botanique en Mattrienne des 13 et 15 avril 1911.

Bull. Soc. bot. Genève, $2^{\circ}$ Série: 195-232.

BEAUVERd (G.). - 1921 - Sur la flore vasculaire des environs de Modane, de Bardonnèche et de Suse (Massif du Cenis).

Bull, Soc. bot. Gendve, 115-184.

Beger (H.). - 1922-1923 - Assoziationsstudien in der Waldstuie des Schanfiggs: Jahresber. Naturl. Ges. Gratenbündens 61 wnd 62 . Chur.

Benevent (E.). - 1926 - Le Climat des Alpes françaises (Mémorial de lO.N.M. de France). 435 p. Paris.

Bertsch (K.). - 1959 - Moosflora von Sudwestdeutschland. Ed, E, Ulmer, 234 p.

Blanchard (R.). - 1943 - Les Alpes occidentales. 3.e Les Grandes Alpes françaises du Nord $\$$. Grenoble.

Blanchard (R.). - 1945 - Les Alpes occidentales. 4. Les Préalpes françaises du Sud. 1 .

561 p. Grenoble - Paris.

Blanchard (R.). - 1956 - Les Alpes occidentales. Essai d'une synthèse. 605 p. Grenoble - Paris.

BLoNDEL (R.). - 1941 - La végétation forestière de la région de SaintPaul, près de Montpellier.

Communication $n^{*} 79$ de la S.I.G.M.A.

Bourt (M.). - 1950 - La pluie en Valais. Bull. de la Murithienne. Fasc. 67: 22 p.

Bover (M.). - 1954 - Notice sur le climat de Sierre. Annales de la Soc, suisse de Bahéologie et de Climatologie, 43: 97 106.

Bouvier, - 1863 - Histoire de la botanique savoyarde. Bull. Soc, bot, Fr. 644-675.

Braun-Blanguet (J.). - 1922 - Une reconnaissance phytosociologique dans le Briançonnais.

Bull. Soc. Bot. de Fr, 69, 5* 5., 78-103.

Braun-Blanguet (R.), Jenny (H.). - (1926-Vegetationsentwicklung und Bodenbildung in der Alpinenstufe der Zentralalpen (Klimaxgebiet des Carcion curvulae).

Ergeb, d. Wissensch. Unters. d. Schweizer Nationalparks. Denkschr. d. Schweiz. Nat, Ges. 2.

Braun-Blangurt (J.) et Susplugas (J.). - 1937 - Reconnaissance phytogéographique dans les Corbières. SIGMA. Comm. 61.

B,aun-Blangue5 (J.), Sissingh (G.), Vlieger (J.). - 1939 - Prodomus der Pflanzengessellschaften. Fase. 6. Klasse der Vaccinio-Piceetea. Comite Intern, du Prodrome phytosociologique.

Braun-Blanguet (J.). - 1948 - La végétation alpine des Pyrénées orientales,

Monograjia de la Estación de Estudios Pirenaicos y del Institufo Español de edafologia, ecologia y fisiologia vegetal.

Barcelone, 306 p. 1948.

Braun-Blanguet (J.) et Richard (R.) - 1949 - Groupements végétaux et sols du bassin de Sierre.

Bull. de la Murithienne. 66: 106-134. 
Braun-Blanguet (J.). - $1950^{\circ}$ - Ubersicht der Pflanzengesellschaften Rätiens.

Vegetatio. Acta Geobotanica. Vol. II, fasc. 2-3.

Braun-Blanquet (J.), Roussine (Mme), NèGre (R.). - 1951 - Les groupements végétaux de la France méditerranéenne. 298 p. C.N.R.S.

Braun-Blanguet (J.) - 1951 - Pflanzensoziologie. Grundzüge der vegetationskunde.

Springer Edit. Wien, $2^{*}$ édit. 631 p.

Braun-Blanguet (J.). - 1953 - Essai sur la végétation du Mont Lozère comparée à celle de l'Aigoual.

Bull. Soc. Bot. Fr., 100, 1953.

$80^{\circ}$ Session extraordinaire (1952). 45-59.

Braun-Blanguet (J.) - 1954 - La végétation alpine et nivale des Alpes françaises. S.I.G.M.A. Comm. n' 125,70 p. Montpellier.

Braun-Blanguet (J.), Pallanas (H.), Bach (R.). - 1954 - Pflanzensoziologische und bodenkundliche Untersuchungen im Schweizerischen Nationalpark und seinen Nachbargebieten. II.

Vegetation und Böden der Wald- und Zwergstrauchgesellschaften (Vaccinio-Piceetalia).

Druck Lüder - A.G. Liestal, 200 p.

Braun-Blanquet (J.). - Zur Vegetation der Nordbündnerischen Föhrentäler. S.I.G.M.A. Comm. n ${ }^{\circ} 146$, p. 235-249. Montpellier.

Braun-Blanguer (J.). - 1961 - Die inneralpine Trockenvegetation von der Provence bis zur Steiermark.

G. Fischer. Edit. 273 p. Stuttgart.

Brny et Will.hite. - 1929 - Ind. Eng. Chem. Anal. 144 p.

Briguet (J.). - 1895 - Notes sur la flore du Massif de Plate. Ed. Burkhardt. Genève.

Cariot et SAINT-LAgrR. - 1897 - Botanique élémentaire, descriptive et usuelle. 21 Flore descriptive du bassin moyen du Rhóne et de la Loire. Vitte. 1027 p. Lyon.

Carles (J.). - 1948 - Géographie botanique. P.U.F. 126 p. Paris.

Carles (J.). - 1953 - A travers le Velay. Bull. Soc, Bot, Fr. 100. 80" session extr. (1952) dans les Cévennes et dans les Causses. 59-67.

Charers. - 1859 - Etudes sur la géographie hotanique de la Savoie. Bull. Soc. bot. Fr. 6.

Chabrert. - 1860 - Esquisse de la végétation de la Savoie.

Bull. Soc. bot. Fr. 7.

Chamert. - 1883 - Recherches botaniques dans les Alpes de la Maurienne. Bull. Soc. bot. Fr. 2-19.

Chatx (G.). - Diplóme d'études supérieures de botanique sur la région du Lautaret.

Lab. Biol. Végét. Marseille. (D.E.S.).

Chodat (R.). - 1894 - Remarques de géographie botanique relatives aux plantes récoltées dans les vallées de Bagnes et de la Viège et au Simplon.

Bull. Soc. bot, Fr. 41, 3n série. I. 278-310.

Christ (H.). - 1879 - Das Pflanzenleben der Schweiz, Zurich.

Chemersts (F.). - 1916 - Plant succesion. An analysis of the development of vegetation. Carnegie Inst. of Washington, $\mathrm{n}^{\circ} 242,512$ p. Washington.

Convert (B.H.). - 1896-1921) - Herborisation en Maurienne et en Tarentaise.

Ann. Soc. bot. Lyon. 103 p. 
Coutagne. - 1942 - L'évaporation du sol et le déficit d'écoulement considérés du point de vue hydrographique, agronomique et elimatologique. La Météorologie, Juillet-décembre 1942, 150-176.

Cussix (G.). - 1964 - Les forèts du versant Sud des Monts Dores, Esquisse phytosociologique.

Ann. Sc. For, 20, fasc. 1. 85-165.

Dansereau (P.). - 1954 - Leíficacité du climat et les inhibitions édaphiques. VIII Cong. inter. Bot. Paris. Section 7. 100.

DEB (B.C.). - 1950 - Journ of Soil Sc. I, p. 212.

Derazac (E.). - 1964 - Manuel des conifères. $172 \mathrm{p}$.

Descolon (A.). - 1952 - Principes dAgronomie. I. La dynamique du Sol. Paris.

Denarié (M.). - 1920 - Observations sur les colonies de Plantes méridionales de la Maurienne.

Bul.. Soc. bot. Fr. 57.

(Session extraordinaire au Mont-Cenis).

Doignon (P.) - 1955 - Flore des mousses de la plaine française. in La Forèt de Fontainebleau. Recherches sur son sol, sa Faune, sa Flore.

Ass, Nat, vallee du Loing. 12, p. 544. Fontainebleau.

Doing (H.). - 1962 - Systematische Ordnung und floristische Zusammensetzung Niederländischer Wald- und Gebuischgesellschaften.

North-Holland publisching Company. 85 p. Amsterdam.

Droutnenu (G, et Gouny (P.). - 1947 - Contribution à létude du dosage de lazone nitrique par la méthode Dewarda. Ann. Agr. 2.

Duchaufour (Ph.). - 1948 - Recherches écologiques sur la Chenaie atlantique française. Ann de IE.N.E.t. et Stat, Rech, et Exp. 332 p. 11, fasc. 1. Nancy.

Duchaufour ( $\mathrm{Ph}$.). - 1950 - Recherches sur l'évolution des sols calcaires en Lorraine.

Ann. de l'E.N.E.F. et Stat. Rech. et Exp. 13, fasc. 2, p. 97-153. Nancy.

Duchaurour (Ph.). - 1952 - Etudes sur l'Fcologie et la Sylviculture du Mélèze (Larix europaea D.C.).

Pédologie et Facteurs biotiques.

Ann. de lEEN.E.F. et Stat. Rech. et Exp. 13, fasc. 1, p. 135-199.

Duchaupour (Ph.). - 1952 - Les espèces calcicoles et calcifuges. Rev. For. Fr., p. 301-305.

Duchaufour (Ph.). - 1957 - Pédologie. Tableaux descriptifs et analytiques des sols.

Ecole Nat. des Eaux et Forêts. 87 p.

Duchaufour (Ph.), Jacamox (M.), Debazac (E.F.), Pardé (J.). - 1958 Un exemple d'utilisation pratique de la cartographie des stations: la forêt du Ban d'Etival (Vosges).

Rev. For. Fr., p. 597-629.

Duchatfour ( $\mathrm{Ph}$.). - 1959 - La dynamique du sol forestier en climat atlantique.

Fonds de Recherches forestières de l'Université Laval-Québec.

Duchaufouk ( $\mathrm{Ph}$.). - 1960 - Stations, types dhumus et groupements écologiques.

Rev. For. Fr., n ${ }^{\circ} 7$, p. $484-494$.

Dhchaufour (Ph.). - 1960- Précis de pédologie. 438 p. Masson et Cie. $2^{*}$ édition. 481 p. 1965.

Duchaufour (Ph.) et Bonneau (M.). - 1960 - Les sols de la hètraie en Europe occidentale.

Bull. Inst. Agro, et des Stat, de Rech. de Gembloux. Série extraordinaire éditée ì l'occasion du centenaire de I'Institut Agronomique de I'Etat. Vol. I, p. 59-74. 
Duvignenu (P.). - 1946 - La variabilité des associations végétales. Bull, de la Soc, Royale de Bot. de Belgique, 78: 107-134.

Duvigneaud (P.). - 1955 - Ftudes écologiques de la végétation en Afrique Tropicale.

Ann, biol. 31, fasc. 5-6, 375-392.

Elitenerkg (H.) - - 1953 - Füht die alpine Vegetations- und Bodenentwicklung auch auf reinen Karbonatgesteinen zum Krumseggenrassen (Caricetum curvulae). Ber. detitsch. Bot. Ges.

Et.1.extr.Rg (H.). - 1954 - Kausalanalyse der Beziehungen zwischen Ackerunkraut Gesellschaften und Boden. VIII $^{e}$ Congr. intern. Bot. Section 7, 39-40, Paris.

Ellennerg (H.), - 1954 - Zur Entwicklung der Vegetationssystematik in Mitteleuropa.

Angetcandte Pflanscrisosiologie, Springer Ed. No spécial, Vienne,

Ellenezkg (H.). - 1963 - Vegetation Mitteleuropas mit den Alpen. Stuttgart.

Elt.exBerger. - 1958 - Etude géologique du pays de Vanoise. Mém. Soc. Carte Géol, 561 p.

Emaerger (L.). - $1930-\mathrm{La}$ végétation de la région méditerranéenne, Essai d'une classification des groupements végétaux. Rev. Gén. Bot. 42.

Fumerger (L.). - 1932 - Sur une formule climatique et ses applications en botanique. La Météorologic. No 92-93. Paris.

Emberger (L.). - 1942 - Un projet dine classification des climats du point de vue phytogéographique.

Bull. Soc. Hist. Nat. Toulouse, 77: 97-124.

Емnekger (L.). - 1943 - Les limites de l'aire de végétation méditerranéenne en France.

Bull. Soc. Hist. Nat. Toulouse. 78

Emarrger (L.). - 1953 - La végétation de l'Italie d'après A. Graccone et remarques sur la méthode en géographie botanique écologique, Recucil des Trawas du Labo. de Boto. Gíld et Zool. de la Fac. de Montpellicy. Série Bota. Fasc. 6.

FмнвкGek (L.). - 1954 - Projet d'une classification biogéographique des climats in \& Les divisions écologiques du monde $>$. Colloques Intern. C.N.R.S., 249-255. Paris.

Farasergr (L.). - 1958 - Principes de la méthode de travail du Service de la Carte des Groupements végétatux du C.N.R.S. Bull. do Ser. Carfe Phytogéographique. Série B. 3, fasc. 2, 91-99.

Emmercen (L.). - 1959 - La cartographie de la végétation et les services qu'elle peut rendre à l'Agronome.

Cahiers des Ing. Agron., $\mathrm{n}^{\circ}$ 130, 17-21. Paris.

FrheNdorger (F.). - 1954 - Gedanken zur Frage der Struktur und Anordnung der Lebensgemeinschaften. Augetiandte Pflansiologie. Springer Verlag. I. 151-167. Vienne.

EтTkк (H.). - 1943 - Pflanzensoziologische und Bodenkundliche Studien an schweizerischen Laubwäldern.

Mitt. der Schtecis. Anst, für forstl. Versuchswesen. 132 p. Zurich.

ETrer (H.). - 1947 - Uber die Waldvegetation am Sudostrand des Schweizerischen Mittellandes.

Milt. der Schwers. Anst. für forst. Versuchswesen.

Evrard et Cuermzzon. - 1917 - Sur la flore de la Haute-Tarentaise. Bull. Soc. bot, Fr.

- 1918 - La végétation de la Haute-Tarentaise. Bull, Soc. bot. Fr. 
Faber, Gatsherg, Hauff, Schlenker, Kreh, Oberdörfer, Schönar, 1954 - Die ökologischen Artengruppen.

Allgemeine Forst- and Jagdseitung, $\mathrm{n}^{\circ} 125$.

Favarger (C.). - 1958 - Flore et végétation des Alpes. Etage subalpin. Delachaux et Niestlé. 275 p.

Favarger (C.). - 1960 - Les enclaves de végétation acidophiles dans le Jura et le problème du climax. Rapports du Sol et de la Végétation. Masson et Cie. Paris, 104-109.

Flahault (Ch.), - 1897 - Rapport sur les herborisations de la Société botanique de France et sur quelques excursions faites hors session, aux mois d'août et de septembre 1897 , dans la vallée de l'Ubaye. Bull. Soc, Bot. Fr. 44, $3^{\circ}$ série, 156-268.

Flahault (Ch.) - 1901 - La flore et la végétation de la France. Introduction à la Flore descriptive et illustrée de la France de labbé H. Costc. 1-52. Paris.

Fourchy (P) - 1944 - Remarques sur la question du déboisement des Alpes.

Revue de Géographie Alpine, 22, fasc. 1, 113-128.

Fourchy (P.) , - 1951 - Les peuplements forestiers de rOisans. Les essences qui les composent et leur répartition. Ann de l'E.N.E.F. et de la Sta. de Rech. et Exp. 12, fasc. 2, 412-465.

Fourchy (P.). - 1952 - Etudes sur l'Ecologie et la Sylviculture du Mélèze (Larix curopaca D.C.). Ecologie du Mélèze particulièrement dans les Alpes françaises. Ann, de PE.N.E.F. et Stat. Rech. et Exp. 13, fasc. 1, 133 p.

Fournier (P.). - 1961 - Les quatre flores de France. Ed. P. Lechevalier. Paris, 1106 p.

FrANz (H.). - 1954 - Die Verschmelzung von Bodenkunde und ökologie in der wissenschaftlichen Erfassung des Gesamtstandortes. Angerwandle Pflansensosiologie. 1. 255-273.

Furrer (E.) - 1955 - Problem um den Rückgang der Arve in den Schweizer Alpen.

Mitt. der Schweis. Anst, für das forstl. Versuchswesen, 31, $3 \mathrm{Heft}$. 669-705. Zurich.

GAMs (H.). - 1939 - Chronica botanica, 133.

Gsus (H.). - 1957 - Kleine Kryptogamenflora. Band IV. Moos und Farnpflansen. $4^{*}$ édit. Stuttgart.

Gandoger (M.). - 1890 - Voyage botanique an Mont-Cenis. Bull. Soc. bot. Fr.

Gaussen (H.) - 1926 - Végétation de la moitié orientale des Pyrénées Sol, climat, végétation.

Thise Fac. Sc. Paris. 560 p. Toulouse.

GAUSSEN (H.). - 1949 - Utilisation des plantes sociales pour établir les conditions de milieu. C.R. Acad. Agri. Fr, 35, n* 12, 547-548,

GAUSSEN (H.) - 1951 - La cartographie des précipitations annuelles des Alpes occidentales. Bull. Soc, Bot. Fr, 98, 10, 117-118.

Gaussen (H.) et Bagnouls (F.). - 1953 - Saison sèche et indice xérothermique. Documents pour les cartes des productions végétales. Série généralités. Cartographie des limites écologiques. Art. VIII. Ed. Privat, $47 \mathrm{p}$. Toulouse.

Gaussen (H.), - 1953 - La Hêtraie sans hêtre. Rev. For. Fr. 650-652.

Guussen (H.). - 1954 - Théories et classification des climats et microclimats.

VIII* Cong. Int. Bot. Section 7, 125-130.

Gaussen (H.). - 1954 - Géographie des plantes. 223p. A. Colin, $2^{\circ}$ édit. Paris. 
Gaussen (H.). - 1955 - Expression des milieux par des formules écologiques. Leur représentation cartographique. Les Divisions Ecologiques du Monde.

C.N.R.S., 257-269.

Gaussen (H.). - 1963 - Ecologic et Phytogéographie. in Précis de Botanique. Masson et Cie. 927-972.

Gonerroy (R.). - 1940 - La nature alpine. Arthaud, Edit. Grenoble, 445 p.

Goonall (D.). - 1954 - Vegetational Classification and Vegetational Continua.

Angewandte Pflansensosiologic. Springer Verlag. $\mathrm{N}^{\circ}$ spécial. 1: 168182. Wien.

Gouxor (M.) - 1956 - A propos de l'homogénéité et du choix des surfaces de relevé.

Bull. du Serv. de la Carte phytogéogr. Série B, 1, fasc. 1, 7-17.

Gros (A.) - 1935 - Dictionnaire étymologique des noms de lieu de la Savoie.

Belley. Impr. Chaduc. 627 p.

Gunfaume (A.). - 1957 - La flore méditerranéenne en France. Bull. Soc. Bot. Fr, 104, 1-15, 106 353-358.

Guinter (Ph.). - 1951 - Savoie: Bornes, lac d'Aunecy, Tarentaise. Bull. Soc, Bot. Fr., 98, 10, $92-97$.

Guinier ( $\mathrm{Ph}$.). - 1951 - Aperçu géobotanique et forestier sur les Alpes françaises.

Bull. Soc. Bot. Fy. 98, 10, 85-92.

Guinier (Ph.) - 1951 - Pour comprendre les forèts des Alpes. Bull. Soc, Bot, Fr., 98, 10, 32-37.

Guivier (Ph.) et Povkter (J.). - 1951 - Les variations du Pinus montana Miller, du Tyrol au Briançonnais.

Bull. Soc, Bot. Fr., 98, 123-127.

Gunocher (M.). - 1938 - Etudes sur la végétation de l'étage alpin dans le bassin supérieur de la Tinée (A.M.).

Thèse. Lyon. $458 \mathrm{p}$.
Gurvocher (M.). - 1939 - végétation des étages montagnard et subalpin dans le bassin du Giffre.

Reve Gén. Bot., 51.

Ginocher (M.). - 1946 - Phytosociologic et Sylviculture. Bull. Soc. For. Franche-Comte. 4. Décembre 1947.

Gtunocher (M.). - 1954 - Nomenclature phytosociologique, Remarques et recommandations. VIII $^{\circ}$ Congrès inter. Bot. Sections 7 et 8. 15-20. Paris.

Guvochis (M.). - 1955 - Logique et dynamique du peuplement végétal. Masson et Cie, 143 p. Paris.

Gunochet (M.) et Casas. (P.). - 1957 - Sur Panalyse différentielle de Czekanowski et son application à la Phytosociologie.

Bull. du Ser. Carte Phytogéegraphique. Série B. 2, fasc. 1, 25-33.

Guyor (H.). - 1921 - Contribution phytogéographique sur le versant méridional des Alpes pennines.

Bull. Soc. bot. Genèze. XIII.

Gvyot (L.). - 1951 - Les excrétions racinaires toxiques chez les végétaux. Bull. techn, d'inform. Ingén. Serv. agricoles. 59. Mai 1951.

Guyor (L.). - 1954 - Effets antibiotiques provoqués par des lichens et des végétaux supérieurs. Répercussion sur l'ćquilibre fungique de profondeur et l'équilibre phanérogamique de surface.

VIII ${ }^{*}$ Congre's Inter. Bot. Sections 21 à 27, 47-52.

Hartmann (F.). - Forstökologie.

Zustanderfassung und standortsgemässe Gestaltung der Lebengrundlagen des Waldes.

Edit. G. Fromme. 460 p. Vienne. 
Hegr. - 1931 - Illustrierte Flora von Mitteleuropa. 12 vol.

Herm (R.). - 1922 - La végétation du bois de la Madeleine et des ilots arbustifs du Col du Lautaret.

Bull. Soc, Bot. Fr., 69, $4^{\circ}$ Série, 61-77.

Hrnis (S.). - 1936 - Idées actuelles sur l'eau du sol et ses rapports avec la plante.

Ann. Agr. 5, 723-741.

HORVAT (L.). - 1959 - Sistematski odonsi termofilnih hrastovih i borovih suma jugoistocne Evrope - Wärmeliebende Eichen - une Kiefernwälder Sudosteuropas in systematischer Betrachtung.

Biol, Galsn. 12, 1-40. Zagreb.

Humert (H.). - 1951 - Dauphiné méridional ; vallée de la Durance, Briançonnais, Gapençais, Dévoluy, Trièves.

Bull. Soc, Bot. Fr. 98, 10, 109-116.

Ім⿱LоKOFF (A.Kh.). - 1953 - Un carrefour biogéographique, Le massif de Fontainebleau. Ecologie des réserves. S.E.D.E.S. 98 p. Paris.

IssLER (E.). - 1931 - Les associations silvatiques haut-rhinoises, Classificatin sociologique des forêts du département du Haut-Rhin, à l'exclusion du Sundgau et Jura alsacien.

Bull, Soc. Bot, Fr, 78, 62-142.

Jover (P.). - 1949 - Le Valois. Phytosociologie et phytogéographie, S.E.D.E.S 389 p. Paris.

Kr.App (E.) - 1952 - Taschenbuch der Gräser.

P. Parey, Ed, 220 p. Berlin.

KLeber.sberg (R.). - 1952 - Die Fehlgebiete der Arve in den Schweizer Alpen.

Beri. Schweis, Bot. Gesellsch, 62.

Kuntens (W.L.). - 1953 - The soils of Europe, illustrated diagnosis and systematics.

Th. Murby, edit. Londres.

Kvoch (R.). - 1954 - Wälder der Schweizer Alpen im Verbreitungsgebiet der Weisstanne.

Sonderabdruck aus den Mitt. der Schweis. Anst. das forstl. Versuchswesen. 233-260. Zurich.

KvocH (R.). - 1957 - I. Vegetationskundliche Schulen. - II. Die Standortstypenbildung.

Mitt. der Schweise. Anst. für das forstl. Vers. Zurich. 32, fasc. 8, 418-456.

LachausséE (E.) - 1947 - La régénération de P'épicéa en haute montagne. Rev. E.F., 281-302, Mai 1947.

Le Brun (P.) et OFFNER (J.). - 1956 - Un siècle de floristique à travers les Alpes françaises.

Bull. Soc, bot. Fr., 5-6, 298-375.

LE Brun (P.). - 1919 - Espèces et localités nouvelles de phanérogames pour les Alpes de la Savoie et du Dauphiné. Bull. Soc, bot. Fr., 66.

LE BRUN (P.). - 1923 - Une graminée nouvelle pour la flore française (Sesleria microcephala D.C.) et quelques localités inédites de plantes peu communes pour le Sud-Est de la France. Bull, Soc, bot. Fr. 70.

Le Brun (P.). - 1923 - Un siècle de floristique à travers les Alpes francaises. Additions et corrections. Bull. Soc. bot. Fr. 104 (5-6).

Larmundatu (H.). - 1951 - Aufbau und waldbauliche Bedeutung der wichtigsten natürlichen Waldgesellchaften in der Schweiz.

104 p. Berne. 
de Lerris (H.). - 1961 - Le Calamagrostis villosa Chaix (Mutel) en HauteSavoie.

Bull. Soc. Bot. Fr. $108^{\circ}$ année, $87^{\circ}$ sess, extra. Savoie.

de Leiris (H.) - 1965 - Stations savoyardes nouvelles de Calamagrostis villosa.

Monde des plantes. $349,8-9$,

LEMÉ (G.). - 1937 - Recherches écologiques sur la végétation du Perche. Thèse Fac, Sc., 389 p. Paris.

Leméx (G.). - 1946 - Etude phytosociologique sur la forêt de la Comté d'Auvergne. Genèse des sols alpins du massif des Monts-Dores, Comm, $n^{\circ} 92$ b S.I.G.M.A. 28 p. Montpellier.

LEMÉE (G.). - 1954 - Phytosociologie et Pédologie. VIII" Congrès inter. Bot. Paris. Section 7, 33-38.

LenobL.. (F.) - 1923 - La légende du déboisement des Alpes. Revue de Géogr. alpine. 11: 5-116.

LENOHL (F.). - 1926 - A propos des associations végétales. Bull, Soc, bot, Fr, 73 : 873-893.

de LitardiÈre. - 1934 - Contribution à létude biogéographique de quelques graminées de Pélément oriental des Alpes de Savoie et du Dauphiné,

Ann. Univers. Grenoble. 261-273.

LOETSCH (F.) - 1953 - Massenzuwachsermittlung durch Bohrspanproben unter Anwendung mathematisch, statisticher Methoden. - Zeitschrift für Weltiorstwirtschaft, 77-98.

Lumi (W.). - 1945 - Besiedlung und Vegetatisentwicklung auf den jungen Seitenmoränen des grossen Aletschgletschers. Veröff der Geob. Inst. Rübel in Zurich, 35-112.

Luguer (A.). - 1926 - Les Associations végétales des Monts-Dores d'Auvergne.

Thèse, 263 p. Paris.

Manil. (G.), Collin (E.), Evrard (R.), Gruber (R.). - 1953 - Les sols forestiers de T'Ardenne. Le plateau de Saint-Hubert - Nassogne. Bull. Inst. Agro. et des Stat. Rech, Gembloux. 21 (3-4).

Mathey (A.). - 1911 - Régénération de l'épicéa dans les Hautes régions. Bull. Sté For. Franche-Comtí, 169 et 5.

Mathos (Cl.Ch.). - 1958 - La vie des plantes. Presses Univers. 125 p.

Mrusfi. (H.) - 1940 - Die Grasheiden Mitteleuropas. Bot. Arch.

Meyer (H.A.). - 1942 - Methods of Forest Growth Determination. Bulletin 435.

Pennsylvania State College - School of Agriculture.

Meyer (H.A.) - 1952 - Accuracy of Forest Growth Determination based on the measurement of Increment Cores.

Bulletin 547. Pennsylvania State College, School of Agriculture.

Mryran (O.). - 1891 - Une herborisation du Mont-Cenis. Revue de bot. - Bull. Soc. Fr. de bot. Toulouse, 1891.

Meyran (O.) - 1900 - Excursion botanique an col de la Vannoise. Ann. Soc. Bot. Lyon, 25 : 720.

Molinirr (René). - 1935 - Etudes phytosociologiques et écologiques en Provence occidentale. Thèse.

Molinier (René), - 1954 - Sur la nature et la signification de divers bois de Pins dans le Sud-Est de la France. 8 Congrès Inter. de Bot. Section 13. Rapports et communications. 35-38. Paris.

Mol.rnIEr (René). - 1954 - La hêtraie sans hẻtre et l'étage du hẻtre sans hêtraie.

Rev. For. Fr., 3: 145-150. 
Moliniek (R.) et Ayasse (L.). - 1955 - Carte des groupements végétaux des environs de la Motte du Caire. Rev. For. Fr. 696-707.

Motrnier (René). - 1959 - Après l'excursion internationale de Phytosociologie en Provence en mai-juin 1958. Imprimerie Générale de Provence. Marseille, $12 \mathrm{p}$.

MOLIsIER (René). - 1954 - Cours de Géobotanique, Office Unizersitaire do Polycopie. Aix-en-Provence, $127 \mathrm{p}$.

Moor (M.). - 1952 - Die Fagion - Gesellschaften im Schweizer Jura. 201 p. Berne.

Моok (M.). - 1960 - Zur Systematik der Querco-Fagetea. Mitt. der Floristisch-sosiolog. Arbeitsgemeinsch. N.F. Heit 8.

Moor (M.). - 1963 - Pflanzengesellschaften als Geologische Zeiger im Jura Regio Basiliensis. 4 (1): 15-38.

Moret (L.). - 1928 - Notice explicative d'une carte géologique de la Savoie et des régions limitrophes à l'échelle du $200000^{\circ}$ (avec unc carte tectonique et une planche de coupes). Imp. Allier. Grenoble. 42 p.

Motgin (P.) - 1931 - La restauration des Alpes (Ministère de l'A friculture - Direction Générale des Eaux et Forêts, Eaux et Génic Rural). Imprimerie Nationale. 584 p. Paris.

NÈGRe (R.). - 1950 - Contribution à l'étude phytosociologique de l'Oisans. La haute vallée du Vénéon (Massif Meije - Ecrins - Pelvoux). Phyton. 2 (1-3): 23-50.

NÈGRe (R.). - 1959 - Recherches phytogéographiques sur T'étage de végétation aride (ss étage chaud) au Maroc occidental. Travaux de I'Institut scieny. Cherifien. Sírie boła. 13. 385 p. Rabat.

Nizenko (A.A.). - 1956 - L'Eole géobotanique franco-stisse à l'époque actuelle.

Rev. Bot, de IU.R.S.S. 41 (6): 880-897.

Notrfalise (A.). - 1954 - Sur les rapports statiques et dynamiques entre le sol et la végétation forestière. Quelques aspects des recherches effectuées en Belgique.

Ronéo.

Orernörfer (E.). - 1956 - Ubersicht der Süddeutschen Pflanzengesellschaften. Beiträge zur naturkundlichen Forschung in Südwestdeutschland. 15 (1): 11-29.

Ofrner (J.). - 1920 - Esquisse botanique de la région du Mont-Cenis et herborisation aux Evettes.

Bull. Soc. Bot. Fr.

ONDE (H.). - 1938 - La Maurienne et la Tarentaise. Thèse. $623 \mathrm{p}$, Arthaud, édit. Grenoble.

ONde (H.). - 1938 - L'homme et la nature intra-alpine. Particularités du paysage végétal et agricole en Maurienne et en Tarentaise. Arthaud. 67 p. Grenoble.

ONDE (H.). - 1941 - L'occupation humaine en Maturienne et en Tarentaise. Rev. Géog. Alpine, 29: 223-264, 391-436.

Ozenda (P.). - 1941 - La température, facteur de répartition de la végétation en montagne.

Les divisions écologiques du monde. 295-312. C.N.R.S.

Ozenda (P.). - 1964 - Biogéographie végétale.

Ed. Doin. 374 p.

Pat.mann (H.). - 1947 - Pédologie et Phytosociologie. Conférence donnée au Congrès international de Pédologie de Montpellier. Alger.

PARdE (J.). - 1958 - Tarière de Pressler sans temps de passage. Rev. For. Fr., 6: 399-409.

PAvillard (J.). - 1935 - Eléments de sociologie végétale (Phytosociologie).

99 p. Hermann et Cie, édit. Paris. 
Prgux (Ch.P.). - 1947 - Haute Durance et Ubaye. Esquisse physique de la zone intra-alpine des Alpes Françaises dit Sud.

Arthaud, 314 p. Grenoble.

Peguy Ch.P.). - 1961 - Précis de climatologie. Masson et Cie, édit. 347 p. Paris.

Perrier de la Bathie et Songeox. - 1863 - Aperçu sur la distribution des espèces végétales dans les Alpes de Savoic.

Bull. Soc. bot. Fr., 675-686.

Perrifr de la Bathie (E.). - 1909 - Introduction à un Catalogue raisonné des plantes vasculaires du District Savoisien des Alpes occidentales. Bull. Soc. Hist. Nat. Sanoic, 1907-1908, 2* Série. 168-194. Chambéry.

Perkier de la Bathie (E). - 1910 - Observations sur l'introduction à un catalogue raisouné des plantes vasculaires du District Savoisien des Alpes occidentales.

Bull. Soc. Hist. Nat, de Savoie. $2^{*}$ Série. 77-71.

Perrier de la Batrie (E.) - 1911 - Additions à la flore de la Maurienne, Bull. Soc, bot. Genève. $2^{n}$ S. 1911.

Perrier de la Bathite (E.). - 1917-1928 - Catalogue raisonné des plantes vasculaires de Savoic, départements de la Savoie et Haute-Savoie, plateau du Mont-Cenis.

2 vol. Klincksieck, édit. Paris.

Pkrs (R.). - 1937 - Recherches sur les durées d'insolation. Thèse Sc. Paris (Bull. Sté Scient. du Dauphiné), 109-258,

Peritamangin (M.). - 1907 - Aperçu sur la géographie hotanique de la Maurienne et de la Tarentaise.

Bull. Acad. Géogr. bot., 16: 310-338. Paris.

Prenant (M.). - 1934 - Adaptation - Ecologie et Biocoenotique, 59 p. Hermann et Cie, édit. Paris.

Quantin (A.). - 1935 - L'évolution de la végétation à l'étage de la chénaie dans le Jura méridional. These. 381 p. Paris.

Quezer. (P.). - 1950 - Les Mégaphorbiaies de l'étage subalpin dans le massif du Mercantour (Alpes-Maritimes). Bull. Sté Bota, de Fr. 97 (10): 192-195.

Quezer (P.) et Rroux (J.A.). - 1950 - La notion de spectre en phytosociologie (spectre zonal réel). Lajeunia, 14: 19-26.

Rauschert (St.). - 1963- Beitrag zur Vereinheitlichung der soziologischen Nomenklatur.

Mitt. flor-soz. Arb. Gemeinsch, N.F. 10, 1963.

Rex (P.). - 1954 - Le Dynamisme de la Végétation et l'intérèt ćconomique de la notion de climax. $V I I I^{\circ}$ Congrès inter. Bot. Section 7, 93-99. Paris.

REY (P.) - 1960 - Essai de phytocinétique biogéographique. C.N.R.S. 399 p. Paris.

Rrynaud-Beauverte (M.A.). - 1936 - Le milieu et la vie en commum des plantes. Lechevalier, édit, 237 p. Paris.

Richard (J.L.). - 1960 - Application pratique de la phytosociologie et de la pédologie à quelques forêts d'épicéas du Jura. Journ. forest, suisse. 4.

Richard (J.L.). - 1960 - Les enclaves de végétation acidophile dans le Jura et le problème du climax. in \& Rapports du sol et de la végétation \$. Masson et Cie, 99-104. Paris.

Richrard (J.L.). - 1961 - Les forêts acidophiles du Jura. Etude phytosociologique et écologique.

These, 164 p. Berne. 
Richard (J.L.). - 1962 - Note complémentaire. Comparaisons entre la Chartreuse et le Jura.

Annales de PE.N.E.F. Stat. Rech. et Exper. 19 (3): 347-377.

Rioux (J.L.). - 1958 - De quelques concepts en Biocenotique. Bull. du Serv. Carte phytogéogr., sétie B. 3 (2): 127-150.

Ronixsox (G.W.). - 1949 - Soils. Th. Murphy. 573 p. Londres,

RoL. (R.). - Cours d'Ecologie. Inédit. Ecole Nat. des $E$. it $F$. Nancy.

Rousseav (L.Z.). - 1959 - De l'influence du type d'humms sur le développement des plantules de Sapin dans les Vosges.

These Fac. Sc. Nancy.

Roussine (M.). - 1949 - L'évolution des théories phytosociologiques en Russie.

Vegetatio. Vol. I (1948) et 3, fasc. 2-3.

Roussine (N.) et Gounor (M.). - 1957 - A propos d'un manuel récent de Phytosociologie: (Aufgaben und Methorlen der Vegetationskunde d'Ellenberg).

Bull. du Serv. Carte Phytogéogra. Série B, 2 (2): 51-61.

SAINT-LAGer, - 1887 - Plantes récoltées en Haute-Maurienne. Ann. Soc. bot. Lyon.

Sanson (J.). - 1945 - Recueil de données statistiques relatives à la climatologie de la France.

Mémorial de la Météo. Nat., 30. Paris. O.N.M. 148 p.

Sauvage (Ch.) - 1950 - Remarques sur la notion de sociabilité. Recueil des Trav. de I"Instit. Bota, de Montpellier. Fasc, 4.

Schlütrk (H.). - 1957 - Ein Beitrag zur Frage ökologischer und soziologischer Artengruppen.

Archiv für Forstwesen. 6 (1): 44-58.

Serald (O.). - 1961 - Die Waldbodenvegetation der Bundsandstein - Standorte des Baar - Schwarzwalds und ihr ökologischer Zeigerwert.

Mitt. des Vereins für forstliche Standortskunde und Forstpflansensüchtung. 2: 79-91.

Schaeffer (R.), Moreac (R.). - 1958-59 - Lalternance des essences. Bull. Soc, For. Franché-Conté. $\mathrm{N}^{\bullet} 1,3-12 ; \mathrm{n}^{\circ} 2,76-83 ; \mathrm{n}^{\circ} 5,277-288$.

Si.avinsky (W.). - 1933 - Les associations végétales de la Haute-Manrienne.

Varsovie. 1933.

Soo (R.). - 1964 - Die rexionalen Fagion. Verbände und Gesellschaften Südosteuropas.

Akadémiai Kiadó. 104 p. Budapest.

SotkatSCHFF (V.N.). - 1956 - Sur certains problèmes actuels de létude de la couverture végétale.

Rev. Bot. U.R.S.S. 4 L. $n^{\circ} 4: 476-486$.

Suswz. (L.). - 1959-1960 - Saggio critico-sperimentale sulla applicabilita del metodo fitosociologico in selvicoltura. 3-137.

Annali del Centro di Economia montana delle Veneste. Padovia. Vol. I. Susprucuas (J.). - $1942-$ Le sol et la végétation dans le Haut-Vallespir
(Pyrénées-Orientales).

Stat. inter. de Géobotanique médit. et alpine. Montpellier, 79, $225 \mathrm{p}$.

Trssier (M.I. ) - 1907 - Note sur la distribution des essences forestières dans les Alpes occidentales.

Bull. Soc, for, Franche-Comte, 3.

TнокNтHWATtE (C.W.). - 1948 - An approach toward arational classification of climate.

Georgraphical Review, 55-94.

Tomasez.ti (R.). - 1956 - Introduzione allo studio della Fitosociologia. Metodi di studio della vegetarione ad usa scientifico, agricolo e forestale.

Industria poligrafica lombardia. Milano, 320 p. + tableaux, 
Trkasbov (S.). - 1934 - Etude forestière sur le Picea Omorica. Pane. Aun. de IE.N.E.F. et de la Stat, de Rech. et Exp. for. V (2): 117178.

Trkasmov (S.) et div. - 1957 - Futaies jardinées du Sneznik. Monographie phytosociologique et forestière.

Inst, de l'Eco, for. et du bois de Slovenic. 163 p. Ljubljana.

Turc (L.). - 1953 - Le bilan d'eat des sols. Thèse.

Inst. Nat. Rech. Agro.

Tüxex (R.). - 1954 - Pflanzengesellschaft und Bodenprofil, VIII $^{\circ}$ Congrès inter. Bot. Section 7. 41-42. Paris.

Tüxex (R.). - 1955 - Das System der nordwestdeutschen Pflanzengesellschaften.

Mitt. der Florist.-sosiologi. Arbeitsgemeinsch. 5: 154-176.

TẽxeN (R.). - 1960 - Zur Systematik der west-und mitteleuropäischen Buchenwälder.

Bull. Inst. Agro, et des Stat. Rech. de Gembloux. Hors série. 1 : 45-58.

UTtiNger (H.). - 1949 - Die Niederschlagsmengen in der Schweiz. 1901-1940. Zurich.

VenLot (B.). - 1863 - Excursion botanique au Mont-Cenis. Revue Horticole.

VerLor (B.). - 1865 - Guide du botaniste herborisant de Chambéry au Mont-Cenis.

Baillières et Fils, édit. 498-510. Paris.

VERLoT (B.). - 1896 - Guide du botaniste herborisant. Herborisation aux Sources de PArc.

Baillières et Fils, édit. 620-628. Paris.

Veyket (P.). - 1944 - Les pays de la Moyenne Durance alpestre. Etude géographique.

Thèse.

Arthaud, edit. 595 p. Grenoble.

VILMORIN (R. de) et GUINET (C). - 1950. Haute-Maurienne. Bull. Soc. bot. Fr. 98 (10): 97-103.

VINEY (R.). - 1955 - Multiplicité des facteurs de production. Rev. For, Fr, 130-137.

WAGNER (H.). - 1954 - Gedanken zur Berücksichtigung der mehrdimensionalen Beziehungen der Pflanzengesellschaiten in der Vegetationssystematik.

VIII Congres inter, Bot. Paris, Section 7. 9-11.

WILDE (S.A.). - 1954 - La symbiose des arbres et des organismes inférieurs: sa nature et son importance en sylviculture. VIII. Congrès inter. Bot. Section 13. 123-126. Paris.

ZLATNIK (A.). - 1957 - Waldtypengruppen der tschechosiwakischen Wälder.

Pour la Science et l'Economie paysanne. Série A. Editions d'Etat (cette référence en russe), 2: 155-210. 


\section{NOTICE}

\section{Tableau général}

Nous avons fait figurer en face de chaque espèce et pour chacune des unités figurant au tableau, deux chiffres:

- le premier correspond à un coefficient de présence

R. Espèces présentes dans moins de $10 \%$ des relevés.

I. Espèces présentes dans 10 à $20 \%$ des relevés.

II. Espèces présentes dans 20 à $40 \%$ des relevés.

III. Espèces présentes dans 40 à $60 \%$ des relevés.

IV. Espèces présentes dans 60 à $80 \%$ des relevés.

V. Espèces présentes dans plus de $80 \%$ des relevés.

- le second correspond à un coefficient d'abondance-dominance qui permet de nuancer le premier.

Pour éviter d'avoir à transcrire de gros chiffres, nous avons renoncé aux degrés de couverture tels qu'on peut les obtenir en observant que le chiffre 5 d'abondance-dominance correspond à 87,5\% de la surface couverte, etc., et avons simplement pris la moyenne des abondances-dominances multipliée par dix, en conservant les coefficients mêmes d'abondance-dominance et en affectant au signe + la valeur 0,5 .

Par exemple, une espèce présentant dans cinq relevés les abondances-dominances ci-après: $5 ; 4 ; 3 ;+; 1$, a une abondance-domi-

nance moyenne de $\frac{(5+4+3+0,5+1) \times 10}{5}=27$.

On pourra observer qu'il n'y a pas correspondance exacte entre les degrés de couverture retenus pour dessiner les spectres floristico-écologiques et les moyennes des coefficients d'abondance-dominance figurant dans le tableau général, par exemple, entre les degrés de couverture 87,5 et 62,5 correspondant aux coefficients 5 et 4 le rapport est de 1,4 alors qu'entre les coefficients eux-mêmes le rapport est de 1,25 .

Ces différences sont minimes et n'entachent en rien, en tout état de cause, "l'homogénéité $n$ de chacune des représentations de la structure des unités phytosociologiques. 


\section{RESUME}

La haute vallée de la Maurienne, dans les Alpes occidentales françaises, présente le plus grand intérêt pour une étude de phytosociologie forestière: l'axe du talweg étant orienté d'est en ouest, les expositions de ses flancs y sont très tranchées; la direction des grandes zones tectoniques et pétrographiques est telle, en outre, qu'une même roche-mère se retrouve sur les deux versants opposés, ce qui permet de suggestives comparaisons; l'influence humaine, enfin, n'a pas été trop écrasante et ne parait pas avoir modifié sensiblement la composition floristique de la plupart des forêts.

La première partie est consacrée à l'étude des méthodes suivies, dans les domaines de la phytosociologie, de la pédologie, de la climatologie et de l'auxométrie.

La plus importante de ces analyses, celle des associations, a été faite en distinguant des groupes écologiques.

Cette méthode permet, en effet, de bien mettre en relief la gradualité des variations de la végétation et les affinités de tous ordres - climatiques et édaphiques - que présentent entre eux certains groupements; elle permet, en outre, par la mise en évidence de caractéristiques stationnelles, de saisir un aspect important de l'écologie de tout le groupement.

Plusieurs tableaux donnent la liste des groupes écologiques et des espèces qui les composent, dont le comportement a été défini à partir de complexes de facteurs écologiques: au premier chef, l'économie de l'eau et la composition et la structure des horizons humifères.

La deuxième partie traite des caractères généraux de la HauteMaurienne: le climat doit son originalité à sa sécheresse, qui explique le régime capricieux des gelées, le grand étalement des températures, le rapide réchauffement au printemps; les roches-mères, dont le róle dans la pédogenèse est important à cause de l'érosion, sont très variées, certaines sont très siliceuses (quartzites) ou très carbonatées (cargneulles, gypses), tandis que d'autres présentent un caractère " mixte " (schistes lustrés); l'action humaine, enfin, est très ancienne et s'est surtout exercée sur les versants exposés au sud. L'analyse des éléments floristiques - essentiellement médioeuropéen et subméditerranéen - met surtout en relief la disparition du Hêtre et d'un nombre important des espèces de son cortège. 
Dans la troisième partie sont analysées successivement les associations forestières et d'abord celles qui trouvent, dans la haute vallée, leur optimum.

Les pineraies de Pin sylvestre peuvent être groupées en deux ensembles: celles reposant sur sols calcimorphes connaissent des formes xérophiles à Coronilla minima et Ononis rotundifolia, mésoxérophiles, mésophiles enfin, où Erica carnea et les Hylocomies jouent le premier ròle; celles reposant sur sols silicatés sont moins variées, car elles sont liées aux expositions sud; elles sont caractérisées par Deschampsio flexuosa et Minuartia laricifolia.

Les pineraies de $\mathrm{P}$ in à crochets qui surmontent en altitude les précédentes et que l'on rencontre, par conséquent, dans l'étage subalpin - à l'exception de certaines d'entre elles qui, sur gypse superficiel, occupent l'étage montagnard - sont liées aux seuls sols calcimorphes et présentent des formes vicariantes de celles des pineraies de Pin sylyestre sur ces mèmes sols.

Les sapinières, sans Hêtre, à Epicéa, sur sol brun plus ou moins lessivé, sont caractérisées autant par l'absence d'un nombre important d'espèces du cortège des Hêtraies montagnardes (Asperula odorata, Festuca silvatica, Elymus Europaeus, etc.) que par l'abondance d'autres espèces, dont Melanpyrum silvaticum. On peut distinguer deux faciès extrêmes: l'un à Carex alba, sur sol sub-calcimorphe; l'autre à Saxifraga cuneifolia sur sol à moder. Elles sont localisées dans l'étage montagnard, sur versants frais.

Les pessières, toutes subalpines, présentent deux types extrêmes: sur les versants exposés at nord, il s'agit de pessières mésophiles à Vaccinium Myrtillus, tandis que sur les versants exposés au sud il s'agit de pessières xérophiles où Vaccinium Vitis idaea et Arctostaphylos Uva-ursi jouent le premier rôle.

Les pineraies de Pin Cembro développées sur sols podzoliques et dans l'étage subalpin supérieur, présentent une localisation analogue, étant noté que, sur les versants secs, elles sont rares et fragmentaires; dans les pineraies mésophiles dominent soit les Vacciniées et Rhododendron ferrugineum, soit une graminée, Calamagrostis villosa.

L'aulnaie verte, sur sol brun colluvial à mull, est caractérisée par l'abondance des espèces des mégaphorbiaies.

A côté de cet ensemble d'associations essentiellement formées d'espèces résineuses, on rencontre deux groupes d'associations marginales: des hêtraies, riches surtout en Polygala Chamaebuxus et Céphalanthères et une chênaie pubescente très appauvrie floristiquement.

Dans la dernière partie, les grands facteurs écologiques qui permettent d" "expliquer " les associations décrites, sont brièvement analysés dans une perspective d'ensemble, d'où il ressort que si, dans chaque étage, à une exposition donnée, apparaissent des phénomènes de convergence d'évolution des sols qui permettent de 
définir un a climax climatique $»$, ces phénomènes sont souvent freinés de façon permanente par l'érosion; à la notion de climax unique doit donc être substituée celle d" " essaim de climax n, et ce, d'autant plus que l'on s'éloigne de l'étage subalpin.

Est ensuite envisagée, dans un esprit de synthèse, et comme conclusion à l'étude critique faite dans la première partie sur la méthode des groupes écologiques, l'importante question de la classification des unités phytosociologiques.

Une brève analyse des problèmes sylvicoles posés aux forestiers termine notre étude des associations forestières de la Haute-Maurienne. 


\section{SUMMARY}

The high valley of the Maurienne, in french western Alps, is most interesting for a study of forest phytosociology: the axis of the valley-bottom has an east-west orientation and subsequently the aspects of the slopes are very contrasted; according to the direction of the great tectonic and petrographic zones, the same parent material is to be found on the opposite slopes, and this enables to make significant comparisons: finally human influence hat not been too marked, and does not seem to have perceptibly altered the floristic grouping of most forests.

The first part deals with the study of the methods which are used for plant sociology, soil science. climatology and auxometry.

The most important analysis concerns communities, and is based on the distinction of ecological groups,

Thus this method enables to make apparent the scaling of vegetation variations and all kinds of affinities - climatic, and between water, soil and root - which exist between some groups. Besides it enables, while showing clearly site characteristics, to hnow an essential aspect of the whole group ecology.

Several tables give the list of ecological groups with the constituent species, the behaviour of which we defined according to the complex sets of ecological factors: essentially the économy of water. and composition and structure of humus enriched horizons.

The second part deals with the general features of " HauteMaurienne $n:$ the originality of the climate is due to its drought which explains the irregularity of frosts, the wide range of temperature, the rapid increas of temperature in spring; the parent materials, which have a great influence in sol formation, owing to erosion, are very diverse - some are very siliceous (quartzite) or very carbonaceoux (" cargneule ", gypsum), whereas others appear as "mixed " material ( schistes lustrés $n$ ); human influence is very old and affected mainly slopes with south aspect. The analysis of floristic element - chiefly medio-european and submediterranean - stresses the disappearance of bech and of numerous species of its suite. 
In the third part are successively examined the forest communities, and first those which live in the high valley with optimum conditions.

We can divide the pineta of Scotch Pine into two groups: on the one hand, those standing on calomorphic soils present xerophilous forms with Coronilla minima and Ononis rotundifolia, mesoxerophilous and laastly "mesophilous " forms, where Erica carnea and Hylocomia pl. sp. take the first part; those standing on silicate soils are not so diverse for they depend on southern aspects; Deschampsia flextosa and Mintartia laricifolia are typical of these pineta.

The pineta of Pinus uncinata, which stay higher than the former ones and for that reason are represented in the subalpine stage except some of them which live in the montane stage on shallow gypsum - depend only on calomorphic soils and present vicarious forms for those of pineta of Scotch Pine on the same soils.

The fir-woods, without beech but with spruce, standing on more or less leached brown soils are characterized as well by the absence of many species belonging to the suite of montane beech-forests (Asperula odorata, Festuca silvatica, Elymus Europaets, etc...) as by the abundance of other species, among which Melampyrum silvaticum. Two extreme aspects can be distinguished: one with Carex alba on sub-calomorphic soil, the other with Saxifraga cuneifolia on surface mull. They are situated in the montane stage, on fresh slopes.

The piceta, which are all subalpine, show two extreme types: mesophilous piceta with Vaccinitu Myrtillus on slopes with northern aspect, and xerophilous piceta on slopes with southern aspect, where Vaccinium Vitis-Idaea and Arctostaphylos Uva-ursi take the first part.

The spruce-stands of Pinus cembra growing on podzolic soils and in the upper subalpine stage show a similar location, but we must remark that, on dry slopes, they are rare and fragmentary: in the "mesophilous " Pineta predominate either the vacciniaceous plants and Rhododendron ferrugineum, or a graminaceous plant, Calamagrostis villosa.

Green alder stand, on colluvial brown soil with soft humus, is characterized by the abundance of the species of " megaphorbiaes $n$.

Besides this set of associations essentially made up with resinous species, there are two groups of marginal associations: beechstands, with mainly Polygala Chamaebuxus and Cephalenteres and stands of Quercus lanuginosa with a very reduced flora.

In the last part are shortly analysed in a comprehensive view the great ecological factors which allow to a explain " the studied 
associations; then it appears that if, in each stage and with a definite aspect, we can notice the convergence of soils formation, which enables to determine à " climatic climax $n$, these occurences are oft continuously minimized by erosion; the idea of "swarm of climax n must be substituted for the idea of single climax, more especially as we move away from the subalpine stage.

Then the important question of the classification of phytosociology units is considered in a synthetic view and as conclusion for the critical study made in the first part and concerning the method of ecological groups.

Our study of forest communities in Haute-Maurienne ends with a short survey of the silvicultural problems faced by the foresters.

Traduction B. Engetmann. 


\section{ZUSAMMENFASSUNG}

Das obere Tal der Maurienne in den französischen Westalpen ist für ein Studium der Waldvegetation von grösstem Interesse: Da die Achse des Flusses von Osten nach Westen verläuft, sind die Expositionen ihrer Abhänge sehr verschieden; die Richtung der grossen tektonischen und petrographischen Zonen ist ausserdem so beschaffen, dass man ein tund dasselbe Muttergestein auf den beiden entgegengesetzten Abhängen vorfindet, was anregende Vergleiche erlaubt; der menschliche Einfluss schliesslich, ist nicht zu stark gewesen und scheint die floristische Zusammensetzung der Mehrzahl der Wälder nicht wesentlich verändert zu haben.

Der erste Teil ist dem Studium der angewandten Methoden auf dem Gebiet der Pflanzensoziologie, der Bodenkunde, der Klimatologie und der Messung des Zuwachses gewidmet.

Die wichtigste dieser Untersuchungen, die der Assoziationen wurde unter Ausscheidung ökologischer Gruppen durchgeführt.

Diese Methode erlaubt es nämlich, die Veränderlichkeit der Vegetation und jede Verwandschaft - klimatische und edaphische die bestimmte Gesellschaften untereinander zeigen, gut zu veranschaulichen; si ermöglicht es ausserdem durch die Aufzeichnung standörtlicher Merkmale eine wichtige Seite der Okologie der ganzen Gesellschaft zu verstehen.

Mehree Tabellen geben die Liste der Okilogischen Gruppen und der Arten, die sie zusammensetzen wieder; ihr Verhalten wurde ausgehend von Komplexen ökologischer Faktoren untersucht: in erster Linie der Wasserhaushalt und die Zusammensetzung und Struktur der Humushorizonte.

Der zweite Teil behandelt die allgemeinen Merkmale der oberen Maurienne: Die Eigentümlichkeit des Klimas ist durch seine Trockenheit bedingt, was das unregelmässige Auftreten der Pröste, den grossen Unterschied der Temperaturen, die schnelle Erwärmung im Frühjahr erklärt; die Muttergesteine, deren Rolle für die Bodenbildung wegen der Erosion wichtig ist, sind sehr verschiedenartig, einige sind sehr silikathaltig (Quarzite) oder sehr kalkreich ( cargnetles », Gipse), während andere gemischten Cha- 
rakter haben (Bundnerschiefer); der Einfluss des Menschen endlich, ist sehr alt und hat sich vor allem auf den Südhängen ausgewirkt. Die Untersuchung der floristischen Elemente - im wesentlichen mitteleuropäisch und submediterran - offenbart vor allem das Verschwinden der Buche und einer bedeutenden Zahl sie begleitender Arten.

Im dritten Teil sind nacheinander die Waldgesselschaften untersucht worden und zwar zuerst die, deren Optimum sich im oberen Tal befindet.

Die Kiefernwälder mit Pinus silvestris können zu zwei Einheiten zusammengefasst werden: diejenigen, die auf Kalziumhaltigen Böden stocken, besitzen xerophile Formen mit Coronilla minima und Ononis rotundifolia, mesoxerophile und schliesslich mesophile Formen in denen Erica carnea und die Hylocomiceten die wichtigste Rolle spielen; diljenigen, di auf silikathaltigem Boden stocken, sind weniger unterschiedlich, denn sie sind an die Südexposition gebunden : sie sind gekennzeichnet durch Deschampsia flexuosa und Minuartia laricifolia.

Die Kiefernwälder mit Pinus uncinata, die die vorhergehenden in grösserer Höhe ablösen und die man also in der subalpinen Stufe findet - mit Ausnahme von einigen unter ihnen, die auf flachgründigem Gips die montane Stufe besiedeln - sind ausschliesslich an Kalzium-haltige Böden gebunden und zeigen Formen, die den Kiefernwäldern mit Pinus silvestris auf denselben Böden entsprechen.

Die Tannenwälder ohne Buche, mit Fichte, auf mehr oder weniger lessivierter Braunerde sind ebenso durch das Fehlen einer bedeutenden Anzahl von montane Buchenwälder begleitende Arten (Asperula odorata, Festuca silvatica, Elymus europaeus, etc...) gekennzeichnet wie durch das reichliche Vorhandensein anderer $\mathrm{Ar}$ ten wie Melampyrum silvaticum. Man kann zwei äusserst verschiedene Facies unterscheiden: eine mit Carex alba auf sub-kalziumhaltigem Boden; die andere mit Saxifraga cuneifolia auf Moder. Sie befinden sich in der montanen Stufe auf frischen Hängen.

Die Fichtenwälder, die alle in der subalpinen Stufe liegen, zeigen zwei äusserst verschieden Typen: auf den Nordhängen handelt es sich um mesophile Fichtenwälder mit Vaccinium Myrtillus, während es sich auf den Südhängen um xerophile Fichtenwälder handelt, wo Vaccinium Vitis-idaea und Arctostaphylos Urva-ursi die wichtigste Rolle spielen.

Die Kiefernwälder mit Pinus cembra, die auf Podsolen und in der oberen subalpinen Stufe ausgebildet sind, zeigen ein entsprechendes Vorkommen, wobei bemerkt sei, dass sie auf den trockenen Hängen selten und unvollständig ausgebildet sind; in den mesophilen Kiefernwäldern überwiegen die Vaccinieten und Rhododendron ferrugineum, oder ein Gras, Calamagrostis viillosa. 
Der Grünerlenwald auf colluvialer Braunerde mit Mull ist durch die Häufigkeit der Arten des Megaphorbiums gekennzeichnet.

Neben dieser Gesamtheit von Associationen, die im wesentlichen von Nadelholzarten gebildet werden, findet man zwei Randgesellschaften: Buchenwälder, die reich vor allem an Polygala chamaebuxus und Cephalanteren sind und einen floristisch sehr verarmten Flaumeichenwald.

Im letzten Teil sind die wesentlichen ökologischen Faktoren, die es erlauben, die beschriebenen Associationen zu erklären, kurz in einer Gesamtïbersicht untersucht worden, woraus deutlich hervorgreht, dass, wenn in jeder Stufe mit gegebener Exposition das Phänomen der Uhereinstimmung der Bodenentwicklung, das erlaubt, einen a klimatischen Klimax o zu definieren, auftritt, diese Entwicklung doch häufig durch die Erosion für immer gebremst ist; an die Stelle des Begriffes eines einzigen Klimax muss also der des "Klimaxschwarm " gesetzt werden und dies umsomehr, als man sich von der stubalpinen Stufe entfernt.

Dann wird als Synthese und als Schlussfolgerung des vergleichenden Studiums, das im ersten Teil über die Methode der ökologischen Gruppen angestellt wurde, die wichtige Frage der Klassifizierung der phytosoziologischen Einheiten geprïft.

Ein kurze Betrachtung der waldbaulichen Probleme, die sich dem Forstmann stellen, beendet unsere Untersuchung der Waldgesellschaften der Haute-Maurienne.

Traduction A. von Daniels. 